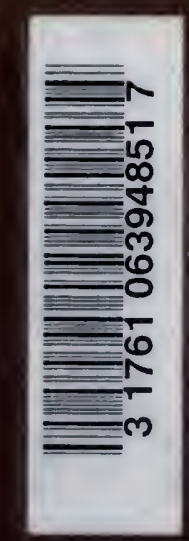

Unnos Toradro Limerary 

Digitized by the Internet Archive in 2007 with funding from Microsoft Corporation 



\title{
BIBLIOGRAPHICAL INDEX
}

OF

\section{North American Fungi}

\author{
BY \\ WILLIAM G. FARLOW \\ Professor of Cryptogamic Botany in Harvard \\ University
}

\begin{abstract}
VOL. I. - PART I.
ABROTHALLUS TO BADHAMIA
\end{abstract}

ISSUED SEPT. 1, 1905

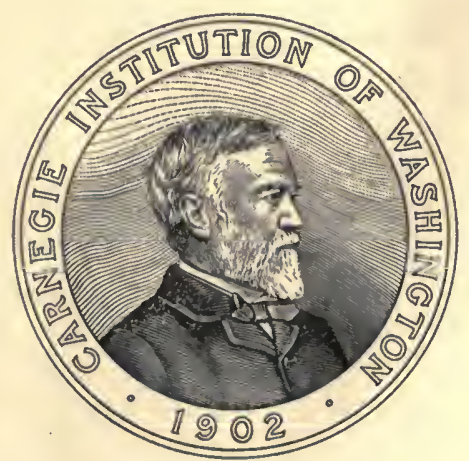

Published by

The Carnegie Institution of Washington $\frac{149504}{16 / 4 / 19}$ 1905 
CARNEGIE INSTITUTION OF WASHINGTON

Publication No, 8

$$
\begin{aligned}
& z \\
& 5356 \\
& F_{97} F_{34}
\end{aligned}
$$

The Zarktuell and Churchill Firs BOSTON, MASS. 


\section{PREFACE.}

THE present Bibliographical Index owes its origin to the fact that in 1874 , when the writer returned from Europe where he had spent a couple of years in the study of mycology, he found it impossible to ascertain what species of fungi were known to occur in the United States. With the exception of the two monographs of Schweinitz, issued in 1822 and 1834 , the accounts of North American fungi were scattered through numerous papers in the publications of learned societies, many of them difficult to obtain, in various State reports and in horticultural and agricultural journals. Berkeley and Curtis' Notices of North American Fungi, begun in Grevillea in 1872 , were then in progress of publication, but the student who desired to find out what was known of our fungi was forced to spend a large amount of time in hunting up in the larger libraries the many scattered and often obscure works from which information could be obtained. The writer then determined to bring together all references to North American species in the form of a card catalogue, not an easy matter at that date, At the same time an authors' catalogue was started including the titles of all the works used in forming the catalogue of species. The authors' catalogue was printed in 1887 as Bibliographical Contribution No. 25 of the Library of Harvard University under the title, "A List of Works on North American Fungi," by W. G. Farlow and William Trelease. This included 653 titles, and was followed in 1888 by a Supplemental List, by W. G. Farlow, being Bibliographical Contribution No. 3 r, bringing up the number of titles to 756 . The authors' catalogue has been continued and a new edition is in preparation, including the parts previously issued with additions up to $\mathbf{1 9 0 5}$.

As originally planned the Index included as species of North American fungi all those known north of Mexico, but in recent years the species of our southern border have proved to be so intimately connected with those of the West Indies, Mexico and Central 
America that it seemed necessary to extend the limit of the Index to species of all countries north of the Isthmus of Panama. In the following pages where a species has been recorded only in countries south of the United States, the name of the region where it occurs is given on the right hand side of the page, but where a species occurs both in the United States and regions farther south no special indication is given. Had it been possible, it would have been desirable to include also species of the northern part of South America, but the line had to be drawn somewhere, and to include South American species would have required an additional amount of work hardly warranted under the circumstances. As it is, we have given all the references known to us to West Indian and Central American species published in recent years, and, to the best of our ability, we have inserted the species recorded from those regions in works issued prior to 1888 , but not included in the List of 1887 and the Supplementary List of 1888 . The references given from works relating especially to the fungi of the West Indies and Central America are probably comparatively complete, but it is probable that some references to species of those regions originally scattered in papers treating also of the fungi of other countries have escaped our notice.

The Sylloge Fungorum of Saccardo, of which the first volume appeared in $\mathbf{I} 882$, has been of the greatest assistance to American mycologists in furnishing a summary of all known species, but naturally in a work covering so large a field it is not possible to give, except in a brief way, special references to the literature of fungi in any particular country, and the Sylloge, although indispensable, does not do away with the necessity of having a special index to the works in which our species are recorded. The need of such an index has been felt in all our universities where the study of descriptive mycology is pursued, as well as in the numerous experiment stations and other government establishments devoted to vegetable pathology. In the absence of such an index it has been necessary for different institutions to spend much time and money in duplicating the work begun by us in 1874 , and for a long time we have wished to publish our index which, as it has been in progress for a longer period than any similar index in this country, is probably therefore more complete, in order that it might be used by all mycologists and prevent an unnecessary duplication of labor and expense. The cost of printing, however, was so great that it has 
been impossible to obtain the means of publication until the Carnegie Institution offered to provide the funds for printing the volume of which the first part is now ready.

The Index, it should be borne in mind, does not purport to be a summary of all references to North American fungi, but is limited to those which concern the systematic mycologist, and does not include-references to papers on fungicides and other technical and agricultural subjects as such, but cites them only when they also contain notes of interest to the systematist. The literature of these branches of applied mycology is already sufficiently fully given in different government publications and technical journals. Nor have we included, in general, references to works treating specially of the physiology of fungi, for the reason that physiological questions are from their nature general and not local. The physiology of North American fungi is not different from that of fungi of other parts of the world, whereas the study of our species from a systematic point of view has a definite local as well as a general importance. What is true of physiological writings is to a great extent true of cytological writings, but we have introduced references to important cytological works published in this country based on material collected here since they have a bearing on morphology and classification. Bacteria and Saccharomycetes are excluded, as those groups at the present day are studied by specialists and not by those interested in what are commonly known as fungi proper.

With these exceptions the Index includes all the references to species of North American fungi known to us, but it should be remarked that in the case of a few species, as Agaricus campestris, which are very common and which from their popular interest have appeared over and over again in journals of every description, we have omitted many references and given only those which are of importance as showing their range or as furnishing good illustrations. As it is, we may perhaps be considered to have been over liberal. It should also be remarked that we have cited only references to North American species as they occur in North America and have not given references to North American species as they occur in Europe. A few perplexing cases have arisen in which new species have been described in Europe parasitic on plants recently imported from America. The supposition is in such cases that the fungi themselves were imported from America and we have accord- 
ingly given notes on some of them, but in other cases it seemed to us doubtful whether the parasites were really American.

In the case of genera we have not thought it necessary to give special references except in the case of those peculiar to North America or first described in North America, or in cases where American writers have given critical notes on certain genera found in Europe as well as America. When we have had any reason for differing from other writers as to limits of genera our opinion is given in the form of notes.

We have endeavored to bring the references as nearly up to date as possible, but it was not practicable to hold in type so large a mass until the whole of the present part was finished. The reader must therefore expect to find the later pages more nearly up to date than the earlier ones, since pages $\mathbf{I}-64$ were struck off in December, 1903, while pages 225 to 312 were not printed until August, I905. It is proposed to give at the end of the volume the addenda to the earlier pages. The progress of the work has been slow, partly owing to delays to be expected in starting a work of this kind, and also to the fact that the two large and very complicated genera, Aecrdium and Agaricus, required a simultaneous arrangement of other genera to appear later.

A word should be said as to the system of classification and the limitations of genera and species which we have followed. In general, while giving as much information as we were able, we have tried as far as possible to avoid changing names in common use for many years. At the present day the Sylloge of Saccardo and the Pflanzenfamilien of Engler and Prantl may be said to be the two works on the classification of fungi in most general use, and we have preferred to follow them as far as possible, making the changes required in consequence of information obtained either from recent critical monographs by those who have made a special study of different orders and genera or from our own examination of American material. We are quite ready to admit that the present classification of fungi is not one which can be called more than temporary, but the time has not arrived when our knowledge of the fungi of the world is sufficiently advanced to make it possible to form a really natural and scientific system.

We have followed the principle of adopting the oldest specific name under which a species was described. We cannot say that 
this method seems to us to be better than any other but, as it is, it is so generally adopted by mycologists and furthermore, since it was voted by the botanists at the recent Congress in Vienna that this plan should be followed in Spermaphytes it seems desirable that all botanists should conform to it. We do not, however, propose in adopting this system to follow it blindly, but in all cases we would accept an older name only when it is quite plain, and not merely possible, or somewhat probable, that the species described by an older writer was the same as that which is generally known under another more recent and more certain name. We have no scruples in declining to accept many of the names of older writers which have of late been substituted for more modern names since from the vagueness of the descriptions and the crudeness of the illustrations it is impossible in the absence of original specimens to be sure that the species were the same as those to which they have since been applied.

There are two categories of botanists: those who believe that nomenclature is an end rather than a means, to whom the changing of names to adapt them to a uniform, automatic system seems to be the important aim in science; and those who regard nomenclature as a necessary evil which can be mitigated by making as few changes as possible. Of these two categories, it is hardly necessary to say that we should prefer to be classed with the latter. Great as is the difficulty of recognizing the identity of the species of early writers in the case of Spermaphytes, it is still worse in the case of fungi, if we accept I 753 as the limit, for the early writers did not make use of the microscopical characters now considered necessary and we must confess that we feel that too much value should not be placed on original specimens of species in which the microscopic characters are important. It has happened in several cases that those who have examined original specimens of certain of the smaller Schweinitzian species do not agree as to their structure, for which the most probable reason is that Schweinitz, not using microscopic characters, did not distinguish between different species of the same general appearance. Such species we have relegated to the limbo of species ignote since we have felt under no moral obligation to make it appear that Schweinitz recognized distinctions which he certainly did not recognize. It is best not to make too violent attempts to interpret the older mycologists but to be content with letting the dead 
bury their dead. 'The business of reviving corpses has been carried altogether too far in mycology. American mycologists, it must be confessed, are peculiarly gullible as far as accepting as authentic much that is offered to them in the way of treatises on the genera and species of earlier mycologists. An examination of some of then, at least, shows that they are as inaccurate as they are useless. No one who uses the Index can complain justly that we have not provided him with names enough, good, bad and indifferent, and those who examine it with care will probably be surprised at the large number of names which have been encumbering the literature of our fungi as nomina nuda or as names of species about which we are and probably always shall be ignorant. At the Vienna Congress it was voted to accept a list of certain genera of Spermaphytes whose names are to be retained regardless of strict priority. 'It is to be hoped that at the next congress a similar list of cryptogams will be presented so that in the case of genera clearly defined and generally recognized under names in use for many years, they may be regarded as fixed and exempt from future changes on the ground of priority.

Although the undersigned must be held responsible for the plan and general character of the Index, and must be held answerable for the defects which those making a practical use of it will undoubtedly detect, it is not to be supposed that a work involving, so large an amount of labor could have been accomplished without the collaboration of several persons, and the writer wishes in this place to state in detail the manner in which the work has been conducted, and to give proper credit to those who have taken part in it. For a few years beginning in 1874 , the indexing was done by the writer himself, but since 1883 his occupations have made it necessary to intrust the main part of the indexing itself to others, while he has had to limit himself to a general supervision of the work, to the examination of authentic specimens involved in settling the romenclature, and to a search through contemporary foreign literature and scattered papers published in the earlier days of mycology bearing directly or indirectly on North American species. Since 1883 the indexing has been done by Mr. A. B. Seymour, with necessary clerical assistance, with the exception of the year $1885-86$, when, during Mr. Seymour's absence, Prof. L. H. Pammel was in charge. By far the greater part of the work of indexing, it will be seen, has been 
done by Mr. Seymour, and to his persistence under difficulties, his care and his interest in the subject, those who use the Index will be indebted. During the considerable number of years when there appeared no immediate prospect that the Index could be printed the accumulation of material went on until it was estimated that about 150,000 references had been brought together. When in 1903 the Carnegie Institution expressed to the writer its willingness under certain conditions to undertake the publication, it was necessary to revise the large mass of data and put it in form for printing since, owing to the numerous cross references, the cards could not be printed directly. Mr. Seymour then brought together the various cards of synonyms and arranged them critically in chronological order, or segregated them, as the case required. They were then revised by the writer to conform to the general plan adopted, and, in cases of disputed or confused synonymy, examinations of authentic specimens were made, and, where it seemed to be of interest, the results of the examinations taken in connection with collateral reading were embodied in the form of notes under different genera and species. Unless otherwise stated, the notes are all by the undersigned, and in cases where Mr. Seymour had made a critical examination the notes were written by him and are signed with his initials. For the arrangement and relations of the aecidia of Gymnosporangia, the Laboulbeniacea, Entomophthoracea and certain genera of other orders specially studied by him, we are indebted to Prof. $\mathrm{R}$. Thaxter as well as for advice on general questions. We are indebted to Mr. A. P. D. Piguet for valuable suggestions made during the proof-reading. We also wish to acknowledge with thanks critical information furnished by the following mycologists : Prof. J. C. Arthur, Prof. G. F. Atkinson, Prof. E: A. Burt, Mr. J. B. Ellis, Prof. E. W. D. Holway, Prof. IV. A. Kellermall, and Prof. C. H. Peck.

\section{W. G. FARLOW.}

Cambridge, Aug. 15, 1905. 



\section{PRINCIPAL ABBREVIATIONS OF NAMES OF AUTHORS AND PUBLICATIONS CITED.}

A. \& $\mathbf{S}$.

Albertini, J. B. von and Schweinitz, L. D. von.

Abh. Nat. Ges. Halle.

Naturforschende Gesellschaft. Halle. Abhandlungen.

Abh. Nat. Verein. Bremen.

Naturwissenschaftlicher Verein. Bremen. Abhandlungen.

Acta Soc. Fauna Fl. Fennica.

Societas pro Fauna et Flora Fennica. Helsingfors. Acta.

Agr. Sci.

Agricultural Science. State College, Pa.

Allg. Forst. \& Jagdzeit.

Allgemeine Forst-und Jagdzeitung. Frankfort a/M .

Am. Florist.

American Florist. Chicago.

Am. Fung.

One thousand American Fungi. C. McIlvaine \& R. K. Macadam.

Am. Agriculturist.

American Agriculturist. New York.

Am. Gardening.

American Gardening. New York.

Am. Jour. Physiol.

American Journal of Physiology. Boston.

Am. Jour. Sci.

American Journal of Science and Arts. New Haven.

Am. Kitchen Mag.

American Kitchen Magazine. Boston.

Am. Mo. Micr. Jour.

American Monthly Microscopical Journal. Washington.

Am. Nat.

American Naturalist. Boston.

Am. Pomol. Soc.

American Pomological Society. Proceedings. 
Am. Quart. Micr. Jour.

American Quarterly Microscopical Journal. New York.

Ann. Bot.

Annals of Botany: London.

Ann. Myc.

Annales Mycologici. Berlin.

Ann. Nat. Hist.

Annals and Magazine of Natural History. London.

Ann. N. Y. Acad.

New York Academy of Sciences. Annals.

Ann. N. Y. Lycoum.

Lyceum of Natural History of New York. Annals .

Ann. Sci. Nat.

Annales des Sciences Naturelles. Botanique. Paris.

Anniv. Mem. Boston Soc.

Anniversary Memoirs of Boston Society of Natural History.

Arth.

Arthur, J. C.

Asc. Lojk.

Ascomycetes Lojkani lecti in Hungaria Transsylvania et Galicia. H. Rehm.

Auct. Amer.

Auctores Americani.

B.

Berkeley, M. J.

B. \& Br.

Berkeley, M. J. and Broome, C. E.

B. \& C.

Berkeley, M. J. and Curtis, M. A.

Barthol.

Bartholomew, E.

Beitr. Biol. Pflanzen.

Beiträge zur Biologie der Pflanzen. Breslau.

Ber. Deutsch. Bot. Ges.

Deutsche botanische Gesellschaft. Berlin. Berichte.

Ber. Schles. Ges.

Schlesische Gesellschaft für vaterländische Cultur. Breslau. Jahresbericht.

Ber. Schweiz. Bot. Ges.

Schweizerische botanische Gesellschaft. Berichte. Berl.

Berlese, A. N. 
Berl. Icon.

Icones Fungorum. A. N. Berlese.

Berl. Icon. Phyc.

Icones Fungorum. Phycomycetes. A. N. Berlese.

Berl. \& Vogl.

Berlese, A. N. and Voglino, P.

Bihang till K. Sv. Vet. Akad. Handl.

Kongliga Svenska Vetenskaps-Akademien. Stockholm. Bihang till Handlingar.

Biv.

Bivona-Bernardi, A. de.

Blox.

Bloxam, A.

Bolt.

Bolton, J.

Bomm. \& Rous.

Bommer, J. E. and Rousseau, M.

Bon.

Bonorden, H. F.

Bot. Centralbl.

Botanisches Centralblatt. Cassel.

Bot. Gaz.

Botanical Gazette. Chicago.

Bot. Notiser.

Botaniska Notiser. Lund.

Bot. Zeit.

Botanische Zeitung. Leipzig.

Bot. 40th Parallel.

United States Geological Exploration of the 4oth Parallel. Botany by Sereno Watson.

Bot. N. Car.

Geological and Natural History Survey of N. Carolina. Part 3, Botany. M. A. Curtis.

Bot. Surv. La.

Report of a Botanical Survey of southern and central Louisiana. Americus Featherman.

Bot. Surv. Nebr.

Botanical Survey of Nebraska. University of Nebraska. Lincoln.

Boud.

Boudier, E.

Bref.

Brefeld, O, 
Bres.

Bresadola, G.

Bres. Henn. \& Magn.

Bresadola, G., Hennings, P., and Magnus, P.

Briosi \& Cav.

Briosi, G. and Cavara, F.

Brit. Columb. Dept. Agr.

Department of Agriculture. British Columbia. Victoria.

British Ured. \& Ust.

British Uredinex and Ustilaginex. C. B. Plowright.

Brond.

Brondeau, L. de.

Bull.

Bulliard, $\mathbf{P}$.

Bull. Ala. Exp. Sta.

Agricultural Experiment Station of the Alabama Polytechnic Institute. Auburn. Bulletin.

Bull. Boston Myc. Club.

Boston Mycological Club. Bulletin.

Bull. Bot. Dept. Jamaica.

Jamaica Botanical Department. Kingston. Bulletin.

Bull. Buf. Soc.

Buffalo Society of Natural Science. Bulletin.

Bull. Bussey Inst.

Bussey Institution. Harvard University. Bulletin.

Bull. Cal. Acad.

California Academy of Sciences. San Francisco. Bulletin.

Bull. Cent. Exp. Farm Can.

Canada. Central Experimental Farm.. Ottawa. Bulletin.

Bull. Colo. Exp. Sta.

Colorado. Agricultural Experiment Station. Fort Collins. Bulletin.

Bull. Conn. Exp. Sta.

Connecticut Agricultural Experiment Station. New Haven. Bulletin.

Bull. Cornell Exp. Sta.

Cornell University, Agricultural Experiment Station. Ithaca. Bulletin.

Bull. Cornell Univ.

Cornell University. Ithaca. Bulletin.

Bull. Del. Exp. Sta.

Delaware College Agricultural Experiment Station. Newark. Bulletin.

Bull. Fla. Exp. Sta.

Florida Agricultural Experiment Station. Lake City. Bulletin. 
Bull. Herb. Bois.

Herbier Boissier. Geneva. Bulletin.

Bull. Idaho Exp. Sta.

University of Idaho Agricultural Experiment Station. Moscow. Bulletin.

Bull. Ill. Lab.

Illinois State Laboratory of Natural History. Urbana. Bulletin.

Bull. Iowa Agr. Coll.

Iowa State College of Agriculture and the Mechanic Arts. Ames. Bulletin.

Bull. Ky. Exp. Sta.

Kentucky Agricultural Experiment Station. Lexington. Bulletin.

Bull. Lab. Bot. Siena.

Laboratorio ed Orto Botanico. Siena. Bulletino.

Bull. Md. Exp. Sta.

Maryland Agricultural Experiment Station. College Park. Bulletin.

Bull. Minn. Acad.

Minnesota Academy of Natural Sciences. Minneapolis. Bulletin.

Bull. Minn. Surv.

Geological and Natural History Survey of Minnesota. Bulletin.

Bull. Miss. Exp. Sta.

Mississippi Agricultural Experiment Station. Bulletin.

Bull. N. Car. Exp. Sta.

North Carolina Agricultural Experiment Station. West Raleigh. Bulletin.

Bull. N. Dak. Exp. Sta.

North Dakota Agricultural College. Government Agricultural Experiment Station for North Dakota. Bulletin.

Bull. Nat. Hist. Soc. N. B.

Natural History Society of New Brunswick. St. Johns. Bulletin.

Bull. Nebr. Exp. Sta.

Nebraska, University of. Agricultural Experiment Station of Nebraska. Lincoln. Bulletin.

Bull. N. Y. Bot. Gard.

New York Botanical Garden. Bulletin.

Bull. N. Y. Mus.

N. Y. State Museum of Natural History. Albany. Bulletin.

Bull. N. Y. Exp. Sta.

New York Agricultural Experiment Station. Geneva. Bulletin.

Bull. Oberlin College Lab.

Oberlin College Laboratory. Oberlin, Ohio. Bulletin.

Bull. Ohio Exp. Sta.

Ohio Agricultural Experiment Station. Wooster. Bulletin. 
Bull. Ohio Univ.

Ohio State University. Columbus. Bulletin.

Bull. Pacific Univ.

Pacific University. Bulletin. Forest Grove, Oregon.

Bull. Penn. Dept. Agr.

Pennsylvania Department of Agriculture. Bulletin.

Bull. S. Dak. Exp. Sta.

South Dakota Agricultural College and Experiment Station. Brookings. Bulletin.

Bull. Soc. Bot. Belg.

Société royale de Botanique de Belgique. Bulletin.

Bull. Soc. Bot. Fr.

Société Botanique de France. Paris. Bulletin.

Bull. Soc. Myc. Fr.

Société Mycologique de France. Paris. Bulletin.

Bull. Tex. Exp. Sta.

Texas Agricultural Experiment Station. Bulletin.

Bull. Torr. Bot. Club.

Torrey Botanical Club. New York. Bulletin.

Bull. Univ. Iowa.

Laboratories of Natural History of the State University of Iowa. Bulletin.

Bull. U. S. Agr. Div. Bot.

United States Department of Agriculture, Division of Botany. Bulletin.

Bull. U. S. Agr. Office Exp. Sta.

United States Department of Agriculture, Office of Experiment Stations. Bulletin.

Bull. U. S. Agr. Pl. Industry.

United States Department of Agriculture, Bureau of Plant Industry. Bulletin.

Bull. U. S. Agr. V. P. P.

United States Department of Agriculture, Division of Vegetable Pathology and Physiology. Bulletin.

Bull. U. S. Agr. Veg. Pathol.

United States Department of Agriculture, Division of Vegetable Pathology. Bulletin.

Bull. Wash. Exp. Sta.

Washington Agricultural Experiment Station. Pullman. Bulletin. Bull. Wash. Lab.

Washburn Laboratory of Natural History. Topeka, Kans. Bulletin. Bull. W. Va. Exp. Sta.

West Virginia Agricultural Experiment Station. Morgantown. Bulletin. 
Bull. Wis. Nat. Hist. Soc.

Wisconsin Natural History Society. Milwaukee. Bulletin.

Buxb.

Buxbaum, J.

C. \& $\mathbf{E}$.

Cooke, M. C. and Ellis, J. B.

C. \& $\mathbf{P}$.

Cooke, M. C. and Peck, C. H.

Can. Nat. \& Geol.

Canadian Naturalist and Geologist. Montreal. Continued as Canadian Record of Science.

Carl.

Carleton, M. A.

Carm.

Carmichael, D.

Cast.

Castagne, L.

Cat. Pac. Fung.

Catalogue of the Pacific coast Fungi. H. W. Harkness and J. P. Moore.

Cat. Pl. Arn: Sept.

Catalogus plantarum Americæ septentrionalis. H. Muhlenberg.

Cat. Pl. Amherst (Hitchcock).

Hitchcock, E. Catalogue of the plants growing without cultivation in the vicinity of Amherst College. Amherst, 1829.

Cat. Pl. Amherst (Frost in).

Tuckerman, E. and Frost, C. C. A catalogue of plants growing without cultivation within thirty miles of Amherst College. Amherst, 1875.

Cat. Pl. Cin.

Catalogue of plants, native and naturalized, collected in Cincinnati, Ohio. T. G. Lea.

Cat. Pl. La.

Catalogue provisoire des plantes de la Basse Louisiane. A. B. Langlcis.

Cat. Pl. Mass.

Report on the Geology . . . of Massachusetts. Catalogue of the animals and plants of Massachusetts. E. Hitchcock.

Cat. Pl. N. J.

Geological Survey of New Jersey, Vol. 2, pt. 1. Catalogue of plants found in New Jersey. N. L. Britton. Fungi, J. B. Ellis. I889.

Cat. Pl. N. Y.

Catalogue of plants of New York. J. Torrey, I8Ig. 
Cat. Pl. R. I.

Plants of Rhode Island. J. L. Bennett, 1888.

Cda.

Corda, A. C. J.

Cent. Am. Fungi.

Central American Fungi (exsiccati). C. L. Smith. Iowa City, Iowa.

Centralbl. Bact.

Centralblatt für Bacteriologie und Parasitenkunde. Jena.

Ces.

Cesati, V.

Champ. Guad. et Martinique.

Enumération méthodique des.Champignons recuellis à la Guadaloupe et a la Martinique. Duss.

Charleston Med. Jour. \& Rev.

Charleston Medical Journal and Review.

Chev.

Chevallier, $\mathrm{F}$.

Circ. U. S. Agr. Div. Bot.

United States Department of Agriculture, Division of Botany. Circular.

Circ. U. S. Agr. Div. Veg. Pathol.

United States Department of Agriculture, Division of Vegetable Pathology. Circular.

Cke.

Cooke, M. C.

Cke. \& B.

Cooke, M. C. and Berkeley, M. J.

Clint.

Clinton, G. P.

Contr. Bot. Dept. Univ. Nebr.

Contributions from the Botanical Department of the University of Nebraska.

Contr. Bot. Vt.

Contributions to the Botany of Vermont. University of Vermont. Burlington.

Contr. U. S. Nat. Herb.

Contributions from the U.S. National Herbarium.

Country Gent.

Cultivator and Country Gentleman. Albany.

Critt. Agr.

Crittogamia Agraria, O. Comes.

Curr.

Currey, F. 
Curt.

Curtis, William.

Cycl. Am. Hort.

Cyclopædia of American Horticulture. Bailey.

D By.

De Bary, A.

D C.

De Candolle, A. P.

De Not.

De Notaris, G.

Desm.

Desmazières, J. B.

Desv. Jour. de Bot.

Desvaux, A. N. Journal de Botanique. Paris.

Dicks.

Dickson, J.

Diet.

Dietel, $P$.

Diet. \& Holw.

Dietel, P. and Holway, E. W. D.

Ditm.

Ditmar, L. P. F.

Dozy \& Molkenb.

Dozy, F. and Molkenboer, J. H.

Dur. \& Mont.

Durieu de Maisonneuve et Montagne, C.

E. F.

Economic Fungi (exsiccati). A. B. Seymour and F. S. Earle.

Ehrb.

Ehrenberg, C. G.

El.

Elenchus Fungorum. E. M. Fries.

Ell.

Ellis, J. B.

Ell. \& Ev.

Ellis, J. B. and Everhart, B. M.

Ell. \& Gall.

Ellis, J. B. and Galloway, B. T.

Endl.

Endlicher, S. L.

Engler, Bot. Jahrb.

Botanische Jahrbücher (Engler). Leipzig. 
Enum. Lichens New Bedford.

Enumeration of the Lichens of New Bedford. H. Willey.

Epicr.

Epicrisis Systematis Mycologici. E. M. Fries.

Eriks.

Eriksson, J.

Essai Tax. Hymen.

Essai Taxonomique des Hymenomycètes. N. Patouillard.

Exp. Sta. Rec.

United States Department of Agriculture, Office of Experiment Stations. Washington. Experiment Station Record.

Farl.

Farlow, W. G.

Farmers' Bull. U. S. Agr.

United States Department of Agriculture. Farmers' Bulletin. Fckl.

Fuckel, L.

F1. Car.

Flora Caroliniana. Thomas Walter.

Fl. Dan.

Flora Danica.

F1. Ind. Occ.

Flora Indiæ Occidentalis. O. Swartz.

F1. Fr.

Flore Française. Lamarck et De Candolle.

Fl. Ludov.

Florula Ludoviciana. C. S. Rafinesque.

Fl. Miami Valley.

The Mycologic flora of the Miami Valley. Ohio. A. P. Morgan.

Fl. Nebr.

Flora of Nebraska. University of Nebraska. Lincoln.

Fres.

Fresenius, G.

Fung. Am.

Fungi Americani exsiccati. H. W. Ravenel.

Fung. Car.

Fungi Caroliniani exsiccati. H. W. Ravenel.

Fung. Columb.

Fungi Columbiani (exsiccati). Ellis \& Everhart, Shear, Bartholomew.

Fung. Dis.

Fungous Diseases of the Grape. F. L. Scribner, 
Fung. Eur.

Fungi Europæi et Extraeuropæi (exsiccati). G. Winter. O. Pazschke.

Fung. Fenn.

Fungi Fenniæ exsiccati. P. A. Karsten.

Fung. Gall.

Fungi Gallici exsiccati. C. Roumeguère.

Fung. Piante Colt.

I Funghi Parassite delle Piante Coltivate. Briosi and Cavara.

Gard. \& For.

Garden and Forest. New York.

Gærtn.

Gærtner, J.

Gall.

Galloway, B. T.

Gard. Chron.

Gardener's Chronicle. London.

Gen. Gast.

Genera of Gastromycètes. C. G. Lloyd.

Gen. Lich.

Genera Lichenum. E. Tuckerman.

Gen. Pl.

Genera plantarum secundum ordines naturales disposita. S. L. Endlicher.

Geol. Wis.

Geology of Wisconsin. W. F. Bundy.

Ges. Nat. Freunde Mag.

Gesellschaft naturforschender Freunde. Berlin. Magazin. 18071818.

Gmel.

Gmelin, J. F.

Gonn. \& Rabh.

Gonnermann; W. and Rabenhorst, L.

Grev. (author).

Greville, R. K.

Grev. (journal).

Grevillea. London.

Hark.

Harkness, H. W.

Hdbk. Med. Sci.

A reference handbook of the Medical Sciences. New York. Wm. Wood \& Co. 
Hdbk. Mushrooms.

Student's Handbook of Mushrooms of America, Edible and Poisonous. T. Taylor.

Hedw.

Hedwigia.

Hedw. Beibl.

Hedwigia . . Beiblatt.

Hedw. Rep.

Repertorium für Kryptogamische Literatur. Beiblatt zur Hedwigia.

Henn.

Hennings, $\mathrm{P}$.

Herb. S.

- Schweinitz Herbarium.

H. I.

A provisional Host Index of the Fungi of the United States. W. G. Farlow and A. B. Seymour.

Hildeb.

Hildebrand, F.

Hoffm.

Hoffmann, G. F.

Holw.

Holway, E. W. D.

Hook.

Hooker, W. J.

Hook. Jour. Bot.

Hooker's Journal of Botany. London.

Horæ Phys. Berol.

Horæ Physicæ Berolinensis. Ed. C. G. Nees von Eseubeck.

Hornem.

Hornemann, J. W.

Huds.

Hudson, W.

Illustr. Fungi.

Illustrative Fungi (exsiccati). L. M. Underwood and O. F. Cook.

Jacq.

Jacquin, N. J.

Jacz.

Jaczewski, A. von.

Jahrb. Wis. Bot.

Jahrbücher für Wissenschaftliche Botanik. Berlin.

Jahresber. Ver. Erdkunde, Dresden.

Verein für Erdkunde. Dresden. Jahresbericht. 
Joh.

Johanson, C. J.

Johnst.

- Johnston, G.

Jour. Appl. Micr.

Journal of Applied Microscopy. Rochester.

Jour. Bot.

Journal of Botany. London.

Jour. Cin. Soc.

Cincinnati Society of Natural History. Journal.

Jour. Columbus Hort. Soc.

Columbus Horticultural Society. Columbus, Ohio. Quarterly Journal of Proceedings.

Jour. de Bot.

Journal de Botanique. Paris.

Jour. Hamilton Assoc.

Hamilton Association. Hamilton, Ontario. Journal and Proceedings.

Jour. Inst. Jamaica.

Institute of Jamaica. Kingston. Journal.

Jour. Linn. Soc.

Linnean Society, London. Journal. Botany.

Jour. Mitch. Soc.

Elisha Mitchell Scientific Society. Chapel Hill, N. Car. Journal.

Jour. Myc.

Journal of Mycology. Columbus, Ohio.

Jour. Quekett Micr. Club.

Quekett Microscopical Club. London. Journal.

Jour. Roy. Hort. Soc.

Royal Horticultural Society. London. Journal.

Jour. Roy. Micr. Soc.

Royal Microscopical Society. London. Journal.

Kalchb.

Kalchbrenner, K.

Karst.

Karsten, P. A.

Kellerm.

Kellerman, W. A.

Kirch.

Kirchner, L.

Kœrn.

Kœrnicke, F. 
Krombh.

Krombholz, J. von.

Krypt. Fl.

Dr. L Rabenhorst's Kryptogamen Flora von Deutschland, (Esterreich und der Schweiz. Leipzig.

Kze.

Kunze, G. H. S.

L.

Linnaus, Carolus.

L. sp. Pl. ed. Willd.

Linnæus, Species Plantarum. Ed. quarta, curante C. L. Willdenow. Tomus vi. H. F. Link.

L. Syst. Veg.

Linnaus, Systema Vegetabilium. Ed. 16. K. Sprengel.

Lagerh.

Lagerheim, G.

Lam. Encyc.

Encyclopédie méthodique botanique. J A. B. de Lamarck.

L'Hér.

L'Héritier de Brutelle, C. L.

Lév.

Léveillé, J. H.

Lib.

Libert, M. A.

Lindgr.

Lindgren, S. J.

Lk.

Link, H. F.

Lindr.

Lindroth, J. I.

Lond. Jour. Bot.

London Journal of Botany.

Macbr.

Macbride, T. H.

Mart.

Martius, C. von.

Mattusch.

Mattuschka, H. G.

Meddel. Stockholms Hœgskolas Bot. Inst.

Stockholms Hoegskolas Botaniska Institutet. Meddelanden.

Meddelelser om Grœnl.

Meddelelser om Grönland. Copenhagen. 
Med. Repos.

Medical Repository. New York.

Mem. Am. Acad.

American Academy of Arts and Sciences. Boston. Memoirs.

Mem. N. Y. Mus.

New York State Museum of Natural History. Albany. Memoirs.

Mem. Soc. Phys, et Hist. Nat.

Société de Physique et d'Histoire Naturelle. Geneva. Mémoires.

Mem. Torr. Bot. Club.

Torrey Botanical Club. New York. Memoirs.

Mer.

Mérat, F. V.

Mex. Fungi.

Pringle's Mexican fungi (exsiccati). A. B. Seymour.

Mich.

Michelia. P. A. Saccardo.

Michx.

Michaux A.

Micr. Sel.

Micromycetes rariores selecti (exsiccati). Tycho Vestergren.

Millsp. \& Nutt.

Millspaugh, C. F. and Nuttall, L. W.

Mittheil. forstl. Versuchs. Oest.

Mittheilungen aus dem forstlichen Versuchswesen Oesterreichs.

Molkenb.

Molkenboer, J. H.

Mon. Ured.

Monographia Uredinearum. Sydow, P. et $\mathrm{H}$.

Mont.

Montagne, C.

Morg.

Morgan, A. P.

Muell.

Mueller, O. F.

Muhl.

Muhlenberg, $\mathrm{H}$.

Myc. Bull.

Mycological Bulletin. Columbus, Ohio.

Myc. Notes.

Mycological Notes. C. G. Lloyd Cincinnati.

Myc. Univ.

Mycotheca Universalis (exsiccati). Thuemen. 
N. A. F.

North American Fungi (exsiccati). Ellis \& Everhart.

N. Am. Fungi.

A list of works on North American Fungi. W. G. Farlow and W. Trelease.

\section{Nees.}

Nees von Esenbeck, C. G.

Neue Denkschr. Schweiz. Ges.

Allgemeine Schweizerische Gesellschaft für die Naturwissenschaften. Neue Denkschriften.

N. Y. Fungi.

New York Fungi (exsiccati). C. L. Shear.

N. Y. Myc. Club.

New York Mycological Club.

Nits.

Nitschke, T.

Nouv. Mem. Soc. Imp. Nat. Mosc.

Nouveaux Mémoires de la Société Impériale des Naturalistes de Moscou.

Nova Acta R. Soc. Sci. Upsal.

Regia Societas Scientiarum Upsaliensis. Upsala. Nova Acta. Nyl.

Nylander, F.

Oefv. K. Vet.-Akad. Foerh.

Konglig Svenska Vetenskaps-Akademien. Stockholm. Oefversigt af Förhandlingar.

Oest. Bot. Zeit.

Oesterreichische botanische Zeitschrift. Vienna.

Ohio Nat.

Ohio Naturalist. Columbus, Ohio.

Ottawa Nat.

Ottawa Naturalist. Ottawa, Canada.

Otth and Wartm.

Otth, C. A. and Wartmann, B.

Oud.

Oudemans, C. A.

Outl.

Outlines of British Fungology. M. J' Berkeley.

P.

Persoon, C. H.

P. in Gmel.

Persoon in J. F. Gmelin, Linnæus, Systema Naturæ. Ed. xiii. 1791. 
P. Syn.

Synopsis methodica Fungorum. C. H. Persoon .

\section{Pass.}

Passerini, G.

Pat.

Patouillard, N.

Paul.

Paulet, J. J.

Pegl.

Peglion, V.

Pk.

Peck, C. H.

Penz.

Penzig, $\mathrm{O}$.

Phil.

Phillips, W.

Pilz. Weinstk.

Die Pilze des Weinstockes. Thuemen.

Pl. Baker.

Plantæ Bakerianæ. E. L. Greene et al.

P1. Bot. Gard. Camb.

Catalogue of the plants of the Botanical Garden of Cambridge. W. D. Peck.

Pl. Cell. Cuba.

Montagne in Ramon de la Sagra. Histoire physique, politique et naturelle de l'lle de Cuba.

Pl. Crypt. Ard.

Plantæ Cryptogamicæ Arduennæ (exsiccatæ). M. A. Libert.

Plowr.

Plowright, C. B.

Poir.

Poiret, J. L.

Pringsh.

Pringsheim, N.

Proc. Am. Acad.

American Academy of Arts and Sciences. Boston. Proceedings.

Proc. Boston Soc.

Boston Society of Natural History. Proceedings.

Proc. Can. Inst.

Canadian Institute. Toronto. Proceedings.

Proc. Davenport Acad.

Davenport Academy of Natural Sciences. Davenport, Iowa. Proceedings. 


\section{xxill}

Proc. Essex Inst.

Essex Institute. Salem, Mass. Proceedings.

Proc. Fla. Hort. Soc.

Florida State Horticultural Society. Proceedings.

Proc. Ind. Acad.

Indiana Academy of Science. Indianapolis. Proceedings.

Proc. Iowa Acad.

Iowa Academy of Sciences. Des Moines. Proceedings.

Proc. La. Soc. Naturalists.

Louisiana Society of Naturalists. Proceedings.

Proc. Miramichi Nat. Hist.

Natural History Association of Miramichi. Chatham, N.B. I'roceedings.

Proc. Nat. Sci. Assoc. Staten Isl.

Natural Science Association of Staten Island. New Brighton, N Y. Proceedings.

Proc. Phil. Acad.

Academy of Natural Sciences of Philadelphia. Proceedings.

Proc. Portland Soc.

Portland Society of Natural History. Proceedings.

Proc. Pough. Soc.

Poughkeepsie Society of Natural Science. Proceedings.

Proc. Rochester Acad.

Rochester Academy of Science. Proceedings.

Proc. Soc. Prom. Agr. Sci.

Society for the Promotion of Agricultural Science. Proceedings.

Proc. S. Cal. Acad.

Southern California Academy of Sciences. Los Angeles. Proceedings.

Prodr.

Nova genera et species plantarum sen prodromus vegetabilium in Indiam occidentalem. O. Swartz.

Pyr. Germ.

Pyrenomycetes Germanici. Nitschke.

Pyren.

North American Pyrenomycetes. Ellis \& Everhart.

Publ. Field Columb. Mus. Bot.

Field Columbian Museum. Chicago. Publications. Botanical series.

Quél.

Quélet, M.

Rabh.

Rabenhorst, L. 
Racib.

Raciborski, M.

Raf.

Rafinesque-Schmaltz, C.

Rav.

Ravenel, H. W.

Reb.

Rebentisch, J.

Relh.

Relhan, R.

Rept. Agr. N. B.

New Brunswick. Department of Agriculture. Report.

Rept. Conn. Agr.

Connecticut Board of Agriculture. Annual Report.

Rept. Conn. Exp. Sta.

Connecticut Agricultural Experiment Station. New Haven. Report.

Rept. Del. Exp. Sta.

Delaware College Agricultural Experiment Station. Newark. Report.

Rept. Exp. Farms Can.

Canada. Experimental Farms. Report. Ottawa.

Rept. Fla. Exp. Sta."

Florida Agricultural Experiment Station. Lake City. Report.

Rept. Ga. Exp. Sta.

State College of Agriculture and Mechanic Arts. Georgia Experiment Station. Report.

Rept. Geol. Canada.

Canada. Geological survey. Report.

Rept. Geol. Ohio.

Ohio. Geological survey. Report.

Rept. Hatch Exp. Sta.

Hatch Experiment Station of the Massachusetts Agricultural College. Amherst. Report.

Rept. Ill. Ind. Univ.

Illinois Industrial University (University of Illinois). Urbana. Report.

Rept. Mass. Agr. Coll.

Massachusetts Agricultural College. Amherst. Report.

Rept. Mass. Exp. Sta.

Massachusetts State Agricultural Experiment Station. Amherst. Report.

Rept. Md. Hort. Soc.

Maryland State Horticultural Society. Report.

Rept. Mich. Acad.

Michigan Academy of Science. Report. 


\section{$\mathrm{Xxx}$}

Ropt. Mich. Agr.

Michigan State Board of Agriculture. Report.

Rept. Minn. Surv.

Minnesota Geological and Natural History Survey. Annual Report.

Rept. Mo. Bot. Gard.

Missouri Botanical Garden. St. Louis. Report.

Rept. Mo. Hort. Soc.

Missouri State Horticultural Society. Report.

Rept. Nebr. Agr.

Nebraska State Board of Agriculture. Report.

Rept. N. Y. Exp. Sta.

Board of Control of the New York Agricultural Experiment Station. Geneva. Annual Report.

Rept. Ohio Acad.

Ohio State Academy of Science. Columbus. Annual Report.

Rept. Ont. Agr. Union.

Ontario Agricultural and Experimental Union. Toronto. Annual Report.

Rept. U. S. Agr.

United States Department of Agriculture. Report.

Rept. U. S. Surv.

United States Geological Survey. Report.

Rept. Vt. Exp. Sta.

Vermont Agricultural Experiment Station. Burlington. Report.

Rept. Wyo. Exp. Sta.

Wyoming Experiment Station. Laramie. Report.

Rev. Gen. de Bot.

Revue générale de botanique. Paris.

Rev. Gen. Pl.

Revisio Generum Plantarum. Otto Kuntze.

Rev. Myc.

Revue Mycologique. C. Roumeguère.

Revista Argent. Hist. Nat.

Revista Argentina de Historia Natural. Buenos Aires.

Riv. Patol. Veg.

Rivista di Patologia Vegetale. Ed. A. and A. N. Berlese.

Rostf.

Rostafinski, J.

Rostr.

Rostrup, E.

Roum.

Roumeguère $\mathrm{C}$. 
S.

Schweinitz, L. D. von.

Sacc.

Saccardo, P. A.

Sacc. \& Cub.

Saccardo, P. A. and Cuboni, J.

Sacc. Cub. \& Manc.

Saccardo, P. A., Cuboni, J., and Mancini, V.

Sacc. \& Syd.

Saccardo, P. A. and Sydow, P.

S. Car. Resources.

South Carolina. Resources and population, institutions and industries. H. W. Ravenel.

Schæff.

Schæffer, J.

Schl.

- Schlechtendal, D. de.

Schm.

Schmidt, J. C.

Schm. \& Kze.

Schmidt,'J. C. and Kunze, G. H. S.

Schrt.

Schrœter, J.

Schulz.

Schulzer von Müggenburg, S.

Schum.

Schuhmacher, C. F.

Scop.

Scopoli, J. A.

Scot. Crypt. F1.

Scottish Cryptogamic Flora. R. K. Greville.

Scrib.

Scribner, F. L.

Secr.

Secretan, Louis.

Sel. Fung. Carp.

Selecta Fungorum Carpologia. Tulasne.

Sfer. Ital.

Sferiacei Italici. G. De Notaris.

Sitz.-Ber. Naturforsch. Freunde.

Gesellschaft naturforschender Freunde. Berlin. Sitzungsberichte. 
S. M.

Systema Mycologicum. E. M. Fries.

\section{Sommf.}

Sommerfelt, C.

S. O. V.

Systema Orbis Vegetabilis. E. M. Fries.

Sow.

Sowerby, J.

Speg.

Spegazzini, C.

Spreng.

Sprengrel, K.

Starb.

Starbäck, K.

Suppl. List Works on N. Am. Fungi.

A supplementary list of works on North American Fungi. W. G. Farlow.

Syd.

Sydow, P. or Sydow, P. et H.

Syll.

Sylloge Fungorum. P. A. Saccardo and others.

Syll. Crypt.

Sylloge generum specierumque Cryptogamarum. J. F. C. Montagne. Symb.

Symbolx Mycologicæ. L. Fuckel.

Syn.

Synopsis methodica Fungorum. C. H. Persoon.

Syn. Am. Bor.

Synopsis Fungorum in America Boreali media degentium. L. D. de Schweinitz.

Syn. Car.

Synopsis Fungorum Carolinæ Superioris. L. D. de Schweinitz.

Syn. N. Am. Lichens.

Synopsis of North American Lichens. E. Tuckerman.

Textbk. Pl. Diseases.

Textbook of Plant Diseases. G. Massee.

Thax.

Thaxter, R.

Thm.

Thuemen, F. von.

Tijdschr. v. Natuur. Geschied.

Tijdschrift voor Natuurlijke Ceschiedenis en Physiologie. Amsterdam and Leiden. 
Tokio Bot. Mag.

Tokio Botanical Magazine.

Torr.

Torrey, J.

Tr. Albany Inst.

Albany Institute. Transactions.

Tr. Am. Hort. Soc.

American Horticultural Society. Transactions.

Tr. Am. Med. Assoc.

American Medical Association. Transactions.

Tr. Am. Phil. Soc.

American Philosophical Society. Philadelphia. Transactions.

Tr. Bot. Soc. Edinb.

Botanical Society of Edinburgh. .Transactions.

Tr. Iowa Hort. Soc.

Iowa State Horticultural Society. Transactions.

Tr. Kans. Acad.

Kansas Academy of Science. Topeka. Transactions.

Tr. Mass. Hort. Soc.

Massachusetts Horticultural Society. Boston. Transactions.

Tr. N. Scot. Inst.

Nova Scotian Institute of Natural Science. Proceedings and Transactions. Halifax.

Tr. St. Louis Acad.

Academy of Science of St. Louis. Transactions.

Tr. Wis. Acad.

Wisconsin Academy of Science, Arts and Letters. Transactions.

Trel.

Trelease, W.

Trent.

Trentepohl, $\mathrm{K}$.

Tuck.

Tuckerinan, E.

Tul.

Tulasne, L R.

Underw.

Underwood, L M.

Ured. Amer.

Uredineae Americanæ exsiccatæ. M. A. Carleton.

Veg. Bahama..

Vegetation of the Bahama Islands. W. C. Coker. 
Verh. Bot. Ver. Brandenb.

Botanischer Verein für die Provinz Brandenburg Berlin. Verhand. lungen.

Vitt.

Vittadini, C.

Vogl.

Voglino, P.

Wakk.

Wakker, J. H.

Wallr.

Wallroth, C. T.

Walt.

Walter, T.

Weinm.

Weinmann, J. A.

West Am. Fung.

West American Fungi (exsiccati). D. Griffiths.

West Indian Bull.

West Indian Bulletin, the Journal of the Imperial agricultural department for West Indies. Barbados.

Westd.

Westendorp, G. D.

Wigg.

Wiggers, H. A.

Willd.

Willdenow, C. L.

Wint.

Winter, G.

Wint. \& Demet.

Winter, G. and Demetrio, C.

With.

Withering, W.

Wulf.

Wulfen, F. X. von.

Yearb. U. S. Agr.

- United States Department of Agriculture. Yearbook.

Zoitschr. Pflanzenkr.

Zeitschrift für Pflanzenkrankheiten. Stuttgart. 


\section{XXXV}

References are arranged chronologically under names of species and synonyms.

BOLD FACE TYPE indicates number of the volume.

Roman NUMERALS (capitals), number of the series.

ROMAN NUMERALS (lower case), page numbers of works in which both Roman and Arabic figures are used in the originals.

d., description; $p l$., plate; $f$., figure.

n.n. Nomen novum.

nom. nud. Nomen nudum.

[ ] Secondary title.

( ) Paging of reprint.

* Before a reference, specimens in exsiccati.

* After a reference, signifies that it relates to foreign, not to American material.

? Before a specific name, signifies that it is doubtful whether the name is properly placed as a synonym.

? After a specific name, that it is doubtful whether in the references cited under it the species has been determined correctly.

Dates on which pages went to press:

$$
\begin{aligned}
& \text { I- } 64 \text {. . . . . . . . . Dec., 1903. } \\
& \text { 65- } 96 \text {. . . . . . . July, I904. } \\
& \text { 97-160 . . . . . . . March, 1905. . } \\
& 161-192 \text {. . . . . . . June, } 1905 . \\
& \text { 193-224 . . . . . . . July, 1905. } \\
& \text { 225-312 . . . . . . . Aug., } 1905 .
\end{aligned}
$$





\title{
BIBLIOGRAPHICAL INDEX OF NORTH AMERICAN FUNGI.
}

\author{
Abrothallus Parmeliarum, (Sommf.) Nyl. \\ Rostr. Meddelelser om Grœnl. 3:613. I891. \\ NoTE. The species of Abrothallus have until recently been considered to be \\ lichens, and references to North American species will be found in works on \\ lichens which cannot be included here. The single species here given is a \\ common parasite of Parmeliea in the North Eastern States and probably \\ throughout the United States. Abrothallus oxysporus, Tul., now placed in \\ Nesolechia, is given as Buellia oxyspora in Tuckerman, Syn. N. Am. \\ Lichens 2 : 29.
}

Acallomyees, Thax.

Thax. Proc. Am. Acad. $38: 23 . d$. 24 June 1902.

Acallomyces Homalotæ, Thax.

Thax. Proc. Am. Acad. $38: 24$. d. 24 June 1902.

Acalyptospora Populi, Pk.

Pk. Rept. N. Y. Mus. 35 : 139. $d . \quad 1885$.

Note. Acalyptospora nervisequia, Desm., the type of the genus, is now considered to be a distortion due to insects and not a fungus. Whether $A$. Populi, Peck is still to be classed with fungi is uncertain, and we are informed by Professor Peck that the original specimens of his species cannot now be found and he is unable to express an opinion on the subject.

Acanthomyees, Thax.

See Rhachomyces, Thax.

NoTE. On grounds of priority Acanthomyces was changed to Rhachomyces. See Thax. Proc. Am. Acad. $30: 467,468$. $d$. 18 Feb. 1895 .

Acanthostigma Alni, Rostr.

Rostr. Meddelelser om Grœnl. 3:619. d. I891.

Sacc. Syll. 11 : 338 . $d$. July 1895 .

Acanthostigma atrobarbum, (C. \& E.) Ell. \& Ev.

Ell. \& Ev. Pyren. 155. $d$. May 1892. Spharia atrobarba, C. \& E.

C. \& E. Grev. 8 : 15. d. Sept. 1879.

*Ell. N. A. F. 590 . I881.

Chxtosphxia atrobarba, Sacc.

Sacc. Syll. $2: 96 . d$. 13 June 1883 .

Cke. Grev. 15: 124. June 1887.

Ell. in Cat. Pl. N. J. 515. 1889 .

Berl. Icon. $1: 27$. d. pl. 16, f. 2. 13 June 1890.

Acanthostigma Berenice, (B. \& C.) Sacc.

Sacc. Syll. $2: 210 . d$. 13 June 1883.

Ell. \& Ev. Pyren. I 54. $d$. May 1892.

Berl. Icon. 1: 105. 1892 . 
Spharia Berenice, B. \& C.

B. Grev. 4 : 108 (178). d. March 1876 .

Venturia Saccardioides, Ell. \& Martin.

Ell. \& Martin, Am. Nat. 18:6\%. d. Jan. I 884.

Berl. \& Vogl. in Sacc. Syll. Add. 1-4: 99. d. 3i Dec. 1886.

Cke. Grev. $16: 17$. Sept. 1887.

Venturia Berenice, Cke.

Cke. Grev. 16: 18. Sept. I887.

Acanthostigma Saccardioides, Sacc.

Sacc. Syll. $9: 854$. $d$. 15 Sept. 1891.

Berl. Icon. 1: 105. 1892 .

Acanthostigma Clintonil, (Pk.) Sacc.

Sacc. Syll. $2: 210 . d$. 13 June 1883.

Ell. \& Ev. Pyren. 155. d. May 1892.

Berl. Icon. 1: 103. d. pl. $100, f_{0}$. 1892.

Spharia Clintonil, Pk.

Pk. Rept. N. Y. Mus. $30: 65$. d. pl. 2, f. $19-23$. Sept. 1878.

Pk. in Day's Cat., Bull. Buf. Soc. $4: 233$ (169). 1883.

Venturia Clintonil, Pk.

Pk. Rept. N. Y. Mus. 28:82. d. pl. 2, f. 22-25. 1876.

Sacc. Syll. $1: 588 . d$. 13 June 1882.

Pk. in Day's Cat., Bull. Buf. Soc. 4: 235 (171). 1883.

Cke. Grev. 16:17, 18. Sept. 1887.

Ell. \& Ev. Pyren. 138. d. May 1892.

Acanthostigma Conocarpi, Tracy \& Earle.

Tracy \& Earle, Bull. Torr. Bot. Club 28: 186. d. 27 March 1901.

Acanthostigma decastylum, Sacc.

See Acanthostigma perpusillum, De Not.

Acanthostigma Ellisil, Sacc. \& Syd.

Sarc. \& Syd. Syll. 14: 591. d. 20 Aug. 1899.

Acanthostigma parasiticum, Ell. \& Ev.

Ell. \& Ev. Proc. Phil. Acad. 1893: 443. d. 27 Feb. 1894.

Sacc. Syll. 11 : 338 . $d$. July 1895 .

Note. The name $A$. parasiticum being preoccupied was changed to $A$. Ellisii by Saccardo \& Sydow.

Acanthostigma Fraxini, Ell.

Ell. Jour. Myc. $7: 276 . d$. 15 May 1893.

Sacc. Syll. $11: 338$. $d$. July 1895 .

Acanthostigma oceidentale, (Ell. \& Ev.) Sacc.

Sacc. Syll. $9: 856$. $d$. 15 Sept. 1891 .

Ell. \& Ev. Pyren. 156. d. May 1892 .

Berl. Icon. $1:$ 105. 1892.

Lindau in Engler \& Prantl, Pflanzen-fam. 1': 398. Feb. 1897.

Chatomella? perforata, Ell. \& Ev.

Kellerm. Bull. Wash. Lab. 1: 76. Jan. 1885 and Tr. Kans. Acad. 9: 82. 1885 .

Ell. \& Ev. Jour. Myc. 1 : 153. d. Dec. 1885.

Kellerm. \& Carl. Tr. Kans. Acad. 10 : 93.1887.

Venturia occidentalis, Ell. \& Ev.

Ell. \& Ev. Jour. Myc. 2: 43. d. April 1886.

Berl. \& Vogl. in Sacc. Syll. Add. 1-4: 102. d. 31 Dec. 1886.

Cke. Grev. 16: 18. Sept. 1887.

*Ell. \& Ev. N. A. F. $214 I$. Feb. 1889.

*Ell \& Ev. Fung. Columb. 1316. May 1899. 
Acanthostigma occidentale, (Ell. \& Ev.) Sacc., var. minor, (Ell. \& Ev.) Sacc.

Sacc. Syll. $9: 856 . d$. 15 Sept. I891.

Ell. \& Ev. Pyren. 156. d. May 1892.

Venturia occidentalis, Ell. \& Ev., var. minor, Ell. \& Ev.

Berl. \& Vogl. in Sacc. Syll. Add. 1-4: 102. d. 31 Dec. 1886.

Ell. \& Ev. Jour. Myc. 2: 43. d. April ז886.

Acanthostigma parasiticum, Ell. \& Ev.

See Acanthostigma Ellisii, Sacc. \& Syd.

Acanthostigma perpusillum, De Not.

De Not. Sfer. Ital. 85. d. pl. 94. I863.*

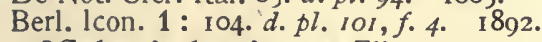
PSpharia longispora, Ell:

Ell. Bull. Torr. Bot. Club $6: 135 . d$. Feb. 1877.

Ell. \& Ev. Pyren. I 55. May 1892 .

Berl. Icon. 2 : I42. Sept. 1899.

Spharia cariosa, C. \& E.

C. \& E. Grev. $6: 94$. d. pl. ioo, f. 28. March 1878.

Langlois, Cat. Pl. La. 34. I887.

Spharia decastyla, Cke.

Cke. Grev. 7 : 52. Dec. 1878. Acanthostigma decastylum, Sacc.

Sacc. Syll. 2 : 210. d. 13 June 1883.

Ell. \& Ev. Pyren. 155. d. pl. $19, f .1-4$. May I 892.

Millsp. \& Nutt. Publ. Field. Columb. Mus. Bot. 1: I42. Jan. I896.

Zignoella cariosa, Sacc.

Sacc. Syll. 2: $215 . d$. 13 June 1883 .

Ell. in Cat. Pl. N. J. 519. 1889.

Ophioceras longisporum, Sacc.

Sacc. Syll. 2: 360 . d. 13 June 1883 .

Ell. in Cat. Pl. N. J. 527.1889. Venturia decastyla, Cke.

Cke. Grev. 16: I8. Sept. 1887.

Lasiospharia subvelutina, Ell. \& Ev.

Ell. \& Ev. Jour. Myc. $3:$ i 17. d. Oct. 1887.

Sacc. Syll. 9: 851. d. I 5 Sept. 1891.

Berl. Icon. 1: 115. 1892.

Psilospharia cariosa, Cke.

Cke. Grev. 16 : 51. Dec. I887.

Ceratostomella longispora, Cke.

Cke. Grev. 17 : 50. March 1889.

Acanthostigma pulchrisetum, Sacc.

See Eriospharia pulchriseta, Sacc.

Acanthostigma Saccardioides, Sacc.

See Acanthostigma Berenice, Sacc.

Acanthostigma Scopula, (C. \& P.) Pk.

Pk. Bull. N. Y. Mus. $1^{2}: 22$. d. Jan. 1888.

Ell. \& Ev. Pyren. 154. d. May I 892.

Berl. Icon. 1 : IO3. d. pl. IOI, f. $l . \quad 1892$.

Spharia Scopula, C. \& P.

C. \& E. Grev. 6: s3. Sept. 1877 .

C. \& E. Grev. $6: 94$. March 1878 .

Cke. Grev. 7: 52. Dec., 1878.

*Ell. N. A. F. $184 . \quad 1878$.

Pk. Rept. N. Y. Mus. 32 : 51. d. 1886.

Langlois, Cat. Pl. La. 34. 887. 
Lasiospharia Scopula, Sacc.

Cke. Grev. 16 : 16. Sept. 1887.

Ell. in Cat. PI. N. J. 515. 1889.

Sacc. Syll. $9: 852$. d. 15 Sept. 1891.

Acanthostigma scopulorum, Ell. \& Ev.

Ell. \& Ev. Proc. Phil. Acad. 1895: 4I4. d. Nov. 1895.

Sacc. Hedw. 35 : xxx. 25 Feb. 1896. [Reportorium no. 7.]

Sacc. \& Syd. Syll. 14: 590. d. 20 Aug. 1899.

Acanthostigma Sequoir, (Plowr.) Sacc.

Sacc. Syll. $2: 208$. d. 13 June 1883.

Ell. \& Ev. Pyren. 156. d. May 1892 .

Berl. Icon. 1 : 105. 1892.

Lindau in Engler \& Prantl, Pflanzen-fam. 1' $: 398$. Feb. 1897.

Venturia Sequoize, Plowr.

Plowr. Grev. 7 : 74. d. pl. 120, f. 3. Dec. 1878 .

Hark. \& Moore, Cat. Pac. Fung. 45. Feb. 1880.

Cke. Grev. 16 : 18. Sept. 1887.

Acanthostigma spinosum, (Hark.) Ell. \& Ev.

Ell. \& Ev. Pyren. I56. d. May 1892.

Rosellinia spinosa, Hark.

Hark. Bull. Cal. Acad. $1: 42$ (22). d. Feb. 1884.

Berl. \& Vogl. in Sacc. Syll. Add. 1-4: 43. d. 3I Dec. I886.

Sacc. Syll. 9: 504. d. I5 Sept. I891.

Coniochata spinosa, Cke.

Cke. Grev. $16: 16$. Sept. 1887.

Acerbia bacillata, (Cke.) Sacc.

PSphzria longispora, Ell. See note in Berl. Icon. 2; 242. Sept. 1899.

Acetabula, Fckl.

Fckl. Symb. 330. d. 1869 .

Note. In Bull. Torr. Bot. Club $30: 93$. 28 Feb. 1903 the name Phleboscyphus is substituted for Acetabula, since there is an Acetabulum L. See also species given under Phleboscyphus.

Acetabula Acetabulum, Underw. \& Earle.

See Acetabula vulgaris, Fckl.

Acetabula cinnamomeolutescens, (S.) Sacc.

Sacc. Syll. 8: 62. d. 20 Dec. 1889.

Peziza cinnamomeolutescens, $\mathrm{S}$.

S. Syn. Car. $117(91)$ no. $1165 . d$. 1822 .

Fr. S. M. 2: 45. 1822 .

Spreng. in ${ }^{\prime}$ L. Syst. Veg. 16 ed. $41^{1}: 499 . d .1827$.

S. Syn. Am. Bor. 170 no. 745.1834.

M. A. Curtis, Bot. N. Car. 131. 1867.

Cke. Bull. Buf. Soc. 2: 287. March 1875 .

Acetabula Mitrula, (S.) Sacc.

Sacc. Syll. $8: 63 . d .20$ Dec. 1889.

Peziza Mitrula, S.

S. Syn. Car. 118 (92) no. 1184. $d$. 1822.

Fr. S. M. 2: 45 . 1822 .

Spreng. in L. Syst. Veg. I6 ed. 41 : 499. d. 1827.

Hitchcock, Cat. Pl. Amherst 62. 1829.

Hitchcock, Cat. PI. Mass. 647. 1833. 2 ed. 648 (128). 1835.

S. Syn. Am. Bor. I 70 no. 746 . 1834 .

M. A. Curtis, Bot. N. Car. 131. 1867.

Cke. Bull. Buf. Soc. 2: 287 . March 1875 .

Acetabula pullula, (Johnson) Sacc. (spelled paulula.)

Sacc. Syll. $8: 63$. d. 20 Dec. 1880. 
Peziza pullula, Johnson.

Johnson, Bull. Minn. Acad. 1 : 342. d. I 878 .

Acetabula sulcata, (P.) Fckl.

Sacc. Syll. $8: 62$. $d$. 20 Dec. I889.

Peziza sulcata, P.

S. Syn. Am. Bor. I70 no. 744. I 834.

Cke. Bull. Buf. Soc. 2: 287. March I 875 .

Cke. Mycographia I08. d. pl. $47, f .185$. Oct. 1876.

Pk. Rept. N. Y. Mus. 30 : 59 . Sept. 1878.

Acetabula vulgaris, Fckl.

Fckl. Symb. 330. d. I 860 .*

Sacc. Syll. $8: 59 . d$. 20 Dec. I 889.

McClatchie, Proc. S. Calif. Acad. Sci. 1 : 364.5 June I897.

Longyear, Rept. Mich. Bd. Agr. 1898: 94 . I 899

Fairman, Proc. Rochester Acad. 3: 214. March 1900.

Durand, Bull. Torr. Bot. Club $27: 473 . d$. 29 Sept. I900:

Peziza Acetabulum, L.

Hitchcock, Cat. Pl. Amherst 62. I 829.

Hitchcock, Cat. Pl. Mass. 647. I833. 2 ed. 648 (128). $\quad$ I8 85.

Lea, Cat. Pl. Cin. 72. 1849 .

James, Jour. Cin. Soc. 2:67 (26). April 1879.

Lea, Jour. Cin. Soc. $5: 2$ I5 (I9). Dec. I 882.

M. A. Curtis, Bot. N. Car. I3I. I867.

Frost, Proc. Boston Soc. 12: 80. I869.

Cke. Bull. Buf. Soc. 2: 287. March 1875.

Frost in Cat. Pl. Amherst 89. 1875 .

Cke. Grev. $6:$ I41. June 1878

C. \& E. Grev. 7 : 40. Dec. 1878.

Cke. Mycographia 107. d. pl. $47, f .183$. Oct. 1876.

Brendel, Flora Peoriana I ed. 362 (94). I 882.

*Rav. Fung. Am. 764. I882.

Cragin, Bull. Wash. Lab. 1: 70. Jan. I885.

Brendel, Fl. Peoriana 2 ed. $69 . \quad$ I 887.

Cobb, List Pl. Amherst, 47. 1887.

Bennett, Cat. PI. R. I. 9 I. I888.

Ell. in Cat. PI. N. J. $544 . \quad$ I 889.

Acetabula Acetabulum, Underw. \& Earle.

Underw. \& Earle, Bull. Ala. Exp. Sta. 80: 200. 28 June 1897.

Phleboscyphus acetabulum, Clements.

Clements, Bull. Torr. Bot. Club 30: 93. 28 Feb. 1903.

Achlya, sp. indet. Hine.

See Achlya racemosa, var. stelligera, Cornu.

Achlya Americana, Humphrey.

Humphrey, Tr. Am. Phil. Soc. 17 : II6. d. pl. 14, f. 9, 10. pl. 15, f. 18, $24,25,29$. pl. 16, f. 30-36. pl. 18, f. 69-73. I 893 .

Schrt. in Engler \& Prantl, Pflanzen-fam. 1': 99. July I893.

Sacc. Syll. 11: 245 . July I 895.

Atk. Bull. Cornell Univ. 3: I4. 25 June I897.

Earle in Mohr, Contr. U. S. Nat. Herb. $6:$ I 5I. 3I July I90I.

Achlya apiculata, D By.

D By. Bot. Zeit. 46 : 635. pl. $10, f_{0} 3-5$. I $888 . *$

Humphrey, Tr. Am. Phil. Soc. 17: I20. d.pl. 15, f. 26, 27. pl. 19, f. 8286. 1803 .

Atk. Bull. Cornell Univ. 3: I4. 25 June I897.

Earle in Mohr, Contr. U. S. Nat. Herb. 6 : I5I. 3I July I9oI.

Achlya cornuta, Archer.

Humphrey, Tr. Am. Phil. Soc. 17 : I26. d.pl. 20, f. 103, 104. I893. 
Achlya megasperma, Humphrev.

Humphrey, Tr. Am. Phil. Soc. 17 : 118. d. pl. 1s, f. 74-77. 1893. Sacc. Syli. 11 : 245. July 1895.

Achlya oblongata, D By.

Humphrey, Tr. Am. Phil. Soc. $17:$ 121. d. pl. 19, f. 87-s9. 1893.

Achlya oblongata, D By., var. globosa, Humphrey.

Humphrey, Tr. Am. Phil. Soc. 17: 122. d. pl. 19, f. 90, 91. 1893. Atk. Bull. Cornell Univ. 3: 14. 25 June I 897.

Earle in Mohr, Contr. U. S. Nat. Herb. 6: 151. 31 July 1901.

Achlya papillosa, Humphrey.

Humphrey, Tr. Am. Phil. Soc. $17:$ I25. d. pl. 20, f. 99-102. 1893. Sacc. Syll. $11: 245$. July 1895 .

Achlya polyandra, Hildeb.

Hine, Am. Quart. Micr. Jour. 1 : 14 I (14). pl. 6, f. 15. Jan. 1879.

Humphrey, Tr. Am. Phil. Soc. 17 : 119. d. pl. $18, f .78-81.1893$.

Achlya prolifera, Nees.

Leidy, Proc. Phil. Acad. 5 : 8.1850.

Bessey, Bull. lowa Agr. Coll. 1884: 139. Jan. 1885.

Achlya prolifera, Pringsh.

See Saprolignia ferax, Kütz.

Achlya racemosa, Hildeb.

Hine, Am. Quart. Micr. Jour. 1 : 20 (3). Oct. I 878.

Humphrey, Rept. Mass. Exp. Sta. 9 : 233. April 1892.

Humphrey, Tr. Am. Phil. Soc. 17 : I22. d. pl. 19, f. 92-95. 1893.

Achlya racemosa, Hildeb., var. stelligera, Cornu.

Humphrey, Tr. Am. Phil. Soc. 17 : 123. d. pl. 19, f. 96-98. 1893. Achlya, sp. indet. Hine.

Hine, Am. Quart. Micr. Jour. 1 : 136 (9). pl. 6, f. 1-14. Jan. 1879.

Achlya racemosa, var. indet. Hine.

Hine, Am. Quart. Micr. Jour. 1: 140 (13). Jan. 1879.

Acinophora, Raf.

See Arachnion, S.

Acinophora aurantiaca, Raf.

See Arachnion? aurantiacum, De Toni.

Acinula candicans, Fr.

S. Syn. Am. Bor. 266 no. 2409. I834.

Acladium, Lk.

Note. This genus is referred by Pound and Clements to Haplaria.

Acladium conspersum, Lk.

S. Syn. Am. Bor. 276 no. 2582 . 1834.

Cke. Bull. Buf. Soc. 3 : 20I. July 1877 .

Acladium densissimum, $\mathrm{S}$.

S. Syn. Am. Bor. 276 no. 2584. d. 1834. ,

Cke. Bull. Buf. Soc. 3 : 20I. July 1877.

Sacc. Syll. $4: 88$. $d$. Io April i 886.

Acladium microspermum, Lk.

S. Syn. Am. Bor. 276 no. 2583 . I 834.

Cke. Bull. Buf. Soc. 3: 201 . July 1877 .

Acompsomyces, Thax.

Thax. Proc. Am. Acad. $37: 37 . d$. June I90I and Bot. Centralbl. 88 : 220. d. 14 Nov. $190 \mathrm{I}$.

Thax. Proc. Am. Acad. 38: 21. d. 24 June 1902. 
Acompsomyces Atomariæ, Thax.

Thax. Proc. Am. Acad. 38:22. d. 24 June 1902.

Acompsomyces Corticariæ, Thax.

Thax. Proc. Am. Acad. $37: 37 . d$. June Igor.

Sacc. \& Syd. Syll. 16:692. d. I Feb. Igoz.

Acompsomyces paupereulus, Thax.

Thax. Proc. Am. Acad. $38: 23 . d .24$ June 1902.

Acontium, Morg.

Morg. Jour. Myc. $8:$ 4. d. 3I May 1902.

Acontium album, Morg.

Morg. Jour. Myc. 8: 4. d. 31 May 1902.

Acontium minus, Morg.

Morg. Jour. Myc. 8: 5. d. 31 May 1902.

Acontium velatum, Morg.

Morg. Jour. Myc. 8 : 5. d. 31 May 1902.

Acremoniella atra, (Cda.) Sacc.

Webber, Rept. Nebr. Agr. 1889: 222 (82). $\quad$ I890.

Acremoniella fusea, (Schm.) Sacc.

Sacc. Syll. 4: 302. d. Io April 1886.* Acremonium fuscum, Schm.

S. Syn. Am. Bor. 283 no. $2697 . \quad 1834$.

Bundy, Geol. Wis. 1873-79 1: 401. 1883.

Acremoniella melanosperma, (S.) Sacc.

Sacc. Syll. 4: 303. d. 10 April i 886.

Acremonium nigrospermum, S.

S. Syn. Am. Bor. 283 no. 2699. d. 1834 .

Acremonium, Lk.

Pound \& Clements, Minn. Bot. Studies 9 : 669. d. 30 Nov. 1896.

Acremonium, sp. indet.

Humphrey, Rept. Mass. Exp. Sta. 9 : 228. April 1892.

Humphrey, Rept. Mass. Exp. Sta. $10: 230$ (22). pl. 4, f. 27-29. 1893.

Sturgis, Rept. Conn. Exp. Sta. 24: 268. 1901 .

Acremonium flexuosum, $\mathrm{Pk}$.

Pk. Rept. N. Y. Mus. 32: 44. d. I 886.

Pk. Bull. N. Y. Mus. $1^{2}: 19 . d$. $p l$. $1, f .16-18$. Jan. 1888.

Sacc. Syll. 10: 53 I. $d$. 30 June 1892 .

Acremonium fuscum, Schm.

See Acremoniella fusca, Sácc.

Acremonium nigrospermum, $\mathrm{S}$.

See Acremoniella melanosperma, Sacc.

Acremonium nitidum, Mart.

S. Syn. Am. Bor. 283 no. 2698 . r 834 .

Acremonium verticillatum, $\mathrm{Lk}$.

S. Syn. Am. Bor. 283 no. 2696.1834 .

Acrocystis, Ell. \& Halsted.

Halsted, Bull. N. J. Exp. Sta. 76 : 14-20. f. $11-13.28$ Nov. 1890.

Sacc. Syll. 9: $339 . \quad 15$ Sept. I89i.

Lindau in Engler \& Prantl, Pflanzen-fam. 11**: 530. Feb. 1900.

Acrocystis Batatas, Ell. \& Halsted.

Halsted, Bull. N. J. Exp. Sta. $76: 14-20 . f_{1} 1_{1-13} .28$ Nov. 1890.

Sacc. Syll. 9: 339. I5 Sept. 1891.

Halsted, Bull. N. J. Exp. Sta. M: 23 Nov. 1891 .

Halsted, Rept. N. J. Exp. Sta. 1894: 359. 1895.

Townsend, Bull. Md. Exp. Sta. 60 : I 56. f. 52, 53. March 1899 . 
Selby, Bull. Ohio Exp. Sta. 121 : 56. Sept. 1900.

Sturgis, Rept. Conn. Exp. Sta. 24: 289. 1901.

Acrospermum album, $\mathrm{Pk}$.

Pk. Rept. N. Y. Mus. 82 : 38. d. 1886.

Pk. Bull. N. Y. Mus. $1^{2}: 24 . d$. Jan. 1888

Sacc. Syll. 9 : 1129. d. I 5 Sept. 1891.

Ell. \& Ev. Pyrell. 728 d. May 1892.

Olson, Bot. Gaz. 23 : 370. 21 May 1897.

Acrospermum compressum, Tode.

Fr. S. M. $2: 245 . d$. 1822.

Grev. Scot. Crypt. Fl. 4: pl. 182. 1826.

S. Syn. Am. Bor. 266 no. 2412.1834.

M. A. Curtis, Bot. N. Car. I I6. 1867.

B. \& C. Jour. Linn Soc. 10: 352. 1869.

Cooke, Handbook 430. $d$. $187 \mathrm{I}$.

Pk. Rept. N. Y. Mus. $25: 85$. Sept. 1873.

B. Grev. 4: 161 (199). June 1876.

Thm. Pilz. Weinstk. 200. $d$. 1878.

Sacc. Syll. $2: 807 . d$. 13 June 1883.

Cke. Grev. 12: 113. June 1884.

*Ell. N. A. F. 1318 . 1885.

Ell. \& Ev. Jour. Myc. 3: 4. d. Jan. 1887.

Langlois, Cat. Pl. La. 27. 1887.

Ell. in Cat. Pl. N. J. 513. 1889 .

Ell. \& Ev. Pyren. 67. d. May 1892.

*Pazschke, Fung. Eur. 3867 . Nov. 1892.

Kellerm. \& Werner, Geol. Ohio $7^{2}$ : 36I. Jan. 1895.

Tracy \& Earle, Bull. Miss. Exp. Sta. 34: 105. May 1895.

Lindau in Engler \& Prantl, Pflanzen-fam. 11 : 278. f. 20I. Dec. I896.

Olson, Bot. Gaz. 23 : 370. 21 May I897.

Clavaria herbarum, $\mathrm{P}$.

Muhl. Cat. Pl. Am. Sept. 2 ed. 108. 1818.

S. Syn. Car. I I3 $(87)$ no. I IOg. 1822.

Clavaria compressa, Purt.

S. Syn. Am. Bor. 182 no. 1039. 1834.

Sacc. Cub. \& Manc. Syll. 6: 709. d. I Aug. 1888.

Ell. in Cat. PI. N. J. 495.1889.

Acrospermum conicum, $P$.

S. Syn. Am. Bor. 266 no. 2413. 1834 .

Acrospermum cornutum, Fr.

S. Syn. Am. Bor. 266 no. 2414. 1834 .

Acrospermum corrugatum, Ell.

Ell. Bull. Torr. Bot. Club $8:$ I24. $d$. Nov. 188I.

Sacc. Syll. $2: 808$. $d$. I3 June 1883.

Cke. Grev. 12: i1 3. June i 884.

Ell. \& Ev. Jour. Myc. 3: 5. d. Jan. 1887.

Hark. Bull. Cal. Acad. 1 : 167. Feb. 1885.

*Ell. \& Ev. N. A. F. 2055. March 1888.

Olson, Bot. Gaz. 23: 370. 21 May 1897.

Acrospermum fultum, Hark.

Hark. Bull. Cal. Acad. 1: 47 (27). d. Feb. 1884.

Cke. Grev. 12: 113 . June 1884.

Cke. Grev. 13: 8. Sept. 1884.

Berl. \& Vogl. in Sacc. Syll. Add. 1-4: 273. d. 31 Dec. 1886.

Sacc. Syll. 9: I129. d. 15 Sept. I 891.

Olson, Bot. Gaz. 23: 370. 21 May 1897. 
Acrospermum foliicolum, B.

*Rav. Fung. Car. 2:65. I853.

M. A. Curtis, Bot. N. Car. I16. I867.

B. Grev. 4: I6I (199) no. 1000. $d$. June I 876.

Cke. Ann. N. Y. Acad. $1: 184$. May 1878.

* Rav. Fung. Am. 734. I882.

Sacc. Syll. $2: 808 . d$. I 3 June 1883.

Cke. Grev. 12: I13. June I884.

Ell. \& Ev. Jour. Myc. 3: 4. d. Jan. I 887.

*Ell. \& Ev. N. A. F. 2149 . Feb. 1880.

*Ell. \& Ev. N. A. F. 2629. Feb. I891.

Ell. \& Ev. Pyren. 67. d. May 1802 .

Olson, Bot. Gaz. 23: 370. 21 May 1897.

Earle in Mohr, Contr. U. S. Nat. Herb. 6: I64. 31 July I9oI. Acrospermum Ravenelii, Cke?

* Cke. in Rav. Fung. Am. 735. I 882.

Note. Probably the same as $A$. foliicolum, B. according to Ell. \& Ev. Pyren. 68.

Acrospermum fultum, Hark.

See Acrospermum corrugatum, Ell.

Acrospermum graminum, Lib.

Lib. Pl. Crypt. Ard. 33. $d$. 1830.*

Pk. Rept. N. Y. Mus. 30: 52 . Sept. 1878.

Hark. Bull. Cal. Acad. 1: 167. Feb. 1885.

Wint. \& Demeţ. Hedw. 24: 198 (22). Oct. 1885.

Ell. \& Ev. Pyren. 67. d. May 1892.

Olson, Bot. Gaz. 23 : 370. 21 May 1897.

Henn. Hedw. $37: 275$. 3I Dec. I 898.

Acrospermum Ravenelii, B. \& C.

B. Grev. 4: 1 161 (199) no. 999. $d$.' June 1876.

Thm. Pilz. Weinstk. 199. $d$. 1878.

Sacc. Syll. 2:808. $d$. I 3 June 1883 .

Cke. Grev. 12: 113. , June 1884 .

Ell. \& Ev. Jour. Myc. 3: 5. d. Jan. I887.

Ell. \& Ev. Jour. Myc. 3 : i16. $d$. Oct. I887.

Ell. \& Ev. Pyren. 68. d. May 1892.

Olson, Bot. Gaz. 23 : 370. 21 May I 897.

Acrospermum Ravenelil, Cke. in Rav. F. Am. 735. See Acrospermum folicolum, B.

Acrospermum urceolatum, Olson.

Olson, Bot. Gaz. 23: 371. d.pl. 29. 21 May I897 and Rev. Myc. 22 :

43. d. pl. $198, f .7-9$. Jan. 1900.

Lindau \& Syd. Hedw. 37 : xxxv. 9 April 1898 . [Beiblatt no 7.]

Sacc. \& Syd. Syll. 14: 724. d. 20 Aug 1899.

Acrospermum viridulum, B. \& C.

M. A. Curtis, Bot.'N. Car. i 6. i 867.

B. Grev. 4: 16I (199) no. 1001. $d$. June 1876.

Cke. Ann. N. Y. Acad. 1 : 184. May 1878.

*Ell. N. A. F. 857. I882.

Sacc. Syll. $2: 808$. d. 13 June 1883.

Cke. Grev. 12: 113. June 1884.

Ell. \& Ev. Jour. Myc. 3: 4. $d$. Jan. 1887.

Ell. in Cat. Pl. N. J. 513 . I 889 .

Ell. \& Ev. Pyren. 67. d. May 1892.

Olsoń, Bot. Gaz. 23: 370. 21 May 1897.

Acrosporium fasciculatum, Grev.*

See Oospora fasciculata, Berl. \& Vogl. 
Acrostalagmus, Cda.

Pound \& Clements, Minn. Bot. Studies 9 : 665. d. 30 Nov, 1896.

Acrostalagmus cinnabarinus, $\mathrm{Cda}$.

Phil. Grev: 5 : i 6. March I877.

Hark. \& Moore, Cat. Pac. Fung. 32. Feb. I880.

Webber, Rept. Nebr. Agr. 1889: 22I (8I). I890.

McClatchie, Proc. S. Calif. Acad. 1: 376. 5 June 1897.

Pk. Rept. N. Y. Mus. 53:848. March I9oI.

Acrostalagmus tetraclados, A. L. Smith.

Smith, A. L., Jour. Linn. Soc. 35: i1. d. pl. 1, f. 9, 10. I April 1901. West Indies.

Acrostalagmus, sp. indet.

MacMillan, Bull. Torr. Bot. Club 19 : 10. pl. 123. Jan. I892.

Pound \& Clements, Minn. Bot. Studies $9: 665$. d. 30 Nov. 1896.

Acrothamnium violaceum, Nees.

S. Syn. Am. Bor. 275 no. 2562 . I834.

Note. See Sacc. Syll. 4: 761. Io April 1886. Is this perhaps an Hypochnus?

Acrothecium, (Preuss) Sacc. emend.

Note. Acrothecium founded by Corda as a subgenus of Trichothecium, Icones $2: 10,1838$, with the species $A$. parasitans, which is placed in the genus Blastotrichum, Sacc. Syll. 4 : I9I, I886, was raised to generic rank by Preuss, Linnæa 24: I IO, 1851, where Corda's generic description was retained and the species $A$. multisporum described. The latter species was again described and figured in Sturm's Deutschlands Flora, 3 Abt., xxx, 85, f. 43 , of whtch the exact date is uncertain, but apparently about 1851 or 1852 . The description of the genus in Sturm is again Corda's original description with the addition of a (?) after one word. In Michelia I : 75, I June I 877 , Saccardo extended the genus to include Cordana, Preuss and Acrotheca Fuckel without generic description. In Michelia 2 : 29, 25 April 1880, the genus was described by Saccardo and the authority given as Corda, but in Sacc. Syll. $4: 483$, I 886, the authority is given as (Preuss) Sacc. emend. Lindau in Engler \& Prantl, Pfanzen-fam. $\mathrm{I}^{1 * *}: 48 \mathrm{I}$, follows Saccardo's limitations, but gives Preuss as the authority.

Acrothecium crustaceum, (S.) Sacc.

Sacc. Syll. $4: 485 . d$. Io April 1886.

Dactylium crustaceum, $\mathrm{S}$.

S. Syn. Am. Bor. 283 no. 2706 . d. 1834 .

Acrothecium delicatulum, B. \& Br.

Johnson, Bull. Minn. Acad. 1: 367. I880.

Acrothecium melanopus, (S.) Sacc.

Sacc. Syll. 4: 485 . $d$. Io April I 886.

Dactylium melanopus, $S$.

S. Syn. Am. Bor. 283 no. 2705 . d. 1834 .

Acrothecium obovatum, C. \& E.

C. \& E. Grev. 5 : 50. d. pl. $80, f .13$. Dec. 1876.

Cke. Jour. Queckett Micr. Club 4: $p l .27, f .30 . \quad$ I 877.

Sacc. Syll. $4: 484$. $d$. Io April 1886.

Ell. in Cat. Pl. N. J. 568.1889.

Acrothecium recurvatum, Morg.

Morg. Jour. Cin. Soc. 18 : 44. d. Dec. 1895.

Sacc. Hedw. 35 : xlviii. 25 Feb. I896. [Reportorium no. 7.]

Sacc. \& Syd. Syll. 14: 1089. d. 20 Aug. I899.

Acryophyton tuberculatum, Leb. See Isaria Sphingum, S. 
Actidium earicinum, $\mathrm{S}$.

S. Syn. Am. Bor. 240 no. 2019. d. 1834 .

Cke. Bull. Buf. Soc. $3: 36$. Nov. 1875 .

Sacc. Syll. $2: 739 . d$. I3 June 1883.

Actidium Crotalariæ, S. MS.

See Parodiella grammodes, Cke.

Actidium ? diatrypoides, Cke.

Cke. Grev. $7: 49$. Dec. 1878.

Sacc. Syll. $2: 739$. I3 June 1883.

Ell. \& Ev. Pyren. 685. May 1892.

Actigea, Raf.

See Scleroderma, $\mathrm{P}$.

Actigea multifida, Raf.

See Scleroderma flavidum, Ell. \& Ev., var.

Actigea sieula, Raf.

See Scleroderma Geaster, Ell. \& Ev., var.

Actiniceps, B. '\& Br.

Pound \& Clements, Minn. Bot. Studies $9: 727 . d$. 3 I May I897.

Actiniceps Besseyi, Macmillan.

See Volutella ciliata, Fr., var. stipitata, (Lib.) Sacc.

NoтE. An authentic specimen of this species on examination seems to us to be the same as Volutella ciliata, Fr., var. stipitata, Sacc.

Actinocladium Penicillus, Fr.

See Sporocybe concentrica, Sacc.

Actinodermium Sterrebeckii, Nees.

See Geaster Linkii, Spreng.

Actinomyees Bovis; Harz.

Note. Information about this species is to be sought in works on animal pathology.

Actinonema Psoraleæ, Ell. \& Ev.

Ell. \& Ev. Proc. Phil. Acad. 1893: 159. d. 28 Feb. I893.

Sacc. Syll. $11: 526$. $d$. July 1895 .

Smyth, Tr. Kans. Acad. 15: 71. March 1898.

Actinonema Rosæ, (Lib.) Fr.

Sacc. Syll. $3: 408$. d. I5 Dec. I 884.

Pound, Bủll. Nebr. Exp. Sta. 1: 357 (87). I8 Dec. I889.

Humphrey, Rept. Mass. Exp. Sta. $7: 229.1889$.

Webber, Rept. Nebr. Agr. 1889: 218 (78). I 890.

Earle, Bull. U. S. Agr. Veg. Pathol. II : 88. 1890.

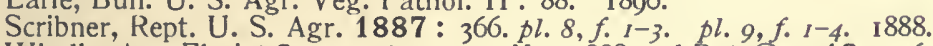

Windle, Am. Florist $3: 424 . f .1-3 . \quad$ I May I 888 and Bot. Gaz. 13 : I 66. July 1888 .

Windle, Am. Mo. Micr. Jour. 9 : I28-130. f. $I-3$. July I 888.

Maynard, Bull. Hatch Exp. Sta. 4: 10. April 1889.

Kelsey, Jour. Myc. 5 : 8I. June 1889.

Jennings, Bull. Tex. Exp. Sta. 9: 23. May I89o.

Scrib. Orchard \& Garden 12: $57 . f^{\circ} 267,268$. March 1890.

Ell. \& Ev. Pyren. 605. May 1802 .

Halsted, Rept. N. J. Exp. Sta. 1891 : 303. 1892.

Halsted, Rept. N. J. Exp. Sta. 1892: 280. 1893.

Underwood, Proc. Ind. Acad. 1893: 43. Aug. I894.

Sturgis, Rept. Conn. Exp. Sta. 17:86. 1894.

*Ell. \& Ev. Fung. Columb. 277. I894.

Halsted, Rept. N. J. Exp. Sta. 1894: 38I. f. 63. I 895. 
Tracy \& Earle, Bull. Miss. Exp. Sta. 34: 109. May 1895.

Halsted, Am. Gardening 16:226. 22 June 1895.

McClatchie in Reid's Hist. Pasadena 616. I 895.

Millsp. \& Nutt. Publ. Field Columb. Mus. Bot. 1: 116. Jan. 1896.

McClatchie, Proc. S. Calif. Acad. $1: 374$. 5 June 1897.

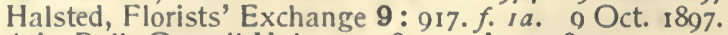

Atk. Bull, Cornell Univ. $3: 28.25$ June 1897.

Underw. \& Earle, Bull. Ala. Exp. Sta. 80: 163. 28 June 1897.

* Seymour \& Earle, E. F. 469. 15 Oct. 1897.

Stevens, Rept. Ohio Acad. $5: 63.1897$.

Orton, Rept. Vt. Exp. Sta. 12 : 17 I. Dec. I899 and Contr. Bot. Vt. 7: 17I. Dec. 1809 .

Lindau in Engler \& Prantl, Pflanzen-fam. 11**: 370. f. 194 F.-L. 1900.

Selby, Bull. Ohio Exp. Sta. 121 : 53. Sept. 1900.

Allescher, Rabh. Krypt.-Fl. $1^{6}:$ 708. d. $f .1900$.

Sturgis, Rept. Conn. Exp. Sta. 24 : 286.2 I 901.

Patterson, Bull. U.S. Agr. Pl. Industry $8: 23$. 3 Feb. 1902.

Ricker, Univ. of Maine Studies 3:25. 30 April 1902.

Asteroma Rosa, Lib.

M. A. Curtis, Bot. N. Car. II 8.1867.

Cooke, Handbook 461. d. f. $174^{\mathrm{a}}$. $187 \mathrm{I}$.

B. Grev. 2:97 (78). Jan. 1874.

Pk. Rept. N. Y. Mus. 28: 58 . I 876.

Cke. Grev. 6 : 135. June 1878.

Vize, Grev. 7 : II. Sept. 1878.

Pk. Rept. N. Y. Mus. $29: 78.1878$.

Hark. \& Moore, Cat. Pac. Fung. 23. Feb. 1880.

*Ell. N. A. F. 536 . I881.

*Rav. Fung. Am. 520. I88I.

Pk. in Day's Cat. Bull. Buf. Soc. 4:202 (138). 1883.

Hark. Bull. Cal. Acad. 1: 160 . Feb. 1885.

Scribner, Rept. U. S. Agr. 1886 : 135. 1887.

Langlois, Cat. Pl. La. 27: 1887.

Ell. in Cat. Pl. N. J. 580.1889.

Dothidea Rosa, S.

S. Syn. Am. Bor. 235 no. 1941. d. 1834.

* Rav. Fung. Car. 1: 68.1852.

Frost, Proc. Boston Soc. 12 : 80. 1860.

Hark. \& Moore, Cat. Pac. Fung. 42. Feb. 1880.

Cke. Grev. 13: 4I. Dec. 1884.

Comes, Critt. Agr. 1 : 365. I891.

Phyllachora Rosa, Sacc.

Sacc. Syll. 2:611. $d$. 13 June 1883.

Tracy \& Earle, Bull. Miss. Exp. Sta. 34: 103. May 1895.

Actinothy rium, Kze.

Allescher, Rabh. Krypt.-Fl. 17 : ${ }_{386}^{86}$ d. f. 1902.

Actinothyrium caulincolum, $\mathrm{S}$.

S. Syn. Am. Bor. 249 no. 2178 . d. 1834 .

Sacc. Syll. $3: 658$. d. 15 Dec. 1884.

Actinothyrium Cubense, B. \& C.

B. \& C. Jour. Linn. Soc. 10: 357. d. 1869.

Sacc. Syll. $3: 658 . d$. 15 Dec. 1884 .

West Indies.

Actinothyrium graminis, Kze.

S. Syn. Am. Bor. 249 no. $2176 . \quad 1834$

Hark. \& Moore, Cat. Pac. Fung. 22. Feb. 1880.

Langlois, Cat. PI. La. 27. 1887.

Rostrup, Meddelelser om Groenl. 18 : 7I (29). 1894. 
Actinothyrium Magnoliæ, S.

S. Syn. Am. Bor. 249 no. 2177. d. 1834.

Sacc. Syll. $3: 658$. d. 15 Dec. 1884 .

ACurtis, Fr.

Fr. Summa 337. 1849 .

Sacc. Cub. \& Manc. Syll. 6 : 691. d. I Aug. I888.

Sacc. Syll. 11 : $139 . d$. July 1895.

ACurtis gigantea, (S.) Fr.

Fr. Summa 337. I849.

Sacc. Cub. \& Manc. Syll. $6: 691$. d. I Aug. I 888.

Cke. Grev. 20: II. Sept. I89I.

Note. The genus ACurtis, Fr. was founded on Clavaria gigantea, S. which by later writers has been recognized as a monstrosity of some species of Lentinus. Writers are not agreed as to whether the species is Lenlinus tigrinus (see B. Gard. Chron. II. 9: 339. I6 March 1878) or Lentinus lepideus (see W. G. Smith, Jour. Bot. 41: 322 . Oct. 1903).

Aecidiella, Ell. \& Kelsey.

See Pucciniosira, Lagerh.

Aecidiella Triumfettæ, Ell. \& Kelsey.

See Pucciniosira pallidula, Lagerh.

Aecidium, P. Including Peridermium, (Lk.) Lev. and Raestelia, Reb.

Note. Under the genus Aecidium are included all our æcidial forms whether their connection with definite teleutosporic forms is known or not. Where such connection is not yet known we have given all the references to the species known to us. Where such connection is known or is strongly suspected we have given under the æcidial name the references to the æcidial form itself with a cross-reference to the uredosporic and teleutosporic forms, where incidental references to the æcidia may also be found.

It should be remarked that the modern method of studying the Uredinex by infection experiments has resulted in the recognition of many races or form-species, and the older characterization and nomenclature of ecidial forms can be regarded only as temporary.

Aecidium abietinum, A. \& S.

NoTE. The species of Albertini and Schweinitz has been shown to include two forms: one connected with Chrysomyxa Rhododendri, the other with Chrysomyxa Ledi. (See DeBary, Bot. Zeit. 37:761 et seq. 1879, and, Schroeter, Beitr. Biol. Pflanzen 3: 51. 1879.) The Aecidium abietinum A. \& S: (Peridermium abietinum, Thm.) as used by American mycologists probably does not include the form connected with Chrysomyxa Rhodo. dendri, as that species has not been reported in America. Some of the references relate to the 'xcidial form of Chrysomyxa Ledi, but uncertainty exists in other cases because under Aecidium abietinum some writers have included as var. decolorans, Thm. the Peridermium decolorans of Peck.

For references to forms probably connected with species of Chrysomyxa on Ledum see Farl. Appalachia 3: 239, Jan. 1884, and Proc. Am. Acad. 20: 320, 1885; also Schroeter, Beitr. Biol. Pflanzen 1. c. and Ber. Schles. Ges. I887: 279 .

Aecidium (Peridermium) abietinum, (Fung. Columb. 1479).

NoTE. The locality and host make it probable that the specimens in this case belong to a species which is not identical with those elsewhere given under Aecidium abietiuum.

Aecidium abietinum, Auct. Amer. p. p.

See Aecidium decolorans, (Pk.).

Aecidium abietinum, f. Engelmanni, Ell. \& Ev. See Aecidium Coloradense, Diet. 
Aecidium abundans, $\mathrm{Pk}$.

Pk. Bot. Gaz. 3: 34. d. April 1878.

*Wint. Fung. Eur. 3016. I 884.

Wint. \& Demet. Hedw. 24: 179 (3). Oct. I 885.

* Roum. Fung. Gall. 3866. I 886.

Roum. Rev. Myc. 8: 197. I Oct. I 886.

Hark. Bull. Cal. Acad. 2 : 440 . I6 June 1887.

Kellerm. \& Carl. Tr. Kans. Acad. 10 : 9I. 1887.

*Ell. \& Ev. N. A. F. 1814. 1887.

De Toni in Sacc. Syll. $7: 797 . d .28$ Oct. 1888.

Kelsey, Jour. Myc. 5 : 80 . June 1889.

Webber, Bull. Nebr. Exp. Sta. 1: 329 (59). 18 Dec. I889.

Gall. Bull. U. S. Agr. Veg. Pathol. 8: 55. 1880.

Williams, Am. Nat. 24: 780 . Aug. I800.

Webber, Rept. Nebr. Agr. 1889: 209 (69). I I yo.

*Ell. \& Ev. Fung. Columb. 873.. April I896.

Ell. in Nelson, Bull. Wyo. Exp. Sta. $28: 202$. May 1896.

Tubeuf-Smith, Diseases of Plants 4II. 1897.

Hume, Proc. Davenport Acad. 7 : 25I. I2 May I899.

Barthol. Tr. Kans. Acad. 16 : 186. June 1899.

Nelson, Rept. Wyo. Exp. Sta. 10 : (34). I I000.

Tracy \& Earle in Greene, PI. Baker. 1: 17. 22 Feb. I90I.

Earle in Greene, Pl. Baker. 2: 2. 25 March IgoI.

Patterson, Bull. U. S. Agr. Pl. Industry 8:8. 3 Feb. I902.

*Griffiths, West Am. Fung. 295. 22 March 1002.

*Barthol., Ell. \& Ev. Fung. Columb. 1701. Feb. 1903.

Aecidium Aconiti-Napelli, (D C.) Wint.

*Ell. \& Ev. N. A. F. 2212. Feb. I889.

Aecidium Actææ, Opiz.

Muhl. Cat. PI. Am. Sept. I ed. 104. 1813. 2 ed. 107. 1818.

Arth. Bull. Iowa Agr. Coll. 1884: I64. Jan. I885.

Burrill, Bull. III. Lab. 2:223. d. I 885 and Rept. Ill. Ind. Univ. 12 : 140. $d$. 1885 .

De Toni in Sacc. Syll. $7: 777$. d. 28 Oct. 1888.

Gall. Bull. U. S. Agr. Veg. Pathol. 8: 55. 1880.

Detmers, Bull. Ohio Exp. Sta. $44: 138$. Sept. 1892.

Davis. Tr. Wis. Acad. 9: 182. 21 Aug. I893.

Small \& Vail, Mem. Torr. Bot. Club 4: 200. A April 1894.

Pk. Rept. N. Y. Mus. 47: 146 (20). Nov. 1894.

Kellerm. \& Werner, Geol. Ohio $7^{2}$ : 345. Jan. 1895.

Tubeuf-Smith, Diseases of Plants $409 . \quad 1897$.

Diet. in Engler \& Prantl, Pflanzen-fam. 11**: 76.1897.

Freeman, Minn. Bot. Studies II. 5 : 555. 20 July I 901.

Kellerm. Jour. Myc. 9: I2. I4 Feb. I903.

NOTE. The form on Actaa spicatn according to Ed. Fischer Bot. Centralbl. 83 : 75, 17 July 1900, and Fortsetzung Entwick. Untersuch. ueber Rostpilze, Ber. Schweitz. Bot. Ges. II : 4-9, I90 I, belongs to Puccinia Actac-Agropyri, Ed. Fisch. The authority for the xcidial name is given as Opiz by most authors, who evidently follow Wallroth (1833), and the date as given by Schroeter (quoting Steudel) is 1824 . It should be noticed that the name Aecidium Actace occurs in Muhlenberg's Catalogue, 1813, where no authority is given.

Aecidium Actææ, Detmers, p. p. See Aecidium cimicifugatum, S

Aecidium Aegopodil, Reb.

See Caoma Aegopodii, Wint. 
Aecidium Aesculi, Ell. \& Kellerm.

Ell. \& Kellerm. Bull. Torr. Bot. Club 11 : I14. d. Oct. 1884.

Kellerm. Bull. Wash. Lab. $1: 74$. Jan. 1885 and Tr. Kans. Acad. 9 : 8I. 1885.

*Ell. N. A. F. 1429.1885.

*Roum. Fung. Gall. 3865 . 1886.

Roum. Rev. Myc. 8: I97. I Oct. I886.

Kellerm. \& Carl. Tr. Kans. Acad. 10:91. 1887.

De Toni in Sacc. Syll. $7: 785 . d$. 28 Oct. 1888.

*Kellerm. \& Swingle Kansas Fungi 1. I May I880.

Webber, Bull. Nebr. Exp. Sta. 1 : 329 (59). 18 Dec. I889.

Webber, Rept. Nebr. Agr. 1889: 209 (69). 1890.

Tubeuf-Smith, Diseases of Plants 4IO. 1897.

* Syd. Uredineen 1198. Feb. I898.

*Ell. \& Ev. Fung. Columb. 1296 . May 1898.

Barthol. Tr. Kans. Acad. 16: 186. June I899.

Aecidium Aethusæ, Ell. \& Ev. non Kirch. See Aecidium Leptotania, Lindr.

Aecidium Aikeni, Syd.

Syd. Ann. Myc. $1: 334$. d. July 1903.

Aecidium Thalictri-flavi, Syd. non Wint.

*Syd. Uredineen, 1397. I899.

Aecidium albescens, Grev.

*Ell. N. A. F. 223. 1879 .

Arth. Bull. lowa Agr. Coll. 1884: : 164. Jan. 1885.

Arth. Bot. Gaz. 10: 369 . Sept. 1885.

Ell. \& Ev. Jour. Myc. 1 : 141. d. Nov. 1885.

Note. The teleutosporic stage of this species is usually understood to be Puccinia Adoxa, D C., but Bubák in Centralbl. Bact. 2 Abt. 10: 574, 1901, considers it to belong to Puccinia argentata, (Schultz) Wint.

Aecidium albiperidium, Arth.

Arth. Jour. Myc. $8: 53 . d$. 30 June 1902.

Note. The above name is given to an Aecidium produced by sowing on Ribes Cynosbati teleutospores obtained in the field on Carex pubescens. The teleutosporic stage is called Puccinia albiperidia, Arth. See also Davis, Tr. Wis. Acad. 14: 86, Sept. 1903, where it is stated that this is probably the same as Aecidium Grossularia, P. See also Klebahn, Pringsh. Jahrb. 34 : 388 and $35: 701$. 1900.

\section{Aecidium album, Clint.}

Note. In Hedw. $36: 297,25$ Oct. 1897, Dietel describes as new the teleutosporic stage of this species, using the name Uromyces albus, (Clint.) Diet. For synonymy of the æcidial stage see Diet. Hedw. 28: 183, June 1889, and Anderson, Jour. Myc. $6:$ เ21, 6 Jan. 1891. See also Aecidium porosum? under Uromyces Coloradensis, Ell. \& Ev. Erythea I : 204, Oct. 1893.

Pk. Bull. Buf. Soc. $1: 68$. d. July 1873 .

Pk. Rept. N. Y. Mus. $26: 78$. d. April 1874 .

Pk. Rept. N. Y. Mus. $29: 76 . \quad 1878$.

Ell. \& Ev. Jour. Myc. 1 : 86 . July 1885.

*Ell. N. A. F. 1431 . 1885.

De Toni in Sacc. Syll. $7: 789 . d .28$ Oct. 1888

Diet. Hedw. 28 : 183 . June 1889.

Anderson, Jour. Myc. 6 : 121. $d$. 6 Jan. 1891.

Tracy \& Earle in Greene, Pl. Baker. 1: 17.22 Feb. I90I. 
Kellerm. Ohio Nat. 2 :'161. d. Dec. 1901.

* Kellerm. Ohio Fungi 2. d. Dec. I901.

* Griffiths, West Am. Fung. 84, 84*. 22 March 1902. Aecidium porosum, $\mathrm{Pk}$.

Pk. Bot. Gaz. 3 : 34. d. April 1878 .

Arth. Bull. lowa Agr. Coll. 1884: 167. Jan. 1885.

Kellerm. Bull. Wash. Lab. $1: 75$. Jan. 1885 and Tr. Kans. Acad. 9 : 81. I 885 .

*Ell. N. A. F. 143 O. 1885 .

Arth. \& Holw. Bull. Minn. Surv. 3: 31. I Oct. 1887.

Kellerm. \& Carl. Tr. Kans. Acad. 10:9r. 1887.

De Toni in Sacc. Syll. $7: 787$. d. 28 Oct. 1888.

Kelsey, Jour. Myc. 5: 80 . June 188 .

Webber, Bull. Nebr. Exp. Sta. $1: 333$ (63). 18 Dec. 1889.

Gall. Bull. U. S. Agr. Veg. Pathol. 8: 56. I889.

Webber, Rept. Nebr. Agr. 1889:211 (71). 1890.

Lagerh. Bot. Notiser 1890: 276. I890.

Davis, Tr. Wis. Acad. 9: 182. 2r Aug. 1893.

*Ell. \& Ev. Fung. Columb. 874. April r 896.

Barthol. Tr. Kans. Acad. 16: 191. June 1889.

Nelson, Rept. Wyo. Exp. Sta. 10: (36). 1900.

Kellerm. Ohio Nat. 2: 136. $d$. Nov. 1901.

* Kellerm. Ohio Fungi 2. $d$. Dec. 1901.

Aecidium Allenii, Clint.

Pk. Rept. N. Y. Mus. 24: 93. d. 106. 1872.

Pk. Rept. N. Y. Mus. $29: 77 . \quad 1878$.

*Ell. \& Ev. N. A. F. 1815.1887

*Roum. Fung. Gall. 4412. 1888.

Roum. Rev. Myc. 10:86. I April I888.

De Toni in Sacc. Syll. $7: 821$. d. 28 Oct. 1888.

*Pazschke, Fung. Eur. 4039. April $\mathrm{s} 895$.

Pound, Clements and others, Bot. Surv. Nebr. $4: 36.20$ Jan. 1896.

Hume, Proc. Davenport Acad. $7: 247,251.12$ May 1899.

Nelson, Rept. Wyo. Exp. Sta. 10 : (34). I900.

Earle in Greene, Pl. Baker. $2: 2.25$ March 1901.

* Griffiths, West Am. Fung. 297. 22 March 1002.

* Barthol., Ell. \& Ev. Fung. Columb. 1702. Feb. 1903.

Aecidium allicolum, Wint.

*Wint. Fung. Eur. 3317. d. 1885.

Wint. Hedw. 24: 260. d. Dec. I885 and Jour. Myc. 2 : I1. d. Jan. 1886.

De Toni in Sacc. Syll. 7 : 830. d. 28 Oct. 1888.

Gall. Bull. U. S. Agr. Veg. Pathol. 8: 55. 1889.

Barthol. Tr. Kans. Acad. 16 : 186 . June 1899.

Aecidium Amorphæ, Cke.

See Uropyxis Amorpha, Schrt.

Aecidium amphigenum, Ell. \& Kellerm.

See Aecidium Kellermannil, De Toni.

Aecidium Anemones, $P$.

Muhl. Cat. Pl. Am. Sept. I ed. 104. 1813. 2 ed. 107. 1818.

Torrey, Cat. Pl. N. Y. 87. I8rg.

*Ell. N. A. F. 225.1879.

Farl. Proc. Am. Acad. 18 : 74. July 1883.

*Ell. \& Ev. N. A. F. 2403 . April 1890.

Aecidium leucospermum, D C.

Watt, Can. Nat. \& Geol. II. 2 : 391. Oct. 1865.

B. Grev. 3 : 60 (102). Dec. 1874 . 
Diet. in Engler \& Prantl, Pflanzen-fam. 11**: 76. f. 50 A, B. I897.

Note, Puccinia fusca, Relh. is considered by some botanists to be the teleutosporic stage of this species, but Magnus, Sitz.-Ber. naturforsch. Freunde $1890: 29$, I $8 \mathrm{Feb}$. I 890, and I890: 145, 21 Oct. 1890, discredits this view and Tranzschel, Centralbl. Bact. 2 Abt. II : 106, 27 Oct. 1903, states that this Aecidium belongs to Ochropsora Sorbi, (Oud.) Diet.

Aecidium Anisacanthi, $\mathrm{Pk}$.

Pk. Bull. Torr. Bot. Club 10 : 75. d. July I 883.

De Toni in Sacc. Syll. 7 : 819. d. 28 Oct. I888.

*Seymour, Pringle's Mex. Fungi 7. I Sept. 1896.

Mexico.

Aecidium Anisotomes, Reichardt.

Pound, Clements and others, Bot. Surv. Nebr. $4: 36.20 \mathrm{~J}$ an. I896.

Barthol. Tr. Kans. Acad. 16: I86. June I899.

Lindr. Acta. Soc. Faunà et Fl. Fennica 221 : 178.1902.

Aecidium Anogræ, Arth.

Arth. Bull. Torr. Bot. Club $28: 664$. d. 30 Dec. Igor.

Aecidium apocynatum, $\mathrm{S}$. See Aecidium Apocyni, S.

Aecidium Apocyni, S.

S. Syn. Car. $68(42)$ no. 448. d. 1822.

Bon. Abh. Nat. Ges. Halle 5 : $208(42)$. i 860.

M. A. Curtis, Bot. N. Car. I24. 1867.

Burrill, Bull. 1ll. Lab. 2: 236. 1885 and,Rept. Ill. Ind. Univ, 12 : I47. 1885 .

Kellerm. \& Carl. Tr. Kans. Acad. 10 : 91. 1887.

De Toni in Sacc. Syll. $7: 808$. $d .28$ Oct. 1888.

Webber, Bull. Nebr. Exp. Sta. 1 : 329 (59). 18 Dec. I889.

Gall. Bull. U. S. Agr. Veg. Pathol. 8: 55. I889.

Webber, Rept. Nebr. Agr. 1889: 209 (69). I890.

Williams, Bull. S. Dak. Exp. Sta. 29:49. Dec. I891.

Cheney, Tr. Wis. Acad. 10: 69. Oct. 1895.

Tubeuf-Smith, Diseases of Plants 4II. 1897.

*Ell. \& Ev. Fung. Columb. 1295. May 1898.

Barthol. Tr. Kans. Acad. 16: I86. June 1899.

Patterson, Bull. U. S. Agr. Pl. Industry 8: 8. 3 Feb. 1902. Croma apocynatum, Lk.

Lk., L. Sp. Pl. ed. Willd. 62: 49. d. 1825 .

S. Syn. Am. Bor. 292 no. 2865. I 834. Uredo Asterum, Spreng. p. p.

Spreng. in L. Syst. Veg. 16 ed. $41^{1}: 572 . d .1827$. Aecidium apocynatum, $\mathrm{S}$.

S. Syn. Am. Bor. 309. 1834 .

*Ell. \& Ev. N. A. F. 1823. I 887.

Aecidium Aquilegiæ, $\mathrm{P}$.

Cockerell, Science Gossip 24: 92. April I888.

Underw. Proc. Ind. Acad. 1893: 49. Aug. I894.

Tubeuf-Smith, Diseases of Plants 409. $\quad$ I897.

. Hume, Proc. Davenport Acad. 7 : 25I. I2 May I899.

Nelson, Rept. Wyo. Exp. Sta. 10 : (34). 1900.

*Shear, Ell. \& Ev. Fung. Columb. 1474. May 1901.

Note. According to Plowright, Gard. Chron. III. 8: 4I, I2 July 1890 , Aecidium Aquilegia on Aquilegia vulgaris and according to Jacky, Ber. Schweiz. Bot. Ges. Heft. 9:(18), 1899, that on Aquilegia alpina are the recidia of Puccinia Agrostidis, Plowr.

Aecidium Ari, Auct. Amer.

See Aecidium Caladil, S. 
Aecidium aroidatum, $\mathrm{S}$.

See Aecidium Caladii, S.

Aecidium Asperifolii, $\mathrm{P}$.

S. Syn. Car. 67 (4I) no. 436.1822.

Kellerm. \& Carl. Tr. Kans. Acad. 10:91. 1887.

Caoma urticatum, S. p. p.

S. Syn. Am. Bor. 294 no. 2898.1834.

Note. The European plant of Persoon is referred to Puccinia R'ubigovera, (D C.) Wint. The American Aecidium called by this name has been seldom found and little studied. See note under Aecidum Impatientis, S.

Aecidium asteratum, S. p. p. See Aecidium Asterum, S.

Aecidium asteratum, S. p. p. See Aecidium Solidaginis, S.

Aecidium asteratum, S. p. p. See Aecidium Verbesinze, S.

\section{Aecidium Asterum, S.}

NorE. In the present state of our knowledge it is impossible to arrange the numerous and confused references to the recidia or Aster, Solidayo and related genera with any degree of definiteness. Arthur, Jour. Myc. 8: 54, 30 June 1902, refers Aecidium Asterum, S. to Puccinia Caricis-Asteris, Arth., but as American writers have used the name Aecidium Asterum to include forms on Solidago and other genera we have here arranged such references according to their host genera without attempting to indicate their real affinities.

S. Syn. Car. 67 (4I) no. 444. d. I 822.

Bon. Abh. Nat. Ges. Halle $5: 208$ (42) d. 1860.

M. A. Curtis, Bot. N. Car. I24. I867.

Edwards, Bull. Torr. Bot. Club 2: 39 . Oct. 1871.

Burrill, Bull. 111. Lab. $2: 230 . d$. I 885 and Rept. 111.Ind. Univ. 12 : I44. d. I885.

Gall. Bull. U. S. Agr. Veg. Pathol. 8 : 55.1889.

Webber, Bull. Nebr. Exp. Sta. 1: 329 (59). 18 Dec. 1889.

Webber, Rept. Nebr. Agr. 1889 : 209 (69). 1890.

Detmers, Bull. Ohio Exp. Sta. 44 : 138. Sept. I 892.

Underw. Proc. Ind. Acad. 1893 : 49. Aug. I 894.

Kellerm. \& Werner, Geol. Ohio $7^{2}$ : 345. Jan. I895.

Underw. \& Earle, Bull. Ala. Exp. Sta. 80 : 208. 28 June 1897.

Stevens, Rept. Ohio Acad. Sci. 5 : 66.1897.

Claassen, Rept. Ohio Acad. 5 : 68.1897.

Tubeuf-Smith, Diseases of Plants 4I I. I 897.

Snyder. Proc. Ind. Acad. 1896: 217. I897.

Orton, Rept. Vt. Exp. Sta. 11 : 210. Sept. 1898 and Contr. Bot. Vt. 2 : I2. Sept. 1898.

*Ell. \& Ev. Fung. Columb. 1391. May I899.

Barthol. Tr. Kans. Acad. 16: I86. June I809.

Nelson, Rept. Wyo. Exp. Sta. 10: (35). 1900.

Freeman, Minn. Bot. Studies I1. $5: 557.20$ July I90 r.

Earle in Mohr, Contr. U.S. Nat. Herb. 6 : 192.31 July Igor.

Ricker, Univ. of Maine Studies 3: 50.30 April 1902.

*Griffiths, West Am. Fung. 370. 21 May 1902. Caoma asteratum, Lk. p. p.

Lk., L. Sp. Pl. ed. Willd. $6^{2}$ : 5 I. d. 1825.

S. Syn. Am. Bor. 292 no. 2870. I 834.

Uredo Asterum, Spreng.

Spreng. in L. Syst. Veg. I6 ed. $4^{1}$ : 572. d. $\quad$ I 827. 
Aecidium asteratum, S. p. p.

S. Syn. Am. Bor. 309. 1834.

Cke. Proc. Portland Soc. 1 : 181. d. 1869.

Pk. Rept. N. Y. Mus. 25: 92 . Sept. 1873.

Frost in Cat. Pl. Amherst 85. 1875.

Pk. Rept. N. Y. Mus. 29: 77. 1878.

*Ell. N. A. F. IOI9. I883.

Arth. Bull. lowa Agr. Coll. 1884: 164. Jan. I885.

Kellerm. Bull. Wash. Lab. $1: 74$. Jan. 1885 and Tr. Kans. Acad. 9 : 81. 1885.

Cobb, List Pl. Amherst 40. 1887.

Kellerm. \& Carl. Tr. Kans. Acad. 10:91. 1887.

Atk. Bull. Cornell Univ. 3: 2I. 25 June 1897.

Aecidium Compositarum, Auct. Amer. p. p.

Trel. Tr. Wis. Acad. 6 : 137 (33). Nov. 1884.

Ell. \& Ev. Jour. Myc. $1: 86$. July 1885.

Cockerell, Zoe 4: 282. Oct. 1893.

Nelson, Rept. Wyo. Exp. Sta. 10: (35). 1900.

Pound \& Clements, Bot. Surv. Nebr. $5: 24.30$ March I9or.

Aecidium Compositarum, forma Asteris, Wint.

Wint. \& Demet. Hedw. 24: 179 (3). Oct. 1885.

Aecidium Asterum, Auct. Amer. p. p. See Aecidium Solidaginis, S.

Aecidium Astragali, Thm.

Cockerell, Zoe $4: 283$. Oct. 1893 .

Note. The host in the single reference to this species in America is said to be possibly Astragalus alpinus. In Northern Europe the Aecidium on that host, Aecidium Astragali, Eriks. non Thm. is connected with Uromyces lapponicus, Lagerh. See Lagerh. Bot. Notiser 1890: 274 and Eriksson, ditto I89I: 40.

Aecidium Atriplicis, Shear.

* Griffiths, West Am. Fung. 32I. 21 May 1902.

Shear, Bull. Torr. Bot. Club 29 : 453. d. 25 July 1902.

Aecidium (sub Rœstelia) aurantiacum, (Pk.)

NoTE. Here are included references to the æcidial form of Gymnosporangium clavipes, C. \& P. See Farlow 1885, I 886 and Thaxter 1887, I 889 given below. See also Gymnosporangium clavipes. There is an Aecidium aurantiacum of Bonorden, 1860, and therefore Aecidium aurantiacum as given here is to be understood to be merely an index name.

Rostelia aurantiaca, $\mathrm{Pk}$.

Pk. Bull. Buf. Soc. $1: 68 d$. July 1873 .

Pk. Rept. N. Y. Mus. 25: 9 I. d. $p l$. $I, f_{0}, I 0-12$. Sept. 1873.

Cke. \& B. Fungi 42. 1875 .

Frost in Cat. Pl. Amherst 84. 1875.

Farl. Bull. Bussey Inst. 2: 225. Jan. 1878 .

Cke. Grev. $7: 45$. Dec. 1878 .

Pk. Rept. N. Y. Mus. 29: $76 . \quad$ I 878.

*Rav. Fung. Am. 27I. 1879.

Farl. Anniv. Mem. Boston Soc. 31. d. 1880.

*EII. N. A. F. 1084. 1883.

Farl. Proc. Am. Acad. 20 : $318 . \quad 1885$.

Farl. Bot. Gaz. 11: 190. July 1886.

Farl. Bot. Gaz. 11 : 236. Sept. 1886.

Thax. Proc. Am. Acad. 22: 264. Jan. 1887 and Centralbl. Bact. 1 : $433,458-460 . \quad 1887$. 
Seymour, Tr. Am. Hort. Soc. 4: 155 (4), I60 (8). 7 April I887.

Cobb, List Pl. Amherst 39. 1887.

*Ell. \& Ev. N. A. F. 2224. Feb. I889.

Thax. Bot. Gaz. 14: 165, 171. July 1889.

Ell. in Cat. PI. N. J. 504. 1889.

Jennings, Bull. Tex. Exp. Sta. 9: 28. May 1890 .

Mayr, Waldungen von Nordamer. 139. 1890.

Gall. Rept. U. S. Agr. 1889 : 416. I890.

Pammel, Bull. lowa Exp. Sta. 1 3: 43. May I891.

Halsted, Rept. N. J. Exp. Sta. 1892: 307. f. 10. I 893.

Bailey, Bull. Cornell Exp. Sta. 80: 626. f. 10-12. Dec. I894.

Fisclier, Hedw. 34: $4 \cdot f$. 7 . I5 Feb. I 895.

Tracy \& Earle, Bull. Miss. Exp. Sta. 34: 91. May 1895.

Sturgis, Rept. Conn. Exp. Sta. 18 : 1 37.1895.

Underw. \& Earle, Bull. Ala. Exp. Sta. 80: 218. 28 June 1897.

Jones, Oberlin College Lab. Bull. $9:$ :. 15 June 1898.

Herbst, Fungal FI. Lehigh Valley 174. $d .1899$.

? Nelson, Rept. Wyo. Exp. Sta. 10 : (36). I I900.

Ricker, Univ. of Maine Studies 3: 53. 30 April 1902.

Roestelia lacerata, B. in Grev. p. p.

B. Grev. 3: 59 (IOI). Dec. I874.

PRostelia cancellata, Auct. Amer. p. p. On Cydonia.

Pk. Rept. N. Y. Mus. 33 : 38 . Oct. 1883.

Aecidium auriellum, $\mathrm{Pk}$.

Pk. Bull. Torr. Bot. Club $10: 74$. $d$. July $188_{3}$

De, Toni in Sacc. Syll. $7: 778 . d$. 28 Oct 1888.

Aecidium Bahiæ, B. \& C.

Billings \& Frost in Bot. 40th Parallel 4I4. I871.

B. Grev. $3: 60$ (102) d. Dec. 1874 .

De Toni in Sacc. Syll. 7 : $801 . d .28$ Oct. 1888.

Aecidium balsameum, (Pk.) Diet.

Note. It has been conjectured that this species is connected with a species of Calyplospora common on Vaccinia in North America since Aecidium columnare A. \& S. closely related morphologically was shown by Hartig, Allg. Forst \& Jagdzeit. I880 (see Bot. Zeit. $38: 618$ ) to helong to Calyplospora Goeppertiana, J. Kuehn. The teleutosporic stage of Aecidium pseudo-columnare, J. Kuehn, Hedw. 23: 168, Nov. 1884, a species still more closely related to Aecidium balsameum is not known. See also notes under Aecidium Peckii, (Thm.) Diet.

Diet. in Engler \& Prantl, Pflanzen-fam. 11**: 78. 1897.

Peridermium balsameum, $\mathrm{Pk}$.

Pk. Rept. N. Y. Mus. $27:$ I04. d. pl. 2, f. 24-26. 1875.

Pk. Rept. N. Y. Mus. $29: 77.1878$.

Thm. Mittheil. forstl. Versuchs. Oest. 2 : 319 (23). d. 1880.

*Ell. N. A. F. 1025 . I883.

Farl. Appalachia 3 : 239, 24I. Jan. I884.

*Thm. Myc. Univ. 2228. I884.

Farl. Proc. Am. Acad. 20:322. I 885.

Arth. \& Holw. Bull. Minn. Surv. 3: 31. I Oct. 1887.

De Toni in Sacc. Syll. $7: 836 . d$. 28 Oct. 1888.

* Roum. Fung. Gall. 4836. I888.

Roum. Rev. Myc. 11:64. I April 1880.

Millsp. Bull. W. Va. Exp. Sta. 24: 509. June I802.

Millsp. \& Nutt. Publ. Field Columb. Mus. Bot. 1 : izo. Jan. I896.

Tubeuf-Smith, Diseases of Plants 417. 1897.

Pk. Bull. N. Y. Mus. 6 : 227. July I $899 . \quad$ [Rept. N. Y. Mus. 52 :] 
Freeman, Minn. Bot. Studies 11. $5: 559 . d .20$ July I 901.

*Barthol., Ell. \& Ev. Fung. Columb. 1629. Oct. 1902.

Aecidium Barbareæ, D C.

B. Grev. 3:60 (102). Dec. I874.

Cke. Grev. 8 : 34 . Sept. I879.

Note. See Sydow, Mon. Ured. I : 5 1 7. 15 May 1903. North American specimens of Aecidium Barbarea probably belong to Puccinia Barbarea, Cke.

\section{Aecidium Berberidis, $P$.}

Note. This form belongs to Puccinia graminis. The following references are all that it seems desirable to insert here, for although the Aecidium has frequently been mentioned, the references are mainly in popular writings or are of slight value to mycologists.

S. Syn. Car. 67 (4I) no. 437. I822.

Grev. Scot. Crypt. Fl. 2:pl. $97 . \quad$ I824.

Sprague, Proc. Boston Soc. 5 : 329. 1856.

M. A. Curtis, Bot. N. Car. 124. I867.

Russell, Am. Nat. 2: 565. $f .1 . \quad$ Jan. I 869.

Cooke, Handbook 538. d. f. $220 . \quad$ I87I.

Pk. Rept. N. Y. Mus. 24: 91, 106. 1872.

Frost in Cat. Pl. Amherst 85. 1875.

Pk. Rept. N. Y. Mus. 29: 76 . 1878 .

Hark. \& Moore, Cat. Pac. Fung. 27. Feb. I880.

Farl. Bull. Torr. Bot. Club 8: 86. Aug. I88I.

Bundy, Geol. Wis. 1873-79, 1: $400 . \quad$ I883.

*Eli. N. A. F. 1010. 1883 .

Arth. Bull. lowa Agr. Coll. 1884: 164. Jan. I 885.

Cobb, List Pl. Amherst 40. I887.

Bennett, Cat. Pl. R. 1. 89. I 888.

Kelsey, Jour. Myc. 5: 80. June 1889.

Bolley, Am. Mo. Micr. Jour. 10 : I 73.f. 6. I78, I79. pl., f. 3. Aug. 1889 and Jour. Myc. $5: 167$. Sept. I 889.

Ell. in Cat. Pl. N. J. 505. 1889.

* Seymour \& Earle, E. F. 85. I Jan. I 891 .

Cockerell, Zoe $4: 283$. Oct. 1893 .

Underw. Proc. Ind. Acad. 1893: 49. Aug. 1894.

Herbst; Fungal Fl. Lehigh Valley i74. $d$. 1899.

Jelliffe, Flora of Long lsland 36. I 899.

Selby, Bull. Ohio Exp. Sta. 121: 17. Sept. 1900.

*Shear, Eli. \& Ev. Fung. Columb. 1472. May IgoI.

\section{Caoma berberidatum, Lk.}

S. Syn. Am. Bor. 293 no: 288I. 1834.

Aecidium Bermudianum, Farl.

Farl. Bot. Gaz. 12: 206. d. Sept. I 887 and Rev. Myc. 10:33. d. I Jan. 1888.

De Toni in Sacc. Syll. $7: 825 . d$. 28 Oct. I 888.

NotE. Gymnosporangium Bermudianum, (Farl.) Earle is the teleutosporic stage of this fungus.

Aecidium biforme, $\mathrm{Pk}$.

Pk. in Parry, Am. Nat. 9: 351. d. June 1875.

De Toni in Sacc. Syll. 7 : 8I I. $d$. 28 Oct. I 888.

Anderson, Jour. Myc. $6: 122.6$ Jan. I891.

Sacc. Syll. 9 : 324. I5 Sept. I 89 I.

Jacz. Hedw. Beibl. 39: (1 33). Io Aug. I900. 
Aecidium Heliotropii, Tracy \& Gall.

Tracy \& Gall. Jour. Myc. 4:21. d. March 1888.

-De Toni in Sacc. Syll. 7 : 81 1. d. 28 Oct. 1888.

Aecidium Bigeloviæ, $\mathrm{Pk}$.

Pk. Bot. Gaz. 3 : 34. d. April 1878.

De Toni in Sacc. Syll. $7: 801 . d .28$ Oct. 1888

* Barthol. Ell. \& Ev. Fung. Columb. 1703. Feb. 1903.

Aecidium Blasdaleanum, Diet. \& Holw.

Diet. Erythea 3: 77. $d$. I May 1895.

*Syd. Uredineen 1149. I897.

Lindau \& Syd. Hedw. 37: xvii. 9 April r 898. [Beiblatt no. 7.]

Sacc. \& Syd. Syll. 14: 376. d. 20 Aug. 1899.

Aecidium Boltoniæ, Arth.

Arth. Bull. Torr. Bot. Club 28 : 664. d. 30 Dec. I90I.

Note. On the same page with the above, the author suggests that Puc. cinia Panicularia, Arth. on Glyceria grandis may be the teleutosporic stage.

Aecidium Borrichiæ, Syd.

Syd. Hedw. Beibl. 40: (129). d. 20 Oct. I901.

Sacc. \& Syd. Syll. 16: i122. I Feb. 1902.

Aecidium Botryapites, (S.) Bennett.

Nore. This is the æcidium of Gymnosporangium biseptatum, Ell. See Farlow 1885, 1886 and Thaxter 1887, 1889 given below and Bull. Conn. Exp. Sta. 107: 15 April 1891 .

Bennett, Cat. PI. R. I. 80. 1888. Caoma Botryapites, S.

S. Syn. Am. Bor. 294 no. 2902. 1834. Roestella Ellis II, Pk.

Pk. Bull. Torr. Bot. Club 6 : 13. d. Feb. 1875 .

*Thm. Myc. Univ. 43i. $d$. 1876 .

Thm. Flora 60 : 170. $d$. II April 1877.

Pk. Rept. N. Y. Mus. $33: 28$. Oct. 1883.

*Roum. Fung. Gall. 4534. 1888.

Roum. Rev. Myc. 10:143. I July I 888.

Roestelia Botryapites, C. \& E.

C. \& E. Grev. 5: 34. Sept. I 876.

Farl. Bull. Bussey Inst. 2:225. Jan. 1878 .

Farl. Anniv. Mem. Boston Soc. 25. d. 1880.

Farl. Proc. Am. Acad. 18: 75 . July I883.

*Ell. N. A. F. 1087 . I883.

Farl. Proc. Am. Acad. $20: 319.1885$ and Jour. Myc. 1: 46. March 1885 .

Farl. Bot. Gaz. $11: 236$. Sept. 1886.

Thax. Proc. Am. Acad. 22: 263, 269. Jan. 1887 and Centralbl. Bact. $1: 432,433,460$. 1887 .

Seymour, Tr. Am. Hort. Soc. 4: 155 (4). 7 April 1887.

Thax. Bot. Gaz. 14: 165, 171. July 1889 .

Ell. in Cat. PI. N. J. 504. 1889.

Fischer, Hedw. 34: 4. f. 10. Jan. I 895.

*Ell. \& Ev. Fung. Columb. 1075. 22 Dec. 1806.

Seymour \& Patterson in Deane's Flora of Reservations 138.1896.

Jelliffe, Flora of Long Island $35 . \quad$ I 899.

Aecidium Bouvardiæ, Diet. \& Holw.

Holw. Bot. Gaz. 24: 36. d. 31 July 1897.

Lindau \& Syd. Hedw. 37 : xvii. 9 April I898. [Beiblatt no. 7.] 
Sacc. \& Syd. Syll. 14: 38 I. d. 20 Aug. I899.

Note. Griffiths in Bull. Torr. Bot. Club. 29: 298, 26 May 1902 suggests that this may be the æcidial stage of his new species Puccinia Bonvardia.

Aecidium Brandegei, Pk.

Pk. Bot. Gaz. 3 : 34 . d. April 1878.

De Toni in Sacc. Syll. $7: 808 . d$. 28 Oct. 1888.

Webber, Bull. Nebr. Exp. Sta. 1 : 329 (59). 18 Dec. 1889.

Webber, Rept. Nebr. Agr. 1889: 209 (69). I8go.

Tubeuf-Smith, Diseases of Plants 4 II. 1897.

Barthol. Tr. Kans. Acad. 16 : I87. June 1899.

Aecidium Brodiææ, Ell. \& Hark.

Ell. \& Hark. Bull. Cal. Acad. 1 : 28 (8) d. Feb. I884.

NoTE. Described as the æcidial stage of Uromyces Brodicec, Ell. \& Hark.

\section{Aecidium Caladii, S.}

Note. This is the æcidial stage of Uromyces Caladii, (S.) Farl. and Aecidium importatum, Henn. Verh. Bot. Ver. Brandenb. 73 : xxv. d. 1896, is probably not distinct.

S. Syn. Car. 69 (43) no. 457. d. 1822.

*Eil. N. A. F. 233 . 1879 .

Arth. Bull. lowa Agr. Coll. 1884: I65. Jan. 1885.

Ell. \& Ev. Jour. Myc. 1: 86. July 1885.

Kellerm. \& Carl. Tr. Kans. Acad. 10: 91. 1887.

Ell. in Cat. Pl. N. J. $505 . \quad 1880$.

* Pazschke, Fung. Eur. 3624.1890.

* Shear, N. Y. Fungi $129 . \quad 1893$.

*Ell. \& Ev. Fung. Columb. 58. I893.

*Ell. \& Ev. Fung. Columb. 659. 24 April 1895.

Stevens, Rept. Ohio Acad. 5: 66.1897.

Barthol. Tr. Kans. Acad. 16: 187. June I899.

Jelliffe, Flora of Long Island $36 . \quad$ I 899. Caoma aroidatum, Lk.

Lk., L. Sp. Pl. ed. Willd. $6^{2} ; 43 . d$. 1825 .

S. Syn. Am. Bor. 292 no. 2860.1834. Uredo Caladii, Spreng.

Spreng. in L. Syst. Veg. 16 ed. $4^{1}: 574 . d .1827$. Cæoma dracontinatum, S.

S. Syn. Am. Bor. 292 no. 2861. $d$. I834.

Nore. The spelling dracontionatum in the index of Syn. Am. Bor. is usually adopted. See below.

\section{Aecidium aroidatum, $\mathrm{S}$.}

S. Syn. Am. Bor. 309. I 834.

* Rav. Fung. Car. 4: 95.1855.

Sprague, Proc. Boston Soc. 5: 329. 1856.

Pk. Rept. N. Y. Mus. 23: 59. 1872.

Pk. Rept. N. Y. Mus. 24: 105. 1872 s

Frost in Cat. $\mathrm{Pl}$. Amherst $85 . \quad 1875$.

Pk. Rept. N. Y. Mus. 29 : $77 . \quad$ I878.

Cobb, List Pl. Amherst 40. 1887.

Bennett, Cat. Pl. R. 1. 89. I 888. Aecidium dracontionatum, S.

S. Syn. Am. Bor. 309. 1834.

Watt, Can. Nat. \& Geol. Il. 2 : 390. Oct. I865.

B. Grev. 3 : 59 (IOI). Dec. I 874 .

Pk. Rept. N. Y. Mus. 28 : 6r. 1876.

Pk. Rept. N. Y. Mus. 29 : 77. I878. 
Wint. \& Demet. Hedw. 24: I80 (4). Oct. I 885.

Tracy \& Earle, Bull. Miss. Exp. Sta. $34: 89$. May 1895 .

Aecidium Ari, Auct. Amer.

M. A. Curtis, Bot. N. Car. I23. 1867.

B. Grev. 3: 59. (101). Dec. 1874.

*Rav. Fung. Am. 728 . 1882 .

De Toni in Sacc. Syll. $7: 831$. d. 28 Oct. I 888.

Bennett, Cat. PI. R. 1. 89. I 888. Aecidium Dracontii, M. A. Curtis.

M. A. Curtis, Bot. N. Car. I24. 1867.

Edwards, Bull. Torr. Bot. Club 2: 39. Oct. 1871 .

Cooke, Handbook 538. $d$. . 1871 .

De Toni in Sacc. Syll. $7: 831$ d. 28 Oct. 1888.

Lung, Bull. Torr. Bot. Club 27 : 586. I4 Nov. 1900.

Pound \& Clements, Bot. Surv. Nebr. 5 : 24. 30 March 1901.

Aecidium Callirrhoes, Ell. \& Kellerm.

Ell. \& Kellerm. Jour. Myc. 2 : 4. d. Jan. I 886.

Kellerm. \& Carl. Tr. Kans. Acad. 10:91. 1887.

De Toni in Sacc. Syll. 7 : $782 . d$. 28 Oct. 1888.

* Kellerm. \& Swingle, Kansas Fungi 26. I Aug. I889.

Jennings, Bull. Tex. Exp. Sta. 9: 23. May 1890.

Webber, Tr. St. Louis Acad. 6: i4. I2 March 1892 and Contr. Bot. Dept. Univ. Nebr. 3: 14. 14 June 1892.

* Svd. Uredineen 1245 . 1898 .

*Ell. \& Ev. Fung. Columb. 1393. May 1899.

Barthol. Tr. Kans. Acad. 16: 187. June 1899.

Aecidium Calthæ, Grev.

Note. In Europe, Aecidium Caltha, Grev. grows in company with Puccinia Caltha, Lk. and is supposed to be its æcidial stage.

Pk. Rept. N. Y. Mus. 25 : 9I. Sept. 1873.

Pk. Rept. N. Y. Mus. $29: 76.1878$.

Ell. in Cat. Pl. N. J. 505. 1889.

Herbst, Fungal Fl. Lehigh Valley 175. d. 1889.

Aecidium Calystegiæ, Desm.

*Ell. N. A. F. 1011 . I883.

Hark. Bull. Cal. Acad. 2: 44I. I6 June 1887.

Arth. Science II. 10: 564 . 20 Oct. 1899.

Aecidium dubium, Clint.

Pk. Rept. N. Y. Mus. $27:$ 104. d. 1875.

Pk. Rept. N. Y. Mus. $29: 77$. 1878.

Note. This Aecidium is the æcidial stage of Puccinia Convolvuli, Cast.

Aecidium eancellatum, (Jacq.) P. in Gmel.

Note. There is good reason for supposing that few if any of the following references to Aecidium cancellatum really relate to the European species of that name which is connected with Gymnosporangium Sabine, (Dicks.) Wint. This Gymnosforangium has been recorded in America on an imported species of juniper, although it is very rare, but, supposing that the determina-

- tion of the species in that case is correct, there is no reason why the true Aecidium cancellatum might not be found also.

Muhl. Cat. Pl. Am. Sept. I ed. 104. 1813. 2 ed. 107. 1818.

S. Syn. Car. 66 (40) no. 43I. I822.

Raẹstella cancellata, Reb.

M. A. Curtis, Bot. N. Car. 123. 1867.

Cke. Proc. Portland Soc. 1 : 180. d. 1869. 
Cooke, Handbook 533. d. I871.

Cke. Grev. 5 : 1 5 I. June 1877.

Farl. Bull. Bussey Inst. 2 : 225. Jan. 1878 .

Vize, Grev. 7 : 12. Sept. 1878.

Hark. \& Moore, Cat. Pac. Fung. 27. Feb. 1880.

Farl. Anniv. Mem. Boston Soc. 27. d. 1880.

Croma Raestelites, S. Am. Bor.

S. Syn. Am. Bor. 294 no. $2900 . \quad 1834$.

\section{Aecidium eandidum, $P$.}

See Cystopus candidus, Lev.

\section{Aecidium carneum, (Bosc.) n. n.}

Note. We have placed under Aecidium carneum references to the foliicolous forms of Aecidium on Pinus palustris and doubtfully on some other species of Pinus of the southern states, for, although the original specimens of Bosc are not to be found in that part of his herbarium, now at Padua, his original figure and description make it probable that his Tubercularia carnea included what has generally been called by American mycologists, Aecidium orientale, Cke. which is an Indian species. It should be added, that although according to Cooke, Bull. Soc. Bot. Fr. 24: 315, Oct. 1877 , Aecidium orientale is a foliicolous species, the name has been extended by collectors to include in some cases corticolous forms. Inasmuch as the corticolous and foliicolous forms of Aecidium Pini have been shown by experiment to belong to several distinct species it is not improbable that the same may prove to be true in the present case. But in the absence of cultures to show the development of the Aecidia of this group, the arrangement here given must be regarded as one of convenience only. See also note under Aecidium Pini, (Willd.) P.

Tubercularia carnea, Bosc.

Bosc, Ges. Nat. Freunde Mag. 5: 88 (6). d.pl. 6, f. 13. 1811.

S. Syn. Car. 56 (30) no. 295.d. $18 z 2$.

Lk., L. Sp. Pl. ed. Willd. 62 : 100.1825.

Spreng. in L. Syst. Veg. 16 ed. $4^{1}:$ : $564 . d$. 1827.

Farlow \& Seymour, H. I. I61. June 1891.

Aecidium Pini, Rav. Fung. Car.

*Rav. Fung. Car. 1: 93.1852.

Peridermium Pini, Auct. Amer. p. p. ?

? M. A. Curtis, Bot. N. Car. I24. I867. p. p.

Peridermium oblongisporium, var. Ravenelii, Thm.

Thm. Mittheil. forstl. Versuchs. Oest. 2: $316(20) . d . \quad{ }_{1} 880$.

* Rav. Fung. Am. 27o. 1879 (sec. Thm.).

Peridermium orientale, Cke. (p. p.?).

Cke. Grev. $7: 45 . \quad$ Dec. 1878.

* Rav. Fung. Am. 270.1879.

Farl. Proc. Am. Acad. 18: 75. July 1883.

*Ell. N. A. F. 1026 b. 1883 .

Wint. \& Demet. Hedw. 24: 182 (6). Oct. I885.

*Wint. Fung. Eur. 3315. I885.

Gall. Bull. U. S. Agr. Veg. Pathol. 8: 57. 1889.

Tracy \& Earle, Bull. Miss. Exp. Sta. 34 : 91. May I 895.

Tracy \& Earle, Bull. Miss. Exp. Sta. 38: 138. May 1806.

Underw. \& Earle, Bull. Torr. Bot. Club 23: 401 . d. 25 Oct. 18,6.

Atk. Bull. Cornell. Univ. $3: 22.25$ June 1897.

Underw. \& Earle, Bull. Ala. Exp. Sta. 80: 213. 28 June 1897.

Tubeuf-Smith, Diseases of Plants 415. 1897.

Earle in Mohr, Contr. U. S. Nat. Herb. 6: 192. 3I July I90I.

Massee, Textblk. Pl. Diseases 267. 1899. 
Peridermium Ravenelii, Klebalın.

Klebahn, Ber. Deutsch. Bot. Ges. 8 : (69). 29 Dec. 1890.

Tubeuf-Smith, Diseases of Plants 416. 1897.

Diet. in Engler \& Prantl, Pflanzen-fam. 11**: 42. 1897.

Peridermium Pini, var. acicolum, E. F. 224.

*Seymour \& Earle, E. F. 224. I Feb. 1893. Aecidium Ravenelii, Diet.

Diet. in Engler \& Prantl, Pflanzen-fam. 11** : 78. 1897. PAecidium orientale, Diet.

Diet. in Engler \& Prantl, Pflanzen-fam. 11*** 78. 1897. Peridermium carneum, Seymour \& Earle.

* Seymour \& Earle, E. F. 550. 7 June 1899.

Aecidium Cassiæ, Ell. \& Kellerm.

Kellerm. \& Carl. Tr. Kans. Acad. 10: 91. 1887.

*Ell. \& Ev. N. A. F. 1825.1887.

Webber, Bull. Nebr. Exp. Sta. 1 : $330(60)$. 18 Dec. I889.

Webber, Rept. Nebr. Agr. 1889: 210 (70). 1890.

Cke. Grev. 20: 110. June 1892.

Sacc. Syll. 11 : 215. July 1895.

Barthol. Tr. Kans. Acad. 16 : $187 . \quad$ June 1899.

Aecidium Ceanothi, Ell. \& Kellerm.

Ell. \& Kellerm. Bull. Torr. Bot. Club 11 : $114 . d$. Oct. 1884.

Kellerm. Bull. Wash. Lab. 1 : 74. Jan. 1885 and Tr. Kans. Acad. 9 : 81. 1885.

*Ell. N. A. F. 1428 . I 885.

Kellerm. \& Carl. Tr. Kans. Acad. 10 : 91. I887.

Webber, Bull. Nebr. Exp. Sta. 1: $330(60)$. 18 Dec. 1889.

Webber, Rept. Nebr. Agr. 1889 : 210 (70). 1890.

Tubeuf-Smith, Diseases of Plants 4II. 1897.

*Ell. \& Ev. Fung. Columb. 1387. May 1899.

Barthol. Tr. Kans. Acad. 16: 187 . June 1899.

Aecidium Cephalanthi, Seymour.

Note. Suspected to belong to Puccinia Seymouriana, Arth. See last reference.

Seymour, Bot. Gaz. 9 : 19I. d. Dec. 1884.

Burrill, Bull. Ill. Lab. 2: 228. $d .1885$ and Rept. Ill. Ind. Univ. 12 : 143. $d$. 1885.

*Roum. Fung. Gall. 3864. 1886.

Roum. Rev. Myc. 8 : 197. I Oct. 1886.

*Wint. Fung. Eur. 3518. 1886.

Kellerm. \& Carl, Tr. Kans. Acad. 10 : 91. 1887.

*Ell. \& Ev. N. A. F. 1817.1887.

De Toni in Sacc. Syll. 7 : $797 . d$. 28 Oct. 1888.

Gall. Bull. U. S. Agr. Veg. Pathol. 8: 55.1889.

*Syd. Uredineen 496. May 1891 .

* Carleton, Ured. Amer. 20. I 894.

Tubeuf-Smith, Diseases of Plants 411 . 1897.

Barthol. Tr. Kans. Acad. 16: 187. June 1899.

Arth. Bot. Gaz. 34: 12.16 July 1902.

Aecidium Cerastil, Wint.

Wint. Jour. Myc. 1 : 1 26. d. Oct. 1885.

Wint. \& Demet. Hedw. 24: 179 (3). d. Oct. 1885.

De Toni in Sacc. Syll. 7 : 780 . d. 28 Oct. 1888.

Gall. Bull. U. S. Agr. Veg. Pathol. 8: 55.1889.

Tubeuf-Smith, Diseases of Plants 4 10. 1897.

Henn. Hedw. 37 : 272. 25 Oct. 1898. 


\section{Aecidium cerebrum, ( $\mathrm{Pk}$.) Diet.}

Note. This species is said by Cooke, Bull. Soc. Bot. Fr. 24: 315, 22 Uct. 1877, to be the same as Aecidium corticolum, (Lk.) but is generally considered distinct. The teleutosporic stage is not known. See also Aecidium piriforme, (Pk.) and Aecidium Pini, (IVilld.) P.

Diet. in Engler \& Prantl, Pflanzen-fam. 11**: 79. I897. ? Caoma pineum, S. Am. Bor, non Lk.

S. Syn. Am. Bor. 294 no. 2903. 1834 (Excl. Syn.).

Peridermium cerebrum, Pk.

Pk. Bull. Buf. Soc. 1 : 68. d. July 1873 .

Pk. Rept. N. Y. Mus. 25 : 91. d. Sept. 1873.

Frost in Cat. Pl. Amherst 84. I875.

Farl. Bull. Bussey Inst. 2: 239. Jan. 1878.

Pk. Rept. N. Y. Mus. $29: 77$. I 878.

Thm. Mittheil. forstl. Versuchs. Oest. 2: 312 (I6). d. 1880.

*Ell. N. A. F. 1022. I883.

Cobb, List Pl. Amherst 39. 1887.

De Toni in Sacc. Syll. 7:837. d. 28 Oct. I 888.

Ell. in Cat. Pl. N. J. 505. I889.

Klebahn, Ber. Deutsch. Bot. Ges. 8: (69). 29 Dec. I 890.

Tracy \& Earle, Bull. Miss. Exp. Sta. 38: I 38. May I 896.

Underw. \& Earle, Bot. Gaz. 22 : 232. 23 Sept. I896.

Underw. \& Earle, Bull. Torr. Bot. Club 23 : 403. d. 25 Oct. I896.

Atk. Bull. Cornell Univ. 3 : 22. 25 June 1897.

Underw. \& Earle, Bull. Ala. Exp. Sta. 80: 2I3. 28 June I897.

Tubeuf-Smith, Diseases of Plants 414. I 897.

Hume, Proc. Davenport Acad. $7: 247,253$. I2 May I899.

Massee, Textbk. Pl. Diseases 267. I 899.

Pound \& Clements, Bot. Surv. Nebr. 5: 24. 30 March Igor.

Earle in Mohr, Contr. U. S. Nat. Herb. 6 : I92. 3 I July I gor.

Schrenk, Yearb. U. S. Agr. 1900:200. I90I.

\section{Peridermium corticolum, Cke.}

Cke. Bull. Soc. Bot. Fr. 24: 315. d. 22 Oct. 1877.

Cke. Grev. $7: 45$. Dec. I 878 .

* Rav. Fung. Am. 269. I879.

Peridermium Pini, var. corticolum, Ell. in N. A. F.

*Ell. N. A. F. I02I, I883.

Aecidium Cestri, Mont.

Carl. in Hitchcock, Rept. Mo. Bot. Gard. 9 : I I 7. 20 April I 898.

Henn. Hedw. $37: 279$. 3I Dec. I 898.

West Indies.

Aecidium Chelonis, Gerard.

Gerard, Bull. Torr. Bot. Club 5: 40. d. Sept. 1874 and Hedw. 13 : 173. $d$. Nov, 1874 .

Pk. Rept. N. Y. Mus. $29: 77$. 1878.

*Wint. Fung. Eur. 3018 . I884.

*Ell. N. A. F. 1433 . I 885 .

Cke. Grev. 20 : I 10. June 1892.

Sacc. Syll. 11 : 219. July I 895.

* Shear, N. Y. Fungi 322. March 1896.

Aecidium Chenopodil-Pruticosi, D C.

D C. Fl. Fr. 6 : 92. d. I8I $5 . *$

Smith \& Pound, Bot. Surv. Nebr. 2: 28. I5 April I893.

*Ell. \& Ev. Fung. Columb. 1294. 1899. (Emended label.)

*Barthol., Ell. \& Ev. Fung. Columb. 150r. Dec. 1901. 
Aecidium Salicornia, F. Columb.

*Ell. \& Ev. Fung. Columb. 1294. May 1898.

Aecidium Chrysopsidis, Ell. \& Anderson.

Kelsey, Jour. Myc. 5 : 80. Jan. 1889.

Ell. \& Anderson, Bot. Gaz. 16 : 48. d. Feb. I891.

Sacc. Syll. $9: 324 . d$. 15 Sept. I89 I.

Aecidium Cimicifugæ, M. A. Curtis. See Aecidium cim icifugatum, $\mathrm{S}$.

Aecidium cimicifugatum, $\mathrm{S}$.

S. Syn. Am. Bor. 309. 1834.

Lea, Cat. Pl. Cin. 71. 1849.

James, Jour. Cin. Soc. 2: 67 (26). April 1879.

*Rav. Fung. Car. 1 : 94.1852.

Watt, Can. Nat. \& Geol. 11. 2: 390. Oct. 1865.

B. Grev. 3: 60 (102). Dec. 1874 .

*Ell. N. A. F. 227.1879.

Pk. Rept. N. Y. Mus. 33 : 28 . Oct. 1883.

*Wint. Fung. Eur. 3420. 1886.

De Toni in Sacc. Syll. $7: 777$. d. 28 Oct. 1888.

Gall. Bull. U. S. Agr. Veg. Pathol. 8: 55. 1880.

Detmers, Bull. Ohio Exp. Sta. 44: 140. Sept. 1892.

Detmers, Bull. Ohio Exp. Sta. tech. ser. I : 174. April 1893.

Kellerm. \& Werner, Geol. Ohio $7^{2}$ : 345. Jan. 1895.

Lagerh. Tromsoe Mus. Aarshefter 17: 100. $d$. 1895.

*Syd. Uredineen 1343. I899."

Kellerm. Jour. Myc. 8 : 57. d. 30 June 1902.

* Kellerm. Ohio Fungi 61 . d. July 1902.

?Freeman, Minn. Bot. Studies II. $5: 555 . d$. 20 July 1901. Caoma cimicifugatum, S.

S. Syn. Am. Bor. 293 no. $2876 . d$. 1834.

Aecidium Cimicifuga, M. A. Curtis.

M. A. Curtis, Bot. N. Car. 123. 1867.

Aecidium Actax, Detmers, p. p.

Detmers, Bull. Ohio Exp. Sta. 44: 138 . Sept. 1892.

Aecidium Cissi, Wint.

Carl. in Hitchcock, Rept. Mo. Bot. Gard. 9 : 117.20 April 1898.

Henn. Hedw. $37: 279$. 3i Dec. I 898.

West Indies.

Aecidium Clarkiæ, Diet. \& Holw.

Diet. Erythea 2: 129. d. 'I Aug. 1894.

Note. This accompanies Puccinia Clarkia, Pk. to which it probably belongs.

\section{Aecidium claytoniatum, S.}

NotE. The teleutosporic stage of this fungus was afterwards described under the name Puciinia Maria.Wilsoni, Clint. See Puccinia claytoniala, (S.) Pk.

S. Syn. Am. Bor. $309 . \quad 1834$.

Watt, Can. Nat. \& Geol. Il. 2 : 390 . Oct. 1865.

Pk. Rept. N. Y. Mus. 23 : 59. 1872.

Pk. Rept. N. Y, Mus. 24: I06. 1872.

B. Grev. 3:61 (103). Dec. I874.

Pk. Rept. N. Y. Mus. 29:76. 1878.

*Ell. N. A. F. 1017 . 1883 .

* Shear, N. Y. Fungi 131. 1803.

Kellerm. \& Werner, Geol. Ohio $7^{2}$ : 345. Jan. 1895 . 
*Ell. \& Ev. Fung. Columb. 875. April i 806.

* Seymour \& Earle, E. F. Suppl. B 27. 5 April 1898.

Syd. Mon. Ured. 1 : 56 I. I 5 May 1903.

Aecidium Claytoniz, Torr.

Torrey, Cat. Pl. N. Y. 87. 1819.

Crooma claytoniatum, $\mathrm{S}$.

S. Syn. Am. Bor. 294 no. 2892. d. I834.

Burrill, Bull. 11l. Lab. 2 : 175 . d. I 885 .

\section{Aecidium Clematidis, D C.}

Note. This is held to be the recidial stage of Puccinia Clematidis, (D C.) Lagerh. (Puccinia Agropyri, Ell. \& Ev.). See Diet. Oest. Bot. Zeit. 42 : 26I (I), Aug. I 892 and Lagerh. Tromsoe Mus. Aarshefter 17: 54. I895:

B. Grev. 3 : 60 (102). Dec. 1874 .

*Thm. Myc. Univ. I22I. 1879.

*Ell. N. A. F. $1005 . \quad$ I 883.

Farl. Appalachia 3 : 239. Jan. I884.

Arth. Bull. lowa Agr. Coll. 1884: I65. Jan. I885.

*Wint. Fung. Eur. $342 I$. I 886.

Halsted, Bull. lowa Agr. Coll. 1886 : $58 . \quad$ I 887.

De Toni in Sacc. Syll. 7: 774 . $d .28$ Oct. I888.

Kelsey, Jour. Myc. $5: 80$. June I 889.

Webber, Bull. Nebr. Exp. Sta. $1: 330$ (60). 18 Dec. I889.

Gall. Bull. U. S. Agr. Veg. Pathol. 8: 55. 1889.

Williams, Am. Nat. 24: 780 . Aug. I 890 .

Webber, Rept. Nebr. Agr. 1889: 2 IO (70). 1890.

Woods, Bot. Surv. Nebr. 2: 38. I5 April 1893.

Davis, Tr. Wis. Acad. 9: I82. 21 Aug. I893.

Carl. Tr. Kans. Acad. 13:5I. 1893.

* Ell. \& Ev. Fung. Columb. 6ra, 6rb. 1893.

* Shear, N. Y. Fungi 80. 1893.

*Pazschke, Fung. Eur. 3934. Feb. 1894.

Small \& Vail, Mem. Torr. Bot. Club 4: 200. April I894.

Pound, Bot. Surv. Nebr. 3: 9. I 8 June I 894 .

*Syd. Uredineen 899. Aug. I894.

McClatchie in Reid's Hist. Pasadena 6I5. I895.

McClatchie, Proc. S. Cal. Acad. 1 : 372. 5 June 1897.

Claassen, Rept. Ohio Acad. 5 ; $68 . \quad$ I 897.

Tubeuf-Smith, Diseases of Plants 409. 1897.

Orton, Rept. Vt. Exp. Sta. 11: 2 10. Sept. 1898 and Contr. Bot. Vt. 2 : 12. Sept. 1808.

Hume, Proc. Davenport Acad. $7: 251$. I2 May I 899.

Barthol. Tr. Kans. Acad. 16: I87. June I 899.

Nelson, Rept. Wyo. Exp. Sta. 10 : (35). I 900.

Tracy \& Earle in Greene, Pl. Baker 1: 17. 22 Feb. I90I.

-Earle in Greene, Pl. Baker 2:2. 25 March 19or.

Freeman, Minn. Bot. Studies I1. 5 : 555.20 July I 90 I.

* Griffiths, West Am. Fung. 292. 22 March 1902.

Ricker, Univ. of Maine Studies 3: 50. 30 April 1902. Aecidium Clematitis, S.

S. Syn. Car. 68 (42) no. 447. d. 1822.

Lk., L. Sp. Pl. ed Willd. $6^{2}: 54$. $d .1825$.

Spreng. in L. Syst. Veg. 16 ed. $41^{1}$ : $571 . d .1827$.

M. A. Curtis, Bot. N. Car. 124. 1867.

Pk. Rept. N. Y. Mus. 23:6I. 1872.

Pk. Rept. N. Y. Mus. 24; I06. I872.

Pk. Rept. N. Y. Mus. 26 :91. April I 874. 
Frost in Cat. Pl. Amherst 85. 1875.

Cobb, List Pl. Amherst 40. 1887 .

Creoma clematitatum, S.

S. Syn. Am. Bor. 293 no. 2874.1834. Aecidium clematitatum, S.

S. Syn. Am. Bor. 309. 1834.

Pk. Rept. N. Y. Mus. $29: 76 . \quad$ เ 878.

Aecidium Ranunculacearum, var. Clematidis, Cke.

Cooke, Handbook 539. $d$. $187 \mathrm{I}$.

Aecidium Cleomis, Ell. \& Anderson.

Anderson, Jour. Myc. $5 ; 82$. June 1889 .

Ell. \& Anderson, Bot. Gaz. $16: 48$. d. Feb. ${ }^{1} 891$.

Sacc. Syll. $9: 325 . d$. 15 Sept. 1891.

Aecidium Clibadii, Syd.

Syd. Ann. Myc. 1: 333. d. July 1903.

Cent. Amer.

Aecidium Collinsiæ, Ell. \& Ev.

Ell. \& Ev. Bull. Wash. Lab. 1 : 4. d. Sept. 1884.

De Toni in Sacc. Syll. $7: 813 . d$. 28 Oct. 1888.

Henn. Hedw. $37: 272$. 25 Oct. 1898 .

Note. Hennings describes an æcidium as part of his plant and Syd. Mon. Ured. I : 246, 15 Nov. 1902, assumes the present Aecidium to be the same.

Aecidium Coloradense, Diet.

Diet. in Engler \& Prantl, Pflanzen-fam. 1 1*** 78. d. 1897.

Sacc. \& Syd. Syll. 14: 388. d. 20 Aug. I899.

Peridermium abietinum, f. Engelmanni, Ell. \& Ev.

*Ell. \& Ev. N. A. F. 2223. Feb. 1889.

Aecidium abietinum, f. Engelmanni, Ell. \& Ev.

*Ell. \& Ev. Fung. Columb. 876. April 1896.

Aecidium Columbiense, Ell. \& Ev.

Ell. \& Ev. Erythea 1 : 206. d. 20 Oct. 1893.

Sacc. Syll. $11: 217$. d. July 1895 .

Aecidium Compositarum, Auct. Amer. p. p.

Note. Very little is known in regard to the development of the Aecidia on Composita in America, but some belong to autœcious and others to hetercecious species. The number of references is so great and in some instances they are so uncertain that for practical use by mycologists it has seemed best under Aecidium Compositarum to group the Aecidia on all the genera of Composita.

Where in the original references no particular host-genus is mentioned, the references are grouped chronologically with the heading of Miscellanea. Where definite genera are mentioned, the hosts are arranged alphabetically, each with its references; e.g., all references to Aecidia on Ambrosia are placed under Aecidium Compositarum, var. Ambrosia.

For information in regard to European Aecidia on Composila see Jacky, Die Compositen-bewohnenden Puccineen von Typus der Fuccinia Hieracii und deren Specialiserung, Inaugural Diss. Bern 1889 and Zeit. Pflanzenkr. 9: 193, 20 Oct. 1899; Klebahn, Bot. Zeit. 58: 133, I May 1900, and Magnus, Hedw. Beibl. 39: (147), Oct. 1900. Also see Magnus, Ber. Deutsch Bot. Ges. II : 453, 29 Aug. 1893, and Lindroth, Ueber einige Compositenbewohnende Puccinien, Acta Soc. Fauna et Fl. Fennica $20^{\circ}: 1901$. 


\section{Miscellanea.}

Lea, Cat. Pl. Cin. 71. 1849.

James, Jour. Cin. Soc. 2 : 67 (26). April 1879.

Sprague, Proc. Boston Soc. 5 : 329. 1856.

* Rav. Fung. Car. 5 : $97 . \quad$ I860.

Watt, Can. Nat. \& Geol. II. 2 : 390. Oct. 1865.

M. A. Curtis, Bot. N. Car. 123. 1867.

Pk. Rept. N. Y. Mus. 22 : 93.1869.

Cooke, Handbook 542. $d$. I87I.

Pk. Rept. N. Y. Mus. 24: $:$ 107. I 872.

Frost in Cat. Pl. Amherst 85. 1875.

Pk. Rept. N. Y. Mus. 29:77. 1878.

Bundy, Geol. Wis. $1873-791 ; 400 . \quad 1883$.

Brendel, Fl. Peoriana 2 ed. $69 . \quad$ I887.

Cobb, List Pl. Amherst 40. 1887.

Halsted, Bull. lowa Agr. Coll. 1886: $58 . \quad$ I887.

Langlois, Cat. Pl. La. 27. 1887.

Bennett, Cat. Pl. R. I. 89. I888.

Ell. in Cat. Pl. N. J. 505. I889.

Tracy \& Earle, Bull. Miss. Exp. Sta. 34: 89. May 1895 .

Aecidium Compositarum, var. Ambrosiæ, Burrill.

Burrill, Bull. Ill. Lab. 2:23ı. d. I885 and Rept. Ill. Ind. Univ. 12 : I44. $d$. 1885 .

De Toni in Sacc. Syll. $7: 798$. d. 28 Oct. 1888.

Cockerell, Zoe $4: 283$. Oct. 1893 .

?Aecidium Compositarum, Auct. Amer. p. p.

Claassen, Rept. Ohio Acad. 5: 68. 1897.

Aecidium Compositarum, var. Artemisiæ, C. \& E.

C. \& E. Grev. $6: 86$. March 1878 .

Aecidium Tanaceti, Cockerell.

Cockerell, Jour. Myc. 5:85. June 1889 .

Aecidium Compositarum, var. Artemisiarum, Ell. \& Ev.

*Ell. \& Ev. N. A. F. 2219. I889.

Aecidium Compositarum, Auct. Amer. p. p.

Patterson, Bull. U. S. Agr. Pl. Industry 8: 8. 3 Feb. I902.

Aecidium Compositarum, var. Asteris, Wint.

See Aecidium Asterum, S.

Aecidium on Baccharis.

See Aecidium fragile, Diet. \& Holw.

Aecidium on Bahia.

See Aecidium Bahize, B. \& C.

Aecidium Compositarum, var. Bidentis, Burrill.

Burrill, Bull. Ill. Lab. 2: 232. d. I885 and Rept. 1ll. Ind. Univ. 12 : I45. $d$. 1885 .

De Toni in Sacc. Syll. $7: 799 . d .28$ Oct. 1888.

PAecidium Compositarum, Auct. Amer. p. p.

Davis, Tr. Wis. Acad. 9 : 16I. 21 Aug. I893.

Davis, Tr. Wis. Acad. 14: 88. Sept. I903.

Aecidium on Bigelovia.

See Aecidium Bigelovia, $\mathrm{Pk}$.

Aecidium on' Boltonia.

See Aecidium Boltoniz, Arth.

Aecidium on Borrichia.

See Aecidium Borrichize, Syd. 
Aecidium on Brickellia.

See Aecidium Guadalajara, Syd.

Aecidium Compositarum, Auct. Amer. p. p. On Cacalia.

*Ell. \& Ev. N. A. F. 1811 . 1887.

Kellerm. \& Carl. Tr. Kans. Acad. 10: 91. 1887.

Davis, Tr. Wis. Acad. 11: 167. Aug. 1897.

Barthol. Tr. Kans. Acad. 16: i87. June 1899. ?Aecidium, sp. indet.

Davis, Bot. Gaz. 19 ! 415. 17 Oct. I894.

Aecidium on Chrysopsis.

See Aecidium Chrysopsidis, Ell. \& Anderson.

Aecidium on Clibadium.

See Aecidium Clibadii, Syd.

Aecidium on Crepis.

See Aecidium crepidicolum, Ell. \& Ev.

Aecidium Compositarum, var. Erigerontis, Wint. See Aecidium erigeronatum, S.

Aecidium Compositarum, var. Eupatoriæ, (S.) B.

Note. For the Aecidium on Eupatorium ageratoides, see Aecidium tenue, S. See also Aecidium roseum, Diet. \& Holw.

B. Grev. 3 : 60 (102). Dec. 1874.

Burrill, Bull. III. Lab. 2: $231 . d$. 1885 and Rept. III. Ind. Univ. 12 : I44. $d . \quad 1885$.

*Ell. N. A. F. 1419.1885.

De Toni in Sacc. Syll. 7: 798. d. 28 Oct. 1888.

Webber, Bull. Nebr. Exp. Sta. 1: $330(60)$. 18 Dec. 1889.

Webber, Rept. Nebr. Agr. 1889: 210 (70). 1890.

Detmers, Bull. Ohio Exp. Sta. 44: 140. Sept. 1892.

* Shear, N. Y. Fungi 127.1893.

Freeman, Minn. Bot. Studies 11. 5: 557. 20 July 1901. Caoma compositarum, var. Eupatoria, S.

S. Syn. Am. Bor. 292 no. 2867. I834. PAecidium Compositarum, Auct. Amer. p. p.

Trel. Tr. Wis. Acad. $6: 137$ (33). Nov. 1884.

Davis, Tr. Wis. Acad. 9 : 16I. 21 Aug. 1893.

Kellerm. \& We ner, Geol. Ohio $7^{2}: 346$. Jan. 1895.

Underw. \& Earle, Bull. Ala. Exp. Sta. 80: 208. 28 June 1897.

Underw. Proc. Ind. Acad. 1894: 151. June 1897.

Snyder, Proc. Ind. Acad. 1896: 217.1897.

Barthol. Tr. Kans. Acad. 16: 187. June 1899.

Earle in Mohr, Contr. U. S. Nat. Herb. 6 : 192. 3I July 1901.

Aecidium on Euthamia.

See under Aecidium Asterum, S. sec. Freeman.

Aecidium on Gnaphalium.

See Aecidium gnaphaliatum, S.

Aecidium on Grindelia.

See Aecidium Grindelia, Syd.

Aecidium Compositarum, Auct. Amer. p. p. On Helenium.

* Seymour \& Earle E. F. Suppl. B 28. 5 April 1898. ?Aecidium Prenanthis, Auct. Amer. p.p.

Tracy \& Earle in Greene, PI. Baker. 1: 19. 22 Feb. 1901?

Earle in Greene, Pl. Baker 2:2. 25 March 1901.

Aecidium on Helianthella.

See Aecidium Helianthella, Arth. 
Aecidium Compositarum, var. Helianthi, Burrill.

Note. See also Aecidium Helianthi-mollis, S. and Aecidium trachelifoliatum, S. See Burrill, 1885 , Barthol. $\mathrm{r} 889$, for notes on Aecidia of Helianthus.

Burrill, Bull. 1ll. Lab. 2 : 232. d. $\quad$ I885 and Rept. Ill. Univ. 12 : 145. d. $\mathbf{1} 885$.

De Toni in Sacc. Syll. 7 : 799. d. 28 Oct. 1888.

Webber, Rept.' Nebr. Agr. 1889: 2 I0 (70). I 890.

Williams, Bull. S. Dak. Exp. Sta. 29 : 49. Dec. I891.

Smith \& Pound, Bot. Surv. Nebr. 2: 28. I5 April 1893.

Detmers, Bull. Ohio Exp. Sta. tech. ser. I : 174. April 1893.

Cockerell, Zoe 4: 282 . Oct. I 893 .

*Syd. Uredineen 1247 . I 898 .

Hume, Proc. Davenport Acad. 7 : 25I. 12 May I 899.

*Ell. \& Ev. Fung. Columb. 1392. May I 899.

Barthol. Tr. Kans. Acad. 16: I 88. June I 899.

*Syd. Uredineen 1394. I899.

Freeman, Minn. Bot. Studies 11. 5: 557. 20 July I90I. Aecidium Helianthi, Pk.

Pk. Rept. N. Y. Mus. 24: 107. I872. ?Aecidium Compositarum, Auct. Amer. p. p.

Arth. Bull. lowa Agr. Coll. 1884: I65. Jan. 1885.

Gall. Bull. U. S. Agr. Veg. Pathol. 8: 55.1889.

Kellerm. \& Werner, Geol. Ohio 72 : 346. Jan. I895.

Underw. \& Earle, Bull. Ala. Exp. Sta. 80: 208. 28 June 1897.

Claassen, Rept. Ohio Acad. 5 : 68. I 897.

Barthol. Tr. Kans. Acad. 16 : 187. June 1899.

Nelson. Rept. Wyo. Exp. Sta. 10 : (35). 1900.

Earle in Molir, Contr. U. S. Nat. Herb. 6 : 192. 31 July Igor.

Patterson, Bull. U. S. Agr. Pl. Industry $8: 8.3$ Feb. I902.

Aecidium Compositarum, var. Heliopsidis, Wint.

Wint. \& Demet. Hedw. 24: 179 (3). Oct. 1885.

PAecidium Compositarum, Auct. Amer. p. p.

Claassen, Rept. Ohio Acad. 5 : $68 . \quad$ I897.

Aecidium on Hieracium.

See Aecidium Columbiense, Ell. \& Ev.

See Aecidium hieraciatum, S.

Aecidium on Iva.

See Aecidium intermixtum, Pk.

Aecidium on Krigia.

See Aecidium Dandelionis, S.

Aecidium Compositarum, var. Lactucæ, Burrill.

Burrill, Bull. III. Lab. 2: 232. d. 1885 and Rept. 11l. Ind. Univ. 12 : I45. $d$. I885.

De Toni in Sacc. Syll. 7 : 799. d. 28 Oct. I888.

Webber, Rept. Nebr. Agr. 1889: 210 (70). I890.

* Seymour \& Earle, E. F. 484. I 5 Oct. I897.

Hume, Proc. Davenport Acad. $7: 252 . \quad$ I2 May 1899.

Tracy \& Earle in Greene, PI. Baker. 1 : 17. 22 Feb. I90I.

Freeman, Minn. Bot. Studies 11. $5: 557.20$ July 1901.

Lindr. Acta Soc. Fauna et Fl. Fennica $20^{9}$ : 2I. IgOI.

* Barthol., Ell. \& Ev. Fung. Columb. 1601. Oct. 1902.

Bubak, Centralbl. Bact. 2 Abt. 9 : 924 . 30 Dec. I902.

PAecidium Compositarum, Auct. Amer. p. p.

Trel. Tr. Wis. Acad. $6: 137$ (33). Nov. I884.

Arth. Bull. lowa Agr. Coll. 1884 : I65. Jan, 1885. 
Ell. \& Ev. Jour. Myc. 1: 86 . July 1885.

Pammel, Agr. Sci. 7 : 26. Jan. 1893.

Underw. Proc. Ind. Acad. 1894: 151. Oct. 1895.

Barthol. Tr. Kans. Acad. $16: 187$. June 1899.

Aecidium on Lactuca.

See also Aecidium hemispharicum, Pk.

See also Aecidium Prenanthis, Auct. Amer. p. p.

Aecidium Compositarum, var. Liatridis, Webber.

See Aecidium Liatridis, Ell. \& Anderson.

Aecidium Compositarum, var. 'Lygodesmiæ, Webber.

See Aecidium Lygodesmize, Shear.

Aecidium Compositarum, Auct. Amer. p. p. On Mikania.

B. Grev. 3:60 (102). Dec. 1874.

Aecidium on Montanoa.

See Aecidium Montanoz, Diet. \& Holw.

Aecidium Compositarum, Auct. Amer. p. p. On Polymnia.

Davis, Tr. Wis. Acad. 9: 161. 21 Aug. 1893.

Aecidium Compositarum, var. Prenanthis, Auct. Amer.

Note. It is uncertain to what species the following references to æcidia on Prenanthes relate, but apparently some are the recidia of Puccinia Prenanthis, (P.) as limited by Lindroth, $q . v$. As far as we know the name Aecidium Prenanthis on Prenanthes does not occur in American literature except incidentally in Pk. Rept. N. Y. Mus. 50: 115. March 1898. See Aecidium Prenanthis, Auct. Amer. p. p.

Freeman, Minn. Bot. Studies II. 5: 556. 20 July I9or.

PAecidium Compositarum, Auct. Amer. p. p.

Trel. Tr. Wis. Acad. 6: 137 (33). Nov. 1884.

Arth. Bull. lowa Agr. Coll. 1884: 165. Jan. 1885.

Orton, Rept. Vt. Exp. Sta. 11 : 210. Sept. 1898 and Contr. Bot. Vt. 2 : 12. Sept. 1898 .

Patterson, Bull. U. S. Agr. PI. Industry 8: 8. 3 Feb. I902.

Aecidium Compositarum, var. Rudbeckiæ, Pk. in litt.

*Ell. N. A. F. ror8a. 1883 .

PAecidium Compositarum, Auct. Amer. p. p.

Arth. Bull. lowa Agr. Coll. 1884: 165. Jan. 1885.

* Syd. Uredineen 97.1889.

Davis, Tr. Wis. Acad. 9: 161. 21 Aug. 1893.

Pound, Bot. Surv. Nebr. $3: 9 . \quad 18$ June 1894 .

Hume, Proc. Davenport Acad. 7: 252. 12 May I899.

Aecidium Compositarum, var. Senecionis, Auct. Amer.

Note. The decilia on Senecio in references here given probably belong to some heterøcious species of Puccinia but their development has not been studied. See also Aecidium sclerothecioides, Ell. \& Ev. For further information see Diet. Hedw. 30: 297. Dec. 1891 and Klebahn, Rostpilze 308. 1904.

Webber, Bull. Nebr. Exp. Sta. 1: 330 (60). 18 Dec. 1880.

Webber, Rept. Nebr. Agr. 1889: 210 (70). 1890. ?Aecidium Compositarum, Auct. Ainer. p. p.

Arth. Bull. lowa Agr. Coll. 1884: 165. Jan. I885.

Davis, Tr. Wis. Acad. 11: 167. Aug. 1897. ?Aecidium Senecionis, Auct. Amer.

*Ell. N. A. F. 1425.1885.

Cockerell, Zoe 4: 283 . Oct. 1893.

Pk. Rept. N. Y. Mus. 50 : I1 5. 28 March 1898. 
PAecidium Jacobere, Auct. Amer.

Freeman, Minn. Bot. Studies 11. 5: 556. 20 July I901.

Aecidium Compositarum, var. Silphii, Burrill.

See Aecidium Silphii, Syd.

Aecidium Compositarum, var. Solidaginis, Ell.

See Aeçidium Solidaginis, S.

Aecidium on Solidago.

See Aecidium recedens, Arth.

See Aecidium solidaginicolum, Ell. \& Ev.

Aecidium on Taraxacum.

See Aecidium Taraxaci, Schm. \& Kze.

Aecidium Compositarum, Auct. Amer. p. p. On Troximon.

Anderson, Jour. Myc. $5: 82$. June I889.

Nelson, Rept. Wyo. Exp. Sta. 10: (35). Igoo.

Aecidium Compositarum, Auct. Amer. p. p. On Vernonia.

Trel. Tr. Wis. Acad. 6 : 137 (33). Nov. I884.

Aecidium on Wedelia.

See Aecidium Wedelia, Earle.

Aecidium Compositarum, var. Xanthii, Ell.

Note. Thought by some botanists to be the aecidial stage of Puccinia Xanthii, S. with which it has been found.

*Ell. N. A. F. 1018 b. 1883 .

Burrill, Bull. Ill. Lab. 2: 23ı. d. 1885 and Rept. 1ll. Ind. Univ. 12 : 145. $d$. 1885 .

De Toni in Sacc. Syll. 7 : 799. d. 28 Oct. 1888.

Pound \& Clements, Bot. Surv. Nebr. 5 : 24. 30 March I9oI.

PAecidium Compositarum, Auct. Amer. p. p.

Davis, Tr. Wis. Acad. 9 : I6I. 2I Aug. I893.

Aecidium conorum-Piceæ, Reess.

Nore. Peridermium conorum-Picea, Pk. in litt. was described by Thuemen, Mittheil. forstl. Versuchs. Oest. 2: 314 (18), 1880 as Peridermium Engelmanni, Thm., q. v., the host being Picea Engelmanni. Farlow, Bull. Bussey Inst. 2 : 239,1878 , given below is probably the same. In Farlow, Bull. Bussey Inst. I : 433, March 1876, the host was Picea excelsa. For further information see Klebahn, Rostpilze 391. 1904.

Farl. Bull. Bussey Inst. 1: 433. March 1876 .

?Farl. Bull. Bussey Inst. 2: 239. Jan. 1878 .

Peridermium conorum, 'Thm.

Thm. Mittheil. forstl. Versuchs. Oest. 2 : 313 (17). d. 1880.

*Seymour \& Earle, E. F. 220. I Feb. I893.

Aecidium Convallariæ, Schum.

NoTE. According to Soppitt, Jour. of Bot. 28: 213, July 1890, this is the xcidial stage of Puccinia Digraphidis, Soppitt. For further information see Klebahn, Rostpilze 265, 267. I904.

Pk. Rept. N. Y. Mus. $25: 92$. Sept. 1873.

Pk. Rept. N. Y. Mus. 29: 77 . 1878.

*Ell. N. A. F. 229. I 879 .

*Wint. Fung. Eur. 3026. I 884.

Trel. Tr. Wis. Acad. 6 : 136 (32). Nov. 1884.

Arth. Bull. lowa Agr. Coll. 1884: 165. Jan. 1885.

Burrill, Bull. 1ll. Lab. 2: 239. d. 1885 and Rept. Ill. Ind. Univ. 12 : 149. d. 1885 . 
*Ell. N. A. F. 1421, I885.

Kellerm. \& Carl. Tr. Kans. Acad. 10 : 9r. 1887.

De Toni in Sacc. Syll. $7: 828$. d. 28 Oct. 1888.

Webber, Bull. Nebr. Exp. Sta. 1 : $330(60)$. 18 Dec. I889.

Gall. Bull. U. S. Agr. Veg. Pathol. 8: $55 . \quad$ I889.

Webber, Rept. Nebr. Agr. 1889: 210 (70). I890.

Davis, Tr. Wis. Acad. 9: 161. 21 Aug. I 893.

Tubeuf-Smith, Diseases of Plants 410. 1897.

Herbst, Fungal FI. Lehigh Valley 176. d. 1809.

Freeman, Minn. Bot. Studies II. 5 : 554. 20 July I 901.

Cxoma convallariatum, Lk.

S. Syn. Am. Bor. 292 no. 2857 . I834.

Aeeidium Convallariæ, Schum., var. Lilii, Farl.

Note. This variety is not connected with Puccinia Digraphidis, Soppitt, but may possibly be the aecidium of Uromyces Holwayi, Lagerh.

*Ell. N. A. F. $228 . \quad 1879$.

Farl. Proc. Am. Acad. 18 : 74. July 1883.

Aecidium convolvulatum, S.

See Cystopus Ipomoze-pandurana, Farl.

Aecidium Convolvuli, M. A. Curtis.

See Cystopus Ipomoe-pandurana, Farl.

Aecidium Cordiæ, Henn.

Magn. in Bres. Henn. \& Magn. in Engler, Bot. Jahrb. 17 : 49I. $d$. 1893.

West Indies.

Aecidium cornutum, P. in Gmel.

NoTE. Since what is believed to be the true Gymnosporangium conicum, D C. occurs in New England and northward on Funiperus communis, the citations of Aecidium cornutum on Pirus Americana in those regions prohably refer to the true Aecidium cornutum of Europe. Citations of Aecidium cornutum in more southern regions and on Pirus arbutifolia and Amelanchier may relate to Ciymnosporangium globosum which has also been cultivated on Pirus Americuna. See also Aecidium globosum, (Farl.) and Aecidium Nidus-avis, (Thax.). R. T.

Roestelia cornuta, Fr.

Pk. Rept. N. Y. Mus. 24:93. $\quad$ I872.

Pk. Rept. N. Y. Mus. 29: 76. 1878.

Farl. Bull. Bussey Inst. 2 : 225. Jan. 1878.

Farl. Anniv. Mem. Boston Suc. 28. I 880.

*EII. N. A. F. 1089 . I883.

Farl. Proc. Am. Acad. 18: 75 . July 1883.

Seymour, Tr. Am. Hort. Soc. 4 : I55 (4). 7 April 1887.

Thax. Bot. Gaz. 14: I69. July I889.

Ell. in Cat. Pl. N. J. 504. 1889.

Tubeuf, Centralbl. Bact. 9 : 93 . 2 Feb. I891.

Thax. Bull. Conn. Exp. Sta. I07: 4. 15 April I891.

* Seymour \& Earle, E. F. 248 x. I Feb. I893.

Ricker, Univ. of Maine Studies 3: 54. 30 April 1902.

On Pirus arbutifolia.

Thax. Bot. Gaz. 14: : 69 . July 1889. Host unknown.

Muhl. Cat. Pl. Am. Sept. I ed. 104. 1813. 2 ed. 107. 1818 (as Aecidium).

Bundy, Geol. Wis. 1873-79, $1: 400 . \quad 1883$.

PRoestelia lacerata, Auct. Amer. p. p. On Pirus Americana.

Ricker. Univ. of Maine Studies 3: 54. 30 April 1902. 
Aecidium eornutum, var. Amelanehieris, (Auct. Amer.).

N.B. . References to Restelia cornuta on Amelanchier alnifolia are to be found under Aecidium Nelsoni, (Arth.)

Aecidium cornutum, Auct. Amer. p. p.

Pk. Rept. N. Y. Mus. $24: 93$. 1872.

Pk. Rept. N. Y. Mus. $29: 76.1878$.

Farl. Anniv. Mem. Boston Soc. 28. 1880.

Seymour, Tr. Am. Hort. Soc. 4 : 155 (4). 7 April 1887.

Thax. Bot. Gaz. 14: 169. July 1889.

Roestelia Amelanchieris, Auct. Amer.

*Ell. \& Ev. N. A. F. 2715. May 1892.

Roestelia cornuta, var. Amelanchieris, Seymour \& Earle.

* Seymour \& Earle, E. F. 248. I Feb. I 893.

Orton, Rept. Vt. Exp. Sta. 11 : 212. Sept. 1898 and Contr. Bot. Vt. 2: 14. Sept. 1898.

Aecidium corticolum, Auct. Amer. p. p.

See Aecidium Pini, (Willd.) P.

Aecidium Corydalis, (Ell. \& Kellerm.) Webber.

Webber, Rept. Nebr. Agr. 1889 : 210 (70). 1890.

Aecidium Dicentra, var. Corydalis, Ell. \& Kellerm.

*Ell. N. A. F. 1435 . 1885.

Aecidium Fumariacearum, Kellerm. \& Swingle.

Kellerm. \& Swingle, Jour. Myc. 4: 95. d. Sept. 1888.

Sacc. Syll. 9: 322. $d$. 15 Sept. I891.

Freeman, Minn. Bot. Studies II. 5: 555. 20 July I90I.

Aecidium erassum, $P$.

See Aecidium Rhamni, P. in Gmel.

Aecidium Cratægi, var. Oxyacanthæ, S.

See Aecidium globosum, (Farl.).

Aecidium erepidicolum, Ell. \& Gall.

Note. This may belong to Puccinia Crepidis-acuminate, Syd. An Aecidium on Crepis is said to belong to a Puccinia on Carex. See Klebahn, Rostpilze 303. 1904.

Ell. \& Gall. Jour. Myc. 6 : 31. d. March 1890.

Sacc. Syll. $9: 324 . d$. 15 Sept. 1891.

Magn. Hedw. Beibl. 40: (32). 20 April I9or.

Aecidium Cressæ, D C.

Note. This. Aecidium accompanies what is supposed to be Puccinia Cresse, (D C.) Lagerh. in Southern California; but compare also Puccinia cretica, Holw.

B. Grev. $3: 60$ (102). Dec. 1874 .

Diet. in Engler \& Prantl. Pflanzenfam. 11**: $34 . \quad$ I 897.

Patterson, Bull. U. S. Agr. Pl. Industry 8: 8. 3 Feb. 1902.

Aecidium Crotonopsidis, Burrill.

Burrill, Bot. Gaz. 9 : 190. d. Dec. 1884.

Burrill. Bull. III. Lab. 2 237. d. 1885 and Rept. Ill. Ind. Univ. 12 : 148. $d$. 1885 .

*Ell. \& Ev. N. A. F. 1824. I 887.

De Toni in Sacc. Syll. $7: 824$. d. 28 Oct. 1888. Aecidium splendens, Wint.

Wint. \& Demet. Hedw. 24: 18 r (5). Oct. 1885.

*Wint. Fung. Eur. 3224. 1885 and Hedw. 24: 256. Dec. I885.

* Roum. Fung. Gall. 3860.1886.

Roum. Rev. Myc. 8: 196. I Oct."i 886. 
De Toni in Sacc. Syll. 7 : 784. d. 28 Oct. 1888.

Gall. Bull. U. S. Agr. Veg. Pathol. 8: 56.1880.

Tubeuf-Smith, Diseases of Plants 410 . I 897.

Aecidium eylindricum, Ell. \& Ev.

Ell. \& Ev. Bull. Torr. Bot. Club 22: 6r. d. 26 Feb. I805.

Sacc. Hedw. 35 : xviii. 25 Feb. 1896. [Reportorium No. 7.]

Smyth, Tr. Kans. Acad. 15: 70. March i 898.

Sacc. \& Syd. Syll. 14:382. d. 20 Aug. I899.

Aecidium Cyparissiæ, Auct. Amer.

See Aecidium Euphorbia, S.

Aecidium Dakotensis, Griffiths.

* Griffiths, West Am. Fung. 389. 21 May 1902.

Griffiths, Bull. Torr. Bot. Club 29 : 300. d. 26 May I902.

Aecidium Dalex, Kellerm. \& Swingle.

Kellerm. \& Swingle, Jour. Myc. 5: 13. d. March I889.

Webber, Bull. Nebr. Exp. Sta. 1 : 33 I (6I). 18 Dec. I889.

Webber, Rept. Nebr. Agr. 1889 : 2 IO (70). I8go.

Sacc. Syll. 9: 320 . d. I 5 Sept. I80 I.

Barthol., Tr. Kans. Acad. 16 : I88. June I 899.

* Syd. Uredineen 1448 . June 1900 .

* Shear, Ell. \& Ev. Fung. Columb. 1473. May I9or.

Aecidium Dandelionis, S. On Krigia.

S. Syn. Car. 66 (40) no. 434. d. I 822 .

Caoma compositatum, var. Prenanthis, S.

S. Syn. Am. Bor. 292 no. 2867. I 834 . Uredo Compositarum, Spreng.

Spreng. in L. Syst. Veg. 16 ed. 41 : 572. d. 1827.

?Aecidium Compositarum, Auct. Amer. p. p.

Trel. Tr. Wis. Acad. $6: 137$ (33). 'Nov. I884.

Freeman, Minn. Bot. Studies II. $5: 556.20$ July I90I.

Aecidium decolorans, (Pk.).

Note. The development of this species is unknown and its relation to other acicolous Aecidia on Abies is uncertain. See note under Aecidium abielinum, A. \& S.

Peridermium decolorans, $\mathrm{Pk}$.

Pk. Rept. N. Y. Mus. 27 : I04. d. pl. I, f. I9-2I. 1875.

Pk. Tr. Albany Inst. $8: 292$ (12). 1876.

Pk. Rept. N. Y. Mus. 29: $77 . \quad 1878$.

Pk. Pl. Summit Mt. Marcy 405, 409. I 880.

Thm. Mitth. forstl. Versuchs. Oest. 2 : 32I (25). I880.

Pk. Rept. N. Y. Mus. 33: 38 . Oct. 1883 .

De Toni in Sacc. Syll. 7 : 837. 28 Oct. I 888.

Pk. Rept. N. Y. Mus. 44: I is (7). Dec. I89 I.

* Seymour \& Earle, E. F. 22I. I Feb. I803.

Orton, Rept. Vt. Exp. Sta. 11 : 212. Sept. I 898 and Contr. Bot. Vt $2:$ I4. Sept. 1898.

Pk. Bull. N. Y. Mus. 6: 227. July I899.

Pk. Rept. N. Y. Mus. $52: 665,672$. Dec. I899. [Bull. 5 : no. 25.]

Davis, Tr. Wis. Acad. 14: 103. Sept. I903.

Peridermium abietinum, var. decolorans, Thm.

Thm. Mittheil. forstl. Versuchs. Oest. 2: 32 I (25). d. I 880.

*Ell. N. A. F. 1024. I883.

Arth \& Holw. Bull. Minn. Surv. 3 : 31. I Oct. 1887.

Freeman, Minn. Bot. Studies II. 5: 559. 20 July I 90 I. Chrysomyxa Ledi, (Aecidium) Wint. Fung. Eur.

*Wint. Fung. Eur. 3607. 1890. 
Aecidium deformans, Mayr.

Note. This species on Pinus mitis was named and figured but not described by the author. The Japanese species, Aecidium giganteum, Mayr, nearly related to the above, is said by Shirai, Tokio Bot. Mag. 13: (I-6) pl. 4, 5, 1899, and Zeit. Pflanzenkr. 10: 218,8 Sept. 1900, to belong to Cronartium Quercuum, (B.) Miyabe, a species found also in North America.

Mayr, Waldungen von Nordamer. I19, 434. pl. 10. I890.

Peridermium deformans, Tubeuf-Smith.

Tubeuf-Smith, Diseases of Plants 416. I897.

Aecidium Delphinil, Barthol.

Note. See also Aecidium Ranunculacearum, Auct. Amer. p. p. on Delphinium. See also Ell. \& Ev. N. A. F. 3480. 1896.

Ell. \& Barthol. Jour. Myc. 8 : 173. d. 20 Dec. 1902.

Aecidium Dicentræ, Trel.

Trel. Tr. Wis. Acad. 6: 136 (32). d. Nov. 1884 and Jour. Myc. 1 : I5. d. Jan. I885.

Kellerm. Bull. Wash. Lab. 1 : 75. Jan. 1885 and Tr. Kans. Acad. 9 : 8 r. 1885 .

Burrill, Bull. Ill. Lab. 2 : 223. d. $\quad$ I 885 and Rept. Ill. Ind. Univ. 12 : I40. $d$. 1885 .

Kellerm. \& Carl, Tr. Kans. Acad. 10 : 91. 1887.

De Toni in Sacc. Syll. $7: 777 . d .28$ Oct. 1888.

*Kellerm. \& Swingle, Kansas Fungi 2. 1 May I889.

Webber, Bull. Nebr. Exp. Sta. 1 : 33I (6I). 18 Dec. I889.

*Pazschke, Fung. Eur. $3634 . \quad$ I 890.

*Syd. Uredineen 497. May I891.

Detmers, Bull. Ohio Exp. Sta. tech. ser. 1 : I74. April 1893.

Underw. Proc. Ind. Acad. 1893: 49. Aug. I894.

Kellerm. \& Werner, Geol. Ohio $7^{2}: 346$. Jan. I895.

Tubeuf-Smith, Diseases of Plants 4 Io. I 897.

Barthol., Tr. Kans. Acad. 16 : I 88. July I 899.

*Pazschke, Fung. Eur. 4335. June 1903.

Aecidium Dicentrze, Burrill.

Burrill, Bot. Gaz. 9 : 189. d. Dec. 1884.

Aecidium Dicentræ, var. Corydalis, Ell. \& Kellerm. See Aecidium Corydalis, Webber.

Aecidium Diodiæ, Burrill.

Burrill, Bot. Gaz. 9 : 189. d. Dec. I884.

Burrill, Buli. lll. Lab. 2 : 228. d. I885 and Rept. Ill. Ind. Univ. 12 : I43. $d$. I885.

De Toni in Sacc. Syll. 7 : 795. d. Oct. 28, 1888.

Tracý \& Earle, Bull. Miss. Exp. Sta. 34: 89. May I 895.

* Barthol., Ell. \& Ev. Fung. Columb. 1704. Feb. I903.

Aecidium Drabæ, Tracy \& Gall.

Note. See also Aecidium monoicum, Pk.

Tracy \& Gall. Jour. Myc. 4 : 21. d. March I 888.

De Toni in Sacc. Syll. 7 : 779. d. 28 Oct. 1888.

Jones, Proc. Cal. Acad. 11. 5: 730.3 Oct. I895.

Tubeuf-Smith, Diseases of Plants 4IO. 1897.

Aecidium Dracontil, M. A. Curtis.

See Aecidium Caladii, S.

Aecidium dracontionatum, $\mathrm{S}$.

See Aecidium Caladii, S. 
Aecidium dubium, Clint.

See Aecidium Calystegia, Desm.

Aecidium elatinum, A. \& S.

Nork. This is the acidium of Melampsorella Caryophyllacearum, (D C.) Schrt. See Ed. Fischer, Ber. Deutsch. Bot. Ges. 19: 397. 26 June 1901, and Zeit. Pflanzenkr. 12: 193. 7 Oct. 1902. It may be remarked that the distribution of Melampsorella Caryophyllacearum, (D C.) Schrt. in the Eastern United States corresponds with that of Aecidium elatinum. For further information see Klebahn, Rostpilze 396. I904.

De Toni in Sacc. Syll. $7: 825$. d. 28 Oct. 1888.

Anderson, A. P., Bot. Gaz. $22: 235.23$ Sept. 1896.

Anderson, A. P., Bot. Gaz. 24: 309. pl. 14, 15. I7 Nov. 1897.

Diet. in Engler \& Prantl, Pflanzenfam. $1^{1 * *}: 78 . f .52,53$ A. 1897.

Tubeuf-Smith, Diseases of Plants 404. f. 24I, 242. 1897.

Anderson, A. P., Bull. Torr. Bot. Club 29:31. 28 Jan. 1902.

Ricker, Univ. of Maine Studies 3: 5I. 30 April I902.

Peridermium eiatinum, Schm. \& Kze.

Pl. Rept. N. Y. Mus. $27:$ 104. 1875.

Pk. Rept. N. Y. Mus. $28: 86$. 1876.

Pk. Rept. N. Y. Mus. $29: 77 . \quad$ I 878.

Thm. Mitth. forstl. Versuchs. Oest. 2: 317 (2I). d. I880.

Farl. Appalachia $3: 243$. Jan. I 884.

*Ell. N. A. F. 1437.1885.

Richards, Proc. Am. Acad. $31: 264$. pl. 1, f. 14. 1896.

Davis, Tr. Wis. Acad. 11 : 174. Aug. 1897.

Pk. Bull. N. Y. Mus. $6: 227$. July 1899.

Nelson, Rept. Wyo. Exp. Sta. $10:(30) .1900$.

Aecidium elegans, B. \& C.

Note. This is understood to be the æcidial stage of Uromyces elegans, Lagerh.

*Rav. Fung. Car. 2 : 88.1853.

*Rav. Fung. Am. 727. 1882 .

Farl. \& Seymour, H. I. 30. Aug. I888.

* Seymour \& Earle, E. F. 50 . 7 June 1899.

Aecidium Orobi, var. elegans, B.

B. Grev. $3: 6$ I $(103)$, d. Dec. I874.

*Thm. Myc. Univ. 1024. 1878.

Aecidium Ellisii, Tracy \& Gall.

Note: In Bot. Gaz. 35: 20. 20 Jan. 1903, Arthur states that this is the rcidium of Puccinia subnitens, Diet. N. B. This species is not to be confounded with Rectelia Ellisii, Pk., a synonym of Aecidium Botryapites, (S.). See also Syd. Mon. Ured. x : 748. I Nov. 1903.

Tracy \& Gall. Jour. Myc. 4 : 2I. d. March 1888.

De Toni in Sacc. Syll. $7: 819$ d. 28 Oct. 1888.

Hume, Proc. Davenport Acad. 7 : 252. 12 May 1899.

Arth. Bot. Gaz. 35 : 20.20 Jan. 1903.

Kellerm. Jour. Myc. $9: 234.31$ Dec. 1903.

Aecidium Engelmanni, (Thm.) Diet.

NotE. See note under Aecidium conorum-Picea, Reess.

Diet. in Engler \& Prantl, Pflanzenfam. 11**: 79. 1897.

Peridermium Engeimanni, Thm.

Thm. Mitth. forstl. Versuchs. Oest. $2: 314$ (18). $d$. I880.

De Toni in Sacc. Syll. $7: 836 . d$. 28 Oct. 1888.

Tubeuf-Smith, Diseases of Plants 417. 1897. 
Pk. Bull. N. Y. Mus. 6 : 227. July 1899.

Pk. Rept. N. Y. Mus. 52: 650. Dec. I899. [Bull. $5:$ no. 25.]

Aecidium Ephedræ, (Cke.) Diet.

Diet. in Engler \& Prantl, Pflanzenfam. 11** : 79. I897.

Aecidium Pini, var. Ephedra, B. \& C.

*Rav. Fung. Car. 3 : 95.1855.

Peridermium Pini, var. minus, B. \& C.

B. Grev. 3 : 59 (IOI) no. 576. d. Dec. 1874. Coleosporium senecionis, var. minus, De Toni. I.

De Toni in Sacc. Syll. 7 : 752 . d. 28 Oct. 1888. Peridermium Ephedra, Cke.

Cke. Ann. N. Y. Acad. 1: 182. May 1878.

Thm. Mitth. forstl. Versuchs Oest. 2: $323(27) \cdot d . \quad$ I 880.

De Toni in Sacc. Syll. $7: 835 . d$. 28 Oct. 1888.

Tubeuf-Smith, Diseases of Plants 417. 1897.

Cockerell, Jour. Myc. 10: 50. I9 March I904.

\section{Aeeidium Epilobil, D C. On Epilobium.}

Note. The true Aecidium Epilobii, D C. belongs to Puccinia Epilobiitetragoni, (D C.) Wint. and we have included here references to Aecidia on species of Epilobium, most of which at least seem really to belong to this species. With regard to the references here given to Aecidia on species of Oenothera there is some doubt, for although by recent writers they are generally placed here, their position cannot be considered certain. Compare also Aecidium Oenothera, Pk.

Farl. Bot. Gaz. 8 : 276 . Aug. I 883.

Farl. Appalachia $3: 239$. Jan. 1884 .

Halsted, Bull. Iowa Agr. Coll. 1888 : i16. April 1888.

*Ell. \& Ev. N. A. F. 3580.24 Jan. I898.

*Ell. \& Ev. Fung. Columb. 1300. May 1898.

Tracy \& Earle in Greene, Pl. Baker. 1 : 17. 22 Feb. I90I.

Trel. in Sacc. Pk. \& Trel., Harrimain Alaska Exp. 5 : 37. 5 April 1904. Caoma Epilobii, Schl.

Ehrb. Horæ Phys. Berol. 96. 1820.

Aecidium Epilobil, Auct. Amer. On Oenothera.

Sprague, Proc. Boston Soc. $6: 319 . \quad 1859$.

Pk. Rept. N. Y. Mus. 23: $58 . \quad 1872$.

Pk. Rept. N. Y. Mus. 24: 106. 1872.

Frost in Cat. Pl. Amherst 85. 1875.

Pk. Rept. N. Y. Mus. 29: 76. 1878.

*Ell. N. A. F. 226 . 1879.

Bundy, Geol. Wis. I873-79 $1: 400.1883$.

Arth. Bull. lowa Agr. Coll. 1884: 165. Jan. I885.

Burrill, Bull. Ill. Lab. 2: 227. $d . \quad$ I 885 and Rept. Ill. Ind. Univ. 12 : 142. $d$. 1885 .

Cobb, List Pl. Amherst 40. 1887.

Kellerm. \& Carl. Tr. Kans. Acad.10 : 91. 1887.

* Seymour \& Earle, E. F. 281. I May I 893.

Halsted, Rept. N. J. Exp. Sta. 1892: 347. I893.

Tracy \& Earle, Bull. Miss. Exp. Sta. 34:89. May 1895.

Atk., Bull. Cornell Univ. 3:2I. 25 June I897.

Underw. \& Earle, Bull. Ala. Exp. Sta. 80:200. 28 June I897.

Halsted, Bull. Torr. Bot. Club $26: 382$. I 8 July I 899 .

Halsted, Rept. N. J. Exp. Sta. 1898: $368 . \quad$ I899.

Earle in Mohr, Contr. U. S. Nat. Herb. 6 : 192. 31 July Igor.

Davis, Tr. Wis. Acad. 14: 103. Sept. 1903. 
Aecidium Oenothera, Fung. Columb. non Pk.

*Ell. \& Ev. Fung. Columb. 1388. May 1899.

Aecidium Epilobil, Auct. Amer. p. p.

See Aecidium Isnardia, Lagerh.

Aecidium erigeronatum, S.

Note. On the basis of cultures Arthur describes this plant in Jour. Myc. 8: 54,30 June, 1902, as the accidium of Puccinia Caricis-Erigerontis, Arth. See also Arth. Bot. Gaz. 35: 16. 20 Jan. 1903.

N.B. Another Aecidium, A. incurvum, Tracy \& Earle, on Erigeron is described.

S. Syn. Am. Bor. 300. 1834 .

Pl. Rept. N. Y. Mus. 24:92, 107. 1872.

*Thm. Myc. Univ. $15 \%$ I875.

Pk. Rept. N. Y. Mus. 29: 77.1878.

Bundy, Geol. Wis. $1873-79,1: 400.1883$.

Arth. Bull. lowa Agr. Coll. 1884: 165. Jan. 1885.

Burrill, Bull. 111. Lab. 2: 229. d. 1885 and Rept. Ill. Ind. Univ. 12 : 143. $d$. 1885 .

Brendel, Flora Peoriana 2 ed. $69 . \quad 1887$.

De Toni in Sacc. Syll. 7 : 801. d. 28 Oct. 1888.

Webber, Bull. Nebr. Exp. Sta. 1: 331 (61). 18 Dec. 1889.

Webber, Rept. Nebr. Agr. 1889:211 (71). 1890.

*Ell. \& Ev. N. A. F. 2402. i89o.

Detmers, Bull. Ohio Exp. Sta. 44 : 138 . Sept. 1892.

Detmers, Bull. Ohio Exp. Sta. tech. ser. 1: 178. April 1893 .

*Ell. \& Ev. N. A. F. 2402 b. Oct. I893. (Issued with Cent. 30.)

*Ell. \& Ev. Fung. Columb. 64. 1893.

* Carl. Ured. Amer. 45. 1894.

Kellerm. \& Werner, Geol. Ohio $7^{2}$ : 346. Jan. 1895.

Underw. Proc. Ind. Acad. 1894: 151 . Oct. 1895.

Tubeuf-Smith, Diseases of Plants 41 I. i 897.

Barthol. Tr. Kans. Acad. 16: I88. June I899.

* Barthol., Ell. \& Ev. Fung. Columb. 1502. Dec. 1901.

Kellerm. Jour. Myc. 9: 237. 31 Dec. 1903.

Caoma erigeronatum, $\mathrm{S}$.

S. Syn. Am. Bor. 292 no. 2869. d. 1834.

PAecidium Compositarum, Auct. Amer. p. p.

Trel. Tr. Wis. Acad. 6: 137 (33). Nov. 1884.

Kellerm. Bull. Wash. Lab. 1: 75. Jan. 1885 and Tr. Kans. Acad. 9 : 81. 1885.

Kellerm. \& Carl. Tr. Kans. Acad. 10 : 91. 1887.

Gall. Bull. U. S. Agr. Veg. Pathol. 8: 55.1889.

Hume, Proc. Davenport Acad. 7:252. 12 May 1899.

Pound \& Clements. Bot. Surv. Nebr. 5: 24. 30 March I9oI.

Aecidium Compositarum, forma Erigerontis, Wint.

Wint. \& Demet. Hedw. 24: 179 (3). Oct. 1885.

Freeman, Minn. Bot. Studies I1. 5: 556. 20 July ío I.

Aecidium Euphorbiæ, S.

Note. Aecidium Euphorbia, P. in Gmel. 1791 was shown by Schroter, Hedw. 14: 98. July 1875 to be the Aecidium of Uromyces Pisi, (P.) D By. This Aecidium on Euphorbia Cyparissias must be rare, even if it occurs at all, in the United States and we find scanty references to Uromyces $P i s i$ in this country unless it has been published under some synonym which we are not able to recognize. See also Jordi, Centralbl. Bact. 2 Abt. 1o: 777. 3 Sept. 1903 where Uromyces Astragali, (Opiz) Sacc. is said to have 
its recidium on Euphorbia Cyparissias. For further information consult Klebahn, Rostpilze 330. 1904.

The Aecidium Euphorbia, S. dates from I 822 and is the æcidium of Uromyces Euphorbia, C. \& P. (see Magnus, Ber. Deutsch. Bot. Ges. 14: 376 . 28 Dec. I 896) and under this name we give all references to Aecidia on different Euphorbia, since evidently the greater part relate to the recidium of Uromyces Euphorbic, C. \& P. . Some references, especially those pertaining to the Western States, may perhaps be distinct but at present their development is unknown. Several species of Uromyces are known on Euphorbia in North America but some of them are believed to be destitute of an recidial stage. See also Arth. Bot. Gaz. 29: 270. 20 April 1900 and Jour. Myc. 8 : 51. 30 June 1902 and Carl. Science II. 13: 250. I5 Feb. I90I.

Muhl. Cat. Pl. Am. Sept. I ed. I04. I8I3. 2 ed. 107. 1818.

S. Syn. Car. 69 (43) no. $455 . \quad$ I 822.

Lea, Cat. Pl. Cin. 71. 1849.

Cke. Handbook 537. I87I.

Frost in Cat. Pl. Amherst 84. $\quad$ I 875.

Johnson, Bull. Minn. Acad. 1: 296. 1877 and Rept. Minn. Surv. 5 : 85. 1877.

Pk. Rept. N. Y. Mus. $29: 77 . \quad$ I 878.

Pk. Rept. N. Y. Mus. $30: 76$. Sept. 1878.

James, Jour. Cin. Soc. $2: 67$ (26).. April 1879.

* Rav. Fung. Am. 484. I881.

Brendel, Flora Peoriana I ed. 362 (94). I882. 2 ed. 69. $\quad$ I 887.

Arth. Bull. lowa Agr. Coll. 1884: I66. Jan. I885.

Burrill, Bull. Ill. Lab. 2: 237. $d . \quad$ I 885 and Rept. Ill. Ind. Univ. 12 : I48. d. 1885 .

*Ell. \& Ev. N. A. F. I820. I887.

Cobb, List Pl. Amherst 4o. $\quad$ I887.

De Toni in Sacc. Syll. $7: 823 . d .{ }_{2} 8$ Oct. I 888.

Bennett, Cat. Pl. R. 1. 89. 1888.

*Ell. \& Ev. N. A. F. 2215. Feb. I889.

Cockerell, Jour. Myc. 5:85. June 1889 .

Seymour, Proc. Boston Soc. 24: I90. 8 Nov. I889.

Webber, Bull. Nebr. Exp. Sta. 1 : 33 I (6I). 18 Dec. I889.

Ell. in Cat. Pl. N. J. 505. I889.

Gall. Bull. U. S. Agr. Veg. Pathol. 8: 55. I889.

Webber, Am. Nat. 24: 178 . Feb. 1890.

Webber, Rept. Nebr. Agr. 1889 : 2I I (7 I). I 890.

Williams, Bull. S. Dak. Exp. Sta. $29: 49$. Dec. I89 I.

* Seymour \& Earle, E. F. 385. I May I893.

Halsted, Rept. N. J. Exp. Sta. 1892 : 349. $\quad$ I 893.

Underw. Proc. Ind. Acad. 1893 : 49. Aug. I894.

*Ell. \& Ev. Fung. Columb. 338. I894.

Kellerm. \& Werner, Geol. Ohio $7^{2}$ : 346. Jan. 1895.

Tracy \& Earle, Bull. Miss. Exp. Sta. 34: 89. May I805.

Ell. \& James in Holzinger, Contr. U. S. Nat. Herb. 3: 275. 23 Nov. 1805 .

McClatchie in Reid's Hist. Pasadena 6I 5. 1895.

McClatchie, Proc. S. Cal. Acad. 1 : 372.5 June I897.

Atk. Bull. Cornell Univ. 3:2I. 25 June 1897.

Underw. \& Earle, Bull. Ala. Exp. Sta. 80: 209. 28 June I897.

Diet. in Engler \& Prantl, Pflanzenfam. 11**: 77. $\quad$ I897.

Snyder, Proc. Ind. Acad. $1896: 217 . \quad$ I897.

Tubeuf-Smith, Diseases of Plants 410 . I897.

Orton, Rept. Vt. Exp. Sta. 11 : 210. Sept. I898 and Contr. Bot. Vt. 2 : 12. Sept. 1808 .

Hume, Proc. Davenport Acad. 7 : 252. 12 May I899. 
Barthol. Tr. Kans. Acad. 16: 188 . June 1809.

Long, Bull. Torr. Bot. Club $27: 586.14$ Nov. 1900.

Nelson, Rept. Wyo. Exp. Sta. 10 : (35). 1900.

Earle in Molır, Contr. U. S. Nat. Herb. 6 : 192. 31 July 1901.

* Barthol., Ell. \& Ev. Fung. Columb. 1503. Dec. 1901.

Patterson, Bull. U. S. Agr. Pl. Industry 8: 8. 3 Feb. 1902.

*Barthol., Ell. \& Ev. Fung. Columb. 1602. Oct. 1902.

Caoma Euphorbia-hypericifolix, S.

S. Syn. Am. Bor. 293 no. 2800. $d$. 1834. Aecidium Euphorbiz-hypericifolia, S.

S. Syn. Am. Bor. 309. 1834.

M. A. Curtis, Bot. N. Car. 123. 1867.

Pk. Rept. N. Y. Mus. 24: 92, 106. 1872. Aecidium Cyparissia, Auct. Amer.

Arth. Bull. lowa Agr. Coll. 1884: 165. Jan. 1885.

Underw. Proc. Ind. Acad. 1896: i7 I. 1897.

Aecidium Euphorbiæ-hypericifoliæ, S. See Aecidium Euphorbia, S.

Aecidium Eurotiæ, Ell. \& Ev.

Ell. \& Ev. Jour. Myc. 6 : 119. d. 6 Jan. 189 I.

Sacc. Syll. $9:$ 319. $d$. I5 Sept. I 801 .

*Ell. \& Ev. N. A. F. 2709 . May 1892.

*Ell. \& Ev. Fung. Columb. 271. 1894.

Nelson, Rept. Wyo. Exp. Sta. 10 : (35). 1900.

Aecidium Fendleri, Tracy \& Earle.

NoTE. Cockerell, 1893, reports Aecidium Berberidis, P. on Berberis Fendleri.

Tracy \& Earle in Greene, Pl. Baker. 1 : 17. d. 22 Feb. 1901.

Earle in Greene, PI. Baker. 2:2. 25 March 1901.

Sacc. \& Syd. Syll. 16: I12I. I Feb. 1902.

Aecidium flamentosum, (Pk.) n. n. Peridermium filamentosum, $\mathrm{Pk}$.

Pk. Bot. Gaz. $7: 56 . d$. May 1882.

De Toni in Sacc. Syll. $7: 837 . d$. 28 Oct. 1888.

Klebahn, Ber. Bot. Ges. 8 : (69). 29 Dec. 1800.

Tubeuf-Smith, Diseases of Plants 415. I897.

Aecidium flmbriatum, (Arth.) n. n.

Roestelia fimbriata, Arth.

Arth. Bull. Torr. Bot. Club 28 : 666. d. 30 Dec. 1901.

Aecidium flaviformis, (Atk.) n. $n$.

Note. The name Rastelia faviformis given below is a misprint for Rastelia foriformis, Thaxter in Herb. Atkinson of which no description has been published. See Gymnosporangium faviforme, Earle.

Ræestelia flaviformis, Atk.

Underw. \& Earle, Bull. Ala. Exp. Sta. 80: 218. 28 June 1897.

*Syd. Uredineen 1194. Feb. 1898 .

Earle in Mohr, Contr. U. S. Nat. Herb. $6: 186.3$ 3I July IgoI.

Aecidium fragile, Diet. \& Holw.

Note. Perhaps the æcidial stage of Puccinia Oaxacana, Diet. \& Holw. which accompanies it. See also Syd. Mon. Ured. I : 24. 30 June 1902.

Holw. Bot. Gaz. 31 : 331. I8 May 190I.

Mexico.

Aecidium Fraseræ, Trel.

Trel. in Sace., Pk.\& Trel., Harriman Alaska Exp. 5 : 36.5 April 1904. 


\section{Aecidium Fraxini, S.}

Note. On the basis of cultures, Arthur 1899, 1900 reports this as the recidial stage of a Puccinia, now Puccinia fraxinata, (Lk.) Arth. $q . v$.

Muhl. Cat. Pl. Am. Sept. I ed. I04. I8I3. 2 ed. I07. I8I8.

S. Syn. Car. 66 (40) no. 430. d. I822.

Fr. S. M. $3: 513$. I832.

Pk. Rept. N. Y. Mus. $23: 59.1872$.

Pk. Rept. N. Y. Mus. 24 : I06. I872.

B. Grev. 3 : 6I (I03). Dec. I874.

Pk. Rept. N. Y. Mus. 29:77. I878.

*Ell. N. A. F. 222 . I879.

Trel. Tr. Wis. Acad. $6:$ I36 (32). Nov. I884.

Arth. Bull. lowa Agr. Coll. 1884 : ı66. Jan. 1885.

Bessey, Am. Nat. $19: 886,887$. Sept. I 885 .

Burrill, Bull. Ill. Lab. 2 : 236. $d . \quad$ I 885 and Rept. III. Ind. Univ. 12 : I48. d. I 885 .

Bessey, Am. Nat. 20 : 8o6. Sept. I 886.

Bessey, Am. Pomol. Soc. 1885 : 43. I 886.

Roum. Rev. Myc. 9: 103. I April I887.

* Roum. Fung. Gall. 4040. 1887.

Bessey, Am. Nat. 21 : 666. July I887.

Halsted, Bull. Iowa Agr. Coll. 1886: 55. 1887.

Kellerm. \& Carl. Tr. Kans. Acad. 10:9I. I887.

Langlois, Cat. Pl. L.a. 27. $\quad$ I887.

De Toni in Sacc. Syll. 7 : 806. d. 28 Oct. I888.

Pound, Am. Nat. 22 : i I 7 . Dec. I 888.

Farl. Proc. Soc. Prom. Agr. Sci. 1888: 26. I 888.

Gall. Jour. Myc. 5:95. June I889.

Webber, Bull. Nebr. Exp. Sta. 1 : 33I (6I). 18 Dec. I889.

Pound, Bull. Nebr. Exp. Sta. 1 : 356 (86). Dec. I889.

Gall. Bull. U. S. Agr. Veg. Pathol. 8: 55. I 889.

Farl. Gard. \& For. 3 : 627. 24 Dec. I800.

Webber, Rept. Nebr. Agr. 1889 : 2 I I (7I). I 890.

Williams, Bull. S. Dak. Exp. Sta. 29:4I. Dec. I89I.

Pammel, Agr. Sci. $7: 25$. Jan. I803.

* Seymour \& Earle, E. F. 209. I Feb. I893.

Carl. Bot. Gaz. 18 : 45I, 454, pl. 37, f. 5, 6. 25 Dec. I 893.

* Carl. Ured. Amer. 33. I894.

*Ell. \& Ev. Fung. Columb. 499. I894.

Tracy \& Earle, Bull. Miss. Exp. Sta. $34: 80 . \quad$ May I 895.

Diet. in Engler \& Prantl, Pflanzenfam. 11**: 77 . I807.

Tubeuf-Smith, Diseases of Plants 4 Io. 1897.

Orton, Rept. - Vt. Exp. Sta. 11 : 2 I I. Sept. 1898 and Contr.'Bot. Vt. 2: I3. Sept. I 898.

Barthol. Ir. Kans. Acad. 16 : I88. June I 899.

Arth. Science II. 10:565. 20 Oct. I 809 .

Jelliffe, Flora of Long Island $36 . \quad$ I899.

Arth. Bot. Gaz. 29 : 275.30 April I000.

Freeman, Minn. Bot. Studies 1I. 5: 559. 20 July I9o I.

Earle in Mohr, Contr. U. S. Nat. Herb. 6 : I92. . 3I July I gor.

Holw. Jour. Appl. Micr. 5 : I655. $f$. I. Feb. I902.

Schrenk, Bull. U. S. Agr. Pl. Industry 32: 9. 28 Feb. 1903. Caoma fraxinatum, Lk.

Lk., L. Sp. Pl. ed. Willd. 62: 62. d. 1825 .

Uredo Fraxini, Spreng.

Spreng. in L. Syst. Veg. I6 ed. 4, : 57I. d. I827. Caoma Fraxinites, S.

S. Syn. Am. Bor. 294 no. 290I. I834. 
Rastelia Fraxini, M. A. Curtis.

M. A. Curtis, Bot. N. Car. 123. 1867. Aecidium, sp. . Mayr.

Mayr, Waldungen von Nordamer. 139, 434. I890.

Aecidium Fumariacearum, Kellerm. \& Swingle.

See Aecidium Corydalis, (Ell. \& Kellerm.) Webber.

Aecidium Galii, P.

Nute. Compare Puccinia Galii, (P.) S. and Puccinia troglodytes, Lindr.

Jolnnson, Bull. Minn. Acad. 1 : 366. 1880.

Arth. Bull. lowa Agr. Coll. 1884: 166. Jan. 1885.

Davis, Tr. Wis. Acad. 14: 87. Sept. 1903.

PAecidium Galiorum, Langl.

Langlois, Cat. Pl. La. 27. 1887.

Aecidium Gauræ, Ell. \& Ev.

Ell. \& Ev. Erythea 1: 205. d. 2 Oct. 1893.

Sacc. Syll. 11: 215. d. July 1805.

Pound \& Clements, Bot. Surv. Nebr. 5: 24. 30 March I9or.

Aecidium gaurinum, $\mathrm{Pk}$.

Note. The teleutosporic stage is Uromyces gaurinus, (Pk.) Snyder.

Pk. Bot. Gaz. 4 : 218. d. Oct. 1879.

De Toni in Sacc. Syll. $7: 790 . d .28$ Oct. 1888.

Kelsey, Jour. Myc. 5 : 80 . June I 889.

*Ell. \& Ev. N. A. F. 2894. March I893.

*Ell. \& Ev. Fung. Columb. 872. April i 896.

* Seymour \& Earle, E. F. Suppl. B 29. 5 April r898r

Barthol. Tr. Kans. Acad. 16: I89. June 1899.

*Griffiths, West Am. Fung. 40. 22 March 1902.

Aecidium Gayophyti, Vize.

Note. Said to belong to Puccinia Gayophyti, Billings.

Vize, Grev. 7 : 12. d. Sept. 1878.

Hark. \& Moore, Cat. Pac. Fung. 27. Feb. I880.

*Ell. N. A. F. 1426.1885.

De Toni in Sacc. Syll. $7:$ 791. d. 28 Oct. 1888.

Ell. \& Varney in Coville, Contr. U. S. Nat. Herb. 4: 232. 29 Nov. 1893 .

Aecidium Geranil, Auct. Amer.

See Aecidium Geranil-maculati, S.

Aecidium Geranil-maculati, S.

Note. Under this name Schweinitz in 1822 described an Aecidium on Geranium which was not recorded again by any other American botanist till 1865. The Schweinitz species had been assumed to be identical with Aecidium Geranii, D C. which is said to belong to Uromyces Geranii, (DC.) Otth \& Wartm. until Lindroth, in 1900, regarding the two as distinct, gave the name of Aecidium sanguinolentum to the common North American form. From the description of Aecidium Geranii-maculati given by Schweinitz we feel justified in regarding Accidium sanguinolentum, Lindr. as a synonym See also Klebahn, Rostpilze 322. 1904. See also Aecidium violascens, Trel, on Geranium erianthus in Alaska.

S. Syn. Car. 67 (4I) no. 443. d. 1822.

Lk., L. Sp. Pl. ed. Willd. $6^{2}$ : 57. d. 1825.

Uredo hemispharica, Spreng.

Spreng. in L. Syst. Veg. I6 ed. 411 : 571. d. 1827.

Cxoma geraniatum, $\mathrm{S}$.

S. Syn. Am. Bor. 293 no. 2879 . 1834. 
Aecidium Geranii, Auct. Amer.

Watt, Can. Nat. \& Geol. 11. 2 : 390. Oct. I865.

M. A. Curtis, Bot. N. Car. I24. I867.

Cke. Proc. Portland Soc. 1: I80. d. I869.

Cke. Handbook 543. I87I.

Pk. Rept. N. Y. Mus. 23: 59. 1872.

Pk. Rept. N. Y. Mus. 24: $107 . \quad$ I 872.

B. Grev. 3 : 60 (IO2). Dec. I874.

Frost in Cat. Pl. Amherst 85. 1875 .

Farl. Bull. Bussey Inst. 2: 226. Jan. I878.

Pk. Rept. N. Y. Mus. $29: 76 . \quad$ I 878.

*Ell. N. A. F. $221 . \quad 1879$.

Johnson, Bull. Minn. Acad. 1: $366 . \quad$ I 880.

Bundy, Geol. Wis. 1873-79 1: 400. I883.

Trel. Tr. Wis. Acad. 6 : I35 (3I). Nov. I884.

Arth. Bull. lowa Agr. Coll. 1884: I66. Jan. I885.

Burrill, Bull. Ill. Lab. 2: 224. d. 1885 and Rept. Ill. Ind. Univ. 12 : I4I. $d$. 1885 .

Cobb, List Pl. Amherst 40. I 887.

Bennett, Cat. PI. R. I. 89. I 888.

Detmers, Bull. Ohio Exp. Sta. 44: 138. Sept. I892.

Underw. Proc. Ind. Acad. 1893: 49. Aug. I894.

Kellerm. \& Werner, Geol. Ohio $7^{2}$ : $346 . \quad J a n . ~ I 895$.

*Ell \& Ev. Fung. Columb. 762.24 April I895.

Claassen, Rept. Ohio Acad. 5 : $68 . \quad$ I 897.

Snyder, Proc. Ind. Acad. 1896 : 217. 1897.

Barthol. Tr. Kans. Acad. 16 : I89. June 1899.

Snyder, Proc. Ind. Acad. 1898 : I88. Dec. I899.

Herbst, Fungal Fl. Lehigh Valley i76. d. I899.

Freeman, Minn. Bot. Studies 11. 5 : 556. 20 July I90I.

Uromyces Geranil, Auct. Amer.

De Toni in Sacc. Syll. $7: 535.28$ Oct. 1888.1.

Detmers, Bull: Ohio Exp. Sta. tech. Ser. 1: 172. April I893. [I ?]

Orton, Rept. Vt. Exp. Sta. 11: 218. Sept. I898 and Contr. Bot. Vt. 2: 20. Sept. 1898.1 .

Aecidium sanguinolentum, Lindr.

Lindr. Bot. Notiser 1900: 24I-246. $d$. Dec. 1900 and Bot. Centralbl. $86: 260 . d$. I5 May I90I.

Sacc. \& Syd. Syll. $16: 33$ I. $d$. I Feb. 1902.

Aecidium Gerardiæ, $\mathrm{Pk}$.

Pk. Bull. Buf. Soc. 1: 68. $d$. July I873.

Pk. Rept. N. Y. Mus. $25: 92$. $d$. Sept. 1873.

Pk. Rept. N. Y. Mus. 29: $77 . \quad 1878$.

*Thm. Myc. Univ. 1225. I879.

De Toni in Sacc. Syll. $7: 81$. $d .28$ Oct. 1888.

*Roum. Fung. Gall. 4618. I888.

Roum. Rev. Myc. 10 : I86. I Oct. I 888.

*Ell. \& Ev. N. A. F. 27 Io. May I802.

Underw. \& Earle, Bull. Ala. Exp. Sta. 80: 209. 28 June 1897.

*Seymour \& Earle, E. F. Suppl. B 3o. 5 April I 898.

Earle in Mohr, Contr. U. S. Nat. Herb. $6:$ i92. 3I July I90I.

Patterson, Bull. U. S. Agr. Pl. Industry $8: 8 .{ }_{3}$ Feb. I902.

Aecidium Giliæ, Pk.

NotE. Although Aecidium Gilia, Pk. and Aecidium Polemonii, Pk. resemble one another, in the absence of cultures to show their identity, they are here kept distinct. Under the first named species we have placed those 
references to Aecitia on Gilia and Phlox which are assumed to belong to Puccinia plumbariu, Pk. including the Aecidium Wilcoxian $u$ of exsiccati, of which, so far as we know, no description was ever published.

Specimens in different exsiccati of Aecidium Phlogis seem to us not to differ from Accidium Gilia, Pk. Aecidium Phlogis, Pk. cited by Arthur, I885, should probably be in part Aecidium Polemonii, Pk. as there is no Aecidium Phlogis, Pk. and Aecidium Phlogis, Ell. \& Ev. dates from 1897. Aecidium Polemonii has also been recorded in several cases on Phlox pilosa, but the name in this case is doubtful and we are not able to refer the Aecidium in question to Aecidizm Gilia, Pk. with certainty nor does it seem to be the true Aecialium Polemonii, Pk.

The 'form on Phlox divaricata called Aecidium Polemonii is probably Aecidium Gilia, Pk.

Pk. Bot. Gaz. 4 : 230. d. Nov. I879.

Thm. Myc. Univ. 2032. I88I. (Note only.)

*Wint. Fung. Eur. 3519. 1886.

*Roum. Fung. Gall. 3926. 1887.

Roum. Rev. Myc. 9: 23. I Jan. 1887 .

*Ell. \& Ev. N. A. F. $1831 . \quad$ I887.

De Toni in Sacc. Syll. $7: 814$. d. 28 Oct. 1888.

*Ell. \& Ev. Fung. Columb. 763. 24 April I 895.

Tubeuf-Smith, Diseases of Plants 4II. I897.

Aecidium Wilcoxianum, Thm.

*Thm. Myc. Univ. $2226 . \quad$ I 884.

*Ell. N. A. F. 1432 . I 885.

*Roum. Fung. Gall. 5213. 1890.

Roum. Rev. Myc. 12:62. April ז 890.

Sacc. Syll. 11: 219 . July 1895. Aecidium Phlogis, Arth. p. p.

Arth. Rull. lowa Agr. Coll. 1884: 167. Jan. 1885. Phlox divaricata. ?Aecidium Polemonii, Auct. Amer. p. p.

Gall. Bull. U. S. Agr. Veg. Pathol. 8: 56. 1889.

Pound \& Clements, Bot. Surv Nebr. 5: 24. 30 March I90I. Aecidium Phlogis, Ell. \& Ev.

Ell. \& Ev. Bull. Torr. Bot. Club 24: 284. d. 29 June 1897.

*Ell. \& Ev. N. A. F. 3582.24 Jan. 1898.

Lindau \& Syd. Hedw. 37 : xvii. 9 April r 898 . [Beiblatt no. 7.]

Sacc. \& Syd. Syll. 14: 383. d. 20 Aug. 1899. .

Nelson, Rept. Wvo. Exp. Sta. 10 : (36). I 1900.

Earle in Greene, Pl. Baker. 2: 2. 25 March rgor.

\section{Aecidium on Gillenia.}

Roestelia lacerata, Wint. \& Demet. p. p.

Wint. \& Demet. Hedw. 24: I83 (7). Oct. I885.

*Wint. Fung. Eur. 3323 . I 885 .

Gall. Bull. U. S. Agr. Veg. Pathol. 8: $58 . \quad$ I889.

Aecidium Glaueis, Dozy \& Molkenb.

Note. Said by Plowright, Grev. 2I: III. June 1893 to belong to Uromyces lineolatus, (Desm.) Schrt. In this connection see also Diet. Hedw. 29: 149. June I890 and Klebahn, Rostpilze 328. I904.

Dozy \& Molkenb. in Tijdschr. v. Natuur. Geschied. 12 : I6. pl. 2, $f$. 1-3. [1846? *

Kelsey, Jour. Myc. 5 : 8o. June 1889.

Nelson, Rept. Wyo. Exp. Sta. 10: (35). I900. 
Aecidium globosum, (Farl.) n. n.

Note: Under this name are given references believed to relate to the acidıum of Gymnosporàngium globosum, Farl. which was by early American writers called Aecilizum Cratagi, var. and by later writers Restelia lacerata. Plants so designated are not the same as European species with these names. Some of the references below are uncertain but it is not unlikely that a majority of citations of Restelia lacerata on Cratagus relate to this species. Móst of the references by Berkeley in Grev. 3: 59 (IOI). Dec. I 874 to Restelia lacerata on Cratagus prove to be Aecidium aurantiacum. This applies to nos. I80, 182, 243 there cited, but no. I6I on Cratagus is Aecidium globosum. For account of culture experiments and results see references below, Farlow I 886 , Thaxter I887, I889, I89 I. It should be noted that the true Accidium laceratum has been produced by cultures on Cratagus (Farlow I 886 p. I 90,238 , Thaxter I 887 p. 266), but aside from this it is not definitely known on this host in America.

Aecidium Cratægi, var. Oxyacanthæ, S.

S. Syn. Car. 66 (40) no. 432. 1822.

Caoma cylindrites, S. Syn. Am. Bor.

S. Syn. Am. Bor. 294 no. 2899. I834.

Reestella lacerata, Auct. Amer. p. p.

* Rav. Fung. Car. 5 : 96.1860.

M. A. Curtis, Bot. N. Car. 123. I867. (p. p. ?)

B. Grev. 3: 59 (IOI). Dec. 1874. p. p.

Farl. Bull. Bussey Inst. 2 : 225. Jan. 1878.

Farl. Anniv. Mem. Boston Soc. 29. d. 1880.

*Rav. Fung. Am. 481. I88I.

*Ell. N. A. F. 1085 . I883.

Burrill, Bull. 11l. Lab. 2 : 240. d. 1885 and Rept. 11l. Ind. Univ. 12 : I 50. d. I 885 .

Seymour, Tr. Am. Hort. Soc. 4 : I55 (4). 7 April I887.

Brendel, $\mathrm{Fl}$. Peoriana 2 ed. 69. 1887.

* Underw. \& Cook, Illustr. Fungi 54. Sept. I889.

Seymour, Proc. Boston Soc. 24: 190. 8 Nov. 1889.

*Syd. Uredineen 296. May 1890.

* Shear, N. Y. Fungi 79. I893.

Ricker, Univ. of Maine Studies 3: 54. 30 April 1902.

Roestelia lacerata $y, z$, Thax.

Farl. Bot. Gaz. 11 : 240. Sept. 1886.

Thax. Proc. Am. Acad. 22: 263, 266, 267. Jan. 1887 and Centralbl. Bact. $1: 432,457,458 . \quad 1887$.

Thax. Bot. Gaz. 14: 165, 167, 168, I71. July 1889.

Thax. Bull. Conn. Exp. Sta. 107: 4. I5 April I891.

? Raestelia lacerata, Auct. Amer. p. p.

Cke. Handbook, 534. I87I.

Frost in Cat. Pl. Amherst 84. 1875.

Pk. Rept. N. Y. Mus. 29: 76. 1878.

Trel. Tr. Wis. Acad. 6 : 138 (34). Nov. 1884.

Arth. Bull. lowa Agr. Coll. 1884: 168. Jan. 1885.

Ell. \& Ev. Jour. Myc. 1: 86 . July 1885.

Wint. \& Demet. Hedw. 24: 183 (7). Oct. 1885 .

Bessey, Am. Pomol. Soc. 1885: 43. I886.

Cobb, List Pl. Amherst 39. I887.

Pound, Bull. Nebr. Exp. Sta. 1: 356 (86). 18 Dec. 1889.

Gall. Bull. U. S. Agr. Veg. Pathol. 8: 58. I 889.

Small \& Vail, Mem. Torr. Bot. Club 4: 200. April 1894.

Underw. Proc. Ind. Acad. 1893: 56. Aug. I894.

Tracy \& Earle, Bull. Miss. Exp. Sta. 34: 91. May 1895. 
Underw. Proc. Ind. Acad. 1894: 153. June 1807.

Snyder, Proc. Ind. Acad. 1896 : 222. 1897.

Barthol. Tr. Kans. Acad. 16: 192. June I 899.

Snyder, Proc. Ind. Acad. 1898 : r88. 'Dec. I 899.

Herbst, Fungal Fl. Lehigh Valley 174. 1899.

Rostelia globosa, Shear.

*Shear, N. Y. Fungi 79. 1893. [Corrected label.]

Fischer, Hedw. 34: 4. f. 6. Jan. I895.

Seymour \& Patterson in Deane's Flora of Reservations 138. 1896.

Stevens, Rept. Ohio Acad. 4: 20. 1896.

NoTE. The following references also probably relate to the present plant, but it may be stated that one herbarium specimen examined bears unduubted xcidium of Gymnosporangium macropus. Compare also note and references to Peck's Reports, 1872, I878 under Aecidium cornutum.

Rostelia cornuta, Auct. Amer. p. p.

Pk. Rept. N. Y. Mus. 24: 93 . I872.

Frost in Cat. Pl. Amherst 84. 1875.

Farl. Anniv. Mem. Boston Soc. 28. I880.

Seymour, Tr. Am. Hort. Soc. 4 : 155 (4). 7 April 1887.

Cobb, List Pl. Amherst 39. I887.

Roestelia penicillata, Auct. Amer. p. p.

Jones, Oberlin College Lab. Bull. 9: 7. I 5 June 1898.

Roestelia pirata, Auct. Amer. p. p.

Barthol. Tr. Kans. Acad. 16: I92. June 1899.

Aecidium gnaphaliatum, $\mathrm{S}$.

Note. Possibly a stage of Puccinia investita, S.

S. Syn. Am. Bor. 309. I834.

Pk. Rept. N. Y. Mus. 22 : 93.1869.

Pk. Rept. N. Y. Mus. 24: 106. 1872.

B. Grev. 3: 60 (102). Dec. 1874.

Pk. Rept. N. Y. Mus. 29: 77. 1878 .

De Toni in Sacc. Syll. $7: 804 . d$. 28 Oct. 1888.

McClatchie, Proc. S. Cal. Acad. 1 : 372.5 June 1897.

Underw. \& Earle, Bull. Ala. Exp. Sta. 80: 209. 28 June 1897.

*Ell. \& Ev. N. A. F. 3569. 24 Jan. 1808 .

*Ell. \& Ev. Fung. Columb. 1285. May I 898.

Orton, Rept. Vt. Exp. Sta. 11 : 2 I r. Sept. 1898 and Contr. Bot. Vt. $2:$ 13. Sept. 1898.

Earle in Mohr, Contr. U. S. Nat. Herb. 6 : 192. 3I July I9oI.

\section{Croma gnaphaliatum, S.}

S. Syn. Am. Bor. 292 no. 2873 . d. I 834.

Wint. Hedw. 24: 255. Dec. I885.

Aecidium Gossypii, Ell. \& Ev.

Ell. \& Ev. Erythea 5 : 6. d. 3i Jan. 1897 .

Lindau \& Syd. Hedw. 37: xvii. 9 April 1898. [Beiblatt no. 7.7

Sacc. \& Syd. Syll. 14 : 371. d. 20 Aug. 1899.

Earle, Bull. Ala. Exp. Sta. 107 : 317. April 1900.

Aecidium gracilens, $\mathrm{Pk}$.

Pk. Bot. Gaz. $4:$ 128. d. Feb. 1879.

De Toni in Sacc. Syll. 7 : 792. d. 28 Oct. I 888.

Aecidium Graebnerianum, Henn.

Note. See also Aecidium Orchidearum, Desm.

Henn. Hedw. 37 : 273. d. 3I Dec. I8g8.

Sacc. \& Syd. Syll. 16:347. d. I Feb. 1902.

*Syd. Uredineen 1799.1903. 
Aecidium Grindeliæ, Syd.

Syd. Hedw. Beibl. 40; (I). d. 2 I Feb. 190 I.

Sacc. \& Syd. Syll. 16 : i122. I Feb. 1902.

Syd. Ann. Myc. 1: 20.30 Jan. 1903.

Kellerm. Jour. Myc. 9 : 117 (-142). 29 May 1903.

Aecidium Grindelia, Griffiths.

* Griffiths, West Am. Fung. 388. 21 May 1902.

Griffiths, Bull. Torr. Bot. Club 29 : 299. d. f. 1o. 26 May i9o2.

* Barthol., Ell. \& Ev. Fung. Columb. 1705. Feb. 1903.

Garrett, Tr. Kans. Acad. 18 : 147. 1903.

Aecidium Grossulariæ, (P. in Gmel.) Schum.

Note. Under the name Aecidizum Grossularia, American writers include a number of æcidial forms, none of which can be connected with Puccinia Ribis, which is a Micropuccinia. See Eriksson, Rev. Gen. de lsot. 10: 506. I5 Dec. 1898. The European Aecidium Grossularia, (P.) Schum. bas been shown to belong to Puccinice on Carex, of which Klebahn has described several form-species. See Klebahn, Zeit. Pflanzenkr. 12: I 44. I5 July 1902 and Pringsheim, Jahrb. $34: 388.1902$. See also Syd. Mon. Ured. 1: 652. I Nov, 1903 and Klebahn, Rustpilze 295 et seq. I904. See also note under Aecidium albiperidium, Arth.

Probably the references given below relate to Aecidia of heterœcious Puccinia, but whether the same as the European species is not known. We have arranged the citations by hosts, beginning with those in which several hosts are given. It should be said that only on Ribes prostratum have been frequently recorded both Aecidium Grossularice and Puccinia Ribis or Puccinia puslchella, $\mathrm{Pk}$., said to be the same.

On Ribes, sp. plur.

Trel. Tr. Wis. Acad. $6: 135$ (31). Nov. 1884.

Arth. Bull. lowa Agr. Coll. 1884: 166. Jan. 1885.

Burrill, Bull. III. Lab. $2: 227 . d$. 1885 and Rept. IIl. Ind. Univ. 12 : I42. $d$. 1885 .

De Toni in Sacc. Syll. $7: 792$. d. 28 Oct. 1888.

Williams, Bull. S. Dak. Exp. Sta. 29:4I. Dec. I89I.

Pammel, Jour. Myc. 7 : ror. April 1892.

Pammel, Agr. Sci. $7: 25 . \quad J a n . ~ 1893$.

Underw. Proc. Ind. Acad. 1893 : 50. Aug. I894.

Pammel, Tr. lowa Hort. Soc. $28: 473 . \quad 1894$.

Pammel, Proc. lowa Acad. 1894: 206.

Pammel \& Carver, Proc. lowa Acad. 3 : 143. 1896.

Tubeuf-Smith, Diseases of Plants 409. I 897.

Orton, Rept. Vt. Exp. Sta. 11 : 2 I I. Sept. I 898 and Contr. Bot. Vt. 2: 13. Sept. 1898.

Barthol. Tr. Kans. Acad. 16 : I89. June 1899.

Freeman, Minn. Bot. Studies 11. 5: 558. 20 July 1901.

Ricker, Univ. of Maine Studies 3 : 5I. 30 April 1902.

On Ribes aureum, Pursh.

*Kellerm. \& Swingle, Kansas Fungi 27. I Aug. I889. On Ribes Cynosbati, L.

Pk. Rept. N..Y. Mus. 22 : 93.1869.

B. Grev. 3:61 (103). Dec. 1874.

Detmers, Bull. Ohio Exp. Sta. 44: 138. Sept. 1892.

Detmers, Bull. Ohio Exp. Sta. tech. ser. $1:$ i78. April 1893 .

* Shear, N. Y. Fungi 132.1893.

* Seymour \& Earle, E. F. 435. I July 1895.

Claassen, Rept. Ohio Acad. 5 :'68. 1897.

Snyder, Prac. Ind. Acad. 1898 : $188 . \quad$ Dec. 1899.

* Kellerm. Ohio Fungi $81, d$. Oct. I902. 
Kellerm. Jour. Myc. $8:$ I20. d. I4 Oct. 1902.

*Kellerm. Ohio Fungl 121. 28 May 1903.

Kellerm. Jour. Myc. 9: i I1. 29 May I903.

Kelsey, Jour. Myc. 5 : 80. June 1889.

On Ribes floridum, L'Her.

* Seymour \& Earle, E. F. 207. I Feb. I893.

Underw. Proc. Ind. Acad. 1894 : 154. Oct. I895.

On Ribes gracile, Michx.

Davis, Tr. Wis. Acad. 14: 87. Sept. 1903.

On Ribes Grossularia, $L$.

Sturgis, Rept. Conn. Exp. Sta. 24: 270. I90I.

Tracy \& Earle, Bull. Miss. Exp. Sta. 34: 90. May 1895.

On Ribes lacustre, Poir.

Halsted, Bull. lowa Agr. Coll. 1886: 58. 1887.

*Ell. \& Ev. Fung. Columb. 357. I 894.

\section{On Ribes oxyacanthoides, L.}

Davis, Tr. Wis. Acad. 9 : I6r. 21 Aug. I893.

On Ribes prostratum, L'Her.

*Ell. N. A. F. 1006 . 1883.

Farl. Appalachia 3: 239 . Jan. 1884.

On Ribes rotundifolium, Michx.

Kellerm. Bull. Wash. Lab: 1 : 75. Jan. 1885 and Tr. Kans. Acad. 9 : 8I. I885.

Kellerm. \& Carl. Tr. Kans. Acad. 10 : 91. 1887.

* Roum. Fung. Gall. 4414. 1888.

Roum. Rev. Myc. 10:86. I April 1888.

Webber, Bull. Nebr. Exp. Sta. 1: 332 (62). 18 Dec. 1889.

Gall. Bull. U. S. Agr. Veg. Pathol. 8: 55 . 1889.

Webber, Rept. Nebr. Agr. 1889:2 I I (7I). I890.

On Ribes, sp. cult.

Bessey, Am. Pomol. Soc. 1885 : 43.1886.

Pound, Bull. Nebr. Exp. Sta. 1: 356 (86). 18 Dec. I 889.

Stone, Rept. Mass. Agr. Coll. 32: 148 (12). 1895.

On Ribes, sp. indet.

M. A. Curtis, Bot. N. Car. 123. 1867.

Frost in Cat. Pl. Amherst 85. 1875.

Cke. Grev. 5 : 151 . June 1877.

Johnson, Bull. Minn. Acad. 1: $365 . \quad$ I 880.

Cragin, Bull. Wash. Lab. 1: 68. Jan. 1885.

Cobb, List. Pl. Amherst 40. I887.

*Underw. \& Cook, Illustr. Fungi 52. Sept. I889.

Kellerm. \& Werner, Geol. Ohio 72: 346 . Jan. 1895.

Hume, Proc. Davenport Acad. $7: 252.12$ May 1899.

Herbst, Fungal Fl. Lehigh Valley I75. d. 1899.

Nelson, Rept. Wyo. Exp. Sta. 10: (35). I900.

Garrett, Tr. Kans. Acad. 18: 147. 1903.

Trel. in Sacc., Pk. \& Trel., Harriman Alaska Exp. 5: 36.5 April 1904. Host not stated.

Watt, Can. Nat. \& Geol. II. 2 : 390 . Oct. 1865.

Edwards, Bull. Torr. Bot. Club 2 : 39. Oct. I871.

Cke. Handbook 54I. $d$. I87I.

Pk. Rept. N. Y. Mus. 24: 107: 1872.

Bundy, Geol. Wis. I873-79 1:400. I 883 .

Ell. \& Ev. Jour. Myc. 1 : 86. July r 885.

Brendel, Fl. Peoriana 2 ed. 69.1887.

Bennett, Cat. Pl. R. I. 89 . I 888. 
Williams, Am. Nat. 24: 780. Aug. I89o.

Humphrey, Rept. Mass. Exp. Sta. $10: 241$ (33). I893.

Cheney, Tr. Wis. Acad. 10:69. Oct. I895.

Aecidium Rumicis, S. Car. p. p.

S. Syn. Car. 66 (40) no. 433.1822

Cacomá grossulariatum, Lk.

S. Syn. Am. Bor. 293 no. 2882. 1834 .

Aecidium Guadalajaræ, Syd.

Syd. Oest. Bot. Zeit. 52 : 183 . d. May 1902.

Mexico.

Aecidium Hamiltoniæ, Thm.

See Aecidium pustulatum, M. A. Curtis.

Aecidium Harknessianum, (Ell. \& Ev.) n. n.

Note. Arthur, I9OI, suggests that this may belong to Gymnosporangium speciosum, $\mathrm{Pk}$.

Rostelia Harknessiana, Ell. \& Ev.

*Ell. \& Ev. N. A. F. 2714. May I892.

*Ell. \& Ev. Fung. Columb. 1293. May 1898.

Arth. Proc. Ind. Acad. 1900: 136. June 1901.

?Ricker Jour. Myc. 8: 127. I4 Oct. I902.

Aecidium Harknessii, (Moore) Diet.

Diet. in Engler \& Prantl, Pflanzenfam. 1 1** : 79. 1897.

Peridermium Harknessii, Moore.

Hark. \& Moore, Cat. Pac. Fung. 27. Fèb. I880,

Thm. Mittheil. forstl. Versuchs Oest. 2: 3I3 (I7). 1880.

Hark. Bull. Cal. Acad. 1: 37 (17). d. Feb. I884.

De Toni in Sacc. Syll. $7: 838$ d. 28 Oct. 1888.

Klebahn, Ber. Deutsch. Bot. Ges. 8: (69). 29 Dec. I89o.

Blasdale, Asa Gray Bulletin $1893^{3}$ : 2. I893.

Tubeuf-Smith, Diseases of Plants 4I5. 1897.

Massee, Textbk. PI. Diseases 264. f. 68. 1899.

Aecidium helianthatum, S.

See Aecidium Helianthi-mollis, S.

Aecidium Helianthellæ, Arth.

NoTe. In Bull. Torr. Bot. Club 31: 5. I9 Jan. 1904 Arthur describes Puccinia Helianthelle suggesting that this Aecidium may belong to it.

Arth. in Earle, Bull. N. Y. Bot. Gard. 2: 348. d. 25 April 1902.

Aecidium Helianthi, Pk.

See Aecidium Compositarum, var. Helianthi, Burrill.

Aecidium Helianthi-mollis, S.

NoTE. For account of cultures showing relationship to teleutosporic stage see Arth. Bot. Gaz. 35: 17. 20 Jan. 1903, Arth. Jour. Myc. 10: I2, 20. 30 Jan. 1904 and Kellerm. Jour. Myc. 9:230-232. 3I Dec. I903.

S. Syn. Car. 68 (42) no. 450. d. I822.

Lk., L. Sp. Pl. ed. Willd. 6² : 52. $d$. $^{2} 825$.

Hitchcock, Cat. Pl. Amherst 59. I829.

Hitchcock, Cat. Pl. Mass. 644. 1833. 2 ed. 645 (125). I835.

M. A. Curtis, Bot. N. Car. I24. I867.

Caoma asteratum, Lk. p. p.

Lk., L. Sp. Pl. ed. Willd. $6^{2}: 5$ I. d. 1825.

Uredo Asterum, Spreng. p. p.

Spreng. in L. Syst. Veg. I6 ed. $4^{1}$ : 572. $d . \quad$ I827.

Croma helianthatum, S.

S. Syn. Am. Bor. 292 no. 2871 . I834. 
Aecidium helianthatum, S.

S. Syn. Am. Bor. 309. 1834.

Kellerm. \& Carl. Tr. Kans. Acad. 10: 9r. I887.

Aecidium Heliotropil, Tracy \& Gall.

See Aecidium biforme, Pk.

Aecidium hemisphæricum, $\mathrm{Pk}$.

Note. This is understood to be the xecidial stage of Puccinia hemispharica, (Pk.) Ell. \& Ev.

Pk. Bot. Gaz. 3: 34. d. April 1878.

Wint. Hedw. 22: 68 (2). May 1883.

De Toni in Sacc. Syll. $7: 805$. d. 28 Oct. 1888.

Kelsey, Jour. Myc. $5: 80$. June 1880 .

*Ell. \& Ev. N. A. F. 2221. I880.

Webber, Am. Nat. 24: 179. Feb. 1890.

*Ell. \& Ev. N. A. F. 2993. Oct. 1893.

Tracy \& Earle in Greene, Pl. Baker. 1: 18. 22 Feb. 1901.

Earle in Greene, Pl. Baker. 2 : 2. 25 March I9OI.

Lindr. Acta Soc. Fauna et Fl. Fennica 209 : 10. 1901.

Aecidium hepaticatum, $\mathrm{S}$. See Aecidium punctatum, $\mathrm{P}$.

Aecidium Hibisei, M. A. Curtis. See Aecidium hibisciatum, $\mathrm{S}$.

Aecidium hibiscíatum, $\mathrm{S}$.

Note. Kellerman in Jour. Myc. 9: I09. 29 May I903, and Jour. Myc. 9:232. 3I Dec. 1903 gives account of cultures by which he produced this Aecidium from teleutospores of Puccinia Muhlenbergic, Arth. See Puccinia hibisciala, (S.) Kellerm. See also Syd. Mon. Ured. I: 765. I Nov. I903.

S. Syn. Am. Bor. 309. I 834 .

B. Grev. 3: 61 (103). Dec. I874.

Wint. \& Demet. Hedw. 24: 180 (4). Oct. 1885

Burrill, Bull. III. Lab. 2: 224. $d .1885$ and Rept. III. Ind. Univ. 12 : I4I. $d$. 1885.

Ludwig. Centralbl. Bact. 1 : 692. June 1887.

*Ell. \& Ev. N. A. F. 1830.1887.

De Toni in Sacc. Syll. 7 : 781. d. 28 Oct. 1888.

Webber, Bull. Nebr. Exp. Sta. 1 : 332 (62). 18 Dec. I 889.

Gall. Bull. U. S. Agr. Veg. Pathol. 8: 55. 1889.

Webber, Rept. Nebr. Agr. 1889: 2 II (7I). 1890.

Lagerh. Tromsœ Mus. Aarshefter 17 : I02. d. 1895.

Underw. \& Earle, Bull. Ala. Exp. Sta. 80: 209. 28 June 1897.

*Ell. \& Ev. Fung. Columb. 1297. May I 898.

Earle in Mohr. Contr. U. S. Nat. Herb. 6 : 192. 31 July rgor.

* Kellerm. Ohio Fungi 82. d. Oct. I902.

Kellerm. Jour. Myc. 8: 120. d. I4 Dec. 1902.

Kellerm. Jour. Myc. 9: 100. 29 May 1903.

Kellerm. Jour. Myc. 9: 170. 29 Oct. I903.

Kellerm. Jour. Myc. $9: 232,238$. 3I Dec. I903.

Cacoma hibisciatum, $\mathrm{S}$.

S. Syn. Am. Bor. 293 no. 2877 . d. 1834. Aecidium Hibisci, M. A. Curtis.

M. A. Curtis, Bot. N. Car. 123. 1867.

Aecidium hieraciatum, $\mathrm{S}$.

S. Syn. Am. Bor. 309. 1834 .

Caoma hieraciatum, S.

S. Syn. Am. Bor. 292 no. 2368. d. 1834. 
PAecidium Compositarum, Auct. Amer. p. p.

Freeman, Minn. Bot. Studies 11. 5: 556. 20 July I $9 \mathrm{CI}$.

Aecidium Holbœllii, Hornem.

See Puccinia Holboellii, Rostr.

Aecidium Holwayi, (Syd.) n. n.

PPeridermium, sp. indet. On Pseudotsuga.

Halsted, Bull. lowa Agr. Coll. 1886 : 59. I887.

Peridermium Holwayi, Syd.

Syd. Ann. Myc. 1: 19. d. Jan. 1903.

Aecidium houstoniatum, S.

S. Syn. Am. Bor. 309.1834 .

Pk. Rept. N. Y. Mus. 22: 93. I 860.

Pk. Rept. N. Y. Mus. 24: I06. I872.

Pk. Rept. N. Y. Mus. 25: rog. Sept. I873.

Frost in Cat. PI. Amherst 85. I875.

Pk. Rept. N. Y. Mus. $29: 77 . \quad$ I 878.

Burrill, Bull. Ili. Lab. 2 : 229. d. I885 and Rept. III. Ind. Univ. 12 : I 43 . $d$. 1885 .

*Ell. N. A. F. 1422 . I 885 .

Cobb, List. Pl. Amherst 40. 1887.

De Toni in Sacc. Syll. $7: 795 . d$. 28 Oct. 1888.

Gall. Bull. U. S. Agr. Veg. Pathol. 8: 55. 1889.

Jennings, Bull. Tex. Exp. Sta. 9: 23. May I890.

Millsp. Bull. W. Va. Exp. Sta. 24 : 508 . June 1892.

Detmers, Bull. Ohio Exp. Sta. 44: I39. Sept. I892.

*Pazschke, Fung. Eur. 3936. Feb. I 894.

*Ell. \& Ev. Fung. Columb. 497. I894.

Kellerm. \& Werner, Geol. Ohio $\mathbf{7 2}^{2}: 346 . \quad J a n . ~ I 895$.

Millsp. \& Nutt. Publ. Field Columb. Mus. Bot. 1 : I $30 . \quad$ Jan. I896.

*Ell. \& Ev. Fung..Columb. 1078.22 Dec. 1896.

Atk. Bull. Cornell Univ. 3: 21. 25 June I 897.

Orton, Rept. Vt. Exp. Sta. 11 : 2 I I. Sept. I 898 and Contr. Bot. Vt. $2:$ I $30^{-}$Sept. I 808.

Barthol. Tr. Kans. Acad. 16 : I89. June 1899.

Pk. Rept. N. Y. Mus. 52:665. Dec. I899. [Bull. 5 : no. 25.].

Earle in Mohr, Contr. U.S. Nat. Herb. 6: 193. 3I July I90I.

Patterson, Bull. U. S. Agr. Pl. Industry 8: 8. 3 Feb. 1902.

Croma houstoniatum, $\mathrm{S}$.

S. Syn. Am. Bor. 293 no. 289 I. $d$. I 834.

Aecidium oldenlandianum, Underw. \& Earle non Ell. \& Tracy.

Underw. \& Earle, Bull. Ala. Exp. Sta. 80: 209. 28 June 1897.

Aecidium hyalinum, (Cke.) n. n.

Raestelia hyalina, Cke.

Cke. Bull. Soc. Bot. Fr. 241 : 315. d. 22 Oct. 1877.

Cke. Hedw. $17: 38$. d. March I878.

Cke. Grev. 6: 137. pl. 10I, f. 15. June 1878 and Grev. $7: 96$. March 1879 .

*Rav. Fung. Am. 37. 1878 .

Farl. Anniv. Mem. Boston Soc. 3I. d. I880.

Seymour, Tr. Am. Hort. Soc. 4 : I 55 (4). 7 April 1887.

Farl. Suppl. List Works on N. Am. Fungi p. 6 no. 687. 1888.

De Toni in Sacc. Syll. $7: 834$. d. 28. Oct. 1888.

Aecidium hydnoideum, B. \& C.

Note. In Jour. Myc. Io: 19, 20. $30 \mathrm{Jan}$. 1904 Arthur gives an account of cultures of this Aecidium producing teleutospores on Bromus cilinlus. See Puccinia hydnoidea, (B. \& C.) Arth. 
*Rav. Fung. Car. 4: 94. 1855.

Pk. Rept. N. Y. Mus. 22 : $93 . \quad$ r 860.

Pl. Rept. N. Y. Mus. 24: 106. 1872.

B. Grev. 3: 6I (103) no. 582. $d$. Dec. 1874.

Frost in Cat. Pl. Amherst 84. $\quad 1875$.

Pk. Rept. N. Y. Mus. $29: 77 . \quad$ I 878.

*Thm. Myc. Univ. 1120 . 1878 .

*Wint. Fung. Eur. 301\%. 1884.

Arth. Bull. lowa Agr. Coll. 1884: I66. Jan. 1885.

Wint. \& Demet. Hedw. 24: 180 (4). Oct. I885.

*Roum. Fung. Gall. 3862.1886.

Roum. Rev. Myc. 8: 196. I Oct. 1886.

*Ell. \& Ev. N. A. F. 1816.1887.

Cobb, List. Pl. Amherst 40. I887.

De Toni in Sacc. Syll. $7: 790$. d. 28 Oct. 1888.

Gall. Bull. U. S. Agr. Veg. Pathol. 8: 55.1880.

Detmers, Bull. Ohio Exp. Sta. 44: 1 39. Sept. 1892.

Davis, Tr. Wis. Acad. 9: 182. 21 Aug. 1893.

Underw. Proc. Ind. Acad. 1893: 50. Aug. I 894.

Kellerm. \& Werner, Geol. Ohio $7^{2}$ : 346. Jan. I895.

Lagerh. Tromsn Mus. Aarshefter 17: I02. $d . \quad$ I 895.

Underw. \& Earle, Bull. Ala. Exp. Sta. 80: 209. 28 June 1897.

Freeman, Minn. Bot. Studies 11. 5 : 558.20 July 1901.

Earle in Mohr, Contr. U.S. Nat. Herb. $6: 193.31$ July 1901.

Patterson, Bull. U. S. Agr. Pl. Industry 8: 8. 3 Feb. 1902.

Ricker, Univ. of Maine Studies 3: 5 I. 30 April 1902.

Aecidium Hydrophylli, $\mathrm{Pk}$.

Pk. Bull. Buf. Soc. 1: 68. d. July 1873 .

Pk. Rept. N. Y. Mus. $26: 78$. $d$. April $\mathbf{1} 874$.

Pk. Rept. N. Y. Mus. 29 : 77.1878.

Arth. Bull. lowa Agr. Coll. 1884: I66. Jan. I885.

Burrill, Bull. III. Lab. $2: 234$. $d$. 1885 and Rept. Ill. Ind. Univ. 12 : I47. $d$. 1885 .

De Toni in Sacc. Syll. $7: 81$ o. d. 28 Oct. 1888

Webber, Bull. Nebr. Exp. Sta. 1: $332(62)$. 18 Dec. 1889.

Webber, Rept. Nebr. Agr. 1889: 2 II (7I). 1890.

Ell. \& James in Holzinger, Contr. U. S. Nat. Herb. $3: 276.23$ Nov. 1895 .

Nelson, Rept. Wyo. Exp. Sta. 10 : (35). 1900.

Tracy \& Earle in Greene, Pl. Baker. 1: 18. 22 Feb. 1901.

Earle in Greene, Pl. Baker. 2 : 2. 25 March Igor.

*Syd. Uredineen 1544. April I90I.

Freeman, Minn. Bot. Studies II. 5: 559. 20 July I 90 I.

Blasdale in Davy, Bull. U. S. Agr. Pl. Industry 12: 75. 1902.

Cockerell, Jour. Myc. 10 : 51. 19 March 1904.

Aecidium hypericatum, S.

See Aecidium Hyperici-frondosi, S.

Aecidium Hyperiei-Prondosi, S.

NoTE. The teleutosporic stage is Uromyces Hyperici-frondosi, (S.) Arth.

S. Syn. Car. 68 (42) no. 451. d. 1822.

M. A. Curtis, Bot. N. Car. 124. 1867.

*Ell. \& Ev. N. A. F. 2209 . Feb. 1889.

Garrett, Tr. Kans. Acad. 18 : 150. 190?.

Caoma hypericatum, Lk.

Lk., L. Sp. Pl. ed. Willd. $6^{2}: 58 . d$. 1825.

S. Syn. Am. Bor. 293 no. 2883 . ${ }_{1} 834$. 
Uredo Hyperici, Spreng.

Spreng. in L/ Syst. Veg. I6 ed. $41^{1}: 572 . d$. 1827. Aecidium hypericatum, S.

S. Syn. Am. Bor. 309. I 834.

Watt, Can. Nat. \& Geol. Il. 2 : 390. Oct. 1865.

Cke. Proc. Portland Soc. $1:$ i 81 . d. 1869.

Pk. Rept. N. Y. Mus. 25 : 92. Sept. 1873.

Pk. Rept. N. Y. Mus. $29: 76 . \quad 1878$.

Arth. Bull. lowa Agr. Coll. 1884: i66. Jan. 1885.

De Toni in Sacc. Syll. $7: 78 \mathrm{I} . d$. 28 Oct. 1888.

*Ell. \& Ev. N. A. F. 2994. Oct. I 893.

Tracy \& Earle, Bull. Miss. Exp. Sta. 34: 90. May 1895 .

Patterson, Bull. U. S. Agr. Pl. Industry 8:8. 3 Feb. 1902.

Ricker, Univ. of Maine Studies 3: 5 I. 30 April 1902.

Aecidium oblongum, Bon.

Bon. Abh. Nat. Ges. Halle 5 : 212 (46). d. 1860.

Aecidium minutissimum, Gerard.

Gerard, Bull. Torr. Bot. Club $5: 40 . d$. Sept. 1874 and Hedw. 13 : 173 . d." Nov. 1874 .

Aecidium Hypericorum, B. \& C.

B. Grev. 3: 6I (103) no. 58I. d. Dec. I874.

De Toni in Sacc. Syll. $7:$ 781. $d$. 28 Oct. 1888.

Underw. \& Earle, Bull. Ala. Exp. Sta. 80: 209. 28 June 1897.

Earle in Mohr, Contr. U. S. Nat. Herb. 6 : 193. 31 July 1901.

Aecidium Hypericorum, B. \& C.

See Aecidium Hyperici=frondosi, S.

Aecidium ilicinum, Ell. \& Ev.

Ell. \& Ev. Bull. Torr. Bot. Club 24: 284. d. 29 June 1897.

Lindau \& Syd. Hedw: 37 : xvii. 9 April r 898. [Beiblatt no. 7.]

Sacc. \& Syd. Syll. 14: 373. $d$. 20 Aug. I899.

Aecidium impatientatum, $\mathrm{S}$.

See Aecidium Impatientis, S.

Aecidium Impatientis, S.

NOTE. As the result of cultures Arthur in Bot. Gaz. 35: 18. 20 Jan. 1903, states this to be the recidial stage of Puccinia Impatientis, (S.) Arth. See also Klebahn, Rostpilze 292. 1904 and Arth. Jour. Myc. 10: 11, 20. 30 Jan. 1904.

S. Syn. Car. 67 (4I) no. 442. d. I822.

Lk., L. Sp. Pl. ed. Willd. 6² : 57. $d$. 1825 .

M. A. Curtis, Bot. N. Car. I24. I867.

Pk. Rept. N. Y. Mus. $23: 59$. 1872.

Pk. Rept. N. Y. Mus. 24: 107. 1872.

*Thm. Myc. Univ. 1121. 1878.

Wint. \& Demet. Hedw. 24: 180 (4). Oct. 1885.

*Wint. Fung. Eur. 3318.1885 .

Burrill, Bull. Ill. Lab. 2 : 224. d. 1885 and Rept. Ill. Ind. Univ. 12 : I 4 I. $d$. I 885 .

Roum. Rev. Myc. 8 : 197. I Oct. 1886.

* Roum. Fung. Gall. $3867 . \quad$ I 886.

De Toni in Sacc. Syll. $7: 784$. d. 28 Oct. 1888.

Webber, Bull. Nebr. Exp. Sta. 1 : 332 (62). 18 Dec. 1889.

Gall. Bull. U. S. Agr. Veg. Pathol. 8: 55. I889.

Webber, Rept. Nebr. Agr. 1889: 2II (7I). I890.

Detmers, Bull. Ohio Exp. Sta. 44 : 1 39. Sept. 1892.

Detmers, Bull. Ohio Exp. Sta. tech. ser. 1: 178. April 1893.

*Ell. \& Ev. Fung. Columb. 196. 1893. 
Underw. Proc. Ind. Acad. 1893: 50. Aug. I 894.

Kellerm. \& Werner, Geol. Ohio 72: 346. Jan. 1895.

Millsp. \& Nutt. Publ. Field Columb. Mus. Bot. Ser. 1 : 1 30. Jan. 1896.

*Shear, N. Y. Fungi 323. March i 896.

Stevens, Rept. Ohio Acad.4: 20. 1896.

Atk. Bull. Cornell Univ. 3:21. 25 June 1897.

Underw. \& Earle, Bull. Ala. Exp. Sta. 80: 209. 28 June 1897.

Claassen, Rept. Ohio Acad. 5 : $68 . \quad$ I897.

Snyder, Proc. Ind. Acad. 1896: 217. 1897.

Orton, Rept. Vt. Exp. Sta. 11:211. Sept. 1808 and Contr. Bot. Vt. 2 : I3. Sept. 1898 .

Barthol. Tr. Kans. Acad. 16 : 189 . June 1899.

*Syd. Uredineen 1346 . I809.

Freeman, Minn. Bot. Studies II. 5 : 556. 20 July 1901.

Earle in Mohr, Contr. U. S. Nat. Herb. 6 : 193. 3I July 1901.

Kellerm. Ohio Nat. 2 : 136. $d$. Nov. I901.

* Kellerm. Ohio Fungi $t$. d. Dec. I901.

Patterson, Bull. U. S. Agr. PI. Industry 8: 8. 3 Feb. 1902.

*Griffiths, West Am. Fung. 299. 22 March 1902.

Arth. Bot. Gaz. 35: $18.20 \mathrm{~J}$ an. 1903.

Syd. Mon. Ured. 1:751. I Nov. 1903.

Uredo Schweinitziana, Spreng.

Spreng. in L. Syst. Veg. 16 ed. $41^{1}$ : $571 . d$. 1827.

Caoma impatientatum, S.

S. Syn. Am. Bor. 293 no. 2880.1834. Aecidium impatientatum, $\mathrm{S}$.

S. Syn. Am. Bor. 309. 1834 .

Pk. Rept. N. Y. Mus. 29: 76. I878.

*Ell. N. A. F. 1001 . I883.

Trel. Tr. Wis. Acad. 6: 135 (31). Nov. 1884.

Arth. Bull. lowa Agrr. Coll. 1884 : ı66. Jan. 1885.

Kellerm. Bull. Wash. Lab. 1 : 75. Jan. 1885 and Tr. Kans. Acad. 9 : 8I. 1885.

Kellerm. \& Carl. Tr. Kans. Acad. 10:91. 1887.

Aecidium incurvum, Tracy \& Earle.

Note. See also Aecidium erigeronatum, $S$.

Tracy \& Earle in Greene, Pl. Baker 1: 18. d. 22 Feb. I901.

Sacc. \& Syd. Syll. 16:1121. I Feb. 1902.

Aecidium intermixtum, $\mathrm{Pk}$.

NOTE. "This occurs in company with Puccinia intermixta, of which it is probably a form." Peck 1879 .

Pk. Bot. Gaz. 4 : 231. d. Nov. 1879.

Langlois, Cat. PI. La. $27 . \quad 1887$.

De Toni in Sacc. Syll. $7: 800$. d. 28 Oct. 1888.

Kelsey, Jour. Myc. 5: 80 . June 1889.

*EIl. \& Ev. N. A. F. 2405. April I 890.

*Ell. \& Ev. Fung. Columb. 63. 1893.

Tracy \& Earle in Greene, Pl. Baker.1 : 18. 22 Feb. 1901

Syd. Mon. Ured. 1 : I01. 30 June 1902.

Aecidium interveniens, (Pk.) n. n.

NotE. Possibly the Aecidium on Malvastrum mentioned by Farlow in Bull. Bussey Inst. 2 : 242. Jan. 1878 as growing with Puccinia Malvastri may be the same as Aecidium interveniens, $1 \mathrm{k}$. but although growing with the Pucrinia there is no proof that it is a stage of it. Compare also Aecidium Malvastri, Ell. \& Tracy and Aecidium Sphoralcee, Ell. \& Ev. 
Roestelia interveniens, $\mathrm{Pk}$.

Pk. Bull. Torr. Bot. Club $10: 74 . d$. July 1883 .

Halsted, Bull. lowa Agr. Coll. 1886 : $58 . \quad$ I887.

De Toni in Sacc. Syll. $7: 834 . d$. 28 Oct. 1888.

Aecidium Ipomœæ, B.

See Cystopus Ipomex-panduranz, Farl.

Aecidium Ipomœæ-panduranæ, S.

See Cystopus Ipomoz-panduranz, Farl.

Aecidium Iridis, Gerard.

Gerard in Pk. Rept. N. Y. Mus. 24: 93. d. 1872.

Pk. Rept. N. Y. Mus. 29: 77. $\quad 1878$.

*Thm. Myc. Univ. 1519. 1880.

*Wint. Fung. Eur. 2927. 1883.

*Ell. N. A. F. 1014. 1883 .

Arth. Bull. lowa Agr. Coll. 1884 : I66. Jan. I885.

De Toni in Sacc. Syll. $7: 827 . d$. 28 Oct. 1888.

* Roum. Fung. Gall. 4917. 1889 .

Roum. Rev. Myc. 11: 129. July 1889.

Webber, Bull. Nebr. Exp. Sta. 1: 332 (62). 18 Dec. 1889.

Webber, Rept. Nebr. Agr. 1889: 211 (71). 1800.

Cheney, Tr. Wis. Acad. 10:69. Oct. I895.

Davis, Tr. Wis. Acad. 11: 174 . Aug. 1897.

Tubeuf-Smith, Diseases of Plants 4II. 1897.

Freeman, Minn. Bot. Studies 11. 5:554. 20 July I9OI.

Aecidium Isnardiæ, Lagerh.

NoTE. This species of Lagerheim is apparently founded on Lea's specimen in Herb. Fries and we have placed under the name Aecidium Isnardia, Lagerh. all the references to Aecidia as quoted on Isnardia. It is not improbable that Aecidium Isnardia should be referred to Aecidium Ludzigia, Ell. \& Ev. published two years earlier ${ }_{2}$ but the specimens of the first named species which we have examined are not sufficient to settle that point.

Lagerh. Tromsoe Mus. Aarshefter 17 : 102. $d$. 1895.

Sacc. Hedw. 35 : xviii. 25 Feb. 1896. [Reportorium No. 7.]

Sacc. \& Syd. Syll. 14: 376. d. 20 Aug. I 899.

Aecidium Epilobii, Auct. Amer. p. p.

Lea, Cat. Pl. Cin. 71. I 849.

M. A. Curtis, Bot. N. Car. 123. 1867.

Cke. Handbook 536. d. 1871 .

B. Grev. 3: 61 (103). Dec. I874.

James, Jour. Cin. Soc. 2: 67 (26). April 1879.

Kellerm. \& Werner, Geol. Ohio $7^{2}$ : 346. Jan. 1895.

Ascidium isomerinum, $\mathrm{Pk}$.

Pk. Bull. Torr. Bot. Club $10: 75 . d$. July 1883 .

Hark. Bull. Cal. Acad. 1: 262 (7). Io Nov. 1885.

De Toni in Sacc. Syll. 7 : 779. d. 28 Oct. 1888.

Aecidium Jacobæ, Auct. Amer.

See Aecidium Compositarum, var. Senecionis. Auct. Amer.

Aecidium Jacquemontir, Ell. \& Ev.

Mexicu.

Ell. \& Ev. Jour. Myc. 8: il. d. 31 May 1902.

Aecidium Jamesianum, Pk.

NotE. As the result of cultures Arthur states this to be the æcidial stage of Puccinia Bartholontai, Diet. = Puccinia Famesiana, (Pk.) Arth. See Arth. Bot. Gaz. 35 : 18.20 Jan. 1903. See also Syd. Mon. Ured. 1 : 734. I Nov, 1903 and Klebahn, Rostpilze 282. 1904. 
Pk. Bot. Gaz. 5 : 34. d. March I 880.

*Ell. N. A. F. 1012. I883.

Trel. Tr. Wis. Acad. 6 : 135 (31). Nov. 1884.

Arth. Bull. lowa Agr. Coll. 1884 : 166. Jan. 1885.

Kellerm. Bull. Wash. Lab. 1: 75. Jan. i 885 and Tr. Kans. Acad. 9 : 81. 1885 .

Burrill, Bull. 11l. Lab. 2 : 236. d. 1885 and Rept. Ill. Ind. Univ. 12 : I47. $d$. 1885 .

Kellerm. \& Carl. Tr. Kans. Acad. 10 : 9I. 1887.

De Toni in Sacc. Syll. $7: 808 . d$. 28 Ohio I 888.

Webber, Bull. Nebr. Exp. Sta. 1 : 332 (62). 18 Dec. I889.

Gall. Bull. U. S. Agr. Veg. Pathol. 8: 56.1880.

Webber, Rept. Nebr. Agr.1889: 2II (7I). I890.

Williams, Bull. S. Dak. Exp. Sta. 29 : 49. Dec. I80I.

Smith \& Pound, Bot: Surv. Nebr. 2: 28. 15 April 1893.

Pound, Bot. Surv. Nebr. 3: 9. 18 June 1894 .

*Carl. Ured. Amer. 32.1894.

*Ell. \& Ev. Fung. Columb. 556. I 894.

Tubeuf-Smith, Diseases of Plants 4II. 1897.

Barthol. Tr. Kans. Acad. 16: 189. June 1899.

Freeman, Minn. Bot. Studies II. 5: 558. 20 July I90I.

*Barthol., Ell. \& Ev. Fung. Columb. 1603. Oct. 1902.

Arth. Bot. Gaz. 35 : 18.20 Jan. 1903.

Garrett, Tr. Kans. Acad. 18 : 147. 1903.

\section{Aecidium Kellermanni, De Toni.}

De Toni in Sacc. Syll. 7 : 788. d. 28 Oct. I888.

Barthol. Tr. Kans. Acad. 16: I90. June I899.

Garrett, Tr. Kanis. Acad. 18: I47. I903.

Aecidium amphigenum, Ell. \& Kellerm.

Ell. \& Kellerm. Jour. Myc. $2: 4$. d. Jan. I 886.

Kellerm. \& Carl. Tr. Kans. Acad. 10: 9I. 1887.

\section{Aecidium laceratum, Sow.}

NoTE. Under Aecidium laceral $n m$ we have included references which belong to the aecidium of Gymnosporangium clavariaforme. The name Aecidium laceratnm has, however, been applied by some American writers also to the aecidia of Gymnosporanginm globosum and some of the references given below may belong to that species.

? Watt, Can. Nat. \& Geol. 11. 2: 391. Oct. 1865.

Roestelia lacerata, Mer.

B. Grev. 3: 59 (IOI). Dec. 1874 .

Farl. Proc. Am. Acad. 20: 318 . 1885.

Farl. Bot. Gaz. 11 : I90. July 1886.

Farl. Bot. Gaz. 11: 238, 241. Sept. I 886.

Thax. Proc. Am. Acad. 22 : 263, 265-267. Jan. I887 and Centralbl. Bact. 1 : $432,458-460 . \quad$ I 887.

Seymour, Tr. Am. Hort. Soc. 4 : 155 (4). 7 April 1887.

Thax. Bot. Gaz. 14: 164, 167. July 1880.

Tubeuf Centralbl. Bact. 9 : 95. 2 Feb. I89I.

Thax. Bull. Conn. Exp. Sta. $107: 5$. I5 April 1891.

Richards, Proc. Am. Acad. 31 : 265. 1806.

? Raestelia lacerata, Auct. Amer. p. p.

Sprague, Proc. Boston Soc. 5 : 329. $\quad{ }_{1} \delta 56$.

Bundy, Geol. Wis. I873-79, $1: 400 . \quad$ I 883.

Bennett, Cat. Pl. R. I. $89 . \quad$ I 888.

Fairman, Proc. Rochester Acad. 1 : 47. Aug. I890.

\section{Host unknown.}


P? Rœestelia lacerata, Auct. Amer. p. p.

Russell, Am. Nat. 2 : 568. f. 2. Jan. 1869.

Cke. Proc. Portland Soc. 1: i80. d. I869.

Pk. Rept. N. Y. Mus. 22: 93. 1869.

? Roestelia lacerata, Auct. Amer. p. p.

NotE. Some of the five following references may relate, at least in part, to Aecidium Nidus-avis, (Thax). R. T.

Pk. Rept. N. Y. Mus. 24: I03. 1872.

Farl. Bull. Bussey Inst. 2: 225. Jan. 1878.

Pk. Rept. N. Y. Mus. $29: 76$. I878.

Farl. Anniv. Mem. Boston Soc. 29. I880.

Somers, Tr. N. Scot. Inst. $7: 465 . \quad$ I 890.

Roestelia penicillata, Auct. Amer. p. p.

Farl. Anniv. Mem. Boston Soc. 30. I880.

*Ell. N. A. F. $1086 c$. 1883 .

PRoestelia penicillata, Auct. Amer. p. p.

Pk. Rept. N. Y. Mus. 34: 46 . Oct. 1883.

Trel. Tr. Wis. Acad. 6 : 138 (34). Nov. 1884.

Farl. Proc. Am. Acad. 20 : $318 . \quad$ I 885.

Bessey, Am. Pomol. Soc. 1885 : 43. I886.

Aecidium on Larix.

Mayr, Waldungen von Nordamer. 221. I8go.

Aecidium lateripes, Kellerm.

Note. This name is given to an æcidium produced on Ruellia strepens by sowing teleutospores of Puccinia lateripes, B. \& Rav. from the same hostplant. See also Kellerm. Jour. Myc. 9: IO7-109. 29 May 1903. See also Carl. Tr. Kans. Acad. 14: 44. 1896. See also Aecidium Tracyanum, Syd.

Kellerm. Jour. Myc. 9 : 234, 238. 31 Dec. 1903.

Aecidium lauratum, Watt.

NoTE. This is a mere catalogue name and there is no clue to its identity.

Watt, Can. Nat. \& Geol. II. 2 : 391. Oct. I865.

Aecidium on Lechea.

NotE. Berkeley in Grev. 3: 60 (IO2). Dec. I874 reports an Aecidium on Lechea which is quoted by Syd., Sacc. Syll. 13:647. 1898. The name given is Aecidium Compositarum. There is probably an error in the name of the host.

Aecidium Ledi, Herb. Auerswald.

Note. This species was never described, but is cited in Beitr. Biol. Pflanzen 3: 52. 1883. There Schroter states that it is the same as the uredo of Chrysomyxa Ledi, (A.\& S.) D By.; but in Ber. Schles. Ges. 1887 : 279 he refers it to Uredo ledicola, Pk.

Aecidia on Leguminosæ.

NOTE. The confusion and uncertainty which prevail among American mycologists in the use of old European names of Aecidia on Leguminose, such as Aecidium Leguminosarum, Aecidium Orobi, etc., is so great that we have here arranged the Aecidia which have been reported on species of that order alphabetically by hosts, giving also for convenience cross references to the species whose literature is comparatively clear.

Aecidium on Leguminosæ, sp. indet. Aecidium Orobi, Auct. Amer. p. p.

Watt, Can. Nat. \& Geol. 11. 2 : 39i. Oct. I865.

Aecidium on Amphicarpæa.

Arth. Jour. Myc. 10: 14. 3o Jan. 1904. 
Aecidium leguminosatum, Auct. Amer. p. p.

Arth. Bull. lowa Agr. Coll. 1884: 166. Jan. I885.

Aecidium Orobi, Auct. Amer. p. p.

Burrill, Bull. III. Lab. 2: 226. d. 1885 and Rept. III. Ind. Univ. 12 : I42. $d$. 1885.

Gall. Bull. U. S. Agr. Veg. Pathol. 8: 56.1880.

Claassen, Rept. Ohio Acad. 5 : 68. 1897.

Freeman, Minn. Bot. Studies 11. 5: 554. 20 July I90I. Aecidium Phaseoiorum, Auct. Amer.

*Ell. N. A. F. 1436 . I 885.

Aecidium on Apios.

Arth. Jour. Myc. 10: 14. 30 Jan. 1904.

Aecidium ieguminosatum, Auct. Amer. p. p.

Arth. Bull. lowa Agr. Coll. 1884: I66. Jan. I885.

Aecidium Orobi, Auct. Amer. p. p.

Freeman, Minn. Bot. Studies 11. 5: 554. 20 July 1901.

Aecidium on Baptisia.

See Aecidium Keliermannii, De Toni.

Aecidium on Cassia.

See Aecidium Cassia, Ell. \& Kellerm.

Aecidium on Dalea.

See Aecidium Daiez, Kellerm. \& Swingle.

Aecidium on Desmodium.

Aecidium Orobi, Auct. Amer. p. p.

B. Grev. 3 : 61 (103). Dec. 1874

Underw. \& Earle, Bull. Ala. Exp. Sta. 80: 209. 28 June 1897.

Earle in Mohr, Contr. U.S. Nat. Herb. 6 : 193. 3I July I9oI.

Aecidium on Lathyrus.

Aecidium Orobi, Auct. Amer. p. p.

Nelson, Rept. Wyo. Exp. Sta. 10: (36). 1900.

Tracy \& Earle in Greene, Pl. Baker. 1: 19. 22 Feb. 190I.

See also Aecidium album, Clint.

Aecidium on Lespedeza.

See Aecidium leucostictum, B. \& C.

Aecidium on Lupinus.

See Aecidium Lupini, Pk.

Aecidium on Phaseolus.

Note. An Aecidium on Phaseolus produced by sowing teleutospores of bean rust is reported by Arthur, Jour. Myc. 10: 14. 30 Jan. 1904. See Uromyces appendiculatus. (P.) Lev. Aecidium Phaseolorum has been reported on Amphicarpaa. See above.

Aecidium on Psoralea.

Aecidium porosum, Auct. Amer. p. p.

Arth. Bull. lowa Agr. Coll. 1884: 167. Jan. 1885.

See also Aecidium Onobrychidis, Burrill.

See also Aecidium Psoraiex, Pk.

Aecidium on Trifolium.

NoTr. In Tr. Wis. Acad. 14: 10I. Sept. 1903 Davis mentions an Aecidium on Trifolium repens as belonging to Uromyces minor, Schrt.

See also Aecidium elegans, B. \& C.

See also Aecidium Trifolit-repent is, Cast.

Aecidium on Vicla.

See Aecidium aibum, Clint. 
Aecidium Leguminosarum, N. A. F. 2216.

See Aecidium Lupini, Pk.

Aecidium leguminosatum, Auct. Amer. p. p.

See on Amphicarpaa and Apios under Aecidium on Leguminosa.

Aecidium Lepidii, Tracy \& Gall.

Tracy \& Gall. Jour. Myc. 4: 2I. d. March 1888.

De Toni in Sacc. Syll. $7: 779$. d. 28 Oct. I888.

Anderson, Jour. Myc. 5: 82. June I889.

Webber, Bull. Nebr. Exp. Sta. 1: 332 (62). I8 Dec. I889.

Webber, Rept. Nebr. Agr. 1889: 2 I I (7I). I 890.

Tubeuf-Smith, Diseases of Plants 4IO. I897.

Cockerell, Jour. Myc. 10: 51. I9 March I904.

Aecidium Leptotæniæ, Lindr.

Lindr. Meddel. Stockholms Hoegskolas Bot. Inst. 4: 8. $d$.

8 March IgoI and Bot. Centralbl. 88: 4. 25 Sept. I90I.

Lindr. Acta Soc. Fauna et Fl. Fennica 22 ${ }^{1}: 167 . d$. Ig02.

- Aecidium Aethusa, Ell. \& Ev. non Kirch.

*Ell. \& Ev. N. A. F. 3581. 24 Jan. I898.

*Ell. \& Ev. Fung. Columb. 1298. May I898.

Aecidium leucospermum, D C.

See Aecidium Anemones, P.

See also under Aecidium Ranunculacearum, Auct. Amer. p. p.

Holw. Jour. Myc. 8: 172.20 Dec. I902.

Klebahn, Rostpilze 357. 1904.

Aecidium leucostictum, B. \& C.

Note. Cultures by Arthur show that this belongs to Uromyces Lespedeza,

Pk. which accompanies and follows it. See Arthur, 1904.

B. \& C. in M. A. Curtis, Bot. N. Car. 123. I867.

Burrill, Bull. IIl. Lab. 2: 226. $d . \quad 1885$ and Rept. III. Ind. Univ. 12 I $42 . d$. I885.

*Ell. \& Ev. N. A. F. 1827 . I887.

De Toni in Sacc. Syll. 7 : $787 . d .28$ Oct. I888.

*Ell. \& Ev. Fung. Columb. 59. 1893.

Underw. \& Earle, Bull. Ala. Exp. Sta. 80: 209. 28 June 1897.

Seymour, Rhodora 2: 186. 8 Sept. 1900.

Earle in Mohr, Contr. U. S. Nat. Herb. 6 : I93. 3I July I9oI.

Arth. Jour. Myc. 10: I4, 20.30 Jan. I904.

Aecidium Orobi, var. leucostictum, B.

B. Grev. 3: 6I (I03). d. Dec. I874.

Aecidium Liatridis, (Webber) Eil. \& Anderson.

Ell. \& Anderson, Bot. Gaz. 16: 47. $d$. Feb. I891.

Ell. \& Anderson, Bot. Gaz. 16 : 85. pl. 10 f. $11-16$. March 1891 .

Sacc. Syll. 9 : 325. $d$. 15 Sept. 1891 .

* Carl. Ured. Amer. 38 . I894.

Barthol. Tr. Kans. Acad. 16 : I9o. June I899. Aecidium Compositarum, var. Liatridis, Webber.

Webber, Bull, Nebr. Exp. Sta. 1: $330(60)$. I8 Dec. I889.

Webber, Rept. Nebr. Agr. 1889 : 2 Io (70). $d .1890$.

Smith \& Pound, Bot. Surv. Nebr. 2: 28. I 5 April 1893.

Freeman, Minn. Bot. Studies II. 5: 557. 20 July I90I.

Aecidium Ligustici, Ell. \& Ev.

Ell. \& Ev. Bull. Torr. Bot. Club. $11: 73 . d$. July I 884.

De Toni in Sacc. Syll. $7: 807 . d$. 28 Oct. I 888.

Lindr. Acta Soc. Fauna et Fl. Fennica $22^{1}: 165$. d. 1902. 
Aecidium Ligustri, Strauss.

Pk. Rept. N. Y. Mus. 56:28. May 1903. [Bull. 67].

Note. For references to the connection of this Aecidium with Puccinia oblusata, Otth on Phragmiles communis see Klebahn, Rostpilze 285. I904.

Aecidium Limonil, Pk.

See Aecidium Statices, Desm.

Aecidium Ludwigiæ, Ell. \& Ev.

Note. See also Aecidium Isnardia Lagerh.

Ell. \& Ev. Proc. Phil. Acad. 1893: 155. d. 28 Feb. I893.

Tracy \& Earle, Bull. Miss. Exp. Sta. 34: 90. May 1895.

Sacc. Syll. 11 : 216. d. July 1895.

McClatchie, Proc. S. Cal. Acad. 1: 372. 5 June 1897.

Patterson, Bull. U. S. Agr. Pl. Industry 8 : 8.• 3 Feb. 1902.

Aecidium luminatum, $\mathrm{S}$.

See Gymnoconia interstitialis, (Schl.) Lagerh.

Aecidium Lupini, Pk.

Pk. Rept. N. Y. Mus. 46 : II 3 (33). d. Dec. I 893.

Sacc. Syll. $11: 214 . d$. July 1895 .

Freeman, Minn. Bot. Studies 11. 5: 554. 20 July I9oI.

Aecidium Leguminosarum, N. A. F.

*Ell. \& Ev. N. A. F. 2216. I889.

Aecidium Lycopi, Gerard.

Note. As the result of cultures, this Aecidium is stated to belong to Puccinia angustata Pk. See Arthur, 1899, 1900, 1902, Kellerman, 1903. Although with one exception the name is spelled $L y c o p i$ in references below, the correct spelling should be Lycopodis as given by Ellis, 1896 .

Gerard in Pk. Bull. Buf. Soc. 1 : 68. d. July 1873 .

Gerard in Pk. Rept. N. Y. Mus. $26: 78$. $d$. April 1874.

Frost in Cat. Pl. Amherst 85. 1875.

Pk. Rept. N. Y. Mus. 29:77. 1878.

Burrill, Bull. 1ll. Lab. 2 : $234 . d$. I 885 and Rept. Ill. Ind. Univ. 12 : I46. $d$. I 885 .

Arth. \& Holw. Bull. Minn. Surv. 3: 31. I Oct. 1887.

Cobb, List PI. Amherst 40. I887.

De Toni in Sacc. Syll. $7: 815 . d$. 28 Oct. 1888.

*Ell. \& Ev. N. A. F. 2996. Oct. I 893.

* Shear, N. Y. Fungi 130.1893.

Underw. Proc. Ind. Acad. 1893: 50. Aug. I894.

Cheney, Tr. Wis. Acad. 10:60. Oct. I895.

*Ell. \& Ev. Fung. Columb. 1077. 22 Dec. 1896.

Underw. \& Earle, Bull. Ala. Exp. Sta. 80: 209. 28 June 1897.

Davis, Tr. Wis. Acad. 11 : 174. Aug. 1897.

Tubeuf-Smith, Diseases of Plants 4 I I. I897.

Arth. Science II. 10: 565 . 20 Oct. I 899.

Snyder, Proc. Ind. Acad. 1898 : 189. Dec. 1899.

Arth. Bot. Gaz. 29 : 273. 30 April 1900.

Freeman, Minn. Bot. Studies II. 5: 546. 20 July I90I.

Earle in Mohr, Contr. U. S. Nat. Herb. 6 : 193. 3I July IgoI.

Patterson, Bull. U. S. Agr. PI. Industry 8: 8. 3 Feb. 1902.

Arth. Jour. Myc. 8 : 56. 30 June 1902.

Syd. Mon. Ured. 1: 690. $d$. I Nov. Ig03.

Kellerm. Jour. Myc. 9: 237. 3I Dec. I903.

Aecidium Lygodesmiæ, (Webber) Shear.

*Shear, Ell. \& Ev. Fung. Columb. 1476 . May I9oI. 
Aecidium Compositarum, var. Lygodesmia, Webber.

Webber Am. Nat. 24: : 177. d. Feb. 1890.

Webber, Rept. Nebr. Agr. 1889: 2 I0 (70). d. 1890.

Sacc. Syll. $9: 323$ d. 15 Sept. 1891.

Smith \& Pound. Bot. Surv. Nebr. 2: 28. I 5 April I893.

PAecidium, sp. indet.

Ell. \& Ev. Proc. Phil. Acad. 1893 : 154. d. 28 Feb. 1893.

\section{Aecidium Lysimachiæ, S.}

Note. Aecidium Lysimachia, (Schl.) Wallr., which dates from 1823 , is considered in Europe to be the æcidium of Puccinia Limosa, Magn. Aecidium Lysinachia, S. dates from 1822 , and, if the two are identical, the latter name has priority; but Schweinitzian specimens which we have seen are not in good condition. We have therefore here inserted the references given by writers as Aecidium Lysimachia, S., and added with doubt American references to Accidium Lysimachia, (Schl.) Wallr.

S. Syn. Car. 67 (4I) nó. 438. d. I822.

Lk., L. Sp. Pl. ed. Willd., 62 : 46.1825.

*Ell. N. A. F. 1424. I885.

*Ell. \& Ev. N. A. F. 1424b. Feb. 1839. [lssued with no. 2300.]

Uredo Lysimachia, Spreng.

Spreng. in L. Syst. Veg. 16 ed. $4^{1}$ : 57I. d. 1827.

Croma lysimachiatum, S.

S. Syn. Am. Bor. 292 no. 2863 . I 1834.

PAecidium Lysimachiae, (Schl.) Wallr.

M. A. Curtis, Bot. N. Car. I $24 . \quad$ I 867.

Pk. Rept. N. Y. Mus. 26: 78 . April 1874 .

Trel. Tr. Wis. Acad. 6 : 134 (30). Nov. 1884.

Burrill, Bull. Ill. Lab. $2: 233$. d. 1885 and Rept. Ill. Ind. Univ. 12 : I46. $d$. 1885 .

De Toni in Sacc. Syll. $7: 817$. d. 28 Oct. 1888.

Webber. Bull. Nebr. Exp. Sta. 1 : 332 (62). 18 Dec. 1889.

Gall. Bull. U. S. Agr. Veg. Pathol. 8: 56. I889.

*Ell. \& Ev. N. A. F. 2404. April r89o.

Webber, Rept. Nebr. Agr. 1889: 2 I I (7r). 1890.

Barthol. Tr. Kans. Acad. 16 : 190. June 1899.

Jelliffe, Flora of Long Island 36.1899.

Nelson, Rept. Wyo. Exp. Sta. 10 : (35). 1900.

Freeman, Minn. Bot. Studies 11.5 : 558. 20 July 1901.

Aecidium lysimachiatum, $\mathrm{Pk}$.

Pk. Rept. N. Y. Mus. $29: 77$. I 878.

Aecidium lysimachiatum, $\mathrm{Pk}$.

See Aecidium Lysimachize, S.

Aecidium macrosporum, $\mathrm{Pk}$.

Note. See note under Aecidium Sinilacis, S.

Pk. Rept. N. Y. Mus. 23: 6r. $d$. 1872.

Pk. Rept. N. Y. Mus. 24: 106. 1872.

Pk. Rept. N. Y. Mus. 29: 77, 1878.

De Toni in Sacc. Syll. $7: 829 . d$. 28 Oct. 1888.

Tubeuf-Smith, Diseases of Plants. 4I I. $\quad: 897$.

Barthol. Tr. Kans. Acad. 16 : 190. June 1899.

Aecidium Smilacis, N. A. F.

*Ell. \& Ev. N. A. F. 2708. May 1892.

Aecidium magnatum, Arth.

Arth. Bull. Torr. Bot. Club 28 : 664 . d. 30 Dec. rgor.

*Griffiths, West Am. Fung. 243. 22 March 1902. 
Aecidium Malvastri, Ell. \& Tracy.

Notr. Hennings alsu describes in Hedw. 36:216. I Aug. I897 an Aecidinm Malvastri on Malvastrum spicatum from Argentine Republic. See note under decilium interveniens, ['k. also note under decidiun Sphe. ralceu, Ell. ¿ Ev.

Ell. \& Tracy, Jour. Myc: $7: 43 . d$. 10 Sept. 1801.

Sacc. Syll. $11: 215 . d$. July 1895 .

Aecidium malvicola, Arth.

Artl. Bull. Torr. Bot. Club 31 : 6. d. I9 Jan. I90.1.

Aecidium Mariæ-Wilsoni, Pli.

See A ecidium pedatatum, S.

Aecidium Mariæ-Wilsoni, Halsted. See Aecidium claytoniatum, S.

Halsted, Bull. Torr. Bot. Club 26: 382. 18 July 1897.

Halsted, Rept. N. J. Exp. Sta. $1898: 368 . \quad$ I899.

Aecidium Maublancii, n. $n$.

Note. Aecidinm Mexicanum Maublanc is anteclated by the species of Diet. \& Holw. of the same name.

Maublanc, Bull. Soc. Myc. Fr. $20: 73$. d. pl. 7, f. 9. 30 April 1904.

\section{Aecidium Menthæ, DC.}

Mexico.

Note. The European plant of this name is called the aecillial stage of Puccinia Mentha, $\mathrm{P}$. The infrequent finding of the Aecidium may be significant, considering the abundance of the mint rust. A. B.S.

Pk. Rept. N. Y. Mus. 24: 92, I06. 1872.

Frost in Cat. PI. Amherst 85. 1875.

Pk. Rept. N. Y: Mus. $29: 77.1878$.

Johnson, Bull. Minn. Acad. 1 : 366. d. 1880.

Arth. Bull. lowa Agr. Coll. 1884: I66. Jan. 1885.

Cobb, List Pl. Amherst 40. 1887.

Bennett, Cat. Pl. R. I. 89. I888.

Cockerell, Jour. Myc. 10: 51. I9 March 1904.

Aecidium Mertensiæ, Arth.

Arth. Bull. Torr. Bot. Club 31 : 6. d. I9 Jan. 1904.

Aecidium Mexicanum, Diet. \& Holw.

Holw. Bot. Gaz. 24: 36. d. 3i July i897. Mexico.

Lindau \& Syd. Hedw. 37 : xvii. 9 April ı 898. [Beiblatt no. 7.]

Sacc. \& Syd. Syll. 14: 373. d. 20 Aug. I 899.

Aecidium Mexicanum, Maublanc.

See Aecidium Maublancil, $n$. $n$.

Aecidium on Micromeria.

Note. In Jour. Myc. Io: 54. I9 March 1904 Dudley and Thompsun describe a new species, Puccinia Micromeric, including acidial stage, on Micromeria in California.

Aecidium micropunctum, Ell. \& Ev.

Ell. \& Ev. Jour. Myc. 6: 119. d. 6 Jan. 1891.

Sacc. Syll. $9: 322 . d$. I 5 Sept. I 891.

Webber. Tr. St. Louis Acad. 6 : 15. 12 March 1892 and Contr. Bot. Dept. Univ. Nebr. 3: 15. 14 June 1892 .

Aecidium minutissimum, Gerard.

See Aecidium Hyperici-frondosi, S. 
Aecidium Mirabilis, Diet. \& Holw.

Holw. Bot. Gaz. 24: 37. d. 3I July I 897.

Lindau \& Syd. Hedw. 37 : xvii. 9 April i898. [Beiblatt no. 7.]

Sacc. \& Syd. Syll. 14: 386. d. 20 Aug. 1899.

Aecidium mirabilissimum, Cockerell.

Note. Thought by the author to belong to Puccinia mirabilissima, Pk.

Cockerell, Journ. Myc. 5:85. June i 889 .

Aecidium on Monardella.

Note. On this host Dudley and Thompson in Jour. Myc. 10: 53. 19 March 1904, describe a new species, Puccinia Monardelle, with aecidial stage.

Aecidium monoicum, $\mathrm{Pk}$.

NoTE. The Aecidium of this name has usually been reported on species of Arabis and Sisymbrium, and it may perhaps include two different forms possibly connected with Puccinia IJolballii, (Hornem.) Rostr. and Puccinia consimilis, Ell. \& Ev. See also Aecidium Draba, Tracy \& Gall. and Aecidium Parryi, Yk.

Pk. Bot. Gaz. 4 : 230. d. Nov. I879.

De Toni in Sacc. Syll. 7 : 779. d. 28 Oct. 1888.

Cockerell, Zoe 4 : 284. Oct. 1893.

Jones, Proc. Cal. Acad. II. 5: 730.3 Oct. 1895.

Tracy \& Earle in Greene, Pl. Baker. $1: 18.22$ Feb. I9or.

Lindr. Acta Soc. Fauna et Fl. Fennica $20^{\circ}$ : 21. I9oI.

\section{On Sisymbrium.}

Anderson, Jour. Myc. 5: 82. June 1889 .

Ell. \& Ev. Jour. Myc. $6:$ I2I. $d$. 6 Jan. I89I.

Ell. in Nelson, Bull. Wyo. Exp. Sta. $28: 203$. May 1896.

Nelson, Rept. Wyo. Exp. Sta. 10 : (35). I 1900.

Cockerell, Jour. Myc. 10: 51. I9 March I904.

On Smelowskia.

Jones, Proc. Cal. Acad. II. 5 : '730. 3 Oct. I 895.

Aecidium Montanoæ, Diet. \& Holw.

Holw. Bot. Gaz. 24: 36. d. 3I July I 897.

Lindau \& Syd. Hedw. 37: xvii. 9 April i $898 . \quad$ [Beiblatt no. 7.]

Sacc. \& Syd. Syll. 14: 379. d. 20 Aug. I899.

Aecidium Myosotidis, Burrill.

Burrill, Bot. Gaz. 9 : 190. $d$. Dec. 1884 .

Burrill, Bull. III. Lab. 2: 234. d. 1885 and Rept. III. Ind. Univ. 12 : I46. $d$. I885.

Wint. \& Demet. Hedw. 24: I80 (4). Oct. I 885.

*Ell. \& Ev. N. A. F. 1832 . 1887.

De Toni in Sacc. Syll. 7 : 81o. d. 28 Oct. 1888.

Gall. Bull. U. S. Agr. Veg. Pathol. 8: 56.1889.

Lagerh. Tromsoe Mus. Aarshefter 17 : I03. d. 1895.

Tubeuf-Smith, Diseases of Plants 4II. 1897.

Aecidium myricatum, S.

S. Sýn. Am. Bor. 300. 1834.

Pk. Rept. N. Y. Mus. 23 : 60. d. 1872.

Pk. Rept. N. Y. Mus. 24: 106. 1872.

Farl. Bull. Bussey Inst. 1: 433. March 1876 .

Pk. Rept. N. Y. Mus. 29: 77. 1878.

*Ell. N. A. F. $230 . \quad$ I879.

* Thm. Myc. Univ. 1224. I879.

Thm. Flora 63: 316. in July I 880.

De Toni in Sacc. Syll. $7: 826 . d .28$ Oct. 1888. 
*Roum. Fung. Gall. 4835. 1880.

Roum. Rev. Myc. 11: 63. I April I88\%.

Ell. in Cat. Pl. N. J. 505. 1880.

*Ell. \& Ev. Fung. Columb. 62. I893. Cacoma myricatum, S.

S. Syn. Am. Bor. 294 no. 2894. d. 1834.

Aecidium Napær, Arth. \& Holw.

Note. In Bull. Torr. Bot. Club 31 : 7. I0 Jan. 1904 Arthur states that Aecidium Callirrhoes, Ell. \& Kellerm. (q. 2\%) is a synonym of Aecidium Napaex, Arth. \& Holw. See also Aecidium Spharalcer, Ell. \& Ev.

Arth. Bull. lowa Agr. Coll. 1884 : ı66. d. Jan. I 885.

De Toni in Sacc. Syll. $7: 783 . d$. 28 Oct. 1888.

*Ell. \& Ev. N. A. F. 2401. April r890.

Detmers, Bull. Ohio Exp. Sta. 44 : 139. Sept. 1892.

Kellerm. \& Werner, Geol. Ohio 72 : 346. Jan. 1895.

Underw. Proc. Ind. Acad. 1894: I5 I. Oct. 1895.

Arth. Bull. Torr. Bot Club 31 : 7. I9 Jan. 1904.

Aecidium Nelsoni, (Arth.) n.n.

NoTr. Arthur, I901 suggests that Gymnosporangium Nelsoni, Arth. may be the teleutosporic stage. See also Klebahn, Rostpilze 354. Ig04.

Roestelia Iacerata, Auct. Amer. p. p.

Hark. Bull. Cal. Acad. 2: 440.16 June I 887.

Seymour, Proc. Boston Soc. 24: 190. 8 Nov. 1889.

Roestelia cornuta, Auct. Amer. p. p.

* Ell. \& Ev. N. A. F. 3146. Nov. 1894.

*Ell. \& Ev. Fung. Columb. 76r. 24 April I 895.

Roestelia EIlisii, Fung. Columb. 1292 non Pk.

*Ell. \& Ev. Fung. Columb. 1292 . May 1898. Roestelia Nelsoni, Arth.

Arth. Bull. Torr. Bot. Club 28 : 665. d. 30 Dec. 1901.

*Griffiths, West Am. Fung. 246, 264. 22 March roo2.

* Barthol., Ell. \& Ev. Fung. Columb. 1676. Oct. 1902.

Aecidium Nesææ, Gerard.

Note. Accompanies Puccinia Nesaa, Ell. \& Ev.

Gerard, Bull. Torr. Bot. Club 4: 47. d. Oct. I873 and Hedw. 13 : 28. d. Feb. 1874 .

Pk. Rept. N. Y. Mus. 27 : 104. 1875.

Pk. Rept. N. Y. Mus. $29: 77$. 1878.

*Ell. N. A. F. 1015. 1883.

*Wint. Fung. Eur. 3019. 1884.

De Toni in Sacc. Syll. 7 : 789. d. 28 Oct. 1888.

Ell in Cat. Pl. N. J. 505. 1889.

Detmers, Bull. Ohio Exp. Sta. tech. ser. 1: 174. April I893.

Davis, Tr. Wis Acad. 9: 182. 21 Aug. 1893.

* Ell \& Ev. Fung. Columb. 197. 1893.

Kellerm. \& Werner, Geol. Ohio $7^{2}$ : 346. Jan. 1895.

Kellerm. Jour. Myc. 9: 12. 14 Feb. 1903.

Aecidium Nidus-avis, (Thax.) n. $n$.

Rastelia cornuta, Auct. Amer. p. p.

Farl. Bot. Gaz. 11: 1 90. July 1886.

Farl. Bot. Gaz. 11 : 238 . Sept. 1886.

Thax. Proc. Am. Acad. 22: 264, 267, 268. Jan. 1887 and Centralbl. Bact. 1 : $433,458-460 . \quad 1887$.

Thax. Bot. Gaz. 14: I65. July 1889 . 
Rastelia Nidus-avis, Thax.

Thax. Bull. Conn. Exp. Sta. 107: 5. 15 April I891,

Thax. Rept. Conn. Exp. Sta. 1891: I64. March I892.

* Seymour \& Earle, E. F. 240. I Feb. I 893.

Stevens, Rept. Ohio Acad. 4: 20. I896.

Aecidium nitens, S.

See Gymnoconia interstitialis, (Schl.) Lagerh.

Aecidium oblongum, Bon.

See Aecidium Hyperici-frondosi, S.

Aecidium occidentale, Arth.

Note. See also Aecidium Clematidis, D C.

Arth. Bull. Torr. Bot. Club 31 : 7. d. I9 Jan. 1904.

Aecidium Oenotheræ, $\mathrm{Pk}$.

Note. The name Aecidium Oenothere, Pk. was changed by De Toni in 1888 to Aecidium Peckii on the supposition that it was different from the earlier described Aecidium Oenothera, Mont. But Peridermium Peckii, Tlim. here given as Aecidium dates from 1880 . It is not certain whether any of the North American Aecidia on Oenothera really belong to Aecidium Oenothere, Mont. but at least the greater part of them are certainly Aecidium Oenothera, Pk. which according to Kellerman is connected with a Puccinia on Carex called by him Puccinia Peckii.

We have therefore thought best to retain as an index name Aecidium Oenothera, Pk. without regard to Montagne's name in order to avoid creating still greater confusion in the synonymy. For further references see also Aecidium Epilobii. See Kellerman, Jour. Myc. 8: 20. 3I May I902 and 9:9. I4 Feb. 1903. Arth. Jour. Myc. 8: 52. 30 June I9O2 and Bot. Gaz. 35: 13. 20 Jan. I903. Syd. Mon. Ured. I: 671. I Nov. 1903. Klebahn, Rostpilze 306. 1904.

Pk. Rept. N. Y. Mus. 23:60. d. $\quad$ I 872.

Pk. Rept. N. Y. Mus. 24: 107. I872.

Frost in Cat. Pl. Amherst 85. 1875.

Pk. Rept. N. Y. Mus. 29: 76. I 878.

Bundy, Geol. Wis. 1873-79 1: 400. I883.

*Ell. N. A. F. 1016. I883.

- Trel. Tr. Wis. Acad. $6:$ i 34 (30). Nov. I 884.

Arth. Bull. lowa Agr. Coll. 1884: I67. Jan. I885.

Burrill, Bull. Ill. Lab. 2 : 227. d. I885 and Rept. Ill. Ind. Univ. 12 : I42. $d .1885$.

Brendel, Flora Peoriana 2 ed. 69. 1887.

Cobb, List Pl. Amherst 40. I 887.

Halsted, Bull. Iowa Agr. Coll. 1886 : 58. I 887.

Kellerm. \& Carl. Tr. Kans. Acad. 10: 91. 1887.

*Roum. Fung. Gall. 44'5. I 888.

Roum. Rev. Myc. 10:86. I April I 888.

Gall. Bull. U. S. Agr. Veg. Pathol. 8: $56 . \quad$ I 889.

Detmers, Bull. Ohio Exp. Sta. 44: 1 39. Sept. I 892.

Detmers, Bull. Ohio Exp. Sta. tech. ser. 1: 178. April 1803.

Underw. Proc. Ind. Acad. 1893 : 50. Aug. I 894.

Kellerm. \& Werner, Geol. Ohio $7^{2}$ : 346. Jan. I 895.

Snyder, Proc. Ind. Acad. 1896 : 2 I 7. I 897.

Herbst, Fungal Fl. Lehigh Valley I75. d. 1899.

Davis, Tr. Wis. Acad. 14: 87 . Sept. 1903. Aecidium Peckii, De Toni.

De Toni in Sacc. Syll. 7 : 790. d. 28 oct. I 888.

Webber, Bull. Nebr. Exp. Sta. 1 : $333(63)$. I8 Dec. I889.

Webber, Rept. Nebr. Agr. 1889: 2 II (7I). I 890. 
*Ell. \& Ev. Fung. Columb. 195. 1893.

McClatchie, Proc. S. Cal. Acad. 1 : 372. 5 June 1897.

*Ell. \& Ev. Fung. Columb. 1191. July 1807 .

Tubeuf-Smith, Diseases of Plants 411 . I897.

Orton, Rept. Vt. Exp. Sta. 11: 21 I. Sept. 1898 and Contr. Bot. Vt. 2: 13. Sept. 1898 .

Barthol. Tr. Kans. Acad. 16: 190. June 1899.

Nelson, Rept. Wyo. Exp. Sta. 10: (36). 1900.

Freeman, Minn. Bot. Studies II. 5: 559. 20 July 1901.

Patterson, Bull. U. S. Agr. Pl. Industry 8: 8. 3 Feb. 1902.

Kellerm. Ohio Nat. 2: 206. d. Feb. 1902.

* Kellerm. Ohio Fungi 17. d. March 1902.

* Griffiths, West Am. Fung. 58. 22 March 1902.

* Barthol., Ell. \& Ev. Fung. Columb. 1604. Oct. 1902.

Arth. Bot. Gaz. 35 : 13. 20 Jan. 1903.

Garrett, Tr. Kans. Acad. 18: 147. 1903.

PAecidium Oenothera, Auct. Amer. p. p.

Ell. in Nelson, Bull. Wyo. Exp. Sta. 28: 203. May 1896.

Tubeuf-Smith, Diseases of Plants 4II. I897.

Nelson, Rept. Wyo. Exp. Sta. 10 : (35). 1900.

Ricker, Univ. of Maine Studies 3: 51. 30 April 1902.

Carl. Jour. Appl. Micr. 6 : 2110.1903.

Aeeidium on Oenothera.

See also under Aecidium Epilobii, D. C.

See also Puccinia Heterantha, Ell. \& Ev.

See also Uromyces Oenothera, Burrill.

Aecidium oldenlandianum, Ell. \& Tracy.

Ell. \& Tracy, Jour. Myc. $7:$ 43. d. Io Sept. I891.

Tracy \& Earle, Bull. Miss. Exp. Sta. 34 : 90. d. May 1895.

Sacc. Syll. 11: 219. d. July 1895 .

Aecidium oldenlandianum, Underw. \& Earle non Ell. \& Tracy. See Aecidium houstoniatum, S.

Aecidium Onobrychidis, Burrill.

Burrill, Bot. Gaz. 9: 189 d. Dec. 1884.

Burrill, Bull. III. Lab. 2: 225. d. I 885 and Rept. Ill. Ind. Univ. 12 : I41. $d$. 1885 .

*Ell. \& Ev. N. A.F. 1826.1887.

De Toni in Sacc. Syll. $7: 776 . d .28$ Oct. 1888.

Gall. Bull. U. S. Agr. Veg. Pathol. 8: 56.1889.

Underw. Proc. Ind. Acad. 1893: 50. Aug. I894.

Tubeuf-Smith, Diseases of Plants 410. 1897.

Aecidium Onosmodil, Arth.

NotE. For Aecidia on Borraginacee see also Aecidium Asperifolii, P. and Aecidium Myosotidis, Burrill.

Arth. Bull. Torr. Bot. Club $31: 6 . d$. 19 Jan. Iy04.

?Aecidium Orchidearum, Desm.

NotE. Compare also Aecidium Grabnerianum, Henn.

Syd. Ann. Myc. 1 : 21. 30 Jan. 1903.

Aecidium orientale, (Cke.) Diet.

Note. Whether the East Indian species of this name occurs in North America is doubtful. For what has been called by that name see Aecidium carneum, (Bosc.)

Diet. in Engler \& Prantl, Pflanzenfam. 11** : 78. $\quad 1897$. 
Aecidium (sub Peridermio) ornamentale, (Arth.)

Note. This species was originally described as a Peridermium, but we have here included that genus under Aecidinm. The species cannot however be called Aecidinm ornamentale as there is a species of that name described by.Kalchbrenner in Thuemen, Myc. Univ. 227. 1875 and in Flora 59:205. I May 1876.

Peridermium ornamentale, Arth.

Arth. Bull. Torr. Bot. Club 28: 665. d. 30 Dec. I90I.

Aecidium Orobi, Auct. Amer.

See under Aecidium on Leguminosa.

See Aecidium Psoralea, Pk.

See Aecidium Trifolil=repentis, Cast.

Aecidium Orobi, var. elegans, B. See Aecidium elegans, B. \& C.

Aecidium Orobi, var. leucostictum, B.

See Aecidium leucostictum, B. \& C.

Aecidium Osmorrhizæ, Pk.

Note. This Aecidium on Osmorrhiza is considered by Lindroth and by Sydow to belong to Puccinia Osmorrhize. C. \& P., a species whose synonymy is far from clear. See Lindr. Acta Soc. Fauna et Fl. Fennica 22': 17. I902. Syd. Mon. Ured. I : 397. I5 May 1903. For convenience we have included Aecidia on Cicuta and on Psezdocymopterus to which authors have applied this name. See lindr. 1. c. 53, I73. 1902.

In Jour. Myc. 9: 10. I4 Feb. 1903 Kellerman gives an account of a culture in which the spores of this Aecidium from Osmorrhiza produced teleutospores on Charophyllum. A. B. S.

Pk. Rept. N. Y. Mus. 24: 92. d. I07. 1872.

Pk. Rept. N. Y. Mus. $29: 77 . \quad$ I 878.

De Toni in Sacc. Syll. 7 : 794. d. 28 Oct. 1888.

Detmers, Bull. Ohio Exp. Sta. 44 : I 40. Sept. I892.

* Syd. Uredineen 1347. 1899.

? Lindr. Acta Soc. Fauna et Fl. Fennica 221 : I73. 1902.

Kellerm. Jour. Myc. 9 : Io. I4 Feb. I903. Aecidium Pimpinella, Auct. Amer.

Arth. Bull. Iowa Agr. Coll. 1884: I67. Jan. 1885.

?Arth. Bull. Iowa Agr. Coll. 1884: I67. Jan. I885. . Cicuta.

Aecidium osmundatum, S.

S. Syn. Am. Bor. 309. ${ }^{8} 834$. Caoma osmundatum, $\mathrm{S}$.

S. Syn. Am. Bor. 294 no. 2895 . d. 1834 .

Aecidium Oxalidis, Thm.

Arth. Bull. lowa Agr. Coll. 1884 : i67. Jan. I885.

*Ell. \& Ev. N. A. F. 2210. Feb. I889.

Webber, Bull. Nebr. Exp. Sta. $1: 333$ (63). $\quad$ i8 Dec. I889.

Webber, Rept. Nebr. Agr. 1889: 2I I (7I). 1890.

Pound, Bot. Surv. Nebr. 3 : Io. 18 June 1894 .

Barthol. Tr. Kans. Acad. 16 : igo. June I899.

Aecidium Palmeri, Anderson.

Note. Holway, in Erythea 7:98. 31 Oct. 1899 associated this as æcidlial stage with the newly described Puccinia Palmeri, (Anderson) Diet. \& Holw. See also Sycl. Mon. Ured. I : 252. 15 Nov. 1902. See also Jones Proc. Cal. Acad. II. 5: 728 . 3 Oct. 1895 where an Aecidum on Pentstemon confertus, var. referred by him to Aecidium Palmeri, Anderson is said to occur with Puccinia Pentstemonis, Pk. See also Blasdale, Jour. Myc. 9: 82. 29 May 1903. 
Anderson, Jour. Myc. 6 : I22. d. 6 Jan. I891.

Sacc. Syll. 9 : 32r. d. i5 Sept. i 891.

Aecidium Palmeri, Anderson.

See Puccinia Pentstemonis, Pk.

Aecidium Pammelii, Trel.

NOTE. Cultures by William Stuart, I902, show this to be the xcidial stage of Puccinia Panici, Diet.

Trel. Tr. Wis. Acad. 6 : 136 (32). d. Nov. 1884 and Jour. Myc. 1 : 15. $d$. Jan. 1885 .

*Ell. N. A. F. 1427.1885.

* Roum. Fung. Gall. $44^{\prime} 3$. 1888.

Roum. Rev. Myc. 10:86. I April I 888.

De Toni in Sacc. Syll. $7: 823$. d. 28 Oct. 1888.

*Ell \& Ev. N. A. F. 2217. Feb. I88\%.

Webber, Bull. Nebr. Exp. Sta. I : 333 (63). 18 Dec. I889).

Gall. Bull. U. S. Agr. Veg. Pathol. 8: 56. I889.

Webber, Rept. Nebr. Agr. 1889: 2I I (7I). I 890.

*Pazschke, Fung. Eur. 4140. 1898.

* Syd. Uredineen 1249.1898.

*Ell. \& Ev. Fung. Columb. 1389. May 1899.

Barthol. Tr. Kans. Acad. 16: 190. June 1809.

Freeman, Minn. Bot. Studies 11. 5: 559. 20 July r 901.

Stuart, Proc. Ind. Acad. 1901: 284. 1902.

* Barthol., Ell. \& Ev. Fung. Columb. 1706. Feb. 1903.

Aecidium Parnassiæ, (Schl.) Gravis.

Trel. in Sacc., Pk. \& Trel., Harriman Alaska Exp. 5 : 37. 5 April 1904.

Aecidium Parryi, Pk:

Nore. See also Aecidium monoicum, Pk.

Pk. in Parry, Am. Nat. 8 : 215 (25). d. April 1874.

Aecidium Peckil, (Thm.) Diet.

Note. This species is the Peridermium Peckii, Thm. I880 first mentioned as Peridermium columnare by Peck, 1876,1878 . It may possibly he connected with Calyptospora Goeppertiana, J. Kuehn, on species of Vaccinium.

Attention should be called to the fact that the name Aecidium Oenothera, Pk. 1872 was changed to Aecidium Peckii by De Toni I 888. The last named species is the æcidium of Puccinia Peckii, (De Toni) Kellerm. q. v.

Diet in Engler \& Prantl, Pflanzenfam. 11**: 78. I897.

Peridermium columnare, Auct. Amer.

Pk. Rept. N. Y. Mus. 28: 6r. 1876.

Pk. Rept. N. Y. Mus. 29: 77. 1878. Peridermium Peckii, Thm.

Thm. Mittheil. forstl. Versuchs. Oest. 2 : 320 (24). d. 1880.

*Ell. N. A. F. 1023.1883.

Farl. Appalachia 3 : 230,242 . Jan. 1884 .

De Toni in Sacc. Syll. $7: 837$. d. 28 Oct. 1888.

Millsp. Bull. W. Va. Exp. Sta. 24: 509. June 1892.

* Seymour \& Earle, E. F. 225. I Feb. I893.

Millsp. \& Nutt. Publ. Field Columb. Mus. Bot. 1 : 130. Jan. I 896.

Tubeuf-Smith, Diseases of Plants 417. 1897.

Orton, Rept. Vt. Exp. Sta. 11: 213 . Sept. 1898 and Contr. Bot. Vt. 2: 15 . Sept. 1898 .

Aecidium Peckil, De Toni.

See Aecidium Oenothera, Pk. 
Aecidium pedatatum, $\mathrm{S}$.

S. Syn. Am. Bor. 309 . I834.

Pk. Rept. N. Y. Mus. 34: 46 . Oct. 1883.

Arth. \& Holw. Minn. Bot. Studies II. 5: 632. $d$. 20 July 1901.

Davis, Tr. Wis. Acad. 14: 87. Sept. I903.

Caoma pedatatum, S.

S. Syn. Am. Bor. 293 no. 2885. d. I 1834. Caoma sagittatum, S.

S. Syn. Am. Bor. 293 no. 2886. $d$. I834. Aecidium sagittatum, S.

S. Syn. Am. Bor. 300. I834. Aecidium Mariz-Wilsoni, $\mathrm{Pk}$.

Pk. Rept. N. Y. Mus. 24: 92. d. 107. I872.

Frost in Cat. Pl. Amherst 85. 1875.

Pk. Rept. N. Y. Mus. 29: 76 . I878.

Bundy, Geol. Wis. 1873-79, 1 : 400. I883.

Burrill, Bull. III. Lab. 2 : 223. $d$. I 885 and Rept. Ill. Ind. Univ. 12 : I 40. $d$. I885.

Cobb, List Pl. Amherst 40. I887.

De Toni in Sacc. Syll. $7: 780 . d .28$ Oct. I 888.

Davis, Tr. Wis. Acad. 9: 161. 21 Aug. 1893.

Underw. Proc. Ind. Acad. 1893 : 50. Aug. 1894.

Tracy \& Earle, Bull. Miss. Exp. Sta. 34: 90. May 1895.

Underw. \& Earle, Bull. Ala. Exp. Sta. 80: 209. 28 June I 897.

Tubeuf.Smith, Diseases of Plants 4 IO. I 897.

Syd. in Sacc. Syll. 13: 1313. 1898.

Arth. \& Holw. Minn. Bot. Studies II. 5 : 639. 20 July I90I.

Earle in Mohr, Contr. U. S. Nat. Herb. 6: 193.31 July I 90 I.

Ricker, Univ, of Maine Studies 3: 5 I. 30 April I 902.

Aecidium Petersii, B. \& C.

B. Grev. 3: 6I (IO3) no. $580 . d$. Dec. I874.

Farl. Proc. Am. Acad. 18: 75 . July I883.

Trel. Tr. Wis. Acad. 6 : I35 (3I). Nov. 1884.

De Toni in Sacc. Syll. $\mathbf{7}: 780 . d$. 28 Oct. 1888.

Diet. Hedw. 28: I85. I889.

Lagerh. Tromsoe Mus. Aarshefter. 17 : I03. d. 1895.

Underw. \& Earle, Bull. Ala. Exp. Sta. 80: 210. $d .28$ June 1897.

Tubeuf-Smith, Diseases of Plants 410. I897.

Sacc. \& Syd. Syll. 14: 372. d. 20 Aug. I899.

Earle in Mohr, Contr. U.S. Nat. Herb. 6: I93. 3I July I90I.

Puccinia Viola, Carl. Ured. Amer. I.

* Carl. Ured. Amer. 28. I894.

Aecidium pentstemoniatum, S.

See Aecidium Pentastemonis, S.

\section{Aecidium Pentastemonis, S.}

NOTE. Aćcording to observations and cultures by Arthur and by Stuart this is the æcidial stage of Puccinia Andropogonis, S. See Arthur 1899, 1900. Arth. \& Holw. I90I. Kellerm. Jour. Myc. 9: 10. 14 Feb. 1903. Arth. Jour. Myc. 10: 11, 20. 30 Jan. I904.

S. Syn. Car. 68 (42) no. 449. d. I 822.

Lk., L. Sp. Pl. ed. Willd. 62: 47. d. 1825 .

M. A. Curtis, Bot. N. Car. I23. I867.

Pk. Rept. N. Y. Mus. 24: 92, 107. 1872.

Trel. Tr. Wis. Acad. 6 : 137 (33). d. Nov. 1884.

Arth. Bull. Iowa Agr. Coll. 1884: I65. d. Jan. I885.

Wint. \& Demet. Hedw. 24: I80 (4). Oct. 1885. 
*Wint. Fung. Eur. 3222. 1885.

Wint. Hedw. 24: 256. Dec. 1885.

Burrill, Bull. 111. Lab. 2: 233. d. 1885 and Rept. 11l. Ind. Univ. 12 : I $46 . d$. I 885 .

Halsted, Bull. lowa Agr. Coll. 1886 : 58.1887.

De Toni in Sacc. Syll. $7: 814$. d. 28 Oct. 1888.

* Kellerin. \& Swingle, Kansas Fungi 28. I Aug. I889.

Webber, Bull. Nebr. Exp. Sta. 1: $333(63)$. I8 Dec. I889.

Webber, Am. Nat. 24: 177. d. Feb. r89o.

Webber, Rept. Nebr. Agr. 1889 : 211 (71). 1890.

* Roum. Fung. Gall. 5514. I891.

Roum. Rev. Myc. 13: 5. I Jan. I891.

Woods, Bot. Surv. Nebr. $2: 38$. I5 April 1893.

Carl. Bot. Gaz. 18 : 453. 25 Dec. 1893.

Tracy \& Earle, Bull. Miss. Exp. Sta. 34: 90. May 1895.

Tracy,\& Earle, Bull. Miss. Exp. Sta. 38 : 138. May 1806.

Underw. \& Earle, Bull. Ala. Exp. Sta. 80: 210. 28 June 1897.

Snyder, Proc. Ind. Acad. 1896: $217 . \quad$ I 897.

Tubeuf-Smith, Diseases of Plants 4I I. I897.

Hume, Proc. Davenport Acad. 7 : 252. 12 May 1899.

Barthol. Tr. Kans. Acad. 16: 190. June 1899.

Arth. Science 11. 10 : 565. 20 Oct. I899.

Arth. Bot. Gaz. 29: 272.30 April 1900.

Bubak, Oest. Bot. Zeit. 50: 320. Sept. $1900^{\circ}$

Arth. \& Holw. Bull. Univ. lowa 5 : 18 I. d. May igor.

Earle in Mohr, Contr. U. S. Nat. Herb. $6: 193.31$ July I9or.

Garrett, Tr. Kans. Acad. 18 : 147. 1903.

Uredo Chelones, Spreng.

Spreng. in L. Syst. Veg. I6 ed. 41 : 572. $d$. 1827.

Caoma pentstemoniatum, $\mathrm{S}$.

S. Syn. Am. Bor. 292 no. 2864. I834.

Aecidium pentstemoniatum, $\mathrm{S}$.

S. Syn. Am. Bor. 309. I834.

Pk. Rept. N. Y. Mus. $29: 77 . \quad 1878$.

*Ell. \& Ev. N. A. F. 1813. 1887.

Aecidium Pentastemonis, S., var. Mimuli, Wint.

Wint. \& Demet. Hedw. 24: 180 (4). Oct. 1885.

*Wint. Fung. Eur. 3223. I 885.

Wint. Hedw. 24: 256 . Dec. 1885.

Gall. Bull. U. S. Agr. Veg. Pathol. 8 : 56.1889.

Aecidium Periclymeni, Schum.

Note. The Aecidium of this name in Europe is considered to belong to Puccinia Fesluca, Plowr. See Syd. Mon. Ured. 1: 752. I Nov. 1903. Klebahn, Rostpilze 290. 1904.

*Ell. N. A. F. IO2O. 1883 .

Arth. Bull. Iowa Agr. Coll. 1884: ı67. Jan. 1885.

Aecidium Petalostemonis, Kellerm. \& Carl.

Note. This is probably the xcidial stage of Uropyxis Pelalostemonis, (Farl.) De Toni. See Ell. \& Ev. N. A. F. 1845.1887.

Kellerm. \& Carl. Tr. Kans. Acad. 10:9I. I887.

Hume, Proc. Davenport Acad. 7 : 252. 12 May 1899.

Aecidium Petersil, B. \& C.

See Aecidium pedatatum, S. 
Aecidium Phaceliæ, Pk.

Note. Sydow 1902 suggests that this may belong to Puccinia Phacelia, Syd. \& Holw.

Pk. Bull. Torr. Bot. Club 11 : 50. d. May 1884.

Hark. Bull. Cal. Acad. 2 : 440. I6 June 1887.

Halsted, Bull. lowa Agr. Coll. 1888 : I13, I16. April 1888.

*Ell. \& Ev. N. A. F. 2218 . Feb. 1889.

Cke. Grev. 20 : I 1o. June 1892.

Sacc. Bull. Soc. Bot. Belg. 312: 225. d. I 892 .

Sacc. Syll. $11: 220 . d$. July I 805 .

McClatchie in Reid's Hist. Pasadena 6r6. 1805.

McClatchie, Proc. S. Cal. Acad. 1:372. 5 June 1897.

Hume, Proc. Davenport Acad. $7: 252.12$ May I 899.

Tracy \& Earle in Greene, Pl. Baker. 1 : $19 .{ }^{2} 2$ Feb. I9oI.

Syd. Mon. Ured. 1 : 314. I5 Nov. 1902.

*Syd. Uredineen 1800.1903.

Cockerell, Jour. Myc. 10:51. I9 March 1904.

Aecidium Phaseolorum, Auct. Amer.

Note. See Aecidium on Amphicarpara and decidium on Phaseolus under Aecidium on Leguminosa.

Aecidium Phlogis, Arth.

See Aecidium Gillia, Pk. Phlox divaricata.

See Aecidium Polemonii, Pk. Phlox pilosa.

Aecidium Phlogis, Ell. \& Ev. See Aecidium Giliz, $\mathrm{Pk}$.

Aecidium Phrymae, Halsted.

Halsted, Jour. Myc. $2:$ 52. d. May I 886.

De Toni in Sacc. Syll. $7: 816$. d. 28 Oct. 1888.

Pound, Bot. Surv. Nebr. 3: 1o. 18 June 1894.

Freeman, Minn. Bot. Studies 11. 5:559. 20 July 1901.

Aecidium Physalidis, Burrill.

See Aecidium Solani, Mont.

Aecidium Physalidis, $\mathrm{Pk}$.

Note. This name which so far as we know occurs only in the citation below probably refers to the aecidium of Puccinia Physalidis, Pk., which is perhaps the same as Aecidizm Solani, Mont. ; but authors are not agreed on that point.

Stuart, Proc. Ind. Acad. $1901: 284 . \quad$ I902.

Aecidium Pimpinellæ, Auct. Amer. See Aecidium Osmorrhiza, Pk.

Aecidium Pini, Auct. Amer. p. p.

Note. Besides citations under cross references given below there are the following references to Aecidium Pini in North America. Some relate to corticolous and some to acicolous forms, but their development has not been studied in this country. In Europe Aeidium Pini has been scparated into a number of forms, the acicolous being in general connected with species of Coleosporium and the corticolous with species of Cronartium. For details see Klebahn, Rostpilze 358, 372, 377. I904.

In this country Coleosporium Senecionis appenrs to be rare and is not accompanied, so far as known, by any Aeciltium on leaves of l'inus; nor has it been ascertained whether the common species of Cronarlium on Myrica is connected with the common form of Aecilium Pini on Pinus rigidla with which it is often found growing. It should be remarked that the common 
Aecidium in the latter case is acicolous and not like the corresponding European form corticolous.

We have arranged the following references under three heads: those where the Aecidium is reported on bark, those on leaves and lastly miscellanea. Probably some of the references properly belong to certain of the species already given here as cross references.

\section{Corticolous forms.}

See also Aecidium cerebrum, (Pk.) Diet. Aecidium deformans, Mayr. Aecidium filamentosum, (Pk.). Aecidium Harknessii, (Moore) Diet. Aecidium piriforme, (Pk.).

Peridermium Pini, Auct. Amer. p. p.

M. A. Curtis, Bot. N. Car. 124. I867. p. p.

Ell. in Cat. PI. N. J. 505. I889. Pinus rigida, $P$. inops.

\section{Acicolous forms.}

See also Aecidium carneum, (Bosc). Aecidium orientale, (Cke.) Diet.

\section{On Pinus rigida.}

Peridermium oblongisporium, Fckl.

Thm. Mitth. forstl. Versuchs. Oest. 2: 315 (19). d. 1880.

Peridermium Pini, var. acicolum, Auct. Amer.

Klebahn, Abh. Nat. Verein Bremen 10: I5I. I887, I889.

* Seymour \& Earle, E. F. 223. I Feb. I893.

Peridermium Pini, Auct. Amer. p. p.

*Ell. \& Ev. N. A. F. 2222. Feb. I889.

*Kellerm. Ohio Fungi 104. d. I4 Feb. I903.

Kellerm. Jour. Myc. 9: 12, 19. d. I4 Feb. 1903.

Peridermium acicolum, Underw. \& Earle.

Underw. \& Earle, Bull. Torr. Bot. Club 23: 400 d. 25 Oct. 1896. On Pinus, sp. indet.

Aecidium Pini, S. Car. 456.

S. Syn. Car. $69(43)$ no. 456. I 822.

Peridermium Pini, Auct. Amer. p. p.

Herbst, Fungal Fl. Lehigh Valley i 74. d. I899.

\section{Miscellanea.}

Aecidium Pini, Auct. Amer. p. p.

Watt, Can. Nat. \& Geol. 11. 2: 391. Oct. 1865.

Peridermium Pini, Auct. Amer., p. p.

Sprague, Proc. Boston Soc. $5: 329 . \quad 1856$.

Russell, Am. Nat. $2: 568,569$. Jan. 1869.

?Frost in Cat. Pl. Amherst 84. 1875. Abies.

?Cobb, List Pl. Amherst 39. 1887.

Vize, Grev. 5: 110. Mar. 1877. P. insignis.

Hark. \& Moore, Cat. Pac. Fung. 27. Feb. I880. P. insignis.

Thm. Mittheil. forstl. Versuchs. Oest. 2: 3 I0 (I4). $d$. I880.

Patterson, Bull. U. S. Agr. Pl. Industry $8:$ 10. 3 Feb. I902. P. mitis.

Aecidium Pini, var. Ephedræ, B. \& C.

See Aecidium Ephedra, Diet. 
Aecidium piratum, $\mathrm{S}$.

Note. Under Aecidium piratum are given the references to the Aecidium of Gymnosporangium macropus, but, as the names Accidium piratum and Aecidium penicillatum, the latter being in fact the name of a European species not found in North America, have been confused by some American writers, some of the following references may belong to other species.

S. Syn. Am. Bor. 309. I 834 .

Am. Agriculturist 27: $454 . f .1,2$. Dec. 1868.

?Pk. in Hayden's Rept. U. S. Surv. $6: 792 . \quad$ I873.

Thax. Proc. Am. Acad. 22: 262, 265. Jan. 1887 and Centralbl. Bact. $1: 432,434 . \quad 1887$.

?Nelson, Rept. Wyo. Exp. Sta. $10:(36) .1900$.

Caoma piratum, $\mathrm{S}$.

S. Syn. Am. Bor. 294 no. $2896 . d$. 1834.

Roestelia lacerata, Auct. Amer. p. p.

B. Grev. 3: 59 (IOI). Dec. I874. p. p.

*Thm. Myc. Univ. 732。 I877.

*Rav. Fung. Am. 481. I88I.

?Pk. Rept. N. Y. Mus. 33 : 38 . Oct. I883.

?Ell. in Cat. Pl. N. J. 504. 1889.

Mayr, Waldungen von Nordamer. I39, 434. I890.

Roestelia penicillata, Auct. Amer. $\mathrm{p}$. $\mathrm{p}$.

Farl. Anniv. Mem. Boston Soc. 30. 1880.

*Ell. N. A. F. $1086 a, b, d$. 1883 .

Trel. Tr. Wis. Acad. 6: 138 (34). Nov. 1884.

Arth. Bull. lowa Agr. Coll. 1884: 168. Jan. 1885.

Burrill, Bull. Ill. Lab. 2 : 240. d. 1885 and Rept. ill. Ind. Univ. 12 : I50. $d$. 1885 .

Farl. Bot. Gaz. 11 : 240, 241. Sept. 1886.

Bessey, Am. Pomol. Soc. 1885: 43. 1886.

Thax. Proc. Am. Acad. 22: 265. Jan. I887 and Centralbl. Bact. 1 : 434. I 1887 .

Brendel, Flora Peoriana 2 ed. $69 . \quad 1887$.

Gall. Rept. Mo. Hort. Soc. $29:$ 295. f. $A$. 1887.

Scribner, Rept. U. S. Agr. 1886 : 135.1887.

Scribner, Bot. Gaz. 13: 16. Jan. I888.

Thax. Bot. Gaz. 14:163, I7 1. July 1889. Roestelia pirata, Thax.'

Thax. Proc. Am. Acad. 22 : 269. Jan. 1887 and Centralbl. Bact. 1 460. 1887.

Seymour, Tr. Am. Hort. Soc. 4: I 55 (4). 7 April 1887.

* Kellerm. \& Swingle, Kansas Fungi 19. I May I889.

Thax. Bot. Gaz. 14: I64, I66, I71. July I889.

Gall. Bull. U. S. Agr. Veg. Pathol. 8: 58, I889.

Gall. Rept. U. S. Agr. 1888: 378. pl. ${ }_{11}, f . I_{\text {. pl. } 12, f .1-3 .} \quad 1889$.

Farl. Gard. \& For. 3: 627. 24 Dec. 1890.

Pearson in Gall. Bull. U. S. Agr. Veg. Pathol. II : 46. 1890.

Gall. Rept. U. S. Agr. $1889: 413$. I 890.

Pammel, Bull. lowa Exp. Sta. 13: 4I.f. x. May 1891.

Pammel, Jour. Mvc. 7 : 102. April 1892.

*Ell. \& Ev. N. A. F. 2213. May I892.

Millsp. Bull. W. Va. Exp. Sta. $24: 508$. June 1892.

Davis, Tr. Wis. Acad. 9 : I62. 21 Aug. I 893.

Jones, Rept. Vt. Exp. Sta. 1892: 83. 1893.

Underw. Proc. Ind. Acad. 1893: 56. Aug. I 894.

Fischer, Hedw. 34: 4. f. 8. I5 Feb. 1895.

?Tracy \& Earle, Bull. Miss. Exp. Sta. 34 : 9i. May 1895. 
Pammel, Proc. lowa Acad. 1894 : 206. 1895.

Pammel \& Carver, Proc. lowa Acad. 3 : 143. 1806.

Underw. \& Earle, Bull. Ala. Exp. Sta. 80: 218. 28 June 1897.

* Syd. Uredineen 1195. Feb. 1898.

Barthol. Tr. Kans. Acad. 16 : 192. June 1899.

Earle, Bull. Ala. Exp. Sta. I06: I68. Nov. I899.

Quaintance, Rept. Ga. Exp. Sta. 13:357. Jan. 190I.

Aecidium piriforme, (Pk.) n. n.

Notk. See also Accidium cerebrum, (Pk.) Diet.

Peridermium piriforme, $\mathrm{Pk}$.

Pk. Bull. Torr. Bot. Club. 6 : 13. d. Feb. 1875.

Thn. Mittheil. forstl. Versuchs. Oest. 2: 31 I (15) d. I880.

De Toni in Sacc. Syll., $7: 835$. d. 28 Oct. 1888.

Klebahn, Ber. Deutsch. Bot. Ges. 8 : (69). 29 Dec. 1890.

Tubeuf-Smith, Diseases of Plants 414. 1897.

Aecidium Pirolæ, P. in Gmel.

See Thecopsora? PIrola, Karst.

\section{Aecidium pirolatum, S.}

See Chrysomyxa Pirola, Rostr.

Aecidium Plantaginis, Ces.

Note. According to cultures by Arthur, Bot. Gaz. 35: 17. 20 Jan. 1903 this is the acidial stage of Uromyces Aristidle, Ell. \& Ev. See also Klebahn, Rostpilze 325. 1904.

*Rav. Fung. Am. 483. I881.

Wint. \& Demet. Hedw. 24 : 180 (4). Oct. 1885.

Burrill, Bull. III. Lab. 2 : 232. d. ${ }_{1} 885$ and Rept. Ill. Ind. Univ. 12 : 145. $d$. 1885 .

*Ell. \&E Ev. N. A. F. 1829.1887.

De Toni in Sacc. Syll. $7: 813 . d$. 28 Oct. 1888.

Kelsey, Jour. Myc. 5 : 80. June 1889.

Gall. Bull. U. S. Agr. Veg. Pathol. 8: 56. I 880.

Tracy \& Earle, Bull. Miss. Exp. Sta. 34: 90. May 1895.

Atk. Bull. Cornell Univ. 3 : 21. 25 June 1897.

Underw. \& Earle, Bull. Ala. Exp. Sta. 80: 2 10. 28 June 1897.

Diet. in Engler \& Prantl, Pflanzenfam. 1 1**: 77.1897.

Tubeuf-Smith, Diseases of Plants 4II. 1897.

Nelson, Rept. Wyo. Exp. Sta. $10:(36) .1900$.

* Shear, Ell. \& Ev. Fung. Columb. 1475. May 19or.

Earle in Mohr, Contr. U. S. Nat. Herb. 6 : 193. 31 July r9or.

Arth. Bot. Gaz. 35 : 18. 20 Jan. 1903.

Aecidium podophyllatum, S.

See Aecidium Podophylli, S.

Aecidium Podophylli, S.

NotE This Aecidium is followed by, and intimately associated with Puccinia Podophylli, S. to which it evidently belongs.

Muhl. Cat. Pl. Am. Sept. I ed. 104. 1813. 2 ed. 107. 1818.

S. Syn. Car. 66 (40) no. 435.d. 1822.

Spreng. in L. Syst. Veg. I6 ed. 41 : 568, $d$. I827.

Fr. S. M. 3: 512. 1832.

Bon. Abh. Nat. Ges. Halle 5 : 216 (50). 1860.

M. A. Curtis, Bot. N. Car. 123. 1867.

Pk. Rept. N. Y. Mus. 23: 59.d. 1872.

Pk. Rept. N. Y. Mus. 24: I07. 1872.

*Ell. N. A. F. 258 . 1879. 
*Rav. Fung. Am. 482. I88I.

Bundy, Geol. Wis. I873-79, $1: 400 . \quad 1883$.

Trel. Tr. Wis. Acad. 6 : I 34 (30). Nov. I 884.

Arth. Bull. lowa Agr. Coll. 1884: I67. Jan. 1885.

Cragin, Bull. Wash. Lab. 1: 68. Jan. I885.

Wint. \& Demet. Hedw. 24: I80 (4). Oct. I885.

Brendel, Flora Peoriana 2 ed. $69 . \quad$ I887.

Tracy \& Earle, Bull. Miss. Exp. Sta. 34: 90. May 1895.

Jones, Oberlin College Lab. Bull. 9: 4. $\quad$ I5 June I808.

Herbst, Fungal Fl. Lehigh Valley I75. $d$. 1899.

Puccinia aurea, Spreng.

Spreng. in L. Syst. Veg. I6 ed. $4^{1}$ : 568. d. 1827. Caoma podophyllatum, S.

S. Syn. Am. Bor. 293 no. 2888 : 1834. Aecidium podophyllatum, $\mathrm{S}$.

S. Syn. Am. Bor. 309. I834.

Lea, Cat. Pl. Cin. 7I. I849.

Watt, Can. Nat. \& Geol. Il. 2 : 391. Oct. I865.

B. Grev. 3: 59 (IOI). Dec. I874.

*Thm. Myc. Univ. 626. I877.

Pk. Rept. N Y. Mus. 29: $76 . \quad 1878$.

James, Jour. Cin. Soc. 2:67 (26). April 1879.

Aecidium Polemonii, Pk.

Note. See note under Aecidium Gilia. Pk.

Pk. Bot. Gaz. 4 : 230. d. Nov. I879.

*Ell. N. A. F. 1008 . 1883.

Trel. Tr. Wis. Acad. $6:$ I 34 (30). Nov. I 884.

Arth. Bull. Iowa Agr. Coll. 1884: I67. Jan. 1885.

Burrill, Bull. Ill. Lab. 2 : $235 . d$. $\quad$ I 885 and Rept. ill. Ind. Univ. 12 : I47. $d$. 1885 .

De Toni in Sacc. Syll. 7 : 8o6. d. 28 Oct. I 888.

Webber, Bull. Nebr. Exp. Sta. 1: $333(63)$. I8 Dec. I889.

Gall. Bull. U. S. Agr. Veg. Pathol. 8: 56. I889.

Webber, Rept. Nebr. Agr. 1889: 2II (7I). I89o.

*Pazschke, Fung. Eur. 3637. 1890.

Tracy \& Earle, Bull. Miss. Exp. Sta. 34: 9o. May 1895.

Tubeuf-Smith, Diseases of Plants 4II. I897.

PAecidium Phlogis, Arth. p. p.

Arth. Bull. lowa Agr. Coll. 1884: I67. Nov. I884.

Phlox pilosa.

Aecidium polygalinum, $\mathrm{Pk}$.

Pk. Bot. Gaz. 6: 275. d. Oct. 1881.

*Ell. N. A. F. 1009.1883.

Trel. Tr. Wis. Acad. 6: I 35 (31). Nov. I884.

Arth. Bull. lowa Agr. Coll. 1884: 167. Jan. I885.

*Wint. Fung. Eur. 3319. I 885.

De Toni in Sacc. Syll. $7: 780 . d .28$ Oct. 1888.

*Syt. Uredineen 1396. I 899.

Aecidium Polygoni, Chev.

Note. Burrill, Bull: Ill. Lab. 2 : $164 . d$. 1885 and Rept. Ill. Ind. Univ, 12: 107. $d$. 1885 describes an Aecidium on Polygonum as the recidial stage of Uromyces Polygoni, (P.) Fckl. It is also described in European works. It is probably more frequent than these references seem to indicate. See also Barthol., Ell. \& Ev. Fung. Columb. 1897. 28 Dec. 1903.

Barthol. Tr. Kans. Acad. 16 : I9I. June I899.

Aecidium porosum, $\mathrm{Pk}$.

See Aecidium album, Clint. and Aecidium on Leguminosa. 
Aecidium Prenanthis, $\mathrm{P}$.

*Ell. \& Ev. N. A. F. 3054. April I804.

Aecidium Prenanthis, Auct. Amer. p. p.

Nore. See note under Aecidium Compositarum, var. Prenanthis, Auct. Aner.

See Aecidium Compositarum on Helenium.

See Aecidium Compositarum, var. Lactuca, Burrill.

Aecidium Proserpinacæ, B. \& C.

B. Grev. 3: 6o (102) no. 577. d. Dec. 1874.

De Toni in Sacc. Syll. 7 : 780 . d. 28 Oct. 1888.

*Ell. \& Ev. N. A. F. 271 . May 1892 .

Underw. \& Earle, Bull. Ala. Exp. Sta. 80: 210. d. 28 June i897.

Tubeuf-Smith, Diseases of Plants 4 10. $189 \%$.

Earle in Mohr, Contr. U. S. Nat. Herb. 6: 193. 31 July r9or.

Aecidium pseudo-balsameum, Diet. \& Holw.

Holw. Erythea $7:$ 98. $d$. 31 Oct. 1899.

Sacc. \& Syd. Syll. 16:346. d. I Feb. 1902.

Blasdale in Davy, Bull. U. S. Agr. PI. Industry 12: 75. 1902.

Aecidium Psoraleæ, $\mathrm{Pk}$.

Pk. in Parry, Am. Nat. 8: 215 (25). d. April 1874.

Burrill, Bull. Ill. Lab. $2: 225 d .1885$ and Rept. Ill. Ind. Univ. 12 : . I42. $d$. 1885.

Kellerm. \& Carl. Tr. Kans. Acad. 10: 92. I887.

Brendel, Flora Peoriana 2 ed. 69. I887.

*Ell. \& Ev. N. A. F. 1818.1887.

De Toni in Sacc. Syll. $7: 785 . d .28$ Oct. 1888.

Webber, Bull. Nebr. Exp. Sta. 1: $333(63)$. 18 Dec. 1889.

Webber, Rept. Nebr. Agr. 1889 : 2 II (7I). I 890.

*Ell. \& Ev. Fung. Columb. 60.1893.

*Ell. \& Ev. Fung. Columb. 557. I894.

Tubeuf-Smith, Diseases of Plants 410. 1897.

Hume, Proc. Davenport Acad. $7: 246,252$. 12 May 1899.

Barthol. Tr. Kans. Acad. 16: 191. June 1899.

* Griffiths, West Am. Fung. 67. 22 March 1902.

* Barthol., Ell. \& Ev. Fung. Columb. 1803. 28 Dec. 1903. Aecidium Orobi, Auct. Amer. p. p.

B. Grev. 3 : 61 (103). Dec. 1874 .

Cke. Ann. N. Y. Acad. 1: 182. May 1878.

Aecidium Pteleæ, B. \& C.

Note. Stated as the result of cultures to be the rcilial stage of Puccinia Windsoria, S. See Arthur, 1899, I900 and Kellerman, Jour. Myc. 9: 10. 14 Fel. 1903. Klebahn, Rostpilze 286.1904.

B. Grev. 3 : 60 (102) no. 579. d. Dec. 1874 .

*Wint. Fung. Eur. 3020. 1884.

Wint. \& Demet. Hedw. 24: i 80 (4). Oct. 1885.

Burrill, Bull. III. Lab. 2: 225. $d . \quad 1885$ and Rept. III. Ind. Univ. 12 : I4I. $d$. 1885 .

Roum. Rev. Myc. 8 : 196. I Oct. 1886.

* Roum. Fung. Gall. 3859 . 1886.

Brendel, Flora Peoriana 2 ed. $69 . \quad$ I 887.

*Ell. \& Ev. N. A. F. 1819.1887.

De Toni in Sacc. Syll. $7: 784 . d$. 28 Oct. 1888.

Gall. Bull. U. S. Agr. Veg. Pathol. 8: 56. 1889.

Underw. Proc. Ind. Acad. 1893: 50. Aug. 1894.

Atk. Bull. Cornell Univ. 3: 2r. 25 June 1897. 
Underw. \& Earle, Bull. Ala. Exp. Sta. 80: 210. d. 28 June I897.

Snyder, Proc. Ind. Acad. 1896 : $217 . \quad$ I897.

Tubeuf-Smith, Diseases of Plants 4IO. I897.

Arth. Science 11. 10 : 565. 20 Oct. 1899.

Earle in Mohr, Contr. U. S. Nat. Herb. 6 : i94. 3i July I 9oI.

Patterson, Bull. U.S. Agr. Pl. Industry $8: 8.3$ Feb. 1902 .

Arth. Bot. Gaz. 35 : 16. 20 Jan. 1903.

Aecidium pulcherrimum, Rav.

See Aecidium Rhamni, P. in Gmel.

\section{Aecidium punctatum, $\mathrm{P}$.}

See also under Aecidium Ranunculacearum, Auct. Amer. p. p.

Muhl. Cat. Pl. Am. Sept. I ed. I04. I813. 2 ed. 107. 1818.

Trel. Tr. Wis. Acad. 6: 136 (32). Nov. I884.

*Wint. Fung. Eur. 3023. 1884.

Arth. Bull. lowa Agr. Coll. 1884: I67. Jan. I885.

Burrill, Bull. III. Lab. 2: 222. $d . \quad$ I 885 and Rept. 11l. Ind. Univ. 12 : I39. $d$. $\mathrm{I} 885$.

EII. N. A. F. I423 (note). Thalictrum.

*Wint. Fung. Eur. 3422 . 1886.

* Roum. Fung. Gall. 3927. 1886.

Roum. Rev. Myc. 9: 23. I Jan. I887.

Wint. Hedw. 26 : 24. Feb. 1887.

Webber, Bull. Nebr. Exp. Sta. $1: 333(63)$. d. I8 Dec. I889. Thalictrum. Gall. Bull. U. S. Agr. Veg. Pathol. 8: 56.1889.

Lagerh. Hedw. 29: 175. Aug. I890.

* Syd. Uredineeñ 300.1890.

Webber, Rept. Nebr. Agr. 1889: 2II (7I). d. I890. Thalictrum.

Detmers, Bull. Ohio Exp. Sta. 44: 139. Sept. 1892.

*Ell. \& Ev. Fung. Columb. 194. I893.

Kellerm. \& Werner, Geol. Ohio $7^{2}: 346 . \quad J a n . ~ I 895$.

Richards, Proc. Am. Acad. 31: 262. pl. $1, f_{.} 12$. 1896.

Underw. \& Earle, Bull. Ala. Exp. Sta. 80: 2 I0. 28 June I897.

Diet. in Engler \& Prantl. Pflanzenfam. 11**: 76. f. 50 C, D. 1897.

Tubeuf-Smith, Diseases of Plants 409. 1897.

Barthol. Tr. Kans. Acad. 16 : I9I. June I899.

Orton, Rept. Vt. Exp. Sta. 12 : 166. Dec. I899 and Contr. Bot. Vt. 7: 166 . Dec. 1899.

Pound \& Clements, Bot. Surv. Nebr. 5 : 24. 30 March igor.

Freeman, Minn. Bot. Studies II. 5: 555.20 July I90I. Thalictrum.

Earle in Mohr, Contr. U. S. Nat. Herb. 6 : I94. 3I July I9OI.

* Barthol., Ell. \& Ev. Fung. Columb. 1504. Dec. I90I.

Aecidium Thalictri, Auct. Amer. p. p.

*Ell. N. A. F. 1004. I883.

Farl. Proc. Am. Acad. 18: 75. July 1883 .

Aecidium quadrifidum, D C.

Pk. Rept. N. Y. Mus. 23: 59. 1872.

Pk. Rept. N. Y. Mus. 24: 105.1872.

Frost in Cat. Pl. Amherst 85. 1875.

Pk. Rept. N. Y. Mus. 29: 76. 1878.

Bundy, Geol. Wis. 1873-79, 1 : 400. I883.

$\mathrm{Cobb}$, List Pl. Amherst 40.1887.

\section{Crooma hepaticatum, S.}

S. Syn. Am. Bor. 293 no. 2878. d. I834.

Aecidium hepaticatum, $\mathrm{S}$.

S. Syn. Am. Bor. 309.1834.

Árth. Bull. Iowa Agr. Coll. 1884: I66. Jan. I885.

*Ell. \& Ev. N. A. F. $1828 . \quad$ I 887. 
Bolley, Am. Mo. Micr. Jour. 10: 175, 176, 178. f. 7. and pl. \%. 5. Aug. I889 and Jour. Myc. 5: 167. Sept. 1889.

*Underw. \& Cook, lllustr. Fungi 53. Sept. I889.

Davis, Tr. Wis. Acad. 9: 16I. 21 Aug. 1893.

Underw. Proc. Ind. Acad. 1893: 50. Aug. 1894.

*Ell. \& Ev. Fung. Columb. 269. 1894.

Pk. Rept. N. Y. Mus. 49: 35 (21). 5 Nov. 1897.

Underw. Moulds 233. pl. 6, f. 17. 1899.

Aecidium Purpusiorum, Henn.

Henn. Hedw. Beibl. 42: (189), d. 4 July I903.

Mexico.

Aecidium pustulatum, M. A. Curtis.

NoTE. Arthur, 1904, gives an account of cultures showing this to be the xcidial stage of Puccinia Andropogi, Auct. Amer. p.p. which he calls Puccinia pustulata, (M. A. Curtis) Arth.

M. A. Curtis in Pk. Rept. N. Y. Mus. 23 : 60. d. 1872.

Pk. Rept. N. Y. Mus. 24: 106. 1872.

Pk. Rept. N. Y. Mus. $29: 77 . \quad 1878$.

*Ell. N. A. F. 224. 1879 .

*Thm. Myc. Univ. 1424.1879.

Trel. Tr. Wis. Acad. 6: 134(30). Nov. I884.

Arth. Bull. lowa Agr. Coll. 1884 : 167. Jan. 1885.

*Wint. Fung. Eur. 3320.1885.

Burrill, Bull. III. Lab. 2 : 237. d. 1885 and Rept. III. Ind. Univ. 12 : I48. d. I 885 .

Kellerm. \& Carl. Tr. Kans. Acad. 10: 92. $\quad$ I887.

De Toni in Sacc. Syll. $7: 822$. d. 28 Oct. 1888.

*Roum. Fung. Gall. 4834. I889.

Roum. Rev. Myc. 11:63. I April 1889.

* Kellerm \& Swingle, Kansas Fungi 29. I Aug. 1889.

Webber, Bull. Nebr. Exp. Sta. 1 : 334 (64). 18 Dec. I889.

Webber, Rept. Nebr. Agr. 1889 : 212 (72). 1890.

Farlow \& Seymour, Host Index 95. Sept. 1890.

Detmers, Bull. Ohio Exp. Sta. 44: 140. Sept. 1892.

Detmers, Bull. Ohio Exp. Sta. tech. ser. 1 : 178. April 1893.

Carl. Bot. Gaz. 18: 454. 25 Dec. I893.

Underw. Proc. Ind. Acad. 1893: 50. Aug. 1894.

* Carl. Ured. Amer. 46.1894.

* Syd. Uredineen 850.1894.

Diet. in Engler \& Prantl, Pflanzenfam. 11** $: 77 . \quad 1897$.

Henn. Hedw. $37: 272.25$ Oct. 1898.

Barthol. Tr. Kans. Acad. 16 : I9I. June 1899.

Freeman, Minn. Bot. Studies II. 5: 558. 20 July 1901.

Arth. Jour. Myc. 10: 17, 20. 30 Jan. 1904.

Aecidium Hamiltonia, Thm.

Thm. Bull. Torr. Bot. Club $6: 350 . d$. Oct. 1879.

De Toni in Sacc. Syll. $7: 822$. d. 28 Oct. 1888.

Aecidium quadrifldum, D C.

See Aecidium punctatum, $P$.

Aecidium Ranunculacearum, Auct. Amer. p. p.

Note. The Aecidia on American species of Ranunculus, with the exception of Aecidium Ranunculi, $\$$., are in great confusion. The name Aecidium Ranunculacearum has been rather indefinitely applied to forms occurring on Ranunculus, Anemone and Thalictrum. In Europe Aecidium Ranunculacearum has been segregated into forms connected with Puccinia perplexans, Plowr., Uromyces Dactylidis, Otth and Uromyces Poe, Rabh. See Syd. Mon. Ured. 1: 719. I Nov. 1903 , Klebahn, Rostpilze 274, 323, 324. 1904. Some of our Aecidia on Ranunculus seem to be the same as European forms 
but as the references in American literature are uncertain we have arranged them by hosts.

The references to Aecidium Ranunculacearum on Anemone with the exception of the form connected with Puccinia simillima, Arth. are also doubtful. An Aecidium on Anemone Caroliniana is described by Sydow as the rcidial stage of Puicinia cohesa, Long, $q . v$.

For the Aecidia on species of Thalictrum see Aecidium Aikeni, Syd., Aecidium Sommerfeltii, Joh., Aecidium Thalictri-favi, (D C.) Wint. and Aecidium punctatum, P.

Aecidium Ranunculacearum, Auct. Amer. p. p. Miscellanea.

Watt, Can. Nat. \& Geol. 11. 2 : 39I. Oct. 1865.

M. A. Curtis, Bot. N. Car. 124. 1867.

Russell, Am. Nat. 2: 565 . Jan. 1869 .

Cke. Handbook 539. $d$. 1871 .

Pk. Rept. N. Y. Mus. 24: 92, 106. 1872.

Frost in Cat. Pl. Amherst 85. 1875.

Pk. Rept. N. Y. Mus. 29: $76 . \quad$ i 878.

Johnson, Bull. Minn. Acad. 1: 366. 1880.

Bundy, Geol. Wis. 1873-79, $1: 400.1883$.

Cobb, List Pl. Amherst $40 . \quad$ I 887.

De Toni in Sacc. Syll. $7: 776 . d$. 28 Oct. 1888 . p. p.

Bennett, Cat. PI. R. 1. 89. 1888.

Caoma ranunculaceatum, S. Am. Bor. p. p.

S. Syn. Am. Bor. 293 no. 2875.1834.

Aecidium Ranunculacearum, Auct. Amer. p. p.

On Anemone cylindrica.

*Ell. \& Ev. N. A. F. 2214. Feb. 1889.

Webber, Bull. Nebr. Exp. Sta. 1 : 334 (64). 18 Dec. 1889.

Webber, Rept. Nebr. Agr. $1889: 212$ (72). 1890.

Cockerell, Zoe $4: 282$. Oct. I 893 .

Patterson, Bull. U. S. Agr. Pl. Industry 8: 8. 3 Feb. 1902.

Aecidium Ranunculacearum, Auct. Amer. p. p.

On Anemone multifida.

Nore. An Aecidium on this host is described by Bubak as the æcidial stage of Puccinia gigantispora, Bubak. See Syd. Mon. Ured. I: $537 . d$. 15 May 1903.

Kelsey, Jour. Myc. 5 : 80. June I889.

Aecidium Ranunculacearum, Auct. Amer. p. p.

On Anemone nemorosa.

Nore. Other references to Aecidia on this host may be found under Aecidium Anemones, P. and Aecidium lezcospermum, DC. also under Aecidium punctatum, P. It is probable that many if not all of the references given below properly belong to one or the other of those species.

*Ell. N. A. F. 1003 a. 1883 .

Arth. Bull. lowa Agr. Coll. 1884: 167. Jan. 1885.

Ell. \& Ev. Jour. Myc. 1 : 86. July 1885.

Kellerm. \& Carl. Tr. Kans. Acad. 10: $92 . \quad 1887$.

Anderson, Jour. Myc. $6:$ 121. d. 6 Jan. I89I.

Freeman, Minn. Bot. Studies 11. 5 : 555. 20 July 1901.

Davis, Tr. Wis. Acad. 14: 88. Sept. 1903.

Aecidium Ranuneulacearum, Auct. Amer. p. p.

On Anemone Pennsylvanica.

NOTE. Cultures by Arthur show an Aecidium on this host to belong to Puccinia simillima, Arth. See Arth. Bot. Gaz. 34: 18. I6 July 1902 and 35 : 20, 23. 20 Jan. 1903. Klebahn, Rostpilze 286. 1904. 
Trel. Tr. Wis. Acad. 6: 136 (32). Nov. 1884 .

Arth. Bull. lowa Agr. Coll. 1884: 167. Jan. 1885.

Burrill, Bull. III. Lab. 2: 221. d. 1885 and Rept. III. Ind. Univ. 12 : I39. d. 1885.

Snyder, Proc. Ind. Acad. 1896 : 217. 1897.

Freeman, Minn. Bot. Studies 11. $5: 555.20$ July 1901.

Arth. Bot. Gaz. 35: 20, 23. 20 Jan. 1903.

Aecidium Ranuneulacearum, Auct. Amer. p. p.

On Anemone Richardsoni.

Trel. in Sacc., Pk. \& Trel., Harriman Alaska Exp. 5 : 36.5 April 1904.

Aecidium Ranunculacearum, Auct. Amer. p. p.

On Anemone Virginiana.

Arth. Bull. lowa Agr. Coll. 1884: 167. Jan. 1885.

Orton, Rept. Vt. Exp. Sta. $11: 218$. Sept. 1898 and Contr. Bot. Vt. 2 : 20. Sept. 1898 .

Aecidium Ranunculacearum, Auct. Amer. p. p. On Anemone, sp. indet.

Hume, Proc. Davenport Acad. 7 : 252. 12 May 1899.

Aecidium Ranunculacearum, Auct. Amer. p. p. On Anemonella. Note. See also under Aecidium Thalictri-flavi, (D C.) Wint.

Detmers, Bull. Ohio Exp. Sta. 44 : 140. Sept. 1892.

Aecidium Ranunculacearum, Auct. Amer. p. p. On Delphinium.

Note. See also Aecidium Delphinii, Barthol.

Ell. \& James in Holzinger, Contr. U. S. Nat. Herb. 3 : 276. 23 Nov. 1895 .

Hume, Proc. Davenport Acad. 7 : 252. 12 May 1899.

Aecidium Ranunculacearum, Auct. Amer. p. p.. On Isopyrum.

Arth. Bull. lowa Agr. Coll. 1884: 167. Jan. 1885.

* Pazschke, Fung. Eur. 3836 . Nov. 1892 . (With note.)

Aecidium Ranunculacearum, Auct. Amer. p. p.

See Aecidium Ranunculi, S. On Ranunculus abortivus.

Aecidium Ranunculacearum, Auct. Amer. p. p. On Ranunculus abortivus.

Freeman, Minn. Bot. Studies 11. 5: 555. 20 July 1901.

Aecidium Ranunculacearum, Auct. Amer. p. p.

On Ranunculus Cymbalaria.

*Ell. \& Ev. N. A. F. 221.3. Feb. 1889.

Kelsey, Jour. Myc. 5:80. June 1889.

Anderson, Jour. Myc. 6 : 121. 6 Jan. 1891.

Carl. Tr. Kans. Acad. 13: 51. 1893.

Hume, Proc. Davenport Acad. $7: 252.12$ May 1899.

Nelson, Rept. Wyo. Exp. Sta. 10: $(36)$. 1900.

*Griffiths, West Am. Fung. 293. 22 March 1902.

Cockerell, Jour. Myc. 10 : 51. 19 March 1904.

Aecidium Ranunculacearum, Auct. Amer. p. p.

On Ranunculus fascicularis.

Tracy \& Earle, Bull. Miss. Exp. Sta. 34: 90. May 1895. 
Aecidium Ranunculacearum, Auct. Amer. p. p.

On Ranunculus, sp. indet.

Halsted, Bull. lowa Agr. Coll. 1888 : I 7 . April I888.

Ell. in Cat. Pl. N. J. 505. I889.

Trel. in Sacc., Pk. \& Trel., Harriman Alaska Exp. 5 : 36. 5 April I904.

Aecidium Ranunculacearum, var. Clematidis, Cke. See Aecidium Clematidis, D C.

\section{Aecidium Ranuneuli, S.}

Note. In Jour. Myc. Io: 18, 20. 30 Jan. 1904, Arthur gives an account of cultures showing this to be the æcidial stage of Puccinia Eatonia, Arth. n. sp. on Eatonia Pennsylvanica.

S. Syn. Car. 67 (4I) no. 440. $d .1822$.

Pk. Rept. N. Y. Mus. $23: 59 . d$. 1872.

Pk. Rept. N. Y. Mus. 24: I05. I872.

Pk. Rept. N. Y. Mus. $29: 76$. I 878.

Wint. Hedw. 22:69. May I883.

Farl. Proc. Am. Acad. 18: 75 . July 1883 .

Trel. Tr. Wis. Acad. 6: I36 (32). Nov. I884.

Arth. Bull. lowa Agr. Coll. 1884 : I68.- Jan. 1885.

Wint. \& Demet. Hedw. 24: I8I (5). Oct. I885.

Burrill, Bull. III. Lab. 2: 222. d. ${ }_{1} 885$ and Rept. 1ll. Ind. Univ. 12 : I39. $d$. I 885 .

Brendel, Flora Peoriana 2 ed. 69. 1887.

De Toni in Sacc. Syll. 7 : 776. d. 28 Oct. 1888. p. p.

Webber, Bull. Nebr. Exp. Sta. 1 : 334 (64). 18 Dec. I889.

Ell. in Cat. Pl. N. J. 505. I889.

Gall. Bull. U. S. Agr. Veg. Pathol. 8 : 56.1889.

Webber, Rept. Nebr. Agr. 1889: 212 (72). 1890.

Anderson, Jour. Myc. 6: I2I. d. 6 Jan. I8gI.

Detmers, Bull. Ohio Exp. Sta. 44: I 39. Sept. 1892.

*Pazschke, Fung. Eur. 3837. Nov. I892.

Detmers, Bull. Ohio Exp. Sta. tech. ser. 1 : I77. April 1893.

Underw. Proc. Ind. Acad. 1893: 50. Aug. I 894.

*Syd. Uredineen 900 . Aug. I 894 .

Kellerm. \& Werner, Geol. Ohio 72 : 346. Jan. 1895.

Tracy \& Earle, Bull. Miss. Exp. Sta. 34: 90. May 1895.

Stevens, Rept. Ohio Acad. 4: 20.1896.

Diet. in Engler \& Prantl, Pflanzenfam. 11** : 76. 1897.

Henn. Hedw. 37 : 272. 25 Oct. I 898.

Herbst, Fungal Fi. Lehigh Valley I76. d. I899.

Kellerm. Jour. Myc. $8: 57 . d$. 30 June I902.

* Kellerm. Ohio Fungi. 62. d. July 1902.

Caoma ranunculaceatum, S. Am. Bor. p. p.

S. Syn. Am. Bor. 293 ńo. 2875. I834.

Aecidium Ranunculacearum, Auct. Amer. p. p.

B. Grev. 3:60 (IO2). Dec. I874.

*Ell. N. A. F. 1003 b. I 883 .

Arth. \& Holw. Bull. Minn. Surv. $3:$ 3I. I Oct. 1887.

Patterson, Bull. U. S. Agr. Pl. Industry 8: 8.3 Feb. 1902.

Aecidium Ravenelii, Diet.

See Aecidium carneum, (Bosc.) n. n.

Aecidium recedens, Arth.

NotE. See also Aecidium Solidaginis, $\mathrm{S}$ and compare notes in Ell. N. A,

F. 1018. 1883 and Burrill, Bull. IIl. Lab. 2: 230.1885.

Arth. Bull, Torr, Bot. Club $31:$ 7. d. I9 Jan. I904. 
Aecidium Solidaginis, Auct. Amer. p. p.

* Barthol., Ell. \& Ev. Fung. Columb. 1804. 28 Dec. 1903.

Aecidium Rhamni, P. in Gmel.

NoTE. This is the acidial stage of Puccinia coronata, Cda. The Acidium crassum reported on Phlox in Forticth Parallel Survey, $\mid \mathrm{I}_{7} 1$, is eviclently an error.

Muhl. Cat. Pl. Am. Sept. I ed. 104. I813. 2 ed. 107. I818. *Ell. \& Ev. N. A. F. 182I. 1887.

Davis, Tr. Wis. Acad. 9: 182. 21 Aug. 1893.

Tracy \& Earle, Bull. Miss. Exp. Sta. 34: 00. May 1805.

Pk. Rept. N. Y. Mus. 50: II 5. 28 March 1808.

*Ell. \& Ev. Fung. Columb. 1299. May I898. Aecidium crassum, $\mathrm{P}$.

* Rav. Fung. Car. 1 : '92. 1852.

Watt. Can. Nat. \& Geol. 11. 2 : 390. Oct. 1865.

M. A. Curtis, Bot. N. Car. 124. 1867.

Cke. Proc. Portland Soc. 1 : I80. d. 1860.

Cke. Handbook 538. d. 1871 .

Pk. Rept. N. Y. Mus, 25 : 9I. Sept. I873.

Pk. Rept. N. Y. Mus. 29: 76.1878.

Arth. Bull. Iowa Agr. Coll. 1884: 165. Jan. 1885.

Ell. in Cat. Pl. N. J. 505.1880.

Gall. Bull. U. S. Agr. Veg. Pathol. 8: 55. 1889.

Anderson, Jour. Myc. 6 : 121. 6 Jan. 1891.

Aecidium pulcherrimum, Rav.

B. Grev. 3 : 6r (103) no. 583. $d$. Dec. 1874.

*Rav. Fung. Am. 726.1882 .

De Toni in Sacc. Syll. $7: 785$. d. 28 Oct. 1888.

*Ell. \& Ev. N. A. F. 2712. May I 802 .

Lagerh. Tromsoe Mus. Aarshefter 17: I04. d. 1805.

Sacc.' \& Syd. Syll. -14: 374. d. 20 Aug. I 899.

Aecidium Rivinæ, B. \& C.

B. \& C. Jour. Linn. Soc: $10: 358$. d. 1869. West Indies.

Bres., Henn. \& Magn. in Engl. Jahrb. 17: 491. 1893.

Heller, Muhlenbergia 1: 20.9 Aug. I90I.

Aecidium roestelioides, Ell. \& Ev.

NotE. See note under Aecidium tuberculatum, Ell. \& Kellerm.

Ell. \& Ev. Jour. Myc. 1 : 93. d. July 1885.

Ludwig, Centralbl. Bact. 1: 692. June I887.

Hark. Bull. Cal. Acad. 2: 440. I6 June i 887.

Halsted, Bull. lowa Agr. Coll. 1888: I I 7. April i 888.

De Toni in Sacc. Syll. $7: 782 . d .28$ Oct. 1888.

Cockerell, Zoe 4: 282 . Oct. 1803.

McClatchie in Reid's Hist. Pasadena 6ı6. 1895.

McClatchie, Proc. S. Cal. Acad. 1: 372.5 June 1897.

Patterson, Bull. U. S. Agr. Pl. Industry 8:8. 3 Feb. 1902.

Arth. Bull. Torr. Bot. Club. 31: 7. I9 Jan. 1904.

Aecidium roseum, Diet. \& Holw.

Holw. Bot. Gaz. 24: 36. d. 31 July 1897.

Lindau \& Syd. Hedw. 37: xvii. 9 April i 8c8. [Beiblatt no. 7.]

Sacc. \& Syd. Syll. 14: 380 . d. 20 Aug. I899.

Aecidium rubellum, $P$. in Gmel.

NOTE. According to accounts by Arthur, 1899, 1900, 1902, this is the acidial stage of Puccinia Phragmitis, (Schum) Koern. now Puccinia rubella, (P.) Arth. See also Bates, Jour. Myc. 9: 220. 3I Dec. 1903 and Klebahn, Rostpilze 284. 1904. 
Cke. Handbook 544. d. I871.

Bennett, Cat. Pl. R. I. 89. 1888.

Arth. Science 11. 10 : 564 . 20 Oct. 1899.

Arth. Bot. Gaz. 29 : 270. 30 April I900.

Arth. Bot. Gaz. 34: I7. f. I6 July 1902.

Aecidium Rumicis, Hoffm.

S. Syn. Car. 66 (40) no. 433. I822.

M. A. Curtis, Bot. N. Car. 124. I867.

Frost in Cat. PI. Amherst 85. 1875.

Cobb, List Pl. Amherst 40. 1887.

Cæoma pubellatum, Lk.

S. Syn. Am. Bor. 292 no. 2862. I 834.

Aecidium Rubi, Torrey.

See Gymnoconia interstitialis, (Schl.) Lagerh.

Aecidium on Ruellia.

Note. See also Aecidium lateripes, Kellerm. See under Puccinia lateripes, B. \& Rav.

Carl. Tr. Kans. Acad. 14: 44. I896.

Aecidium Rumicis, S. Syn. Car. p. p.

See Aecídium rubellum, P.

Aecidium Rumieis, S. Syn. Car. p. p.

See Aecidium Grossularia, $\mathrm{D}$ C.

Aecidium Rusbyi, Gerard.

Gerard, Bull. Torr. Bot. Club. 8: 34. d. March $188 \mathrm{I}$.

De Toni in Sacc. Syll. $7: 832 . d$. 28 Oct. I888.

Aecidium rutilum, Bon.

See Cystopus Ipomez-pandurani, Farl.

Aecidium sagittatum, $\mathrm{S}^{\prime}$.

See Aecidium pedatatum, S.

Aecidium Salicorniæ, Fung. Columb.

See Aecidium Chenopodilifruticosi, D C. .

Aecidium on Salvia.

Nore. In Jour. Myc. 9: 227. 31. Dec. I903 Kellerman describes Aecidium caulicolum, Kellerm. as the xcidial stage of Puccinia caulicola, Tracy \& Gall. $q . v$.

Aecidium sambuciatum, $\mathrm{S}$.

See Aecidium Sambuci, S.

Aecidium Sambuci, S.

Note. According to cultures by Arthur and by Kellerman this is the recidial stage of a Puccinia, now Puccinia Sambuci, (S) Arth. See Arth. Jour. Myc. 8: 55. 30 June 1902. Arth. Bot. Gaz. 35: 15. 20 Jan. 1903. Kellerm. Jour. Myc. 9: 7-9. I4 Feb. 1903. Syd. Mon. Ured. I: 669. I Nov. 1903. Klebahn, Rostpilze 316., I904.

Muhl. Cat. PI. Am. Sept. I ed. I04. I8I3. 2 ed. I07. 1818.

S. Syn. Car. 67 (4I) no. 44I. d. I822.

Lk., L. Sp. Pl. ed. Willd. 6²: 53. d. 1825 .

M. A. Curtis, Bot. N. Car. 124. I 867.

Pk. Rept. N. Y. Mus. 22: 93. I869.

Pk. Rept. N. Y. Mus. 24: I06. I872.

Frost in Cat. Pl. Amherst 85. $\quad$ I 875 .

Farl. Bull. Bussey lnst. 2 : 226. Jan. I 878.

*Ell. N. A. F. 1002 . I 883 .

Trel. Tr. Wis. Acad. 6: I 36 (32). Nov. I 884. 
Arth. Bull. lowa Agr. Coll. 1884: I68. Jan. I885.

Burrill, Bull. 1ll. Lab. 2 : 228. $d . \quad$ I885 and Rept. Ill. Ind. Univ. 12 : I43. $d$. I 885 .

Bessey, Am. Pomol. Snc. 1885: 43. 1886.

Cobb, List Pl. Amherst 40. 1887.

Kellerm. \& Carl. Tr. Kans. Acad. 10 : 92. 1887.

De Toni in Sacc. Syll. $7: 796$. d. 28 Oct. 1888.

Webber, Bull. Nebr. Exp. Sta. 1 : 334 (64). 18 Dec. 1889.

Gall. Bull. U. S. Agr. Veg. Pathol. 8: 56 . 1889.

Webber, Rept. Nebr. Agr. 1889: 212 (72). 1890.

Humphrey, Rept. Mass. Exp. Sta. 8: 223. I891.

Pammel, Agr. Sci. 7: 26. Jan. 1893.

Detmers, Bull. Ohio Exp. Sta tech. ser. 1 : 174. April 1893.

*Pazschke, Fung. Eur. 3937. Feb. I 894.

Small \& Vail, Mem. Torr. Bot. Club. 4: 200. April 1894.

Underw. Proc. Ind. Acad. 1893: 50. Aug. 1894.

Kellerm. \& Werner, Geol. Ohio 72: 346 . Jan. I 895.

Stevens, Rept. Ohio Acad. 4: 20. 1896.

Stevens, Rept. Ohio Acad. 5 : 66. 1897.

Atk. Bull. Cornell Univ. 3: 21. 25 June 1897.

Underw. \& Earle, Bull. Ala. Exp. Sta. 80: 2II. 28 June 1897.

Davis, Tr. Wis. Acad. 11: 167. Aug. 1897.

* Seymour \& Earle, E. F. 475. I5 Oct. 1897.

Tubeuf-Smith, Diseases of Plants 411. 1897.

Orton, Rept. Vt. Exp. Sta. 11 : 21 I. Sept. 1898 and Contr. Bot. Vt. 2: 13. Sept. 1898.

Barthol. Tr. Kans. Acad. 16 : 191. June 1899.

Herbst, Fungal FI. Lehigh Valley 175. $d . \quad 1809$.

Earle in Mohr, Contr. U. S. Nat. Herb. 6 : 194. 31 July 1901.

Kellerm. Ohio Nat. 2 : i 36. $d$. Nov. igor.

* Kellerm. Ohio Fungi 3. d. I901.

Patterson, Bull. U. S. Agr. PI. Industry 8: 8. 3 Feb. 1902.

\section{Cxoma sambuciatum, S.}

S. Syn. Am. Bor. 294 no. 2897. 1834. Aecidium sambuciatum, S.

S. Syn. Am. Bor. 300.1834 .

Watt, Can. Nat. \& Geol. 11. 2 : 391. Oct. 1865.

B. Grev. 3 : 60 (102). Dec. 1874 .

Pk. Rept. N. Y. Mus. 29: 77 . 1878.

*Rav. Fung. Am. 725. 1882.

Aecidium sanguinolentum, Lindr. See Aecidium Geranil-maculati, S.

Aecidium Saniculæ, Carm.

NotE. Aecidium Sanicula, Carm. of Europe is considered to be the xcidium of Puccinia Sanicula, Grev. In North America the latter spccies, at least according to later European mycologists, does not occur; but the Aecidium called by American mycologists Aecidium Sanicula may be connected with Puccinin Marylandica, Lindr. or possibly with Puccinia microica, Ell.

Pk. Rept. N. Y. Mus. $30: 54$. Sept. 1878.

Underw. \& Earle, Bull. Ala. Exp. Sta. 80: 2II. 28 June 1897.

Earle in Mohr, Contr. U. S. Nat. Herb. 6 : 194. 31 July Igor.

Aecidium Sareobati, Pk.

Pk. Bot. Gaz. 6 : 240. d. July 1881.

Hark. Bull. Cal. Acad. 1 : 162.7 March I885.

*Ell. \& Ev. N. A. F. 1822.1887.

De Toni in Sacc. Syll. 7 : $820, d .28$ Oct. 1888. 
Nelson, Rept. Wyo. Exp. Sta. 10: (36). 1900.

*Griffiths, West Am. Fung. 359. 21 May 1902.

Ricker, Jour. Myc. 8 : 126. I4 Oct. 1902.

Peridermium gracile, Hark.

Hark. Bull. Cal. Acad. 1 : 36 (16). d. Feb. I884.

Aecidium sclepothecioides, Ell. \& Ev.

Note. See also Aecidium Compositarum, var. Senecionis, Auct. Amer.

Ell. \& Ev. Am. Nat. 31 : 428. d. May I 897.

Lindau \& Syd. Hedw. 37 : xvii. 9 April I898. [Beiblatt no. 7.]

Sacc. \& Syd. Syll. 14: 377. d. 20 Aug. 1899.

Aecidium Serophulariæ, N. A. F. See Aecidium tenue, S.

Aecidium Senecionis, Auct. Amer.

See Aecidium Compositarum, var. Senecionis, Auct. Amer.

Aecidium Sil-latifolii, Wint.

NoTE. It is uncertain with what teleutosporic form the Aecidium in refer. ences given below is connected. For a statement of the connection of the Aecidium on Sium latifolium of Europe with Uromyces Scirpi, (Cast.) Lagerh., = Uromyces lineolatus, (Desm.) Schrt, see Klebahn, Rostpilze 325 . I904.

Trel. Tr. Wis. Acad. $6: 137$ (33). Nov. 1884.

Lindr. Acta Soc. Fauna et Fl. Fennica $22^{1}$ : 177. 1902.

Aecidium Silphii, (Burrill) Syd.

*Syd. Uredineen 1546. April I90 I.

Aecidium Compositarum, Auct. Amer. p. p.

Trel. Tr. Wis. Acad. $6: 137$ (33). Nov. I884.

Arth. Bull. lowa Agr. Coll. 1884: 165. Jan. I885.

Kellerm. \& Carl. Tr. Kans. Acad. 10:91. 1887.

Gall. Bull. U. S. Agr. Veg. Pathol. 8: 55. I 889.

Davis, Tr. Wis. Acad. 9 : 16I. 21 Aug. 1893.

Underw. \& Earle, Bull. Ala. Exp. Sta. 80: 208. 28 June 1897.

Barthol. Tr. Kans. Acad. 16 : 187. June 1899.

* Shear, Ell. \& Ev. Fung. Columb. 1478. May I9oı.

Earle in Mohr, Contr. U. S. Nat. Herb. 6: I92. 31 July I9or. Aecidium Compositarum, var. Silphii, Burrill.

Burrill, Bull. Ill. Lab. 2: 231. d. 1885 and Rept. III. Ind. Univ. 12 : 144: $d$. 1885 .

Wint. \& Demet. Hedw. 24: 179 (3). Oct. 1885.

De Toni in Sacc. Syll. $7: 798 . d$. 28 Oct. 1888.

* Vestergren, Micr. sel. 626. May igo3.

Aecidium smilacinatum, $\mathrm{S}$.

See Aecidium Smilacis, S.

\section{Aecidium Smilacis, S.}

Nore. The Accidium on Smilax herbacea and $S$. hispida is said by Arthur to belong to Puccinia amphigena, Diet. It differs from the authentic specimen of Aecidium Smilacis, S. which we have examined, in not causing verruciform distortions of the leaves but, as far as the microscopic characters of the spores are concerned, they appear to be the same; for although in the Schweinitzian specimen they were hardly mature, they at least were smaller and wanting in the thickening of the apical wall found in Aecidium macrosporum, Pk. See also Syd. Mon. Ured. I : 742. I Nov. 1903. Klebahn, Rostpilze 276. 1904. Arth. Jour. Myc. I0: II. 30 Jan. I904.

S. Syn. Car. 69 (43) no. 452. 1822.

M. A. Curtis, Bot. N. Car. 124. 1867. 
Kellerm. Bull. Wash. Lab. 1 : 75. Jan. 1885 and Tr. Kans. Acad. 9 : 8I. 1885 .

Burrill, Bull. III. Lab. 2: 238. d. $188_{5}$ and Rept. III. Ind. Univ. 12 : 149. $d$. 1885 .

Kellerm. \& Carl. Tr. Kans. Acad. 10 : 92. 1887.

De Toni in Sacc. Syll. $7: 829 . d .28$ Oct. 1888.

Webber, Bull. Nebr. Exp. Sta. 1: 334 (64). 18 Dec. 1889.

Webber, Rept. Nebr. Agr. 1889 : 212 (72). 1800.

Tracy \& Earle, Bull. Miss. Exp. Sta. 34: 90. May 1895.

Tubeuf-Smith, Diseases of Plants 4I1. 1897.

Barthol. Tr. Kans. Acad. 16 : I9I. June I 899.

Patterson, Bull. U. S. Agr. Pl. Industry 8: 8. 3 Feb. 1902.

Arth. Bot. Gaz. 35 : 20. 20 Jan. 1903.

Caoma smilacinatum, $\mathrm{S}$.

Lk., L. Sp. Pl. ed. Willd. $6^{2}: 43 . d .1825$.

S. Syn. Am. Bor. 292 no. 2859. 1834.

Uredo Smilacis, S.

Spreng. in L. Syst. Veg. 16 ed. $4^{1}$ : 572. $d . \quad 1827$. Aecidium smilacinatum, $\mathrm{S}$.

S. Syn. Am. Bor. 309.1834 .

Aecidium verrucosum, Bon.

Bon. Abh. Nat. Ges. Halle 5: 210 (44) d. 1860.

Aecidium Smilacis, N. A. F. 2708.

See Aecidium macrosporum, $\mathrm{Pk}$.

Aecidium Solani, Mont.

NótE. Whether American writers are correct in referring the Aecidium on Physalis in North America to Aecidium Solani, Mont. 1852, is duubtful. 'The Aecidium on Physalis, whatever it may be called, is generally considered to belong to Puccinia Physalidis, Pk.

In Harkness, 1885, and Seymour, 1896, given below, the host is Solanum. Harkness considered his Aecidium to belong to Puccinia Solani, Cke. 1878, but there is an earlier Puccinia Solani, B. \& C. 1853 , based on specimens from Surinam in Herb. Schweinitz.

Trel. Tr. Wis. Acad. $6: 134$ (30). Nov. 1884.

Hark. Bull. Cal. Acad. 1 : 162. 7 March I885.

Burrill, Bull. III. Lab. 2 : 235. $d$. 1885 and Rept. III. Ind. Univ. 12 : 147. $d$. 1885 .

*Ell. \& Ev. N. A. F. 3147. Nov. 1894.

*Ell. \& Ev. Fung. Columb. 558. 1894.

* Seymour, Pringle's Mex. Fungi 6. I Sept. 1896. Aecidium Physalidis, Burrill.

Burrill, Bot. Gaz. 9 : 190. d. Dec. 1884.

Aecidium solidaginicolum, Ell. \& Ev.

Ell. \& Ev. Erythea 1: 206. d. 2 Oct. 1893.

Aecidium Solidaginis, S.

Note. For connection of Aecidium on Solidago with Puccinia CaricisSolidaginis, Arth. see Arth. 1903, Kellerm. 1903. See also Kellerm. Jour. Myc. 9: 228, 237. 31 Dec. 1903. See also Syd. Mon. Ured. I; 666. I Nov. 1903 and Klebahn, Rostpilze 305. 1904.

Likewise for Uromyces Solidagini-Caricis, Arth. see Arth. Jour. Myc. 10: $15,20.30$ Jan. 1904.

The greater part of the references given below properly belong to Schweinitz's species but some, especially from older writings, cannot now be verified.

See also Aecidium 'solidiginicolum, Ell. \& Ev. and Aecidium recedens, Arth. 
Muhl. Cat. Pl. Am. Sept. I ed. I04. I813. 2 ed. I07. I818.

S. Syn. Car. 68 (42) no. 446. $d$. 1822.

Lk., L. Sp. Pl. ed. Willd. $6^{2}:$ 52. $d$. 1825.

Hitchcock, Cat. Pl. Amherst 59. I829.

Hitchcock, Cat. PI. Mass. 644. I833. 2 ed. 645 (I25). 1835.

* Griffiths, West Am. Fung. 362. 2i May 1902.

Arth. Bot. Gaz. 35 : 21. 20 Jan. 1903.

* Barthol. Ell. \& Ev. Fung. C Jlumb. 1707, 1708. Feb. I903.

Garrett, Tr. Kans. Acad. 18; 147. 1903.

Cacoma asteratum, Lk. p. p.

Lk., L. Sp. Pl. ed. Willd. $6^{2}$ : 51, $d . \quad$ I825.

S. Syn. Am. Bor. 292 no. 2870.1834.

Aecidium asteratum, S. p. p.

S. Syn. Am. Bor. 300. I 834 .

Atk. Bull. Cornell Univ. 3: 21. 25 June 1897. Aecidium Compositarum, Auct. Amer. p. p.

Trel. Tr. Wis. Acad. 6 : 137 (33). Nov. 1884.

Arth. Bull. lowa Agr. Coll. 1884: I65. Jan. I 885.

Arth. \& Holw. Bull. Minn. Surv. 3: 3I. I Oct. I887.

Anderson, Jour. Myc. $5: 82$. June I 889.

Detmers, Bull. Ohio Exp. Sta. 44: 140. Sept. I 802.

Detmers, Bull. Ohio Exp. Sta. tech. ser. 1: 178. April I893.

Stevens. Rept. Ohio Acad. 4: 20. 1896.

Herbst, Fungal Fl. Lehigh Valley 176. Dec. I899.

Pound \& Clements, Bot. Surv. Nebr. 5 : 24. 30 March I9oI,

Patterson, Bull. U.S. Agr. Pl. Industry 8: 8. 3 Feb. 1902.

Aecidium Asterum, Auct. Amer. p. p.

Burrill, Bull. 1ll. Lab. 2: 230. d. 1885 and Rept. Ill. Ind. Univ. 12 : I44. $d$. I885.

Gall. Bull. U. S. Agr. Veg. Pathol. 8: 55. 1889.

Detmers, Bull. Ohio Exp. Sta. $44: 138$. Sept. I 892.

Halsted, Rept. N. J. Exp. Sta. 1892: 347. I893.

* Shear, N. Y. Fungi 128 . I893.

Underw. Proc. Ind. Acad. 1893 : 49. Aug. I 894.

Kellerm. \& Werner, Geol. Ohio $7^{2}: 345$. Jan. I 895.

Underw. Proc. Ind. Acad. 1894: i 54 . Oct. i 895.

Stevens, Rept. Ohio, Acad. 4: $20 . \quad$ I896.

Underw. \& Earle, Bull. Ala. Exp. Sta. 80: 208. 28 June 1897.

Tubeuf-Smith, Diseases of Plants 4ri. 1897.

Claassen, Rept. Ohio Acad. 5 : 68. I 897.

*Ell. \& Ev. Fung. Columb. 1391. May I899.

Barthol. Tr. Kans. Acad. 16: I86. June I 899.

Orton, Rept. Vt. Exp. Sta. 12: I65. Dec. I 899 and Contr. Bot. Vt. I65. Dec. I899.

Claassen, Rept. Ohio Acad. 7 : 43. 1899.

Earle in Greene, Pl. Baker. 2: 2. 25 March I9oI.

Freeman, Minn. Bot. Studies 11. $5: 557.20$ July I90 I.

Earle in Mohr, Contr. U. S. Nat. Herb. 6 : 192. 3I July I9oI.

Aecidium Compositarum, var. Solidaginis, Ell.

*Ell. N. A. F. 1018c. I883. (With note.)

Burrill, Bull. 1Il. Lab. 2:230. 1885 and Rept. 111. Ind. Univ, 12: I44. I885. (Note.)

Wint. \& Demet. Hedw. 24 : I80 (4). Oct. I885.

Aecidium Solidaginis, Auct. Amer. p. p.

See Aecidium recederis, Arth. 
Aecidium Sommerfeltii, Joh.

Notk. There are at least two species of Aecidium on species of Thalictrum in Nurth America; Aecidium Sommer/eltii, Juh, $189+$ based on Caroma Thalictri, Sommf. 1826 and Aecidium Thalictri-favi, Wint. based on Accidium K'anuncnlacearum, var. Thalictri-flavi, DC. 1815, from which Aecidium Thalictri, Grev. 1823 appears not to differ.

IVe use the name Aecidium Sommerfeltii, Joh. for the species which occurs on Thalictrum alpinum in Greenland and the northern and higher parts of America and possibly on sume other hosts.

American mycologists have as a rule followed De Toni who refers to Aecid. ium Sommerfeltii all Aecidia on Thalictrum in Europe and North America, but others have used the name Aecidium Thalictri in the sense in which we here use the name Aecidinm Thalictri-flavi.

Of the Aecidia on Thalictrum Fendleri, referred variously to Aecidium Sommerfeltii and Accidinm Thalictri, the specimens we have seen seem to be Aecidium Thalictri-flavi; but in some cases genuine Aecidium Sommerfeltii appears to have been found on that host.

According to Juel Oefv. K. Vet.-Akad. Foerh. 52: 383.1885 , Aecidium Sommerfeltii is the Aecidium of Puccinia septentrionalis, Juel on Polygonum viviparum. See also Syd. Mon. Ured. I: 575. I5 May 1903, Klebahn, Rostpilze 321. I904.

\section{On Thalictrum alpinum.}

Rostr. Meddelelser om Groenl. 3 : 536.1888.

De Toni in Sacc. Syll. $7: 775 . d$. 28 Oct. 1888.

Rostr. Meddelelser om Groenl. 3 : $604 . \quad$ I 891.

\section{On Thalictrum Fendleri.}

?Cockerell, Zoe. $4: 283$. Oct. 1893.

Henn. Hedw. $37: 272$. 25 Oct. 1898.

Hume, Proc. Davenport Acad. $7: 252$. 12 May 1899.

Cockerell, Jour. Myc. 10 : 51. 19 March 1904.

\section{Miscellanea.}

?Hume, Proc. Davenport Acad. 7 : 252. 12 May I899. T. sparsiflorum. ?Blasdale in Davy, Bull. U. S. Agr. Pl. Industry 12: 75. 1902. Aquilegia.

Aecidium Sommerfeltil, Auct. Amer. p. p.

See Aecidium Thalictri-flavi, (D C.) Wint.

Aecidium Sphæralceæ, Ell. \& Ev.

NOTE. The species of Accidium on Malvacex, some of which were described from dried specimens, appear to have been unduly multiplied. See Aecidium Malvastri, Ell. \& Tracy, Aecidium interveniens, (Pk.), Aecidium Napaa, Arth. \& Holw. and Aecidinm Callirrhoes, Ell. \& Kellerm. See Arth. Bull. Torr. Bot. Club 31: 7. I9 Jan. I904. See also Syd. Mon. Ured. I : 478. I5 May 1903.

Ell. \& Ev. Bull. Torr. Bot. Club 22 : 364. d. 31 Aug. 1895.

Sacc. Hedw. 35 : xvii. 25 Feb. 1896. [Reportorium no. 7.]

*Ell. \& Ev. N. A. F. 3345. Feb. I896.

*Ell. \& Ev. Fung. Columb. 871. April I896.

Aecidium splendens, Wint.

See Aecidium Crotonopsidis, Burrill.

Aecidium Spragueæ, Hark.

NoTE. Described by the author as the acidial stage of Uromvces Spraguea, Hark. 
Hark. Bull. Cal. Acad. 1 : 36,16$), d$. Feb. 1884.

De Toni in Sacc. Syll. 7 : 557. $d$. 28 Oct. 1888 .

Aecidium Statices, Desm.

Note. The teleutosporic stage is Uromyces Limonii, (D C.) Lev.

Pk. Rept. N. Y. Mus. 29 : 77. $\quad$ I 878.

*Ell. N. A. F. 242. 1879 .

Hark. Bull. Cal. Acad. 1 : I62. 7 March 1885.

*Ell. \& Ev. Fung. Columb. 658. 24 April 1895.

Tracy \& Earle, Bull. Miss. Exp. Sta. 34: 91. May 1895.

Aecidium Limonil, Pk.

Pk. Rept. N. Y. Mus. 23: 60. d. 1872.

Pk. Rept. N. Y. Mus. 24: 106. 1872.

Aecidium Stillingiæ, Tracy \& Earle.

Tracy \& Earle, Bull. Torr. Bot. Club $26: 493 . d$. 22 Sept. 1899.

Sacc. \& Syd. Syll. 16 : 346. $d$. I Feb. I902.

Aecidium Tanaceti, Cockerell.

See Aecidium Compositarum, var. Artemisia, C. \& E.

Aecidium Taraxaci, Kze. \& Schm.

Note. Compare Aecidium Compositarum on Krigia, Iactuca, Troximon. For association of Aecidium Taraxaci, Kze. \& Schm. with Puccinia silvatica, Schrt., see Klebahn, Rostpilze 302. 1904. See also Oscar Mayus, Centralbl. Bact. 10: 714. 19 Aug. 1903.

Trel. Tr. Wis. Acad. 6 : 137 (33). Nov. 1884.

Aecidium Tenellae, Diet. \& Holw.

Note. Originally printed Aecidium Tonella, D. \& H.

Diet. Erythea $3:$ 77. $d$. I May I 895 .

Lindau \& Syd. Hedw. 37 : xviii. 9 April I898. [Beiblatt no. 7.]

Sacc. \& Syd. Syll. 14: 384. d. 20 Aug. I899.

Aecidium tenue, $\mathrm{S}$.

Note. This is believed to be the rcidial stage of Puccinia tenuis, (S.) Burrill, with which it is intimately associated on leaves of Eupatorium ageratoides. See Burrill 1885 and Syd. Mon. U̦red. I : 82. 30 June 1902

Specimens reported under this name on several other species of Eupato. rium, grouped below under Miscellanea, are presumed to belong more properly to Aecidium Compositarum, var. Eupatorii, (S.) B. The teleutospores of Puccinia tenuis, Burrill, are reported only on Eupatorium ageratoides. There is a Puccinia tenuis, Bon. 1860. A. B. S.

S. Syn. Am. Bor. 309. I834.

Pk. Rept. N. Y. Mus. 24: 92, I07. 1872.

Pk. Rept. N. Y. Mus. 25: Io9. Sept. I873.

Pk. Rept. N. Y. Mus. $29: 77$. 1878.

*Ell. N. A. F. 1420 . I 885.

Burrill, Bull. Ill. Lab. 2: 18 I. d. 1885 and Rept. 1ll. Ind. Univ. 12 : II 7. $d$. I 885 .

*Pazschke, Fung. Eur. 4141。 1898.

Caoma tenue, $\mathrm{S}$.

S. Syn. Am. Bor. 293 no. 2889. d. 1834.

Aecidium Compositarum, var. Eupatoria, Auct. Amer. p. p.

* Ell. \& Ev. Fung. Columb. 498 . I 894.

Freeman, Minn. Bot. Studies II. $5: 557.20$ July 1901 .

Aecidium Scrophularia, N. A. F.

*Ell. \& Ev. N. A. F. 1810. ${ }_{1} 887$. 


\section{Miscellanea.}

Aecidium tenue, Auct. Amer. p. p.

Arth. Bull. lowa Agr. Coll. 1884: 168. Jan. 1885.

Wint. \& Demet. Hedw. 24: 18r (5). Oct. 1885.

Gall. Bull. U. S. Agr. Veg. Pathol. 8: 56. 1889.

Aecidium Thalictri, Grev.

See Aecidium Thalictri-flavi, (D C.) Wint.

Aecidium Thalletri, Auct. Amer. p. p.

See Aecidium punctatum, $\mathrm{P}$.

Aecidium Thalietri-Plavi, (D C.) Wint.

NoTE. Assuming it to be correct that Aecidium Thalictri-Ravi, (D C.) Wint. and Aecidium Thalictri, Grev. are synonyms, it should be remarked that the form on Thalictrum flavmm is considered by Plowright, British Ured. \& Ust. 181. 1889 to belong to Puccinia persistens, Plowr. on Poa nemoralis, while the form on Thalictrum alpinum is considered Juel, Oefv. K. Vet. Akad. Foerh. 51:413. 1894 to belong to Puccinia borealis, Juel on Agrostis borealis. See also Syd. Mon. Ured. I : 718, 719. I Nov. 1903, Klebahn, Rostpilze 276, 291. 1904.

Some of our references to Accidia on Thalictrum alpinum are given under Aecidium Sommerfeltii, Joh. The references given below to Aecidium Thalictri-flavi, in absence of cultures made in this country, must be regarded as provisional. See also Aecidium punctatum, P. Compare also Aecidium Aikeni, Syd. especially with reference to citations of Aecidium on Thalictrum purpurascens.

For anatomical details of. Aecidia on Thalictrum see Oscar Mayus, Centralb. Bact. 2 Abt. 1o: 649, 706 et seq. 1903

\section{On Thalictrum.}

Kelsey, Jour. Myc. $5: 80$. June 1889.

Freeman, Minn. Bot. Studies 11. 5: 555. 20 July 1901 .

Patterson, Bull. U. S. Agr. Pl. Industry 8:8. 3 Feb. 1902.

\section{On Anemonella.}

Noтع. See also under Aecidium Ranunculacearum, Auct. Amer. p. p.

Wint. \& Demet. Hedw. 24: 181 (5). Oct. 1885.

*Wint. Fung. Eur. 3322 . I 885.

Gall. Bull. U. S. Agr. Veg. Pathol. 8: 56. 1889.

Underw. Proc. Ind. Acad. 1894 : 151. Oct. 1895. Aecidium Thalictri, Grev.

\section{On Thalictrum.}

Farl. Appalachia $3:$ 239. Jan. 1884.

Trel. Tr. Wis. Acad. 6: 136 (32). Nov. 1884.

Arth. Bull. lowa Agr. Coll. 1884: i68. Jan. 1885.

*Ell. N. A. F. 1423 . 1885.

Arth. \& Holw. Bull. Minn. Surv. 3: 31. I Oct. 1887.

Halsted, Bull. lowa Agr. Coll. 1886: 58 . 1887.

Halsted, Bull. lowa Agr. Coll. 1888: i i6. April 1888.

Rostr. Meddelelser om Groenl. 3 : 604. 1801 .

Davis, Tr. Wis. Acad. 9 : 161. 21 Aug. I893.

? Cockerell, Zoe. 4: 283 . Oct. 1893.

*Ell. \& Ev. Fung. Columb. 193. I893.

* Griffiths, West. Am. Fung. 35. 22 March 1902.

\section{Miscellanea.}

No7e. In Jour. Myc 8: 126. I4 Oct. 1902 Rịcker mentions an Aecidium on Thalictrum occidentale from Idaho.

Watt, Can. Nat. \& Geol. 11. 2 : 391. Oct. 1865. 
Pk. Rept. N. Y. Mus. 24: 92, 106. 1872.

B. Grev. 3 : 60 (I02). Dec. 1874.

Pk. Rept. N. Y. Mus. 29: $76 . \quad$ I 878 .

*Ell. \& Ev. Fung. Columb. 1390. May 1899.

Aecidium Sommerfeltii, Auct. Amer. p. p.

On Thalietrum.

Webber, Bull. Nebr. Exp. Sta. 1 : 334 (64). I8 Dec. I889.

Webber, Rept. Nebr. Agr. 1889: 2 I2 (72). I89o.

Nelson, Rept. Wyo. Exp. Sta. 10 : (36). 1900.

Tracy \& Earle in Greene, Pl. Baker. 1: 19. 22 Feb. I90I.

Earle in Greene, Pl. Baker. 2: 3. 25 March I9oI.

\section{On Anemonella.}

Detmers, Bull. Ohio Exp. Sta. 44: 139. Sept. I892.

Kellerm. \& Werner Geol. Ohio 72 : 347 . Jan. I895.

Aecidium Tissæ, Ell. \& Ev.

McClatchie, Proc. S. Calif. Acad. 1 : 372. 5 June 1897.

Ell. \& Ev. Bull. Torr. Bot. Club 24: 284. $d$. 29 June 1897.

Lindau \& Syd. Hedw. 37 : xviii. 9 April 1898. [Beiblatt no. 7.]

Sacc. \& Syd. Syll. 14: 374. d. 20 Aug. I 899.

Aecidium Tonellæ, Diet. \& Holw.

See Aecidium Tenella, Diet. \& Holw.

Aecidium trachelifoliatum, S.

Note. On Helianthus authors report also Aecidium Compositarum, var. Helianthi, Burrill, and Aecidium Helianthi-mollis, $\mathrm{S}$.

S. Syn. Am. Bor. 309. I834.

Pk. Rept. N. Y. Mus. $29: 77 . \quad 1878$.

Cke. Grev. 20 : I 10. June I 892.

Croma trachellfoliatum, $\mathrm{s}$.

S. Syn. Am. Bor. 292 no. $2872 . d$. I 834 .

Aecidium Traeyanum, Syd.

Note. See also Aecidium lateripes, Kellerm.

Syd. Hedw. Beibl. 40 : (129). d. 20 Oct. I90I.

Sacc. \& Syd. Syll. 16: i122. I Feb. I902.

Aecidium transformans, (Ell.) Pazschke.

* Pazschke, Fung. Eur. 4238 . 1901 .

Roestelia transformans, Ell.

Ell. in Pk. Bull. Torr. Bot. Club 5 : 3. d. Jan. 1874 .

Farl. Bull. Bussey Inst. 2: 225. Jan. 1878.

Farl. Anniv. Mem. Boston Soc. 27. d. 1880.

*Ell. N. A. F. 1088 . I 883.

Farl. Proc. Am. Acad. $20: 315 . \quad$ I 885.

Farl. Bot. Gaz. 11: 238 . Sept. I 886.

Thax. Proc. Am. Acad. 22: 269. Jan. 1887 and Centralbl. Bact. 1 : 460. 1887.

Seymour, Tr. Am. Hort. Soc. $4:$ i55 (4). 7 April 1887.

Ell. in Cat. Pl. N. J. 504. I889.

* Seymour \& Earle, E. F. 247. I Feb. I893.

Hicks, Asa Gray Bull. 1893"3 : $12 . \quad 1893$.

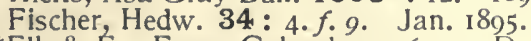

*Ell. \& Ev. Fung. Columb. 1076. 22 Dec. 1896.

Diet. Ann. Myc. 1:9. $30 \mathrm{Jan} .1903$.

Rostelia transformans, Ell., var. fructigena, Thm.

*Thm. Myc. Univ. I029. 1878.

Thm. Flora $62:$ i04. I March i879. 
Roum. Rev. Myc. 10 : 143. I July 1888.

* Roum. Fung. Gall. 4533. 1888.

Aecidium Trifolli-repentis, Cast.

Notr. Under this name we have placed references to Aecidia on Trifolium repens which should properly be considered to be the Aecidium of Uromyces minor, Schrt. but whether this is strictly true in all cases cannot be stated.

Thm. Nat. Can. $10: 9$. Jan. 1878 .

?Aecidium Orobi, Auct. Amer. p. p.

Pk. Rept. N. Y. Mus. $23: 59.1872$.

Pk. Rept. N. Y. Mus. 24: 107. 1872.

B. Grev. 3: 61 (103). Dec. 1874.

Pk. Rept. N. Y. Mus. $29: 76 . \quad$ i 878.

Johnson, Bull. Minn. Acad. 1: 365. 1880.

Arth. Bull. lowa Agr. Coll. 1884: 167. Jan. 1885.

Aecidium Triglochinis, Diet. \& Holw.

Holw. Erythea $7: 98$. d. 31 Oct. 1899.

Sacc.\& Syd. Syll. $16: 348 . d$. I Feb. 1902.

Aecidium Trillil, Burrill.

Burrill, Bot. Gaz. 9 : 100. d. Dec. 1884.

Burrill, Bull. III. Lab. 2: 238. d. 1885 and Rept. III. Ind. Univ. 12 : 149. $d$. 1885 .

De Toni in Sacc. Syll. $7: 828 . d .28$ Oct. 1888.

Claassen, Rept. Ohio Acad. 5 : 68.1897.

Snyder, Proc. Ind. Acad. 1896: 218., 1897.

Aecidium tuberculatum, Ell. \& Kellerm.

Note. We are unable to distinguish some specimens in exsiccati issued as Aecidium tuberculatum, Ell. \& Kellerm. from Aecidium roesteitoides, Ell. \& Ev. The original descriptions of the two differ, however. See Arth. Bull. Torr. Bot. Club 3I : 7. 19 Jan. 1904.

Ell. \& Kellerm. Jour. Myc. 4 : 26, d. March 1888.

Swingle, Jour. Myc. 4:27. March 1888.

De Toni in Sacc. Syll. $7: 827$. d. 28 Oct. 1888.

*Kellerm. \& Swingle, Kansas Fungi 30. I A'ug. 1889.

*Ell. \& Ev. N. A. F. 2893. March 1893.

Carl. Bot. Gaz. 18 : 453. 25 Dec. 1803.

*Ell. \& Ev. Fung. Columb. 500. 1894.

* Carl. Ured. Amer. 31, 1894.

* Syd. Uredineen 1199 . Feb. 1898.

Barthol. Tr. Kans. Acad. 16 : 191. June 1899.

Nelson, Rept. Wyo. Exp. Sta. 10: (36). 1000.

Carl. Science II. 13 : 250. I5 Feb. 1901.

*Pazschke, Fung. Eur. 4239. 1901.

Carl. Jour. Appl. Micr. 6: 2111. 1903.

Arth. Bull. Torr. Bot. Club 31: 7. 19 Jan. 1904.

Aecidium Tussilaginis, Muhl. Cat.

Muhl. Cat. PI. Am. Sept. I ed. 104. 1813. 2 ed. 107. 1818.

Aecidium Urticæ, Schum.

Note. The teleutosporic stage is Puccinia Caricis, (Schum.) Reb. as established in Europe by Magnus, Schroeter. and others and verified in America by Arthur, 1899, 1900, 1902, 1903 and Kellerman, 1903. See also Syd. Mon. Ured. I : 648. I Nov. 1903 and Klebahn, Rustpilze 293. I904.

M. A. Curtis, Bot. N. Car. 124. 1867.

Cke. Handbook 541. $d$. 1871. 
Pk. Rept. N. Y. Mus. 24: 92, 106. 1872.

Frost in Cat. Pl. Amherst 85. 1875.

Johnson, Bull. Minn. Acad. 1 : 296. 1877 and Rept. Minn. Surv. 5 :

85. 1877.

Pk. Rept. N. Y. Mus. $29: 77 . \quad 1878$.

*Ell. N. A. F. 267.1879.

Arth. Bull. Iowa Agr. Coll. 1884: 168. Jan. 1885.

Burrill, Bull. 1ll. Lab. $2: 238 . d .1885$ and Rept. Ill. Ind. Univ. 12 : 149. $d$. 1885.

Cobb, List Pl. Amherst 40. 1887.

De Toni in Sacc. Syll. $7: 821 . d$. 28 Oct. 1888. p. p.

Bennett, Cat. Pl. R. 1. 89. $\quad$ I 888.

Kelsey, Jour. Myc. 5 : 80 . June 1889.

Anderson, Jour. Myc. 5: 82. June 1889 .

Webber, Bull. Nebr. Exp. Sta. 1: 334 (64). 18 Dec. 1889.

Gall. Bull. U. S. Agr. Veg. Pathol. 8: 56. 1889.

Webber. Rept. Nebr. Agr. 1889: 212 (72). 1890.

Williams, Bull. S. Dak. Exp. Sta. 29: 49. Dec. I891.

* Seymour \& Earle, E. F. 388. I May 1893.

McClatchie in Reid's Hist. Pasadena 6I6. 1895.

McClatchie, Proc. S. Cal. Acad. 1 : 372. 5 June 1897.

Hume, Proc. Davenport Acad. 7 : 249. 12 May 1899.

Barthol. Tr. Kans. Acad. 16: 192. June 1899.

Arth. Science 11. 10: 565. 20 Oct. 1899.

Arth. Bot. Gaz. 29: 270. 30 April 1900.

Tracy \& Earle in Greene, PI. Baker. 1: 19. 22 Feb. 1901.

Freeman, Minn. Bot. Studies 11. 5: 546. 20 July 1901.

*Griffiths, West Am. Fung. 52. 22 March 1902.

Arth. Jour. Myc. 8: 52, 56. 3o June 1902.

Kellerm. Jour. Myc. 8 : 59. $d$. 30 June 1902.

*Kellerm. Ohio Fungi 69. d. July 1902.

Arth. Bot. Gaz. 35: 16, 22. 20 Jan. 1903.

Kellerm. Jour. Myc. $9:$ 9, 10, 12, 13. 14 Feb. 1903.

Cæoma urticatum, Lk.

S. Syn. Am. Bor. 294 no. 2898. 1834.

Aecidium Uvulariæ, S.

S. Syn. Car. 69 (43) no. 453.d. 1822.

M. A. Curtis, Bot. N. Car. 123. 1867.

Pk. Rept. N. Y. Mus. 26: 78 . April 1874.

Wint. \& Demet. Hedw. 24 : I81 (5). Oct. 1885.

De Toni in Sacc. Syll. 7 : 830. d. 28 Oct. 1888.

Freeman, Minn. Bot. Studies 11. 5: 554. 20 July I90I.

Croma alliatum, Lk. p. p.

Lk., L. Sp. Pl. ed. Willd. $6^{2}: 43$. d. 1825.

Uredo Uvularia, Spreng.

Spreng. in L. Syst. Veg. IO ed. $4^{1}: 572 . d . \quad$ I 827.

Caoma uvulariatum, S.

S. Syn. Am. Bor. 292 no. 2858 . d. 1834.

Aecidium uvulariatum, $\mathrm{S}$.

S. Syn. Am. Bor. 309. 1834 .

Pk. Rept. N. Y. Mus. $29: 77 . \quad 1878$.

Aecidium uvulariatum, $\mathrm{S}$.

See Aecidium Uvulariz, S.

Aecidium Valerianearum, Duby.

*Ell. \& Ev. N. A. F. 2211 . Feb. 1889.

Aecidium Valerianellæ, Biv: Bernh.

Diet. Bot. Gaz. 18: 256. d. 15 July 1893 . 
Blasdale, Asa Gray Bull $1893^{3}$ : I. $\quad$ I 893.

Henn. Hedw. 37 : 272. d. 25 Oct. I 898.

Aecidium verbenicolum, Ell. \& Kellerm.

Norf. According to cultures by Arthur this is the recidial stage of Puccinia Vilfa, Arth. \& Holw. which is renamed Puccinia verbenicola, Arth. See Arthur, 1899, 1900, 1903. See also Klebahn, Rostpilze 275. 1904.

Ell. \& Kellerm. Bull. Torr. Bot. Club 11 : I I4. d. Oct. I884.

Kellerm. Bull. Wash. Lab. 1 : 75. Jan. 1885 and Tr. Kans. Acad. 9 : 81. 1885 .

Wint. \& Demet. Hedw. 24: 181 (5). Oct. 1885.

*Ell. N. A. F. I434. I885.

Roum. Rev. Myc. 8 : Ig6. I Oct. I 886.

*Roum. Fung. Gall. 3861. I886.

Kellerm. \& Carl. Tr. Kans. Acad. 10:92. 1887.

Gall. Bull. U. S. Agr. Veg. Pathol. 8:,56. I 889.

* Pazschke, Fung. Eur. 3939. Feb. I 894.

Diet. in Engler \& Prantl, Pflanzenfam. 11**: 77. 1897.

Barthol. Tr. Kans. Acad. 16 : 192. June I899.

Arth. Science 11. 10 : 565. 20 Oct. I 899.

Arth. Bot. Gaz. 29 : 274. 30 April 1900.

Arth. Bot. Gaz. 35 : 16. 20 Jan. 1903.

Garrett, Tr. Kans. Acad. 18 : 147. 1903.

Aecidium Verbena, Auct. Amer. non Speg.

De Toni in Sacc. Syll. 7 : 816. d. 28 Oct. 1888.

Webber, Bull. Nebr. Exp. Sta. 1 : 334 (64). 18 Dec. 1889.

Webber, Rept. Nebr. Agr. 1889: $212(72)$. I 890.

Williams, Bull. S. Dak. Exp. Sta. 29: 49. Dec. I891.

*Ell. \& Ev. Fung. Columb. 270. I 894.

* Carl. Ured. Amer. 2I. I 894 .

Snyder, Proc. Ind. Acad. 1896 : 2 I8. I897.

* Syd. Uredineen 1200 . Feb. 1808 .

* Seymour \& Earle, E. F. 523. 7 June I 899.

Freeman, Minn. Bot. Studies 11. 5 : 556. 20 July I90 I.

Patterson, Bull. U. S. Agr. Pl. Industry 8: 8. 3 Feb. 1902.

Aecidium Verbesinæ, S.

Note. Said by Lagerheim to belong to Puccinia Verbesina, S. See Lagerh. Tromsoe Mus. Aarshefter 17: 78. 1895.

S. Syn. Car. 68 (42) no. 445. d. 1822.

Lk., L. Sp. Pl. ed. Willd. $6^{2}:$ 52. d. 1825.

Underw. \& Earle, Bull. Ala. Exp. Sta. 80: 21 I. 28 June 1897. Cama asteratum, Lk. p. p.

Lk., L. Sp. PI. ed. Willd. 62: 51. d. 1825.

S. Syn. Am. Bor. 292 no. $2870 . \quad$ I 834 .

Uredo Asterum, Spreng.

Spreng. in L. Syst. Veg. I6 ed. 4 1 : 572. d. 1827. Aecidium asteratum, S. p. p.

S. Syn. Am. Bor. 309. I834.

Aecidium Vernoniæ, Henn.

Duss. Champ. Guad. \& Martinique 7. 1903.

Aecidium verrucosum, Bon. See Aecidium Smilacis, S.

Aecidium Violæ, Schum.

Note. The references given here belong, at least in the majority of cases, to the Aecidium of Puccinia Viola, (Schum.) D C. For details see Arth. \& Holw. Minn. Bot. Studies II. 5: 633. 20 July I90I. Gertain references 
may belong to other species, but at the present time there is no means of ver ifying them.

S. Syn. Car. 67 (4I) no. 439. I822.

Watt, Can. Nat. \& Geol. II. 2 : 391. Oct. I865.

M. A. Curtis, Bot. N. Car. I 24. 1867.

Cke. Handbook 543. d. I87I.

Edwards, Bull. Torr. Bot. Club 2 : 39. Oct. I87 1.

Pk. Rept. N. Y. Mus. 23 : 59.1872.

Pk. Rept. N. Y. Mus. 24: I06. 1872.

B. Grev. 3: 6I (I03). Dec. I 874 .

Frost in Cat. Pl. Amherst 85. $\quad$ 875.

Cke. Grev. 6 : I37. June 1878.

Pk. Rept. N. Y. Mus. $29: 76 . \quad$ I878.

* Rav. Fung. Am. 38. I878.

Johnson, Bull. Minn. Acad. 1 : 366. 1880.

Farl. Proc. Am. Acad. 18: 75. July 1883.

*Ell. N. A. F. 1007. 1883.

Bundy, Geol. Wis. I $873-79,1: 400.1883$.

Arth. Bull. lowa Agr. Coll. 1884: I68. Jan. 1885.

Cragin, Bull. Wash. Lab. 1 : 68. July I 885 .

Ell. \& Ev. Jour. Myc. 1 : 86. July I 885.

Cobb, List. Pl. Amherst 40. I887.

Bennett, Cat. Pl. R. I. 89. I 888.

Ell. in Cat. Pl. N. J. 505. I 889.

Williams, Am. Nat. 24: 779. Aug. 1800.

Small \& Vail, Mem. Torr. Bot. Club. 4: 200. April I894.

*Ell. \& Ev. Fung. Columb. $496 . \quad$ I894.

Herbst, Fungal Fl. Lehigh Valley I75. d. $\quad$ I899.

Caoma violatum, Lk.

S. Syn. Am. Bor. 293 no. $2884 . \quad$ I834.

Aecidium Violarum, D C.

Sprague, Proc. Boston Soc. 6 : 319. $\quad$ I859.

Aecidium Violarum, DC.

See Aecidium Viola, Schum.

Aecidium violascens, Trel.

Note. Compare also Aecidium Geranii-maculati, S.

Trel. in Sacc., Pk. \& Trel., Harriman Alaska Exp. 5 : 37. d. 5 April I904.

Aecidium Wedeliæ, Earle.

Earle, Muhlenbergia $1:$ i6. $d$. July igor. West Indies.

Aecidium Wilcoxianum, Thm.

See Aecidium Giliz, Pk.

Aecidium Williamsi, Ricker.

Ricker, Jour. Myc. 10: I65. $d$. 24 June 1904.

Aecidium Xanthoxyli, $\mathrm{Pk}$.

Pk. Bot. Gaz. 6 : 275. d. Oct. I88I.

*Wint. Fung. Eur. 2928. 1883.

*Ell. N. A. F. 1013. I883.

Arth. Bull. lowa Agr. Coll. 1884: I68. Jan. I885.

Kellerm. Bull. Wash. Lab. 1: 75. Jan. I885 and Tr. Kans. Acad. 9 : 8I. I 885 .

Kellerm. \& Carl. Tr. Kans. Acad. 10: 92. I887.

De Toni in Sacc. Syll. 7 : 784. d. 28 Oct. I 888.

Webber, Bull. Nebr. Exp. Sta. 1: 335 (65). 18 Dec. I889.

Gall. Bull. U. S. Agr. Veg. Pathol. 8: 56. I889.

Jennings, Bull, Téx, Exp. Sta. 9: 23. May 1890. 
Webber, Rept. Nebr. Agr. 1889: 212 (72). 1890.

* Carl. Ured. Amer. 6. I894.

Tubeuf-Smith, Diseases of Plants 410.1897.

Barthol. Tr. Kans. Acad. 16 : 192. June 1809.

Long, Bull. Torr. Bot. Club. 27 : $586 .{ }_{14}$ Nov. 1900.

*Syd. Uredineen 1548. April 1901.

* Shear, Ell. \& Ev. Fung. Columb. 1477. May 1901.

Patterson, Bull. U. S. Agr. PI. Industry 8: 8. 3 Feb. 1902.

Garrett, Tr. Kans. Acad. 18: 147. 1903.

\section{Aecidium on Zauschneria.}

See Puccinia Zauschneriz, Syd.

Aecidiam Zephyranthis, Shear.

Shear, Bull. Torr. Bot. Club. $29: 454 . d$. 25 July I902. Mexico.

Aedycia, Raf.

Note. See also Corynites, B. \& C.

Raf. Med. Repos. II. hex. 5: 358. $d$. and Desv. Jour. de Bot. 1: 222. I808.

Endl. Gen. Pl. 31. 1840.

Gerard, Bull. Torr. Bot. Club. 7 : 30. March I880.

Fischer in Sacc. Syll. $7:$ 12. 15 March 1888.

Aedyeia alba, Raf.

Raf. Med. Repos. Il. hex. 5: 358. d. and Desv. Jour. de Bot. 1: 222. 1808.

Aedycia rubra, Raf.

Raf. Med. Repos. II. hex. 5: 358.d. and Desv. Jour. de Bot. 1: 222. I808.

Aegerita, P.

Pound \& Clements, Minn. Bot. Studies $9:$ 732. d. 31 May 1897.

Aegerita cæsia, $\mathrm{P}$.

S. Syn. Car. I24 (98) no. I275. I 822.

S. Syn. Am. Bor. 303 no. 304I. d. I834.

M. A. Curtis, Bot. N. Car. I53. I867.

Cke. Bull. Buf. Soc. 3 : 195 . July 1877.

Aegerita candida, $\mathrm{P}$.

Note. Schweinitzian specimens of Algerita candida show only small white globular masses which are probably what he understood to be this species. They have not the structure of Aegerita, but are mainly inorganic matter.

Muhl. Cat. PI. Am. Sept. I ed. I06. I813, 2 ed. 108. I818.

S. Syn. Car. 124 (98) no. 1274. 1822.

Grev. Scot. Crypt. Fl. $5: p l .268$. ${ }^{2} 827$

Fr. S. M. 3: 220. $d$. 1832 .

S. Syn. Am. Bor. 303 no. 3040. I834.

Sprague, Proc. Boston Soc. 6 : 321. 1859.

M. A. Curtis, Bot. N. Car. 153. 1867.

Cke. Handbook 56r. d. f. 235. I87I.

B. Grev. 3 : 100 (I I0). March 1875.

Frost in Cat. PI. Amherst 86. 1875.

Cke. Bull. Buf. Soc. 3 : 195 . July 1877.

C. \& E. Grev. $6: 87$. March I 878 .

Pk. Rept. N. Y. Mus. 29 : 5I. 1878.

*EII. N. A. F. 361. I879.

Pk. in Day's Cat., Bull. Buf. Soc. 4 : 210 (146). 1883.

Sacc. Syll. 4: 66r. d. Io April i 886.

Cobb, List Pi. Amherst 40. 1887.

Ell. in Cat. Pl. N. J. 575.1889.

Lindau in Engler \& Prantl, Pflanzenfam. 11**: 500. Feb. I900. 
Aegerita Cordæ, Sacc. ?

Ricker, Univ. of Maine Studies 3: 24. 30 April 1902.

Aegerita decolorans, (S.) Sacc.

Sacc. Syll. 4 : 664. $d$. Io April I 886.

Lindau in Engler \& Prantl, Pflanzenfam. 11** : 500. Feb. I900.

Dermosporium decolorans, S.

S. Syn. Am. Bor. 302 no. 3033. d. 1834.

Cke. Bull. Buf. Soc. 3: 194. July 1877.

Aegerita lutescens, S.

Note. Aegerita lutescens of Herb. Schweinitz has not the structure of the genus if Aegerita candida, P. is to be regarded as the type. The $A$. lutescens of Herb. Schweinitz is a fungus certainly near Penicillium or Coremium, but the material we have examined does not warrant assigning the species a definite place, although it is certainly not an Aegerita.

S. Syn. Am. Bor. 303 no. 3042. d. 1834.

Cke. Bull. Buf. Soc. 3 : I95. July I877.

Sacc. Syll. $4: 663$. $d$. 10 April 1886.

Aegerita 0vulum, S.

S. Syn. Am. Bor. 303 no. 3043. d. 1834.

M. A. Curtis, Bot. N. Car. I53. 1867.

Cke. Bull. Buf. Soc. 3 : 195. July 1877.

Sacc. Syll. 4: 663. $d$. Io April I886.

Aegerita rosea, (S.) Sacc.

Sacc. Syll. $4: 664$. $d$. Io April 1886.

Dermosporium roseum, $\mathrm{S}$.

S. Syn. Am. Bor. 302 no. 3034. I 834 .

Cke. Bull. Buf. Soc. 3 : 194. July I 877.

Aethalium candidum, Schl. in Spreng.

See Fuligo septica, Gmel.

Aethalium ferpincola, S.

See Fuligo septica, Gmel.

Aethalium fiavum, Lk.

See Fuligo septica, Gmel.

Aethalium geophilum, $\mathrm{Pk}$.

See Hyphelia terrestris, Fr.

Aethalium muscorum, S.

See Fuligo septica, Gmel.

See Fuligo muscorum, A. \& S.

Aethalium septicum, (L.) Fr.

See Fuligo septica, Gmel.

Aethalium vaporarium, (P.) S.

See Fuligo septica, Gmel.

Aethalium violaceum, Spreng. See Fuligo violacea, $\mathrm{P}$.

Agaricus, L.

NoTE. In assigning the authority to the specific combinations of species here cross-referred from Agaricus to other genera we have in general cited the author by whom the species were placed under the names given, regardless of the question whether he considered the names to represent genera or subgenera, the plan followed in Saccardo's Sylloge and by a large number of writers. This we have found to be the only practical method, for since we believe that the present classification of Agaricacea is of necessity only provisional and temporary. it would be a waste of time to attempt to search through the innumerable scattered lists, monographs and other small papers of Euro- 
pean writers of the last century, to settle, points in regard to species common to both countries which could at the liest have only a slight value An examination of the writings of those who have attempted to give as authorities for the species of Agaricucea only those who accepted the genera now adopted as genera rather than subgenera has shown so many inconsistencies and inaccuracies that we have not been able to follow them.

For uniformity's sake we have followed the same plan in regard to the purely North American species, but in this case, as all American references known to us are given, with dates, the reader has the means of settling the question of authority for himself.

As used here in its limited sense we have kept under Agraricus the species placed by mycologists in Psallioti and Pratella, the type of the genus being Agaricus campestris, $\mathrm{L}$.

Agaricus abietinus, Bull.

See Lenzites abietina, (Bull.) Fr.

Agaricus abortivus, B. \& C.

See Clitopilus abortivus, B. \& C.

Agaricus abruptus, Pk.

Pk. Mem. N. Y. Mus. 3: 163. d. pl. 59, f. 8-14. Nov. 1900.

White, Bull. Torr. Bot. Club. 29: 561. 26 Sept. 1902.

Longyear, Rept. Mich. Acad. 4: I I5. I904. Agaricus silvicola, Pk. non Vitt.

Pk. Rept. N. Y. Mus. 23 : 97.d. 1872.

Pk. Rept. N. Y. Mus. 36: 47. d. 1885.

Pk. Country Gent. 59 : 518 (3I). 12 July 1894. Agaricus arvensis, var. abruptus, Pk.

Pk. Rept. N. Y. Mus. 46: 135 (55).d. Dec. I893 and Rev. Myc. 16 : 126. I July I 894 .

Pk. Country Gent. 59: 518 (30). I 2 July I 894.

Pk. Bull. N. Y. Mus. 6 : 213. July I 899 . Agaricus silvicola, var. abruptus, (Pk.) Conant.

Conant, Bull. Boston Myc. Club. 19. 5 Feb. 1903.

Agaricus abseondens, $\mathrm{Pk}$. See Pleurotus lignatilis, (P.) Fr.

Agaricus abundans, $\mathrm{Pk}$.

See Collybia abundans, Pk.

Agaricus acericolus, $\mathrm{Pk}$.

See Pholiota acericola, Pk.

Agaricus acervatus, $\mathrm{Fr}$.

See Collybia acervata, Fr.

Agaricus Achimenes, B. \& C.

B. \& C. Am. Jour. Sci. II. $8: 403$. d. Nov. 1849.

B. \& C. Hook. Jour. Bot. 1: 98 (2). $d .1849 . \quad[$ no. 202.]

Rav. Charleston Med. Jour. \& Rev. 6: 192. March 1851.

M. A. Curtis, Bot. N. Car. 90.1867.

Sacc. Cub. \& Manc. Syll. 5: 995. d. 28 May 1887.

Mcllvaine \& Macadam, Am. Fung. 340. d. 1900.

Psalliota Achimenes, Lloyd.

Lloyd, Myc. Notes 29. Nov. 1899.

Agaricus Acicula, Schæff. See Mycena Acicula, (Schaff.) Fr.

Agaricus acicularis, Muhl.

NotE. This is possibly Mycena capillaris, (Schum.) Fr.

Muhl. Tr. Am. Phil. Soc. 3: 184. 1793.

Agaricus acris, Bolt.

See Lactarius acris, (Bolt.) Fr. 
Agaricus acutesquamosus, Weinm.

See Lepiota acutesquamosa, (Weinm.) Fr.

Agaricus acutus, $P$.

See Cortinarius acutus, (P.) Fr.

Agaricus adiposus, Fr.

See Pholiota adiposa, Fr.

Agaricus Adirondackensis, Pk.

See Clitocybe Adirondackensis, Pk.

Agaricus admirabilis, $\mathrm{Pk}$.

See Pluteus admirabilis, $\mathrm{Pk}$.

Agaricus adnatus, W. G. Smith.

See Amanitopsis adnata, (W. G. Smith) Sacc.

Agarieus Adonis, Búll.

See Mycena Adonis, (Bull.) Fr.

Agarieus adstringens, $P$.

See Tricholoma adstringens, (P.) Fr.

Agaricus adustus, $P$.

See Russula adusta, (P.) Fr.

Agaricus æruginosus, Curt.

See Stropharia aruginosa, (Curt.) Fr.

Agaricus æthalus, B. \& C.

See Pluteus athalus, B. \& C.

Agaricus affinis, Frost.

See Amanita Frostiana, Pk.

Agaricus affricatus, Fr.

See Omphalia affricata, Fr.

Agaricus aggericolus, $\mathrm{Pk}$.

See Pholiota aggericola, $\mathrm{Pk}$.

Agaricus agglutinatus, B. \& C.

See Amanitops is agglutinata, (B. \& C.) Sacc.

Agaricus aggregatus, Schæff.

See Clitocybe aggregata, (Schæff.) Fr.

Agaricus albellus, Fr.

See Tricholoma albellum, Fr.

Agaricus albissimus, $\mathrm{Pk}$.

See Clitocybe albissima, Pk.

Agaricus albobrunneus, $P$.

See Tricholoma albobrunneum, (P.) Fr.

Agaricus albocrenulatus, Pk.

See Pholiota albocrenulata, Pk.

Agaricus alboflavidus, $\mathrm{Pk}$.

See Tricholoma alboflavidum, $\mathrm{Pk}$.

Agaricus alboflavus, Morg.

See Omphalia alboflava, Morg.

Agaricus albogriseus, $\mathrm{Pk}$.

See Clitopilus albogriseus, $\mathrm{Pk}$.

Agaricus alboides, $\mathrm{Pk}$.

See Tricholoma album, (Schæff.) Fr.

Agaricus alborubellus, Mont.

See Pluteus alborubellus, (Mont.) Pat.

Agaricus alboviolaceus, $P$.

See Cortinarius alboviolaceus, (P.) Fr. 
Agaricus albus, $\mathrm{P}$.

Note. This is possibly Tricholoma Columbetta, Fr.

S. Syn. Car. 84 (58). no. 659. 1822.

Agaricus albus, Schreff.

See Tricholoma album, (Schxff.) Fr.

Note. For note on Tricholoma album, Shear, N. Y. Fungi 5. 1893 see Romell under Agaricus ( Tricholoma) lentus, Post. in Bot. Notiser 1895 : 65.

Agaricus alealinus, $\mathrm{Fr}$.

See Mycena aicalina, Fr.

Agaricus aleuriatus, Fr.'

See Pluteolus aleuriatus, Fr.

Agaricus algidus, Herb. S. and S. Am. Bor.

Note. In Berkeley \& Curtis, Commentary, Jour. Phil. Acad. 11. 3: 206. 1856 the Agaricus algidus of Herb. Schweinitz is said to be Agaricus atro. caruleus, Fr. The other references to Agaricus algidus will be found under Pleurotus algidus, Fr.

Agaricus algidus, $\mathrm{Fr}$.

See Pleurotus algidus, Fr.

Agaricus alliaceus, Jacq.

See Marasmius alliaceus, (Jacq.) Fr.

Agarleus alliatus, Schæff.

See Marasmius scorodonius, Fr.

Agaricus allosporus, B.

Note. The only reference to this species known to us is that given below in which there is no description.

B. Jour. Linn. Soc. 5: 86. 1861 and Arctic Manual 2: 527. 1875.

Agaricus alluvilinus, $\mathrm{Pk}$.

See Lepiota ailuviina, Pk.

Agaricus alneas, L.

See Schizophyilum commune, Fr.

Agaricus alnicola, Fr.

See Fiammuia ainicoia, Fr.

Agaricas alphitophorus, B.

See Mycena aiphitophora, B.

Agaricus (Clitocybe) altus, Frost.

Note. Never described so far as we know.

Frost in Cat. PI. Amherst 63.1875.

Cobb, List Pl. Amherst 31. 1887.

Agaricus alutaceus, $P$.

See Russuia alutacea, P.

Agaricus alveolatus, Cragin.

See Piuteus alveolatus, (Cragin) Sacc. Syll.

See also Pleurotus subpaimatus, Fr.

Agaricus Alveolus, Lasch.

See Crepidotus Aiveoius, ( Lasch) Fr.

Agaricus amabilipes, Pk.

See Collybia amabilipes, Pk.

Agaricus amabillissimus, $\mathrm{Pk}$.

See Mycena amabilissima, $\mathrm{Pk}$.

Agaricus Americanus, Pk.

See Lepiota Americana, Pk. 
Agaricus amethysteus, $\mathrm{P}$.

See Laccaria laccata, (Ścop.) B. \& Br.

Agaricus amianthinus, Scop.

See Lepiota amianthina, (Scop.) Fr.

Agaricus amictus, Fr.

See Mycena amicta, Fr.

Agaricus amplus, $\mathrm{P}$.

See Clitocybe ampla, (P.) Fr.

Agaricus amygdalinus, $M$. A. Curtis.

See Agaricus fabaceus, B.

Agaricus androsaceus, $L$.

See Marasmius androsaceus, (L.) Fr.

Agaricus anepsius, Mont.

See Flammula anepsia, Mont.

Agaricus angustilamellatus, Mont.

See Clitocybe angustilamellata, Mont.

Agaricus angustipes, $\mathrm{Pk}$.

See Pholiota angustipes, $\mathrm{Pk}$.

Agaricus angustissimus, Lasch.

See Clitocybe angustissima, (Lasch) Fr.

Agaricus anisarius, $\mathrm{Pk}$.

See Clitocybe anisaria, $\mathrm{Pk}$.

Agaricus anomalus, Frost.

NoTE. There is no means of ascertaining to what species the two following references belong, but they do not belong to Cortinarius anomalus, $\mathrm{Fr}$. which is also cited by Frost in his catalogue on another page.

Frost in Cat. Pl. Amherst 63. 1875.

Cobb, List. Pl. Amherst 31. I887.

Agaricus anomalus, Fr.

See Cortinarius anomalus, Fr.

Agaricus Anthiceps, B. \& C.

See Omphalia Anthiceps, B. \& C.

Agaricus Antillarum, Fr.

See Psilocybe Antillarum, Fr.

Agaricus apertus, $\mathrm{Pk}$.

See Clitocybe aperta, Pk.

Agaricus appendiculatus, Bull.

See Hypholoma appendiculatum, (Bull.) Fr.

Agaricus applicatus, Batsch.

See Pleurotus applicatus, (Batsch) Fr.

Agaricus aquatilis, Fr.

See Galera aquatilis, Fr.

Agaricus aqueus, Batsch.

Note. Possibly Coprinus deliquescens, (Bull.) Fr.

Muhl. Tr. Am. Phil. Soc. 4: 240. 1799.

Agaricus ardosiacus, Bull.

See Entoloma ardosiacum, (Bull.) Fr.

Agaricus arenulinus, Pk.

See Psilocybe arenulina, $\mathrm{Pk}$.

Agaricus areolatus, Klotzsch.

See Psilocybe areolata, (Klotzsch) Fr. 
Agaricus argentatus, $\mathrm{P}$.

See Cortinarius argentatus, (P.) Fr.

Agaricus argenteus, Brændle.

Brændle in Pk. Bull. Torr. Bot. Club. $26: 68$. d. 18 Feb. 1899 and Asa Gray Bull. 7 : 38. April 1899.

Mcllvaine \& Macadam, Am. Fung. 339. d. 1900.

Brown \& Fernekes, Bull. Wis. Nat. Hist. Soc. Il. 2 : 46. Jan. 1902.

Sacc. \& Syd. Syll. $16: 118 . d$. I Feb. 1902.

Psalliota argentea, Lloyd.

Lloyd, Myc. Notes 29. Nov. 1899.

Agaricus argillaceus, S. Syn. Car. 670. See Inocybe geophylla, (Sow.) Fr.

Agaricus argillosus, B. \& C.

See Naucoria argillosa, B. \& C.

Agaricus armeniacus, Schæff.

See Cortinarius armeniacus, (Schæeff.) Fr.

Agaricus Artemisiæ, Pass.

See Hypholoma Artemisia, (Pass.) Fr.

Agaricus arvensis, Schrff.

M. A. Curtis, Bot. N. Car. 90. 1867.

Pk. Rept. N. Y. Mus. 22:78. I860.

Curtis, Gard. Chron. I066. 9 Oct. 1869 and Jour. Roy. Hort. Soc. 11. $2: 72,77,78$. 1870 .

Winder, Mushrooms of Canada 20. I871.

Parker, Proc. Poughk. Soc. $1: 84$. I Nov. 1875.

Frost in Cat. Pl. Amherst 65. 1875.

Seaman, Field \& Forest 1 : 71. Jan. 1876.

Morg. Fl. Miami Valley 59. 1878.

Pk. Rept. N. Y. Mus. 31: 54. 1879.

Hark. \& Moore, Cat. Pac. Fung. 7. Feb. 1880.

Somers, Tr. N. Scot. Inst. 5 : 249. 1882.

Morg. Jour. Cin. Soc. $6:$ i1 1 . $d$ : July 1883.

Rav. S. Car. Resources 355. I 883.

Pk. Rept. N. Y. Mus. 36: $46 . d$. 1885.

Palmer, Mushrooms pl. I. I 885.

Sacc. Cub. \& Manc. Syll. 5 : 994. d. 28 May 1887.

Cobb, List Pl. Amherst 32. 1887.

Bennett, Cat. PI. R. I. $77 . \quad 1888$.

Ell. in Cat. Pl. N. J. 475.1889.

McBride, Bull. Univ. lowa 1: 187. d. 198. $d$. June 1890.

Taylor, Rept. U.S. Agr. 1890 : 368 (7). pl. i, f. 6. 1890. [repr. 1893.]

Forster, Mushrooms 16. 1890 .

Pk. Country Gent. 59 : 518 (29). f. 12 July 1894.

Eby, Fungi of Lancaster Co. 8. 1894.

Kellerm. \& Werner, Geol. Ohio 72: 315. Jan. 1895.

Gibson, Mushrooms 93. 1895.

Sturgis, Rept. Conn. Agr. 1895 : 80 (10).' Jan. 1896.

Bates, Tr. Mass. Hort. Soc. $1896:$ i 74.1896.

Webster, Am. Kitchen Mag. 6: 217. Feb. 1897.

Taylor, Hdbk. Mushrooms 2: 24. 1897.

Pk. Rept. N. Y. Mus. $48: 238$ (140). pl. 8. March 1897.

Coville, Circ. U. S. Agr. 13: 7. f. 2-5. I Dec. 1897.

Taylor, Hdbk. Mushrooms 3: 2. pl. E, f. 3. 1897.

Farl. Yearb. U. S. Agr. $1897: 460$. pl. $^{24}$. 1898. [Bull. 15.]

Smith, C. O., Rhodora 1 : 161. I Sept. 1899.

Underw. Moulds 122.1899. 
Atk. Mushrooms 21. d. f. 18. 20 Oct. 1900.

Hyams, Bull. N. Car. Exp. Sta. 177; 30.d. Dec. 1900.

Atk. in Bailey's Cycl. Am. Hort. ro43. 1900.

Mcllvaine \& Macadam, Am. Fung. 341. d. 1900.

Trel. Rept. Mo. Hort. Soc. 43: 227. 1900.

Brown \& Fernekes, Bull. Wis. Nat. Hist. Soc. Il. $2: 46$. Jan. 1902.

Conant, Bull. Boston Myc. Club i9. 5 Feb. 1903.

Longyear, Rept. Mich. Acad. 4 : I I 5.1904. Agaricus edulis, Auct. Amer.

Muhl. Cat. Pl. Am. Sept. I ed. I04. I8I3. 2 ed. 107. I8I8.

S. Syn. Car. $86(60)$ no. 719. I 822.

Psalliota arvensis, Taylor.

Taylor, Food Products 2: 21. $\quad 1893$.

Lloyd, Myc. Notes 29, 30. Nov. I 899

Perry, Agr. Student 6: 179 and Ohio Univ. Bull. IV. 30 : 6. May I900. Psalliota edulis, Lloyd.

Lloyd, Myc. Notes 30 . Nov. 1899.

Agaricus arvensis, var. abruptus, $\mathrm{Pk}$.

See Agaricus abruptus, Pk.

Agaricus ascophorus, $\mathrm{Pk}$.

Note. Originally placed in Hebeloma by Peck, 1872 , and said by Berkeley, Grev. 1875 to be a Flammula. Under the name Marasmius ascophorus the same fungus appears to be referred to by Cooke, 1891. See also Flammula Highlandensis, Pk.

Pk. Rept. N. Y. Mus. 24: 68. d. pl. 3, f. 1-6. 1872.

B. Gard. Chron. II. 3 : 503. $f$. 106. I7 April 1875.

De Seynes, Grev. 3 : 169. d. pl. 46. June 1875.

B. Grev. 3 : 170 . June 1875 .

Johnson, Bull. Minn. Acad. 1: 230. 1877 and Rept. Minn. Surv. 5 : 71. 1877.

Marasmius ascophorus, Cke.

Cke. Grev. 19 : 104. June 1891 .

Agaricus asemus, Fr.

See Collybia asema, Fr.

Agaricus asper, Fr. See Amanita aspera, P.

Agaricus aspratus, B. See Lepiota asprata, B.

Agaricus asprellus, Fr. See Leptonia asprella, Fr.

Agaricus assularum, B. \& C.

See Leptonia assularum, B. \& C.

Agaricus atomatoides, $\mathrm{Pk}$. See Psilocybe atomatoides, Pk.

Agaricus atomatus, Fr. See Psathyrella atomata, Fr.

Agaricus atramentarius, Bull. See Coprinus atramentarius, (Bull.) Fr.

Agaricus atratoldes, $\mathrm{Pk}$. See Collybia atratoides, $\mathrm{Pk}$.

Agaricus atratus, Fr. See Collybia atrata, Fr.

Agaricus Atrides, Lasch. See Eccilia Atrides, (Lasch) Fr. 
Agaricus atroalboides, $\mathrm{Pk}$.

See Mycena atroaiboides, Pk.

Agaricus atroalbus, S. Syn. Car. 68r. See Mycena amicta, Fr.

Agaricus atrocæruleus, Fr. See Pleurotus atroczeruleus, Fr.

Agaricus atrocinereus, S. Syn. Car. 649. See Tricholoma cuneifolium, Fr.

Agaricus atrocyaneus, Batsch. See Mycena atrocyanea, (Batsch) Fr.

Agaricus atrorufus, Sihæff. See Psilocybe atrorufa, (Schæff.) Fr.

Agaricus atrotomentosus, Batsch. See Paxilius atrotomentosus, (Batsch) Fr.

Agaricus aurantius, Schæff. See Armiilaria aurantia, (Schæff.) Fr.

Agaricus auratocephalus, Ell. See Ciitocybe chrysocephala, Sacc. Syll.

Agaricus aureobrunneus, B. \& C. See Flammula aureobrunnea, B. \& C.

Agaricus (Mycena) aureosquamosus, Frost.

Note. Apparently not described. Placed by Frost in subgenus Mycena.

Frost in Cat. Pl. Amherst 64. 1875.

Cubb, List Pl. Amherst 32.1887.

Agaricus aureus, S. Syn. Car. 751.

Note. This is possibly Russula aurata, Fr. but the identity cannot he. certainly established.

Agaricus aureus, Mattusch. See Phoilota aurea, (Mattusch.) Fr.

Agaricus auricomus, Batscl. See Inocybe auricoma, (Batsch) Fr.

Agaricus aurivellus, Batsch. See Pholiota auriveiia, (Batsch) Fr.

Agaricus Austini, Pk. See Omphalia Austini, Pk.

Agaricus autumnalis, $\mathrm{Pk}$. See Naucoria autumnaiis, $\mathrm{Pk}$.

Agaricus Babingtonil, Blox. See Noianea Babingtonii, (Blox.) Fr.

Agaricus Badhami, B. \& Br. See Lepiota Badhami, B. \& Br.

Agaricus badipus, $P$. See Naucoria badipus, (P.) Fr.

Agaricus badius, Schæff. See Amanitopsis vaginata, (Bull.) Rcze.

Agaricus Balanus, Batsch.

Note. This name mentioned only by Muhlenberg probably relates to Agaricus Balinus, Batsch, Elenchus p. 63 , no. 62 , referred by Streinz to Coprinus micacens, (Bull.) Fr. but not cited by Fries nor other authors which we have consulted.

Muhl. Tr. Am. Phil. Soc. 4: 240. 1799. 
Agaricus bambusigena, B. \& C.

B. \& C. Jour. Linn. Soc. $10: 291 . d . \quad$ I 869.
Sacc. Cub. \& Manc. Syll. 5: 1004. d. 28 May ı 887.

Psalliota bambusigena, B. \& C.

Henn. in Engler \& Prantl, Pflanzenfam. 11** : 240. 1898.

Agaricus barbatulus, B. \& C.

See Pleurotus barbatulus, B. \& C.

Agaricus Batschianus, Fr.

See Entoloma Batschianum, Fr.

Agarieus Behringensis, B. \& C.

See Omphalia Behringensis, B. \& C.

Agaricus Belliæ, Johnst.

See Omphalia Bellia, (Johnst.) Fr.

Agaricus Bellotianus, B.

See Naucoria Bellotiana, B.

Agaricus bellulus, $\mathrm{Pk}$.

See Naucoria bellula, Pk.

Agaricus bellus, $P$.

See Clitocybe bella, (P.) Fr.

Agaricus Benzonii, Fr.

See Hiatula Benzonii, Fr.

Agaricus betulinus, L.

See Lenzites betulina, (L.) Fr.

Agarieus bicolor, $\mathrm{P}$.

See Tricholoma personatum, Fr.

Agaricus bifrons, B.

See Psathyra bifrons, B.

Agaricus bivelus, Fr.

See Cortinarius bivelus, Fr.

Agaricus Blakei, B. \& C.

See Pleurotus Blakei, B. \& C.

Agaricus blattarius, Fr.

See Pholiota blattaria, Fr.

Agaricus bombyeinus, Schæff.

See Volvaria bombycina, (Schæff.) Fr.

Agaricus Bongardii, Weinm.

See Inocybe Bongardii, (Weinm.) Fr.

Agarieus Boryanus, Berk. \& Mont.

See Collybia Boryana, Berk. \& Mont.

Agaricus brevipes, Bull.

See Tricholoma brevipes, (Bull.) Fr.

Agaricus Brownei, Banning.

See Tricholoma Brownei, Banning.

Agarieus brumalis, Fr.

See Clitocybe brumalis, Fr.

Agarieus brunnescens, $\mathrm{Pk}$.

Pk. Bull. Torr. Bot. Club 27 : I6. d. 24 Jan. I000.

Sacc. \& Syd. Syll. 16 : I13. d. I Feb. 1902.

Agaricus bulbigera, A. \& S.

See Armillaria bulbigera, (A. \& S.) Fr.

Agaricus bulbillosus, Fr.

See Psathyra bulbillosa, Fr. 
Agaricus Bulbillus, S.

Note. As far as we know this name occurs first in Fr. Epicr. 118. 1836. as a synonym of Agaricus echinipes, Lasch but although quoted since by other writers, we have not been able to trace the name in Schweinitz.

Agaricus bulbosús, Bull.

See Amanita Mappa, Fr.

Agaricus bulbosus, McClatchie non Bull.

McClatchie, Proc. S. Cal. Acad. $1:$ 382. d. 5 June 1897.

Sacc. \& Syd. Syll. 16: 113.d. I Feb. 1902.

Psalliota bulbosa, Lloyd.

Lloyd, Myc. Notes 29. Nov. 1899.

Agarieus bullaceus, Bull.

See Psilocybe bullacea, (Bull.) Fr.

Agaricus butyraceus, Bull.

See Collybia butyracea, (Bull.) Fr.

Agaricus byssisedus, $P$.

See Claudopus byssisedus, (P.) Fr.

Agaricus eacaophyllus, B. \& C.

See Crepidotus cacaophyllus, B. \& C.

Agaricus eærulipes, Pk.

See Psilocybe carulipes, Pk.

Agaricus cæsariatus, Fr.

See Inocybe cresariata, Fr.

Agaricus cæsarius, Scop.

See Amanita casarea, (Scop.) P.

Agaricus eæsius, S. Syn. Car. \& S. Am. Bor.

See Entoloma turbidum, Fr.

Agaricus eæspitosus, (B.) M. A. Curtis, Bot. N. Car.

See Pleurotus caspitosus, Sacc. Syll.

Note. This is Agaricus caspitosus, M. A. Curtis, Bot. N. Car. 85 where it is placed under the subgenus Clitocybe. The plant referred to was Lentinus caspitosus, 13. Decades 120 in Lond. Jour. Bot. 6: $317(6)$. 1847, a species the synonymy of which is complicated. See notes under Lentinus caspitosus, B. and Pleurotus caspitosus, Sacc. Syll.

Agaricus ealamistratus, Fr.

See Inocybe calamistrata, Fr.

Agaricus Calathus, Buxb.

See Clitocybe Calathus, (Buxb.) Fr.

Agaricus Californicus, $\mathrm{Pk}$.

Pk. Bull. Torr. Bot. Club 22 : 203. d. 15 May 1895.

McClatchie in Reid's Hist. Pasadena 621. 1895.

McClatchie, Proc. S. Calif. Acad. 1 : 382. 5 June 1897.

Sacc. Hedw. 35 : vi. 25 Feb. 1896. [Reportorium No. 7.]

Sacc. \& Syd. Syll. 14 : 150. d. 20 Aug. 1899.

Mcllvaine \& Macadam, Am. Fung. 338. d. 1900.

Psalliota Californica, Lloyd.

Lloyd, Myc. Notes 29. Nov. 1899. .

Agaricus Californiensis, B. \& C.

See Mycena Californiensis, B. \& C.

Agaricus callistus, $\mathrm{Pk}$.

See Galera callista, Pk.

See Pluteolus callistus, Pk. 
Agaricus ealochrous, $\mathrm{P}$.

See Cortinarius calochrous, (P.) Fr.

Agaricus calopus, $\mathrm{P}$.

See Marasmius calopus, (P.) Fr.

Agaricus Campanella, Batsch.

See Omphalia Campanella, (Batsch) Fr.

Agaricus eampanulatus, L.

See Chalymotta campanulata, (L.) Karst.

See Panaolus campanulatus, (L.) Fr.

Agaricus eampestris, L.

NoTE. We can give here only a part of the very numerous North American references to. this common species, selecting those which show its range or which contain illustrations or economic notes of value.

Walt. Fl. Car. 260. $d$. 1788.

Muhl. Tr. Am. Phil, Soc. 3: 184. 1793.

Muhl. Cat. Pl. Am. Sept. I ed. I04. I8I3. 2 ed. I07. 1818.

Raf. Fl. Ludov. I2. I8I7.

Peck, W. D., Pl. Bot. Gard. Camb. 49. I8I8.

Torr. Cat. Pl. N. Y. 88. I8I9.

Fr. S. M. $1: 281$. I82I.

S. Syn. Car. $86(60)$ no. $720 . \quad 1822$.

Dewey in Hist. Berkshire Co. 85. 1829.

Hitchcock, Cat. Pl. Amherst 59. I829.

Hitchcock, Cat. Pl. Mass. I ed. 644. 1833.2 ed. 646 (I26). 1835.

S. Syn. Am. Bor. I5I no. $237 . \quad 1834$.

Lev. Ann. Sci. Nat. III. 5 : I I 5. 1846.

Lea, Cat. PI. Cin. 53. 1849 and Jour. Cin. Soc. $5: 200$ (4). Dec. 1882 .

Rav. Charleston Med. Jour. \& Rev. 6: 192. March I851.

B. Ann. Nat. Hist. II. 9: I93. March I 852 .

Sprague, Proc. Boston Soc. 5 : 322, 326.1856.

Porcher, Resources Southern fields I ed.594. I863. 2 ed. 694. I869.

Curtis, M. A., Bot. N. Car. 90. 1867.

Curtis, M. A., Gard. Chron. I066. 9 Oct. 1869 and Jour. Roy Hort. Soc. II. 2: $72,74,78$. 1870 .

Pk. Rept. N. Y. Mus. 22: 78 . 1869.

Featherman, Bot. Surv. La. 1870: I23. I871.

Winder, Mushrooms of Canada, 6, 7, 20. $f . \quad$ I87I.

Cke. Handbook 137. $d$. pl. 5, f. 26. 1871 .

Pk. Rept. N. Y. Mus. 23 : $97 . d$. I872.

Parker, Proc. Poughk. Soc. 1 : 84. I Nov. 1875.

Frost in Cat. PI. Amherst 65. I875.

Seaman, Field \& Forest 1: 71. Jan. 1876.

Farl. Bull. Bussey Inst. 1: 436 . March I 876 .

Cke. Field \& Forest 2: 1 46. Feb. 1877.

Johnson, Bull. Minn. Acad. 1 : 235 and Rept. Minn. Surv. 5 : 72.1877.

Morg. Fl. Miami Valley 67. I878.

James, Jour. Cin. Soc. 2: 64 (23). April 1879.

Hark. \& Moore, Cat. Pac. Fung. 7. Feb. I880.

Brendel, Fl. Peoriana I ed. 392 (94). 1882.

Somers, Tr. N. Scot. Inst. 5: I80. I 1882.

Morg. Jour. Cin. Soc. 6 : i1 2 . $d$. July 1883.

Rav. S. Car. Resources $355 . \quad$ I 883.

Bundy, Geol. Wis. I873-79, 1: 397. 1883.

Cragin, Bull. Wash. Lab. 1 : 21. Sept. 1884.

Bessey, Bull. lowa Agr. Coll. 1884: I46. Jan. I885. 
Palmer, Mushrooms pl. I. $\quad 1885$.

Pk. Rept. N. Y. Mus. 36: 42. d. I 885.

Taylor, Rept. U. S. Agr. 1885: I04 (Io). d. pl. I, f. 5. 1886 and I894. Brendel, FI. Peoriana 2 ed. 68 . 1887.

Cobb, List Pl. Amherst 32. 1887.

Arthur, Rept. N. Y. Exp. Sta. 6: $464 . \quad 1888$.

Ladd, Rept. N. Y. Exp. Sta. 6: 464. ${ }_{1} 888$.

Ell. in Cat. PI. N. J. 475.1889.

McBride, Bull. Univ. lowa 1: 188. $d$. 197. $d$. June 1890.

Somers, Tr. N. Scot. Inst. $7: 466 . \quad 1890$.

Webber, Rept. Nebr. Agr. 1889: 229 (89). $\quad$ I 890.

Banning in Pk. Rept. N. Y. Mus. 44: 182 (70). Dec. 1891.

Falconer, Mushrooms i 16. f. 24. 1891 .

Fairman, Proc. Rochester Acad. 2 : 159. Feb. 1803.

Atk. \& Schrenk, Jour. Mitch. Soc. 9 : 100 (6). April 1893.

Cockerell, Zoe 4: 285. Oct. i 893 .

Pk. Country Gent. 59 : 518 (26). $f$. $\quad$ I2 July 1894.

Underw. Proc. Ind. Acad. 1893: 6I. Aug. 1894.

Kellerm. \& Werner, Geol. Ohio 72: 315 . Jan. 1895.

Beach, Bull. N. Y. Exp. Sta. 88: 350. pl. 2, f. 1-13. March I 895.

Graves, Asa Gray Bull. 3 : 43. I Oct. 1895.

Gibson, Mushrooms 80. d. pl. 5, 6. 1805 .

McClatchie in Reid's Hist. Pasadena 621. 1895.

Millsp. \& Nutt. Publ. Field Columb. Mus. Bot. 1 : 160 . Jan. 1896.

Sturgis, Rept. Conn. Agr. 1895 : 79 (9). pl. 4, f. 2. Jan. 1896.

Underw. Bull. Ala. Exp. Sta. $73: 337,339 . f$. $l$. Oct. 1896.

Bates, Tr. Mass. Hart. Soc. 1896 : 170, 174. d." 1896.

Webster, Am. Kitchen Mag. 6: 217. Feb. 1897 and Taylor, Hdbk. Mushrooms 2: 24 . I897.

Falconer, Farmers' Bull. U. S. Agr. 53: I-20. f. 1-14. March 1897.

Pk. Rept. N. Y. Mus. 48: 232 (134), pl. 6, f. March I 897.

McClatchie, Proc. S. Calif. Acad. 1: 382. 5 June 1897.

Atk. Bull. Cornell Univ. 3 : 25. 25 June 1897.

Underw. \& Earle, Bull. Ala. Exp. Sta. 80: 253. 28 June 1897.

Atk. Bull. Cornell Exp. Sta. 138: 340-352. d. f. 87-100, 110 \& 112 . Sept. I 897.

Coville, Circ. U. S. Agr. I3: 4. f. I. I Dec. I 897.

Beal, Rept. Mich. Agr. 49. 1897.

Taylor, Hdbk. Mushrooms i : pl. A. 1897.

Mendel, Am. Jour. Physiol. 1: 220. I March I 898.

Pk. Rept. N. Y. Mus. 50: 80. 28 March 1898.

Farl. Yearb. U.S. Agr. 1897: 456. pl. 21. 1898. [Bull. 15.]

Atk. Bull. Cornell Exp. Sta. I68: 5 IO. May 1899.

Smith, C. O., Rhodora 1: 16i. I Sept. I899.

Herbst. Fungal FI. Lehigh Valley 72. d. I809.

Knox, Sketchbook, $p l$. '. 1899.

Longyear, Rept. Mich. Bd. Agr. 1898 : 92. I899.

Atk. Mushrooms, I, 3, I8. d. f. 1-8, 12. 243, 248. 20 Oct. 1900. 2 ed. 289, 294. April 1902.

Atk. in Bailey's Cycl. Am. Hort. 1042. f. 1440-1442. 1900.

Mcllvaine \& Macadam, Am. Fung. 332. d.pl. $91 . f .4$, 5. 1900.

Nelson, Rept. Wyo. Exp. Sta. 10: (38). I900.

Trel. Rept. Mo. Hort. Soc. 43: 238 (4). 1900.

Henderson, Bull. Idaho Exp. Sta. $27: 30$, 31, 48. pl. 1, 2. March I90I.

Sweetser, Bull. Pacific Univ. 14: 12. $f . F_{1}$, 2. May I 901.

Earle in Mohr, Contr. U. S. Nat. Herb. 6 : 224. 3I July 1901.

Garman, Bull. Ky. Exp. Sta. $96: 221 . f .9-11$. Nov. 1901.

Williams, Plant World 4:207. Nov. 1901. 
Hay, Bull. N. H. Soc. N. B. 4: 343. 5 : IIo, II I, II9. I90I, I903.

Williamson, Proc. Ind. Acad. 1900: I65. I90I.

Brown \& Fernekes, Bull. Wis. Nat. Hist. Soc. 11. 2 : 45. Jan. I902.

Webster, Rhodora 4: 77, 79. 4 April 1902.

Ricker, Univ. of Maine Studies 3: 55. 30 April 1902.

Ferguson; Bull. U. S. Agr. Pl. Industry 16: 15, 17, 32.

Hess, Bull. lowa Exp. Sta. 61 : 152. $f$. 10 . July 1902.

White, Bull. Torr. Bot. 'Club 29: 562.26 Sept. 1902.

Webster, Rhodora 4: i99. 18 Oct. 1902.

Chesnut, Contr. U.S. Nat. Herb. 7 : 30I. f. 66.1902.

Weems \& Hess, Proc. Soc. Prom. Agr. Sci. 23: 167. 1902.

Duss, Champ. Guad. \& Martinique, 56. 1903.

Cockerell, Jour. Myc. 10: 50. I9 March I904.

Bres. in Sacc. Pk. \& Trel. Harriman Alaska Exp. 5: 45. 5 April igo4.

Pk. Rept. N. Y. Mus. 57: 4. July i904. [Bull. 75.]

Duggar, Farmers' Bull. U. S. Agr. 204: 5, 20. f. I, 2. I904.

Longyear, Rept. Mich. Acad. 4: i I 5. I 904.

Psalliota campestris, Fr.

Rostr. Meddelelser om Groenl. 3 : 529. I888. 3 : 598. I89I.

Taylor, Food Products 2: 2I. I893.

Rostrup, Meddelelser om Groenl. 18 : 50 (8). I 894.

Pat. \& Hariot, Jour. de Bot. 10: 250. I Aug. I896.

Henn. in Engler \& Prantl, Pflanzenfam. 1 1**: 238. d. f. ${ }_{116, \text { A. }} 1898$.

Lloyd, Myc. Notes 26. d. Nov. 1899 .

Jelliffe, Flora of Long Island 4I. I890.

Langl. Proc. La. Soc. Naturalists 1897-9 : 47, 59. 1900.

Perry, Agricultural Student 6: i79. May i 900 and Ohio Univ. Bull. IV. 30: 6. May 1900.

Agaricus eampestris, L., var. albus, B.

Pk. Rept. N. Y. Mus. 36 : 44. d. 1885.

Pk. Country Gent. 59 : 5 I8 (27). I2 July I 894.

Mcllvaine \& Macadam, Am. Fung. 332. d. 1900.

Agaricus eampestris, L., var. Buchanani, Pk.

Pk. Rept. N. Y. Mus. $36: 44$. d. I 885 .

Mcllvaine \& Macadam, Am. Fung. 333. d. 1900.

Agaricus campestris, L., var. elongatus, $\mathrm{Pk}$.

Pk. Rept. N. Y. Mus. 36: 44. $d$. I 885.

Mcllvaine \& Macadam, Am. Fung. 333. d. 1900.

Agaricus campestris, L., var. griseus, Pk.

Pk. Rept. N. Y. Mus. 44: I 50 (38). Dec. I89I.

Pk. Country Gent. 59: 518 (27). I2 July I894.

Mcllvaine \& Macadam, Am. Fung. 332. d. 1900.

Agaricus campestris, L., var. hortensis, Cke.

Pk. Rept. N. Y. Mus. 36 : 44. d. $\quad$ I 885.

Pk. Country Gent. 59: 5 I8 (27). I2 July I 894.

Mcllvaine \& Macadam, Am. Fung. 333. d. 1900.

Longyear, Rept. Mich. Acad. 4: II 5. 1904.

Psalliota campestris, Fr., var. hortensis, Lloyd.

Lloyd, Myc. Notes 27. $d$. Nov. I899.

Agaricus campestris, L., var. pratensis, Vitt.

Johnson, Bull. Minn. Acad. 1: 235 and Rept. Minn. Surv. 5 : 72.1877.

Longyear, Rept. Mich. Bd. Agr. 1898: $92 . \quad$ I899.

Agaricus campestris, L., var. praticola, Vitt.

Pk. Rept. N. Y. Mus. 36: 44. d. 1885.

Mcllvaine \& Macadam, Am. Fung. 332. d. 1900. 
Agaricus campestris, L., var. rufescens, B.

Pk. Rept. N. Y. Mus. $36: 44$. d. 1885.

Ell. in Cat. Pl. N. J. 475. 1889.

Mcllvaine \& Macadam, Am. Fung. 332. d. 1900.

Agaricus campestris, L., var. silvicola, Vitt.

Bon. Zweite Deutsche Nordpolarfahrt 2:80. 1874.

Johnson, Bull. Minn. Acad. 1: 235. 1877 and Rept. Minn. Surv. 5 : 72. 1877 .

Longyear, Rept. Mich. Bd. Agr. 1898 : 92. 1899.

Agaricus campestris, L., var. umbrinus, Vitt.

Pk. Rept. N. Y. Mus. 36: 44. $d$. 1885.

Mcllvaine \& Macadam, Am. Fung. 332. d. 1900.

Agaricus campestris, L., var. vaporarius, Vitt.

Pk. Rept. N. Y. Mus. 36: 44. d. 1885 :

Mcllvaine \& Macadam, Am. Fung. 333. d. 1900.

Agaricus campestris, L., var. villaticus, Brond.

Pk. Rept. N. Y. Mus. 25: 109. Sept. 1873.

Pk. Rept. N. Y. Mus. $36: 44 . d .1885$.

Mcllvaine \& Macadam, Am. Fung. 333. d. 1900. Agaricus villaticus, Brond.

Winder, Mushrooms of Canada 20. 1871.

Agaricus camphoratus, Bull.

See Lactarlus camphoratus, (Bull.) Fr.

Agaricus camptophyllus, B.

See Omphalla camptophylla, B.

Agaricus camptopus, Pk.

See Psllocybe camptopus, Pk.

Agaricus candicans, $P$.

See Clitocybe candicans, (P.) Fr.

Agaricus candidissimus, B. \& C.

See Pleurotus candidiss/mus, B. \& C.

Agaricus Candolleanus, Fr.

See Hypholoma Candolleanum, Fr.

Agaricus Cantharellus, S.

See Hygrophorus Cantharellus, (S.) Fr.

Agaricus caperatus, $P$.

See Phollota caperata, (P.) Fr.

Agaricus capillaris, Schum.

See Mycena caplliarls, (Schum.) Fr.

Agaricus capistratus, Cke.

See Pholiota capistrata, Cke.

Agaricus capnoides, Fr.

See Hypholoma capnoldes, Fr.

Agaricus Caput-Medusæ, Fr.

See Stropharla Caput-Medusa, Fr.

Agaricus carbonarius, Fr.

See Flammula carbonarla, Fr.

Agaricus Carcharias, $P$.

See Leplota Carcharlas, P.

Agaricus carecomœis, B. \& C.

See Collybla carecomis, B. \& C.

Agaricus carneo-albus, With.

See Clitopllus carneo-albus, (With.) Fr. 
Agaricus carneogriseus, B. \& Br.

See Eccilia carneogrisea, B. \& Br.

Agaricus carneolus, Fr.

See Omphalia carneola, Fr.

Agaricus earneus, Bull.

See Tricholoma carneum, (Bull.) Fr.

Agaricus carnosior, $\mathrm{Pk}$.

See Clitocybe clavipes, (P.) Fr.

Agaricus castaneus, Bull.

See Cortinarius castaneus, (Bull.) Fr.

Agaricus castus, M. A. Curtis.

See Tricholoma castum, M. A. Curtis.

Agaricus catephes, B.

See Pleurotus catephes, B.

Agaricus eaudicinus, $P$.

See Pholiota mutabilis, (Schæff.) Fr.

Agaricus caulicinalis, Bull.

See Collybia stipitaria, Fr.

Agaricus caveatus, B. \& C.

See Pleurotus caveatus, B. \& C.

Agarieus Ceciliæ, B. \& Br.

See Amanitopsis strangulata, (Fr.) Roze.

Agaricus cellaris, Banning.

See Tricholoma cellare, Banning.

Agaricus centenarius, B. \& C.

See Omphalia centenaria, B. \& C.

Agaricus cepæstipes, Sow.

See Lepiota cepzstipes, (Sow.) Fr.

Agaricus ceraceus, Wulf.

See Hygrophorus ceraceus, (Wulf.) Fr.

Agaricus cerasinus, $\mathrm{Pk}$.

See Pholiota cerasina, Pk.

Agaricus cerinus, Morg. non P.

See Collybia tenuipes, (S.) Sacc. Syll.

Agaricus cerinus, $\mathrm{P}$.

See Tricholoma cerinum, (P.) Fr.

Agaricus cernuus, Vahl.

See Psilocybe cernua, (Vahl) Fr.

Agaricus cerodes, Fr.

See Naucoria cerodes, Fr.

Agaricus cerussatus, Fr.

See Clitocybe cerussata, Fr.

Agaricus cervinus, Schæff.

See Pluteus cervinus, (Schæff.) Fr.

Agaricus chalybrus, $P$.

See Leptonia chalybzea, (P.) Fr.

Agarieus Chama, Bosc.

See Lentinus Chama, (Bosc) Fr.

Agaricus cheimonoceps, B. \& C.

See Lepiota cheimonoceps, B. \&. C.

Agarieus cheimonophilus, Pk. non B. \& C.

See Crepidotus versutus, Pk. 
Agaricus cheimonophilus, B. \& C.

See Armillaria cheimonophila, B. \& C.

See Muciduia cheimonophila, (B. \& C.) Pat.

Agaricus chioneus, $P$.

See Pleurotus chioneus, (P.) B.

Agaricus chlorinosmus, $\mathrm{Pk}$.

See Amanita chlorinosma, Pk.

Agaricus chondripes, B. \& C.

See Omphalia chondripes, B. \& C.

Agaricus chrysenteroides, Pk.

See Tricholoma chrysenteroides, Pk.

Agarieus chryseus. Pk.

See Omphalia chrysophylla, Fr., var. chrysea, Pk.

Agaricus chrysocephalus, Henn.

See Ciltocybe chrysocephala, Sacc. Syll.

NoTE. Originally spelled auratocephalus and name changed in Saccardo's

Sylloge to Clitocybe chrysocephala, q. v.

Agarieus ehrysodon, Batsch.

See Hygrophorus chrysodon, (Batsch) Fr.

Agaricus chrysoleucus, Fr.

See Omphalia chrysoleuca, Fr.

Agarleus chrysopellus, B. \& C.

See' Fiammula chrysopella, B. \& C.

Agaricus chrysophæus, Schæff.

See Pluteus chrysophæus, (Schæff.) Fr.

Agaricus chrysophlebius, B. \& Rav.

See Piuteus chrysophlebius, B. \& Rav.

Agaricus chrysophyllus, Fr.

See Omphalia chrysophylla, Fr.

Agaricus chrysotrichus, B. \& C.

See Flammula chrysotricha, B. \& C.

Agaricus cicatricatus, Ell. \& Ev.

See Inocybe cicatricata, Ell. \& Ev.

Agaricus ciliatulus, Fr.

See Hiatula clilatuia, Fr.

Agaricus cinereus, Bull.

See Coprinus fimetarius, (L.) Fr.

Agaricus cinnabarinus, $\mathrm{S}$.

See Hygrophorus cinnabarinus, (S.) Fr.

Agaricus cinnamomeus, $L$.

See Cortinarius cinnamomeus, (L.) Fr.

Agaricus eireinatus, Secr.

See Pleurotus circinatus; (Secr.) Fr.

Agaricus cirrhatus, Schum.

See Collybia cirrhata, (Schum.) B.

Agaricus eitricolor, B. \& C.

See Mycena citricolor, B. \& C.

Agaricus citrinellus, $\mathrm{P}$.

See Mycena citrinella, (P.) Fr.

Agaricus clavicularis, Fr.

See Mycena clavicularis, Fr. 
Agaricus elavipes, $\mathrm{P}$.

See Clitocybe clavipes, (P.) Fr.

Agaricus Clavus, L.

See Collybia Clavus, (L.) Fr.

Agaricus Clintonianus, $\mathrm{Pk}$.

See Nolanea Clintoniana, Pk.

Agaricus clusilis, Fr.

See Collybia clusilis, Fr.

Agaricus clypeatus, L.

See Entoloma clypeatum, (L.) Fr.

Agaricus elypeolarius, Bull.

See Lepiota clypeolaria, (Bull.) Fr.

Agaricus coceineus, Schæff.

See Hygrophorus coccineus, (Schæff.) Fr.

Agaricus eochleatus, S. Syn. Car. 766.

See Lentinus cochleatus, Fr.

Agaricus cohærens, P.

See Mycena coharens, (P.) Fr.

See Marasmius coharens, (P.) Cke.

Agaricus Coldwellii, McKay.

See Pleurotus Coldwelli, McKay.

Agaricus collariatus, Fr.

See Mycena collariata, Fr.

Agaricus collinitus, $\mathrm{P}$.

See Cortinarius collinitus, (P.) Fr.

Agaricus collinus, $\mathrm{P}$.

See Marasmius oreades, (Bolt.) Fr.

Agaricus coloreus, Pk.

See Collybia colorea, Pk.

Agaricus Colossus, Fr.

See Tricholoma Colossus, Fr.

Agaricus colubrinus, S. Syn. Car. 556.

See Lepiota clypeolaria, (Bull.) Fr.

Agaricus Columbanus, Mont.

See Clitocybe Columbana, Mont.

Agaricus Columbetta, Fr.

See Tricholoma Columbetta, Fr.

Agaricus Colvini, Pk.

See Hebeloma Colvini, Pk.

Agaricus comaropsis, Mont.

See Hypholoma comaropsis, Mont.

Agaricus comatellus, Pk.

See Inocybe comatella, Pk.

Agaricus comatus, Fl. Dan.

See Coprinus comatus, (Fl. Dan.) P.

Agaricus commiscibilis, B. \& C.

See Pleurotus commiscibilis, B. \& C.

Agarieus communis, $\mathrm{Pk}$.

See Cortinarius communis, $\mathrm{Pk}$.

Agaricus comosus, Fr.

See Pholiota comosa, Fr. 
Agaricus compactus, Fr.

See Trlcholoma compactum, Fr.

Agaricus compressipes, Pk.

See Clltocybe compressipes, Pk.

Agaricus comtulus, Fr.

Pk. Rept. N. Y. Mus. 41:60. 1888.

Pk. Rept. N. Y. Mus. 44: 182 (70). Dec. 1891.

Smith, C. O., Rhodora 1: I62. d. I Sept. 1899.

Atk. Mushrooms 24. d. f. 24. 20 Oct. 1900.

Mcllvaine \& Macadam, Ain. Fung. 334. d. 1900.

Atk. in Bailey's Cycl. Am. Hort. I043. 1900.

Psalllota comtula, Fr.

Lloyd, Myc. Notes 28. $d$. Nov. I899.

Agaricus conchatus, Bull.

See Panus conchatus, (Bull.) Fr.

Agaricus conferruminatus, B. \& Mont.

See Mycena conferruminata, B. \& Mont.

Agaricus confertus, Bolt.

See Galera conferta, (Bolt.) Fr.

Agaricus confluens, $P$.

See Collybla confluens, (P.) Fr.

Agaricus conicus, Scop.

See Hygrophorus conlcus, (Scop.) Fr.

Agaricus conicus, $\mathrm{Pk}$.

See Nolanea conica, Pk.

Agaricus conigenoides, Ell.

See Collybla conigenoides, Ell.

Agaricus conigenoides, Pk. non Ell.

See Collybla alblpllata, Pk.

Agaricus conigenus, $P$.

See Collybia conlgena, (P.) Fr.

Agaricus connatipes, B. \& C.

See Mycena connatlpes, B. \& C.

Agaricus connatus, B. \& C.

See Pleurotus connatus, B. \& C.

Agaricus connexus, $\mathrm{Pk}$.

See Clltocybe connexa, Pk.

Agaricus conocephalus, Bull.

See Bolbltlus conocephalus, (Bull.) Fr.

Agaricus conopileus, $\mathrm{Fr}$.

See Psathyra conopllea, Fr.

Agaricus conopus, $P$.

See Cortlnarius sublanatus, Fr.

Agaricus consobrinus, B. \& Mont.

See Trlcholoma consobrlmum, B. \& Mont.

Agaricus consociatus, M. A. Curtis.

NotE. This species, first mentioned by Curtis, 1867 , under the subgenus Tricholoma has, as far as we can ascertain, never been described. It is cited in the references given below.

M. A. Curtis, Bot. N. Car. 85. 1867.

Cke, \& B. Fungi 89.1875.

Taylor, Food Product 2: 21. 1893.

Hyams, Bull. N. Car. Exp. Sta. $177 ; 49 . \quad$ Dec. 1900. 
Agaricus conspersus, $\mathrm{P}$.

See Naucoria conspersa, (P.) Fr.

Agaricus constans, $\mathrm{Pk}$.

See Mycena constans, $\mathrm{Pk}$.

Agaricus constrictus, Fr.

See Armillaria constricta, Fr.

Agaricus contrarius, $\mathrm{Pk}$.

See Naucoria contraria, Pk.

Agaricus coprinoceps, B. \& C.

See Naucoria coprinoceps, B. \& C.

Agaricus coprinoides, Pk.

See Galera coprinoides, Pk.

Agaricus coriaceus, Bull.

See Lenzites betulina, (L.) Fr.

Agaricus corneipes, Fr.

See Psilocybe corneipes, Fr.

Agaricus coronatus, Fr.

See Hypholoma coronatum, Fr.

Agaricus corrugis, $P$.

See Psathyra corrugis, (P.) Fr.

Agaricus corticatus, Fr.

See Pleurotus corticatus, Fr.

Agaricus corticola, Schum.

See Mycena corticola, (Schum.) Fr.

Agaricus costatus, Fr.

See Entoloma costatum, Fr.

Agaricus cothurnatus, $\mathrm{Pk}$.

Pk. Bull. Torr. Bot. Club. 31 : 18I. d. 28 April 1904.

Agaricus craspedius, Fr.

See Pleurotus craspedius, Fr.

Agaricus crassifolius, B.

See Tricholoma crassifolium, B.

Agaricus crassipes, Schæff.

See Collybia fusipes, (Bull.) B.

Agaricus cremoraceus, $\mathrm{Pk}$.

See Collybia cremoracea, $\mathrm{Pk}$.

Agaricus crenulatus, Fr.

See Hiatula crenulata, Fr.

Agaricus cretacellus, Atk.

Atk. Jour. Myc. 8: 1 10. d. I4 Oct. 1902.

Agaricus cretaceus, Auct. Amer.p. p.

Note. The references to Agaricus cretaceus in American literature are all uncertain 'and even European writers disagree as to the characters of the European species of this name. Some of the references given below probably relate to Lepiota naucina, Fr. but others, especially those of an early date, cannot now be recognized with any degree of certainty.

S. Syn. Car. $84(58)$ no. $667 . \quad$ I 822.

S. Syn. Am. Bor. 147 no. $75 . \quad 1834$.

M. A. Curtis, Bot. N. Car. $90 . \quad 1867$.

Pk. Rept. N. Y. Mus. 22: 78. I 869.

Cke. \& B. Fungi 89. 1875.

Frost in Cat. Pl. Amherst 64. 1875 , 
Gerard, Proc. Poughk. Soc. $1: 84$. I Nov. 1876.

Johnson, Bull. Minn. AcaJ. 1 : 223. 1877 and Rept. Minn. Surv. 5 : 69. 1877.

Hark. \& Moore, Cat. Pac. Fung. 7. Feb. 1880.

Palmer, Mushrooms pl. 4: 1885.

Cobb, List'Pl. Amherst 32.1887.

Webster, Am. Kitchen Mag. 6: 217. Feb. 1897.

Taylor, Hdbl. Mushrooms 2: 24. 1897 .

Mcllvaine \& Macadam, Am. Fung. 344. d. 1900.

Psalliota cretacea, Lloyd.

Lloyd, Myc. Notes 29. Nov. I 899.

Agaricus cretatus, B. \& Br.

See Clitopilus cretatus, B. \& Br.

Agaricus crinitus, L.

See Lentinus crinitus, (L.) Fr.

Agaricus crinitus, S.

See Lentinus Lecomtei, Fr.

Agaricus cristatellus, Pk.

See Lepiota cristatella, Pk.

Agaricus eristatus, A. \& S.

See Lepiota cristata, A. \& S.

Agaricus crocatus, Schrad. See Mycena crocata, (Schrad.) Fr.

Agaricus croceocoruleus, $P$.

See Cortinarius croceocoeruleus, (P.) Fr.

Agaricus eroceotinctus, $\mathrm{Pk}$.

See Crepidotus croceotinctus, $\mathrm{Pk}$.

Agaricus croceus, Schæff.

See Cortinarius cinnamomeus, var. croceus, Fr.

Agaricus crocophyllus, B.

See Crepidotus crocophyllus, B.

Agaricus crocosporus, B. \& C.

See Galera crocospora, B. \& C.

Agaricus erustuliniformis, Bull.

See Hebeloma crustuliniforme, (Bull.) Fr.

Agaricus Cubensis, B. \& C.

See Amanitopsis Cubensis, (B. \& C.) Sacc. Syll.

Agaricus cultorum, B. \& C.

See Lepiota cultorum, B. \& C.

Agaricus cuneifolius, $\mathrm{Fr}$.

See Tricholoma cuneifolium, Fr.

Agaricus Curcuma, B. \& C.

See Naucoria Curcuma, B. \& C.

Agaricus Curtisil, B.

See Pluteus Curtisi, B.

Agaricus curvipes, $P$.

See Pholiota tuberculosa, Fr.

Agaricus curvomarginatus, $\mathrm{Pk}$.

See Naucoria curvomarginata, Pk.

Agaricus cuspidatus, $\mathrm{Pk}$.

See Entoloma cuspidatum, $\mathrm{Pk}$.

Agaricus cyaneus, Pk.

See Entoloma cyaneum, Pk. 
Agaricus eyaneus, S. Syn. Car. 572.

See Cortinarius cærulescens, (Schæff.) Fr.

Agaricus eyanipes, Frost.

NoTE. Apparently no description has ever been published.

Frost in Cat. Pl. Amherst 64. 1875.

Cobb, List Pl. Amherst 32. 1887.

Agaricus eyanoxanthus, Schæff.

See Russula cyanoxantha, (Schæff.) Fr.

Agaricus eyanoxanthus, S. Syn. Car. 757.

See Russula emetica, Fr.

Agaricus eyathiformis, Bull.

See Clitocybe cyathiformis, (Bull.) Fr.

Agaricus eyathoides, Bolt.

See Clitocybe cyathiformis, (Bull.) Fr.

Agaricus cymbaliferus, Mont.

See Mycena cymbalifera, Mont.

Agaricus cyphellæformis, B.

See Pleurotus cyphellaformis, B.

Agaricus dactyliotus, B. \& Mont.

See Pholiota dactyliota, B. \& Mont.

Agaricus daucipes, B. \& Mont.

See Amanitopsis daucipes, (B. \& Mont.) Sacc.

Agaricus dealbatus, Sow.

See Clitocybe dealbata, (Sow.) Fr.

Agaricus debilis, Bull.

See Mycena debilis, (Bull.) Fr.

Agaricus decipiens, $\mathrm{P}$.

See Cortinarius decipiens, (P.) Fr.'

Agaricus decolorans, $\mathrm{P}$.

See Cortinarius decolorans, (P.) Fr.

Agaricus decolorans, S. Syn. Car. 580.

See Cortinarius calochrous, (P.) Fr.

Agaricus decorosus, Pk.

See Tricholoma decorosum, Pk.

Agaricus decumbens, $\mathrm{P}$.

See Cortinarius decumbens, (P.) Fr.

Agaricus deglubens, Fr.

See Inocybe deglubens, Fr.

Agaricus delectabilis, $\mathrm{Pk}$.

See Mycena delectabilis, $\mathrm{Pk}$.

Agaricus delicatellus, $\mathrm{Pk}$.

See Collybia delicatella, $\mathrm{Pk}$.

Agaricus delicatulus, $\mathrm{Pk}$.

See Nolanea delicatula, $\mathrm{Pk}$.

Agaricus delicatus, Fr.

See Lepiota delicata, Fr.

Agaricus deliciosus, L.

See Lactarius deliciosus, (L.) Fr.

Agaricus demetriacus, B. \& Mont.

See Entoloma demetriacum, B. \& Mont. 
Agaricus dentatus, $P$.

See Lentinus cochleatus, Fr.

Agaricus denticulatus, $\mathrm{S}$.

See Lentinus Schweinitzii, Fr.

Agaricus depallens, P.

See Russula depallens, (P.) Fr.

Agaricus depluens, Batsch.

See Claudopus depluens, (Batsch) Fr.

Agaricus destrictus, $\mathrm{Fr}$.

See Inocybe destricta, Fr.

Agaricus detersibilis, B. \& C.

See Collybia detersibilis, B. \& C.

Agaricus detersibilis, Pk.

See Pholiota detersibilis, Pk.

Agaricus dichromus, B. \& C.

See Psilocybe dichroma, B. \& C.

Agaricus dichropus, Fr.

See Tricholoma dichropus, Fr.

Agaricus digitalis, Batsch.

See Coprinus digitalis, (Batsch) Fr.

Agaricus dilatatus, Fr.

See Mycena dilatata, Fr.

Agaricus dilutus, $P$.

See Cortinarius dilutus, (P.) Fr.

Agaricus diminutivus, $\mathrm{Pk}$.

Pk. Bull. Buf. Soc. 1: 53. d. July 1873 and Rept. N. Y. Mus. 26 : 59. d. April 1874.

Pk. Rept. N. Y. Mus. $36: 49 . d . \quad$ I 885.

Sacc. Cub. \& Manc. Syll. 5 : 1009. d. 28 May 1887.

Smith, C. O., Rhodora 1: 162. d. I Sept. 1899.

Atk. Mushrooms 26. d. 20 Oct. 1900.

Mcllvaine \& Macadam, Am. Fung. 335. d. I 1900.

Pk. Rept. N. Y. Mus. 541: 184. d. pl. $74, f .1-8$. Dec. 1901.

Conant, Bull. Boston Myc. Club I9: 5 Feb. I903.

Longyear, Rept. Mich. Acad. 4 : I1 15.1904.

Psalliota diminutiva, $\mathrm{Pk}$.

Henn. in Engler \& Prantl, Pflanzenfam. 11** : 238. 1898.

Lloyd. Myc. Notes 29. Nov. I899.

Agaricus discoideus, $P$.

See Hygrophorus discoideus, (P.) Fr.

Agaricus discolor, $\mathrm{Pk}$.

See Pholiota discolor, Pk.

Agaricus discomorbidus, $\mathrm{Pk}$.

See Naucoria discomorbida, Pk.

Agaricus discopus, Lev.

?See Mycena discopus, Lev.

Agaricus discretus, Fr.

See Hiatula discreta, Fr.

Agaricus dispersus, Fr.

See Hypholoma dispersum, $\mathrm{Fr}$.

Agaricus disseminatus, $P$.

See Psathyrella disseminata, (P.) Fr.

Agaricus ditopus, Fr.

See Clitocybe ditopus, Fr. 
Agaricus domesticus, $\mathrm{P}$.

See Coprinus domesticus, P.

Agaricus dorsalis, Bosc.

See Panus dorsalis, (Bosc) Fr.

Agaricus dorsalis, Pk.

See Crepidotus dorsalis, $\mathrm{Pk}$.

Agaricus dryinus, $P$.

See Pleurotus dryinus, (P.) Fr.

Agaricus dryophilus, Bull.

See Collybia dryophila, (Bull.) Fr.

Agarieus dulcamarus, A. \& S.

See Inocybe dulcamara, (A. \& S.) Fr.

Agarieus duleamarus, S. Syn. Car. 628.

See Cortinarius illopodius, (Bull.) Fr.

Agaricus durus, Bolt.

See Pholiota dura, (Bolt.) Fr.

Agaricus dysthales, $\mathrm{Pk}$.

See Entoloma dysthales, Pk.

Agaricus eburneus, Bull.

See Hygrophorus eburneus, (Bull.) Fr.

Agaricus echinatus, Roth.

NoTe. The wpecies of this name is referred to by American authors as both Psalliota echinata, Fr. and Inocybe echinata, Sacc. Syll. The generic position is perhaps doubtful. For references see Inocybe echinata, (Roth) Sacc. Syll.

Agarieus eehinipes, Lasch.

See Mycena echinipes, (Lasch) Fr.

Agaricus echinocarpus, Ell.

See Inocybe Gaillardi, Gillet.

Agaricus ectypoides, $\mathrm{Pk}$.

See Clitocybe ectypoides, Pk.

Agaricus edulis, Auct. Amer.

See Agaricus arvensis, Schæff.

Agaricus egenulus, B. \& Br.

See Hypholoma egenulum, B. \& Br.

Agaricus elatinus, $P$.

See Panus violaceofulvus, (Batsch) Quel.

Agaricus elatior, Pk.

See Naucoria elatior, $\mathrm{Pk}$.

Agaricus elegans, $P$.

See Mycena elegans, (P.) Fr.

Agaricus elixus, Sow.

See Clitocybe elixa, (Sow.) Fr.

Agaricus elodes, Fr.

See Entoloma elodes, Fr.

Agaricus elongatipes, $\mathrm{Pk}$.

See Psilocybe elongatipes, Pk.

Agaricus elvensis, B. \& Br.

Hark. \& Moore, Cat. Pac. Fung. 7. Feb. I880.

Mcllvaine \& Macadam, Am. Fung. 338. d. 1900. 
Psalliota elvensis, B. \& Br.

Lloyd, Myc. Notes 29. Nov. 1899.

Agaricus emendatior, B. \& C.

See Volvaria emendatior, B. \& C.

Agaricus emeticus, Fr.

See Russula emetica, Fr.

Agaricus ephemeroides, Bull.

See Coprinus ephemeroides, (Bull.) Fr.

Agaricus ephemerus, Bull.

See Coprinus ephemerus, (Bull.) Fr.

Agaricus epibates, Fr.

See Psathyra epibates, Fr.

Agaricus Epichysium, P.

See Omphalia Epichysium, (P.) Fr.

Agaricus epigæus, S. Syn. Car. 816.

See Pleurotus applicatus, (Batsch) Fr.

Agaricus epimyces, $\mathrm{Pk}$.

See Panzolus epimyces, Pk.

Agaricus epiphyllus, Fr.

See Marasmius epiphyllus, Fr.

Agaricus epipterygius, Scop.

See Mycena epipterygia, (Scop.) Fr.

Agaricus epixanthus, Fr.

See Hypholoma epixanthum, Fr.

Agaricus equestris, L.

See Tricholoma equestre, (L.) Fr.

Agaricus ericaceus, S.

See Psilocybe ericæa, (P.) Fr.

Agaricus ericæus, $P$.

See Psilocybe ericaa, (P.) Fr.

Agaricus ericetorum, $\mathrm{P}$.

See Omphalia umbellifera, (L.) Fr.

Agaricus erinaceëllus, $\mathrm{Pk}$.

See Pholiota detersibilis, $\mathrm{Pk}$.

Agaricus erinaceus, $\mathrm{Fr}$.

See Naucoria erinacea, Fr.

Agaricus erubescens, Fr.

See Hygrophorus erubescens, Fr.

Agaricus erubescens, Mont.

See Clitocybe erubescens, Mont.

Agaricus erysibodes, Mont.

See Hebeloma erysibodes, Mont.

Agaricus esculentoides, $\mathrm{Pk}$.

See Collybia esculentoides, $\mathrm{Pk}$.

Agaricus esculentus, Wulf.

See Collybia esculenta, (Wulf.) Fr.

Agaricus Estensis, Morg.

See Collybia Estensis, Morg.

Agaricus eugrammus, Mont.

See Panus eugrammus, (Mont.) Fr.

Agaricus eumorphus, S. Syn. Car. 644.

See Cortinarius anomalus, Fr. 
Agaricus euosmus, B.

See Pleurotus euosmus, B.

Agaricus euspeireus, B. \& C.

See Mycena euspeirea, B. \& C.

Agaricus eutheles, B. \& Br.

See Inocybe eutheles, B. \& Br.

Agaricus eutheloides, $\mathrm{Pk}$.

See Inocybe eutheloides, $\mathrm{Pk}$.

Agaricus euthygrammus, B. \& C.

See Naucoria euthygramma B. \& C.

Agaricus excavatus, B. \& C.

See Pleurotus excavatus, B. \& C.

Agaricus excedens, $\mathrm{Pk}$.

See Hebeloma excedens, Pk.

Agaricus excelsus, Fr.

See Amanita excelsa, Fr.

Agaricus excoriatus, Schæff.

See Lepiota excoriata, (Schæff.) Fr.

Agaricus eximius, $\mathrm{Pk}$.

See Pilosace eximia, Pk.

Agaricus expallens, $P$.

See Clitocybe expallens, (P.) Fr.

Agaricus expansus, $\mathrm{Pk}$.

See Galera expansa, $\mathrm{Pk}$.

Agaricus exsculptus, Fr.

See Collybia exsculpta, Fr.

Agaricus exsertus, Fr.

Note. This is considered by Fries to be a variety of Agaricus campestris, L.

Psalliota exserta, Fr.

Lloyd, Myc. Notes 28. $d$. Nov. 1899.

Agaricus fabaceus, B.

NOTE. We have placed here the references to Agaricus amygdalinus, M. A. Curtis of which no description was ever published by Curtis but which he later considered, as shown by notes in his herbarium, to be really Agaricus fabaceus, B. Agaricus subrufescens, Pk. is certainly closely related to what is here classed under Agaricus fabaceus and inasmuch as it is not possible at present to settle the points of distinction, all references to these species are placed together since one studying the subject should examine them all.

B. Lond. Jour. Bot. 6 : 3 I4 (3). d. I847. [No. II 4.]
[

B. \& C. Am. Jour Sci. II. 8 : 403 . Nov. 1849.

Lea, Cat. Pl. Cin. 53. d. 1849 and Jour. Cin. Soc. 5 : 200 (4). d.

Dec. I 882.

Rav. Charleston Med. Jour. \& Rev. 6 : I92. March 185 I.

* Rav. Fung. Car. 3: 3. 1855.

Sprague, Proc. Boston Soc. 6 : 3i6. 1859.

B. Outl. 80 . I 860 .

James, Jour. Cin. Soc. 2 : 64 (23). April 1879.

Morg. Jour. Cin. Soc. 6 : i I I. $d$. July 1883.

Sacc. Cub. \& Manc. Syll. 5 : 994. $d .28$ May 1887.

Farl. Proc. Boston Soc. 26: 358. 8 Sept. I 894.

Kellerm. \& Werner, Geol. Ohio $7^{2}$ : 31 5. Jan. I 895.

Atk. Mushrooms 23. I900. 
Atk. in Bailey's Cycl. Am. Hort. I043. I900.

Mcllvaine \& Macadam, Am. Fung. 341. d. 1900.

Agaricus amygdalinus, M. A. Curtis.

M. A. Curtis in Rav. Fung. Car. 3: 3. I855. (MS. note.)

M. A. Curtis, Bot. N. Car. 90 . I 867.

M. A. Curtis, Jour. Roy. Hort. Soc. Il. $2: 72,78$. I870.

Featherman, Bot. Surv. La. 1870: 123." I87I.

Cke. \& B. Fungi 88. I875.

Rav. S. Car. Resources 355. I883.

Atk. Mushrooms 23. 20 Oct. 1900.

Trel. Rept. Mo. Hort. Soc. $43: 228$ (5). 1900.

Trel. Rept. Mo. Bot. Gard. 15: 84. pl. 35, 36. 30 April I904.

Memminger, Jour. Myc. 11: 12. 4 Jan. 1905. Agaricus subrufescens, $\mathrm{Pk}$.

Pk. N. Y. Tribune. d. 25 Dec. 1892 and Gard. \& For. 5:624d. 28 Dec. 1802.

Pk. Rept. N. Y. Mus. 46: 105 (25) d. Dec. 1893 and Rev. Myc. 16 : I25. $d$. I July I 894 .

Falconer, Am. Florist 9 : 657. 22 Feb. 1894.

Taylor, Rept. U. S. Agr. 1885 : (22). I894. [In reprint.only.]

Taylor, Rept. U. S. Agr. 1893: pl. $4^{\mathrm{a}}$. I894.

Sacc. Syll. 11 : 70. d. July 1805 .

Pk. Rept. N. Y. Mus. $48: 236$ (138). pl. 7. f. March 1897.

Underw. Moulds i22. $\quad 1899$.

Atk. Mushrooms 23. d. 20 Oct. I900.

Mcllvaine \& Macadam, Am. Fung. 344. d. I900.

Williams, Plant World 4 : 207. Nov. I901.

Trel. Rept. Mo. Hort. Soc. 43: 227. (4). Igor.

Psalliota amygdalina, M. A. Curtis.

M. A. Curtis, Bot. N. Car. 9o. 1867.

Taylor, Food Products $2: 21$. I 893 .

Lloyd, Myc. Notes 29. Nov. I89y.

Hyams, Bull. N. Car. Exp. Sta. I77: 45. Dec. 1900.

Psalliota fabacea, Lloyd.

Lloyd, Myc. Notes 29. Nov. I899.

Perry, Agricultural Student 6 : I79. May 1900 and Ohio Univ. Bull. IV. $30: 7$. May 1900.

Psalliota subrufescens, Lloyd.

Lloyd, Myc. Notes 29. Nov. I899.

Agaricus facifer, B. \& C.

See Pleurotus facifer, B. \& C.

Agaricus fagicola, Lasch.

See Psathyra fagicola, (Lasch) Fr.

Agaricus faleifolius, Mont.

See Psathyrella falcifolia, Mont.

Agaricus fallax, Pk:

See Tricholoma fallax, Pk.

Agaricus Familia, Pk.

See Collybia Familia, Pk.

Agaricus farinaceus, Huds.

See Laccarla laccata, (Scop.) B. \& Br.

Agaricus farinaceus, Schum. See Panus farinaceus, (Schum.) Fr.

Agaricus farinosus, S.

See Amanitopsis farinosa, (S.) Atk. 
Agaricus Pascicularis, Huds.

See Hypholoma fasciculare, (Huds.) Fr.

Agaricus fastibilis, $P$.

See Hebeloma fastibile, (P.) Fr.

Agaricus fastigiatus, Schæff.

See Inocybe fastigiata, (Schæff.) Fr.

Agaricus Feildeni, B.

See Stropharia Feildeni, B.

Agaricus felinus, $P$.

See Lepiota felina, P.

Agaricus Fenzlii, Schulz.

See Annularia Fenzlii, Schulz.

Agaricus Perrugineus, S. Syn. Car. 703.

See Coprinus micaceus, (Bull.) Fr.

Agaricus fertilis, A. \& S.

See Entoloma clypeatum, (L.) B.

Agaricus fibrillosus, P.

See Psathyra fibrillosa, (P.) Fr.

Agaricus Fibula, Bull.

See Omphalia Fibula, (Bull.) Fr.

Agaricus Fibula, var. Swartzii, Fr.

See Omphalia Swartzii, (Fr.) Pk.

Agaricus fibuloides, Pk.

See Omphalia fibuloides, $\mathrm{Pk}$.

Agaricus filipes, Bull.

See Mycena fillpes, (Bull.) Fr.

Agaricus fimetarius, L.

See Coprinus fimetarius, (L.) Fr.

Agaricus Pimicola, Fr.

See Panzolus fimicola, Fr.

Agaricus fimiputris, Bull.

See Anellaria fimiputris, (Bull.) Karst.

Agaricus Plabelliformis, Bolt.

See Lentinus flabelliformis, (Bolt.) Fr.

Agaricus flaccidus, Sow.

See Clitocybe flaccida, (Sow.) Fr.

Agaricus flammans, Fr.

See Pholiota flammans, Fr.

Agaricus flavellus, B. \& C.

See Omphalia flavella, B. \& C.

Agaricus flavescens, Pk.

See Tricholoma flavescens, $\mathrm{Pk}$.

Agaricus flavidellus, Pk.

See Clitocybe flavidella, $\mathrm{Pk}$.

Agaricus flavidus, $P$.

See Flammula flavida, (P.) Fr.

Agaricus (Mycena) flavipes, S. Svn. Car. 685.

Note. It is impossible to say what plant this name is intended to desig. nate.

S. Syn. Car. 85 (59) no. 685. I822.

Agaricus flavobrunneus, Fr.

See Tricholoma flavobrunneum, $\mathrm{Fr}$. 
Agaricus Plavolanatus, B. \& C.

See Pleurotus flavolanatus, B. \& C.

Agaricus Plavorubens, B. \& Mont.

See Amanita flavorubens, B. \& Mont.

Agaricus flavovirens, $\mathrm{P}$.

See Tricholoma equestre, (L.) Fr.

Agaricus flavus, Johnson.

See Entoloma flavum, Johnson.

Agaricus flexipes, Fr.

See Cortinarius flexipes, Fr.

Agaricus flexuosus, Fr.

See Lactarius flexuosus, Fr.

Agaricus floccosus, Schrff.

See Phollota squarrosa, (Muell.) Fr.

Agaricus (Galera) flocculentus, Fr.

Note. In the reference given below Fries cites his Epicrisis p. 209 but in that place the name Agaricus flocculentus, Fr. does not occur; instead is Agaricus frustulentus, $\mathrm{Fr}$. which is not said to occur in Central America.

Fr. Nova Acta R. Soc. Sci. Upsal. 111. 1 : 24. $1851 . \quad$ Cent. Amer.

Polakowsky, Jahresber. Ver. Erdkunde Dresden 16: 74. 1879.

Agaricus flocculentus, Poll.

See Inocybe lanuginosa, (Bull.) Fr.

Agaricus flocculosus, B.

See Inocybe flocculosa, B.

Agaricus floralis, B. \& Rav.

See Lepiota floralis, B. \& Rav.

Agaricus fluxilis, Fr.

See Pleurotus fluxilis, Fr.

Agaricus foederatus, B. \& Mont.

Mont. Syll. Crypt. 121. $d$. 1856.

Sacc. Cub. \& Manc. Syll. 5 : 1003. d. 28 May 1887.

Mcllvaine \& Macadam, Am. Fung. 339. d. 1900. Psalliota foederata, B. \& Mont.

Henn. in Engler \& Prantl, Pflanzenfam. 11** : 240. 1898.

Lloyd, Myc. Notes 29. Nov. 1899.

Agaricus fœnisecii, P.

See Psilocybe foenisecii, (P.) Fr.

Agaricus lœtens, $P$.

See Russula foetens, (P.) Fr.

Agaricus foliomarginatus, $\mathrm{Pk}$.

See Leptonia foliomarginata, Pk.

Agaricus fragilior, Pk.

See Hebeloma fragilius, Pk.

Agaricus Pragilis, Schæff.

See Omphalia Campanella, (Batsch) Fr.

Agaricus fragilis, $P$.

See Russula fragilis, $P$.

Agaricus fragilissimus, Henn.

See Hiatula fragil ssima, Rav.

Agaricus Pragrans, Sow.

See Clitocybe fragrans, (Sow.) Fr.

Agaricus Friesii, Lasch.

See Lepiota Friesil, (Lasch) Fr. 
Agaricus Frostianus, $\mathrm{Pk}$.

See Amanita Frostiana, Pk.

Agaricus frumentaceus, Bull.

See Tricholoma frumentaceum, (Bull.) Fr.

Agaricus (Galera) Prustulentus, Fr.

Note. See note under Agaricus flocculentus, Fr.

Agaricus fulgens, A. \& S.

See Cortinarius fulgens, (A. \& S.) Fr.

Agaricus fuliginarius, Batsch.

See Collybia plexipes, Fr.

Agaricus Puliginosus, Fr.

See Lactarius fuliginosus, $\mathrm{Fr}$.

Agaricus fulvaster, B. \& C.

See Lepiota fulvastra, B. \& C.

Agaricus fulvellus, Fr.

See Tricholoma fulvellum, $\mathrm{Fr}$.

Agaricus fulvofuligineus, A. \& S.

See Cortinarius scaurus, Fr.

Agaricus fulvotomentosus, $\mathrm{Pk}$.

See Crepidotus fulvotomentosus, $\mathrm{Pk}$.

Agaricus fulvus, Fr.

See Tricholoma flavobrunneum, Fr.

Agaricus fulvus, $\mathrm{Pk}$.

See Naucoria fulva, Pk.

Agaricus fumescens, $\mathrm{Pk}$.

See Tricholoma fumescens, $\mathrm{Pk}$.

Agaricus fumidellus, $\mathrm{Pk}$.

See Tricholoma fumidellum, $\mathrm{Pk}$.

Agaricus fumosoluteus, $\mathrm{Pk}$.

See Tricholoma fumosoluteum, Pk.

Agaricus fumosus, $P$.

See Clitocybe fumosa, (P.) Fr.

Agaricus furcatus, $P$.

See Russula furcata, P.

Agaricus furfuraceus, $P$.

See Tubaria furfuracea, (P.) Fr.

Agaricus furnus, Batsch.

NoTE. This is possilly a misprint for Agaricus firmus but in any case it is impossible to say what species the author intended to designate.

Muhl. Tr. Am. Phil. Soc. 4: 240. 1799.

Agaricus fuscescens, Schæff.

See Coprinus fuscescens, (Schæff.) Fr.

Agaricus fuscifolius, $\mathrm{Pk}$.

See Nolanea fuscifolia, $\mathrm{Pk}$.

Agaricus fuscifrons, B. \& C.

See Pleurotus fuscifrons, B. \& C.

Agaricus fuscocarneus.

NotE. It is impossible to determine what species is intended in the citation given.

Bennett, Cat. PI. R. 1. 77. 1888. 
Agaricus fuseodiseus, Pk.

See Inocybe fuscodlsca, (Pk.) Massee.

Agaricus Puscogriseëllus, Pk.

See Nolanea fuscogrlseélla, Pk.

Agaricus fuscolilacinus, $\mathrm{Pk}$.

See Collybia fuscolilacina, $\mathrm{Pk}$.

Agaricus fuscosquameus, $\mathrm{Pk}$.

See Leplota fuscosquamea, Pk.

Agaricus fusipes, Bull.

See Collybla fusipes, (Bull.) B.

Agaricus Fusus, Batsch.

See Flammula Fusus, (Batsch) Fr.

Agaricus galericulatus, Scop.

See Mycena galericulata, (Scop.) Fr.

Agaricus galopus, P.

See Mycena galopus, P.

Agaricus gambosus, Fr.

See Tricholoma gambosum, Fr.

Agaricus gelatinosus, Gmel.

See Crepldotus mollls, (Schæff.) Fr.

Agaricus geminellus, $\mathrm{Pk}$.

See Naucoria geminella, Pk. .

Agarieus gentilis, Fr.

See Cortinarius gentills, Fr.

Agaricus geophyllus, Sow.

See Inocybe geophylla, (Sow.) Fr.

Agaricus geotropus, Bull.

See Clitocybe geotropa, (Bull.) Fr.

Agaricus Gerardianus, $\mathrm{Pk}$.

See Omphalla Gerardiana, $\mathrm{Pk}$.

Agaricus gibbus, $P$.

See Clltocybe Infundibullformis, (Schæff.) Fr.

Agarleus giganteus, Leys.

See Clitocybe maxima, (Gaertn. \& Meyer) Fr.

Agaricus gilvus, Fr.

See Clitocybe gllva, $\mathrm{Fr}$.

Agarieus glandulosus, Bull.

See Pleurotus glandulosus, (Bull.) Fr.

Agaricus glaucipes, B. \& C.

See Clltocybe glauclpes, B. \& C.

Agaricus glaucopus, Schæff.

See Cortlnarlus glaucopus, (Schæff.) Fr.

Agaricus gliodermus, Fr.

See Lepiota glioderma, Fr.

Agaricus gloiocephalus, D C.

See Volvaria glolocephala, (D C.) Fr.

Agaricus glutinosus, Lindgr.

See Hebeloma glutlnosum, (Lindgr.) Fr.

Agaricus Gordoni, B. \& Br.

See Psathyra Gordonl, B. \& Br.

Agaricus gossypinus, Johnson.

See Hebeloma gossypinum, Johnson. 
Agaricus gracilentus, Kromblh.

See Leplota gracilenta, (Krombh.) Fr.

Agaricus gracilis, Fr.

See Psathyrella gracills, Fr.

Agaricus gracillimus, Weinm.

See Omphalia graclllima, (Weinm.) Fr.

Agaricus graciloides, $\mathrm{Pk}$.

See Psathyrella graclloides, $\mathrm{Pk}$.

Agaricus grammopodius, Bull.

See TrIcholoma grammopodium, (Bull.) Fr.

Agaricus granosus, Morg.

See Lepiota granosa, Morg.

Agaricus granularis, $\mathrm{Pk}$.

See Pluteus granularls, Pk.

Agaricus granulosus, Batsch.

See Leplota granulosa, (Batsch) Fr.

Agaricus granulosus, Batsch, var. amianthinus, $\mathrm{Pk}$. See Lepiota amlanthlna, (Scop.) Fr.

Agaricus graveolens, $\mathrm{P}$.

See Tricholoma graveolens, (P.) Fr.

Agaricus Grayanus, Pk. See Entoloma Grayanum, $\mathrm{Pk}$.

Agaricus Greigensis, $\mathrm{Pk}$.

See Claudopus Grelgens/s, Pk.

Agaricus griseoscabrosus, $\mathrm{Pk}$.

See Inocybe griseoscabrosa, (Pk.) Earle.

Agaricus Guadelupensis, Pat.

Pat. Bull. Soc. Myc. Fr. 15: 197. d. 31 July 1899.

Sacc. \& Syd. Syll. Fung. 16 : I14. d. I Feb. 1902.

West Indies.

Duss, Champ. Guad. \& Martinique 56. 1903.

Agaricus gummosus, Lasch. See Flammula gummosa, (Lasch) Fr.

Agaricus gyroflexus, Fr. See Psathyra gyroflexa, Fr.

Agaricus hædinus, B. \& C. See Pleurotus hredinus, B. \& C.

Agaricus hæmatocephalus, Mont. See Marasmlus hæmatocephalus, (Mont.) Fr.

Agaricus hæmatopus, P. See Mycena hrematopus, (P.) Fr.

Agaricus hæmorrhoidarius, Schulzer.

Pk. Rept. N. Y. Mus. 45: 79 (19) d. Dec. 1893.

Pound, Bot. Surv. Nebr. 3: 12. 18 June 1894.

Pk. Bull. Torr. Bot. Club 26: 67. d. 18 Feb. 1899.

Underw. Moulds I22. I 899.

Mcllvaine \& Macadam, Am. Fung. 336. d. 1900.

Williams, Plant World 4: 207. Nov. 1901.

Pk. Rept. N. Y. Mus. 54i : 183. d. pl. $75 f .1-13$. Dec. I901. Psalllota hamorrholdarla, Schulzer.

Lloyd, Myc. Notes 29. Nov. I899.

Agaricus hærens, Pk. See Crepldotus harens, $\mathrm{Pk}$.

Agaricus Hallianus, $\mathrm{Pk}$.

See Flammula Halllana, Pk. 
Agaricus háriolorum, Bull.

See Collybia hariolorum, (Bull.) Fr.

Agaricus haustellaris, Fr.

See Crepidotus haustellaris, Fr.

Agaricus Hebeloma, Pk.

See Tricholoma Hebeloma, Pk.

Agaricus helictus, B.

See Nolanea helicta, B.

Agaricus helodes, Fr.

See Entoloma helodes, Fr.

Agaricus helvoliceps, B. \& C.

See Flammula helvoliceps, B. \& C.

Agaricus helvolus, $\mathrm{P}$.

See Cortinarius gentilis, Fr.

Agaricus helvus, Fr.

See Lactarius helvus, Fr.

Agaricus hemileucus, B. \& C.

See Collybia hemileuca, B. \& C.

Agaricus hemiphlebius, B. \& C.

See Pleurotus hemiphlebius, B. \& C.

Agaricus hemisclerus, B. \& C.

See Lepiota hemisclera, B. \& C.

Agaricus hemispilus, Lev.

See Clitocybe? hemispila, (Lev.) Sacc. Syll.

Agaricus hemitrichus, P.

See Cortinarius hemitrichus, (P.) Fr.

Agaricus hepaticus, Batsch.

See Omphalia hepatica, (Batsch) Fr.

Agaricus herbarum, Pk.

See Crepidotus herbarum, Pk.

Agaricus hereynicus, var. brunneus, S. Syn. Car. 574. See Cortinarius vioiaceus, (L.) Fr.

Agaricus heteroclitus, Fr.

See Pholiota heteroclita, Fr.

Agaricus hiascens, Fr.

See Psathyrella hiascens, Fr.

Agaricus hiemalis, Osbeck.

See Mycena hiemalis, (Osbeck) Fr.

Agaricus Highlandensis, $\mathrm{Pk}$.

See Flammula Highlandensis, $\mathrm{Pk}$.

Note. Compare also Agaricus ascophorus, Pk.

Agaricus hirtipes, Schum.

See Nolanea hirtipes, (Schum.) Fr.

Agaricus hirtosquamulosus, $\mathrm{Pk}$.

See Hypholoma hirtosquamulosum, Pk.

Agarieus Hoffmani, Pk.

See Clitocybe Hoffmani, Pk.

Agaricus hololepis, Fr.

See Pllosace hololepis, Fr.

Agaricus holoporphyrus, B. \& C.

See Clitocybe holoporphyra, B. \& C. 
Agaricus holosericeus, Fr.

See Lepiota holosericea, Fr.

Agaricus hordus, Pk. nec Fr.

'See Collybia platyphylla, (P.) Fr.

Agarieus hormophorus, Mont.

See Pholiota hormophora, Mont.

Agaricus Hornemanni, Fr.

See Stropharia depilata, (P.) Fr.

Agaricus Howeanus, Pk.

See Pholiota Howeana, (Pk.) Earle.

Agaricus humilis, $P$.

See Tricholoma humile, (P.) Fr.

Agaricus hyacinthus, Batsch.

See Hygrophorus conicus, (Scop.) Fr.

Agáricus hydrophorus, Bull.

See Psathyrella hydrophorus, (Bull.) Fr.

Agaricus hygrophoroides, Pk.

See Collybia hygrophoroides, $\mathrm{Pk}$.

Agaricus hymenocephalus, $\mathrm{Pk}$.

See Hypholoma hymenocephalum, Pk.

Agaricus hypnophilus, B.

See Pleurotus hypnophilus, B.

Agaricus Hypnorum, Batsch.

See Galera Hypnorum, (Batsch) Fr.

Agaricus hypopithyus, M. A. Curtis.

NotE. This species is mentioned in the citations given below under the subgenus Tricholoma but it apparently was never described. Under the genus Tricholoma it is referred to but again without description.

Curtis, M. A., Bot. N. Car. 84. 1867.

Cke. \& B. Fungi 89. 1875.

Agaricus hypoporphyrus, B. \& C.

See Leptonia hypoporphyra, B. \& C.

Agaricus hypothejus, Fr.

See Hygrophorus hypothejus, Fr.

Agaricus ianthinus, Batsch.

See Mycena pura, (P.) Fr.

Agaricus iliopodius, Bull.

See Cortinarius iliopodius, (Bull.) Fr.

Agaricus illicitus, $\mathrm{Pk}$.

See'Hebeloma illicitum, $\mathrm{Pk}$.

Agaricus illinitus, L.

See Lepiota illinita, (L.) Fr.

Agaricus illudens, S.

See Clitocybe illudens, S.

Agaricus imbricatus, Fr.

See Tricholoma imbricatum, Fr.

Agaricus immaculatus, Pk.

See Mycena immaculata, Pk.

Agaricus impolitoides, $\mathrm{Pk}$.

See Tricholoma scalpturatum, Fr.

Agarieus ineanus, Fr.

See Leptonia incana, Fr. 
Agaricus incertus, $\mathrm{Pk}$.

See Hypholoma appendlculatum, (Bull.) Fr.

Agaricus inconstans, Fr.

Note. This species, mentioned by Muhlenberg, 1813,1818 , Torrey 1819 , is uncertain. It is referred by Schweinitz, 1834, to Panus conchatus, Fr.

Agaricus indecens, Pk.

See Phollota Indecens, Pk.

Agarieus Indigo, S.

See Lactarius Indlgo, (S.) Fr.

Agaricus indigoferus, Ell.

See Entoloma Indigoferum, Ell.

Agaricus infelix, $\mathrm{Pk}$.

See Inocybe infellx, $\mathrm{Pk}$.

Agaricus infidus, $\mathrm{Pk}$.

See Inocybe inflda, (Pk.) Earle.

Agaricus infractus, Fr.

See Cortinarlus infractus, Fr.

Agaricus infundibullformis, Schæeff.

See Clltocybe infundibullformis, (Schæff.) Fr.

Agaricus inopus, Fr.

See Flammula inopus, Fr.

Agaricus inquilinus, $\mathrm{Fr}$.

See Tubarla Inquillna, Fr.

Agraricus integrellus, $P$.

See Omphalia Integrella, (P.) Fr.

Agaricus intertextus, B. \& C.

See Mycena Intertexta, B. \& C.

Agaricus involutus, Batsch.

See Paxillus Involutus, (Batsch) Fr.

Agaricus locephalus, B. \& C.

See Mycena locephala, B. \& C.

Agaricus Iris, B.

See Mycena Irls, B.

Agaricus jejunus, Fr.

Fr. Nova Acta R. Soc. Sci. Upsal. III. 1 : 24. d. 1851. West Indies. Sacc. Cub. \& Manc. Syll. 5 : I00I. d. 28 May I887.

Agaricus Johnsonianus, $\mathrm{Pk}$.

See Pholiota Johnsoniana, (Pk.) Atk.

Agaricus jubatus, Fr.

See Entoloma jubatum, Fr.

Agaricus juncicola, Fr.

See Mycena junclcola, Fr.

Agaricus kermesinus, B. \& C.

See Omphalia kermesina, B. \& C.

Agaricus laccatus, Scop.

See Laccaria laccata, (Scop.) B. \& Br.

Agaricus laceratus, S. Syn. Car. 637.

NOTE. It is uncertain to what plant this name belongs, but it apparently is not Collybia lacerata, (Lasch). See P. Syn. 336. 1801 and Streinz, Nomenclator 61. 1862.

S. Syn. Car. $82(56)$ no. 637. I822. 
Agaricus lacerus, Fr.

See Inocybe lacera, Fr.

Agaricus lachnophyllus, B.

See Collybia lachnophylla, B.

See Marasmius cohærens, (P.) Cke.

Agarieus lachrymabundum, Bull.

See Hypholoma lachrymabundum, (Bull.) Fr.

Agaricus lacteus, $\mathrm{P}$.

See Mycena lactea, P.

Agaricus lacteus, S. Syn. Car. $745 \cdot$

See Russula furcata, P.

Agaricus lactifluus, Schæff.

See Lactarius volemus, Fr.

Agaricus lacunosus, $\mathrm{Pk}$.

See Collybia lacunosa, Pk.

Agaricus lætifrons, B. \& C.

See Pluteus latifrons, B. \& C.

Agaricus lætus, P.

See Hygrophorus lætus, (P.) Fr.

Agaricus lævis, Krombh.

See Annularia lavis, (Krombh.) Fr.

Agaricus lamellirugus, D C.

See Paxillus panuoides, Fr.

Agaricus (Pleurotus) lamellosus, S.

Note. The only reference we have to this species is that given below, but no description appears to have been given, although Schweinitz gives himself as authority.

S. Syn. Am. Bor. I49 no. 16r. I834.

Agaricus lampropus, Fr.

See Leptonia lampropus, Fr.

Agaricus lanuginosus, Bull.

See Inocybe lanuginosa, (Bull.) Fr.

Agaricus laseivus, Fr.

See Tricholoma lascivum, Fr.

Agaricus laterarius, Pk.

See Tricholoma laterarium, Pk.

Agaricus latericolor, Mont.

See Hebeloma latericolor, Mont.

Agaricus lateritius, Schæff.

See Hypholoma sublateritium, Fr.

Agaricus lateritius, Fr. non Schæff.

See Galera lateritia, Fr.

Agaricus latifolius, $\mathrm{Pk}$.

See Mycena latifolia, Pk.

Agaricus Leaianus, B.

See Mycena Leaiana, B.

Agaricus Leightoni, B.

See Pleurotus Leightoni, B.

Agarieus leiopus, S. Syn. Car. 658.

See Collybia asema, Fr.

Agaricus leiphæmius, Mont.

See Clitocybe leiphamia, Mont. 
Agaricus lenticeps, Pk.

See Naucoria lenticeps, Pk.

Agaricus lenticularis, Lasch.

See Leplota ienticuiaris, (Lasch) Gillet.

Agaricus lentinoides, Pk.

See Coliybia ientinoldes, Pk.

Agaricus lentus, $P$.

See Fiammula ienta, (P.) Fr.

Agaricus leoninus, Schæff.

See Pluteus ieoninus, (Schæff.) Fr.

Agaricus leoninus, var. b., S. Syn. Car. 638.

See Pluteus ieoninus, (Schæff.) Fr.

Agaricus lepideus, Fr.

See Lentinus lepideus, Fr.

Agaricus lepidomyces, A. \& S.

See Cortinarius phoildeus, Fr.

Agaricus leptocephalus, P.

See Mycena alcaina, Fr.

Agaricus leptolomus, Pk.

See Ciltocybe leptoioma, Pk.

Agaricus leptophyllus, $\mathrm{Pk}$.

See Mycena leptophyila, Pk.

Agaricus leucocephalus, $\mathrm{Fr}$.

See Trichoioma leucocephaium, Fr.

Agaricus leucophanes, B. \& Br.

See Panzolus ieucophanes, B. \& Br.

Agaricus leucophyllus, A. \& S.

See Tricholoma ustale, Fr.

Agaricus leucoxanthus, P.

See Trichoioma sejunctum, (Sow.) Fr.

Agarieus lignatilis, $P$.

See Pleurotus ilgnatills, (P.) Fr.

Agaricus lignicola, Pk.

See Naucoria ilgnicoia, Pk.

Agaricus lilaoifolius, $\mathrm{Pk}$.

See Omphalia illacifoila, $\mathrm{Pk}$.

Agaricus lilacinus, $\mathrm{Pk}$.

See Omphaila lilacifolla, Pk.

Agaricus lilacinus, Mont.

See Mycena Illacina, Mont.

Agaricus limicola, $\mathrm{Pk}$.

See Psilocybe ilmicola, Pk.

Agaricus limonellus, $\mathrm{Pk}$.

See Phollota limonella, Pk.

Agaricus Limonium, $\mathrm{Pk}$.

See Collybia scorzonerea, (Batsch) Fr.

Agaricus limophilus, Pk.

See Psllocybe IImophiia, Pk.

Agaricus limpidus, Fr.

See Pleurotus limpidus, $\mathrm{Fr}$.

Agaricus lineatus, Bull.

See Mycena lineata, (Bull.) P. 
Agaricus liratus, B. \& C.

See Galera lirata, B. \& C.

Agaricus lividus, S. Syn. Car. 758.

. See Russula furcata, P.

Agaricus longicaudus, $P$.

See Hebeloma longicaudum, (P.) Fr

Agaricus lorigipes, Bull.

See Collybia longipes, (Bull.) Fr.

Agaricus longistriatus, $\mathrm{Pk}$.

See Pluteus longistriatus, $\mathrm{Pk}$.

Agaricus lucifugas, Fr.

See Inocybe lucifuga, Fr.

Agaricus lupinus, Fr.

See Flammula lupina, Fr.

Agaricus luridus, Schæff.

See Tricholoma luridum, (Schæff.) Fr.

Agaricus (Mycena) luteo-alba?

Somers, Tr. N. Scot. Inst. 6 : 286. $\quad$ I 887.

Agaricus luteofolius, $\mathrm{Pk}$.

See Pholiota luteofolia, Pk.

Agaricus luteo-olivaceus, B. \& C.

See Collybia luteo=olivacea, B. \& C.

Agaricus luteopallens, Pk.

See Mycena luteopallens, $\mathrm{Pk}$.

Agaricus luteovirens, A. \& S.

See Armillaria luteovirens, (A. \& S.) Fr.

Agaricus luteus, Huds.

See Russula lutea, (Huds.) Fr.

Agaricus lycoperdoides, Bull.

See Nyctalis lycoperdoides, (Bull.) Schrt.

Agaricus macromastes, Fr.

See Galera macromastes, Fr.

Agaricus macropus, Fr.

See Cortinarius macropus, Fr.

Agaricus macrosporus, Mont.

See Lentinus Lecomtei, Fr.

Agaricus macrourus, Scop.

See Collybia radicata, (Relh.) Fr.

Agaricus maculatus, A. \& S.

See Collybia maculata, (A. \& S.) Fr.

Agaricus maculatus, Scop.

See Gomphidius maculatus, (Scop.) Fr.

Agaricus maculosus, $\mathrm{Pk}$.

See Clitocybe maculosa, Pk.

Agaricus madeodiscus, $\mathrm{Pk}$.

See Hypholoma madeodiscum, Pk.

Agaricus madidus, Fr.

See Entoloma madidum, Fr.

Agaricus magnifleus, $\mathrm{Pk}$.

Pk. Bull. Torr. Bot. Club. 26 : 67. d. 8 Feb. 1899.

Mcllvaine \& Macadam, Am. Fung. 342. d. pl. 94. 1900.

Trel. Rept. Mo. Hort. Soc. $43: 228$ (5). 1900. 
Sacc. \& Syd. Syll. 16 : i19. d. I Feb. 1902.

Psalliota magnifica, Lloyd.

Lloyd, Myc. Notes 29. Nov. 1899.

Agaricus magnivelaris, $\mathrm{Pk}$.

See Armillaria ponderosa, $\mathrm{Pk}$.

Agarieus magnus, Frost.

Note. This species, so far as we know, is mentioned only in places cited below and no description has been published.

Frost in Cat. PI. Amherst 65. 1875.

Cobb, List Pl. Amherst 32. 1887.

Agaricus majalis, Fr.

See Entoloma najaie, Fr.

Agaricus malachius, B. \& C.

See Crepidotus malachius, B. \& C.

Agaricus mammosus, L.

See Nolanea mammosa, (L.) Fr.

Agaricus Mappa, Batsch.

See Amanita Mappa, (Batsch) Fr.

Agaricus marginatus, Batsch.

See Phollota marginata, (Batsch) Fr.

Agaricus maritimoides, $\mathrm{Pk}$.

See Inocybe maritimoides, $\mathrm{Pk}$.

Agaricus maritimus, Fr.

See Inocybe maritima, Fr.

Agaricus maritimus, $\mathrm{Pk}$.

Pk. Bull. Torr. Bot. Club 26 : 66. d. 8 Feb. I899.

Underw. Moulds I22. 1899 .

Atk. Mushrooms I42. 20 Oct. 1900.

Mcllvaine \& Macadam, Am. Fung. 337. d. 1900.

Pk. Rept. N. Y. Mus. 541: 184. d. Dec. I901.

Sacc. \& Syd. Syll. $16:$ i I4. d. I Feb. I002.

Conant, Bull. Boston Myc. Club ig. 5 Feb. I903. Psailiota maritima, Lloyd.

Lloyd, Myc. Notes 29. Nov. I899.

Agaricus marmoreus, $\mathrm{Pk}$.

See Ciltocybe marmorea, Pk.

Agaricus Martianus, B. \& C.

See Galera Martiana, B. \& C.

Agaricus mastiger, B. \& Br.

See Psathyra mastigera, B. \& Br.

Agarieus mastoideus, Fr.

See Lepiota mastoidea, Fr.

See Lepiota umbonata, (Schum.) Schrt.

Agaricus mastrucatus, Fr.

See Pleurotus mastrucatus, Fr.

Agaricus maximus, Gærtn. \& Meyer.

See Clitocybe maxima, (Gærtn. \& Meyer) Fr.

Agaricus melaleucus, $\mathrm{P}$.

See Tricholoma melaleucum, (P.) Fr.

Agaricus meliigena, B. \& Cke.

See Mycena meliigena, B. \& Cke.

Agaricus Melilotus, B. \& C.

See Clitopilus Melilotus, B. \& C. 
Agaricus melinoides, $P$.

See Naucoria melinoides, (P.) Fr.

Agaricus melleorubens, B. \& C.

See Armillaria melleorubens, B. \& C.

Agaricus melleus, Vahl.

See Armillaria mellea, (Vahl) Fr.

Agaricus mesomorphus, A. \& S.

See Lepiota delicata, Fr.

Agaricus mesophæus, P.

See Hebeloma mesophæum, (P.) Fr.

Agaricus metachrous, Fr.

See Clitocybe metachroa, Fr.

Agaricus metulisporus, B. \& Br.

See Lepiota metulispora, B. \& Br.

Agaricus Miamensis, Morg.

See Lepiota Miamensis, Morg.

Agaricus micaceus, Bull.

See Coprinus micaceus, (Bull.) Fr.

Agaricus Michelianus, Fr.

See Collybia Micheliana, Fr.

Agaricus Micheneri, B. \& C.

See Pleurotus Micheneri, B. \& C.

Agaricus micropus, $\mathrm{Pk}$.

See Clitopilus micropus, $\mathrm{Pk}$.

Agaricus microsporus, ElI.

See Tricholoma microsporum, Ell.

Agaricus miculatus, Fr.

See Tricholoma miculatum, Fr.

Agaricus miniatus, Fr.

See Hygrophorus miniatus, Fr.

Agaricus minutulus, $\mathrm{Pk}$.

See Mycena minutula, Pk.

Agaricus minutus.

No're. This name recorded by Muhlenberg without authority is impossible to trace.

Muhl. Tr. Am. Phil. Soc. 4: 240. 1799.

Agaricus miratus, $\mathrm{Pk}$.

See Mycena mirata, Pk.

Agaricus mitis, $P$.

See Pleurotus mitis, (P.) Fr.

Agaricus modestus, $\mathrm{Pk}$.

See Hypholoma modestum, Pk.

Agaricus mollis, Schæff.

See Crepidotus mollis, (Schæff.) Fr.

Agaricus monadelphus, Morg.

See Clitocybe monadelpha, Morg.

See Armillaria mellea, (Vahl) Fr.

Agaricus (Tricholoma) monstrosus, Sow.

Note. We are unable to say what this species is.

Johnson, Bull. Minn. Acad. 1 : 354. 1880. 
Agaricus montanus, $P$.

See Psilocybe atrorufa, (Schæff.) Fr.

Agaricus montanus, $\mathrm{Pk}$.

See Omphaila montana, Pk.

Agaricus monticulosus, B. \& C.

See Amanita monticulosa, B. \& C. .

Agaricus Morgani, Pk.

See Lepiota Morgani, Pk.

Agaricus mucidolens, B.

See Gaiera mucidoiens, B.

Agaricus mucidus, Schrad.

See Armiliaria mucida, (Schrad.) Fr.

Agaricus mucifer, B. \& Mont.

See Tricholoma muciferum, B. \& Mont.

Agaricus Mucor, Batsch.

See Mycena Mucor, (Batsch) Fr.

Agaricus multipunctus, Pk.

See Pleurotus decorus, Fr.

Agaricus muralis, Sow.

See Omphalia murais, (Sow.) Fr.

Agaricus muricatus, Fr.

See Phoilota muricata, Fr.

Agaricus murinaceus, Bull.

See Trichoioma murinaceum, (Bull.) Fr.

Agaricus murinolilacinus, Ell. \& Ev.

See Inocybe murinoliacina, Ell. \& Ev.

Agaricus Murrayi, B. \& C.

See Entoloma Murrayi, B. \& C.

Agaricus musæcola, B. \& C.

See Crepidotus musacoia, B. \& C.

Agaricus muscarius, L.

See Amanita muscaria, (L.) P.

Agaricus muscarius, var. major, $\mathrm{Pk}$.

See Amanita strobiliformis, (Vitt.) Fr.

Agaricus muscarius, var. minor, $\mathrm{Pk}$.

See Amanita Frostiana, Pk.

Agaricus muscigenus, Schum.

See Coliybia muscigena, (Schum.) Fr.

Agaricus muscorum, Hoffm.

See Tubaria muscorum, (Hoffm.) Fr.

Agaricus mutabilis, Schæff.

See Phoilota mutabilis, (Schæff.) Fr.

Agaricus mutatus, $\mathrm{Pk}$.

See Inocybe mutata, (Pk.) Massee.

Agaricus mutilus, Fr.

See Pieurotus mutilus, Fr.

Agaricus mycenoides, $\mathrm{Fr}$.

See Pholiota mycenoides, Fr.

Agaricus myomyces, A. \& S. no. 503.

See Trichoioma terreum, (Schæeff.) Fr.

Agaricus myriadophyllus, Pk.

See Coliybia myriadophyila, Pk. 
Agaricus nanus, $P$.

See Pluteus nanus, (P.) Fr.

Agaricus narcoticus, Batsch.

See Coprinus narcoticus, (Batsch) P.

Agaricus nardosmius, Ell.

See Armillaria nardosmia, Ell.

Agaricus naucinoides, $\mathrm{Pk}$.

See Lepiota naucina, Fr.

Agaricus naucinus, Fr.

See Lepiota naucina, Fr.

Agaricus nauseosus, $P$.

Note. The species by this name in S. Syn. Car. 759 is later referred by Schweinitz to Russula nitida, P.q. v. Other references to this species seem doubtful.

Agaricus (Entoloma) nauseosus, M. A. Curtis.

Nore. This occurs only in Curtis's Catalogue and no description has ever been published.

M. A. Curtis, Bot. N. Car. 88. I867.

Agaricus nebularis, Batsch.

See Clitocybe nebularis, (Batsch) Fr.

Agaricus necator, $P$.

See Lactarius turpis, Fr.

Agaricus nephrodes, B. \& C.

See Crepidotus nephrodes, B. \& C.

Agaricus nephrodes, Pk. 26 Rept. non B. \& C.

See Crepidotus applanatus, Fr.

Agaricus Nicotiana, B. \& C.

See Naucoria Nicotiana, B. \& C.

Agaricus nidorosus, Fr.

See Entoloma nidorosum, Fr.

Agaricus nidulans, $P$.

See Claudopus nidulans, (P.) Pk.

Agaricus niger, $\mathrm{S}$.

See Pleurotus niger, (S.) Fr.

Agaricus nigrella, S. Syn. Car. 785 .

See Collybia atrata, Fr.

Agaricus nigripes, S.

See Marasmius nigripes, (S.) Fr.

Agaricus nigrita, B. \& C.

See Collybia nigrita, B. \& C.

Agaricus niphetus, Ell.

See Pleurotus niphetus, Ell.

Agaricus nitidipes, $\mathrm{Pk}$.

See Hypholoma nitidipes, Pk.

Agaricus nitidus, Fr.

See Amanita nitida, Fr.

Agaricus nitidus, $P$.

See Russula nitida, $\mathrm{P}$.

Agaricus nitidus, S. Syn. Car. 716.

See Psilocybe ericaa, (P.) Fr. 
Agaricus nivalis, Pk. non Grev.

See Amanitopsis albocreata, Atk.

Agaricus niveus, P. Syn. p. 400.

See Coprlnus niveus, P.

Agarieus niveus, P. Syn. p. 438.

See Russula fragllls, P.

Agaricus noctiphilus, Ell.

See Lepiota noctiphlla, Ell.

Agaricus nodulosporus, $\mathrm{Pk}$.

See Inocybe nodulispora, $\mathrm{Pk}$.

Agaricus nolitangere, Fr.

See Psathyra nolltangere, Fr.

Agaricus Noveboracensis, Pk.

See Clitopllus Noveboracensls, Pk.

Agaricus nuceus, Bolt.

See Naucorla nucea, (Bolt.) Fr.

Agaricus nudus, Bull.

See Trlcholoma nudum, (Bull.) Fr.

Agaricus oblitus, $\mathrm{Pk}$.

See Leplota oblita, Pk.

Agaricus obscuratus, B. \& Br.

See Marasmlus obscuratus, B. \& Br.

Agaricus obscurus, P.

See Inocybe obscura, (P.) Fr.

Agaricus obtusatus, Fr.

See Psathyra obtusata, Fr.

Agaricus ocellatus, Fr.

See Collybia ocellata, Fr.

Agaricus ochraceus, A. \& S.

See Russula ochracea, A. \& S.

Agaricus ochroleucus, $\mathrm{P}$.

See CortInarlus ochroleucus, (P.) Fr.

Agaricus ochroleucus, S. Syn. Car. 754.

See Russula emetlca, Fr.

Agaricus oehropurpureus, B.

See Clltocybe ochropurpurea, B.

Agaricus Oculus, $\mathrm{Pk}$.

See Omphalla Oculus, Pk.

Agaricus odoratus, $\mathrm{Pk}$.

See Psathyrella odorata, Pk.

Agaricus odorifer, Pk.

See Mycena odorifera, Pk.

Agaricus odorus, Bull.

See Clitocybe odora, (Bull.) Fr.

Agarieus Ohiensis, Mont.

See Clltocybe Ohiensis, Mont.

Agaricus olnodes, B. \& C.

See Naucorla olnodes, B. \& C.

Agaricus oligosarcus, Rav.

See Lepiota ollgosarca, Rav.

Agaricus olivæsporus, Ell. \& Ev.

See Hypholoma ollvesporum, Ell. \& Ev. 
Agaricus olivarius, $\mathrm{Pk}$.

See Omphalia olivaria, $\mathrm{Pk}$.

Agaricus olivascens, S. Syn. Car. 762.

See Russula alutacea, Fr.

Agaricus Oniseus, Fr. ¿See Omphalia Oniscus, Fr.

Agaricus onustus, Howe.

See Amanita onusta, Howe.

Agaricus opacus, With.

See Clitocybe opaca, (With.) Fr.

Agaricus Orcella, Bull.

See Clitopilus Orcella, (Bull.) Fr.

Agaricus oreades, Bolt.

See Marasmius oreades, (Bolt.) Fr.

See Marasmius caryophylleus, (Schæff.) Schrt.

Agaricus ornellus, $\mathrm{Pk}$.

See Hypholoma ornellum, Pk.

Agaricus ostreatus, Jacq.

See Pleurotus ostreatus, (Jacq.) Fr.

Agaricus ovalis, Fr.

See Galera ovalis, Fr.

Agaricus pallidipes, Ell. \& Ev.

See Inocybe pallidipes, Ell. \& Ev.

Agaricus pallidomarginatus, $\mathrm{Pk}$.

See Hebeloma pallidomarginatum, $\mathrm{Pk}$.

Agaricus pallidus, $P$.

See Lactarius pallidus, (P.) Fr.

Agaricus palmigena, B. \& C.

See Psilocybe palmigena, B. \& C.

Agaricus paludinellus, $\mathrm{Pk}$.

See Inocybe trechispora, (B.) Karst.

Agaricus palustris, $\mathrm{Pk}$.

See Mycena palustris, $\mathrm{Pk}$.

Agaricus panæolus, Fr.

See Tricholoma panzolum, Fr.

Agaricus pantherinus, DC.

See Amanita pantherina, (D C.) Fr.

Agaricus papilionaceus, Bull.

See Chalymotta papilionacea, (Bull.) Karst.

See Panzolus papilionaceus, (Bull.) Fr.

Agaricus papillatus, Batsch.

See Coprinus papillatus, (Batsch) $\mathrm{P}$.

Agaricus papularis, Fr.

See Naucoria papularis, Fr.

Agaricus papyraceus.

Nore. We have no means of knowing what species this is.

Dewey in Hist. Berkshire Co. 85. I 829.

Hitchcock, Cat. Pl. Mass. 644. $1833 . \quad 2$ ed. 646 (126). 1835.

Agaricus parilis, Fr.

See Clitocybe parilis, Fr.

Agaricus parvifructus, $\mathrm{Pk}$.

See Hebeloma parvifructum, $\mathrm{Pk}$. 
Agaricus parvulus, Weinm.

See Volvarla pusllla, (P.) Fr.

Agaricus pascuensis, $\mathrm{Pk}$.

See Clitopllus pascuensis, $\mathrm{Pk}$.

Agaricus pascuus, $P$.

See Nolanea pascua, (P.) Fr.

Agaricus patuloides, $\mathrm{Pk}$.

See Tricholoma patulum, Fr.

Agaricus pauperculus, $B$.

See Mycena paupercula, B.

Agaricus Peckil, Howe.

See Armlllarla aurantla, (Schæff.) Fr.

Agaricus Pecten, B. \& C.

See Crepidotus Pecten, B. \& C.

Agaricus pectinatus, S.

See Lentlmus pectinatus, (S.) Fr.

Agaricus pectinatus, B. \& C.

See Naucorla pectinata, B. \& C.

Agaricus pediades, Fr.

See Naucorla pediades, Fr.

Agaricus pelianthinus, $\mathrm{Fr}$. See Mycena pellanthina, Fr.

Agaricus pelidnus, B. \& Mont. See Leplota pelldna, B. \& Mont.

Agaricus pelliculosus, $\mathrm{S}$. See Lentinus pelliculosus, (S.) Fr.

Agaricus pellucidus, Bull. See Tubarla pelluclda, (Bull.) Fr.

Agaricus peltigerinus, $\mathrm{Pk}$. See Clitocybe peltlgerlna, Pk.

Agaricus penetrans, Fr. See Flammula penetrans, Fr.

Agaricus Pennsylvanicus, B. \& C. See Naucorla Pennsylvanlca, B. \& C.

Agaricus perbrevis, Weinm. See Inocybe perbrevls, Weinm.

Agaricus peregrinus, $\mathrm{Fr}$. See Flammula peregrina, Fr.

Agaricus perforans, Hoffm.

See Marasmlus perforans, (Hoffm.) Fr.

Agaricus periporphyrus, B. \& C.

Note. In Engler \& Prantl, Pflanzenfam. ${ }^{1} 1^{1 * *}: 267.1898$ this species credited to Peck is quoted from North America, evidently by error. The species is described by Berkeley and Curtis from the Bonin Islands. See B. \& C. Proc. Am. Acad. 4: 111.14 Dec. 1858 and Sacc. Syll. 5: 115.

Agaricus perplexus, Pk.

See Hypholoma perplexum, Pk.

Agaricus perpusillus, Fr. See Pleurotus perpusillus, Fr.

Agaricus personatus, Fr.

See Tricholoma personatum, Fr. 
Agaricus pessundatus, Fr.

See Tricholoma pessundatum, Fr.

Agaricus petaloides, S. Am. Bor. 147.

See Panus angustatus, B.

Agaricus petaloides, Bull.

See Pleurotus petaloides, (Bull.) Fr.

Agaricus petasatus, Fr.

See Pluteus petasatus, Fr.

Agaricus Phalænarum, Fr.

See Panzolus Phalznarum, Fr.

Agaricus phalloides, Fr.

See Amanita phalloides, Fr.

Agaricus phlebophorus, Ditm.

See Pluteus phlebophorus, (Ditm.) Fr.

Agaricus pholideus, $\mathrm{Fr}$.

See Cortinarius pholideus, Fr.

Agaricus pholidotus, Mont.

See Psathyra pholidota, Mont.

Agaricus phyllogenus, $\mathrm{Pk}$.

See Hypholoma phyllogenum, $\mathrm{Pk}$.

Agaricus phyllophilus, $P$.

See Clitocybe phyllophila, (P.) Fr.

Agaricus physcopodius, Mont.

See Collybia physcopodia, Mont.

Agaricus picreus, $\mathrm{P}$.

See Flammula picrea, (P.) Fr.

Agaricus pilularius, Mont.

See Collybia pilularia, Mont.

Agaricus pineus, Spreng.

See Clitocybe piniaria, (Bosc) Fr.

Agaricus piniarius, Bosc.

See Clitocybe piniaria, (Bosc) Fr.

Agaricus pinophilus, $\mathrm{Pk}$.

See Clitocybe pinophila, $\mathrm{Pk}$.

Agaricus pinsitus, $\mathrm{Fr}$.

See Pleurotus pinsitus, Fr.

Agaricus Pinus, Frost.

See Clitocybe clavipes, (P.) Fr.

Agaricus piperatus, Scop.

See Lactarius piperatus, (Scop.) Fr.

Agaricus pirinus, B. \& C.

See Eccilia pirina, B. \& C.

Agaricus piriodorus, $\mathrm{P}$.

See Inocybe piriodora, (P.) Fr.

Agaricus pithyophilus, $\mathrm{Fr}$.

See Clitocybe pithyophila, Fr.

Agaricus Placenta, Batsch.

See Entoloma Placenta, (Batsch) Fr.

Agaricus placomyces, $\mathrm{Pk}$.

Nore. This species is nearly related to Agaricus silvaticus, Schreff. of which it is considered by some to be merely a variety. 
Pk. Rept. N. Y. Mus. $29: 40$. d. 1878.

Pk. Rept. N. Y. Mus. $36: 48 . d$. 1885 .

Sacc. Cub. \& Manc. Syll. 5: 1003. d. 28 May 1887 .

Fairman, Proc. Rochester Acad. 2: 159. Feb. 1893.

Pk. Country Gent. 59: $518(31) d$. f. 12 July 1894 .

Pk. Rept. N. Y. Mus. 48: 240 (142). pl. 9. f. 7-12. March 1897.

Atk. Bull. Cornell Univ. 3: 25. 25 June 1897.

Underw. \& Earle, Bull. Ala. Exp. Sta. 80:253. 28 June 1897.

[Underw.] N. Y. Myc. Club i6. 1898.

Smith, C. O., Rhodora 1: $163 . d$. I Sept. I 899.

Underw. Moulds I22. 1899.

Atk. in Bailey's Cycl. Am. Hort. 1043. 1900.

Atk. Mushrooms 23. d. $p l .4, f .21,22, p l .5, f .23 .20$ Oct. 1900.

Mcllvaine \& Macadam, Am. Fung. 345. d. pl. $91, f .3 .1900$.

Sweetser, Bull. Pacific Univ. 14: 12. May 1901.

Earle in Mohr, Contr. U. S. Nat. Herb. 6 : 224. 31 July 1901.

Ferguson, Bull. U. S. Agr. Pl. Industry 16: $5,17.14$ June 1902. Psalliota placomyces, $\mathrm{Pk}$.

Henn. in Engler \& Prantl, Pflanzenfam. 11** $: 240 . \quad 1898$.

Lloyd, Myc. Notes 27. d. Nov. 1899.

Agaricus planus, $P$. See Pleurotus planus, (P.) Fr.

Agaricus platyphyllus, $P$. See Collybia platyphylla, (P.) Fr.

Agaricus plexipes, Fr. See Collybla plexIpes, Fr.

Agaricus plicatellus, $\mathrm{Pk}$. See Galera coprlnoldes, Pk.

Agaricus plicatilis, Curt. See Coprlnus plicatills, (Curt.) Fr.

Agaricus plumatus, Walt. See Lentinus plumatus, (Walt.) Fr.

Agaricus plumbeus, Schæff. See Amanitopsls vaginata, (Bull.) Roze.

Agaricus plumiger, B. \& C. See Psathyra plumigera, B. \& C.

Agaricus Pluteus, $P$. See Pluteus cervinus, (Schæff.) Fr.

Agaricus Plutonius, B. \& C. See Psilocybe Plutonius, B. \& C.

Agaricus Poculum, Pk. See Clitocybe Poculum, Pk.

Agaricus polyehrous, $B$. See Flammula polychroa, B.

Agaricus polychrous, Pk. Rept. See Flammula spumosa, Fr.

Agaricus polygrammus, Bull. See Mycena polygramma, (Bull.) P.

Agaricus polymyees, $P$. See Armillarla mellea, (Vahl.) Fr.

Agaricus poly pyramis, B. \& C. See Amanita solltarla, (Bull.) Fr. 
Agaricus polystictus, B.

See Lepiota polysticta, B.

Agaricus polytrichophilus, $\mathrm{Pk}$.

See Psathyra polytrichophila, Pk.

Agaricus pometi, Paul.

See Pleurotus pometi, (Paul.) Fr.

Agaricus ponderosus, $\mathrm{Pk}$.

See Armillaria ponderosa, $\mathrm{Pk}$.

Agarieus popinalis, Fr.

See Clitopilus popinalis, Fr.

Agaricus porcellanus, Schæff.

See Coprinus comatus, (FI. Dan.) P.

Agaricus porphyrellus, B. \& C.

See Clitocybe porphyrella, B. \& C.

Agaricus porrigens, $P$.

See Pleurotus porrigens, (P.) Fr.

Agarieus portentosus, $\mathrm{Fr}$.

See Tricholoma portentosum, Fr.

Agaricus præceps, B. \& C.

See Collybia praceps, B. \& C.

Agaricus præcox, $\mathrm{P}$.

See Pholiota przecox, (P.) Fr.

Agarieus præfoliatus, $\mathrm{Pk}$.

See Collybia platyphylla, (P.) Fr.

Agaricus prælongus, $\mathrm{Pk}$.

See Mycena polygramma, (Bull.) P.

Agaricus prætorius, Fr. p. p.

See Amanita cæsarea, (Scop.) P.

Agaricus pratensis, Schæff.

S. Syn. Car. 81 (55) no. 605. 1822.

S. Syn. Am. Bor. 147 no. 77.1834.

Hark. \& Moore, Cat. Pac. Fung. 7. Feb. 1880.

Webber, Rept. Nebr. Agr. 1889: 229 (89). 1890.

Mcllvaine \& Macadam, Am. Fung. 340. d. 1900.

Agaricus procerus, Scop.

See Lepiota procera, (Scop.) P.

Agaricus proliferus, Sow.

See Mycena prolifera, (Sow.) Fr.

Agaricus pronus, Fr.

See Psathyrella prona, Fr.

Agaricus pruinatipes, Pk.

See Naucoria pruinatipes, Pk.

Agaricus pruinosus, Lasch.

See Clitocybe pruinosa, (Lasch) Fr.

Agaricus prunuloides, Fr.

See Entoloma prunuloides, Fr.

Agaricus Prunulus, Scop.

See Clitopilus Prunulus, (Scop.) Fr.

Agaricus Prunulus, var. Oreella, Auct. Amer.

See Clitopilus Orcella, (Bull.) Fr.

Agaricus pseudotener, Fr.

See Psathyra pseudotenera, $\mathrm{Fr}$. 
Agaricus psilopus, Rav.

See Lepiota psilopus, Rav.

Agaricus psittacinus, Schæff.

See Hygrophorus psittacinus, (Schæff.) Fr.

Agaricus pterigenus, Fr.

See Mycena pterigena, Fr.

Agaricus pubescens, S.

See Amanitopsis pubescens, (S.) Sacc.

Agaricus pubescentipes, $\mathrm{Pk}$.

See Voivaria pubescentipes, $\mathrm{Pk}$.

Agaricus pulcher, $P$.

See Coprinus pilicatilis, (Curt.) Fr.

Agaricus pulcherrimus, $\mathrm{Pk}$.

See Mycena puicherrima, Pk.

Agaricus pulicosus, Mont.

See Psilocybe pulicosa, Mont.

Agaricus pulvinatus, $\mathrm{P}$.

See Pieurotus puivinatus, (P.) Fr.

Agaricus pumilus, $P$.

See Phoilota pumila, (P.) Fr.

Agaricus punctatus, Fr.

See Hebeloma punctatum, Fr.

Agaricus purpurascens, A. \& S.

See Hygrophorus purpurascens, (A. \& S.) Fr.

Agaricus purpureofuscus, $\mathrm{Pk}$.

See Mycena purpureofusca, $\mathrm{Pk}$.

Agaricus purus, $P$.

See Mycena pura, (P.) Fr.

Agaricus pusillomyces, $\mathrm{Pk}$.

See Lepiota pusiliomyces, $\mathrm{Pk}$.

Agaricus pusillus, (P.) $\mathrm{Fr}$.

See Voivaria pusilia, (P.) Fr.

Agaricus pusillus, Fr. non $\mathrm{P}$.

See Agaricus pusiolus, Fr.

Agarleus pusillus, $\mathrm{Pk}$.

Note. This species is assigned to Agaricus proper. It should be borne in mind that there is also Agaricus (Volvaria) pusillus, (P.) Fr. S. M. 1: 279 found in America which is here placed under Volvaria pusilla, (P.) Fr.

Pk. Rept. N. Y. Mus. 54 ${ }^{1}$ : 152. d. Dec. I90I.

Longyear, Rept. Mich. Acad. 3: 60. 1902. and 4: 115. 1904.

Conant, Bull. Boston Myc. Club 19: 5 Feb. 1903.

Agaricus pusiolus, Fr.

See Naucoria pusiola, Fr.

Agaricus putredinis, B. \& C.

See Pleurotus putredinis, B. \& C.

Agaricus putrigenus, $\mathrm{Pk}$.

See Crepidotus putrigenus, $\mathrm{Pk}$.

Agaricus pyrrholepis, Mont.

See Hebeioma pyrrholepidum, Mont.

Agaricus pyrrhus, B. \& C.

See Crepidotus pyrrhus, B. \& C. 
Agaricus pyxidatus, Bull.

See Omphalia pyxidata, (Bull.) P.

Agaricus quadratus, B. \& C.

See Nolanea quadrata, B. \& C.

Agaricus quercinus, L.

See Drdalea quercina, (L.) P.

Agaricus racemosus, $\mathrm{P}$.

See Collybia racemosa, (P.) Fr.

Agaricus radiatus, Bolt.

See Coprinus radiatus, (Bolt.) P.

Agaricus radicatellus, $\mathrm{Pk}$.

See Mycena radicatella, Pk.

Agaricus radicatus, Relh.

See Collybia radicata, (Relh.) Fr.

Agaricus radicosus, Bull.

See Pholiota radicosa, (Bull.) Fr.

Agaricus radiozonarius, Johnson.

See Clitocybe radiozonaria, Johnson.

Agaricus Radius, Johnson.

See Mycena Radius, Johnson.

Agaricus ramealis, Bull.

See Marasmius ramealis, (Bull.) Fr.

Agaricus ramentaceus, Bull.

- See Armillaria ramentacea, (Bull.) Fr.

Agaricus ramosus, Bull.

See Collybia ramosa, (Bull.) Fr.

Agaricus Ravenelii, B. \& C.

See Amanita Ravenelii, B. \& C.

Agaricus recutitus, Fr.

See Amanita recutita, Fr.

Agaricus reflexus, Muhl.

Note. This is probably Pholiota squarrosa, (Muell.) Fr.

Muhl. Cat. Pl. Am. Sept. I ed. 104. 1813. 2 ed. 107. 1818.

Agaricus repandus, Bull.

See Entoloma repandum, (Bull.) Fr.

Agaricus resplendens, $\mathrm{Fr}$.

See Tricholoma resplendens, Fr.

Agaricus reticeps, Mont.

See Clitocybe reticeps, Mont.

Agaricus reticulatus, Johnson.

See Tricholoma reticulatum, Johnson.

Agaricus retirugis, Fr.

See Panzolus retirugis, Fr.

Agaricus rhacodes, Vitt.

See Lepiota rhacodes, (Vitt.) Fr.

Agaricus rhacodes, Pk. 22 Rept.

See Lepiota Americana, Pk.

Agaricus Rhacodium, B. \& C.

See Pleurotus Rhacodium, B. \& C.

Agaricus rhagadiosus, Fr.

See Armillaria rhagadiosa, Fr. 
Agaricus rheicolor, B.

See Collybia rhelcolor, B.

Agaricus rhinarius, B. \& C.

See Tricholoma rhinarium, B. \& C.

Agaricus rhodocylix, Lasch.

See Eccilia rhodocyilx, (Lasch) Fr.

Agaricus Rhododendri, Pk.

See Omphaila Rhododendri, Pk.

Agaricus rhodophaeus, Mont. See Psilocybe rhodophaea, Mont.

Agaricus rhodopolius, Fr. See Entoloma rhodopolium, Fr.

Agaricus rhodoxanthus, $\mathrm{S}$. See Paxillus rhodoxanthus, (S.) Pk.

Agaricus rhyssosporus, Mont. See Omphaila rhyssospora, Mont.

Agarieus rhytopilus, Mont.

Massee, Jour. of Bot. 30: 196. July 1892.

West Indies.

Agaricus Ricensis, Fr. See Flammula RIcensis, Fr.

Agaricus rigens, S. Am. Bor. no. 157. Misprint for Agaricus rlngens.

Agaricus rimosus, Bull. See Inocybe rimosa, (Bull.) Fr.

Agaricus ringens, var. tenuissimus, Fr. See Lentlnus tenuissimus, (S.) Fr.

Agaricus rivulosus, $P$. See Ciitocybe rivuiosa, (P.) Fr.

Agaricus Robinsonil, B. \& Mont. See Entoioma Robinsonil, B. \& Mont.

Agaricus robustus, A. \& S. See Armlliaria robusta, (A. \& S.) Fr.

Agaricus Rodmani, Pk.

Pk. Rept. N. Y. Mus. $36: 45 . d$. I 885.

Macoun, Ottawa Nat. 2 : 142. Feb. 1889.

Forster, Boston Med. \& Surg. Jour. 123: 454 (I). 6 Nuv. 1890.

Pk. Country Gent. 59: 5 I8 (28) f. I2 July 1894.

Millsp. \& Nutt. Publ. Field Columb. Mus. Bot. 1 : I6c. Jan. 1896.

Pk. Rept. N. Y. Mus. 48: 235 (I 37) pl. 9, f. I-6. March 1897.

[Underw.] N. Y. Myc. Club I6. I898.

Smith, C. O., Rhodora 1: 161. d. I Sept. 1899.

Longyear, Rept. Mich. Bd. Agr. 1898: 92. 1899.

Underw. Moulds 122. 1899.

Atk. in Bailey's Cycl. Am. Hort. 1043. 1900.

Atk. Mushrooms 20 d. $f .17 .20$ Oct. 1900.

Mcllvaine \& Macadam, Am. Fung. 336. d. 1900.

Kellerm. Ohio Myc. Bull. 1 : 22. f. 28. 24 June 1903: Psaliiota Rodmani, Pk.

Taylor, Food Products 2: 21. 1893.

Lloyd, Myc. Notes 29. Nov. 1899.

Agaricus roridulus, B. \& C.

See Mycena roriduia, B. \& C. 
Agaricus roridus, Fr.

See Mycena rorida, Fr.

Agaricus rosaceus, $P$.

See Russula rosacea (P.) Fr.

Agaricus rosaceus, S. Syn. Car. 746.

See Russula rubra, Fr.

Agaricus rosellus, Fr.

See Mycena rosella, Fr.

Agaricus roseoalbus, Fr.

See Pluteus roseoalbus, Fr.

Agaricus roseocandidus, $\mathrm{Pk}$.

See Mycena roseocandida, $\mathrm{Pk}$.

Agaricus roseus, Bull.

See Mycena pura, (P.) Fr.

Agaricus Rotula, Scop.

See Marasmius Rotula, (Scop.) Fr.

Agaricus ruber, S. Syn. Car. 738.

See Lactarius helvus, Fr.

Agaricus ruber, Fr.

See Russula rubra, Fr.

Agaricus rubescens, Fr.

See Amanita rubescens, P.

Agaricus rubescentifolius, $\mathrm{Pk}$.

See Collybia colorea, Pk., var. rubescentifolia, Pk.

Agaricus Rubi, B.

See Crepidotus Rubi, B.

Agaricus rubicundus, $\mathrm{Pk}$.

See Tricholoma Russula, (Schæff.) Fr.

Agaricus rubiginosus, $\mathrm{P}$.

See Galera rubiginosa, (P.) Fr.

Agaricus rubromarginatus, $\mathrm{Fr}$.

See Mycena rubromarginata, ${ }^{\circ} \mathrm{Fr}$.

Agaricus rubrotinetus, $\mathrm{Pk}$.

See Lepiota rubrotincta, Pk.

Agarieus rubrotinctus, B. \& C.

See Clitocybe rubrotincta, B. \& C.

Agaricus rufocarneus, B.

See Nolanea rufocarnea, B.

Agaricus rufo-olivaceus, $\mathrm{Fr}$.

See Cortinarius rufo=olivaceus, Fr.

Agaricus rufo-violaceus.

? Error for Agaricus rufo-olivaceus, $\mathrm{Fr}$.

Agaricus rufulus, B. \& Br.

See Omphalia demissa, Fr.

Agaricus rugosodiscus, Pk.

See Omphalia rugosodisca, $\mathrm{Pk}$.

See Mycena rugosodisca, (Pk.) Sacc. Syll.

See Collybia rugosodisca, $\mathrm{Pk}$.

Agaricus rugosus, Fr.

See Mycena rugosa, $\mathrm{Fr}$.

Agaricus rugulosus, S. 
S. Syn. Car. 83 (57) no. 653. d. 1822.

Agaricus rupincola, Mont.

See Psathyrella ruplncola, Mont.

Agaricus Russula, Scheff.

See Tricholoma Russula, (Schæff.) Fr.

Agaricus russuloides, Pk.

See Amanita russuloides, Pli.

Agaricus rusticus, Fr.

See Omphalia rustica, Fr.

Agaricus rutilans, Schæeff.

See Trlcholoma rutllans, (Schæff.) Fr.

Agaricus rutilescens, Pk.

Pk. Bull. Torr. Bot. Club 31 : 179. d. 28 April 1904.

Agaricus sabuletorum, B. \& C.

See Inocybe lanuginosa, (Bull.) Fr.

Agaricus sacchariferus, B. \& Br.

See Mycena saccharifera, B. \& Br.

Agaricus saccharinophilus, $\mathrm{Pk}$.

See Hypholoma saccharinophllum, Pk.

Agaricus salignus, $\mathrm{P}$.

See Pleurotus sallgnus, (P.) Fr.

Agaricus Sallei, B.

B. Ann. Nat. Hist. II. 9 : 193. d. March 1852. West Indies.

Sacc. Cub. \& Manc. Syll. 5: 995. d. 28 May 1887.

Agaricus salmoneus, Pk.

See Entoloma salmoneum, Pk.

Agaricus sambucinus, Fr.

See Inocybe sambucina, Fr.

Agaricus sandicinus, Fr.

See Clitocybe sandiclna, Fr.

Agaricus sanguineus, Wulf.

See Cortinarlus sanguineus, (Wulf.) Fr.

Agaricus sanguineus, B. \& C.

See Omphalla sangulnea, B. \& C.

Agaricus sanguinolentus, A. \& S.

See Mycena sanguinolenta, A. \& S.

Agaricus sapidus, Kalchbr.

See Pleurotus sapidus, Kalchbr.

Agaricus sapineus, Fr.

See Flammula saplnea, Fr.

Agaricus saponaceus, Fr.

See Tricholoma saponaceum, Fr.

Agaricus sarcophyllus, $\mathrm{Pk}$.

See Hebeloma sarcophyllum, Pk.

Agaricus scabellus, A. \& S.

See Inocybe scabella, (A. \& S.) Fr.

Agaricus scabrinellus, $\mathrm{Pk}$.

See Entoloma scabrinellum, $\mathrm{Pk}$.

Agaricus seabriusculus, Pk.

See Omphalla umbellifera, (L.) Fr., var. scabriuscula, Pk.

Agaricus scabrosus, Fr.

See Leptonia scabrosa, Fr. 
Agaricus scalpturatus, Fr.

See Tricholoma scalpturatum, Fr.

Agaricus seatigena, B. \& C.

See Psilocybe scatigena, B. \& C.

Agaricus scaurus, Fr.

See Cortinarius scaurus, Fr.

Agaricus Schumacheri, Pk. 24 Rept.

See Clitocybe nebularis, (Batsch) Fr.

Agaricus scobifer, B. \& C.

See Pholiota scobifera, B. \&,C.

Agaricus scorodonius, Fr.

See Marasmius scorodonius, Fr.

Agaricus scorpioides, Fr.

See Naucoria scorpioides, Fr.

Agaricus seyphoides, Fr.

See Omphalia scyphoides, Fr.

Agaricus sejunetus, Sow.

See Tricholoma sejunctum, (Sow.) Fr.

Agaricus semicaptus, B. \& C.

See Pleurotus semicaptus, B. \& C.

Agaricus semiglobatus, Batsch.

See Stropharia semiglobata, (Batsch) Fr.

Agaricus semihærens, B. \& C.

See Collybia semihzerens, B. \& C.

Agaricus semilanceatus, Fr.

See Psilocybe semilanceata, Fr.

Agaricus semiorbicularis, Bull.

See Naucoria semiorbicularis, (Bull.). Fr.

Agaricus semiovatus, Sow.

See Anellaria separata, (L.) Karst.

Agarieus semitectus, B. \& C.

See Pleurotus semitectus, B. \& C.

Agaricus semivestitus, B. \& Br.

See Psathyra semivestita, B. \& Br.

Agaricus separatus, L.

See Anellaria separata, (L.) Karst.

Agaricus sepiarius, Wulf.

See Lenzites sepiaria, (Wulf.) Fr.

Agaricus septicus, Fr.

See Pleurotus septicus, Fr.

Agaricus sericellus, Fr.

See Entoloma sericellum, Fr.

Agaricus sericeus, S. Syn. Car. 665.

See Entoloma sericellum, Fr.

Agaricus sericeus, Bull.

See Entoloma sericeum, (Bull.) Fr.

Agaricus serotinoides, Pk.

See Pleurotus petaloides, (Bull.) Fr.

Agaricus serotinus, Schrad.

See Pleurotus serotinus, (Schrad.) Fr.

Agaricus serotínus, Pk. in Day's Cat.

See Pleurotus petaloides, (Bull.) Fr. 
Agaricus'serrulatus, Fr.

See Leptonia serrulata, Fr.

Agaricus (Pleurotus) sestonoides, Cke.

NotE. Known to us only at the place cited below.

Hark. \& Moore, Cat. Pac. Fung. 4. Feb. I880.

Agariéus setipes, Fr.

NorE. The only reference is the one given below which is probably referable to Omphalia setipes, $\mathrm{Fr}$.

Curtis, M. A., Bot. N. Car. 87. $\quad$ I86\%.

Agaricus setisedus, $\mathrm{S}$.

See Clitocybe setiseda, (S.) Fr.

Agaricus setosus, (Sow.)

See Mycena setosa, (Sow.) Fr.

Agaricus Seymourianus, Pk.

See Clitopilus Seymourianus, Pk.

Agaricus siceus, $\mathrm{S}$.

See Marasmius siccus, (S.) Fr.

Agaricus sideroides, Bull. See Naucoria sideroides, (Bull.) Fr.

Agaricus Sienna, Pk.

See Tricholoma Sienna, Pk.

Agaricus silaceus, S. Syn. Car. 726.

See Flammula spumosa, Fr.

Agaricus siligineus, Fr.

See Galera siliginea, Fr.

Agaricus silvaticus, Schæff.

Note. See note under Agaricus placomyces, $\mathrm{Pk}$.

Lea, Cat. Pl. Cin. 53. $\quad$ I 849.

M. A. Curtis, Bot. N. Car. 90. I867.

Cke. Handbook i38. d. I87I.

Pk. Rept. N.Y. Mus. 26 : 59. April 1874.

Cke. \& B. Fungi 89. 1875 .

Farl. Bull. Bussey Inst. 1: 436 . March 1876 .

Pk. Rept. N. Y. Mus. 28: 85. 1876 .

Johnson, Bull. Minn. Acad. 1 : 235. 1877 and Rept. Minn. Surv. 5 : 72. 1877 .

James, Jour. Cin. Soc. $2: 64$ (23). April 1879.

Hark. \& Moore, Cat. Pac. Fung. 7. Feb. 1880.

Morg. Jour. Cin. Soc. 6: 112 . $d$. July 1883.

Bundy, Geol. Wis. 1873-79, 1: 397.1883.

Pk. in Day's Cat. Bull. Buf. Soc. 4: 179 (115). 1883.

Pk. Rept. N. Y. Mus. $36: 48$. d. I 885.

Sacc. Cub. \& Manc. Syll. 5 : 1000. d. 28 May 1887.

Macbride, Bull. Univ. lowa 1: I88. $d$. June 1890 .

Banning in Pk. Rept. N. Y. Mus. 44: $182(70)$. Dec. I891.

Pk. Country Gent. 59: 518 (32). 12 July 1894.

Underw. Proc. Ind. Acad. 1893: 6r. Aug. I 894.

Kellerm. \& Werner, Geol. Ohio $7^{2}$ : 315. Jan. I895.

Millsp. \& Nutt. Publ. Field Columb. Mus. Bot. 1 : I60. . Jan. 1896.

McClatchie, Proc. S. Calif. Acad. 1: 382.5 June 1897.

Atk. Bull. Cornell Univ. 3 : 25. 25 June 1897.

Pk. Bull. N. Y. Mus. 6 : 213 . July 1899. 
Smith, C. O., Rhodora 1: 163. I Sept. I899.

Underw. Moulds I22. 1899.

Herbst, Fungal Fl. Lehigh Valley 73. d. I899.

Atk. in Bailey's Cycl. Am. Hort. 1043. I900.

Mcllvaine \& Macadam, Am. Fung. 334. d. I900.

Nelson, Rept. Wyo. Exp. Sta. 10: (38). I I 900.

Pound \& Clements, Bot. Surv. Nebr. $5: 25$. 30 March I90I.

Sweetser, Bull. Pacific Univ. 14: 12. May 1901.

Earle in Mohr, Contr. U. S. Nat. Herb. $6: 224$. 3I July rgor.

Williamson, Proc. Ind. Acad. 1900: 165. I901.

Psalliota silvatica, Fr.

Henn. in Engler \& Prantl, Pflanzenfam. 11**: 239. d. 1898.

Lloyd, Myc. Notes 27. $d$. Nov. I899.

Perry, Agricultural Student 6: 179. May 1900. and Ohio Univ. Bull. IV. $30: 7$. May Igoo.

Hyams, Bull. N. Car. Exp. Sta. $177: 45$. d. Dec. 1900.

Agaricus silvicola, Vitt.

Note. See Agaricus abruptus, Pk. to which some of the following references may belong rather than to Agaricus silvicola, Vitt.

Millsp. \& Nutt. Publ. Field Columb. Mus. Bot. 1 : 160. Jan. 1896.

Webster, Am. Kitchen Mag. 6:217. Feb. 1897 and Taylor, Hdbk. Mushrooms 2: 24. 1897.

Smith, C. O., Rhodora 1: I63. I Sept. 1899.

Atk. Mushrooms $22 d$. $f .19,20.20$ Oct. 1900.

Pk. Mem. N. Y. Mus. 3 : 164. d. pl. 59, $f .1-7$. Nov. 1900.

Mcllvaine \& Macadam, Am. Fung. 343. d. pl. gr, f. 2. Igco.

Atk. in Bailey's Cycl. Am. Hort. 1043. $d$. 1900.

Hay, Bull. N. H. Soc. N. B. 4: 343 . I90I and Bull. N. H. Soc. N. B. 5 : 110.1903.

Brown \& Fernekes, Bull. Wis. Nat. Hist. Soc. 11. 2. 46. Jan. I902.

Conant, Bull. Boston Myc. Club i9: 5 Feb. 1903.

Psalliota silvicola, Fr.

Taylor, Food Products 2: 21. 1893.

Lloyd, Myc. Notes 28. $d$. Nov. 1899 .

Perry, Agricultural Student 6: I79. May 1900 and Ohio Univ. Bull. IV. 30: 7. May 1900.

Hyams, Bull. N. Car. Exp. Sta. 177 : 45. d. Dec. 1900.

Agaricus silvieola, Pk. non Vitt.

See Agaricus abruptus, Pk.

Agaricus silvicola, var. abruptus, Conant.

See Agaricus abruptus, Pk.

Agaricus simillimus, $\mathrm{Pk}$.

See Collybia acervata, Fr.

Agaricus sinapizans, Fr.

See Hebeloma sinapizans, Fr.

Agaricus sinopicus, Fr.

See Clitocybe sinopica, Fr.

Agaricus sinuatus, Bull.

See Entoloma sinuatum, (Bull.) Fr.

Agarieus siparioides, B. \& C.

See Naucoria siparioides, B. \& C.

Agaricus soleatus, Howe.

See Amanitopsis volvatus, ( $\mathrm{Pk}$.) Sacc.

Agaricus solidipes, $\mathrm{Pk}$.

See Panzolus solidipes, $\mathrm{Pk}$. 
Agaricus solidipes, $\mathrm{Pk}$.

Pk. Bull. Torr. Bot. Club 31 : 179. d. 28 April 1904.

Agarieus solitarius, Bull.

See Amanita solitaria, (Bull.) Fr.

See also Amanita strobiliformis, (Vitt.) Fr.

Agaricus sordarius, $\mathrm{P}$.

Note. Agaricus sordarius, P. is referred by authors to Clitocybe obtexta, (Lasch) Fr. but we do not know whether the citation below relates to the same or not.

S. Syn. Car. 84 (58) no. 668.1822.

Agaricus sordescens, B. \& C.

See Lepiota sordescens, B. \& C.

Agaricus sordidulus, Pk.

See Hebeloma sordidulum, $\mathrm{Pk}$.

Agaricus sordidus, Schum.

See Tricholoma sordidum, (Schum.) Fr.

Agaricus spadiceus, Schæff.

See Psilocybe spadicea, (Schæff.) Fr.

Agaricus sparteus, Fr.

See Galera spartea, Fr.

Agaricus spathulatus, S. Syn. Car. 807.

See Panus angustatus, B.

Agaricus spathulatus, $\mathrm{P}$.

See Pleurotus petaloides, (Bull.) Fr., var, spathulatus, (P.) Fr.

Agaricus speciosus, Fr.

See Volvaria speciosa, Fr.

Agaricus spectabilis, Fr.

See Pholiota spectabilis, Fr.

Agaricus speireus, Fr.

See Mycena speirea, Fr.

Agaricus spermaticus, (Paul.) Fr.

See Tricholoma spermaticum, (Paul.) Fr.

Agaricus sphærosporus, B.

See Omphalia spharospora, B.

Agaricus sphærosporus, $\mathrm{Pk}$.

Pk. Bull. Torr. Bot. Club. 31 : 181. d. 28 April 1904.

Agaricus Sphagnorum, P.

See Galera Sphagnorum, (P.) Fr.

Agaricus spinulifer, $\mathrm{Pk}$.

See Marasmius cohzrens, (P.) Cke.

Agaricus spissus, Fr.

See Amanita spissa, Fr.

Agaricus Spraguei, B. \& C.

See Collybia Spraguei, B. \& C.

Agaricus spretus, Pk.

See Amanita spreta, Pk.

Agaricus spumosus, $\mathrm{Fr}$.

.See Flammula spumosa, Fr

Agaricus squalens, Fr.

See Psilocybe squalens, Fr. 
Agarieus squalidellus, $\mathrm{Pk}$.

See Psilocybe squalidella, Pk.

Agaricus squamosus, $P$.

See Stropharia squamosa, (P.) Fr.

Agaricus squarrosoides, $\mathrm{Pk}$.

See Pholiota squarrosoides, Pk.

Agaricus squarrosus, Muell.

See Pholiota squarrosa, (Muell.) Fr.

Agaricus stellatosporus, $\mathrm{Pk}$.

See Inocybe stellatospora, (Pk.) Earle.

Agaricus stellatus, Fr.

See Omphalia stellata, Fr.

Agarieus stereorarius, Schum.

See Stropharia stercoraria, (Schum.) Fr.

Agarieus stereocephalus, B. \& C.

See Collybia stereocephala, B. \& C.

Agarieus sterilomarginatus, $\mathrm{Pk}$.

See Pluteus sterilomarginatus, $\mathrm{Pk}$.

Agaricus Sterlingii, Pk.

Pk. Bull. Torr. Bot. Club 29 : 73. d. 25 Feb. 1902.

Agaricus sterquilinus, $\mathrm{Fr}$.

See Coprinus sterquilinus, Fr.

Agarieus stipatus, Auct.

Note. It is impossible to say to what species the following references belong but they probably belong to some species of Hypholoma and perhaps to Hypholoma appendiculatum, (Bull.) Fr.

S. Syn. Car. 86-(60) no. 727. I822.

S. Syn. Am. Bor. I5I no. 247. 1834.

Lea, Cat. PI. Cin. 53. 1849.

James, Jour. Cin. Soc. $2: 64$ (23). April 1879.

Agarieus stipitarius, Fr.

See Collybia stipitaria, Fr.

Agaricus stipticus, Bull.

See Panus stipticus, (Bull.) Fr.

Agaricus stipularis, Fr.

See Mycena stipularis, Fr.

Agaricus strangulatus, Fr.

See Amanitopsis strangulata, (Fr.) Roze.

Agarieus striatellus, Batsch.

See Psathyrella disseminata, (P.) Fr.

Agaricus striatifolius, $\mathrm{Pk}$.

See Tricholoma striatifolium, $\mathrm{Pk}$.

Agarieus striatulus, $P$.

See Pleurotus striatulus, (P.) Fr.

Agaricus striatus, Swartz.

See Lenzites striata, (Swartz) Fr.

Agaricus strietior, $\mathrm{Pk}$.

See Entoloma strictius, $\mathrm{Pk}$. 
Agaricus strigosus, S.

See Lentinus Lecomtei, Fr.

See Lentinus strigosus, (S.) Fr.

Agaricus stroblliformis, Vitt.

See Amanita strobiliformis, (Vitt.) Fr.

Agaricus strobilinus, P.

See Mycena strobilind, $\mathrm{P}$.

Agarleus strombodes, B. \& Mont.

See Omphalia strombodes, B. \& Mont.

Agaricus stupparius, B. \& C.

See Collybia stupparia, B. \& C.

Agaricus stylobates, P.

See Mycena stylobates, $\mathrm{P}$.

Agarleus subareolatus, Pk.

See Pleurotus subareolatus, Pk.

Agaricus subatratus, Fr.

See Psathyrella subatrata, Fr.

Agaricus subbarbatus, B. \& C.

See Pleurotus subbarbatus, B. \& C.

Agaricus subeæruleus, Pk.

See Mycena subcærulea, Pk.

Agaricus subelypeolarius, B. \& C.

See Lepiota subclypeolaria, B. \& C.

Agaricus subdecurrens, Ell. \& Ev.

See Inocybe subdecurrens, Ell. \& Ev.

Agaricus subduleis, Bull.

See Lactarius subdulcis, (Bull.) Fr.

Agaricus subexilis, $\mathrm{Pk}$.

See Inocybe subexilis, Pk.

Agaricus subglobosus, A. \& S.

See Naucoria subglobosa, (A. \& S.) Fr.

Agaricus subhirtus, $\mathrm{Pk}$.

See Clitocybe subhirta, Pk.

Agarieus subinearnatus, $\mathrm{Pk}$.

See Mycena subincarnata, Pk.

Agaricus subinvolutus, Batsch.

See Clitocybe subinvoluta, (Batsch) Fr.

Agaricus sublateritius, Fr.

See Hypholoma sublateritium, Fr.

Agaricus subochraceus, $\mathrm{Pk}$.

See Inocybe subochracea, Pk.

Agaricus subpalmatus, Fr.

See Pleurotus subpalmatus, $\mathrm{Fr}$.

Agaricus subpellueidus, B. \& C.

See Omphalia subpellucida, B. \& C.

Agaricus subremotus, B. \& C.

See Lepiota subremota, B. \& C.

Agaricus subrufescens, Pk.

See Agaricus fabaceus, B.

Agaricus subrufeseens, Ell. \& Ev.

See Tricholoma subrufescens, Ell. \& Ev. 
Agaricus subtortus, $\mathrm{P}$.

See Cortinarius subtortus, (P.) Fr.

Agaricus subversus, Schum.

See Pleurotus perpusillus, Fr.

Agaricus subviridis, B. \& C.

See Psilocybe subviridis, B. \& C.

Agaricus subzonalis, $\mathrm{Pk}$.

See Clitocybe subzonalis, $\mathrm{Pk}$.

Agarieus succosus, $\mathrm{Pk}$.

See Mycena succosa, (Pk.) Sacc. Syll.

Agaricus sudus, Fr.

See Tricholoma sudus, Fr.

Agaricus suleatipes, $\mathrm{Pk}$.

See Galera sulcatipes, Pk.

Agaricus suleatus, L.

See Mycena galericulata, (Scop.) Fr.

Agaricus Sullivantii, Mont.

See Psilocybe Sullivantii, Mont.

Agaricus sulphureoides, $\mathrm{Pk}$.

See Pleurotus sulphureoides, Pk.

Agaricus sulphureus, Bull.

See Tricholoma sulphureum, (Bull.) Fr.

Agaricus tabularis, $\mathrm{Pk}$.

Pk. Bull. Torr. Bot. Club 25 : 325. $d$. 8 June 1898.

Mcllvaine \& Macadam, Am. Fung. 347. d. I900.

Sacc. \& Syd. Syll. 16 : 1 I4. d. I Feb. 1902. Psalliota tabularis, Lloyd.

Lloyd, Myc. Notes 30. Nov. I899.

Agaricus Tagetes, B. \& Mont.

See Omphalia Tagetes, B. \& Mont.

See Collybia Tagetes, (B. \& Mont.) Sacc.

Agaricus tardus, S. Syn. Car. 782.

See Clitocybe cyathiformis, (Bull.) Fr.

Agaricus Taylori, B.

See Volvaria Taylori, B.

Agaricus temnophyllus, $\mathrm{Pk}$.

See Pholiota temnophylla, Pk.

Agaricus tenacellus; $P$.

See Collybia tenacella, (P.) Fr.

Agaricus tener, Schæff.

See Galera tenera, (Schæff.) Fr.

Agaricus teneroides, $\mathrm{Pk}$.

See Galera teneroides, Pk.

Agaricus tenerrimus, $\mathrm{B}$.

See Mycena tenerrima, B.

Agaricus tenuipes, S.

See Collybia tenuipes, (S.) Sacc. Syll.

Agaricus tenuis, Bolt.

See Mycena tenuis, (Bolt.) Fr.

Agaricus tenuissimus, $\mathrm{S}$.

See Lentinus tenuissimus, (S.) Fr. 
Agaricus tephroleueus, $\mathrm{P}$.

See Hygrophorus tephroleucus, (P.) Fr.

Agaricus tephromelas, $\mathrm{P}$.

See Pleurotus tremulus, (Schæff.) Fr.

Agaricus tephrostictus, B. \& C.

See Pluteus tephrostictus, B. \& C.

Agaricus terræolens, Pk.

See Tricholoma terraolens, Pk.

Agaricus terreus, Schæff.

See Tricholoma terreum, (Schæff.) Fr.

Agaricus tessellatus, $\mathrm{P}$.

See Pleurotus tessellatus, (P.) Fr.

Agaricus testaceus, A. \& S.

See Lactarius volemus, Fr.

Agaricus Texensis, B. \& C.

See Collybia Texensis, B. \& C.

Agaricus theiogalus, Bull.

See Lactarius theiogalus, (Bull.) Fr.

Agaricus thujinus, $\mathrm{Pk}$.

See Tricholomia thujinum, $\mathrm{Pk}$.

Agarieus tigrinus, Bull.

See Lentinus tigrinus, (Bull.) Fr̀.

Agarieus tiliophilus, $\mathrm{Pk}$. See Crepidotus tiliophilus, $\mathrm{Pk}$.

Agaricus Tintinabulum, Fr.

See Mycena Tintinabulum, Fr.

Agaricus titubans, Bull.

See Bolbitius titubans, (Bull.) Fr.

Agaricus tomentellus, Auct. Amer.

See Lentinus plumatus, (Walt.) Fr.

Agaricus tomentosulus, $\mathrm{Pk}$.

See Pleurotus tomentosulus, Pk.

Agaricus tomentosus, Ell. \& Ev.

See Inocybe subdecurrens, Eli. \& Ev.

Agaricus torminosus, Schæff.

See Lactarius torminosus, (Schæff.) Fr.

Agaricus tortipes, Mont.

See Galera tortipes, Mont.

Agaricus torulosus, P.

See Lentinus carneotomentosus, (Batsch) Schrt.

Agaricus torulosus, P. sec. S. Syn. Car. 797.

See Panus conchatus, (Bull.) Fr.

Agaricus trabeus, $P$.

See Lenzites trabeus, (P.) Fr.

Agaricus transmutans, $\mathrm{Pk}$.

See Tricholoma transmutans, Pk.

Agaricus trechisporus, B.

See Inocybe trechispora, B.

Agarieus tremulus, Schæff.

See Pleurotus tremulus, (Schæff.) Fr.

Agaricus Trentonensis, Pk.

See Tricholoma Trentonense, $\mathrm{Pk}$. 
Agaricus trepidus, Fr.

See'Psathyrella trepida, Fr.

Agaricus tricholepis, Fr.

See Pilosace tricholepis, Fr.

Agaricus trichopus, A. \& S.

See Tricholoma flavobrunneum, Fr.

Agaricus tridens, Moore.

Note. This is possibly sterile Lentinus lepiacus, (Schæeff.) Fr.

Hark. \& Moore, Cat. Pac. Fungi 46. d. Feb. 1880 and Bull. Torr. Bot. Club. 7 : 58. May i 880.

Agaricus triscopus, Fr.

See Naucoria triscopus, Fr.

Agaricus trullæformis, Fr.

See Clitocybe trullaformis, Fr.

Agaricus trullisatus, Ell.

See Clitocybe trullisata, Ell.

Àgaricus truncicola, $\mathrm{Pk}$.

See Clitocybe truncicola, Pk.

Agaricus tubælormis, S.

See Lentinus friabilis, Fr.

Agaricus tuberculosus, $\mathrm{Fr}$.

See Pholiota tuberculosa, Fr.

Agaricus tuberosus, Bull.

See Collybia tuberosa, (Bull.) Fr.

Agaricus tumulosus, Kalchb.

See Clitocybe tumulosa, (Kalchb.) Fr.

Agaricus turbidus, Fr.

See Entoloma turbidum, Fr.

Agaricus turbinatus, Bull.

See Cortinarius turbinatus, (Bull.) Fr.

Agaricus udus, $\mathrm{P}$.

See Psilocybe uda, (P.) Fr.

Agaricus ulmarius, Bull.

See Pleurotus ulmarius, (Bull.) Fr.

Agaricus umbelliferus, L.

See Omphalia umbellifera; (L.) Fr.

Agaricus umbilicatus, Scheff.

See Omphalia umbilicata, (Schæff.) Fr.

Agaricus umbonatescens, Pk.

See Stropharia umbonatescens, Pk.

Agaricus umboninotus, $\mathrm{Pk}$.

See Inocybe umboninota, Pk.

Agaricus umbrinus, $P$.

See Lactarius umbrinus, (P.) Fr.

Agaricus umbrosus, $P$.

See Pluteus umbrosus, (P.) Fr.

Agaricus undatus, Fr.

See Clitopilus undatus, Fr.

Agaricus undulatellus, $\mathrm{Pk}$.

See Leptonia undulatella, Pl. 
Agaricus unicolor, Vaht.

See Phoilota unicoior, (Vahl.) Fr.

Agaricus unitinctus, $\mathrm{Pk}$.

See Ciitopilus unitinctus, Pk.

Agaricus ustalis, Fr.

See Trichoioma ustale, Fr.

Agaricus vaceinus, $P$.

See Trichoioma vaccinum, (P.) Fr.

Agaricus vaginatus, Bull.

See Amanitopsis vaginata, (Bull.) Roze.

Agaricus variabilis, $P$.

See Ciaudopus variabiiis, (P.) Cke.

Agaricus variegatus, Scop.

See Trichoioma variegatum, (Scop.) Fr.

Agaricus variicolor, B. \& C.

See Leptonia variicolor, B. \& C.

Agaricus vellereus, Fr.

See Lactarius vellereus, Fr.

Agaricus velutinus, $P$.

See Hyphoioma velutinum, (P.) Fr.

Agaricus velutipes, Curt.

See Coilybia velutipes, (Curt.) Fr.

Agaricus venifer, B. \& C.

See Hebeloma venifer, B. \& C.

Agaricus ventricosus, Bull.

See Collybia ventricosa, (Bull.) Fr.

Agaricus vermifluus, $\mathrm{Pk}$.

See Pholiota vermifiua, Pk.

Agaricus vernalis, $\mathrm{Pk}$.

See Naucoria vernalis, Pk.

Agaricus vernus, Auct. Amer.

See Amanita phalloides, Fr.

Agaricus versicolor, Bull.

See Lenzites variegata, Fr.

Agaricus versipellis, Fr.

See Hebeloma versipellis, $\mathrm{Fr}$.

Agaricus versutus, $\mathrm{Pk}$.

See Crepidotus versutus, $\mathrm{Pk}$.

Agaricus vervacti, Fr.

See Naucoria vervacti, Fr.

Agaricus vexans, $\mathrm{Pk}$.

See Mycena vexans, Pk.

Agarieus vialis, S. Syn. Car. 609.

Nore. It is impossible to state to what species the following citation relates.

S. Syn. Car. $82(56)$ no. 609 . 1822 .

Agaricus vilescens, Pk.

See Clitocybe vilescens, Pk.

Agaricus villaticus, Brond.

See Agaricus campestris, L., var. villaticus, Brond. 
Agaricus villosus, $\mathrm{Fr}$.

See Phoilota villosa, Fr.

Agaricus violaceocinereus, $P$.

See Cortinarius violaceocinereus, (P.) Fr.

Agaricus violaceofulvus, S. Am. Bor. 230.

See Pleurotus pinsitus, Fr.

Agaricus violaceus, $L$.

See Cortinarius vioiaceus, (L.) Fr.

Agaricus virescens, Schæeff.

See Russula virescens, (Schæff.) Fr.

Agaricus virescens, S. Syn. Car. 76r.

See Russula furcata, P.

Agaricus virescens, Fr.

See Amanita virescens, $\mathrm{P}$.

Agaricus virescens, $\mathrm{Pk}$.

See Trichoioma virescens, $\mathrm{Pk}$.

Agaricus virgineus, Wulf.

See Hygrophorus virgineus, (Wulf.) Fr.

Agaricus viridis, With.

See Ciitocybe viridis, (Wulf.) Fr.

Agaricus viriditinetus, $\mathrm{Pk}$.

See Trichoioma virescens, $\mathrm{Pk}$.

Agaricus virosus, $\mathrm{Fr}$ :

See Amanita virosa, Fr.

Agaricus viscidus, $L$.

See Gomphidius viscidus, (L.) Fr.

Agaricus vitellinus, $\mathrm{P}$.

See Bolbitius vitellinus, (P.) Fr.

Agaricus vitilis, $\mathrm{Fr}$.

See Mycena vitilis, Fr.

Agaricus vitreus, Fr.

See Mycena vitreus, Fr.

Agaricus Vittadini, Farl. non Morett.

See Lepiota subremota, B. \& C.

Agaricus volemus, $\mathrm{Fr}$.

See Lactarius volemus, Fr.

Agaricus volvaceus, Bull.

See Voivaria volvacea, (Bull.) Fr.

Agaricus volvatus, $\mathrm{Pk}$.

See Amanitopsis volvata, (Pk.) Sacc.

Agaricus vulgaris, $P$.

See Mycena vulgaris, (P.) Fr.

Agaricus Watsoni, Pk.

See Eccilia Watsoni, Pk.

Agarieus Woodianus, Pk.

See Clitopiius Woodianus, $\mathrm{Pk}$.

Agaricus xanthophyllus, B. \& C.

See Omphalia xanthophylla, B. \& C.

Agaricus xanthopilus, Mont.

See Collybia xanthopila, Mont.

Agaricus xanthopus, Fr.

See Collybia xanthopus, Fr. 
Agaricus xylogenus, Mont.

Mont. Syll. Crypt. 122. d. 1856.

Sacc. Cub. \& Manc. Syll. 5 : Ioro. d. 28 May 1887.

Mcllvaine \& Macadam, Am. Fung. 339. d. 1900.

Psalliota xylogena; Mont.

Lloyd, Myc. Notes 30. Nov. I 899.

Agaricus Yucatanensis, Ell. \& Ev.

Ell. \& Ev. in Ell. Publ. Field Columb. Mus. Bot. $1: 285 . d . p l .8$. Dec. 1806. [Publ. no. 15.]

Sacc. \& Syủ. Syll. 14: I 50. d. 20 Aug. I899.

Agaricus Zippelii, Lev.

See Pleurotus Zippelii, (Lev.) Sacc. Syll.

Agaricus zonatus, $\mathrm{Pk}$.

See Collybia zonata, Pk.

Aglaospora Betulæ, (Schum.) O. Kuntze.

Note. The Spharia melasperma of Fries S.M. $2: 389$. a. 1823 and Scl. Suec. 157 is a synonym of this species but the Sphceria melasperma of Schweinitz Am. 13or. which we have examined is not the same as Fr. Scl. Suec. 157.

Kuntze, O., Rev. Gen. Pl. $3^{2}$ : 441. I898. Spharia lanciformis, Fr.

S. Syn. Am. Bor. 196 no. 1263 . 1834 . Melanconis lanciformis, Tul.

Cke. Proc. Phil. Acad. 1877 : 127. d. 22 May 1877. Pseudovalsa lanciformis, Ces. \& De Not.

Berl. Icon. 1: 47. d.pl. 35, f. 2. 1 Jan. 1891.*

Rostr. Meddelelser om Groenl. 3 : 619. 1891.

Ell. \& Ev. Pyren. 540. d. May i 892 .

Harvey, Bull. Torr. Bot. Club 23: 56. 29 Feb. 1896.

Ricker, Univ. of Maine Studies 3: 36. 30 April 1902.

Melanconls elliptlca, $\mathrm{Pk}$.

Pk. Rept. N.Y. Mus. 25 : 102. Sept. 1873.

Frost in Cat. Pl. Amherst 94. I 875.

Cke. Proc. Phil. Acad. 1877: 127.d. 22 May 1877.

Cobb, List Pl. Amherst 45. 1887.

Diatrype elliptica, $\mathrm{Pk}$.

Pk. Rept. N.Y. Mus. 28:87. 1876.

Pseudovalsa lanclformis, (Fr.) De Not., var. elliptica, (Pk.) Sacc.

Sacc. Syll. 2 : 135. $d$. 13 June 1883.

Aglaospora bicornis, (Cke.) O. Kuntze.

Kuntze, O., Rev. Gen. Pl. $3^{2}$ : 44I. 1898. Melanconis blcornIs, Cke.

Cke. \& B. Fungi 1 34. $f$. 72. 197. 1875 .

Plk. Rept. N.Y. Mus. 28: 72. d. 1876.

Cke. Proc. Pliil. Acad. $1877:$ 127. d. 22 May 1877.

Pk. in Day's Cat., Bull. Buf. Soc. 4: 229 (I65). 1883. Pseudovalsa bicornis, Sacc.

Sacc. Syll. 2 : 139. d. I3 June 1883.

Berl. lcon. $1: 49 . d$. pl. $36, f .4$. I Jan. I89I.

Ell. \& Ev. Pyren. 539. d. May 1892.

Aglaospora Canadensis, (Ell. \& Ev.) n. n.

Pseudovalsa Canadensis, Ell. \& Ev.

Ell. \& Ev. Jour. Myc. 9 : 224. d. 31 Dec. 1903. 
Aglaospora eaudata, (Ell. \& Ev.) n. n.

Massariovalsa caudata, Ell. \& Ev.

Ell. \& Ev. Pyren. 408. $d$. May 1892 .

Ell. \& Ev. Proc. Phil. Acad. 1893: 1 38. d. 28 Feb. 1893.

*Ell. \& Ev. N. A. F. 2928 . Oct. I 893.

Lindau in Engler \& Prantl, Pflanzenfam. 1' ${ }^{1}: 445$. Feb. 1897.

Pseudovalsa caudata, Sacc.

Sacc. Syll. 11 : 330. $d$. July 1895.

Aglaospora convergens, (Tode) O. Kuntze.

Kuntze, O., Rev. Gen. Pl. 32: 441. 1898.

Spharia convergens, Tode.

Muhl. Cat. Pl. Am. Sept. I ed. 103. I813. 2 ed. 106. I818.

S. Syn. Car. 38 (12) no. I12. 1822.

S. Syn. Am. Bor. 203 no. 1390.1834.

Lea, Cat. Pl. Cin. $75 . \quad 1849$.

Pseudovalsa convergens, Sacc.

Sacc. Syll. $2:$ 1 36. $d$. 13 June 1883.

Ell. \& Ev. Pyren. 542. $d$. May 1892.

Kellerm. \& Werner, Geol. Ohio 72: 358. Jan. 1895.

Aglaospora Fairmani, (Ell. \& Ev.) O. Kuntze.

Kuntze, O., Rev. Gen. Pl. 32 : 44I. 1898.

Pseudovalsa Fairmani, Ell. \& Ev.

Fairman, Proc. Rochester Acad. $1: 49 . d . p l .3, f_{.1-3}, 10,11$. Aug. 1890.

Berl. Icon. 1: 49. I Jan. I89I.

Sacc. Syll. 9 : 820. $d$. 15 Sept. 189 I.

Ell. \& Ev. Pyren. 540. d. May 1892 .

Aglaospora hapaloeystis, (B. \& Br.) O. Kuntze.

Kuntze, O., Rev. Gen. Pl. 32 : 441. 1898.

Valsa hapalocystis, B. \& Br.

Pk. Rept. N.Y. Mus, 25: 104. Sept. 1873.

Frost in Cat. Pl. Amherst 95. 1875.

Cke. Proc. Phil. Acad. 1877 : 123. d. 22 May 1877.

Cobb, List Pl. Amherst 46. 1887.

Pseudovalsa hapalocystis, Sacc.

Sacc. Syll. 2 : 138. d. 13 June 1883.

*Ell. \& Ev. N. A. F. 2119.1889.

Berl. Icon. $1: 48$. d. pl. $36, f . r$. I Jan. 1891 .

Ell. \& Ev. Pyren. 538. d. May 1892.

Lindau in Engler \& Prantl, Pflanzenfam. ${ }^{1}{ }^{1}: 472$. Feb. 1897.

*Rehm, Ascomyceten 1479.1003.

* Barthol. Ell. \& Ev. Fung. Columb. 1843. 28 Dec. 1903.

Aglaospora irregularis, (DC.) O. Kuntze.

Kuntze, O., Rev. Gen. Pl. $3^{2}$ : 44I. 1808.

Spharia profusa, Fr.

S. Syn. Am. Bor. 201 no. I346. I834.

Spharia anomia, Fr.

S. Syn. Am. Bor. 199 no. 1316. 1834. Spharia amorphostoma, S.

S. Syn. Am. Bor. 201 no. 1334. $d .1834$.

Sacc. Syll. 2 : $383 . d$. I 3 June 1883. Spharia ocellata, S.

S. Syn. Am. Bor. 217 no. 1638.1834.

B. Grev. 4: 155 (193). June 1876 .

Valsa profusa, Fr.

Pk. Rept. N. Y. Mus, 25 : 103. Sept. 1873.

Cke. Proc. Phil. Acad. 1877: 123. 22 May 1877. 
C. \& E. Grev. 6 : 12. Sept. 1877.

* Ell. N. A. F. 172. 1878.

Valsa amorphostoma, Clie.

Clie. Proc. Phil. Acad. 1877: 124. 22 May 1877.

Stevenson, Proc. Pliil. Acad. 1878: 87. 9 April 1878. Aglaospora profusa, De Not.

Rehm, Asc. Lojk. 41. d. I882.

Sacc. Syll. 2 : 133. d. 13 June 1883.

Clie. Grev. 13: 39. Dec. 1884.

Tubeuf-Smith, Diseases of Plants 220. $\quad 1807$.

Schrenk, Rept. Mo. Bot. Gard. 12 : 21. Io Feb. I901.

Ricker, Univ. of Maine Studies 3: 32. 30 April 1902. Valsa anomia, Strauss.

B. Grev. 4 : 99 (169). d. March 1876.

Che. Proc. Phil. Acad. 1877: 116. it April 1877.

Sacc. Syll. 1 : 126. d. 13 June 1882.

Cke. Grev. 14: 51. Dec. 1885.

Ell. \& Ev. Pyren. 474. d. May I 892. Spharia Sartwellii, B. \& C. nom. nud.

Sprague, Proc. Bostoil Soc: 6 : 320. 1859. Massaria macrospora, B. \& C. nom. nud.

Pk. Rept. N.Y. Mus. 25 : 103. Sept. 1873. Massaria seiridia, B. \& C.

Curtis, M. A., Bot. N. Car. 150. 1867.

B. Grev. 4: 155 (193) no. 976. d. June 1876. Valsa Sartwellii, B. \& C. nom. nud.

Cke. Proc. Phil. Acad. 1877: 123. 22 May 1877. Pseudovalsa profusa, Clie.

Cke. Grev. 14: 55. Dec. 1885.

Ell. in Cat. Pl. N. J. 534. 1880.

Berl. Icon. 1 : 47. d. pl. 34, f. 6. I Jan. 1891.

Ell. \& Ev. Pyren. 538. d. May 1802.

Starb. Bihang till K. Sv. Vet.-Akad. Handl. 193 : no. 2 : 36.1894.

*Ell. \& Ev. Fung. Columb. 523. 1894.

Harvey, Bull. Torr. Bot. Club 23: 55. 29 Feb. 1896.

* Barthol., Ell. \& Ev. Fung. Columb. 1566. Dec. 1901. Pseudovalsa irregularis, Schrt.

Lindau in Engler \& Prantl, Pflanzenfam. 1' ${ }^{1}$ 472. d. f. $280 \mathrm{~K}$. Feb. 1897.

Aglaospora minima, (Ell. \& Ev.) n. n.

Pseudovalsa minima, Ell. \& Ev.

Ell. \& Ev. Jour. Myc. 9: 224. d. 3i Dec. 1903.

Aglaospora occulta, (Ell.) n. n. Pseudovalsa occulta, Ell.

Ell. Proc. Phil. Acad. 1895: 27 (8). d. April 1895.

Sacc. Hedw. 35 : Rep. 7 : xxx. d. 25 Feb. 1806.

Sacc. \& Syd. Syll. 14: 578. d. 20 Aug. 1899.

Aglaospora profusa, De Not. See Aglaospora irregularis, (DC.) O. Kuntze.

Aglaospora ribesia, (Sacc. \& Scalia) n. n. Pseudovalsa ribesia, Sacc. \& Scalia.

Sacc. Pk. \& Trel., Harriman Alaska Exp. 5 : 29. d.pl. 5, f. 14. 5 April I904.

Aglaospora sambucina, (Pk.) O. Kuntzt.

Kuntze, O., Rev. Gen. Pl. $3^{2}: 441$. 1898. Valsa sambucina, $\mathrm{Pk}$.

Pk. Rept. N. Y. Mus. $28: 75$. d. 1876. 
Pseudovalsa sambucina, Sacc.

Sacc. Syll. 2 : 137. d. 13 June 1883.

Cke. Grev. 14: 56. Dec. 1885.

Berl. Icon. 1 : 49. I Jan. I89 I.

Ell. \& Ev. Pyren. 539. d. May 1892.

Aglaospora sigmoidea, (C. \& E.) O. Kuntze.

Kuntze, O., Rev. Gen. Pl. 3² : 441. 1898.

Melanconis sigmoidea, C. \& E.

C. \& E. Grev. 6: 92. d.pl. I0o, f. 26. March 1878.

*Eli. N. A. F. 1566 . I886.

Pseudovalsa sigmoidea, Sacc.

Sacc. Syll. $2:$ 136. $d$. 13 June 1883.

Cke. Grev. 14 : 56. Dec. 1885.

Ell. in Cat. Pl. N. J. 534. 1889.

Berl. Icon. $1: 47 . d . p l .35, f_{.}$. . I J Jan. I891.

Ell. \& Ev. Pyren. 541. d. May I 892 .

*Ell. \& Ev. N. A. F. 2758 . May 1892.

Millsp. \& Nutt. Publ. Field Columb. Mus. Bot. 1 : ı16, 142. d. Jan. I 896.

*Ell. \& Ev. Fung. Columb. 949. April 1806.

*Shear, Ell. \& Ev. Fung. Columb. 1423. May 1901.

Aglaospora Smithii, (Ell. \& Ev.) n. n.

Pseudovalsa Smithii, Elt. \& Ev.

Ell. \& Ev. in Smith, C. L., Bull. Univ. lowa 2: 404. d. Nov. 1893. Sacc. Syll. 11 : 331. d. July 1895.

*Smith, C. L., Cent. Amer. Fungi i6. 1896.

Aglaospora stylospora, (Ell. \& Ev.) O. Kuntze.

Kuntze, O., Rev. Gen. Pl. 32: 44I. 1898.

Pseudovalsa stylospora, Ell. \& Ev.

Ell. \& Ev. Proc. Phil. Acad. 1890: 223. d. I89o.

Berl. Icon. 1 : 48 . d. pl. 35, f. 5 . I Jan. I89 I.

Sacc. Syll. $9: 821 . d$. 15 Sept. 1891.

Ell. \& Ev. Pyren. 539. d. pl. 35, f. 9-1I. May 1892.

Aglaospora subrufa, (Ell. \& Ev.) n. n. Pseudovalsa subrufa, Ell. \& Ev.

Ell. \& Ev. Erythea 1 : 146. $d$. I July 1893.

Sacc. Syll. 11 : $330 . d$. July 1895 .

Aglaospora taleola, Tul. See Diaporthe taleola, (Fr.) Sacc.

Aglaospora Titan, O. Kuntze.

See Titania Berkeleyi, Berl.

Aglaospora tubulosa, (B. \& C.) O. Kuntze.

NotE. The name of this fungus was originally Valsa tubulosa, B. \& C. as in Curtis, M. A., Bot. N. Car. 142. 1867 and in the Curtis herbarium. In Grevillea, 1876 it is Valsa tribulosa, B. \& C. but the original spelling has been followed by subsequent authors.

Kuntze, O., Rev. Gen. PI. 32 : 441. 1898.

Valsa tubulosa, B. \& C.

Curtis, M. A., Bot. N. Car. 142. 1867.

Cke. Proc. Phil. Acad. 1877: 123. $d .22$ May 1877.

Valsa tribulosa, B. \& C.

B. Grev. 4 : 102 (172). no. 877. d. March 1876.

Pseudovalsa tubulosa, Sacc.

Sacc. Syll. 2 : 137. $d$. 13 June 1883.

Cke. Grev. 14 : 48, 56 . Dec. I885.

Berl. \& Vogl. in Sacc, Syll, Add, 1-4 : 151. 31 Dec. 1886. 
Berl. Icon. 1: 47.*d. pl. 34, f. 5. I Iall. ISol.

Ell. \& Ev. Pyren. 542. d. May IS̉oz.

Calospora tribulosa, Sacc.

Sacc. Syll. $2: 232$. d. I 3 June I883.

Aglaospora ulmicola, (Ell. \& Ev.) n. n.

Pseudovalsa ulmicola, Ell. \& Ev.

Ell. \& Ev. Proc. Phil. Acad. 1893: 450. d. 1893.

Sacc. Syll. 11 : 330. d. July 1895.

Aglaospora viticola, (Ell. \& Ev.) n. n.

Pseudovalsa viticola, Ell. \& Ev.

Ell. \& Ev. Proc. Phil. Acad. 1894: 343. d. 30 Nov. i894.

Sacc. Syll. 11 : 330. d. July 1895.

Smyth, Tr. Kans. Acad. 15: 67. March 1898.

Ell. \& Ev. Bull. Torr. Bot. Club 27: 63. d. 17 Feb. 1900.

Sacc. \& Syd. Syll. 16 : 527. I Feb. 1002.

Lophiostoma pustulatum, Ell. \& Ev.

Ell. \& Ev. Bull. Torr. Bot. Club 25 : 503. d. Io Sept. 1808.

Smyth, Tr. Kans. Acad. 16: 165. June 1899.

Aglaospora Xanthoxyli, O. Kuntze.

See Fenestella Xanthoxyli, (Pk.) Sacc.

Agyriella, Ell. \& Ev.

See Agyriopsis, Sacc. \& Syd.

Agyriella Betheli, Ell. \& Ev.

See Agyriops is Betheli, (Ell. \& Ev.) Sacc. \& Syd.

Agyriopsis, Sacc. \& Syd.

Sacc. \& Syd. Syll. 14: 805. d. 20 Aug. I 800.

Lindau in Engler \& Prantl, Pflanzenfam. 11**: 533. d. Feb. I900. Agyriella, Ell. \& Ev.

Ell. \& Ev. Bull. Torr. Bot. Club. 24 : 470. d. 30 Oct. I 897.

Lindau \& Syd. Hedw. 37 : Beibl. 7 : li. d. 9 April 1898.

Agyriopsis Betheli, (Ell. \& Ev.) Sacc. \& Syd.

Sacc. \& Syd. Syll. 14: 805. d. 20 Aug. I 890.

Lindau in Engler \& Prantl, Pflanzenfam. 11**: 533. Feb. 1900. Agyriella Betheli, Ell. \& Ev.

Ell. \& Ev. Bull. Torr. Bot. Club 24: 470. d. 30 Oct. I897.

Lindau \& Syd. Hedw. 37 : Beibl. 7 : li. 9 April 1898.

Agyrium, Fr.

Note. The genus Agyrium was by some of the earlier writers regirded as belonging to Lichens while others regarded it as belonging to Fungi. Fur notes as to its relation to Lichens see Tuck. Gen. Lich. 225. 1872.

Agyrium atrovirens, Fr.

NoTE. The reference in Schweinitz below is to the Agyrium atrovirens of Fr. S.M. 2: 232 . I 822 which was later considered by Fr. El. $2: 25$. 1830 to be an abortive form of Stictis Pupula, Fr. Other references to Agyrium atrovirens are to be found under Cryptodiscus atrovirens, (Fr.) C.la. and Propolidium atrovirens, (Fr.) Rehm.

S. Syn. Am. Bor. 187 no. I141. I834.

Agyrium brunneolum, B. \& C.

B. Grev. 4: 6 (144) no. 774. $d$. Sept. 1875 .

Sacc. Syll. $8: 635 . d$. 20 Dec. 1880.

Underw. \& Earle, Bull. Ala. Exp. Sta. 80: 200. d. 28 June 1897.

Earle in Mohr, Contr. U. S. Nat. Herb. 6 : 159. 3I July IgoI.

Agyrium cæsium, Fr.

S. Syn. Am. Bor. I87 no. I1 139 . I834. 
Agyrium earneolum, Tuck.

Tuck. Syn. N. Am. Lichens 2: 111. d. 1888.

Agyrium herbarum, Fr.

S. Syn. Am. Bor. I 87 no. 1144.1834 .

C. \& E. Grev. 6 : 9. Sept. 1877 .

*Ell. N. A. F. 45I. I880.

Sacc. Syll. $8: 635 . d$. 20 Dec. 1889.

Ell. in Cat. Pl. N. J. 556. 1889.

?Tremella Stictis, A. \& S. non P.

S. Syn. Car. i16 (90) no. 1146. 1822.

Agyrium lacteum, Fr.

NOTE. The only information we have of this species is a description contained in a short account in Fries, Summa $480 . \quad 1846$ where it occurs in a foot note under Diaphanium. This is repeated in Sacc. Syll. 4:672. Io April I 886. The specimen labeled Agyrium lacteun from Herb. Schweinitz which we have examined is on the stem of some unknown herbaceous plant and is Cylindrocolla Urtica, (P.) Bon.

S. Syn. Am. Bor. 187 no. 1143 . d. 1834 .

Agyrium nigrieans, Fr.

See Epidochium nigricans, Fr.

Agyrium rufum, (P.) Fr.

Nore. Under Tuckerman and Willey are given the principal references to this species in North American lichenological literature.

Grev. Scot. Crypt. Fl. 41: pl. 232. 1826.

S. Syn. Am. Bor. 187 no. I1 40.1834.

M. A. Curtis, Bot. N. Car. 135. 1867.

Cke. Handbook 733. d. f. 340. i871.

B. Grev. 4: 6 (144): Sept. 1875.

C. \& E. Grev. 4: 180. June 1876 .

Cke. Grev. $7: 48$. Dec. 1878 .

* Rav. Fung. Am. 314. 1879.

*EII. N. A. F. 450. 1881 .

Rehm, Asc. Lojk. 24. d. 1882.

Willey, Intr. to study of Lichens $54 . \quad 1887$.

Tuck. Syn. N. Am. Lichens 2: i1 1. d. 1888.

Sacc. Syll. 8 : 634. $d$. 20 Dec. 1889.

Ell. in Cat. Pl. N. J. $556 . \quad 1889$.

Willey, Enum. Lichens New Bedford 28. 1892.

Lindau in Engler \& Prantl, Pflanzenfam. 11: 219. f. 170 A, B. Dec. 1894 .

Underw. \& Earle, Bull. Ala. Exp. Sta. 80: 200. 28 June 1897.

Earle in Mohr, Contr. U. S. Nat. Herb. 6 : 159. 31 July 1901.

Ricker, Univ. of Măine Studies 3: 47. 30 April 1902.

Agyrium sexdecimsporum, Fckl.

NoTE. This species is referred by Rehm in Rahh. Krypt.-Fl. $x^{3}: 307$. 1890 to Biatorella geophana, (Nyl.) Rehm, q.v. It was furmerly considered to be a lichen and it appears in the lichenological literature of North America as Biatora and Biatorella. See-Tuck. Syn. N. Am. Lichens 2: $50 . d .1888$. Willey, Intr. to study of Lichens 48. 1887 and Enum. Lichens New Bedford 25. 1892 .

C. \& E. Grev. 8 : 13. Sept. 1879 .

Sacc. Syll. 8: $636 d$. 20 Dec. 1889 .

Ell. in Cat. Pl. N. J. 556.1889.

Agyrium Tuekermanii, B. \& Rav.

B. Grev. 4: 6 (144) no. 773. d. Sept. 1875.

Sacc. Syll. 8 : 635. d. 20 Dec. 1889. 


\section{Allographum.}

See Aulographum.

Albugo, (P.) S. F. Gray.

Sivingle, Jour. Myc. 7: 109. d. April 1892.

Magn. Hedw. 32 : 66. April 1893.

Albugo Amaranti, O. Kuntze.

See Albugo Bliti, (Biv.) O. Kuntze.

Albugo Bliti, (Biv.) O. Kuntze.

Kuntze, O., Rev. Gen. PI. 2: 6581891.

Schrt. in Engler \& Prantl, Pflanzenfam. $1^{1}:$ 112. d. Aug. 1893.

Clements, FI. Nebr. 1: 57. $d$. 15 Aug. 1894.

McClatchie, Proc. S. Cal. Acad. 1: 351 . 5 June 1897.

Stevens, F. L., Bot. Gaz. 28 : 149. pl. 11-15. 25 Sept. 1899 and 28 : 233. 21 Nov. 1899 and Rev. Myc. 22: 117. pl. 206, f. 1-8. pl. $207, f .14^{-22}$. Oct. 1900 .

Davis, B. M., Bot. Gaz. 29 : '297. 25 May 1900.

Stevens, Bot. Gaz. 32 : 254. pl. $4, f .47,51,52$. 21 Oct. 1901.

Swingle, Bull. U.S. Agr. Pl. Industry $37: 35.27$ June 1903.

\section{Czoma Amaranti, S.}

S. Syn. Ain. Bor. 292 no. 2853. d. 1834 . Uredo Amaranti, B.

B. Jour. Roy. Hort. Soc. 3 : 270 (8). f. 4. Aug. 1848 . Cystopus Bliti, Lev.

Farl. Bull. Bussey lnst. 1 : $429 . d$. 432 . March 1876 .

Burrill, Bull. III. Lab. 1' ${ }^{1}: 57$. Dec. 1876 .

*Thm. Myc. Univ. 619. 1877 .

Pk. Rept. N. Y. Mus. $29: 76 . \cdot 1878$.

*Ell. N. A. F. 206.1879.

Farl. Bot. Gaz. 8: 335. d. Nov. 1883.

Pk. in Day's Cat., Bull. Buf. Soc. 4: 213 (149). 1883.

Farl. Bot. Gaz $9: 40$. March I884.

Trel. Tr. Wis. Acad. 6 : 108 (4). Nov. 1884.

Bessey, Bull. lowa Agr. Coll. 1884: 140. Jan. 1885.

Kellerm. Bull. Wash. Lab. 1: 78. Jan. 1885 and Tr. Kans. Acad. $9: 83.1885$.

Wint. \& Demet. Hedw. 24: 201 (25). Oct. 1885.

Arth. \& Holw. Bull. Minn. Surv. 3: $36 . \quad$ I Oct. 1887.

Brendel, Fl. Peoriana 2 ed. $60 . \quad 1887$.

Kellerm. \& Carl. Tr. Kans. Acad. 10: 94. 1887.

Berl. \& De Toni in Sacc. Syll. $7: 236 . d$. 15 March 1888.

Halsted, Bot. Gaz. 13: 57. March 1888.

Halsted, Bull. lowva Agr. Cdll. 1888 : 100. April 1888.

*Roum. Fung. Gall. 4551. 1888 .

Roum. Rev. Myc. 10: 145. I July 1888.

Tracy \& Gall. Jour. Myc. 4: 36. May 1888.

Macbr. \& Hitchcock, Bull. Univ. lowa 1: 51. Nov. 1888.

Halsted, Jour. Myc. 5 : 10. March 1889.

Seymour, Proc. Boston Soc. 24: 184. 8 Nov. 1889.

Jennings, Bull. Tex. Exp. Sta. $9: 25$. May 1890.

Webber, Rept. Nebr. Agr. 1889: 190 (50). 1890.

Pammel, Bull. lowa Exp. Sta. 15: 236 (3). pl. 6, f. 1-4. Nov. 1891. and Am. Mo. Micr. Jour. 14: 191. pl. 1, f. 1-4. July 1893 .

Williams, Bull. S. Dak. Exp. Sta. 29: 46. Dec. 1891.

Williams, Bull. Torr. Bot. Club 19:83. 5 March 1892.

Pammel, Jour. Myc. 7: 102. April 1802.

* Seymour \& Earle, E. F. 352, 354. I May 1893.

*Eli. \& Ev. Fung. Columb. 46. I893. 
Halsted, Rept. N.' J. Exp. Sta. 1892 : 348. 1893.

* Shear, N. Y. Fungi 198.1893.

Halsted, Bull. N: J. Exp. Sta. Io7 : 11. Io Jan. 1895.

Halsted, Rept. N. J. Exp. Sta. 1894: 345.1895.

Millsp. \& Nutt. Publ. Field Columb. Mus. Bot. 1: 83. Jan. 1896.

Pammel \& Carver, Proc. lowa Acad. 3 : 144. 1896.

Atk. Bull. Cornell Unıv. 3 : 14. 25 June 1897.

Tubeuf-Smith, Diseases of Plants 127. 1897.

Orton, Rept. Vt. Exp. Sta. 11: 202. Sept. 1898 and Contr. Bot. Vt. $2: 4$. Sept. 1898.

Halsted, Bull. Torr. Bot. Club 25 : 575. 7 Nov. 1898.

Berl. Icon. Phyc. 8. d. pl. 4. 1898 .

Hume, Proc. Davenport Acad. 7 : 253. 12 May 1899.

Selby, Bull. Ohio Exp. Sta. 121 : 18. Sept. 1900.

Berl. Riv. Patol. Veg. 9: 20. d. f. $5.20 \mathrm{Feb}$. I 201.

Selby \& Ames, Bull. Ohio Exp. Sta. 126: 173. March 1901.

Sturgis, Rept. Conn. Exp. Sta. 24: 261. 1001.

*Griffitlis, West Am. Fung. 33. 22 March 1902.

Cystopus candidus, Pk. p.p. non Lev.

Pk. Rept. N. Y: Mus. 22: 93. 1869.

Pk. Rept. N. Y. Mus. 24: 103. 1872. Cystopus Amaranti, B.

B. Grev. $3: 58$ (100) no. 571. d. Dec. 1874 .

Pk. Rept. N. Y. Mus. 28 : 6i. I876.

Berl. \& De Toni- in Sacc. Syll. 7 : 236. d. 15 March 1888.

Swingle, Tr. Kans. Acad. 11 : 69. I Nov. 1889.

Swingle, Tr. Kans. Acad. 12 : 131. 1890.

Halsted, Rept. N. J. Exp. Sta. 1892 : 278.1893.

Cystopus Cyathula, Wint. in litt. ad Roum.

*Roum. Fung. Gall. 4863. 1888.

Roum. Rev. Myc. 11:66. I April 1889.

Albugo Amaranti, $\mathrm{O}$. Kuntze.

Kuntze, O., Rev. Gen. Pl. 2 : 658. 1891.

Swingle, Jour. Myc. 7 : 115. April 1892.

Davis,.J. J., Tr. Wis. Acad. 9 : 155. 21 Aüg. 1893.

Underw. Proc. Ind. Acad. 1893 : 31. Aug. I894.

Kellerm. \& Werner, Geol. Ohio $7^{2}$ : 348. Jan. 1895.

Tracy \& Earle, Bull. Miss. Exp. Sta. 34: 94. May 1895.

Underw. \& Earle, Bull. Ala. Exp. Sta. 80: 136. 28 June 1897.

Stevens, Rept. Ohio Acad. 6 : 38.1898.

Long, Bull. Torr. Bot. Club 27: 586.14 Nov. 1900.

Earle in Mohr, Contr. U. S. Nat. Herb. 6: 151. 31 July 190r.

Patterson, Bull. U. S. Agr. Pl. Industry 8: 17. 3 Feb. 1902.

*Barthol., Ell. \& Ev. Fung. Columb. 1709. Feb. 1903.

Albugo Bliti, Vestergren.

See Albugo Platensis, (Speg.) Swingle.

Albugo eandidus, (P.) O. Kuntze.

Kuntze, O., Rev. Gen. Pl. 2 : 568 . 1891 .

Swingle, Jour. Myc. 7 : 110. April 1892.

Davis, J. J., Tr. Wis. Acad. 9 : 154. 21 Aug. 1893.

Schrt. in Engler \& Prantl, Pflanzenfam. 1': I Io. d. f.94,95. Aug. 1893 .

Underw. Proc. Ind. Acad. 1893 : 31, 32. Aug. 1894.

Clements, Fl. Nebr. 1: 57. $d$. 1894.

Kellerm. \& Werner, Geol. Ohio $7^{2}: 348$. Jan. 1895 .

Tracy \& Earle, Bull. Miss. Exp. Sta. 34: 94. May 1895.

Underw. Proc. Ind. Acad. $1894: 153$. Oct. 1895.

Ellis \& James in Holzinger, Contr. U. S, Nat, Herb, 3 : 276. 23 Nov. 
- Pammei, Proc. lowa Acad. 2 : 203. 1895.

Pound, Clements and others, Bot. Surv. Nebr. 4: 34. 20 Jan. 1896.

Stevens, Rept. Olıio Acad. 4: 20. 1896.

Mc Clatchie, Proc. S. Cal. Acad. 1: 351. 5 June 1807.

Underw. \& Earle, Bull. Ala. Exp. Sta. 80: 136. 28 June 1897.

Stevens, Rept. Ohio Acad. 5: 66. 1897.

Stevens, Rept. Ohio Acad. 6 : $38 . \quad$ I 898

Underw. Moulds 32. pl. 3, f. 6. 1899 .

Davis, B. M., Bot. Gaz. 29 : 297. pl. 22.' 25 May 1900.

Long, Bull. Torr. Bot. Club 27: 586 . 14 Nov. 1900.

Nelson, Rept. Wyo. Exp. Sta. 10: (20). 1900.

Tracy \& Earle in Greene, PI. Baker 1: 16. 22 Feb. 190r.

Stevens, Ber. Deutsch. Bot. Ges. 19: 174. 24 April 1901.

Earle in Mohr. Contr. U.S. Nat. Herb. 6: 152. 31 July 1901.

Stevens, Bot. Gaz. 32:91, 98. pl. 2. 20 Aug. I9ol and $32: 254$ 21 Oct. I901.

Patterson, Bull. U. S. Agr. Pl. Industry 8: 17. 3 Feb. 1902.

*Griffiths, West. Am. Fung. 335. 21 May 1902.

Kellerm. Jour. Myc. $8: 57$. d. 30 June 1902.

* Kellerm. Ohio Fungi 63. $d$. July 1902.

*Barthol. Ell. \& Ev. Fung. Columb. 1710. Feb. 1903.

*Kellerm. Ohio Fungi 122. 1903.

Kellerm. Jour. Myc. 9: i II. 29 May 1903.

* Barthol., Ell. \& Ev. Fung. Columb. 1805, 1806. 28 Dec. 1903.

Clint. Rept. Conn. Exp. Sta. 27 : 353. May 1904.

Kellerm. Jour. Myc. 10: I15. 31 May I904.

* Barthol., Ell. \& Ev. Fung. Columb. 200\%. 20 March 1905. Aecidium candidum, $\mathrm{P}$.

P. in Gmel. Syst. Nat. 2 : 1473. d. 1791.* Uredo candida, $\mathrm{P}$.

S. Syn. Car. 71 (45) no. 48 I. I 822.

Grev. Scot. Crypt. FI. 5 : pl. 251. 1827.

Lea, Cat. Pl. Cin. 7 I. 1849 .

Watt, Can. Nat. \& Geol. 11. 2 : 392. Oct. 1865. Uredo Cheiranthi, P.

S. Syn. Car. 71 (45) no. 482.1822. Caoma candidum, S. Am. Bor. p.p.

S. Syn. Am. Bor. 292. no. 2852.1834. Cystopus candidus, Lev.

* Rav. Fung. Car. 4: 93.1855.

Sprague, Proc. Boston Soc. 6: $319 . \quad 1859$.

Watt, Can. Nat. \& Geol. II. 2 : 39r. Oct. 1865.

Curtis, M. A., Bot. N. Car. 124. 1867.

Russell, Am. Nat. 2 : 633.f. 4. Feb. 1869.

Pk. Rept. N. Y. Mus. 24: 103. 1872.

B. Grev. $3: 58$ (100). Dec. 1874 .

Farl. Bull. Bussey Inst. 1: 329. f. 5. 1875.

Frost in Cat. Pl. Amherst 84. 1875.

Farl. Bull. Bussey Inst. 1: 429. d. 432. March 1876 .

Burrill, Bull. 111. Lab. 1: 57. pl. $1, f .1-4$. Dec. 1876.

Pk. Rept. N. Y. Mus, 29: 76.1878.

James, Jour. Cin. Soc. 2: 67 (26). April 1879.

* Ell. N. A. F. 204. I879.

Hark \& Moore, Cat. Pac. Fung. 26. Feb. 1880.

Johnson, Bull. Minn. Acad. 1 : 365. I 880.

Farl. Bot. Gaz. 8 : 334. d. Nov. 1883 .

Bundy, Geol. Wis. 1873-79. $1: 400$. $1883^{\circ}$.

Pk. in Day's Cat., Bull. Buf. Soc. 4: 213 (149). 1883: 
Farl. Bot. Gaz. 9 : 40. March 1884

Trel. Tr. Wis. Acad. 6 : 108 (4). Nov. I884.

Barnes in Bot. Gaz. 9 : 194. Dec. I884.

Bessey, Bull. lowa Agr. Coll. 1884 : I40. Jan. I885.

Kellerm. Bull. Wash. Lab. 1 : 78. Jan. I885 and Tr. Kans. Acad. 9 : 83. 1885.

Hark. Bull. Cal. Acad. 1: 163. 7 March 1885.

Wint. \& Demet. Hedw. 24: 201 (25). Oct. I 885.

Arth. \& Holw. Bull. Minn. Surv. 3: 36. I Oct. I887.

Brendel, FI. Peoriana 2 ed. 60.1887.

Halsted, Bull. lowa Agr. Coll. 1886 : 54. 1887.

Kellerm. \& Carl. Tr. Kans. Acad. 10: 94. I887.

Langlois, Cat. Pl. La. 29. 1887.

Scribner, Rept. U. S. Agr. 1886 : I34. 1887.

Berl. \& De Toni in Sacc. Syll. $7: 234$. d. 15 March 1888.

Halsted, Bot. Gaz. 13: 56 . March 1888.

Halsted, Bull. lowa Agr. Coll. 1888 : 99. April 1888.

Tracy \& Gall. Jour. Myc. 4: 36. May 1888.

Macbr. \& Hitchcock, Bull. Univ. lowa 1: 51. Nov. 1888.

Halsted, Jour. Myc. 5: 9. March I889.

Kelsey,-Jour. Myc. 5:8I. June I889.

Swingle, Tr. Kans. Acad. 11: 66. I Nov. 1889.

Ell. in Cat. Pl. N. J. 594. 1880.

*Underw. \& Cook, Illustr. Fungi 9ı. 1889.

Humphrey, Rept. Mass. Exp. Sta. 8: 222. Jan. I 890.

*Ell. \& Ev. N. A. F. 2420.1890.

Webber, Rept. Nebr. Agr. 1889: 190 (50). I 890.

Swingle, Tr. Kans. Acad. 12 : 130. 30 March I891.

Halsted, Rept. N. J. Exp. Sta. 1890 : 350. June 1891.

Halsted, Bot. Gaz. 16 : 340. 15 Dec. I89I.

Williams, Bull. S. Dak. Exp. Sta. 29: 46. Dec. I891.

Williams, Bull. Torr. Bot. Club 19:83. 5 March 1892 .

Atk. Bull. Ala. Exp. Sta. 4I : 44. Dec. I 892.

McCarthy, Bull. N. Car. Exp. Sta. 84: 15. 21 April I892.

Millsp. Bull. W. Va. Exp. Sta. 24 : 5 Io. June 1892 .

Halsted, Rept. N. J. Exp. Sta. 1891 : $249 . \quad 1802$.

* Seymour \& Earle, E. F. 256, 257, 259. I May I893.

*Ell. \& Ev. Fung. Columb. 133. I893.

Halsted, Rept. N. J. Exp. Sta. 1892: 277, 346. I893.

* Shear, N. Y. Fungi 199. I 893.

Halsted, Rept. N. J. Exp. Sta. 1893 : 326. 1894.

* Seyınour \& Earle, E. F. 40ı. I July I 895.

Halsted, Rept. N. J. Exp. Sta. 1894: 356.1895.

Millsp. \& Nutt. Publ. Field Columb. Mus. Bot. 1:82. Jan. 1806.

Wager, Ann. Bot. 10: 297. pl. 15, 16 . Sept. 1806.

Pammel \& Carver, Proc. lowa Acad. 3 : I44. 1896.

Atk. Bull. Cornell Univ. 3 : I4. 25 June i 897.

Davis, J. J., Tr. Wis. Acad. 11 : 165. Aug. 1897.

* Seymour \& Earle, E. F. 454. I5 Oct. I 897.

Beal, Proc. Soc. Prom. Agr. Sci. 18: 37.1897.

Ell. in Hitchcock, Rept. Mo. Bot. Gard. 9 : 120. 20 April 1898.

Orton, Rept. Vt. Exp. Sta. 11: 202. Sept. 1898 and Contr. Bot. Vt. 2: 4. Sept. 1898 .

Berl. Icon. Phyc. 6. d. pl. I.pl. 2, f. I. 1808.

Halsted, Bull. Torr. Bot. Club 26: I8. I6 Jan. I809.

Halsted, Bull. Torr. Bot. Club 26: $382 . \quad$ i 8 July I899.

Orton, Rept. Vt. Exp. Sta. 12: I65. Dec. I899 and Contr. Bot. Vt. 7: 165. Dec. 1899. 
Halsted, Rept. N. J. Exp. Sta. 1898 : $368 . \quad$ I 899.

Herbst, Fungal Fl. Lehigh Valley 205. d. 1899.

Selby, Bull. Ohio Exp. Sta. 121 : 21, 37. Sept. 1900.

Hume, Rept. Fla. Exp. Sta. 1900: 39.1900.

Berl. Riv. Patol: Veg. 9: 9. d. f. 1. 20 Feb. 1901.

Halsted, Rept. N. J. Exp. Sta. $1900: 462$. pl. 1l. 1901

Sturgis, Rept. Conn. Exp. Sta. 24 : 262, 285, 291. 1901.

*Griffitlis, West Am. Fung. 46. 22 March 1902.

Ricker, Univ. of Maine Studies 3: 18. 30 April 1902.

Albugo Convolvulacearum, Henn.

See Albugo Ipomoex-pandurana, (S.) Swingle.

Albugo Ipomœæ-panduranæ, (S.) Swingle.

Nort. For notes on the structure of Aecidium Ipomare-pandurana, S. which some writers even at the present date quote incorrectly as a true Accidium, see Farl. Bot. Gaz. 14: 187-189. 16 Aug. 1889.

Whether Cystopus Convolvulacearum, Speg. is specifically distinct from this species as supposed hy some mycologists seems to us doubtful and at least the no. 1809 N. A. F. and 501 Rav. Fung. Am. cited by Berlese in Riv. Patol. Veg. 9: 25 under $C$. Convolvulacearum seem to us to be Albugo Ipomace-pandurane as is probably also Alhugo Convolvulaciarum of Hennings given below.

Swingle, Jour. Myc. 7 : 112 . April 1892.

Schrt. in Engler \& Prantl, Pflanzenfam. 1' : 112. d. Aug. 1893.

Underw. Proc. Ind. Acad. 1893: 32. Aug. I894.

Clements, Fl. Nebr. I : 58. d. I804.

Tracy \& Earle, Bull. Miss. Exp. Sta. 34 : 94. May 1805.

Underw. \& Earle, Bull. Ala. Exp. Sta. 80: 136. 28 June 1897.

Underw. Proc. Ind. Acad. 1896: 172. 1897.

Long, Bull. Torr. Bot. Club 27 : 586.14 Nov. 1900.

Earle in Mohr, Contr. U. S. Nat. Herb. 6: 152. 31 July I9oI.

Patterson, Bull. U. S. Agr. PI. Industry 8: 17. 3 Feb. 1902.

Stevens, Bot. Gaz. 38 : 300. f. 1, 2. 20 Oct. 1904.

* Barthol, Ell. \& Ev. Fung. Columb. 2002, 2003, 2004. 20 March 1905. Aecidium Ipomoex-pandurana, S.

S. Syn. Car. 69 (43) no. 454. d. 1822. Uredo Convolvuli, Spreng.

Spreng. in L. Syst. Veg. 16 ed. $41{ }^{1}$ : 572. d. 1827.

Hark. \& Moore, Cat. Pac. Fung. 26. Feb. 1880. Caoma convolvulatum, Lk.

Lk., L. Sp. Pl. ed. Willd. 62 : 49. d. 1825.

S. Syn. Am. Bor. 292 no. 2866.1834. Aecidium rutilum, Bon.

Bon. Abh. Nat. Ges. Halle 5 : $212(46)$. d. 1860. PAecidium Convolvuli, M. A. Curtis.

Curtis, M. A., Bot. N. Car. 123. 1867. Cystopus cubicus, Auct. Amer. p.p.

B. \& C. Jour. Linn. Soc. $10: 357.1869$.

B. Grev. 3: 58 (100). Dec. I874.

Farl. Bull. Bussey Inst. 1: 429. $d$. March 1876.

*Thm. Myc. Univ. 815. 1877.

Cke. Grev. $7: 46$. Dec. 1878.

* Rav. Fung. Am. 501. I881.

Kellerm. Bull. Wash. Lab. 1 : 78. Jan. 1885 and Tr. Kans. Acad. 9: 83. 1885.

ElI. in Cat. PI. N. J. 594. 1889.

Aecidium Ipomøa, B.

B. Grev. 3 : 60 (102). Dec. 1874 . 
Cystopus convolvulacearum, Zalewski.

Zalewski, Bot. Centralbl. 15: 223. I883.

*Ell. \&Ev. N. A. F. 1809 . 1887.

Kellerm. \& Carl. Tr. Kans. Acad. 10: 94. I887.

Langlois, Cat. PI. La. 29. I887.

Farl. Bot. Gaz. 14: 187, 188. Aug. I889.

Tubeuf-Smith, Diseases of Plants I27. I 897.

Ell. in Hitchcock, Rèpt. Mo. Bot. Gard. 9 : 120. 20 April 1898.

Berl. Riv. Patol. Veg. 9 : 25. d. f. 4. 20 Feb. 1901.

Cystopus Tragopogonis, Auct. Amer. p.p.

Berl. \& De Toni in Sacc. Syll. $7: 234 . d$. I 5 March 1888.

Bomm. \& Rouss. in Durand \& Pittier, Bull. Soc. Bot. Belg. $35^{1}$ : 156. I 806.

Berl. Icon. Phyc. 8. d.pl. 5. 1898.

Berl. Riv. Patol. Veg. 9 : I9. d.f. 3. 20 Feb. I90ı.

Cystopus Ipomex-pandurana, Farl.

Farl. Bot. Gaz. 14: 187, 189. Aug. I889.

Swingle, Tr. Kans. Acad. 11: 67, 68. $d$. I Nov. I889.

Halsted, Jour. Myc. 5: 203. Dec. I889.

Halsted, Bot. Gaz. 15: 323. Dec. I890.

* Seymour \& Earle, E. F. 47. I Jan. I890.

Halsted, Rusts, Smuts, Ergots and Rots 19. June 1890.

Halsted, Bull. N. J. Exp. Sta. 76: 28-30. f. 19. I890.

Swingle, Tr. Kans. Acad. 12: I3I. 30 March I89I.

Sacc. Syll. 9 : 34 I. $d$. I 5 Sept. I 89 I.

Halsted, Bot. Gaz. 16 : 340. Dec. 189 I.

Webber, Tr. St. Louis Acad. 6 : I I. I2 March 1892 and Contr. Bot. Dept. Univ. Nebr. 3: II. I4 June I892.

Halsted, Rept. N. J. Exp. Sta. 1891: 249. 1892.

* Seymour \& Earle, E. F. 334, 336, 337. I May 1893.

Halsted, Rept. N. J. Exp. Sta. 1892: 277, 348. I893.

Atk. Bull. Cornell Univ. $3:$ i 4.25 June I 897.

Townsend, Bull. Md. Exp. Sta. 60: 166. f. 60. March 1899.

Selby, Bull. Ohio Exp. Sta. I2 I : 56. Sept. I900.

Hume, Rept. Fla. Exp. Sta. 1900: 39. I900.

Berl. Riv. Patol. Veg. 9 : I4. $d$. 20 Feb. I90I.

Sturgis, Rept. Conn. Exp. Sta. 24: 289. I9OI.

Cystopus candidus, var. Convolvuli, Berl.

Berl. Icon. Phyc. 7. d. I 898 .

PAlbugo Convolvulacearum, Henn.

Henn. Hedw. 37 : 277.' 3I Dec. I898.

Albugo Tragopogonis, Auct. Amer. p.p.

Ell. \& Kelsey in Millsp. Publ. Field Columb. Mus. Bot. 1: 465. Nov. 1902. [Publ. 68.]

Albugo Platensis, (Speg.) Swingle.

Swingle, Jour. Myc. 7 : I1 3 . April 1892.

Long, Bull. Torr. Bot. Club. 27: 586. i4 Nov. 1900.

Patterson, Bull. U. S. Agr. Pl. Industry 8: 17. 3 Feb. I902.

*Barthol., Ell. \& Ev. Fung. Columb. 1605. Oct. 1902.

Cystopus Platensis, Speg.

Speg. Revista Argent. Hist. Nat: 1: 32 (7).d. I Feb. 1891 and Jour. Myc. $7:$ II5. $d$. April I892.*

Berl. Riv. Patol. Veg. 9 : 27. d. 20 Feb. I9oI.

Albugo Bliti, Vestergren.

*Vestergren, Micr. Sel. 586. Dec. I902. 
Albugo Portulacæ, (D C.) O. Kuntze.

Kuntze, O., Rev. Gen. PI. 2: 658. I891.

Swingle, Jour. Myc. 7: III. April 1892.

Schrt. in Engler \& Prantl, Pflanzenfam. $1^{1}:$ i12. d. f. 96. Aug. I893.

Underw. Proc. Ind. Acad. 1893: 32. Aug. I894.

Clements, FI. Nebr. I : 57. $d . p l . ~ 16, f .3 a, b .1894$.

Kellerm. \& Werner. Geol. Ohio 7" : 348. Jan. 1895.

Tracy \& Earle, Bull. Miss. Exp. Sta. 34: 94. May I 895.

Pammel, Proc. lowa Acad. 2 : 203. 1895.

Stevens, Rept. Ohio Acau. 4: 20. I806.

Underw. \& Earle, Bull. Ala. Exp. Sta. 80: 136. 28 June 1897.

Long, Bull. Torr. Bot. Club. 27; 586 . I4 Nov. 1900.

Earle in Mohr, Contr. U.S. Nat. Herb. 6 : 152.31 July 1901.

Stevens, F. L., Bot. Gaz. 32 : 79, 97. pl. 1. pl. 4, f. 44-46, 4s-50. 20 Aug. I90I and $32: 254$. 2I Oct. I90I.

Patterson, Bull. U. S. Agr. Pl. Industry 8: 17. 3 Feb. 1902.

* Kellerm. Ohio Fungi $\varepsilon_{3}$. d. Oct. 1902 .

Kellerm. Jour. Myc. 8 : I 20. $d$. I4 Oct. I902.

Cama candidum,'S. Am. Bor. p.p.

S. Syn. Am. Bor. 292 no. $2852 . \quad$ I 834.

Cystopus candidus, Pk. p.p. non Lev.

Pk. Rept. N. Y. Mus. 24: 103. 1872. Cystopus Portulaca, Lev.

Cke. Grev. $7: 46$. Dec. I 878.

Pk. Rept. N. Y. Mus 29: $51,76.1878$.

* Rav. Fung. Am. 500. I88I.

Farl. Bot. Gaz. 8: 336. d. Nov. 1883 .

Bundy, Geol. Wis. 1873-79, $1: 400.1883$.

Pk. in Day's Cat., Bull. Buf. Soc. 4: 213 (149). 1883.

Trel. Tr. Wis. Acad. 6 : I08 (4). Nov. 1884.

Bessey, Bull. lowa Agr. Coll. 1884: I40. Jan. $1885 . \quad[83 . \quad 1885$.

Kellerm: Bull. Wash. Lab. 1: 78. Jan. 1885 and Tr. Kans. Acad. 9:

Wint. \& Demet. Hedw. 24: 201 (25). Oct. 1885.

*Ell. \& Ev. N. A. F. 1808.1887.

Kellerm. \& Carl. Tr. Kans. Acad. 10 : 94. 1887.

Berl. \& De Toni in Sacc. Syll. 7: 235. d. 15 March 1888.

Halsted, Bot. Gaz. 13: 57. March I 888.

Halsted, Bull. lowa Agr. Coll. 1888: IOI. April i 888.

Tracy \& Gall. Jour. Myc. 4: 36. May 1888.

Macbr. \& Hitchcock, Bull. Univ. Iowa 1: 5I. Nov. 1888.

Halsted, Jour. Myc. 5 : I0. March I889.

Swingle, Tr. Kans. Acad. 11: 68. I Nov. I889.

Webber, Bull. Nebr. Exp. Sta. 1: 36I (91). I8 Dec. I 889.

Ell. in Cat. PI. N. J. 594.1889.

Jennings, Bull. Tex. Exp. Sta. 9: 25. May 1890.

Swingle, Tr. Kans. Acad. 12 : 131. 1890.

Webber, Rept. Nebr. Agr. 1889 : Igo (50). I 890.

Halsted, Bot. Gaz. 16 : 340. Dec. 1891.

Williams, Bull. S. Dak. Exp. Sta. 29: 46. Dec. I891.

Halsted, Rept. N. J. Exp. Sta. 1891: 249. 1892.

* Seymour \& Earle, E. F. 264. I May 1893.

*Ell. \& Ev. Fung. Columb. 45. 1893.

Halsted, Rept. N. J. Exp. Sta. 1892 : 278, 346. I 893.

Halsted, Rept. N. J. Exp. Sta. 1893: 327. 1894.

Millsp. \& Nutt. Publ. Field Columb. Mus. Bot. 1: 83. Jan. 1896.

Pammel \& Carver Proc. lowa Acad. 3 : $144 . \quad$ i 896.

- Atk. Bull. Cornell Univ. 3: 14. 25 June 1897.

Tubeuf-Smith, Diseases of Plants I 27. I 897. 
Halsted, Bull. Torr. Bot. Club. 25 : 333. 8 June I 898.

Orton, Rept. Vt. Exp. Sta. 11: 203. Sept. 1898 aníd Contr. Bot. Vt. 2 :- 5. Sept. 1898.

Halsted, Bull. Torr. Bot. Club. 25 : 577. 7 Nov. I898.

Berl. Icon. Phyc. 7. d. pl. 2, f. 2. pl. 3. I898.

Hume, Proc. Davenport Acad. $7: 253 . \quad$ I2 May 1899.

Berl. Riv. Patol. Veg. 9 : I6. d. f. 2 . 20 Feb. 1901.

* Griffiths, West Am. Fung. 31. 22 March I902.

Albugo pulverulentus, (B. \& C.) O. Kuntze.

NotE. This species, said to grow on some Composite in Cuba, cannot be traced in Herb. Curtis by the numbers cited in the original description and it is impossible to recognize the fungus from the description. All the species of Cystopus in Herb. Curtis from Cuba are labeled Cystopus cubicus.

Kuntze, O., Rev. Gen. Pl. 2 : 658. I89I.

Cystopus pulverulentus, B. \& C.

B. \& C. Jour. Linn. Soc. 10:357. d. I 869.

Berl. \& De Toni in Sacc. Syll. 7: 237. d. I5 March 1888.

Albugo Tragopogonis, (P.) S. F. Gray.

NotE. See note under Albugo pulverulentus, (B. \& C.) O. Kuntze.

Swingle, Jour. Myc. 7 : I12. April 1892.

Davis, J. J., Tr. Wis. Acad. 9 : 154.21 Aug. I 893.

Schrt. in Engler \& Prantl, Pflanzenfam. 1 1: I I2. d. Aug. I893.

Clements, FI. Nebr. I : 58. d. 1894.

Kellerm. \& Werner, Geol. Ohio $7^{2}$ : 348. Jan. I895.

Underw. \& Earle, Bull. Ala. Exp. Sta. 80: 136. 28 June 1897.

Stevens, F. L., Ber. Deutsch. Bot. Ges. 19 : 17I. pl. 8. 24 April I90I.

Earle in Mohr. Contr. U. S. Nat. Herb. $6:$ i 52.31 July I 901.

Stevens, F. L., Bot. Gaz. $32: 85,97$. pl. 3, pl. 4, f. 43, 53. 20 Aug. I901 and $32: 254$. 21 Oct. I901.

Patterson, Bull. U. S. Agr. Pl. Industry 8: 17. 3 Feb. I902.

* Griffiths, West Am. Fung. 336. 21 May 1902.

* Vestergren, Micr. Sel. $705^{b}$. Oct. I903.

Clint. Rept. Conn. Exp. Sta. 27 : 357. May I 904.

Cystopus cubicus, (Strauss) Lev.

Pk. Rept. N. Y. Mus. 25 : 9I. Sept. 1873.

Farl. Bull. Bussey Inst. 1: 429, 432. March 1876.

Burrill, Bull. III. Lab. $1^{1}$ : 57. Dec. 1876.

Pk. Rept. N. Y. Mus. $29: 76 . \quad$ I878.

Pk. Rept. N. Y. Mus. 30:77. Sept. I878.

*Ell. N. A. F. 205. 1879.

Farl. Bot. Gaz. 8 : 335. d. Nov. I 883.

Pk. in Day's Cat., Bull. Buf. Soc. $4: 212$ (I48). $188_{3}$.

Farl. Bot. Gaz. 9 : 4 o. March 1884.

Trel. Tr. Wis. Acad. 6 : 108 (4). Nov. 1884

Hark. Bull. Cal. Acad. 1: I63. 7 March I885.

Wint. \& Demet. Hedw. 24: 201 (25). Oct. I 885.

Pk. Rept. N. Y. Mus. 38 : II I. I885.

Arth. \& Holw. Bull. Minn. Surv. 3: 36. I Oct. I 887.

?Langlois, Cat. Pl. La. 29. 1887.

?Brendel, FI. Peoriana 2 ed. 69. I887.

Halsted, Bot. Gaz. 13: 57 . March 1888.

Halsted, Bull. lowa Agr. Coll. 1888: ı00. April 1888.

Tracy \& Gall. Jour. Myc. 4: 36. d. May i 888.

Macbr. Bull. Univ. lowa 1 : 5 I. Nov. 1888.

*Ell. \& Ev. N. A. F. 2208 . Feb. 1889.

Halsted, Jour. Myc. 5: io. March 1889. 
Farl. Bot. Gaz. 14: 188. Aug. 1889.

*Ell. \& Ev. N. A. F. 2421. April I 890. Cystopus spimulosus, D By.

Farl. Bull. Bussey Inst. 1: 429. March 1876.

Pk. Rept. N. Y. Mus. 29: 51, 76. I878.

Pk. in Day's Cat., Bull. Buf. Soc. 4: 213 (149). 1883.

Halsted, Rept. N. J. Exp. Sta. 1892: 278.1893. Cystopus Tragopogonis, Schrt.

Kelsey, Jour. Myc. 5 : 81. June 1889.

Swingle, Tr. Kans. Acad. 11: 68. I Nov. 1880.

Webber, Rept. Nebr. Agr. 1889: I 190 (50). 1890.

Williams, Bull. S. Dak. Exp. Sta. 29: 46. Dec. I891.

Webber, Tr. St. Louis Acad. 6: 3. 12 March 1892 and Contr. Bot. Dept. Univ. Nebr. 3: 4. 14 June 1892.

Williams, Bull. Torr. Bot. Club $19: 83.5$ March 1892.

* Seymour \& Earle, E. F. 291, 302.0 I May 1893.

Halsted, Rept. N. J. Exp. Sta. 1892: 278, 347. 1893.

* Sevinour \& Earle, E. F. 445. I July I 895.

Halsted, Rept. N. J. Exp. Sta. 1894: 355.1895.

*Ell. \& Ev. Fung. Columb. 878 . April 1806.

Pammel \& Carver Proc. lowa Acad. 3 : 144. 1896.

Atk. Bull. Cornell Univ. 3 : 14. 25 June 1897.

Tubeuf-Smith, Diseases of Plants 127. 1897.

Orton, Rept. Vt. Exp. Sta. 11: 203. Sept. 1898 and Contr. Bot. Vt. 2: 5. Sept. 1808 .

Berl. Icon. Phyc. 8. d. pl. 5. 1808.

Hume, Proc. Davenport Acad. 7: 253. 1899.

Berl. Riv. Patol. Veg. 9 : 19. d. $f .3 .20$ Feb. 1901.

Sturgis, Rept. Conn. Exp. Sta. 24: 286. I90I.

Cystopus Tragopogonis, var. spinulosus, J. J. Davis.

Davis, J. J., Tr. Wis. Acad. 11 : 165. Aug. 1897.

Albugo Tragopogonis, Auct. Amer. p.p. See Albugo Ipomoex-pandurana, (S.) Swingle.

Aleurina, Sacc.

Rehm, Ann. Myc. 1 : 514.10 Dec. 1903.

Aleurina Puscocarpa, (Ell. \& Holw.) Sacc. \& Syd.

Sacc. \& Syd. Syll. 16 : 739. I Feb. 1902.

Rehm, Ann. Myc. 1 : 514. d. 10 Dec. 1903.

Peziza fuscocarpa, Ell. \& Holw.

Ell. \& Holw. Jour. Myc. 1 : 5. $d$. Jan. 1885.

Arth. \& Holw. Bull. Minn. Surv. 3: 35. I Oct. I887.

Langlois, Cat. PI. La. 32. I887.

*Ell. \& Ev. N. A. F. 2325.1890.

Phxopezia fuscocarpa, Sacc.

Sacc. Syll. $8: 474 . d$. 20 Dec. 1889.

Durand, Bull. Torr. Bot. Club $27: 479 . d .29$ Sept. 1900.

Humaria fuscocarpa, Morg.

Morg. Jour. Myc. 8 : 189. 20 Dec. 1902.

Aleurina lignicola, (Rostr.) Sacc. \& Syd.

Sacc. \& Syd. Syll. 16: 739. I Feb. 1902.

Rehm, Ann. Myc. 1: 514. Io Dec. 1903.

Phroopezia lignicola, Rostr.

Rostr. Meddelelser om Groenl. 18 : 58 (16). d. 1894.

Sacc. Syll. $11: 415 . d$. July'1 895 .

Aleurina Lloydiana, Rehm.

Rehm, Ann. Myc. 1: 515. Io Dec. 1903.

Rehm, Ann. Myc. 2: 35.d. I5 Feb. 1904. 
Aleurina Novæ-Terræ, (Ell. \& Ev.) Sacc. \& Syd.

Sacc. \& Syd. Syll. 16: 738. $d$. I Feb. I902.

Rehm, Ann. Myc. 1: 5 I4. Io Dec. I903. Phaopeziza Nova-Terra, Ell. \& Ev.

Ell. \& Ev. Bull. Torr. Bot. Club 25 : 507. d. Io Sept. I 898.

Aleurina vinacea, (Clements) Sacc. \& Syd.

Sacc. \& Syd. Syll. 16: 740. I Feb. 1902.

Rehm, Ann. Myc. 1 : 515. Io Dec. 1903.

Phaopezia vinacea, Clements.

Pound, Clements and others, Bot. Surv. Nebr. $4:$ I6. $d$. 20 Jan. I 896.

Sacc. \& Lindau, Hedw. 36 : Rep. 7 : xxxi. 30 April 1897.

Sacc. \& Syd. Syll. 14: 78I. d. 20 Aug. I899.

Aleurina Wisconsinensis, Rehm.

Rehm, Ann. Myc. $2: 34$. $d$. I5 Feb. 1904.

Aleurodiscus amorphus, Rabh.

See Corticium amorphum, (P.) Fr.

Aleurodiscus disciformis, Pat.

See Peniophora disciformis, (D C.) Cke.

Aleurodiscus Mancinianus, Pat.

See Stereum Mancinianum, Sacc. \& Cúb.

Aleurodiscus Micheneri, (B. \& C.) Massee.

Note. Under the name Michenera Artocreas, B. \& C. this was made the type of the genus Michenera, a genus which, so far as the type species at least is concerned, cannot be accepted. Michenera Artocreas as shown by cultures is undoubtedly merely a conidial form of a Corticium, probably $C$. subgiganteum, $\mathrm{B}$. and the references to the conidial stage as given below are inserted here provisionally for convenience.

Massee, Jour. Linn. Soc. 25 :. I20. 8 June I 889.

Michenera Artocreas. B. \& C.

B. \& C. Jour. Linn. Soc. 10:333. d. I869.

Pk. Rept. N. Y. Mus. 28: 52 . 1876.

B. \& Br. Jour. Linn. Soc. $15: 83$. 3 March 1876.

Sacc. Cub. \& Manc. Syll. 6: 653. d. I Aug. I 888.

Peirce, Bull. Torr. Bot. Club 17: 306. pl. rlo, f. $k-n$. Dec. I 890

Pat. Bull. Soc. Myc. $7: 42$ (3). pl. 4, f. 1-5. I89I.

*Ell. \& Ev. N. A. F. 3102. Nov. I894.

Henn. in Engler \& Prantl, Pflanzenfam. 11** : I20. d.f. 67 H.J. I898.

Pat. Essai Tax. Hymen. 67. d. $f .46$. I900. Artocreas Micheneri, B. \& C.

B. \& C. in B. \& Br. Jour. Linn: Soc. 14: 73. d. 1875 .

*EII. N. A. F. 105 . I 878 .

Massee, Jour. Roy. Micr. Soc. 1888 : I73 (I). April I 888.

Ell. in Cat. PI. N. J. 492. I889.

Aleurodiscus Oakesii, Cke.

See Corticium Oakesii, B. \& C.

Allantonectria, Earle.

Earle in Greene, PI. Baker. 2 : I I. $d .25$ March I9or.

Sacc. \& Syd. Syll. 16 : I I40. $d . \quad$ I Feb. 1902.

Allantonectria Yuceæ, Earle.

Earle in Greene, PI. Baker. 2: 12. d. 25 March I9oI.

Sacc. \& Syd. Syll. 16: I 140. I Feb. 1902.

Sacc. Syll. 17:: 778. d. 25 May I905. 
Allantospora radiclcola, Wakk.

Howard, Bull. Bot. Dept. Jamaica 11. 8: 18I. I90I " and Exp. Sta. Rec. 14: 58. I902.

West Indies.

Allescherina, Berl.

Note. This genus is certainly very closely related to Cryptovalsa, being distinguished from it only by the fact that the perithecia are separated and not collected in definite groups.

Berl. in Abbado, Malpighia 16: 300. d. 1902 and Jour. Myc. 10: 205. $d .24$ June I904.

Berl. \& Sacc. Icon. 3: 108, d. March 1905.

Allescherina deusta, (Ell. \& Martin) Berl.

Berl. in Abbado, Malpighia $16: 303 . d .1902$.

Berl. \& Sacc. Icon. 3: 109. d. pl. 136, f. 1. March 1905.

Diatrypella deusta, Ell. \& Martín.

Ell. \& Martin, Am. Nat. 16: 809. d. Oct. I 882.

Sacc. Syll. 2 : Add. p. ix. d. 13 Jan. I 883.

*Ell. N. A. F. 1184 . 1884 .

Calkins, Jour. Myc. $3: 70$. June 1887

* Relım, Ascomyceten 935. June 1888.

Rehm, Hedw. 27: 172. d. June 1888.

Sacc. Syll. $9: 478$. d. 15 Sept. I8g1.

Ell. \& Ev. Pyren. 593. d. May I 892 .

*Ell. \& Ev. Fung. Columb. 1242. May 1808 .

Diatrype deusta, Cke.

Cke. Grev. 14: 15. Sept. I885.

Allescherina eutypæformis, (Sacc.) Berl.

Berl. in Abbado, Malpighia 16: 306. d. 1902.

Berl. \& Sacc. Icon. 3: I1 o. d. pl. 137,f. 2. March 1905.

Diatrype quercina, (P.) Fr., var. lignicola, C \& E.

C. \& E. Grev. 5 : 54. d. Dec. 1876. Cryptovalsa entypaformis, Sacc.

Sacc. Mich. 2: 569. d. Dec. I882.

Sacc. Syll. 2: Add. p. viii. d. I3 June 1883.

Sacc. Syll. $9: 471$. d. 15 Sept. I891.

Ell. \& Ev. Pyren. 517. d. May 1892.

Pk. Rept. N. Y. Mus. 54 ${ }^{1}$ : I 57. Dec. 1901.

Allescherina sparsa, (Ell. \& Ev.) Berl.

Berl. in Abbado, Malpighia 16: 303. d. 1902.

Berl. \& Sacc. Icon. 3: 100. d. pl. 135, f. 2. March 1905.

Cryptovalsa sparsa, Ell. \& Ev.

Ell. \& Ev. Proc. Phil. Acad. 1890 : 224. d. I890.

Sacc. Syll. $9: 472 . d . \quad 15$ Sept. I891.

Ell. \& Ev. Pyren. 515. d. May I 892 .

Valsa sparsa, Lindau.

Lindau in Engler \& Prantl, Pflanzenfam. 1 $: 459 . \quad$ Feb. 1897.

Allophylaria Senecionis, Clements.

Clements, Bull. Torr. Bot. Club. 30 : 88. d. 28 Feb. 1903.

Alternaria.

Note. The references to species of this genus have been arranged by $\mathbf{A}$.

B. Seymour.

Alternaria Brassicæ, (B.) Sacc.

*Ell. \& Ev. N. A. F. 3506. 24 Jan. I 898.

* Seymour \& Earle, E. F. 515. 7 June I899.

Ricker, Univ. of Maine Studies 3: 2I. 30 April 1902.

Clint. Rept. Conn. Exp. Sta. 27 : 31 I , 332. May 1904. 
Alternaria Brassicæ, (B.) Sacc., var. Citri, Penz.

Langlois, Cat. PI. La. 27. 1887.

Alternaria Brassicæ, (B.) Sacc., var. macrospora, Sacc.

Clint. Rept. Conn. Exp. Sta. 27: 31 I, 353. May 1904.

Alternaria Brassicæ, (B.) Sacc., var. nigrescens, Pegl.

Note. The synonymy of this species is stated by Clinton, 1904. For other references to cucurbitaceous hosts see Alternaria, sp. indet.

Selby, Jour. Columbus Hort. Soc. 11 : 114 (2) pl. 1. Dec. I896.

Selby, Bull. Ohio Exp. Sta. 73 : 235. f. 3. Dec. 1896.

Selby, Rept. Ohio Acad. 5 : 70 (4). 1897.

Sturgis, Rept. Conn. Exp. Sta. 22 : 235. 1899.

Sturgis, Rept. Conn. Exp. Sta. 24: 275, 292. 1901.

Chester, Rept. Del. Exp. Sta. 13: 38 . f. 2-4. d. -1902.

Clint. Rept. Conn. Exp. Sta. 27 : 331, 370. May 1904.

Macrosporium cucumerinum, Ell.' \& Ev.

Ell. \& Ev. Proc. Phila. Acad. 1895 : 440. d. Nov. 1895.

Sacc. Hedw. 35 : Rep. 7 : xlix. 25 Feb. 1896.

*Ell. \& Ev. N. A. F. 3396. Feb. 1896.

Sacc. \& Syd. Syll. 14: 1095. d. 20 Aug. 1899.

Quaintance, Rept. Ga. Exp. Sta. 13: 357. Jan. I9or.

Griffin, Bull. Colo. Exp. Sta. 62: 7. April I90I.

Griffin, Bull. Colo. Exp. 64: 12. June I901.

Hume, Rept. Fla. Exp. Sta. 1901 : 86. d.f. I90I.

Norton, Rept. Md. Hort. Soc. 4 : 3I. I902.

Chester, Bull. Del. Exp. Sta. $57:$ 3. d. $f .1,2$. ' June 1902.

Chester, Rept. Del. Exp. Sta. 13: 36. d. f. 1, 5-7. 1902.

Alternaria chartarum, Preuss.

Pk. Rept. N. Y. Mus. 33 : 28 . Oct. 1883.

Langlois, Cat. PI. La. 27. I887.

*Ell. \& Ev. N.'A.'F. 2192 . Feb. I889.

Alternaria Citri, Pierce.

Pierce, Bot. Gaz. 33 : 234. d. 22 March 1902.

Orton, Yearb. U. S. Agr.1902: 717. I903.

Alternaria fasciculata, (C. \& E.) Jones \& Grout.

Note. This species and Alternaria Solani have been confused by various writers, some considering them to be the same, others writing so vaguely that it is impossible to state with certainty what species is implied. The present arrangement of these two species depends chiefly on the results of cultures and the synonymy given by Jones, $1896,1897,1898,1899$.

Jones \& Grout, Bull. Torr. Bot. Club 24: 257.d. pl. 308, f. 4-6. 29 May 1897.

Jones, L. R.. Rept. Vt. Exp. Sta. 10: 51. 1898.

*Ell. \& Ev. Fung. Columb. 1368. May 1899.

* Seymour \& Earle, E. F. 522. 7 June 1899.

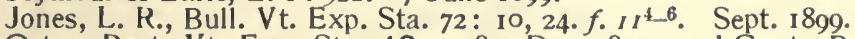

Orton, Rept. Vt. Exp. Sta. 12: 178. Dec. 1899 and Contr. Bot. Vt. 7: 178. Dec. 1899.

Macrosporium chartarum, Pk.

Pk. Bull. Buf. Soc. 1: 69. d. July 1873 .

Pk. Rept. N. Y. Mus. $25:$ 93. d. Sept. 1873 .

Cke. Bull. Buf. Soc. 3 : 200 . July 1877.

Johnson, Bull. Minn. Acad. 1: 367. 1880.

*Ell. N. A. F. 648 . 188 I.

Pk. in Day's Cat., Bull. Buf. Soc. 4: 211 (147). I883.

Sacc. Syll. 4: 539. d. Io April 1886. 
Ell. in Cat. PI. N. J. 570. $188 \%$.

Webber, Rept. Nebr. Agr. 1889 : 225 (85). I800.

* Ell. \& Ev. Fung. Columb. 396. I894.

Lindau in Engler \& Prantl, Pflanzenfam. I 1**: 484. Feb. 1900. Macrosporium fasciculatum, C. \& E.

C. \& E. Grev. $6: 6 . d . p l .96, f .30$. Sept. I 877.

C. \& E. Grev. 6 ; 88. March 1878 .

* Ell. N. A. F. 52.1878.

*Thm. Myc. Univ. 1870. 1881.

Sacc. Syll. 4: 525. d. Io April 1880.

Ell. in Cat. Pl. N. J. 570. I889.

* Ell. \& Ev. Fung. Columb. 399. I894.

Pound, Clements and others, Bot. Surv. Nebr. 4 : 39. 20 Jan. 1896. Macrosporium Maydis, C. \& E.

C. \& E. Grev. 6 : 87. d. March 1878.

Kellerm. Bull. Wash. Lab. 1: 78. Jan. 1885 and Tr. Kans. Acad. 9 : 83. 1885 .

Sacc. Syll. 4: 536. d. Io April 1886.

*Wint. Fung. Eur. 3592. 1886.

Kellerm. \& Carl. Tr. Kans. Acad. 10: 94. 1887.

Langlois, Cat. PI. La. 31. 1887.

Ell. in Cat. PI. N. J. 570. I880.

*Ell. \& Ev. N. A. F. 3098 . April I894.

*Ell. \& Ev. Fung. Columb. 383. 1894.

Ell. Rept. Del. Exp. Sta. 6 : 32. Jan. I 895

McClatchie in Reid's Hist. Pasadena 6I8. I 895.

Pound, Clements and others, Bot. Surv. Nebr. $4: 39.20$ Jan. 1896. Millsp. \& Nutt. Publ. Field Columb. Mus. Bot. 1 : 96. Jan. 1896.

* Shear, N. Y. Fungi 393. March I806.

McClatchie, Proc. S. Cal. Acad. 1:376. 5 June 1897. Macrosporium Tomato, Cke.

*Rav. Fung. Am. 603. I882.

Cke. Grev. 12 : 32. d. Sept. 1883.

Sacc. Syll. $4:$ :534. d. Io April I 886.

Pk. Rept. N. Y. Mus. 40: 65. May 1888.

Gall. Rept. U. S. Agr. 1888: 341. d. pl. 3, f. 1-7. I889.

*Ell. \& Ev. N. A. F. 2484 . April I 890.

Thax. Rept. Conn. Exp. Sta. 14: 95 (17). 180I.

Millsp. Bull. W. Va. Exp. Sta. 24: 516. June 1892.

Halsted, Rept. N. J. Exp. Sta. 1894: 360. I 895.

Millsp. \& Nutt. Publ. Field Columb. Mus. 1 : 96. Jan. 1896.

Underw. \& Earle, Bull. Ala. Exp. Sta. 60: 262. Feb. 1896.

Stewart, Rept. N. Y. Exp. Sta. 14: 531. 1806.

Stewart, Bull. N. Y. Exp. Sta. I25: 305. pl. 8. July 1897.

Halsted, Bull. Penn. Dept. Agr. 28 : 17. I897.

Selby, Jour. Columbus Hort. Soc. 11 : I I 5 (3). I 897.

Anderson, J. R., Brit. Columb. Dept. Agr. 1897 : 54. 1897.

Jones, H. L., Oberlin College Lab. Bull. 9: 6 . I 5 June I 898.

Beach, Rept. N. Y. Exp. Sta. $16: 27$ I. pl. $9 . \quad$ I 898.

Massee, Textbk. Pl. Diseases 325. d. f. 89 . 1899.

Earle, Bull. Ala. Exp. Sta. I08: 21. April 1900.

Quaintance, Rept. Ga. Exp. Sta. 13: 358 . pl. 7. Jan. Igor.

Clint. Rept. Conn. Exp. Sta. $27: 365$. May 1904.

Halsted, Rept. N. J. Exp. Sta. 1903: 546. Ig04.

Alternaria Solani, Sorauer, p. p.

Sorauer, Zeitschr. Pflanzenkr. 6 : 6. 1896.

Alternaria Tomato, Jones.

Jones, Bull. Torr. Bot. Club $23: 1353$. 25 Sept. I 896. 
Alternaria hispidula, Ell.

Ell. Bull. Torr. Bot. Club 10 : 52. d. May 1883 .

Sacc. Syll. 4 : $546 . d$. Io April 1886.

Alternaria lancipes, Ell. \& Ev.

Ell. \& Ev. Jour. Myc. 4: 45. d. May I 888.

Sacc. Syll. $10: 679 . d$. 30 June 1892.

Alternaria Solani, (Ell. \& Martin) Jones \& Grout.

NotE. See note under Alternaria fasciculata.

Jones, L. R., Bull. Torr. Bot. Club 23 : 353. 25 Sept. 1896.

Selby, Bull. Ohio Exp. Sta. 73 : 241. Dec. 1896.

* Seymour \& Earle, E. F. 521. 7 June I899.

Jones, L. R., Bull. Vt. Exp. Sta. 72: I6. f. 8-10, I I $^{1-3}$. Sept. I 899.

Orton, Rept. Vt. Exp. Sta. 12 : 179. Dec. 1899 and Contr. Bot. Vt. 7: 179. Dec. 1899.

Earle, Bull. Ala. Exp. Sta. I08: 32. d. April 1900.

Selby, Bull. Ohio Exp. Sta. I2 I : 50. f. 44. Sept. 1900.

Sturgis, Rept. Conn. Exp. Sta. 24: 283, 290. 1901.

Norton, Rept. Md. Hort. Soc. $4: 31.1902$.

Halsted, Rept. N. J. Exp. Sta. 1902 : 422.1903.

Jones, Tr. Mass. Hort. Soc. 1903: 150.1903.

Macoun, Rept. Ont. Agr. Union 1902: 42. 1903.

Clint. Rept. Conn. Exp. Sta. $27: 320,349,365$. May 1904.

Orton, Yearb. U. S. Agr. 1903: 552, 553.1904.

Macrosporium Solani, Ell. \& Martin.

Ell. \& Martin, Am. Nat. 16 : I003. d. Oct. 1882.

*Ell. N. A. F. 1265 . I884.

Kellerm. Bull. Wash. Lab. 1 : 78. Jan. 1885 and Tr. Kans. Acad. 9 : 83. I 885.

Arth. Rept. N. Y. Exp. Sta. 4: 250. Jan. 1886.

Kellerm. \& Carl. Tr. Kans. Acad. 10: 94. 1887.

Langlois, Cat. PI. La. 31. I887.

Ell. in Cat. PI. N. J. 570. 1880.

*Ell. \& Ev. N. A. F. 2485 . April 1800.

Halsted, Rusts, Smuts, Ergots and Rots, 19. June 1890.

Gall. Bull. U. S. Agr. Veg. Pathol. I I : 88. 1890.

Webber, Rept. Nebr. Agr. 1889 : 225 (85). 1800.

Chester, Bull. Del. Exp. Sta. 15: 14. Jan. I 892.

Briosi \& Cav. Fung. Piante Colt. Igo. d.f. April I892.

Chester, Rept. Del. Exp. Sta. 4: 58. d. f. 4-7. 1892.

Woolverton Jour. Hamilton Assoc. 8 : 86. $f .11 .1892$.

Pammel, Bull. lowa Exp. Sta. 20: 719. Feb. 1893.

* Seymour \& Earle, E. F. 339, 340. I May 1893.

Gall. Agr. Sci. 7: 370 . Sept. 1803.

Rolfs, Bull. Fla. Exp. Sta. 21 : 36. Oct. 1893.

Burrill, Proc. Soc. Prom. Agr. Sci. 12: 29. 1893.

Chester, Rept. Del. Exp. Sta. 5: 67. 1893.

Fletcher, Rept. Exp. Farms Can. 1892: 2I. I 893.

Gall. Proc. Soc. Prom. Agr. Sci. 14: 46. I 893.

Halsted, Rept. N. J. Exp. Sta. $1892: 348.1893$.

Jones, L. R., Rept. Vt. Exp. Sta. 6: 67. pl. 4. 1893.

Piper, Bull. Wash. Exp. Sta. 8 : 137. June 1893.

Lamson, Bull. N. H. Exp. Sta. 22: 3. d. May 1894.

*Ell. \& Ev. Fung. Columb. 398.1894.

Gall. Rept. U. S. Agr. 1893: 260, 26I. f. I. 1894.

Sturgis, Rept. Conn. Exp. Sta. 17: Ior. 1894.

Goff, Rept. Wis. Exp. Sta. 10: 245. I894.

Clint. Bull. III. Exp. Sta. 40 : 137. f. 2. April 1895. 
Fletcher, Bull. Cent. Exp. Farm Can. 23: 24. f. 5. April 1895.

Taft \& Davis, Bull. Mich. Exp. Sta. 121: 65. April 1895.

Tracy \& Earle, Bull. Miss. Exp. Sta. 34: 121. May 1895.

Jones, L. R., Bull. Vt. Exp. Sta. 49 : 91, 93. $f$. 1. 94. $f .2$. Dec. 1895.

Selby, Jour. Columbus Hort. Soc. 10 : 140 (2). Dec. 1895.

Halsted, Rept. N. J. Exp. Sta. 1894: 354, 360. f. 42.1895.

M.Clatchie in Reid's Hist. Pasadena 618. 1895.

Sturǵis, Rept. Conn. Exp. Sta. 18: 127. 1895.

Stewart, Bull. N. Y. Exp. Sta. 101: 72, Feb. 1806.

Garman, Bull. Ky. Exp. Sta. 61 : 18. March 1806.

*Ell. \& Ev. Fung. Columb. 891 . April 1806.

Jones, L. R., Rept. Vt. Exp. Sta. 9: 74, 79, 85. text-f. 12. pl. 1, f. $1-3$. pl. $2, f_{.} 8$. pl. $3, f_{.}$10. 1806 .

McClatchie, Proc. S. Cal. Acad. 1 ! 376. 5 June 1897.

Atk. Bull. Cornell Univ. 3: 40.25 June 1897.

Roberts \& Clint. Bull. Cornell Exp. Sta. 140: 59. $f .123$. Nov. 1897.

Halsted, Bull. Penn. Dept. Agr. 28: 10. 1897.

Stewart, Rept. N. Y. Exp. Sta. 15: $498 . \quad 1897$.

Tubeuf-Smith, Diseases of Plants 517.1897.

Anderson, J. R., Brit. Columb. Dept. Agr. 1897 : 54.1897.

Rolfs, Bull. Fla. Exp. Sta. $47: 124 . d$. $p l .1, f .1$. Sept. 1898.

Stewart, Rept. N. Y. Exp. Sta. 16: 378 . 1898.

Tillinghast \& Adams, Bull. R. 1. Exp? Sta. 52: 11. Feb. 1899.

Lindau in Engler \& Prantl, Pflanzenfam. 11**: 484 . Feb. 1900.

Hume, Rept. Fla. Exp. Sta. 1900: 43. 1900.

Earle in Mohr, Contr. U. S. Nat. Herb. $6: 247,31$ July 1901.

Ricker, Univ. of Maine Studies 3: 23. 30 April 1902.

* Barthol., Ell. \& Ev. Fung. Columb. 1831. 28 Dec. 1903. Macrosporium Solanl, Cke.

Cke. Grev. 12 : 32. d. Sept. 1883.

*Ell. N. A. F. 1265 b. I885. [Issued with Cent. XIV.]. Macrosporium Cookei, Sacc.

Sacc. Syll. 4: 530.d. 10 April 1886.

Stevens, F. L., Rept. Ohio Acad. 4: 23. 1896.

Atk. Bull. Cornell Univ. 3 : 40. 25 June 1897.

Underw. \& Earle, Bull. Ala. Exp. Sta. 80: 155. 28 June 1897.

Stevens, F. L., Rept. Ohio Acad. 5 : 64. 1897.

Earle in Mohr, Contr. U. S. Nat. Herb. $6: 246$. 31 July 1901.

Patterson, Bull. U. S. Agr. Pl. Industry 8: 30. 3 Feb. 1902.

Macrosporlum Solani, "Rav." sec. Gall.

Gall. Rept. U. S. Agr. 1888: $400 . p l$. $3, f .1-7.1880$.

Fairchild, Bull. U. S. Agr. Veg. Pathol. 6: 47, 48. 1894. Alternarla Solani, Sorauer, p. p.

Sorauer, Zeitschr. Pflanzenkr. 6; 6. 1806.

Jones, L. R., Rept. Vt. Exp. Sta. $9: 85,86.1896$.

Jones \& Grout, Bull. Torr. Bot. Club 24: 257. d. pl. 308, f. 1-3. 29 May 1897.

Jones, L. R., Rept. Vt. Exp. Sta. 10 : 45. d. 1897.

Gall. Farmers' Bull. U. S. Agr. 91 : 5. f. $t$. 1899.

Patterson, Bull. U. S. Agr. Pl. Industry 8: 27. 3 Feb. 1902.

Alternaria Solani, Sorauer, p. p.

See Alternarla fasciculata, (C. \& E.) Jones \& Grout.

Alternaria Solani, Sorauer, p. p.

See Alternaria SolanI, (Ell. \& Martin) Jones \& Grout.

Alternaria tenuis, Nees.

S. Syn. Am. Bor. 286. no. 2753. 1834.

Wint. \& Demet. Hedw. 24: 201 (25). Oct. 1885. 
Pk. Ŕept. N. Y. Mus. 35 : I $39 . \quad$ I 885.

Sacc. Syll. 4: $545 . d$. Io A pril 1886.

Ell. in Cat. Pl. N. J. 57I. I880.

?Atk. Bull. U. S. Agr. Office Exp. Sta. 33 : 282. $\quad$ I8g6.

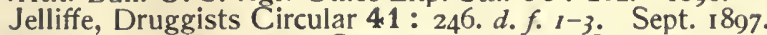

Jelliffe, Bull. Torr. Bot. Club 24: 48I. 30 Oct. 1897.

Jones, H. L., Oberlin College Lab. Bull. 9: 4. 15 June 1898.

Earle, Bull. Ala. Exp. Sta. 99: 285. Dec. I 898.

Berl. Fungi Moricolæ fasc. 7 : no. I4. pl. $4, f .4-6.20$ July I 899.

Jelliffe, Flora of Long Island 33. 1899.

Earle, Bull. Ala. Exp. Sta. 107: 317. April 1900.

Lindau in Engler \& Prantl, Pflanzenfam. 11** : 486. $f .252 \mathrm{H}$. Feb. I000.

Pound \& Clements, Bot. Surv. Nebr. 5: 19. 30 March I90I.

*Barthol., Ell. \& Ev. Fung. Columb. 1505. Dec. 190I.

Alternaria tenuis, Nees, var. condensata, Sacc.

Sacc. Mich. 2: 578. I Dec. I 882.

Alternaria Tomato, Jones.

See Alternaria fasciculata, (C. \& E.) Jones \& Grout.

Alternaria Violæ, Gall. \& Dorsett.

Dorsett, Bull. U.S. Agr. V. P.P. 23: is d.pl. 1-7. 28 Nov. I900 and Jour. Roy. Hort. Soc. 11. 26 : 49I. Dec. I9oI.

Sturgis, Rept. Conn. Exp. Sta. 24: 292. I90I.

Sacc. \& Syd. Syll. 16 : I080. $d$. I Feb. 1902.

Lewton-Brain, West Indian Bull. 4: 57. 1903.

Clint. Rept. Conn. Exp. Sta. 27: 368. pl. 28, f. d. May'1904.

Orton, Yearb. U. S. Agr. 1903 : 555. I904.

Alternaria, sp. indet.

Note. On account of their possible importance from an agricultural point of view the following references to unnamed species of Alternaria are here inserted. For addition references to cucurbitaceous hosts see Alternaria Brassica, (B.) Sacc, var. nigrescens, Pegl.

\section{On Aralia.}

Van Hook, Bull. Cornell Exp. Sta. 219: 183.f. 39-41. June I904.

\section{On Cucurbitaceæ.}

Webber, Rept. Nebr. Agr. 1889 : 225 (85). 1890.

Sturgis, Rept. Conn. Exp. Sta. 20: 267. 1897.

Selby, Bull. Ohio Exp. Sta. 121 : 39. $f$. 30. Sept. 1900.

Sturgis, Rept. Conn. Exp. Sta. 23: 272.1900.

Hedgcock, Science II. 16 : 136, 137. 25 July 1902.

Stone \& Smith, Rept. Hatch Exp. Sta. 14: 63. 1902.

Stone \& Smith, Rept. Hatch Exp. Sta. 15: 28, 3I. Jan. 1903.

\section{On Gossypium herbaceum.}

Atk. Bull. Ala. Exp. Sta. 27 : 168 (6). pl. 2, f. 5. May I89I.

Earle, Bull. Ala. Exp. Sta. 107: 302, 307, 312, 317. April 1900.

\section{On Linum.}

Bolley, Proc. Soc. Prom. Agr. Sci. $23: 85$ (4). 1902.

Bolley, Bull. N. Dak. Exp. Sta. 55: 187. March 1903.

\section{On Nicotiana.}

Sturgis, Rept. Conn. Exp. Sta. 23 : 265, 268. 1900. 


\section{On Olea.}

Sturgis, Rept. Conn. Exp. Sta: 24: 277. 1901.

Bioletti, Rept. Cal. Exp. Sta. 1898-1901 : 306. 1902.

\section{On Troprolum majus.}

Halsted, Rept. N. J. Exp. Sta. 1891 : 299. I 892.

Halsted, Rept. N. J. Exp. Sta. 1892: 290. d. 1893.

Sturgis, Rept. Conn. Exp. Sta. 24: 276. I90I.

\section{On various garden erops.}

Pound \& Clements, Minn. Bot. Studies 9: 649. d. 30 Nov. 1896.

Jones, L. R., Rept. Vt. Exp. Sta. 9: 85. pl. $l, f .4^{-6 .} p l .2, f .7 . p l$. 3, f. 9. I 896 .

Hedgcock, Science 11. 16 : 136, 137. 25 July 1902.

Alwisia bombarda, B. \& Br.

Lister, Jour. of Bot. 42: 135. d. May 1904. West Indies.

Alysidium, Kze.

Note. This genus was founded on Alysidium fulvum, Kze. of which the North American references are given below. As linited by some authors it includes certain species referred to Oidium, Oospora, etc. Since all the fungi of this group are either known or suspected to be imperfect forms of higher fungi it seems to us unnecessary to attempt to settle the question as to what is the oldest name of the genus to which they might be referred and we have preferred to retain most of them in Ospora, the genus under which several of our species were first described and under which they are to be found in most recent works.

Alysidium albipes, Pound \& Clements. See Oospora albipes, (Pk.) Sacc.

Alysidium candidulum, Pound \& Clements. See Oospora candidula, Sacc.

Alysidium compactum, Pound \& Clements. See Oospora compacta, (C. \& E.) Sacc. \& Vogl.

Alysidium cuboideum, Pound \& Clements. See Oospora cuboidea, Sacc. \& Ell.

Alysidium Cucumeris, Pound \& Clements. See Oospora Cucumeris, Pk.

Alysidium fasciculatum, Pound \& Clements. See Oospora fasciculata, (B.) Sacc. \& Vogl.

Alysidium fulvum, Kze.

Pound \& Clements, Minn. Bot. Studies 9 : 650. 30 Nov. 1896. Oidium fulvum, Lk.

S. Syn. Am. Bor. 285 no. 2745. 1834.

Pk. Rept. N. Y. Mus. 26 : 79. April 1874.

Frost in Cat. PI. Amherst 87. 1875.

Cobb, List Pl. Amherst 48. 1887.

Bennett, Cat. PI. R. I. 90. 1888. Oospora fulva, Sacc. \& Vogl.

Sacc. Syll. 4: 22. d. Io April 1886.

Lindau, Rabh. Krypt.-FI. 18: 46. d. I6 May 1904.

Alysidium hyalinulum, Pound \& Clements. See Oospora hyalinula, Sacc.

Alysidium insulare, Pound \& Clements. See Oospora insularis, (Thm.) Sacc. \& Vogl.

Alysidium lactis, Pound \& Clements. See Oospora lactis, (Fres.) Sacc. 
Alysidium ovalisporum, Pound \& Clements.

See Oospora ovalispora, (B.) Sacc. \& Vogl.

Alysidium pallidum, Pound \& Clements.

See Oospora paliida, (B. \& Rav.) Sacc. \& Vogl.

Alysidium pulvinatum, Pound \& Clements.

See Oospora puivinata, (B. \& C.) Sacc. \& Vogl.

Alysidium Tulipiferæ, Pound \& Clements.

See Oospora Tulipiferz, Ell. \& Martin.

Alytosporium, Ehrb.

NOTE. A number of the species of this genus are probably no more than the sterile forms of some higher fungi such as Thelephoracea. Certain other species have been referred to other genera of Fung $i$ Imperfecti.

Alytosporium croceum, Lk. See Sporotrichum croceum, Kze.

Aly tosporium fulvum, Lk.

S. Syn. Am. Bor. 274 no. 2555. I 834 .

Alytosporium fuseum, Lk.

See Trichosporium fuscum, (Lk.) Sacc.

Alytosporium murinum, Lk.

See Trichosporium murinum, (Lk.) Sacc.

Alytosporium psittacinum, Lk.

S. Syn. Am. Bor. 274 no. 2553. 1834.

Aly tosporium pteridicola, S. nom. nud.

NoTE. This name is cited at the place referred to below but we have not been able to trace it elsewhere.

Cke. Grev. 20 : 22. Sept. 1891.

Amanita abrupta, Pk.

Pk. Bull. Torr. Bot. Club 24: 138. d. 30 March I897.

Underw. \& Earle, Bull. Ala. Exp. Sta. 80: 253. d. 28 June 1897.

Lindau \& Syd. Hedw. 37 : Beibl. 7 : I. 9 April I 898.

Lloyd, Volvæ 7, I4. $d . \quad$ I 898 and Rev. Myc. 21:93. July I 899.

Sacc. \& Syd. Syll. 14: 63. d. 20 Aug. 1899.

Williams, Asa Gray Bull. 7 : $79 . \quad$ Aug. I 899.

Mcllvaine \& Macadam, Am. Fung. 23. d. 1900.

Earle in Mohr, Contr. U. S. Nat. Herb. 6 : 228. 3r July 190 I.

Amanita adnata, W. G. Smith.

See Amanitopsis adnata (W. G. Smith) Sacc.

Amanita affinis, Frost.

See Amanita Frostiana, Pk.

Amanita agglutinata, Lloyd.

See Amanitopsis agglutinata, (B. \& C.) Sacc.

Amanita aspera, $P$.

Muhl. Cat. Pl. Am. Sept. I ed. 104. I813, 2 ed. 107. I8I8.

S. Syn. Car. 79 (53) no. 552. 1822.

Sacc. Cub. \& Manc. Syll. 5 : 19. d. 28 May I 887.

Lloyd, Volvæ 7. $d$. I 898 and Rev. Myc. $21: 93$. July 1899.

Mcllvaine \& Macadam, Am. Fung. 23. d. 1900.

Agaricus asper, Fr.

S. Syn. Am. Bor. I45 no. Io. I 834 .

Curtis, M. A., Bot. N. Car. 83. 1867.

Cooke, Handbook ı. d.. I 871 .

Johnson, Bull. Minn. Açad. 1; 209. 1877 and Rept. Minn. Surv. 5:

67. 1877 . 
Bundy, Geol. Wiš. 1873-79, 1 : 396. 1883.

Morg. Jour. Myc. 3: 30. d. March 1887.

Amanita aterrima, Raf.

Raf. Précis 49. $d$. 1814 .

NotE. It is impossible to recognize from the original descriptions either this species or Amanita asurea, Raf. It may be said, however, that neither of them appears to be an fmanita in the modern sense.

Amanita aurantiaca, S. See Amanita casarea, (Scop.) P.

Amanita azurea, Raf.

Raf. Précis 49. $d$. I814.

Amanita baccata, Fr. See Amanitopsis baccata, (Fr.) Sacc.

Amanita badia, $P$. See Amanitopsis vayinata, (Bull.) Roze.

Amanita bulbosa, P.

NOTE. It is difficult to say with certainty to what species of later writers the Amanitu bulbosa of Persoon is to be referred but as far as we can ascertain it is considered to be the same as Amanita Mappa, (Batsch.) Fr. The Agaricus bulbosus of Bulliard as represented by his plates no. 2 and 577 is in part Amanita phalloides and in part Amanita Mappa.

In Lloyd, Volvæ 21. 1898 Amanita bulbosa, Rav, is said to be the same as Amanita Ravenelii, B. \& C. but we have no note as to where an Amanita bulbosa, Rav. was ever printed.

See also the references given under Amanita phalloides to Amanita bulbosa, Auct. Amer. and note under Amanita solitaria, (Bull.) Fr.

Amanita cæsarea, (Scop.) P.

S. Syn. Car. $79(53)$ no. 546.1822.

Hitchcock, Cat. PI. Amherst 59. 1829.

Hitchcock, Cat. Pl. Mass. 644. 1833. 2 ed. 646 (126). 1835.

Sacc. Cub. \& Manc. Syll. 5: 8. . 28 May 1887.

Banning in Pk. Rept. N.Y. Mus. 44: 178 (66). Dec. 189I.

* Shear, N. Y. Fungi $1 . \quad 1803$.

Taylor, Rept. U. S. Agr. 1892 : 284. d. pl. I. 1893.

Atk. \& Schrenk, Jour. Mitch. Soc. 9 : 98 (4). April 1893.

Taylor, Food Products 2: 2I. 1893.

Pk. Country Gent. 59: 566 (42) f. 2 Aug. 1894.

Pk. Rept. N. Y. Mus. 47: 135 (0). Nov. 1804.

Kellerm. \& Werner, Geol. Ohio 72: 300. Jan. 1805 .

Underw. Bull. Ala. Exp. Sta. 73 : 341 . d. f. 2. Oct. 1896.

Webster, Am. Kitchen Mag. 6: 215 . Feb. 1897 and Taylor, Hdbk. Mushrooms 2: 24. 1807 .

Pk. Rept. N. Y. Mus. 48: 253 (155). pl. 15. March 1897.

Atk. Bull. Cornell Univ. $3: 24.25$ June 1897.

Underw. \& Earle, Bull. Ala. Exp. Sta. 80: 253. 28 June 1897.

Williams, Asa Gray Bull. $6: 83 \cdot f .13^{4}$. Oct. 1898 .

[Underw.] N. Y. Myc. Club 16.1898.

Lloyd, Volvæ I, 3.d. 13. d. 1898 and Rev. Myc. 21 : 91. July 1899.

Farl. Yearb. U.S. Agr. 1897: 458. I898. [Bull. 15.]

Henn. in Engler \& Prantl, Pflanzenfam. 11**: 275. d. 1898.

Williams, Asa Gray Bull 7 : 78 . Aug. 1899.

Child, Jour. Hamilton Assoc. 15: 105.1809.

Herbst, Fungal FI. Lehigh Valley 27.d. 1899.

Underw. Moulds 120. 1899 . 
Atk. Mushrooms $54,70 . d . p l .18, f .72 . p l .19, f .2 .20$ Oct. 1900.

Williams, Asa Gray Bull. 8 : 103. Oct. 1900.

Hyams, Bull. N. Car. Exp. Sta. I77 : 30. Dec. 1900.

Atk. in Bailey's Cycl. Am. Hort. 1044. 1900.

Mcllvaine \& Macadam, Am. Fung. 12. d. 1900.

Perry, Agricultural Student 6: 179. May 1900 and Ohio Univ. Bull. IV. $30: 8$. May 1900.

Trel. Rept. Mo. Hort. Soc. 43 : 236 (13). 1900.

Earle in Mohr, Contr. U. S. Nat. Herb. $6: 228.31$ July I9oI.

Conant, Bull. Boston Myc. Club 19: 5 Feb. I903.

MacKay, Tr. N. Scot. Inst. 11: 124. Aug. 1904.

Agaricus czesareus, Scop.

\section{S. Syn. Am. Bor. 145 no. 3. I834.}

Rav. Charleston Med. Jour. \& Rev. 6 : 190. March 1851.

B. \& C. Ann. Nat. Hist. III. $4: 284$ (i). d. Oct. 1859 .

Curtis, M. A., Bot. N. Car. 83. 1867.

Curtis, M. A., Jour. Roy. Hort. Soc. II. 2 : 72, 74. 1870.

Pk. Rept. N. Y. Mus. 23 : 68. $d$. 1872.

Cke. \& B. Fungi 88. 1875.

Frost in Cat. Pl. Amherst 62. 1875.

* Rav. Fung. Am. $406 . \quad 1878$.

Morg. Jour. Cin. Soc. $6: 57$. d. April 1883.

Pk. Rept. N. Y. Mus. 33: 4 I. $d$. Oct. I883.

Rav. S. Car. Resources 354. 1883.

Porcher in Wood's Hdbk. Med. Sci. 3 : 275. pl. 12, f. 3 Er 5.1886.

Morg. Jour. Myc. 3: 26. d. March 1887 .

Cobb, List Pl. Amherst 31. I887.

Ell. in Cat. PI. N. J. 468.1889.

Eby, Fungi of Lancaster Co. 7. I 894.

Taylor, Hdbk. Mushrooms $5:$ 5. d. pl. 14, f. 1-4. 1898.

Amanita aurantiaca, S. nom. nud.

S. Syn. Car. 79 (53) no. 547. 1822.

Lloyd, Volvæ 2I. I 898.

Agaricus pratorius, Fr. p.p.

NoTF. Under this name in the citation below reference is made to an unpublished description by Schweinitz. Agaricus pratorius, Fr. however, is an Amanitopsis and therefore the Agaricus pratorius of Fries's Epicrisis does not belong here. As far as the reference to Schweinitz is concerned it is probable but not certain that it relates to the Amanita aurantiaca of S. Syn. Car. 547.

Fr. Epicr. I1. 1836-38.

Amanita calyptrata, Pk.

Pk. Bull. Torr. Bot. Club 27 : 14. d. 24 Jan. 1900.

Mcllvaine \& Macadam, Am. Fung. 26. $d$. 1000.

Sweetser, Bull. Pacific Univ. $1^{4}:$ 12. $f . c^{4}$. May 1901.

Sacc. \& Syd. Syll. 16 : I. $d$. I Feb. 1902.

Amanita calyptrata, Pk., var. albescens, Pk.

Pk. Rept. N. Y. Mus. 53: 840. d.pl. A, f. $1-5$. I9 March 1901.

\section{Amanita candida, Pk.}

Pk. Bull. Torr. Bot. Club 24: 137. d. 30 March 1897.

Underw. \& Earle, Bull. Ala. Exp. Sta. $80: 253 . d$. 28 June 1897.

Lindau \& Syd. Hedw. 37 : Beibl. 7 : 1. 9 April i 898.

Lloyd, Volvæ 6. d. 14. $d$. 1898 and Rev. Myc. 21: 93 . July 1899.

Sacc. \& Syd. Syll. 14: 63. d. 20 Aug. I 899.

Williams, Asa Gray Bull. 7 : 79. Aug. 1899 . 
Williams, Asa Gray Bull. 8 : 103. Oct. 1900.

Mcllvaine \& Macadam, Anı. Fung. 20. d. 1900.

Earle in Mohr, Contr. U. S. Nat. Herb. 6 : 228. 31 July Igor.

Amanita Ceciliæ, B. \& Br.

See Amianitops is strangulata, (Fr.) Roze.

Amanita chlorinosma, Pk.

Pk. in Austin, Bull. Torr. Bot. Club 6 : 278. d. Dec. 1878.

Underw. \& Earle, Bull. Ala. Exp. Sta. 80: 254. 28 June 1897.

Lloyd, Volvat 7. d. I 5. d. I 898 and Rev. Myc. 21 : 93 . July 1899.

Williams, Asa Gray Bull. 7 : 79. Aug. 1899.

Williams, Asa Gray Bull. 8: 103. Oct. 1900.

Mcllvaine \& Macadan, Am. Fung. 25. d. pl. 8, f. 1. 1900.

Earle in Mohr, Contr. U. S. Nat. Herb. 6 : 228. 3I July I9oI. Agaricus chlorinosmus, Pk.

Pl. in Austin, Bull. Torr. Bot. Club $6: 278$. d. Dec. 1878 .

Austin, Bull. Torr. Bot. Club $6: 208$. March 1879.

Pk. Bot. Gaz. 4: 137. d. March I879.

Ell. in Cat. Pl. N. J. 468.1889.

Tricholoma chlorinosmum, Sacc. Syll.

Sacc. Cub. \& Manc. Syll. 5 : Ior. d. 28 May I 887.

Amanita clreinata, Schum.

See Amanita rubescens, P., var. circinata, P.

Amanita citrina, S. Syn. Car. 544.

See A manita phalloides, Fr.

Amanita cothurnata, Atk.

Atk. Mushrooms 66. d. pl. $17, f .68, f .69,70.20$ Oct. 1900 and Rhodora 3: 40. I Feb. IgoI.

Amanita crenulata, $\mathrm{Pk}$.

Pk. Bull. Torr. Bot. Club $27:$ 15. d. 24 Jan. 1900.

Mcllvaine \& Macadam, Am. Fung. 27. d. 1900.

Sacc. \& Syd. Syll. 16: 2. d. I Feb. I902.

White, Bull. Torr. Bot. Club 29: 562. 26 Sept. 1902.

Conant, Bull. Boston Myc. Club I9: 5 Feb. I903.

Amanita daucipes, B. \& Mont.

See Amanitopsis daucipes, (B. \& Mont.) Sacc. Syll.

Amanita excelsa, Fr.

Sacc. Cub. \& Manc. Syll. 5 : I4. $d .28$ May I 887.

Lloyd, Volvæ 6. d. 13. I 898 and Rev. Myc. 21 : 92. July I 899.

Mcllvaine \& Macadam, Am. Fung. 17. d. 1900.

Agaricus excelsus, Fr.

S. Syn. Am. Bor. 145 no. 7. 1834.

Rav. Charleston Med. Jour. \& Rev. 6 : 190. March 1851 .

Andrews, Proc. Boston Soc. 5: 322 . April 1856.

Curtis, M. A., Bot. N. Car. 83. 1867.

Cooke, Handbook 8. $d$. 1871 .

Frost in Cat. Pl. Amherst 62. I 875.

Johnson, Bull. Minn. Acad. 1 : 209. 1877 and Rept. Minn. Surv. 5 : 67. 1877 .

Hark. \& Moore, Cat. Pac. Fung. 2. Feb. 1880.

Morg. Jour. Myc. $3: 28$. d. March 1887.

Cobb, List Pl. Amherst 31. I887.

Bennett, Cat. PI. R. I. 76. I 888 .

Eby, Fungi of Lancaster Co. 7. 1894.

Amanita farinosa, S.

See Amanitopsis farinosa, (S.) Atk. 
Amanita flavoconia, Atk.

NotE. It is possible that some of the earlier notices given under Amanita Frostiana, Pk. may refer to this species.

Atk. Jour. Myc. 8 : I IO. d. I4 Oct. 1902.

Hay, Bull. Nat. Hist. Soc. N. B. 5 : 114.1903.

Pk. Rept. N. Y. Mus. 564: 2I. d. May 1903. [Bull.67.]

Sacc. Syll. $17:$ I. d. 25 May 1905.

Amanita flavorubens, Mont.

Mont. Syll. Crypt. 96. $d .1856$.

Sacc. Cub. \& Manc. Syll. 5 ? I7. d. 28 May I887.

Kellerm. \& Werner, Geol. Ohio $7^{2}$ : 300. Jan. I895.

Millsp. \& Nutt. Publ. Field Columb. Mus. Bot. 1: 1 57. Jan. I 890. Henn. in Engler \& Prantl, Pflanzenfam. $11 * *: 275 . \quad$ I 898.

Lloyd, Volvæ 7. d. I5. d. I 898 and Rev. Myc. 21: 93 . July I899. Agaricus flavorubens, B. \& Mont.

Mont. Syll. Crypt. 96. $d$. I 856.

Amanita flavorubescens, Atk.

Atk. Jour. Myc. 8 : i I I. d. I4 Oct. I902.

Sacc. Syll. 17 : I. d. 25 May I905.

Amanita floceocephala, Atk.

Atk. Mushrooms 62. d. f. 63 . 20 Oct. I900 and Rhodora $3: 40$. I Feb. I90I.

White, Bull. Torr. Bot. Club 29: 562. 26 Sept. 1902.

Amanita formosa, Gonn. \& Rabh.

See Amanita muscaria, (L.) P., var. formosa, P.

Amanita Frostiana, Pk.

NotE. See note under Ámanila favoconia, Atk.

Pk. Rept. N. Y. Mus. 33: I8, 44. d. Oct. 1883.

Sacc. Cub. \& Manc. Syll. 5 : 14. d. 28 May I887.

Underw. \& Earle, Bull. Ala. Exp. Sta. 80: 254. 28 June I 897.

Williams, Asa Gray Bull. 6 : 83. Oct. 1898 .

Henn. in Engler \& Prantl, Pflanzenfam. 11** : 275. I898.

Lloyd, Volvæ 5.d. I3. d. I898 and Rev. Myc. 21 : 92 . July I899.

Taylor, Hdbk. Muslirooms 5: Io. 1898.

Pk. Bull. N. Y. Mus. 6 : I9I. July I899.

Williams, Asa Gray Bull. 7 : 78. Aug. I 899 .

Underw. Moulds I20. I899.

Atk. Mushrooms 54. d. 20 Oct. I000.

Williams, Asa Gray Bull. 8 : 103. Oct. I900.

Mcllvaine \& Macadam, Am. Fung. I6. d. pl. 6, f. 5. I900 and Am. Kitchen Mag. 14: I 22. Dec. I900.

Atk. in Bailey's Cycl. A m. Hort. ro44. d. I900.

Earle in Mohr, Contr. U. S. Nat. Herb. 6 : 220. 3 I July Igor.

Brown \& Fernekes, Bull. Wis. Nat. Hist. Soc. 11. 2 : 46. Jan. I902.

Ricker, Univ. of Maine Studies $3: 55$. 30 April 1902.

White, Bull. Torr. Bot. Club 29 : 562. 26 Sept. 1902.

Longyear, Rept. Mich. Acad. 3 : 55. 1902.

Conant, Bull. Boston Myc. Club I9: 5 Feb. I903.

Hay, Bull. N. H. Soc. N. B. 5 : I I4. I903.

Longyear, Rept. Mich. Acad. 4: 115.1904.

Agaricus affinis, Frost, nom. nud.

Frost in Cat. PI. Amherst 62. I875.

Cobb, List Pl. Amherst 31. I 887.

Amanita affinis, Frost, nom. nud.

Frost in Cat. PI. Amherst 62. 1875 .

Lloyd, Volvæ 2I. $\quad$ I 898 . 
Agaricus muscarius, var. minor, $\mathrm{Pk}$.

Pk. Rept. N. Y. Mus. 23: 69. d. 1872.

Bundy, Geol. Wis. I873-79, 1 : 396. I 1883.

Amanita muscaria, var. minor, $\mathrm{Pk}$.

Pk. Rept. N. Y. Mus. 23: 69. $d$. 1872.

I. loyd, Volva 2I. I 898.

Agaricus Frostianus, Pk.

Pk. Rept. N. Y. Mus. 33: 18, 44. d. Oct. 1883.

Morg. Jour. Myc. $3: 28$. d. March 1887.

Amanita Frostiana, Pk., var. pallidipes, $\mathrm{Pk}$.

Pk. Rept. N. Y. Mus. 53: 855. $d$. 19 March 1901.

Sacc. \& Syd. Syll. 16 : 1110. I Feb. 1902.

Sacc. Syll. $17: 2$. d. 25 May 1905.

Amanita hyperborea, Karst.

See Amanitops is hyperborea, Karst.

Amanita incarnata, $P$.

See Volvaria bombycina, (Schreff.) Fr.

Amanita lenticularis, Fr.

See Lepiota ienticularis, (Lasch) Gillet.

Amanita livida, $P$.

See Amanitopsis vaginata, (Bull.) Roze, var. IVida, (P.) Pk.

Amanita magnivelaris, $\mathrm{Pk}$.

Pk. Rept. N. Y. Mus. 50: $96 . d .28$ March 1898.

Lloyd, Volvæ 4. $d$. 13. $d$. 1898 and Rev. Myc. 21 : 92 . July 1899.

Sacc. \& Syd. Syll. 14:64. d. 20 Aug. I899.

Mcllvaine \& Macadam, Am. Fung. 10. d. 1900.

Amanita Mappa, (Batsch) Fr.

Lloyd, Volvæ 4. d. 13. 1898 and Rev. Myc. 21 : 92. July 1899.

Williams, Asa Gray Bull. 8 : 104. Oct. 1900.

Mcllvaine \& Macadam, Am. Fung. 10. d. 1900.

White, Bull. Torr. Bot. Club 29 : 562.26 Sept. 1902.

Hay, Bull. Nat. Hist. Soc. N. B. 5 : 114.1903.

Schaffner, Ohio Nat. 4: 17. Nov." 1903.

Agaricus Mappa, Batsch.

Curtis, M. A., Bot.' N. Car. 83. 1867.

Pk. Rept. N. Y. Mus. $22: 76$. 1869.

Frost in Cat. PI. Amherst 62. 1875 .

Johnson, Bull. Minn. Acad. 1 : 209. 1877 and Rept. Minn. Surv. 5 : 67. 1877.

Palmer, Mushrooms pl. 1o, f. 4. 1885.

Morg. Jour. Myc. 3: 27. d. March 1887.

Cobb, List PI. Amherst 3I. 1887.

Eby, Fungi of Lancaster Co. 7. $\quad$ I894.

Taylor, Hdbk. Mushrooms 5: i1. d. pl. 15, f. 9. 1898.

Amanita monticulosa, B. \& C.

B. \& C. Ann. Nat. Hist. II. 12: 418 (2). d. Dec. 1853.

Sacc. Cub. \& Manc. Syll. 5 : I8. d. 28 May I887.

Lloyd, Volvæ 7. $d$. 14. $d$. 1898 and Rev. Myc. 21 : 93. July 1899.

Henn. in Engler \& Prantl, Pflanzenfam. 11**: 275. 1898.

Williams, Asa Gray Bull. 8 : 103. Oct. 1900.

Mcllvaine \& Macadam, Am. Fung. 25. d. 1900.

Ricker, Univ. of Maine Studies $3: 55$. 30 April 1902.

Agaricus monticulosus, B. \& C.

B. \& C. Ann. Nat. Hist. II. 12: 418 (2). d. Dec. 1853 .

Curtis, M. A., Bot. N. Car. 83. 1867.

Morg. Jour. Myc. 3 : 30. d. March 1887. 
Amanita multisquamosa, $\mathrm{Pk}$.

Pk. Rept. N. Y. Mus. 53: 840. d. pl. B, f. $I-7$. I9 March IgoI.

Sacc. \& Syd. Syll. 16: i I10. I Feb. I902.

Sacc. Syll. $17: 2$. d. 25 May I905.

Amanita muscaria, (L.) P.

Grev. Scot. Crypt. Fl. 1 : pl. 54 . I823.

Porcher, Tr. Am. Med. Assoc. $2: 854 . \quad$ I 849.

Sacc. Cub. \& Manc. Syll. 5 : 13. d. 28 May 1887.

Banning in Pk. Rept. N. Y. Mus. 44: 177 (65). Dec. 1891 .

Rostr. Meddelelser om Groenl. 3 : $596 . \quad$ I 801 .

Millsp. Bull. W. Va. Exp. Sta. 24 : 502. June 1892.

Atk. \& Schrenk, Jour. Mitch. Soc. 9: 98 (4). April I 893.

*Shear, N. Y. Fungi I01. 1893.

Taylor, Rept. U. S. Agr. 1892: 284. d. pl. 2. 1893.

Pk. Country Gent. 59: 566 (43). 2 Aug. 1894.

Underw. Proc. Ind. Acad. 1893: 6r. Aug. I894.

Kellerm. \& Werner, Geol. Ohio $7^{2}$ : 300 . Jan. I 895.

Graves, Asa Gray Bull. 3 : 44. I Oct. I 895.

Gíbson, Mushrooms 287. pl. 37. I895.

Pound, Clements and others, Bot. Surv. Nebr. $4: 43$. 20 Jan. I 896.

Millsp. \& Nutt. Publ. Field Columb. Mus. Bot. $1:$ i 56. Jan. 1896.

Underw. Bull. Ala. Exp. Sta. 73: 344. f. 3. Oct. I 896.

Bates, Tr. Mass. Hort. Soc. $1896:$ i $73 . d$. 1896.

Webster, Am. Kitchen Mag. 6: 21 5. Feb. 1807.

Pk. Rept. N. Y. Mus. 48: 3 Io (2I 2). pl. 42. March 1897.

Underw. \& Earle, Bull. Ala. Exp. Sta. 80: 254. 28 June 1897.

Coville, Circ. U. S. Agr. I 3: 15. f. 14-20. I Dec. I897. [2 ed. f. 18-24.]

Farl. Gard. \& Forest 10: 469 . I Dec. I 897.

Chesnut, Yearb. U. S. Agr. 1896 : I44. I897.

Taylor, Hdbk. Mushrooms $3: 2 . p l$. E, f. 2. 1897.

Williams, Asa Gray Bull. 6: 82. pl. $1, f .3$. Oct. 1898 .

Chesnut, Bull. U. S. Agr. Div. Bot. 20: I2. f. I. 1898 and Farmer's Bull. U. S. Agr. 86: 4. f. 1. 1898.

Farl. Yearb. U.S. Agr. 1897: 457. d. pl. 22. I898. [Bull. 15.]

Henn. in Engler \& Prantl, Pflanzenfam. 11** : 275. d. I898.

Lloyd, Volvæ 5. $d$. 13. I898 and Rev. Myc. 21: 92 . July I 899.

Taylor, Hdbk. Mushrooms 5 : $6 . \quad$ I 898.

Halsted, Bull. N. J. Exp. Sta. I35: I 2. f. 6. 8 Feb. I 899.

Pk. Bull. N. Y. Mus. 6 : IgI. July I 899.

Williams, Asa Gray Bull. 7 : 78. Aug. 1899.

Chesnut, Rept. Bureau of Animal Industry 15: 393. f. $38 . \quad 1899$.

Child, Jour. Hamilton Assoc. 15: 105. I899.

Herbst, Fungal Fl. Lehigh Valley 23. d. pl. 1899.

Jelliffe, Flora of Long Island $42 . \quad 1899$.

Longyear, Rept. Mich. Agr. 1898: 92. 1899.

Underw. Moulds I I9. I899.

Atk. Mushrooms 52. d. pl. 12, f. 52. pl. 13, f. 53, 54. 249. 20 Oct. 1900. 295, 297. April I 902. [2 ed.]

Williams, Asa Gray Bull. 8 : 103. Oct. 1900.

Atk. in Bailey's Cycl. Am. Hort. I044. $d$. I900.

Mcllvaine \& Macadam, Am. Fung. I4. d. pl. 6, f. 4. pl. .9 .623 .1900$.

Perry, Agricultural Student 6 : r79. May 1900 and Ohio Univ. Bull. IV. 30: 8. May I0do.

Trel. Rept. Mo. Hort. Soc. 43 : 236 (13). 1900.

Henderson, Bull. Idaho Exp. Sta. $27: 53$. pl. I2. March I9or.

Carter, Am. Jour. Physiol. 5 : 158, I68. I April I90I.

Sweetser, Bull. Pacific Univ. 14: I2. May I90I.

Earle in Mohr, Contr. U. S. Nat. Herb. 6 : 229. 31 July I90I. 
Hay, Bull. Nat. Hist. Soc. N. B. 4 : 342., r90I and 5 : I19. 1903.

Ricker, Univ. of Maine Studies 3: 55. 30 April 1902.

White, Bull. Torr. Bot. Club. 29 : 562. 26 Sept. 1902.

Chesnut, Contr. U. S. Nat. Herb. 7: 302. f. 67.1902.

Conant, Bull. Boston Myc. Club 19: 5 Feb. 1903.

Woodworth, Birds \& Nature 14: 95. pl. 592. Sept. 1903.

Schaffner, Ohio Nat. 4: 17. Nov. 1903.

Gibson, Mushrooms 51, 53.pl. 4, d. 58. $f ., 73,287$. pl. 37. 1903. [2 ed.]

Kellerm. Mvc. Bull. 2: 53. f. 51, 52. 26 March 1904.

Longyear, Rept. Mich. Acad. 4 : II 5.1904.

Mackay, Tr. N. Scot. Inst. 11 : 124. Aug. 1904.

Agaricus muscarius, L.

S. Syn. Am. Bor. 145 no. 4. 1834 .

Rav. Charleston Med. Jour. \& Rev. 6 : 190. March 1851.

Andrews, Proc. Boston Soc. 5 : 322. April 1856.

Sprague, Proc. Boston Soc. 6 : 316.1859.

Curtis, M. A., Bot. N. Car. 83. 1867.

Cooke, Handbook 8. pl. 2, f. l. d. $187 \mathrm{I}$.

Winder, Mushrooms of Canada 20. I871.

Pk. Rept. N. Y. Mus. 23: 68, d. 1872 .

Pk. Rept. N. Y. Mus. 24: I02. I 872.

Frost in Cat. Pl. Amherst $62 . \quad 1875$.

Farl. Bull. Bussey Inst. 1: 436 . March 1876.

Pk. Tr. Albany Inst. 8 : 163.1876.

Johnson, Bull. Minn. Acad. 1 : 209. 1877 and Rept. Minn. Surv. 5 : 67. 1877.

Fowler, Rept. Agr. N. B. 1878 : Append. B : 63. 1879.

Banning, Bot. Gaz. 5 : 6. d. Jan. 1880.

Somers, Tr. N. Scot. Inst. 5 : 189.1882.

Morg. Jour. Cin. Soc. 6 : 58. $d$. April 1883.

Pk. Rept. N. Y. Mus. 33 : 43. d. 44. Oct. I 883.

Rav. S. Car. Resources 354. I883.

Bessey, Bull. lowa Agr. Coll. 1884 : 146. Jan. I885.

Palmer, Mushrooms pl. 10, $f . t .1885$.

Morg. Jour. Myc. $3:$ 28. d. March 1887.

Calkins, Jour. Myc. 3: 58. May 1887.

Arth. \& Holw. Bull. Minn. Surv. 3: 26. I Oct. 1887.

$\mathrm{Cnbb}$, List Pl. Amherst 31. 1887.

Bennett. Cat. PI. R. I. 76. I 888.

Ell. in Cat. Pl. N. J. 468.1889.

Taylor, Rept. U. S. Agr. 1890: 373 (7). pl. 2, f. 8. 1890 [reprint 1893].

Mcllvaine, Therapeutic Gaz. $17: 296$ (2). 15 May 1893.

Eby, Fungi of Lancaster Co. $7 . \quad$ I894.

Sturgis, Rept. Conn. Agr. 1895: 76, 77 (6). pl. I, f. ı. Jan. 1896.

Taylor, Hdbk. Mushrooms 5: 9. $d . p l .15, f, 1-7.1898$.

Fowler, Proc. Miramichi Nat. Hist. 2: 28. 1901.

Amanita muscaria, (L.) P., var. alba, Pk.

Pk. Rept. N. Y. Mus. 46: 133 (53). d. , Dec. 1893.

* Shear, N. Y. Fungi 3. 1893.

Millsp. \& Nutt. Publ. Field Columb. Mus. Bot. 1 : 157. d. Jan. 1896.

Agaricus muscarius, $\mathrm{L}$., var. albus, $\mathrm{Pk}$.

Pk. Rept. N. Y. Mus. 33: $44 . d$. Oct. 1883.

Amanita muscaria, (L.) P., var. coecinea, Beardslee.

Beardslee, Amanitas S. Appalach. 8. d. p!. 3. . Sept. 1902.

Amanita musearia, (L.) P., var. formosa, P.

Pk. Bull. N. Y. Mus. 6 : I9I. July 1899.

Mcllvaine \& Macadam, Am. Fung. 14. d. 1900. 
White, Bull. Torr. Bot. Club 29 : 562. 26 Sept. 1902.

Pk. Rept. N. Y. Mus. 55: 961. d. Nov. I902. [Bull. 54.]

Hay, Bull. Nat. Hist. Soc. N. B. 5 : II4. 1903.

Pk. Rept. N. Y. Mus. 57: 22. d. July 1904. [Bull. 75.] Agaricus muscarius, L., var. formosus, Fr.

Pk. Rept. N. Y. Mus. $33: 44$. d. Oct. 1883.

Macbr. Bull. Univ. Iowa $1: 35 . d$. Nov. 1888.

Amanita formosa, Gonn. \& Rabh.

Lloyd, Volvæ 21. I898.

Amanita muscaria, var. major, $\mathrm{Pk}$.

See Amanita strobiliformis, (Vitt.) Fr.

Amanita muscaria, var. minor, $\mathrm{Pk}$.

See Amanita Frostiana, Pk.

Amanita muscaria, (L.) P., var. Puella, P.

S. Syn. Car. 79 (53) no. $548 . \quad$ I 822.

Atk. Mushrooms 55. 20 Oct. 1900.

Amanita muscaria, (L.) P., var. regalis, Fr.

Mcllvaine \& Macadam, Am. Fung. I4. d. 1900.

Agaricus muscarius, var, regalis, Fr.

Pk. Rept. N. Y. Mus. 33:44. Oct. 1883.

Amanita muscaria, var. umbrina, (P.) Sacc. Syll.

Mcllvaine \& Macadam, Am. Fung. 14. d. 1900.

Agaricus muscarius, var, umbrinus, $\mathrm{Pl}$.

Pk. Rept. N. Y. Mus. 33: 44. $d$. Oct. I883.

A manita nitida, Fr.

Pk. Rept. N. Y. Mus. 43: 62 (I6). Nov̂. 1800.

Banning in Pk. Rept. N. Y. Mus. 44: $177(65)$. Dec. I89I.

Lloyd, Volvæ 7. $d . \quad$ I 898 and Rev. Myc. 21 : 93. July I 899.

Mcllvaine \& Macadam, Am. Fung. 24. d. I900. Agaricus nitidus, Fr.

Banning, Bot. Gaz. 5:6. d. Jan. 1880.

Hark. \& Moore, Cat. Pac. Fung. 2. Feb. 1880.

Morg. Jour. Myc. 3: 30. d. March 1887.

Amanita nivalis, Grev.

See Amanitopsis vaginata (Bull.) Roze, var, alba, Sacc. Syll.

Amanita nivalis, $\mathrm{Pk}$.

See Amanitopsis albocreata, Atk.

Amanita onusta, Howe.

Howe Bull. 'Torr. Bot. Club $5: 42$ d. Oct. I 874 .

Sacc. Syll. $9:$ I. $d$. 15 Sept. 1891.

Lloyd, Volvæ 9, I6. d.21. 1808.

Pk. Rept. N. Y. Mus. 53: 839. d. I9 March I90I. Agaricus onustus, Howe.

Howe, Bull. Torr. Bot. Club $5: 42$. d. Oct. 1874 .

Pk. Rept. N. Y. Mus. $33: 49$. Oct. I883.

Morg. Jour. Myc. $3:$ 32. $d$. March I 887.

Amanita pantherina, (D C.) Fr.

Sacc. Cub. \& Manc. Syll. 5 : I4. d. 28 May 1887.

Pk. Bull. N. Y. Mus. 12: 25. Jan. I 888.

Fairman, Proc. Rochester Acad. 2: 154. Feb. I 893.

Atk. \& Schrenk, Jour. Mitch. Soc. 9 : 98 (4). April 1893.

Underw. Proc. Ind. Acad. 1893: 61. Aug. 1894.

Kellerm. \& Werner, Geol. Ohio $7^{2}$ : 300. Jan. 1895.

Atk. Bull. Cornell Univ. 3: 24. 25 June 1897.

Williams, Asa Gray Bull. 6: 83. pl. $1, f .2$. Oct. 1898 . 
Lloyd, Volvit 4. $d$. I3. 1808 and Rev. Myc. 21 : 92. July I899.

Williams, Asa Gray Bull. 7 : 78. Aug. I809.

Herbst, Fungal Fl. Lehigh Valley 28. d. 1899.

Atk. Mushrooms 69, 249. 20 Oct. 1900. 295. April 1902. [2 ed.]

Williams, Asa Gray Bull. 8 : 103. Oct. 1900.

Mcllvaine \& Macadain, Am. Fung. 17. d. 1900.

Sweetser, Bull. Pacific Univ. 14: 12. May IgoI.

Earle in Mohr, Contr. U. S. Nat. Herb. 6 : 229. 3I July I9or.

Amanita umbrina, $\mathrm{P}$.

Muhl. Cat. Pl. Am. Sept. I ed. 104. I813. 2 ed. 107. 1818.

S. Syn. Car. 79 (53) no. 549. 1822.

Lloyd, Volva 21. 1898.

Henn. in Engler \& Prantl, Pflanzenfam. 11** : 275. d. 1808.

Perry, Agricultural Student 6 : 179. May 1900 and Ohio Univ. Bull. iV. 30: 8. May 1900.

Schaffner, Ohio Nat. 4: 18. Nov. 1903.

Agaricus pantherinus, D C.

S. Syn. Am. Bor. 145 no. 5. 1834 .

Lea, Cat. Pl. Cin. 48.1849.

Curtis, M. A., Bot. N. Car. 83. 1867.

Cke. Handbook 9. $d$. I87I.

Johnson, Bull. Minn. Acad. 1 : 209. 1877 and Rept. Minn. Surv. 5 : 67. 1877.

James, Jour. Cin. Soc. $2: 63$ (22). April I879.

Hark. \& Moore, Cat. Pac. Fung. 2. Feb. 1880.

Bundy, Geol. Wis. 1873-79, 1 : 396. 1883.

Morg. Jour. Cin. Soc. 6: 58. d. April 1883.

Morg. Jour. Myc. 3: 28, d. March 1887.

Macbr. Bull. Univ. lowa 1: 182. June I89o.

Amanita pellueidula, Banning.

Banning in Pk. Rept. N. Y. Mus. 44: 178 (66). d. Dec. I891.

\section{Amanita phalloides, $\mathrm{Fr}$.}

Notk. By some writers Amanita verna is considered to be distinct from $A$. phalloides but by others a variety of that species. It is not certain that all the references to $A$. verna given below relate to the European form and they are here placed under $A$. phalloides as they should be cunsulted in cunnection with that species.

Sacc. Cub. \& Manc. Syll. 5 : 9. d. 28 May 1887.

Banning in Pk. Rept. N. Y. Mus. 44: $177(65)$. Dec. I89 r.

Fairman, Proc. Rochester Acad. 2 : 154. Feb. 1893.

Atk. \& Schrenk, Jour. Mitch. Soc. 9: 98 (4). April 1893.

* Shear, N. Y. Fungi 2. I 893.

Underw. Proc. Ind. Acad. 1893 : 6r. Aug. 1894.

Pk. Rept. N. Y. Mus. 47 : I35 (9), I 37 (II). Nov. I894.

McClatchie in Reid's Hist. Pasadena 619. 1895.

Pk. Rept. N. Y. Mus. 48: $313(215)$. pl. $40,41, f .1-3$. March 1897.

McClatchie, Proc. S. Cal. Acad. 1: 385.5 June 1897.

Atk. Bull. Cornell Univ. 3: 24. 25 June 1897.

Underw. \& Earle, Bull. Ala. Exp. Sta. 80: 255. 28 June 1897.

Atk. Bull. Cornell Exp. Sta. 138: 358-362. (24-28) d. f. 106-109. Sept. I897.

Coville, Circ. U. S. Agr. Div. Bot. 13: 20. f. 21. I Dec. 1897. [2 ed. 21. f. 25-27.]

Chesnut, Yearb. U. S. Agr. 1896 : 144. d. f. 28.1897.

Jones, H. L., Oberlin College Lab. Bull. $9: 4$. I 5 June 1898.

Williams, Asa Gray Bull. 6: 8I. pl. $I, f$. $I$. Oct. 1898 .

Chesnut, Bull. U. S. Agr. Div. Bot. 20: 14. f. 2. 1898. 
Chesnut, Farmers' Bull. U. S. Agr. 86: 6. f. 2.1898.

Farl. Yearb. U. S. Agr. $1897:$ 458. d. pl. 23. I 898 . [Bull. I 5.]

Henn. in Engler \& Prantl, Pflanzenfam. 1.1** : 275. d. f. 125 B. I 898. Lloyd, Volvæ 3. $d$. f. 3. I3. I 898 and Rev. Myc. 21 : 92 . July I 899.

Halsted, Bull. Torr. Bot. Club 26: I6. I6 Jan. I899.

Williams, Asa Gray Bull. 7: 17. Feb. 1899.

Halsted, Bull. N. J. Exp. Sta. I35: 1 3. f. 7. 8 Feb. 1890.

Pk. Bull. N. Y. Mus. 6 : 191. July 1899.

Sterling, Toadstools. Aug. I899.

Williams, Asa Gray Bull. $7: 78$. Aug. I899.

Child, Jour. Hamilton Assoc. 15: 105. 1899.

Herbst, Fungal FI. Lehigh Valley 27. d. 1899.

Underw. Moulds I IO, I I9, 235. pl. 8, f. I, 2. I 899 .

Chesnut, Plant World 3: 8 . Jan. 1900.

Atk. Mushrooms $3, \mathrm{I} 4,55 . d . f_{.55} . \quad p l .14, f .56 . f .57,58.249,25 \mathrm{I}$. 20 Oct. 1900. 295, 297. April 1902. [2 ed.]

Williams, Asa Gray Bull. 8 : 100. 103. Oct. 1900.

Atk. in Bailey's Cycl. Am. Hort. 1044. d. f. I443. I900.

Mcllvaine \& Macadam, Am. Fung. 7. d. pl. 6, f. 2, 3. 629. 1900.

Trel. Rept. Mo. Hort. Soc. 43: 224 (I) f. 236 (I3). 1900.

Henderson, Bull. Idaho Exp. Sta. 27: 30. f. March IgoI.

Carter, Am. Jour. Physiol. 5 : I63, I7I, I73. I April 1901.

Earle in Mohr, Contr. U. S. Nat. Herb. $6: 229.3$ J July I9oI.

Garman, Bull. Ky. Exp. Sta. $96: f .5$. Nov. I9oI.

Williamson, Proc. Ind. Acad. 1900 : I63. 1901.

Brown \& Fernekes, Bull. Wis. Nat. Hist. Soc. II. 2: 46. Jan. I902.

Ricker, Univ. of Maine Studies 3: 55. 30 April 1902.

White, Bull. Torr. Bot. Club 29: 562. 26 Sept. 1902.

Conant, Bull. Boston Myc. Club i9: 5 Feb. 1903.

Schaffner, Ohio Nat. 4: i 7. Nov. 1903.

Gibson, Mushrooms 73. $f$. 1903. [2 ed.]

Hay, Bull. Nat. Hist. Soc. N. B. 5 : 1 I I, I I4. I903.

Kellerm. Myc. Bull. 2 : 56. $f .53 .26$ March 1904.

Trel. Rept. Mo. Bot. Gard. 15: 84. 30 April I904.

Longyear, Rept. Mich. Acad. 4: i I 5. I904.

MacKay, Tr. N. Scot. Inst. 11 : I24. Aug. I904.

Amanita bulbosa, Auct. Amer.

S. Syn. Car. $79(53)$ no. 543 . 1822.

Mcllvaine, Therapeutic Gaz. 17: 297 (4). 15 May I893.

Lloyd, Volvæ 2I. I 898.

Carter, Am. Jour. Physiol. 5: I6I, I70, 172, I74. I April I90I.

Agaricus phalloides, Fr.

S. Syn. Am. Bor. 145 no. I. 1834 .

Sprague, Proc. Boston Soc. 6 : $316 . \quad 1859$.

Curtis, M. A., Bot. N. Car. 83. I867.

Cke. Handbook 7. $d$. $187 \mathrm{I}$.

Winder, Mushrooms of Canada 20. 1871 .

Pk. Rept. N. Y. Mus. 23: 69. 1872.

Frost in Cat. Pl. Amherst 62. 1875.

Johnson, Bull. Minn. Acad. 1 : 209. 1877 and Rept. Minn. Surv. 5 : 67.1877.

Hark. \& Moore, Cat. Pac. Fung. 2. Feb. 1880.

Pk. Rept. N. Y. Mus. 33: 42. d. Oct. 1883.

Bundy, Geol. Wis. $1873-79,1: 396.1883$.

Pk. in Day's Cat., Bull. Buf. Soc. 4 : I 74 (IIO). I 883.

Palmer, Mushrooms $p l$. Iо, $f .2,3 . \quad$ I 885.

Morg. Jour. Myc. $3:$ 27. d. March I887.

Cobb, List Pl. Amherst 31. I887. 
Macbr. Bull. Univ. lowa 1 : 36. d. Nov. 1888.

Bennett, Cat. PI. R. I. $76 . \quad 1888$.

Somers, Tr. N. Scot. Inst. $7: 464 . \quad 1800$.

Eby, Fungi of Lancaster Co. 7. I894.

Sturgis, Rept. Conn. Agr. 1895 : 76, 77 (6). Jan. 1896.

Taylor, Hdbk. Mushrooms 5: 10. d.pl. 15, f. 8. 1898 .

Amanita citrina, S. Syn. Car. 544.

Muhl. Cat. Pl. Am. Sept. I ed. 104. 1813. 2 ed. 107. 1818.

S. Syn. Car. 79 (53) no. 544. 1822.

Lloyd, Volvæ 21. 1808.

Amanita viridis, var, fuliginea, S. Syn. Car. 545.

S. Syn. Car. 79 (53) no. 545. 1822.

Lloyd, Volvæ 21. 1808 .

Agaricus vernus, Auct. Amer.

Curt's, M. A., Bot. N. Car. 83. 1867.

Winder, Mushrooms of Canada 20. 1871 .

Pk. Rept. N. Y. Mus. 23:69. d. 1872.

Frost in Cat. Pl. Amherst 62.1875 .

Johnson, Bull. Minn. Acad. 1: 209. 1877 and Rept. Minn. Surv. 5 : 67. 1877.

Hark. \& Moore, Cat. Pac. Fung. 2. Feb. 1880.

Morg. Jour. Cin. Soc. 6: 57. d. April 1883.

Bundy, Geol. Wis. $1873-79,1: 396.1883$.

Palmer, Mushrooms pl. 9. d. 1885.

Cobb, List PI. Amherst 31. 1887.

Macbr. Bull. Univ. lowa 1: 36. Nov. 1888.

Ell. in Cat. PI. N. J. 468 . 1880 .

Somers, Tr. N. Scot. Inst. 7: 464. d. 1890.

Taylor, Rept. U. S. Agr. 1890: 372 (7). pl. 2, f. 6. 1890. [reprint I893.]

Eby, Fungi of Lancaster Co. 7. 1894.

Taylor, Hdbk. Mushrooms 5: i1. d. pl. 16; f. $1-4 . \quad 1898$.

Amanita verna, Auct. Amer.

Banning in Pk. Rept. N. Y. Mus. 44: 177 (65). Dec. 1891.

Atk. \& Schrenk, Jour. Mitch. Soc. 9: 98 (4). April 1893.

Mcllvaine, Therapeutic Gaz. 17: 297 (4). 15 May 1893.

Pk. Country Gent. 59: $582(48)$. d. 9 Aug. I 894 .

Kellerm. \& Werner, Geol. Ohio $7^{2}$ : 300. Jan. 1895.

Graves, Asa Gray Bull. 3: $44 . \quad$ I Oct. 1895.

Millsp. \& Nutt. Publ. Field Columb. Mus. Bot. 1: 156 . Jan. 1896.

Bates, Tr. Mass. Hort. Soc. 1896: 172, 173. d. 1896.

Pk. Rept. N. Y. Mus. 48: 313 (215). pl. 4l,f. 4-7. March 1897.

Taylor, Hdbk. Mushrooms 4: $15 . \quad 1897$.

Halsted, Bull. N. J. Exp. Sta. 135: 14. 8 Feb. 1899.

Jelliffe, Flora of Long Island $42 . \quad 1899$.

Longyear, Rept. Mich. Bd. Agr. 1898: 92. 1899.

Underw. Moulds 119. 1899 .

Atk. Mushrooms 60. d. $f .59-61.20$ Oct. 1900.

Atk. in Bailey's Cycl. Am. Hort. 1044. d. 1900.

Mcllvaine \& Macadam, Am. Fung. 9. d. 627. 1900.

Perry, Agricultural Student 6: 179. May 1900 and Ohio Univ. Bull. 1V. 30: 8. May 1900 .

?Carter, Am. Jour. Physiol. 5 : 161, 172, 174. I April 1901.

Brown \& Fernekes, Bull. Wis. Nat. Hist. Soc. 11. 2 : 46. Jan. 1902.

White, Bull. Torr. Bot. Club. 29: 562. 26 Sept. 1902.

Schaffner, Ohio Nat. 4: 18. Nov. 1903.

Gibson, Mushrooms 44, 49. pl. 3. 1903. [2 ed.]

Hay, Bull. Nat. Hist. Soc. N. B. 5: 114.1903. 
Longyear, Rept. Mich. Acad. 4 : II 5.1904.

MacKay, Tr. N. Scot. Inst. 11: 123. Aug. I904.

Amanita phalloides, var. verna, Auct. Amer.

Williams, Asa Gray Bull. 7 : 78. Aug. I899.

Amanita phalloides, S. Am. Bor. I excl. var. bulbosa.

See Amanita phalloides, Fr.

Amanita phalloides, var. bulbosus, S. Am. Bor. I.

See Amanita Mappa, (Batsch) Fr.

Amanita phalloides, Fr., var. striatula, Pk.

Pk. Rept. N. Y. Mus. 55: 96ı. $d$. Nov. 1902. [Bull. $10:$ no. 54.]

Sacc. Syll. $17: 2 . d$. 25 May I905.

Amanita polypyramis, B. \& C.

See Amanita solitaria, (Bull.) Fr.

Amanita prairiicola, $\mathrm{Pk}$.

Pk. Bull. Torr. Bot. Club 24 : 138. d. 30 March I 897 .

Lindau \& Syd. Hedw. 37: Beibl. 7: I. 9 April I 898.

Lloyd, Volvæ 7, 15. $d$. I898 and Rev. Myc. 21: 93. July I899.

Smyth, Tr. Kans. Acad. 16 : I64. July 1899.

Mcllvaine \& Macadam, Am. Fung. 24. d. I900.

Amanita praticola, Sacc. \& Syd.

Sacc. \& Syd. Syll. 14: 63. d. 20 Aug. I899.

Amanita praticola, Sacc. \& Syd.

See Amanita prairilicola, Pk.

Amanita pubescens, $\mathrm{S}$.

See Amanitopsis pubescens, (S.) Sacc.

Amanita pusilla, Llovd.

See Amanitopsis pusilia, Pk.

Amanita pusilla, $\mathrm{P}$.

See Volvaria pusilla, (P.) Fr.

Amanita pustulata, Schrt.

See Amanita rubescens, P.

Amanita radicata, $\mathrm{Pk}$.

Pk. Bull. Torr. But. Club 27 : 600. d. 29 Dec. 10 jos.

Shear, Plant World 4: 18 . Jan. I901.

Sacc. \& Syd. Syll. $16: 2$. d. I Feb. I902.

Amanita Ravenelii, B. \& C.

Nore. There is an Agaricus (Galera) Ravenelii, B. \& C. which is not this species and which occurs only as a catalogue name in Curtis, M. A., liot, N. Car. 89. 1867. It is probably Galera lateritia, Fr. or nearly related to it.

B. \& C. Ann. Nat. Hist. III. 4 : 284.(I). d. Oct. I859.

Sacc. Cub. \& Manc. Syll. 5 : 15. d. 28 May 1887.

Sacc. Syll. 9:2. I5 Sept. I891.

Atk. Bull. Cornell Univ. 3: 24. 25 June 1877.

Henn. in Engler \& Prantl, Pflanzenfam. 11**: 275. 189 \$.

Lloyd, Volva 6, I4. $d$. I898 and Rev. Myc. 21 : 93. July I89\%.

Mcllvaine \& Macadam, Am. Fung. I8. $d$. 1900.

Earle in Mohr, Contr. U. S. Nat. Herb. 6 : 229. 3I July Igor.

Agaricus Ravenelii, B. \& C.

B. \& C. Ann. Nat. Hist. III. 4: 284 (I). d. Oct. 1859.

Morg. Jour. Myc. 3: 27. d. March I 887 .

Amanita recutita, Fr.

Atk. \& Schrenk, Jour. Mitch. Soc. $9: 98$ (4). April 1893.

Lloyd, Volvæ 3. d. 13. d. I 898 and Rev Myc. 21: 92. July i899. 
Williams, Asa Gray Bull. 8 : 104. Oct. 1900.

Mcllvaine \& Macadam, Am. Fung. 12. d. 1900.

Agaricus recutitus, $\mathrm{Fr}$.

Curtis, M. A., Bot. N. Car. 83. 1867.

Morg. Jour. Myc. 3: 27. d. March 1887.

Amanita rubescens, $P$.

S. Syn. Car. 79 (53) no. 550. 1822.

Sacc. Cub. \& Manc. Syll. 5 : I6. d. 28 May 1887.

Banning in Pk. Rept. N. Y. Mus. 44: 177 (65). Dec. I891.

Taylor, Food Products 2: 21. 1893.

Pk. Country Gent. 59 : 566 (43). d. f. 2 Aug. 1894.

Kellerm. \& Werner, Geol. Ohio $7^{2}: 300$. Jan. 1805 .

Pk. Rept. N. Y. Mus. 48: 255 (157). pl. 16, f. March I897.

Underw. \& Earle, Bull. Ala. Exp. Sta. 80: 255. 28 June I897.

Williams, Asa Gray Bull. 6 : 84. $f .13^{6}$. Oct. 1898.

Farl. Yearb. U. S. Agr. $1897: 458,46$ I. I 898. [Bull. 15.]

Lloyd, Volvæ 7. d. 15. 1898 and Rev. Myc. 21 : 93 . July I 899.

[Underw.] N. Y. Myc. Club i6. I898.

Pk Bull. N. Y. Mus. 6 : I92. July I899.

Williams, Asa Gray Bull. 7 : 79. Aug. 1899 .

Herbst, Fungal Fl. Lehigh Valley 30. d. 1899.

Jelliffe, Flora of Long Island 42. 1890.

Longyear, Rept. Micl. Bd. Agr. 1898: 92. 1899.

Underw. Moulds I 20.1899.

Webster, Rhodora 2 : 193. 8 Sept. 1900.

Atk. Mushrooms 7I. d. $f_{\cdot} 73 . \quad p l .19, f .1 . \quad p l .20, f .74 .20$ Oct. 1900.

Williams, Asa Gray Bull. 8: 103. Oct. 1900.

Hyams, Bull. N. Car. Exp. Sta. I77: 31. Dec. 1900.

Atk. in Bailey's Cycl. Am. Hort. 1044. I900.

Mcllvaine \& Macadam, Am. Fung. 2I. d. pl. 8, f. 2. pl. 12, f. 4. 1900.

Pk. Rept. N. Y. Mus. $53: 855$. I9 March I90I.

Earle in Mohr, Contr. U. S. Nat. Herb. 6 : 229. 3I July 1901.

White, Bull. Torr. Bot. Club 29 : 562. 26 Sept. 1902.

Conant, Bull. Boston Myc. Club i9: 5 Feb. 1903.

Kellerm. Myc. Bull. 2 : 84. f. 75.5 Sept. 1904.

Longyear, Rept. Mich. Acad. 4: II5. 1904.

Agaricus rubescens, Fr.

S. Syn. Am. Bor. I45 no. 8. I834.

Lea, Cat. Pl. Cin. 48.1849.

Curtis, M. A., Bot. N. Car. 83. 1867.

Cke. Handbook 9. $d$. 1871 .

Pk. Rept. N. Y. Mus. 24: 59. 1872.

Parker, Proc. Poughk. Soc. 1: 84. I Nov. 1875.

Frost in Cat. Pl. Amherst 62. 1875.

James, Jour. Cin. Soc. 2: 63 (22). April 1879.

Banning, Bot. Gaz. 5 : 6. d. Jan. 1880.

Banning, Bot. Gaz. 5 : 23. Feb. 1880.

Morg. Jour. Cin. Soc. 6 : 59. d. April 1883.

Pk. Rept. N. Y. Mus. 33 : 44. d. Oct. 1883.

Morg. Jour. Myc. 3: 29. d. March 1887.

Cobb, List PI. Amherst 3I. I887.

Bennett, Cat. PI. R. I. 76.1888.

Ell. in Cat. PI. N. J. 468.1889.

Taylor, Hdbk. Mushrooms 5: 7. d. pl. 14, f. 5-9. 1898.

A manita pustulata, (Schæff.) Schrt.

Henn. in Engler \& Prantl, Pflanzenfam. 11**: 274. d. f. 125 A. I 898.

Perry Agricultural Student 6 : 179. May 1900 and Ohio Univ. Bull. iv. $30: 8$. May 1900 . 
Amanita rubescens, P., var. eireinata, P. Amanita circinata, Schum.

Taylor, Food Products 2: 2I. I893.

Amanita russuloides, $\mathrm{Pk}$.

Pk. Bull. Buf. Soc. 1: 41. d. July 1873.

Pk. Rept. N. Y. Mus. $25: 72$. d. Sept. $18730^{\circ}$

Sacc. Cub. \& Manc. Syll. 5: 13. d. 28 May 1887.

Henn. in Engler \& Prantl, Pflanzenfam. 11**: 275. 1898.

Lloyd, Volvæ 5. d. 14. $d$. I 898 and Rev. Myc. 21 : 92. July I899.

Williams, Asa Gray Bull. $7: 79$. Aug. 1899.

Williams, Asa Gray Bull. 8 : 103. Oct. 1900.

Mcllvaine \& Macadam, Am. Fung. I8, $d$. 1900.

Agaricus russuioides, $\mathrm{Pk}$.

Pk. Bull. Buf. Soc. 1 : 41 . d. July 1873.

Pk. Rept. N. Y. Mus. $25: 72 . d$. Sept. I873.

Pk. Rept. N. Y. Mus. 33: 43. d. Oct. 1883 .

Morg. Jour. Myc. 3: 28. d. March I887.

Amanita soleata, Howe.

See Amanitopsis volvata, (Pk.) Sacc.

Amanita solitapia, (Bull.) Fr.

NotF. The Agaricus bulbosus, Schæff., listed by Muhlenberg, 1799 is referred by Streinz to Amanita solitaria, (Bull.) Fr. The American references to Amanita solitaria and Amanita strobiliformis are very much confused and in fact the distinctions between these two species as given by European authors are far from clear. It seems to us that it may be assumed that some of the references here given under both these species are open to question. See note under Amanita bulbosa, P. Is not ilitocybe solitaria, Jelliffe, Fl. L. I. 42. 1899 a misprint for Amanita solitaria?

Banning in Pk. Rept. N. Y. Mus. 44: 177 (65). Dec. I 891.

Atk. \& Schrenk, Jour. Mitch. Soc. 9 : 98 (4). April 1893.

Atk. Bull. Cornell Univ. 3: 24. 25 June 1897.

Underw. \& Earle, Bull. Ala. Exp. Sta. 80: 255. 28 June 1897.

Lloyd, Volvæ 6. d. I4. $d$. I 898 and Rev. Myc. 21: 93 . July I899.

Williams, Asa Gray Bull. 7 : 79. Aug. 1899.

Herbst, Fungal FI. Lehigh Valley 29.d. 1899.

Atk. Mushrooms $72 . d . p l .2 I, f .75, p l .22, f .76 .20$ Oct. 1900.

Williams, Asa Gray Bull. 8 : 103. Oct. 1900.

Atk. in Bailey's Cycl. Am. Hort. 1044. 1900.

Mcllvaine \& Macadam, Am. Fung. I9. $d$. I 1900.

Earle in Mohr, Contr. U. S. Nat. Herb. $6: 229.3$ 3 July I9or.

Conant, Bull. Boston Myc. Club i9: 5 Feb. 1903.

Agaricus sointarius, Bull.

Banning, Bot. Gaz. 5 : 7.d. Jan. 1880.

Pk. Rept. N. Y. Mus. 33: $18,45 . d$. Oct. 1883.

Morg. Jour. Myc. $3:$ 29. d. March 1887.

Agaricus buibosus, Schæff.

Muhl. Tr. Am. Phil. Soc. 4: 239. 1799.

Agaricus polypyramis, $\mathrm{B}$. \& $\mathrm{C}$.

B. \& C. Ann. Nat. Hist. II. 12: 4I7 (I). d. Dec. 1853.

Curtis, M. A., Bot. N. Car. 83. I867.

Morg. Bot. Gaz. 8 : i56. d. Jan. 1883. Amanita poiypyramis, B. \& C.

B. \& C. Ann. Nat. Hist. 11. $12: 417$ (I). d. Dec. 1853.

Sacc. Cub. \& Manc. Syll. 5 : i8. $d .28$ May i 887.

Millsp. \& Nutt. Publ. Field Columb. Mus. Bot. 1: 157. d. Jan. I896.

Henn. in Engler \& Prantl, Pflanzenfam. 11**: 275. I898. 
Lloyd. Volva 6, 14. d. 1898 and Rev. Myc. $21: 93$. July 1899. Atk. Muslirooms 74. 20 Oct. 1900.

Amanita spadicea, $P$.

See Amanitopsis vaginata, (Bull.) Roze, var. Iivida, (P.) Pk.

Amanita spissa, Fr.

Banning in Pk. Rept. N. Y. Mus. 44: 177 (65). d. Dec. I89 I.

Lloyd, Volva 7. d. I 5.1898 and Rev. Myc. $21: 93$. July 1899.

Mcllvaine \& Macadam, Am. Fung. 22. d. 1900.

MacKay, Tr. N. Scot. Inst. 11 : 124. Aug. I904.

Agaricus spissus, Fr.

Somers, Tr. N. Scot. Inst. 5 : 247. 1882.

Amanita spreta, Pk.

Pk. Rept. N. Y. Mus. $33: 42$. d. Oct. 1883.

Pk. Rept. N. Y. Mus. 32: 24. d. 1886.

Sacc. Cub. \& Manc. Syll. 5: 12. d. 28 May 1887.

Atk. Bull. Cornell Univ. 3:24. 25 June 1897.

Underw. \& Earle, Bull. Ala. Exp. Sta. 80: 255. 28 June 1807.

Lloyd, Volva 3. d. I 3. d. I 898 and Rev. Myc. 21: 92 . July I 899.

Henn. in Engler \& Prantl, Pflanzenfam. 11**: $276 . \quad$ I808.

Williams, Asa Gray Bull. 7: 78. Aug. 1899.

Herbst, Fungal Fl. Lehigh Valley 26. d. I 899.

Atk. Mushrooms 69. d. $f .7 I .20$ Oct. 1900.

Mcllvaine \& Macadam, Am. Fung. I I. d. pl. 6, f. 1.1900.

Earle in Mohr, Contr. U. S. Nat. Herb. 6: 229. 31 July igor.

Ricker, Univ. of Maine Studies 3: 55. 30 April I902.

White, Bull. Torr. Bot. Club 29: 562. 26 Sept. 1902.

Agaricus spretus, Pk.

Pk. Rept. N. Y. Mus. 33: 42. d. Oct. 1883.

Pk. Rept. N. Y. Mus. $32: 24$. d. 1886.

Morg. Jour. Myc. 3: 26. d. March 1887.

Amanita strangulata, Fr.

See Amanitopsis strangulata, (Fr.) Roze.

Amanita strobiliformis, (Vitt.) $\mathrm{Fr}$.

Banning in Pk. Rept. N. Y. Mus. 44: 177 (65). Dec. I89I.

Taylor, Food Products 2: 21. 1893.

Pk. Country Gent. 59 : 566 (44). 2 Aug. 1894.

Underw. \& Earle, Bull. Ala. Exp. Sta. 80: 255. 28 June 1897.

Williams, Asa Gray Bull. $6: 83 \cdot f \cdot 13^{5}$. Oct. 1898 .

Lloyd, Volvæ 6. d. 14. I808 and Rev. Myc. 21: 93. July I899.

Williams, Asa Gray Bull. 7 : 79. Aug. 1899.

[Williams?] Asa Gray Bull. $7: 87 . d$. pl. 6. Oct. 1899.

Herbst, Fungal Fl. Lehigh Valley 29. d. 1899.

Williams, Asa Gray Bull. 8: 104. Oct. 1900.

Atk. in Bailey's Cycl. Am. Hort. 1044. I900.

Mcllvaine \& Macadam, Am. Fung. 19. d. pl. 8, f. 3. 1900.

Earle in Mohr, Contr. U. S. Nat. Herb. 6 : 229. 3i July Igor.

Conant, Bull. Boston Myc. Club 19: 5 Feb. I903.

Kellerm. Ohio Myc. Bull. 1 : 26. $f .32$. 31 July 1903.

MacKay, Tr. N. Scot. Inst. 11: 124. Aug I904.

Agaricus strobiliformis, Vitt.

Curtis, M. A., Bot. N. Car. 83. 1867.

Frost, Proc. Boston Soc. $12: 77 . \quad 1869$.

Frost in Cat. Pl. Amherst 62. 1875 .

Farl. Bull. Bussey Inst. 1: 436. March 1876.

Morg. Fl. Miami Valley 67. 1878.

Banning, Bot. Gaz. 5: 5. d. Jan. 1880.

Hark. \& Moore, Cat. Pac. Fung. 2. Feb. 1880. 
Pk. Rept. N. Y. Mus. 33 : I8, 46. d. Oct. I883.

Morg. Jour. Myc. $3: 29$. . March 1887.

Cobb, List Pl. Amherst 3 . 1887.

Taylor, Hdbk. Mushrooms 5: 8. d. pl. 14 t. I 898 .

Hyams, Bull. N. Car. Exp. Sta. 177: 30. Dec. 1900. Agaricus muscarius, var. major, Plk.

Pk. Rept. N. Y. Mus. 23:69. d. I872. Amanita muscaria, var. major, PK.

Pk. Rept. N. Y. Mus. 23 : 69. d. 1872.

Lloyd, Volvæ 21. 1898.

Amanita submaeulata, Pk.

Pk. Bull. Torr. Bot. Club 27 : 609. d. 29 Dec. I9oo.

Shear, Plant World, 4: 18. Jan. I9ò. .

Sacc. \& Syd. Syll. 16: I. d. I Feb. I902.

Amanita umbrina, $P$.

See Amanita pantherina, (DC.) Fr.

Amanita vaginata, Lam.

See Amanitopsis vaginatus, (Bull.) Roze.

Amanita velatipes, Atk.

Atkinson, Mushrooms 61, 63. d. pl. 15, f. 64. f.65, 66. pl. 16, f. 67. 20 Oct. 1900 and Rhodora $3: 40$. I Feb. I 90 I.

Amanita velosa, Lloyd.

See Amanitopsis velosa, Pk.

Amanita verna, Auct. Amer.

See Amanita phalloides, Fr.

Amanita vireseens, $P$.

Note. Schweinitz states that his Amanita virescens, var, alba, S. Syn. Car. $55 \mathrm{I}$ is the same as Agaricus virescens, Fr. S. M. I: 19, which is Amanita virescens, $\mathbf{P}$. The species is considered doubtful by Fries and is said to differ from Amanita rubescens, P. only in color and in the presence of poly. edral warts.

Lloyd, Volvæ 2I. $\quad$ I 898. Agaricus virescens, Fr.

S. Syn. Am. Bor. 145 no. 9. I834.

Amanita virescens, var. alba, S. Syn. Car. $55 \mathrm{I}$.

S. Syn. Car. 79 (53) no. 551. 1822.

Amanita virgata, $\mathrm{S}$.

See Volvaria volvacea, (Bull.) Fr.

Amanita viridis, var. Puliginea, S. Syn. Car. 545.

See Amanita phalloides, Fr.

Amanita virosa, Fr.

Underw. Proc. Ind. Acad. 1893: 6r. Aug. I894.

Kellerm. \& Werner, Geol. Ohio $7^{2}$ : 300 . Jan. 1805 .

Underw. \& Earle, Bull. Ala. Exp. Sta. 80: 255. 28 June 1897.

Lloyd, Volvæ 4. d. 13. 1898 and Rev. Myc. 21: 92. July I899.

Williams, Asa Gray Bull. $7: 78$. Aug. I899.

Atk. Mushrooms 6r. $d . f .62 .20$ Oct. 1900.

Williams, Asa Gray Bull. 8 : 104. Oct. 1900.

Mcllvaine \& Macadam, Am. Fung. 6. d. 1900.

Atk. in Bailey's Cycl. Am. Hort. 1044. I900.

Earle in Mohr, Contr. U. S. Nat. Herb. 6: 229. 3I July I9or.

Schaffner, Ohio Nat. 4: 18. Nov. 1903.

Hay, Bull. Nat. Hist. Soc. N. B. 5 : II 4.1903.

MacKay, Tr. N. Scot. Inst. 11: 124. Aug. 1904. 
Agarlcus virosus, $\mathrm{Fr}$.

Lea, Cat. Pl. Cin. $48 . \quad{ }_{1} 840$.

Sprague, Proc. Boston Soc. 6 : 316.1859.

Curtis, M. A., Bot. N. Car. 83. 1867.

James, Jour. Cin. Soc. 2: 63 (22). April 1879.

Hark. \& Moore, Cat. Pac. Fung. 2. Feb. 1880.

Morg. Jour. Cin. Soc. 6 : 60. d. April 1883.

Morg. Jour. Myc. 3: 26. d. March I887.

Macbr. Bull. lowa Univ. 1 : $182 . d$. 1890.

Amanita volvata, Pk.

See Amanitopsis volvata, (Pk.) Sacc.

Amanitopsis adnata. (W. G. Smith) Sacc.

Mcllvaine \& Macadam, Am. Fung. 30. d. 1900.

Agaricus adnatus, W. G. Smith.

Johnson, Bull. Minn. Acad. 1: 209. 1877 and Rept. Minn. Surv. 5 :

67. 1877.

Hark. \& Moore, Cat. Pac. Fung. 2. Feb. 1880.

Somers, Tr. N. Scot. Inst. 5 : $189 . \quad 1882$.

Bundy, Geol. Wis. $1873-79,1: 396.1883$.

Morg. Jour. Myc. 3 : 3ı. d. March 1887.

Amanita adnata, Smith, W. G.

Lloyd, Volvæ 9. d. 16. I 898 and Rev. Mvc. 21: 94. July 1899.

Herbst, Fungal Fl. Lehigh Valley 32. d. 1899.

MacKay, Tr. N. Scot. Inst. 11: 123. Aug. 1904.

Amanitopsis agglutinata, (B. \& C.) Sacc.

Sacc. Cub. \& Manc. Syll. 5 : 23. d. 28 Mav 1887.

Atk. Bull. Cornell Univ. 3 : 24. 25 June 1897.

Henn. in Engler \& Prantl, Pflanzenfam. 11**: 273. 1898.

Mcllvaine \& Macadam, Am. Fung. 32. d. 1900.

Earle in Mohr, Contr. U. S. Nat. Herb. 6 : 228. 31 July 1901.

Agaricus agglutinatus, B. \& C.

B. \& C. Hook. Jour. Bot. 1: 97 (1). d. 1849. [Decades 201.]

B. \& C. Am. Jour. Sci. 11. $8: 401$. Nov. 1849.

Curtis, M. A., Bot. N. Car. 83.1867.

Morg. Jour. Myc. 3 : 31. d. March 1887.

Amanita agglutinata, B. \& C.

B. \& C. Hook. Jour. Bot. 1: 97 (I). d. 1849. [Decades 201.]

Lloyd, Volva 9, 15.d. 1898 and Rev. Myc. 21: 94. July I898.

Amanitopsis albocreata, Atk.

Atk. Jour. Myc. 8 : i I I. d. 14 Oct. 1902.

Sacc. Syll. $17: 2 . d .25$ May 1905.

Agaricus nivalis, $\mathrm{Pk}$. non Grev.

Pk. Rept. N. Y. Mus. 33: 48. d. Oct. 1883.

Morg. Jour. Myc. $3:$ 32. $d$. March 1887.

Amanita nivalls, Pk.

Pk. Rept. N. Y. Mus. 33 : 48. d. Oct. 1883.

Fairman, Proc. Rochester Acad. $2: 154$. Feb. 1893.

Lloyd, Volvæ 9, 16. $d_{0} .1898$ and Rev. Myc. 21: 94. July I899.

Beardslee, Amanitas S. Appalach. 6. d. Sept. 1902.

Amanitopsls nivalis, Sacc. Syll. p. p. non Grev.

Sacc. Cub. \& Manc. Syll. 5: 22. May 28, 1887.

Atk. Bull. Cornell Univ. 3: 24. 25 June 1897.

Earle in Mohr, Contr. U. S. Nat. Herb. $6: 228.31$ July 1901.

Amanitopsis baceata, (Fr.) Sacc.

Amanita baccata, Fr.

Beardslee, Amanitas S. Appalach. 2. d. pl. 1. Sept. 1902. 
Amanitopsis Cubensis, (B. \& C.) Sacc. Syll.

Sacc. Cub. \& Manc. Syll. 5 : 25. d. 28 May I887. West Indies. Agaricus Cubensis, B. \& C.

B. \& C. Jour. Linn. Soc. $10: 282 . d$. 1869.

Amanitopsis daucipes, (B. \& Mont.) Sacc. Syll.

Sacc. Cub. \& Manc. Syll. 5 : 26. d. 28 May I887.

Henn. in Engler \& Prantl, Pflanzenfam. 11**: 273. 1898. Agaricus daucipes, B. \& Mont.

Mont. Syll. Crypt. 96. $d$. 1856. Amanita daucipes, B. \& Mont.

Mont. Syll. Crypt. 96. $d$. 1856.

Lloyd, Volvæ 7. I4. d. 1898 and Rev. Myc. 21 : 93. July I 899.

Williams, Asa 'Gray Bull. 7 : 79. Aug. 1899.

Mcllvaine \& Macadam, Am. Fung. 25. d. 1900.

Amanitopsis farinosa, (S.) Atk.

Atk. Mushrooms 76. $d$. f. 78 . 20 Oct. 1900.

Mcllvaine \& Macadam, Am. Fung. 3I. d. I900.

Earle in Mohr, Contr. U. S. Nat. Herb. 6: 228. 3i July Igor.

Atk. in Coker, Veg. Bahama 246. 1905.

\section{Amanita farinosa, $\mathrm{S}$.}

S. Syn. Car. 79 (53) no. 553. d. I 822.

Lloyd, Volvæ 9, I6. $d$. 1898 and Rev. Myc. 21 : 94 . July I899.

Williams, Asa Gray Bull. 7 : 79 . Aug. I 899.

Herbst, Fungal Fl. Lehigh Valley 3I. d. I899.

Williams, Asa Gray Bull. 8 : 104. Oct. 1900.

Beardslee, Amanitas S., Appalach. 4. d. Sept. "ígoz.

Agaricus farinosus, $\mathrm{S}$.

S. Syn. Am. Bor. I45 no. 6. I834.

Fr. Epicr. II. I836-38.

Curtis, M. A., Bot. N. Car. 83. 1867.

Pk. Rept. N.Y. Mus. 23 : $70 . d$. 1872.

Pk. Rept. N. Y. Mus. 33: 49. $d$. Oct. I883.

Morg. Jour. Myc. 3: 32. d. March 1887.

Ell. in Cat. Pl. N. J. $468 . \quad$ I889.

Amanitopsis hyperborea, Karst. Amanita hyperborea, Karst.

Rostr. Meddelelser om Groenl. 3 : 528. 1888.

Rostr. Meddelelser om Groenl. 3: 596 . I89I.

Amanitopsis lenticularis, (Lasch) Massee. See Lepiota lenticularis, (Lasch) Gillet.

Amanitopsis nivalis, (Grev.) Sacc. p. p. See Amanitopsis vaginata, (Bull.) Roze var. alba, Sacc.

Amanitopsis nivalis, Sacc. Syll. p. p. See Amanitopsis albocreata, Atk.

Amanitopsis parcivolvata, $\mathrm{Pk}$.

Pk. Bull. Torr. Bot. Club 27 : 6ro. d. 29 Dec. 1900.

Sacc. \& Syd. Syll. 16:2. d. I Feb. Igoz.

Amanitopsis plumbea, Schrt. See Amanitopsis vaginata, (Bull.) Roze.

Amanitopsis pubescens, (S.) Sacc.

Sacc. Cub. \& Manc. Syll. 5 : 25. d. 28 May 1887.

Henn. in Engler \& Prantl, Pflanzenfam. 11**: 273. I898.

Mcllvaine \& Macadam, Am. Fung. 32. d. 1900.

Amanita pubescens, $\mathrm{S}$.

S. Syn. Car. 79 (53) no. 554. d. I 822. 
Lloyd, Volva 9, 16. d. 1898 and Rev. Myc. 21 : 94 . July 1899.

Beardslee, Amanitas S. Appalach. 6. Sept. 1902.

Agaricus pubescens, $\mathrm{S}$.

S. Syn. Am. Bor. I45. no. II. 1834.

Fr. Epicr. 12. I836-38.

Curtis, M. A., Bot. N. Car. 83. 1867.

Morg. Jour. Myc. 3 : 33. d. March 1887.

Amanitopsis pusilla, $\mathrm{Pl}$.

Pk. Rept. N. Y. Mus. 50: $96 . d$. 28 March 1898.

Sacc. \& Syd. Syll. 14: 64. d. 20 Aug. 1899.

Mcllvaine \& Macadam, Am. Fung. 32. d. I900.

Amanita pusllla, Lloyd.

Lloyd, Volvæ 9, 16. d. 1898 and Rev. Myc. 21 : 94. July 1899.

Amanitopsis strangulata, (Fr.) Roze.

Underw. \& Earle, Bull. Ala. Exp. Sta. So: 255. 28 June 1897.

Pk. Rept. N. Y. Mus. 51' : 300. d. pl. 50, $f{ }^{\circ},-10 . \quad$ II Feb. 1899 and Rev. Myc. 22: 51. April 1900.

Pk. Mem. N. Y. Mus. 3 : 134. d. pl. 44, f. $1-10$. Nov. 1900.

Mcllvaine \& Macadam, Am. Fung. 30. d. pl. 10, f. 4. 1900.

Earle in Mohr, Contr. U. S. Nat. Herb. $6: 228$. 31 July I 90 I.

Brown \& Fernekes, Bull. Wis. Nat. Hist. Soc. II. 2 : 46. Jan. 1902.

Conant, Bull. Boston Myc. Club 19: 5 Feb. 1903.

Pk. Rept. N. Y. Mus. 564: 35. May 1903. [Bull. 67.] Agaricus strangulatus, Fr.

Hark. \& Moore, Cat. Pac. Fung. 2. Feb. 1880.

Pk. Rept. N. Y. Mus. $33: 48$. d. Oct. 1883.

Morg. Jour. Myc. $3:$ 32. . March 1887.

Ell. in Cat. Pl. N. J. 468. 1889. Amanita strangulata, $\mathrm{Fr}$.

Lloyd, Volvæ 9, I6. 1898 and Rev. Myc. 21 : 94. July 1899.

Williams, Asa Gray Bull. 7 : 79 . Aug. 1899.

Beardslee, Amanitas S. Appalach. 6. d. pl. 2. Sept. 1902.

Agaricus Cecilia, B. \& Br.

Pk. Rept. N. Y. Mus. 23: 70. d. 1872.

Frost in Cat. Pl. Amherst 62.1875 .

Johnson, Bull. Minn. Acad. 1 : 208. 1877 and Rept. Minn. Surv. 5 : $67 . \quad 1877$.

Bundy, Geol. Wis. $1873-79,1$ : $3960^{\circ} 1883$.

Cobb, List Pl. Amherst 31. 1887.

Amanlta Cecilia, B. \& Br.

Webster, Am. Kitchen Mag. 6 : 215. Feb. 1897.

Lloyd, Volvæ 2I. I898.

Amanitopsis vaginata, (Bull.) Roze.

Sacc. Cub. \& Manc. Syll. 5 : 21. d. 28 May 1887.

Banning in Pk. Rept. N. Y. Mus. 44: 178 (66). Dec. I 891.

Atk. \& Schrenk, Jour. Mitch. Soc. 9: 98 (4). April 1893.

Pk. Country Gent. 59 : 566 (44). f. 2 Aug. I 894.

Kellerm. \& Werner, Geol. Ohio $7^{2}$ : 300. Jan. 1895.

Pk. Rept. N. Y. Mus. 48: 257 (159). pl. $17, f_{1}, t-4$. March 1897.

Atk. Bull. Cornell Univ. 3: 24. 25 June 1897.

Underw. \& Earle, Bull. Ala. Exp. Sta. 80: 255. 28 June I897.

Taylor, Hdbk. Mushrooms 5: 9. d. 1898 .

[Underw.] N. Y. Myc. Club 16. 1898.

Pk. Bull. N. Y. Mus. 6: 192 . July 1899.

Longyear, Rept. Mich. Bd. Agr. 1898 : 92. 1899.

Underw. Moulds i20. I899.

Mcllvaine \& Macadam, Am. Fung. 28. d.pl. 1o, f. 1, 2. 1900. 
Atk. Mushrooms 74. d. pl. 23, f. 77. 20 Oct. I900.

Sweetser, Bull. Pacific Univ. 14: 12. May I90I.

Earle in Mohr, Contr. U. S. Nat. Herb. 6 : 228. 31 July roor.

Brown \& Fernekes, Bull. Wis. Nat. Hist. Soc. Il. $2: 46$. Jan. 1902.

Webster, Rhodora 4: 78. 4 April I9o2.

White, Bull. Torr. Bot. Club 29: 562. 26 Sept. I9o2.

Conant, Bull. Boston Myc. Club 19: 5 Feb. I903.

Longyear, Rept. Mich. Acad. 4: 1 I 5.1904.

Kellerm. Myc. Bull. 2 : 89. f. 79. 20 Nov. rgo4.

Pseudofarinaceus speciosior. Batt.

Pound, Clement and others, Bot. Surv. Nebr. $4: 43$. 20 Jan. I896. Agaricus vaginatus, Bull.

S. Syn. Am. Bor. I45 no. 2. 1834 .

Lea, Cat. Pl. Cin. 48. I849.

Rav. Charleston Med. Jour. \& Rev. 6 : Igo. March I851.

Sprague, Proc. Boston Soc. $6: 316 . \quad 1859$.

B. Jour. Linn. Soc. 5: 86. r86 $\mathrm{I}$ and Arctic Manual 2: $526 . \quad$ I 875.

Curtis, M. A., Bot. N. Car. 83. I867.

Cke. Handbook 5. $d$. I87I.

Pk. Rept. N. Y. Mus. 23: 69. d. 1872.

Bon. Zweite Nordpolarfahrt 2: 90. I874 and Arctic Manual 2 : 585. I 875 .

Frost in Cat. Pl. Amherst 62. 1875.

Smith, W. G., Tr. Bot. Soc. Edinb. $9: 465$ (37). 1868 and Arctic Manual 2: 283. I875.

Farl. Bull. Bussey Inst. 1: 436. March 1876.

Johnson, Bull. Minn. Acad. 1: 208. 1877 and Rept. Minn. Surv. 5 : $67 . \quad 1877$.

Morg. Fl. Miami Valley 67. 1878.

Cke. Grev. 7 : 43. Dec. I 878.

* Rav. Fung. Am. 397. I879.

James, Jour. Cin. Soc. $2: 63$ (22). April 1879.

Banning, Bot. Gaz. 5 : 6. d. 9. Jan. I880.

Hark. \& Moore, Cat. Pac. Fung. 2. Feb. I88o.

Somers, Tr. N. Scot. Inst. 5 : I 89. I 882.

Morg. Jour. Cin. Soc. 6: 59. d. April 1883.

Pk. Rept. N. Y. Mus. $33: 47$. d. Oct. I883.

Bundy, Geol. Wis. 1873-79, 1 : 396.1883.

Pk. in Day's Cat., Bull. Buf. Soc. 4 : 174 (110). I 883.

Bessey, Bull. lowa Agr. Coll. 1884: I46. Jan. r885.

Cobb, List Pl. Amherst 31. 1887.

Morg. Jour. Myc. 3: 31. d. March 1887.

Macbr. Bull. Univ. lowa 1: 36. d. Nov. 1888.

Ell. in Cat. Pl. N. J. 468.1889. Agaricus plumbeus, Schreff.

Muhl. Tr. Am. Phil. Soc. 4: 239. 1799. Agaricus badius, Schæff.

Muhl. Tr. Am. Phil. Soc. 4: 239. 1799. Amanita vaginata, Lam.

Lam. Encyc. 1: 109. d. 1783.*

Rostr. Meddelelser om Groenl. 3 : 528. I 888.

Rostr. Meddelelser om Groenl. 3 : 596. I89I.

* Shear, N. Y. Fungi 4. I893.

Taylor, Food Products 2: 21. I893.

Webster, Am. Kitchen Mag. 6: 215. Feb. 1897.

Lloyd, Volvæ 8. d. f. 7. I 5. I 898 and Rev. Myc. 21 : 93 . July I899.

Williams, Asa Gray Bull. 7 : 79. Aug. 1899.

Herbst, Fungal Fl. Lehigh Valley 31.d. 1899. 
Williams, Asa Gray Bull. 8 : 104. Oct. 1900.

Lloyd, Myc. Notes 61. May 1901.

Beardslee, Amanitas S. Appalach. 2. d. Sept. 1902.

MacKay, Tr. N. Scot. Inst. 11: 123. Aug. 1904.

Amanita badia, $\mathrm{P}$.

Lloyd, Volvæ 21. iso8.

Amanitopsis piumbea, Schrt.

Henn. in Engler \& Prantl, Pflanzenfam. 11** : 273. d. 1898.

Perry, Agricultural Student $6:$ 179. May 1900 and Ohio Univ. Bull.

IV. 30:8. May 1900.

Amanitopsis vaginata, (Bull.) Roze, var, alba, Sacc. Syll.

Pk. Country Gent. 59: 567 (45). d. 2 Aug. 1894.

Pli. Rept. N. Y. Mus. 48: 258 (160). d. March 1897.

Taylor, Hdbk. Mushrooms 5: 9. d. 1898 Agaricus nivaiis, Grev.

Morg. Jour. Myc. $3:$ 32. d. March 1887. Amanita nivaiis, Grev.

Lloyd, Volvæ 9, 16. d. 1898. Amanitopsis nivalis, (Grev.) Sacc. p. p.

Sacc. Cub. \& Manc, Syll. 5 : 22. d. 28 May 1887.

Amanitopsis vaginata, (Bull.) Roze, var. crassivolvata, Pk.

Pk. in White, Bull. Torr. Bot. Club 29 : 562. d. 26 Sept. 1902.

Sacc. Syll. $17:$ 3. d. 25 May I 905.

Amanitopsis vaginata, (Bull.) Roze, var. Pulva, (Schæff.) Sacc. Syll. Pk. Country Gent. 59: 567 (45). d. 2 Aug. 1894.

Pk. Rept. N. Y. Mus. 48: 258 (i60). d. March 1897.

Taylor, Hdbk. Mushrooms 5: 9. d. 1898.

Mcllvaine \& Macadam, Am. Fung. 29. d. 1900.

Hay, Bull. Nat. Hist. Soc. N. B. 4: 342. 1901 and $5: 119.1903$.

Brown \& Fernekes, Bull. Wis. Nat. Hist. Soc. II. $2: 46$. Jan. 1902.

White, Bull. Torr. Bot. Club 29: 562. 26 Sept. 1902.

Agaricus fuivus, Schæff.

Muhl. Tr. Am. Phil. Soc. 4: 239. 1799.

Amanitopsis vaginata, (Bull.) Roze, var. livida, (P.) Pk.

Pk. Country Gent. 59: $567(45) . d$. 2 Aug. I 894.

Pk. Rept. N. Y. Mus. 48: 258 (160). d. pl. 17, f. 5-9. March 1897.

Taylor, Hdbk. Mushrooms 5: 9. $d$. 1898.

Hay, Bull. Nat. Hist. Soc. N. B. 4: 342. 1901 and 5: 119.1903. Amanita livida, $\mathrm{P}$.

Muhl. Cat. Pl. Am. Sept. I ed. 104. 1813. 2 ed. 107. 1818.

Torrey, Cat. PI. N. Y. $88 . \quad 1819$.

S. Syn. Car. $78(52)$ no. 538 . 1822 .

Hitchiock, Cat. Pl. Amherst 59. 1829.

Hitchcock, Cat. Pl. Mass. 644. 1833. 2 ed. 646 (126). 1835.

Lloyd, Volvæ 21. 1898.

Amanita spadicea, $\mathrm{P}$.

Muhl. Cat. PI. Am. Sept. 1 ed. 104. 1813. 2 ed. 107. 1818.

S. Syn. Car. 78 (52) no. 539. 1822.

Lloyd, Volva 21. 1898 .

Amanitopsis velosa, $\mathrm{Pk}$.

NOTE. The above name originally given by Peck in 1895 was changed by Saccardo and Sydow, apparently on etymological grounds to Amanitopsis villosa.

Pk. Bull. Torr. Bot. Club 22 : 485. d. 30 Dec. 1895.

McClatchie in Reid's Hist. Pasadena 619. 1895.

McClatchie, Proc. S. Cal. Acad. 1: 385. 5 June 1897.

Mcllvaine \& Macadam, Am. Fung. 29. d. 1900. 
Amanita velosa, Lloyd.

Lloyd, Volvæ 9, 15. $d .1898$ and Rev. Myc. 21 : 93. July 1899.

Williams, Asa Gray Bull. 8 : 103. Oct. 1900.

Amanitopsis villosa, Sacc. Syll.

Sacc. Hedw. 35. Rep. 7: 1. 25 Feb. 1896.

Sacc. \& Syd. Syll. 14: 64. d. 20 Aug. 1899.

Amanitopsis villosa, Sacc. Syll.

See Amanitopsis velosa, Pk.

Amanitopsis volvata, (Pk.) Sacc.

Sacc. Cub. \& Manc. Syll. 5 : 23. d. 28 May 1887.

Banning in Pk. Rept. N. Y. Mus. 44: 178 (66). Dec. I 89I.

Atk. \& Schrenk, Jour. Mitch. Soc. 9 : 98 (4). April I893.

Kellerm. \& Werner, Geol. Ohio $7^{2}$ : 301. Jan. I 895.

Underw. \& Earle, Bull. Ala. Exp. Sta. 80 : 255. 28 June 1897.

Henn. in Engler \& Prantl, Pflanzenfam. 11**: 273. 1898.

Mcllvaine \& Macadam, Am. Fung. 31. d. 1900.

Atk. Mushrooms 76.20 Oct. 1900.

Earle in Mohr, Contr. U. S. Nat. Herb. 6 : 228. 31 July I9or.

White, Bull. Torr. Bot. Club. 29: 563. 26 Sept. 1902.

Conant, Bull. Boston Myc. Club i9: 5 Feb. I903.

Pk. Rept. N. Y. Mus. 564: 35. d. May 1903. [Bull. 67.] Agaricus volvatus, $\mathrm{Pk}$.

Pk. Rept. N. Y. Mus. 24: 59. d. 1872.

Frost in Cat. Pl. Amherst 62. 1875 .

Banning, Bot. Gaz. 6: 20I. April 188I.

Morg. Jour. Cin. Soc. 6 : 59. d. April 1883.

Pk. Rept. N. Y. Mus. $33: 47 . d$. Oct. 1883.

Morg. Jour. Myc. 3: 31. d. March 1887.

Cobb, List Pl. Amherst 31. 1887.

Amanita volvata, $\mathrm{Pk}$.

Pk. Rept. N. Y. Mus. 24: 59. d. 1872.

Lloyd, Volvæ 9. d. 15. $d$. 1898 and Rev. Myc. 21 : 94. July 1899.

Williams, Asa Gray Bull. $7: 79$. Aug. 1899.

Herbst, Fungal Fl. Lehigh Valley 30. d. 1899.

Williams, Asa Gray Bull. 8 : I04. Oct. 1900.

Agaricus soleatus, Howe.

Howe, Bull. Torr. Bot. Club 5 : 42. d. Oct. 1874 .

Pk. Rept. N. Y. Mus. 33 : 49. Oct. 1883.

Amanita soleata, Howe.

Howe, Bull. Torr. Bot. Club 5 : 42. d. Oct. 1874 .

Lloyd, Volvæ 9, I 5. d. 21. I898.

Amanitopsis volvata, (Pk.) Sacc., var. elongatus, $\mathrm{Pk}$.

Pk. Rept. N. Y. Mus. $53: 856 . d_{0} p l$. $A, f .6-10$. I 9 March 190 I.

Sacc. \& Syd. Syll, 16 : iा Io. I Feb. 1902.

Sacc. Syll. 17 : 3. d. 25 May 1905.

Amaurochrte atra, (A. \& S.) Rostf.

Note. This name we have adopted rather than $A$. fuliginosa since it is accepted by nearly all writers and is based upon Lycogala atrum, A. \& S. of which the description and figure are clearly characteristic whereas those of Licea fuliginosa, Sowerhy are vague and uncertain. See also Dermodium.

Cke. Ann. N. Y. Lyceum 11 : 398 . June 1877.

Pk. Rept. N. Y. Mus. 30: 50. Sept. 1878.

Pk. Rept. N. Y. Mus. 31: 5 G. I 879.

Berl. in Sacc. Syll. 7 : 401. d. 15 March, 1888.

Schrt. in Engler \& Prantl, Ptlanzenfam. 11: 25. d. June I889. 
Ell. in Cat. PI. N. J. 598. 1889.

Massee, Mon. Myx. 89. d. Dec. I892.

Lister, Mycetozoa 134. d. pl. $5 I \mathrm{~A} .1894$.

Harvey, Bull. Torr. Bot. Club 23: 3 10. 20 Aug. 1896.

Ricker, Univ. of Maine Studies 3: 15. 30 April 1902.

Lycogala atrum, A. \& S.

S. Syn. Car. 6I (35) no. 35I. I822.

Reticularia atra, Fr.

Curtis, M. A., Bot. N. Car. II I. 1867.

Che. Handbook 379. $d$. I871.

Johnson, Bull. Minn. Acad. 1: 289. 1877 and Rept. Minn. Surv. 5 : 82. 1877 .

Morg. Jour. Cin. Soc. 15 : 138 (12). d. March 1893.

Kellerm. \& Werner, Geol. Ohio 7*: 400. Jan. 1895.

Underw. \& Earle, Bull. Ala. Exp. Sta. 80: 135. 28 June 1897.

Earle in Mohr, Contr. U. S. Nat. Herb. 6 : 14I. 3I July I90I. Reticularia Strongylium, S. Am. Bor.

S. Syn. Am. Bor. 262 no. 2374. 1834.

Rostf. Mycetozoa 242. 1875 .

Cke. Ann. N. Y. Lyceum 11 : 402 . June 1877. Amaurochate fuliginosa, (Sow.) Macbr.

Macbr. Slime-moulds 109. d. pl. 5, f. 8, 8a. Aug. 1899 .

Amaurochrte fuliginosa, (Sow.) Macbr. See Amaurochate atra, (A. \& S.) Rostf.

Amaurochæte minor, Sacc. \& Ell.

Sacc. Mich. $2: 566$. Dec. 1882.

Berl. in Sacc. Syll. 7 : 40I. d. 15 March 1888.

Lister, Mycetozoa 135. d. 1894.

Amerosporium Caricum, (Lib.) Sacc.

*Ell. \& Ev. N. A. F. 2575 . Feb. I891.

Tracy \& Earle, Bull. Miss. Exp. Sta. 34 : 112. May 1895.

Amerosporium cinctum, Ell. \& Ev.

Ell. \& Ev. Proc. Phil. Acad. 1895: 435. d. Nov. 1895.

McClatchie in Reid's Hist. Pasadena 6i6. 1895.

Sacc. Hedw. 35 : Rep. 7 : xliv. 25 Feb. 1896.

Sacc. \& Syd. Syll. 14: 1001. d. 20 Aug. 1899.

Amerosporium Dolichi, Ell. \& Ev. See Amerosporium aeconomicum, Ell. \& Tracy.

Amerosporium Equiseti, (Pk.) Sacc.

Sacc. Syll. 3: 682. $d$. I5 Dec. 1884.

Excipula Equiseti, Pk.

Pk. Rept. N. Y. Mus. 26: 77. d. April 1874.

Pk. in Day's Cat., Bull. Buf. Soc. 4: 202 (138). 1883.

Amerosporium Geranil, Cke. \& Hark.

Cke. \& Hark. Grev. 13: I 12. $d$. June 1885.

Hark. Bull. Cal. Acad. 1 : 269 (14). I9 Nov. I885.

Berl. \& Vogl. in Sacc. Syll. Add. 1-4 : 359.d. 31 Dec. 1886.

Sacc. Syll. $10: 436 . d$. 30 June 1892.

Amerosporium ilicinum, Ell. \& Ev.

Langlois, Cat. Pl. La. 27. 1887.

Ell. \& Halsted, Jour. Myc. 4:9. Jan. 1888 .

Ell. \& Ev. Jour. Myc 4: 50. d. June 1888.

Sacc. Syll. 10: 437. d. 30 June 1892 .

Ell. \& Ev. N. Am. Phyllostictas 40. Dec. 1900. 
Amerosporium ineurvum, (Cke.) Sacc.

Sacc. Syll. 3:68I. $d$. 15 Dec. I884. Excipula incurva, Cke.

Cke. Grev. 5 : I5I. $d$. June 1877.

Ell. in Cat. Pl. N. J. 589. 1889.

Amerosporium leueotrichum, (Pk.) Sacc.

Sacc. Syll. $3: 682$. $d$. I 5 Dec. I884. Excipula leucotricha, Pk.

Pk. Rept. N. Y. Mus. $29: 49 . d$. 1878.

Amerosporium macrochæta, Ell. \& Ev.

Langlois, Cat. Pl. La. 27. 1887.

Ell. \& Ev. Jour. Myc. 4: 50. d. June I 888.

Sacc. Syll. 10: 438. d. 30 June 1892 .

Amerosporium microsporum, (C. \& E.) Sacc.

Sacc. Syll. 3 : 68I. $d$. . I 5 Dec. I 884 .

Excipula microspora, C. \& E.

C. \& E. Grev. $6: 4 . d . p l .95, f .18$. Sept. 1877 .

Ell. in Cat. Pl. N. J. 589 . 1889.

Amerosporium œconomicum, Ell. \& Tracy.

Note. The names Amerosporium aconomicum and $A$. Dolichi are founded on material of the same host, locality, and collector and J. B. Ellis states in a letter that they are synonyms.

Ell. \& Ev. Jour. Myc. 4: 102. d. Oct. 1888.

Sacc. Syll. $10: 437 . d$. 30 June 1892.

Tracy \& Earle, Bull. Miss. Exp. Sta. 34 : r12. d. May 1895.

Tracy \& Earle, Bull. Miss. Exp. Sta. $38:$ 148. May 1896.

Atk. Bull. Cornell Univ. 3 : 33. 25 June 1897.

Underw. \& Earle, Bull. Ala. Exp. Sta. 80: 175. 28 June 1897.

Earle in Mohr, Contr. U. S. Nat. Herb. $6: 261$. 31 July 1901;

Amerosporium Dolichi, Ell. \& Ev.

*Ell. \& Ev. N. A. F. 2574. I891.

Amerosporium punetiforme, (B.) Sacc.

Sacc. Syll. $3: 68$ I. $d$. 15 Dec. I884.

Excipula punctiformis, B.

B. Grev. 2: 97 (78) no. 453. $d$. Jan. 1874 .

Amerosporium sabalinum, Ell. \& Ev.

Langlois, Cat. Pl. La. 27. 1887.

Ell. \& Ev. Jour. Myc. 4 : 50. d. June 1888.

Sacc. Syll. 10: 437. d. 30 June 1892 .

Amerosporium subclausum, Ell. \& Kellerm.

Ell. \& Kell. Jour. Myc. 5 : 143. d. Sept. I889.

Sacc. Syll. $10: 437 . d$. 30 June 1892.

Lindau in Engler \& Prantl, Pflanzenfam. 11**: 395. Jan. 1900.

Amorphomyces, Thax.

Thax. Proc. Am. Acad. 28: 158. d. 20 June 1893 and Jour. Roy. Micr. Soc. 1894: 96. d. Feb. 1894 and Bot. Centralbl. Beiheft.

4: $111, d$. I894.

Thax. Proc. Am. Acad. 29 : 106. II Jan. I894.

Thax. Mem. Am. Acad. $12: 295$. d. pl. $5, f_{1}$ 17-29. Dec. I896.

Lindau in Engler \& Prantl, Pflanzenfam. 11: 501. $d$. April 1897.

Sacc. \& Syd. Syll. 14: 728. 20 Aug. 1899.

Amorphomyees Falagriæ, Thax.

Thax. Proc. Am. Acad. 28 : 158. d. 20 June 1893.

Thax. Proc. Am. Acad. 29: I06. II Jan. I894.

Sacc. Syll. 11 : 449 . July 1895. 
Thax. Mem. Am. Acad. 12 : 296. d. pl. 5, f. 17-29. Dec. 1896. Lindau in Engler \& Prantl, Pflanzemfam. 11 : 501. f. 291 C. April 1897. Sacc. \& Syd. Syll. 14: 728. d. 20 Aug. 1899.

Ricker, Univ. of Maine Studies 3: 41. 30 April 1902.

Amorphomyces Floridanus, Thax. See Dioicomyces Fioridanus, Thax.

\section{Ampelomyces quisqualis, Ces.}

NoTE. The name Ampelomyces quisqualis, Ces. given in Klotzsch Herb. Viv. Myc. 1660. 1852 and also in Bot. Zeit. 10: 301. 23 April 1852 certainly antedates Cicinnobolus, Ehrb. which did not appear until the following year (Bot. Zeit. 11: 16. 7 Jan. 1853) and then in a very vague way.

The true structure and position of the genus Cicinnobolus were first given by De Bary in Beitr. Morph. und Phys. der Pilze $3: 53-75$. pl. $11,12.1870$ where he called the species Cicinnobolus Cesatii. "In our opinion the name Cicinnobolus Cesatii, DBy. is the name which should be preferred to any other but we do not agree with De Bary in referring the genus to Ehrenberg but consider that he is himself the real author of the genus.

Griffiths, Bull. Torr. Bot. Club. $26:$ I 84, 186. d. pl. 358. I2 April I899. Underw. Moulds 232. pl. $5, f .3 .1899$.

Nelson, Rept. Wyo. Exp. Sta. 10 : (29). 1900.

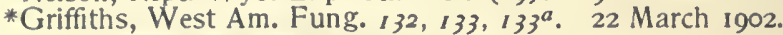
Cicinnoboius Cesatii, D By.

Hark. Bull. Cal. Acad. 1 : 258 (3). ig Nov. I885.

Burrill \& Earle, Bull. IIl. Lab. 2 : 390,39 I. f. 2 . July 1887.

Halsted, Bull. lowa Agr. Coll. 1888: 110. April 1888.

Pammel, Jour. Myc. 4: 38. d. May 1888.

Scribner, Rept. U. S. Agr. $1887: 375,378,395 . p l .11, f_{\bullet} 2.1888$.

Kellerm. \& Swingle, Rept. Kans. Exp. Sta. 1: 312. d. Feb. 1889.

Gall. Rept. U.S. Agr. 1888 : 355. d. pl. 7. f. 6. 1889.

Anderson, Jour. Myc. $5: 84$. June 1889.

* Sevmour \& Earle, E. F. 145. I Sept. 1892.

Underw. Proc. Ind. Acad. 1893 : 43. Aug. 1894.

*Ell. \& Ev. Fung. Columb. 960 . April 1896.

*Ell. \& Ev. N. A. F. 3445. Io Dec. I896.

Stevens, F. L., Rept. Ohio Acad. 4: 2I. 1896

Pammel \& Carver, Proc. lowa Acad. 3: 146. 1896.

Atk. Bull. Cornell Univ. 3 : 29. 25 June 1897.

Underw. \& Earle, Bull. Ala. Exp. Sta. 80: 163. 28 June 1897.

Stevens, F. L., Rept. Ohio Acad. 5: 64. 1897.

Orton, Rept. Vt. Exp. Sta. 11: 205. Sept. 1898 and Contr. Bot. Vt. $2: 7$. Sept. 1898.

Brannon, Proc. Ind. Acad. 1898 : 293. Dec. I 899.

Orton, Rept. Vt. Exp. Sta. 12: i72. Dec. 1899 and Contr. Bot. Vt. 7:172. Dec. 1899.

Patterson, Bull. U. S. Agr. Pl. Industry 8: 23. 3 Feb. 1902.

* Barthol. Ell. \& Ev. Fung. Columb. 1815. 28 Dec. 1903.

\section{Amphiphytum, S.}

Note. Where this genus was described and whether it is a North American genus we are unable to ascertain. It is referred to in the citations given below and also in Reichenbach, Conspect. Reg. Veg. 1828 as a doubtful genus but we have been unable to trace the name in Schweinitz, the author designated by Endlicher and Corda.

Endl. Gen. Pl. 35. 1840.

Cda. Icon. 5 : 44 . 1842. 
Amphisphærella aperta, Cke.

See Rosellinia aperta, (S.) Sacc.

Amphisphærella Diderma, Cke.

See Rosellinia Diderma, (S.) Sacc.

Amphisphæria acicola, Sacc.

See Neopeckia Couiteri, (Pk.) Sacc.

Amphisphæria æthiops, (B. \& C.) Sacc.

Sacc. Syll. $1:$ 722. $d$. I3 June I882.

Cke. Grev. 16: 89. March I888.

"Ell. \& Ev. Pyren. 204. d. May 1892.

Spharia zthiops, B. \& C.

Curtis, M. A., Bot. N. Car. I46. I867.

B. Grev. 4: I 43 (I8I) no. 9I8. d. June I876.

Amphisphæria albomaculans, (S.) Cke.

Cke. Grev. 16: 89, 9I. $d$. March I888.

Sacc. Syll. $9: 747 . d$. I 5 Sept. I89 .

Ell. \& Ev. Pyren. 204. d. May 1892.

Sphreria albomaculans, S.

S. Syn. Am. Bor. 215 no. I592. 1834.

Sacc. Syll. 2: 413. d. I3 June 1883 .

Amphisphæria aperta, Cke.

See Roselinia aperta, (S.) Sacc.

Amphisphæria apiosporioides, Rehm.

Rehm, Hedw. 39 : 323. d. f. 7. 28 Dec. 1900.

Sacc. \& Syd. Syll. 16: 504. d. I Feb. I902.

Amphisphæria applanata, (Fr.) Ces. \& De Not.

Farl. Appalachia 3 : 247. Jan. I884.

Ell. \& Ev. Pyren. 206. d. May 1892.

Harvey, Bull. Torr. Bot. Club 23 : 53. 29 Feb. I896.

Ricker, Univ. of Maine Studies 3: 32. 30 April 1902.

Sphæria applanata, Fr. p. p.

S. Syn. Am. Bor. 214 no: 1574. 1834.

Amphisphæria applanata, (Fr.) Ces. \& De Not., var. Alaskæ, Sacc.

Sacc. Syll. $17: 685 . d$. 25 May 1905.

Amphispharia applanata, Sacc. in Alaska Exp.

Sacc., Pk. \& Trel., Harriman Alaska Exp. 5 : 30. d. 5 April 1904.

Amphisphæria aquatica, (Ell. \& Ev.) Berl. \& Vogl.

Berl. \& Vogl. in Sacc. Syll. Add. 1-4: I25. d. 3I Dec. 1886.

Cke. Grev. 16: 89. March 1888.

Sacc. Syll. $9: 744 . d$. I 5 Sept. I 89 I.

Ell. \& Ev. Pyren. 205. d. May I 892 .

Sphria aquatica, Ell. \& Ev.

Ell. \& Ev. Torr. Bull. $11:$ 42. d. April 1884.

Didymospharia aquatica, Ell. \& Ev.

Ell. in Cat. Pl. N. J. 523. 1889.

Amphisphrria aspera, Ell. \& Ev.

Ell. \& Ev. Bull. Torr. Bot. Club 27 : 52. d. 17 Feb. 1900.

Sacc. \& Syd. Syll. 16 : 505. d. I Feb. 1902.

Amphisphæria aterrima, Pat.

Pat. in Duss Champ. Guad. \& Martinique 7I. d. 1903. West Indies.

Sacc. Syll. $17: 684$. d. 25 May 1905. 
Amphisphæria atrograna, (C. \& E.) Sacc.

Sacc. Syll. $1: 722$. d. 13 June 1882.

Ell. in C.at. Pl. N. J. 518.1889.

Ell. \& Ev. Pyren. 20I. d. May 1892.

Spharia atrograna, C. \& E.

C. \& E. Grev. 8: 15. d. Sept. 1879.

Melanomma atrogranum, Cke.

Cke. Grev. 16 : 52. Dec. 1887.

Amphisphæria bisphærica, (C. \& E.) Sacc.

Sacc. Syll. 1: 721. $d$. I3 June I882.

Cke. Grev. 16: 89. March 1888 .

Ell. in Cat. PI. N. J. 518.1889.

Ell. \& Ev. Pyren. 20I. d. May I 892.

*Ell. \& Ev. Fung. Columb. 1318. May 1899. Spharia bispharica, C. \& E.

C. \& E. Grev. 7. 4I. d. Dec. 1878.

*EII. N. A. F. 1339. 1885 .

Amphisphæria botulispora, (Cke.) Sacc.

Sacc. Svll. 1: 719. $d$. 13 June I882.

Ell. \& Ev. Pyren. 203. d. . May 1892.

Spharia botulispora, Cke.

C. \& E. Grev. 6: 96. pl. 100, f. 31. March 1878 .

Cke. Ann. N. Y. Acad. 1: 186. d. May 1878.

Cke. Jour. Linn. Soc. 17: 143. d. I880. Xylospharia botulispora, Clie.

Cke. Grev. $17: 86$. June i 889.

Amphisphæria caryophaga, Cke. See Trematospharia caryophaga, (S.) Sacc.

Amphisphæria conferta, (S.) Sacc.

Note. The Spharia conferla of Schweinitz, Syn. Car. 187 was said by - Schweinitz to be the same as the Spharia confertula, S. Am. Bor. 1508. The Spharia conferta of S. Am. Bor. 1477 according to Ellis and to our own examination of an original specimen is immature Spharella maculiformis, (P.) Awd.

With regard to Spharia conferla, S. Syn. Car. 187 , the spores are said by Cooke, Grev. 15: 8I. $d$. March 1887 to be two-parted and on this authority it forms the basis of Amphispharia conferta, (S.) Sacc. Starbaeck, 1894, found no spores in Schweinitzian specimens but noted a marked resemblance externally to Spheria euomphala, B. \& C. Ellis in Proc. Phil. Acad. 1895: 25 (6). 21 Feb. 1895 gives details of spores of Spharia confertula from Pennsylvania and expresses a doubt whether it is the same as the North Carolina specimens of that name.

We have examined a specimen of $S$. conferlula (S. conferta, S Syn. Car.) from Carolina and the external resemblance to $S$. euomphala is marked, as stated by Starbaeck but the spores do not agree either with those described by Cooke or with those described by Ellis. Two kinds of spores were seen; one dark-colored, elliptic, $8-9 \mu$, by $2.8 \mu$, filling the perithecium but no asci were seen and in one case slender, clavate asci $84 \mu$ long by $7 \mu$ broad with hyaline acicular spores $14-16 \mu$ by $2.8 \mu, 6$ - to 8 -guttulate but not clearly septate.

From the above it will be seen that it is hopeless to expect to be able to recognize what Schweinitz understood by $S$. conferla and $S$. confertula. We have given below the synonomy based on Cooke's notes on the spores but we do not believe that the species is an Amphispharia nor that any one at the present day can say to what genus it belongs. It should be placed permanently under species ignola. 
Sacc. Syll. $9: 747 . d$. I 5 Sept. I 89 I.

Ell. \& Ev. Pyren. 206. d. Mav I892.

Spharia conferta, S. Syn. Car. 187.

S. Syn. Car. 45 (I9) no. I87. d. I822.

Fr. S. M. 2 : 444 . d. 1823.

Spreng. in L. Syst. Veg. 16 ed. $41^{1}: 388 . d$. 1827.

Sacc. Syll. $2: 401 . d$. I3 June I883.

Ell. \& Ev. Pyren. 745. May 1892.

Starb. Bot. Notiser 1893: 28. I5 Feb. 1893.

Starb. Bihang till K. Sv. Vet. Akad. Handl. 193 : no. 2 : 25.1894. Spharia confertula, S.

S. Syn. Am. Bor. 211 no. 1508.1834.

Curtis, M. A., Bot. N. Car. I44. I867.

Byssospharia (Amphisphæria) conferta, Cke.

Cke. Grev. 15: 81. d. March 1887.

Cke. Grev. 15: i23. June i 887.

Trematospharia confertula, Ell.

Ell. Proc. Phil. Acad. 1895: 25 (6). d. 2I Feb. r895.

Sacc. Hedw. 35 : Rep. 7 : xxix. d. 25 Feb. 1896.

Sacc. \& Syd. Syll. 14: 576. d. 20 Aug. I 899.

Amphisphæria confertissima, Ell. \& Ev.

Ell. \& Ev. Proc. Phil. Acad. 1895: 418 . d. Nov. 1895.

Sacc. Hedw. 35 : Rep. 7 : xxviii. 25 Feb. 1896.

Sacc. \& Syd. Syll. 14: 557. d. 20 Aug. 1899.

Amphisphæria conica, (Lév.) Ces. \& De Not.

Langlois, Cat. Pl. La. 27. I887.

Amphisphæria decorticata, (Cke \& Hark.) Berl. \& Vogl.

Berl. \& Vogl. in Sacc. Syll. Add. 1-4: 1 124. d. 3 I Dec. I886.

Sacc. Syll. 9: $742 . d$. I5 Sept. I891.

Ell. \& Ev. Pyren. 202. d. May 1892.

Spharia decorticata, Cke. \& Hark.

Cke. \& Hark. Grev. 13 : 19. d. Sept. 1884.

Hark. Bull. Cal. Acad. 1 : i75. 7 March I885.

Melanomma decorticatum, Cke.

Cke. Grev. 16 : 52. Dec. 1887.

Amphisphæria deerrata, Cke.

See Rosellinia deerrata, (C. \& E.) Sacc.

Amphisphæria deformis, Ell. \& Langlois.

Ell. \& Ev. Jour. Myc. 4: 123. $d$. Dec. 1888.

Sacc. Syll. $9: 744 . d$. is Sept. I89I.

Ell. \& Ev. Pyren. 202. d. May 1892 .

Sacc. Syll. 11 : 316. d. July I 895.

Amphisphæria Despreauxii, Cke.

See Rosellinia Despreauxii, (Mont.) Sacc.

Amphisphæria Diderma, Cke.

See Rosellinia Diderma, (S.) Sacc.

Amphisphæria diffusa, Sacc.

See Neopeckia diffusa, (S.) Starb.

Amphisphæria dothideospora, Cke. \& Hark.

Cke. \& Hark. Grev. 14: 9. d. Sept. 1885.

Hark. Bull. Cal. Acad. 1: 27 I (I6). in Nov. 1885.

Berl. \& Vogl. in Sacc. Syll. Add. 1-4 : I25. d. 3 I Dec. I886.

*Ell. \& Ev. N. A. F. 1662.1886.

*Wint. Fung. Eur. 3553. I 886.

Sacc. Syll. $9: 746 . d$. 15 Sept. I 891.

Ell. \& Ev. Pyren. 202. d. May 1892. 
Melanomma dothideasporum, Cke.

Cke. Grev. 16 : 53. Dec. I 887.

Amphisphreria Eckfeldtil, Cke.

See Melanopsamma Eckfeldtii, (Ell.) Ell. \& Ev.

Amphisphæria fallax, De Not.

Ell. \& Ev. Proc. Phil. Acad. 1893 : 129. 28 Feb. 1893.

Amphisphæria granulosa, Ell. \& Ev.

Ell. \& Ev. Jour. Myc. 10: 169. d. 24 June 1904.

Sacc. Syll. $17: 685 . d$. 25 May 1905.

Amphisphæria Hypoxylon, Ell. \& Ev.

NoTE. This belungs to the genus Otthia. See Otthia Hypoxylon, the name which is here given to retain the original specific name rather than Othia hypoxyloides to which it was changed by Ellis and Everhart, Pyren. 249. May 1892.

Amphisphæria inerustans, Ell. \& Ev.

Webber, Rept. Nebr. Agr. 1889 : 193 (53). 1890.

Ell. \& Ev. Pyren. 201. d. May 1892 .

Sacc. Syll. 11: 316. d. July 1895.

Amphisphæria inspersa, Cke.

See Leptospharia inspersa, (S.) Sacc.

Amphisphæria Juniperi, Tracy \& Earle.

Tracy \& Earle in Greene, Pl. Baker. 1: 30. d. 22 Feb. I901.

Sacc. \& Syd. Syll. 16: I134. I Feb. I902.

Sacc. Syll. $17: 684$. d. 25 May 1905.

Amphisphæria Langloisil, Ell. \& Ev.

Ell. \& Ev. Pyren. 205. d. May 18902.

Sacc. Syll. 11: 315. d. July 1895 .

Amphisphæria megalospora, (Mont.) Sacc.

Sacc. Syll. 1: 724. $d$. I J June 1882 .

Sphzria megalospora, Mont.

B. \& C. Jour. Linn. Soc. $10: 388.1869$.

West Indies.

Amphisphæria melantera, Ell. \& Ev.

Ell. \& Ev. Bull. Torr. Bot. Club 24: 278. d. 29 June 1897.

*Ell. \& Ev. N. A. F. 3521.24 Jan. I 898.

Lindau \& Syd. Hedw. 37 : Beibl. 7 : xxvii. 9 April 1898.

Ell. \& Ev. Bull. Torr. Bot. Club $25: 513 . d$. 10 Sept. 1898.

Sacc. \& Syd. Syll. 14: 556. d. 20 Aug. I 899.

Amphisphæria melasperma, (Cke.) Sacc.

Sacc. Syll. 1: 725. $d$. I3 June 1882 .

Ell. \& Ev. Pyren. 203. d. May 1892 .

Psilospharia melasperma, Cke.

Cke. Grev. 8: i18. d. March 1880 .

Melanomma melaspermum, Cke.

Cke. Grev. $16: 52$. Dec. 1887.

Amphisphæria mobilis, (S.) Ell.

NOTE. The Spharia mobilis of the Schweinitz herbarium according to Ellis is an Amphispharia and not the Spharia mobilis of Fr. S. M. 2: 461 or of Tode which is a Nectria. All American references belong to the Amphispharia. It should be called to mind that in several instances the name has been misspelled nobilis.

Ell. \& Ev. Proc. Phil. Acad. 1895: 26 (7) d. April 1895.

Sacc. Hedw. 35: Rep. 7 : xxviii. d. 25 Feb. 1896.

Sacc. \& Syd. Syll. 14: 557. d. 20 Aug. 1899. 
Spharia mobilis, S. non Tode.

S. Syn. Car. 45 (I9) no. I98. I822.

Fr. S. M. 2: 46 I. d. I 823 .

S Syn. Am. Bor. 214 no. I569. 1834.

Curtis, M. A., Bot. N. Car. I45. 1867.

Nectria mobilis, Auct. Amer.

Sacc. Syll. 2: 5 Io. I3 June I883.

Ell. \& Ev. Pyren. IIo. d. May I 892 .

Amphisphæria myeophila, Cke.

See Rosellinia mycophila, (Fr.) Sacc.

Amphisphæria nuda, Ell. \& Ev.

Ell. \& Ev. Erythea 2: I8. $d$. 9 Feb. I 894.

*Ell. \& Ev. N. A. F. 30II. April I 894.

Sacc. Syll. 11 : 315. d. July I 895.

*Ell. \& Ev. Fung. Columb. 1036. 22 Dec. 1896.

Amphisphæria obtusissima, Cke.

See Rosellinia obtusissima, (B. \& C.) Sacc.

Amphisphæria Oronoensis, (Ell. \& Ev.) Sacc.

Sacc. Syll. 9 : 746. $d$. I 5 Sept. I 89 I.

Ell. \& Ev. Pyren. 203. d. May 1802.

Harvey, Bull. Torr. Bot. Club 23: 53. 29 Feb. 1896.

Ricker, Univ. of Maine Studies 3: 32. 30 April 1902.

Spharia Oronoensis, Ell. \& Ev.

Ell. \& Ev. Jour. Myc. 3: i 17. d. Oct. I887.

Amphisphæria Papilla, (S.) Sacc.

Sacc. Mich. 2: 138. $d$. April 1880.

Cke. Grev. 16: 89. March I888.

*Ell. \& Ev. N. A. F. 3012. April I894.

Ell. Proc. Phil. Acad. 1895: 23 (4).d. April 1895.

Sacc. Hedw. 35 : Rep. 7 : xxviii. 25 Feb. 1896.

Sacc. \& Syd. Syll. 14: 557. d. 20 Aug. I 899. Sphzria Papilla, S.

S. Syn. Car. 42 (I6) no. I59. d. I822.

Fr. S. M. $2: 462$. d. I823.

S. Syn. Am. Bor. 214 no. 1572.1834.

Sprague, Proc. Boston Soc. 6: $320 . \quad 1859$.

Curtis, M. A., Bot. N. Car. I45. I867.

Pk. Rept. N. Y. Mus. 22: 99.1869.

B. Grev. 4: I44 (182). d. June 1876.

Melanopsamma Papilla, Sacc.

Sacc. Syll. $1: 580 . d$. I3 June I 882.

Ell. \& Ev. Pyren. 178. $d$. May I892.

Amphisphæria papillata, (Schum.) De Not.

Ell. \& Ev. Pyren. 206. $d$. May I 892 .

Spharia papillata, Schum.

Fr. S. M. $2: 46$ I. $d$. I $823 .^{*}$

S. Syn. Am. Bor. 214 no. $1570 . \quad 1834$.

Amphisphæria peniophora, Cke.

See Melanomma callicarpum, (Sacc.) Berl.

Amphisphæria phileura, (C. \& P.) Sacc.

Sacc. Syll. $1: 725 . d$. I 3 June I 882 .

Cke. Grev. 16: 89. March I 888.

Ell. \& Ev. Pyren. 203. d. 'May i 892 '.

Sphæria phileura, C. \& P.

C. \& P. in C. \& E. Grev. 5: 55. d.pl. 81, f.6. Dec. 1876. 
Amphisphæria pllosella, Ell. \& Ev.

Ell. \& Ev. Proc. Phil. Acad. 1895: 418. d. Nov. 1895.

Sacc. Hedw. 35 : Rep. 7 : xxviii. 25 Feb. 1806.

Sacc. \& Syd. Syll. 14: 557. d. 20 Aug. 1899.

Amphisphreria pinicola, Rehm.

*Ell. \& Ev. N. A. F. 3113. Nov. 1804.

Millsp. \& Nutt. Publ. Field Columb. Mus. Bot. 1 : 141. Jan. 1896.

Amphisphæria Platani, Ell. \& Ev.

Ell. \& Ev. Pyren. 205. d. May I 892.

Sacc. Syll. $11: 315 . d$. July 1895.

Amphisphæria Populi, Tracy \& Earle.

Tracy \& Earle in Greene, Pl. Baker. 1 : 30. d. 22 Feb. I901.

Sacc. \& Syd. Syll. 16: 1134. I Feb. I902.

Sacc. Syll. $17: 685$. d. 25 May I905.

Amphisphæria prinicola, Cke.

See Rosellinia prinicola, (B. \& C.) Sacc.

Amphisphæria pseudo-umbrina, Sacc.

Pound, Bot. Surv. Nebr. 3: 7. I8 June 1894.

Amphisphæria putaminum, Cke.

See Caryospora putaminum, (S.) Sacc.

Amphisphæria quercetis, Cke. \& Massee.

Cke. Grev. $16: 89,92 . d$. March I 888.

Sacc. Syll. $9: 742 . d$. I 5 Sept. I891.

Ell. \& Ev. Pyren. 205. d. May i 892 .

Amphisphæria salebrosa, (C. \& P.) Sacc.

Sacc. Syll. $1:$ 726. $d$. 13 June I 882.

Ell. \& Ev. Pyren. 202. d. May I 892.

Sphzeria salebrosa, C. \& P.

Pk. Rept. N. Y. Mus. 29: 6ı, d. 1878.

Melanomma salebrosum, Cke.

Cke. Grev. 16: 52. Dec. I887.

Amphisphærla separans, Ell. \& Ev.

Ell. \& Ev. Bull. Torr. Bot. Club 24: г 30. d. 30 March 1897 :

Lindau \& Syd. Hedw. 37: Beibl. 7 : xxvii. 9 April 1898.

Smyth, Tr. Kans. Acad. 16 : 164 . July 1899 .

Sacc. \& Syd. Syll. 14: 555. d. 20 Aug. I899.

Earle, Bull. Ala. Exp. Sta. 107 : 323. April I 900.

Amphisphæria subiculosa, Ell. \& Ev.

See Neopeckia diffusa, (L.) Starb., var. Rhodomphalos, (B.) n. n.

Amphisphæria thujina, ( $\mathrm{Pk}$.) Sacc.

Sacc. Syll. 1: 726. $d$. I J June 1882 .

Cke. Grev. 16: 89. March 1888.

Ell. \& Ev. Pyren. 204. d. May 1892.

Spharia thujina, Pk.

Pk. Rept. N. Y. Mus. 27 : 110. d. 1875.

Amphisphæria tumulata, (Cke.) Sacc.

Sacc. Syll. 1: 727. $d$. 13 June 1882.

Ell. \& Ev. Pyren. 204. d. May 1892.

Sphreria tumulata, Cke.

Cke. Grev. $7:$ 4. $d$. Sept. .1878.

Hark. \& Moore, Cat. Pac. Fung. 44. Feb. 1880.

Xylospharia tumulata, Cke.

Cke. Grev. $17: 86$. June 1889. 
Amphisphæria umbrina, (Fr.) De Not.

*Ell. \& Ev. N. A. F. 2922 . Oct. I 893.

Jelliffe, Flora of Long Island 30. I899.

Amphisphæria Wellingtoniæ, (Cke. \& Hark.) Berl. \& Vogl.

Berl. \& Vogl. in Sacc. Syll. Add. 1-4: 124. d. 3 I Dec. I886.

Sacc. Syll. 9: 743. $d$. I5 Sept. I89 I.

Ell. \& Ev. Pyren. 206. $d$. May I892. Spharia Wellingtonia, Cke. \& Hark.

Cke. \& Hark. Grev. 13: 19. d. Sept. 1884.

Hark. Bull. Cal. Acad. 1: i74. 7 March I885. Xylospharia Wellingtonia, Cke.

Cke. Grev. $17: 86$. June I889.

Amphitrichum albocoronatum, O. Kuntze.

See Ceratostomella albocoronata, (Ell.) Sacc.

Amphitrichum Araliæ, Spreng.

See Meliola Aralia, (Spreng.) Mont.

Amphitrichum brevirostre, O. Kuntze.

See Ceratostomella brevirostre, (Duf.) Sacc.

Amphitrichum capillare, O. Kuntze.

See Ceratostomella capillare, (Ell.) Sacc.

Amphitrichum cirrhosum, $O$. Kuntze.

See Ceratostomella cirrhosa, (P.) Sacc.

Amphitrichum echinellum, $\mathrm{O}$. Kuntze. See Ceratostomella echinella, Ell. \& Ev.

Amphitrichum hystricinum, O. Kuntze. See Ceratostomella hystricina, (Cke.) Sacc.

Amphitrichum investitum, $O$. Kuntze. See Ceratostomella investita, (S.) Starb.

Amphitrichum Mali, O. Kuntze. See Ceratostomella Mali, Ell. \& Ev.

Amphitrichum microsporum, O. Kuntze. See Ceratostomella microspora, Ell. \& Ev.

Amphitrichum nigrum, (Tode) O. Kuntze. See Ceratostomella rostrata, (Fr.) Sacc.

Amphitrichum nyssicola, O. Kuntze. See Ceratostomella nyssicola, (B. \& C.) Sacc.

Amphitrichum Sacchari, Spreng. See Meliola amphitricha, Fr.

Amphitrichum strictum, O. Kuntze.

See Ceratostomella stricta, (P.) Sacc.

Androsaceus aciculaformis, Duss.

See Marasmius aciculaformis, B. \& C.

Androsaceus atrorubens, Duss. See Marasmius atrorubens, B.

Androsaceus Bulliardi, Duss. See Marasmius Bulliardi, Quel.

Androsaceus castaneus, Duss. See Marasmius castaneus, Mont.

Androsaceus corrugatus, Pat. See Marasmius corrugatus, (Pat.) Sacc. \& Syd.

Androsaceus dispar, Duss. See Collybia dispar, (Mont.) Sacc.

Androsaceus ferrugineus, Duss. See Marasmius ferrugineus, $\mathrm{B}$. 
Androsaceus glaucopus, Pat.

See Marasmius glaucopus, (Pat.) n. n.

Androsaceus hæmatocephalus, Pat.

See Marasmius hamatocephalus, (Mont.) Fr.

Androsaceus Myricre, Pat.

See Marasmius Myrica, (Pat.) Sacc. \& Syd.

Anellaria fimiputris, (Bull.) Karst.

Sacc. Cub. \& Manc. Syll. 5 : II26. d. 28 May 1887.

Kellerm. \& Werner, Geol. Ohio $7^{2}: 317$. Jan. I 895.

Henn. in Engler \& Prantl, Pflanzenfam. 11**: 234. ${ }^{1 .} 1898$. Agaricus fimiputris, Bull.

Lea, Cat. Pl. Cin. 53. 1849.

Andrews, Proc. Boston Soc. 5 : 322. April 1856.

Frost, Proc. Boston Soc. 12: 77. 1869.

Cke. Handbook 156. $d$. I871.

Frost in Cat. Pl. Amherst 66. 1875 .

Johnson, Bull. Minn. Acad. 1: 239. 1877 and Rept. Minn. Surv. 5 : 12. 1877 .

James, Jour. Cin. Soc. 2 : 64 (23). April 1879.

Hark. \& Moore, Cat. Pac. Fung. 8. Feb. 1880.

Morg. Jour. Cin. Soc. 6 : II6. d. July I 883.

Cobb, List Pl. Amherst 33. 1887.

Pansolus fimiputris, Fr.

Underw. Proc. Ind. Acad. 1893: 62. Aug. I894.

Anellaria separata, (L.) Karst.

Mcllvaine \& Macadam, Am. Fung. 388. d.pl. 109. 1900. Agaricus separatus, L.

Curtis, M. A., Bot. N. Car. 9r. 1867.

Winder, Mushrooms of Canada 20. 1871 . $[1877$.

Johnson, Bull. Minn. Acad. 1 : 239. and Rept. Minn. Surv. 5: 72.

Somers, Tr. N. Scot. Inst. $5: 249.1882$. Agaricus semiovatus, Sow.

S. Syn. Car. 85 (59) no. 71 1. 1822.

S. Syn. Am. Bor. 151 no. 249.1834.

Andrews, Proc. Boston Soc. 5 : 322. April 1856.

Panzeolus separatus, Fr.

Banning in Pk. Rept. N. Y. Mus. 44: 187 (75). Dec. 1891.

Herbst, Fungal FI. Lehigh Valley 76. $d . \quad 1899$.

Angelina $\mathrm{Fr}$.

Fr. Summa 358. d. 1849 .

Duby, Mem. Soc. Phys. et Hist. Nat. 16: 51 (39). d. 1862.

Durand, Jour. Myc. 8: 108, 109. $d$. 14 Oct. 1902.

Angelina Leprieuril, Mont.

See Triblidiella Leprieurii, (Mont.) Sacc.

Angelina maura, Ell.

See Hysteropatella Prostii, (Duby) Rehm.

Angelina nigrocinnabarina, B. \& C.

See Triblidiella nigrocinnabarina, (S.) Durand.

Angelina rufescens, (S.) Duby.

Duby, Mem. Soc. Phys, et Hist. Nat. 16 : 51 (39). $d_{0} \quad 1862$.

Cke. Bull. Buf. Soc. 3 : 35 . Nov. 1875 .

*Thm. Myc. Univ. 72.1875.

*Ell. N. A. F. 466 . 1880.

*Rehm, Ascomyceten 36r. 1877.

Rehm, Ascomyceten 89. d. I88I.

Sacc. Syll. 2 : 740. d. ${ }_{13}$ June 1883. 
Pk. in Day's Cat., Bull. Buf. Soc. 4: 222 (158). I883.

Wint. \& Demet. Hedw. 24: 198 (22). Oct. I 885.

Ell. in Cat. Pl. N. J. 542.1889.

Ell. \& Ev. Pyren. 686. d. May 1892.

Harvey, Bull. Torr. Bot. Club 23: 57. 29 Feb. I 896.

Lindau in Engler \& Prantl, Pflanzenfam. 11: 268. May 1896.

Underw. \& Earle, Bull. Ala. Exp. Sta. 80: I06. 28 June 1897.

Earle in Mohr, Contr. U. S. Nat. Herb. 6: I62. 31 July IgoI.

Ricker, Univ. of Maine Studies 3: 40. 30 April 1902.

Durand, Jour. Myc. 8 : 109. d. I4 Oct. 1902.

Hysterium rufescens, S.

S. Syn. Car. 50 (24) no. 252. d. 1822.

Grev. Scot. Crypt. Fl. 1 : pl. 60.1823.

Fr. S. M. $2: 583$. d. 1823.

Spreng. in L. Syst. Veg. I6 ed. $4^{1}: 413$. d. 1827.

Fr. El. 2 : I4I. I828.

Hitchcock, Cat. Pl. Amherst 6r. I829.

S. Syn. Am. Bor. 244 no. 208I. I 834 .

Hitchcock, Cat. Pl. Mass. 646. I833. 2 ed. 647 (127): 1835.

De Not. Prime Linee Isterini 5 I (47). d. 1847.

Fr. Nova Acta R. Soc. Sci. Upsal. 111. 1 : 122. I85I.

Sprague, Proc. Boston Soc. 5: 331. 1856.

Duby, Mem. Soc. Phys, et Hist. Nat. 16 : 5I (39). d. 1862.

Billings, Am. Nat. 5 : 630. d. pl. $11, f . w$. Oct. 187 I.

Frost in Cat. Pl. Amherst 92. 1875.

Rehm, Hedw. 25 : I96 (42). Oct. I 886.

Cobb, List Pl. Amherst 45. I 887.

Ascobolus conglomeratus, $\mathrm{S}$.

Fr. El. 2 : I6. I828.

S. Syn. Am. Bor. 178 no. 960. d. 1834.

Sprague, Proc. Boston Soc. 5 : $330 . \quad 1856$.

* Rav. Fung. Car. 5 : 44. I860.

Curtis, M. A., Bot. N. Car. I 35.1867.

Pk. Rept. N. Y. Mus. $22: 95$. I 860

B. Grev. 4 : 6 (144). d. Sept. 1875 .

Frost in Cat. Pl. Amherst 9I. I875.

Cobb, List. Pl. Amherst 47. I887.

Angelina conglomerata, $\mathrm{Fr}$.

Fr. Summa 358. I 849.

Fr. Nova Acta R. Soc. Sci. Upsal. 111. 1 : I2I. d. I85I.

Boud. Ann. Sci. Nat. V. 10: 213. d. 1860.

Morg. Jour. Myc. 8: I82. 20 Dec. I902.

Angioridium sinuosum, Grev.

See Physarum sinuosum, (Bull.) Fr.

Angioridium valvatum, B.

See Physarum bogoriense, Racib.

See Physarum pallidum, (B. \& C.) Lister.

Anixia.

Note. The gehus Anixia was originally defined by Fries. If the modern genus is to be credited to Fries it can be only to Fries in Hoffmann, Icones Anal. 3: 70. 1863 as is done by Lindau in Engler \& Prantl, Pflanzenfam. $x^{1}: 333$. Jan. 1897 . Since the genus as at present understood is based on Didymium parietinum, Schrader, it may be questioned whether it would not be better to retain the genus Anixia for the ill-defined species first proposed by Fries and, following Rostafinski, to raise to generic rank the subgenus Myogala of Fries in Summa Veg. Scand. 448. I 846 which included only the original Didynium parietinum of Schrader but one could 
not follow Rostafinski in substituting for Schrader's name that of bicolor taken from the later Licea bicolor of Persoon.

Of the two North American species referred to Anixia, A. inlerrupta can not belong to the genus in the modern sense, judging by the description of Schweinitz. We have seen no specimen however. Of the second species we know but a single puhlished record although it has been collected several times in this country. It is the same as Anixia parielina, (Schrad.) Lindau.

The spelling of Myogala has been changed to Mycogala in certain references, whether on etymological grounds or not we do not know; but the Mycogala parietinum of Sacc. Syll. 3: 185. d. I5 Dec. I 884 placed in Fungi Imperfecti can not be as stated by him the M/yogala bicolor of Rostafinski, Mycetozoa 30I. I875 since Rostafinski included the A. truncigena of Hoffmann, which is ascosporic.

Anixia interrupta, S.

S. Syn. Am. Bor. 264 no. 2395. d. 1834.

Sacc. Syll. 1: $36 . d$. I June 1882 .

Anixia parietina, (Schrad.) Lindau. Lycogala parietinum, Fr.

Sprague, Proc. Boston Soc. 5 : 328.1856.

Berl. in Sacc. Syll. 7 : 386. d. 15 March 1888.

Annularia Fenzlii, Schulz. Agaricus Fenzlii, Schulz.

Morg. Bot. Gaz. 8: 156. Jan. 1883.

Annularia lævis, (Krombh.) Fr.

Pound \& Clements, Bot. Surv. Nebr. 5: 25. 30 March 190 I. Agaricus lavis, Krombh.

Morg. Bot. Gaz. 8 : 156. Jan. 1883.

Annularia mammillata, Longyear.

Longyear, Rept. Mich. Acad. 3: 59. d.f. 4. 1902.

Longyear, Rept. Mich. Acad. 4: i1 $5 . d .1904$.

Anonymos Caroliniensis, Walt.

NoTE. Following his peculiar custom this author has used the name Anonymos for species in various groups which are uncertain. The plant here referred to is apparently one of the Gasteromycetes but it can not be determined with certainty.

Walt. Fl. Car. 263. d. 1788.

Antennaria, Lk.

See Antennularia, Reichenbach.

Antennaria aretica, Sacc.

See Antennularia arctica, (Rostr.) n. n.

Antennaria elæophila, Mont.

See Antennularia elzophila, (Mont.) n. n.

Antennaria Guava, Cke.

See Antennularia Guava, (Cke.) n. n.

Antennaria pinophila, Nees.

- See Antennularia pinophila, (Nees) n. n.

Antennaria rectangularis, Sacc.

See Antennularia rectangularis, (Sacc.) n. n.

Antennaria Robinsonii, B. \& Mont.

See Antennularia Robinsonii, (B. \& Mont.) Wildeman.

Antennaria semiovata, B. \& Br.

See Antennularia semiovata, (B. \& Br.) n. n.

Antennariella, sp. indet.

Henn. Hedw. Beibl. 41: (102). d. 23 June 1902. Cent. Amer. 
Antennatula arctica, Rostr.

See Antennularia arctica, (Rostr.) n. n.

Antennularia, Reichenbach.

Note. Unless one adopts the principle that the same name may be applied to genera both of fungi and of phænogams - a principle in favor of which something might be said - the genus Antennaria of Fungi can not stand since Antennaria, Lk. 1809 is antedated by Antennaria, Gærtner I 791, a genus of Composito.

Reichenbach in 1828 substituted for Antennaria, Lk. the name Antennularia which he afterwards changed to Antennataria, there being an earlier genus of animals of the former name. According to modern views the change was not required and therefore the oldest name for the heterogeneous collection of species classed by mycologists under Antennaria is Antennularia rather than the more recent Antennina, Fr., Antennula, Strauss and Antennatula, Strauss.

The species of Antennaria are placed by some writers, as Rostrup, in Hyphomycetece and by others, as Saccardo and Lindau, in Perisporiacea, although of the thirteen species recognized by Lindau only one is known to have asci. As it is, the genus is merely a temporary resting place for a number of imperfect forms and for the sake of uniformity in generic nomenclature and to avoid the use of a name better known in phænogams than in fungi we have adopted the name Antennularia.

Antennularia aretica, (Rostr.) n. n.

Antennatula arctica, Rostr.

Rostr. Meddelelser om Groenl. 3 : 577. d. 1888 and Bot. Centralbl. $36: 7 . d$. 1888.

Rostr. Meddelelser om Groenl. 3 : 629. I891.

Rostr. Meddelelser om Groenl. $18: 72$ (30). I 894.

Antennaria arctica, Sacc.

Sacc. Syll. $9: 442 . d$. I 5 Sept. I891.

Antennularia elæophila, (Mont.) n. n. Antennaria elreophila, Mont.

Halsted, Bull. lowa Agr. Coll. 1888 : i18. April 1888.

Antennularia Guava, (Cke.) n. n.

NorE. This species was described without special locality in a paper on California fungi. The material is apparently the same as that cited ly Harkness and Moore, I880, where the locality given is Hawaii not California. Whether the species really occurs in the United States is not certain. The spelling given by Harkness and Moore is Guave and the spelling Guava is probably a printer's error.

Antennaria Guava, Cke.

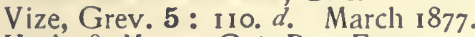

Hark. \& Moore, Cat. Pac. Fung. 31. Feb. I880. Hawaii.

Sacc. Syll. $1: 8$ I. $d$. I 3 June I882.

Antennularia pinophila, (Nees) n. n.

NOTE. This name was changed to pithyophila by Fries and Saccardo places the species in the genus Hormiscium.

Antennaria pinophila, Nees.

Lea, Cat. Pl. Cin. 77. d. I849 and Jour, Cin. Soc. 5:217 (21). d. Dec. I882.

James, Jour. Cin. Soc. 2 : 68 (27). April I879.

Kellerm. \& Werner, Geol. Ohio 72 : 353. Jan. 1895.

Antennularia rectangularis, (Sacc.) n. n.

Antennaria rectangularis, Sacc.

Sacc. Pk. \& Trel., Harriman Alaska Exp. 5 : 34. d. pl. 3, f.

Sacc. Syll. 17 : 559. d. 25 May 1905. 
Antennularia Robinsonil, (B. \& Mont.) Wildeman.

Bomm. \& Rous. in Durand \& Pittier, Bull. Soc. Bot. Belg. 351 : 158. 1806.

Antennaria Robinsonii, B. \& Mont.

Cockerell, Bull. Torr. Bot. Club 20:296. 15 July 1893. West Indies.

Antennularia semiovata, (B. \& Br.) n, n.

Antennaria semiovata, $\mathrm{B}$. \& $\mathrm{Br}$.

B. Grev. 4: 161 (109). June 1876.

Underw. \& Earle, Bull. Ala. Exp. Sta. 80: 182. 28 June 1897.

Earle in Mohr, Contr. U.S. Nat. Herb. 6: 168. 31 July 1901.

Antennularia? tenuis, Earle.

Earle, Bull. N. Y. Bot. Gard. 3 : 302. d. 30 June 1904. West Indies.

Anthina ceranoides, Lk.

NoTE. The following is the only reference we have. The species is assigned by Schweinitz to Link but we do not know where it was published.

S. Syn. Am. Bor. 305. no. 3073. 1834.

Anthina flammea, $\mathrm{Fr}$.

Eby, Fungi of Lancaster Co. 10. 1894.

Anthina plumosa, Fr.

See Pterula plumosa, (S.) Fr.

Anthostoma Aceris, Cke.

See Valsaria Aceris, (C. \& E.) Sacc.

Anthostoma adustum, (C. \& P.) Sacc.

Sacc. Syll. 1 : 307. d. 13 June 1882.

Ell. \& Ev. Pyren. 580. d. May 1892. Diatrype adusta, C. \& P.

Pk. Rept. N. Y. Mus. 29: 58. d. 1878.

Cke. Grev. 14: 17. Sept. 1885. Fuckelia adusta, Cke.

Cke. Grev. 12: 52. Dec. 1883.

Anthostoma amplisporum, (Cke.) Berl. \& Vogl.

Berl. \& Vogl. in Sacc. Syll. Add. 1-4: 46. d. 31 Dec. 1886.

Sacc: Syll. 9: 522. $d$. I 5 Sept. 1891.

Ell. \& Ev. Pyren. 583. d. May 1892. Fuckelia amplispora, Cke.

Cke. Grev. 12: 51.d. Dec. 1883.

Anthostoma amygdalinum, (Cke.) Sacc.

Sacc. Syll. $1:$ 308. $d$. 13 June 1882.

Ell. \& Ev. Pyren. 580. d. May 1892.

Lindau in Engler \& Prantl, Pflanzenfam. 11 ${ }^{1}$ 456. Feb. 1897. Melanconis amygdalina, Cke.

C. \& E. Grev. 5: 55. d. pl. 81, f. 4. Dec. 1876.

Cke. Proc. Phil. Acad. 1877: 127. d. 22 May 1877.

Fuckelia amygdalina, Cke.

Cke. Grev. 12: 52. Dec. 1883.

Anthostoma atrofuscum, Berl. \& Vogl.

See Rosellinia atrofusca, (B. \& C.) Starb.

Anthostoma atropunetatum, (S.) Sacc.

NOTE. Although we retain this species in the genus Anthostoma its generic position seems to us to be uncertain. It does not seem to us to be a Iyppoxylon and it is very doubtful whether it is an Anthostoma. In some respects it resembles Nummularia.

Sacc. Syll. 1 : 295. $d$. 13 June 1882.

Rehm, Hedw. 21: 133. d. Sept. 1882. 
Cke. Grev. 11 : 132. June 1883.

*Wint. Fung. Eur. 3159. I884.

Wint. \& Demet. Hedw. 24: I9I (15). Oct. I885.

Kellerm. \& Werner, Geol. Ohio $\mathbf{7}^{2}$ : 354 . Jan. I 895.

Underw. \& Earle, Bull. Ala. Exp. Sta. 80: 186. 28 June I897.

Earle in Mohr, Contr. U.S. Nat. Herb. 6 : 177. 3I July IgoI.

*Rehm, Ascomyceten 1596. May I905.

Spharia atropunctata, S.

S. Syn. Car. 3I (5) no. 44. $d_{0}$ I 822

Fr. S. M. 2: 351. d. 1823 .

Spr. in L. Syst. Veg. I6 ed. $4^{1}:$ 388. d. I827.

S. Syn. Am. Bor. I94 no. 1232. I834.

Lea, Cat. Pl. Cin. 74. I849.

*Rav. Fung. Car. 1: 62 . 1852.

Curr. Tr. Linn. Soc. 22 : 269. pl. 46, f. 70. 1859.

Curr. Tr. Linn. Soc. 25: 243. 1866.

Spharia tephromelas, Spreng. Herb.

Fr. S. M. 2 : 35 I. 1823.

Diatrype atropunctata, M. A. Curtis.

Curtis, M. A., Bot. N. Car. 140. I867.

Featherman, Bot. Surv. La. 1871 : I59. 1872.

Pk. Rept. N. Y. Mus. 25 : Ior. Sept. 1873.

B. Grev. 4: 95 (165). March 1876 .

Cke. Ann. N. Y. Acad. 1 : 185. May 1878.

Cke. Grev. 6 : I44. June 1878.

* Rav. Fung. Am. 9o. I878.

James, Jour. Cin. Soc. 2 : 68 (27). April 1879.

Cke. Grev. 8 : I 8. March I880.

Pk. in Day's Cat., Bull. Buf. Soc. 4 : 229 (165). 1883.

Hypoxylon atropunctatum, Frost's Cat.

Frost in Cat. Pl. Amherst 94. 1875.

*Ell. N. A. F. 576 . I88I.

Cke. Grev. $11:$ i32. d. I38. June 1883.

Sacc. Syll. 2 : Add. p. Xxiv. I3 June 1883.

Eke. Grev. 13: I 5. Sept. 1884.

Cragin, Bull. Wash. Lab. 1: 7I. Jan. 1885.

Calkins, Jour. Myc. 2 : 127. Nov. 1886.

Cobb, List Pl. Amherst 45. I887.

Langlois, Cat. Pl. La. 30. I887.

Ell. \& Ev. Jour. Myc. 4: 89. d. Sept. 1888.

Ell. in Cat. Pl. N. J. 538 . 1889.

Sacc. Syll. 9: 564 . I 5 Sept. I89I.

Ell. \& Ev. Pyren. 650. d. May 1892 .

Underw. Proc. Ind. Acad. 1893 : 41. Aug. I894.

Kellerm. \& Werner, Geol. Ohio 72: 355 . Jan. 1895.

*Ell. \& Ev. Fung. Columb. 1135. July 1897.

Rehm, Ann. Myc. 2: i 76. d. I5 April I904.

Anthostoma cercidicolum, (B. \& C.) Sacc.

Sacc. Syll. $1: 306 . d$. I 3 June 1882 .

Ell. \& Ev. Pyren. 582. d. May 1892.

Diatrype cercidicola, B. \& C.

Pk. Rept. N. Y. Mus. 25: IoI. d. Sept. I873.

Cke. Grev. 14: I6. Sept. 1885.

Hypoxylon suborbiculare, $\mathrm{Pk}$.

Pk. Rept. N. Y. Mus. 30: 63. $d$. Sept. 1878.

Sacc. Syll. $1: 356 . d$. I3 June I 882.

Pk. in Day's Cat., Bull. Buf. Soc. $4: 227$ (I63). $188_{3}$. 
Ell. \& Ev. Jour. Myc. $4: 67$. d. July 1888.

Ell. \& Ev. Pyren. 638. d. May 1892.

Fuckelia cercidicola, Cke.

Cke. Grev. 12 : 52. Dec, 1883.

Anthostoma Closterium, B. \& C.

See Anthostomella Closterium, (B. \& C.) Sacc.

Anthostoma diseineolum, (S.) Sacc.

Nore. There is no reason to suppose that this species founded on Spharia discincola, S. is really an Anthostoma but whether it is the same as Spheria discreta, S. is open to doubt. On the strength of Schweinitzian material examined by Currey and Cooke, $S$. discincola and $S$. discreta are the same and in that case the former name has priority. Cooke, however, retains both names whence it might be inferred that he had doubts as to whether the material examined by him was really the $S$. discincola of Schweinitz Syn. Car. Tulasne places the S. discincola of Herb. Mus. Paris received from Schweinitz in Nummularia discreta but believes that it is not the true $S$. discincola of S. Syn. Car.

We are obliged therefore to consider the $S$. discincola, S. as a doubtful species which on the authority of Schweinitz and Fries is distinct from $S$. discreta but whose exact characters can not be ascertained at the present time. It is here referred to Nummularia discreta as a doubtful synonym since the commonly accepted name discreta should not be superseded by the very uncertain discincola published earlier.

Anthostoma dryophilum, (Curr.) Sacc.

Ell. \& Ev. Pyren. 581.d. May 1892.

Pk. Rept. N. Y. Mus. 55 : 957. Nov. 1902. [Bull. 10 : no. 54.]

Diatrype dryophila, Curr.

Cke. Ann. N. Y. Acad. 1 : 185. May 1878.

Anthostoma dryophilum, (Curr.) Sacc. var. minor, (Cke.) Rehm.

Rehm, Hedw. 21 : 134. Sept. I882.

Ell. \& Ev. Pyren. 58I. d. May 1892.

Diatrype dryophila, Curr., var. minor, Cke.

C. \& E. Grev. 5 : 32. d. pl. 75, f. 13. Sept. 1876.

*Ell. N. A. F. 87. 1878 .

Anthostoma Ellisil, Sacc.

See Anthostoma microsporum, Karst.

Anthostoma Ellisil, Sacc., var. exudans, Pk.

See Anthostoma microsporum, Karst., var. exudans, (Pk.) n. n.

Anthostoma exutans, Sacc.

See Nummularia exutans, Cke.

Anthostoma flavo-viride, Ell. \& Holw.

Arth. \& Holw. Bull. Minn. Surv. 3: 32. d. 1 Oct. 1887.

Sacc. Syll. 9 : $515 . d$. is Sept. 1891.

Ell. \& Ev. Pyren. 578. d. May I892.

Lindau in Engler \& Prantl, Pflanzenfam. 11 : 456. Feb. I897.

Anthostoma formosum, Ell. \& Ev.

Ell. \& Ev. Proc. Phil. Acad. 1894 : 344. d. 30 Nov. 1894.

Sacc. Syll. 11: 283 . d. July 1895 .

Smyth, Tr. Kans. Acad. 15: 68. March 1898.

Rehm, Ann. Myc. 2: 176. d. I5 April 1904. 
Anthostoma gastrinum, (Fr.) Sacc.

Sacc. Syll. 1: 303. d. 13 June 1882.

*Ell. \& Ev. N. A. F. 2513. Feb. I891.

Sacc. Syll, 9 : $523 . \quad 15$ Sept. 1891.

Ell. \& Ev. Pyren. 579. d. May I892.

*Ell. \& Ev. Fung. Columb. 124. 1893.

Lindau in Engler \& Prantl, Pflanzenfam. 11 $: 456$. Feb. 1897. Spharia irregularis, Sow.

S. Syn. Am. Bor. 196 no. 1259. 1834. Spharia gastrina, Fr.

S. Syn. Am. Bor. I99 no. I3II. I 834. Diatrype gastrina, Rav. Fung. Car.

* Rav. Fung. Car. 5 : 58.1860. Melogramma gastrinum, Tul.

Cke. Handbook 803. d. I87I.

B. Grev. 4: 97 (167). March 1876 . Valsa gastrina, M. A. Curtis.

Curtis, M. A., Bot. N. Car. 141. 1867. Fuckelia gastrina, Cke.

Cke. Grev. 13 : Io8. June 1885.

Anthostoma? gigasporum, (Cke. \& Hark.) Berl. \& Vogl.

Berl. \& Vogl. in Sacc. Syll. Add. 1-4: 47. d. 3I Dec. I 886.

Sacc. Syll. 9: 520. $d$. I 5 Sept. I891.

Ell. \& Ev. Pyren. 578. d. May I 892 . Spharia gigaspora, Cke. \& Hark.

Cke. \& Hark. Grev. 13: I8. $d$. Sept. I884.

Hark. Bull. Cal. Acad. 1: i74. 7 March 1885. Xylospharia gigaspora, Cke.

Cke. Grev. $17: 85$. June I889.

Anthostoma grandineum, (B. \& Rav.) Sacc.

Sacc. Syll. 1: $299 . d$. I J June I 882.

Ell. \& Ev. Pyren. 577. d. May 1892.

*Ell. \& Ev. Fung. Columb. 324. I894. Diatrype grandinea, B. \& Rav.

* Rav. Fung. Car. $4: 40.1855$.

B. Grev. 4: 95 (165) no. 85 $\mathrm{I}^{b} . d$. March 1876 .

*Ell. N. A. F. 494 . 1880.

Ell. in Cat. Pl. N. J. 535.1889. Fuckelia grandinea, Cke.

Cke. Grev. 12: 53. Dec. I883. Camarops grandinea, Cke.

Cke. Grev. 13: 108. June 1885.

Anthostoma hiascens, (Fr.) Nits.

Ell. \& Ev. Pyren. 742. d. May I892. Spharia hiascens, Fr.

S. Syn. Am. Bor. 216 no. I623. I834.

Anthostoma hypophlæum, Sacc.

See Nummularia hypophloea, (B. \& Rav.) Cke.

Anthostoma insidens, (S.) Berl.

See Hypoxylon insidens, (S.) Ell. \& Ev.

Anthostoma melanotes, (B. \& Br.) Sacc.

Farl. \& Seymour, H. l. 123. Sept. 1890.

Ell. \& Ev. Pyren. 577. d. May 1892. 
Spharia meianotes, B. \& Br.

C. \& E. Grev. 4 ; 179 . June 1876.

Anthostoma microcium, Ell. \& Ev.

Ell. \& Ev. Proc. Phil. Acad. 1894: 344. d. 30 Nov. 1894.

Sacc. Syll. 11: 283. d. July I895.

Millsp. \& Nutt. Publ. Field Columb. Mus. Bot. 1 : 135. d. Jan. 1896.

Anthostoma microplacum, Sacc.

See Nummularia micropiaca, (B. \& C.) Cke.

Anthostoma microsporum, Karst.

* Karst. Fung. Fenn. 860. d. I869.

Pk. Rept. N. Y. Mus. 43: 80 (34). Oct. 1890.

Ell. \& Ev. Pyren. 582. $d$. May 1892 .

Harvey, Bull. Torr. Bot. Club $23: 56.29$ Feb. 1896.

Ricker, Univ. of Maine Studies 3: 32. 30 April 1902.

Diatrype microspora, Ell.

Ell. Bull. Torr. Bot. Club $8: 74$. d. July I88I.

Cke. Grev. 14 : 16. Sept. 1885.

Anthostoma Eliisii, Sacc.

Sacc. Syll. 1: 308. $d$. 13 June 1882.

Ricker, Univ. of Maine Studies 3: 32. 30 April 1902.

Fuckelia Ellisil, Cke.

Cke. Grev. 12 : 52. Dec. 1883.

Anthostoma microsporum, Karst., var. exudans, (Pk.) n. n.

Note. In Peck's Report, 188 , the name for this species was originally printed Valsa exudans but in copies of the Report sent out later the name was corrected to Anthostoma Ellisii, Sacc., var. exzdans.

Valsa exudans, $\mathrm{Pk}$.

Pk. Rept. N. Y. Mus. 40:67. d. May 1888.

Anthostoma Eilisil, Sacc., var. exudans, $\mathrm{Pk}$.

Pk. Rept. N. Y. Mus. 40:67. d. May 1888.

Sacc. Syll. 9 : 52I. $d$. I5 Sept. I 89 I.

Anthostoma mortuosum, (Ell.) Sacc.

Sacc. Syll. 2 : Add. p. xiii. $d$. 13 June 1883.

Hark. Bull. Cal. Acad. 1: 165. 7 March I 885.

Sacc. Syll. 9 : 5 19. $d$. 15 Sept. 1891.

Sphzria mortuosa, Ell.

Ell. Bull. Torr. Bot. Club 9: 73. $d$. June 1882.

*Ell. N. A. F. 897 . 1882 . Xylospharia mortuosa, Cke.

Cke. Grev. 17 : 86. June 1889.

Anthostoma Ontariense, Ell.

Ell. \& Ev. Proc. Phil. Acad. 1890 : 228. d. 1890.

Sacc. Sylt. 9 : $521 . d$. 15 Sept. 1891.

Ell. \& Ev. Pyren. 581. d. pl. $34, f_{0} 13-16 . \quad$ May 1892.

Pk. Rept. N. Y. Mus. 46 : I I6'(36). Dec. 1893.

* Shear, N. Y. Fungi 347. March 1896.

Lindau in Engler \& Prantl, Pflanzenfam. ${ }^{11}: 456 . f .275$ C, D. Feb. 1897.

Anthostoma Oreodaphnes, Cke.

See Anthostomella Oreodaphnes, (Cke. \& Hark.) Berl. \& Vogl.

Anthostoma ostiolatum, Cke.

See Anthostomeila ostiolata, Ell. \& Ev. 
Anthostoma phæospermum, (Ell.) Sacc.

Sacc. Syll. 2 : Add. p. xiv. d. 13 June 1883.

Sacc. Syll. $9:$ 520. $d$. I 5 Sept. I891.

Ell. \& Ev. Pyren. 581. d. May I892.

Diatrype phrosperma, Ell.

*Ell. \& Ev. N. A. F. 1557 . I886.

Fuckelia phrosperma, Cke.

Cke. Grev. 12 : 52 . Dec. I883.

Anthostoma pholidigena, Cke.

See Anthostomella pholidigena, Ell.

Anthostoma picaceum, (C. \& E.) Cke.

NotE. The Spharia vexata, C. \& E. of N. A. F. 183 is referred by Ellis in index to N. A. F. to Spharia picacea, C.\& E. and again in North American Pyrenomycetes 578 to Anthostoma picaceztm.

The specimen no. 183 in our copy of N. A. F. however has hyaline, allanboid spores and belongs rather to Spharia vexata, C. \& E. than to Spharia picacea, C. \& E.

Cke. Grev. 17 : 90. June 1889.

Ell. \& Ev. Pyren. 578. d. May 1892 . Spharia picacea, C. \& E.

C. \& E. Grev. $7:$ :.$d$. Sept. 1878 . Anthostomella picacea, Sacc.

Sacc. Syll. 1 : 293. $d$. I3 June 1882.

Ell. in Cat. P!. N. J. 527.1889. Xylospharia picacea, Cke.

Cke. Grev. $17: 86$. June I889.

Anthostoma polynesia, (B. \& C.) Sacc.

Sacc. Syll. 1: 298. d. I3 June 1882.

Ell. \& Ev. Pyren. 579. $d$. May 1892. Diatrype polynesia, B. \& C.

Curtis, M. A., Bot. N. Car. I4I. I867. Spharia polynesia, B. \& C.

B. Grev. 4: I46 (I84) no. 931. d. June 1876. Xylospharia polynesia, Cke.

Cke. Grev. $17: 85$. Jan. 1889.

Anthostoma pulviniceps, $\mathrm{Pk}$.

Pk. in Ell. \& Ev. Pyren. 578. d. May I892.

Sacc. Syll. $11: 283$. d. July 1895 .

Anthostoma rumpens, Sacc.

See Nummularia rumpens, Cke.

Anthostoma saprophilum, Ell. \& Ev.

Martin, Jour. Myc. 3: 43. d. April 1887.

Sacc. Syll. 9: 518. d. 15 Sept. I891.

Ell. \& Ev. Pyren. 583. d. May 1892. Xylospharia saprophila, Cke.

Cke. Grev. 17: 86. June I889.

Anthostoma stomaticum, (Cke.) Berl. \& Vogl.

Berl. \& Vogl. in Sacc. Syll. Add. 1-4: 47. d. 31 Dec. 1886.

Sacc. Syll. 9 : 5I6. $d$. 15 Sept. I 89 I. Botryospharia stomatica, Cke.

Cke. Grev. 13 : 102. d. 108. June 1885.

Ell. \& Ev. Pyren. 548. d. May I892.

Sphæria stomatica, S. in Herb. B.

Berl. \& Vogl. in Sacc. Syll. Add. 1-4 : 47. 31 Dec. 1886. 
Anthostoma sustentum, (Plowr.) Sace.

Sacc. Syll. 1: 300. d. I3 June 1882.

Ell. \& Ev. Pyren. 579. $d$. May 1892. Spharia sustenta, Plowr.

Cke. Grev. $7:$ 73. . Dec. 1878.

Plowr. Grev. 8 : 73. Dec. 1879.

Hark. \& Moore, Cat. Pac. Fung. 44. Feb. 1880.

Xylospharia sustenta, Cke.

Cke. Grev. $17: 85$. June 1889 .

Anthostoma tuberculosum, (S.) Ell. \& Ev.

Ell. \& Ev. Pyren. 582. d. May I892.

Spharia tuberculosa, S.

S. Syn. Car. 43 (17) no. 164. d. 1822.

Fr. S. M. 2: 477 . d. 1823 .

S. Syn. Am. Bor. 216 no. 1624. 1834.

Curtis, M. A., Bot. N. Car. 148. 1867.

Sacc. Syll. 2: $419 . d$. I3 June I 883. Xylospharla tuberculosa, Cke.

Cke. Grev. 17: 85. June I889.

Anthostoma turgidum, (P.) Nits.

Pk. Rept. N. Y. Mus. 42: 130 (34). Dec. I889.

Ell. \& Ev. Pyren. 580. d. May 1892.

Rehm, Hedw. Beibl. 34: (165). I9 Oct. I89.5.

Spharla turglda, P.

Fr. S. M. $2: 400 . d$. 1823 .

S. Syn. Am. Bor. 202 no. 1359. I834. Valsa turgida, Fr.

Curtis, M. A., Bot. N. Car. 142. 1867.

Cke. Handbook 836. d. I871.

Cke. Proc. Phil. Acad. 1877 : 122. d. 22 May 1877.

Anthostomella abdita, (B. \& C.) Sacc.

Sacc. Syll. 1: 290. $d$. 13 June 1882.

Sphzeria abdita, B. \& C.

B. \& C. Jour. Linn. Soc. $10: 388 . d$. 1869.

West Indies.

Anthostomella albocineta, Ell. \& Ev.

Ell. \& Ev. Proc. Phil. Acad. 1895 : 424. d. Nov. 1895.

Sacc. Hedw. 35 : Rep. 7 : xxvi. 25 Feb. 1896.

Sacc. \& Syd. Syll. 14: 502. $d$. 20 Aug. 1899.

Anthostomella Baptisiæ, (Cke.) Sacc.

Sacc. Syll. 1: 285. $d$. I3 June 1882 .

Cke. Grev. 18 : 28. Dec. 1889.

Ell. \& Ev. Pyren. 420. d. May 1892. Spharia Baptisiae, Cke.

Cke. Grev. 6 : 145. d. June 1878.

*Rav. Fung. Am. 200. 1878.

Anthostomella brachystoma, Ell. \& Ev.

Ell. \& Ev. Bull. Wash. Lab. 1: 5. d. Sept. 1884.

Berl. \& Vogl. in Sacc. Syll. Add. 1-4: 45. d. 31 Dec. 1886.

Sacc. Syll. 9 : 506. d. 15 Sept. 1891.

Ell. \& Ev. Pyren. 419. d. May 1892.

Lindau in Engler \& Prantl, Pflanzenfam. ${ }^{1}{ }^{1}: 452$. Feb. 1897. Xylospharla brachystoma, Cke.

Cke. Grev. 17: 86. June I889.

Anthostomella Cacti, Sacc.

See Vermicularla Cacti, (S.) Starb. 
Anthostomella Closterium, (B. \& C.) Sacc.

Sacc. Syll. $1: 287 . d$. 13 June 1882.

Sacc. Syll. 2 : Add. p. xiii. $d$. 13 June 1883.

Sacc. Syll. 9 : 512. d. 15 Sept. I8gr.

Ell. \& Ev. Pyren. 422. $d$. May I892.

Lindau in Engler \& Prantl, Pflanzenfam. 11 : 452. Feb. 1897. Spharia Closterium, B. \& C.

Curtis, M. A., Bot. N. Car. 147. 1867.

B. Grev. 4: I47 (185) no. 933. d. June 1876.

Pk. Rept. N. Y. Mus. $30: 66$. Sept. 1878. Anthostoma Closterium, B. \& C.

Cke. Grev. 17 : 90. June 1889.

Anthostomella Coffer, Delacroix.

Delacroix, Bull. Soc. Myc. Fr. 20: 145. d.pl. g, f. Bı pe, 2, 3. 20 July I904.

Sacc. Syll. 17 : 594. d. 25 May I905.

Anthostomella confusa, Sacc.

Sacc. Syll. $1:$ 287. $d$. I 3 June 1882.

Cke. Grev. 18: 28. Dec. I 889.

Ell. \& Ev. Pyren. 421. d. May 1892 . Spharia appendiculosa, B. \& C.

B. Grev. 4: 153 (I9I) no. 967. d. June 1876 .

Cke. Ann. N. Y. Acad. 1 : 187. May 1878.

Anthostomella cornicola, Ell. \& Ev.

Ell. \& Ev. Erythea 1 : 198. d. 2 Oct. 1893.

Sacc. Syll. 11: 283 . $d$. July I 895 .

Anthostomella eliminata, (B. \& C.) Sacc.

Sacc. Syll. 1 : 281. $d$. I3 June I882.

Cke. Grev. 18: 28 . Dec. 1889 .

Ell. \& Ev. Pyren. 418. d. May 1802 .

Underw. \& Earle, Bull. Ala. Exp. Sta. 80: 186. $d .28$ June 1897.

Earle in Mohr. Contr. U. S. Nat. Herb. 6 : 177. 31 July Igor.

Spharia eliminata, B. \& C.

B. Grev. 4: 148 (186) no. $943 . d$. June 1876.

Anthostomella eructans, Ell. \& Ev.

Fairman, Proc. Rochester Acad. 1: 49. d. pl. 4, f. 7. and 8. Aug. I89o.

Sacc. Syll. $9:$ 505. $d$. I 5 Sept. I 891 .

Ell. \& Ev. Pyren. 420. d. May I892.

Anthostomella hypsophila, Ell. \& Ev.

Ell. \& Ev. Proc. Phil. Acad. 1894: 338. d. 30 Nov. 1894.

Sacc. Syll. $11: 282$. $d$. July 1895 .

Anthostomella leucobasis, (Ell. \& Martin) Sacc.

Sacc. Syll. 2 : Add. p. xii. d. I 3 June 1883.

Cke. Grev. 18 : 28. Dec. 1889 .

Sacc. Syll. 9: 509. d. is Sept. I891.

Ell. \& Ev. Pyren. 418. d. May I892.

*Ell. \& Ev. Fung. Columb. 722. 24 April I895. Spharia leucobasis, Eil. \& Martin.

Ell. \& Martin, Am. Nat. 16 : 809. d. Oct. I882.

*Ell. N. A. F. 1199. I884.

Ell. \& Martin, Jour. Myc. 1 : 99. d. Aug. 1885 and Hedw. $25: 42$. Oct. I 886.

Calkins, Jour. Myc. 3 : 58. May 1887.

Anthostomella limitata, Sacc.

Pk. Rept. N. Y. Mus. 42: 130 (34). Dec. I889. 
Anthostomella Ludoviciana, Ell. \& Langlois.

Ell. \& Ev. Proc. Phil. Acad. 1890 : 228. d. I89o.

Sacc. Syll. $9: 509 . d$. 15 Sept. 1891.

Ell. \& Ev. Pyren. 419. d. May 1892.

Anthostomella Magnoliæ, Ell. \& Ev.

Ell. \& Ev. Jour. Myc. 4: 122. d. Dec. I 888.

Sacc. Sull. 9 : 51 I. $d .15$ Sept. I891.

Ell. \& Ev. Pyren. 418. d. pl. 31, f. $1-3$. May 1892.

Anthostomella mammoides, Ell. \& Ev.

Ell. \& Ev. Proc. Phil. Acad. 1893: 139. d. 28 Feb. 1893.

Sacc. Syll. 11 : 282. $d$. July 1895 .

Ell. \& Ev. Bull. Torr. Bot. Club 27 : 63. d. 17 Feb. 1900.

Anthostomella melanosticta, Ell. \& Ev.

Ell. \& Ev. Jour. Myc. 3 : 44. d. April 1887.

Langlois, Cat. PI. La. 27.1887.

Sacc. Syll. $9: 510 . d$. 15 Sept. 1801.

Ell. \& Ev. Pyren. 418. d. May 1892.

LIndau in Engler \& Prantl, Pflanzenfam. 1': 452. Feb. I897.

Anthostomella minor, Ell. \& Ev.

Ell. \& Ev. Jour. Myc. 3: 43. d. April 1887.

Calkins, Jour. Myc. $3: 70$. June 1887.

*Ell. \& Ev. N. A. F. 1965. March i 888.

Sacc. Syll. 9: $510 . d$. 15 Sept. 1891.

Ell. \& Ev. Pyren. 417. d. May I892.

Anthostomella nigro-annulata, (B. \& C.) Sacc.

Sace. Syll. 1 : 279. d. I 3 June 1882.

Cke. Grev. 18: 28 . Dec. 1889.

Ell. \& Ev. Pyren. 417. d. May 1892 . Sphreria nigro-annulata, B. \& C.

*Rav. Fung. Car. 5 : 73. I860.

Curtis, M. A., Bot. N. Car. 148: 1867.

B. \& C. Jour. Linn. Soc. 10: 388. d. I869.

B. Grev. 4: $14^{8}$ (186). June 1876.

*Ell. \& Ev. N. A. F. 1672 . 1886.

Calkins, Jour. Myc. 3: 58 . May 1887.

Langlois, Cat. Pl. La. 34. 1887.

Anthostomella nigrotecta, (B. \& Rav.) Sacc.

Sacc. Syll. $1: 284$. $d$. 13 June 1882 .

Cke. Grev. 18: 28 . Dec. I889.

Ell. \& Ev. Pyren. 421. d. May 1892.

Spharla nigrotecta, B. \& Rav.

B. Grev. 4: 155 (193) no. 972. d. June 1876.

Anthostomella Oreodaphnes, (Cke. \& Hark.) Berl. \& Vogl.

Berl. \& Vogl. in Sacc. Syll. Add. 1-4: 44. d. 31 Dec. I 886.

Sacc. Syll. 9 : 508. $d .15$ Sept. I 891.

Ell. \& Ev. Pyren. 419. d. May 1892. Spharla Oreodaphnes, Cke. \& Hark.

Cke. \& Hark. Grev. 13: 18. d. Sept. I884.

Hark. Bull. Cal. Acad. 1: 174. 7 March 1885. Anthostoma Oreodaphnes, Cke.

Cke. Grev. 17 : 90. June I 889.

Anthostomella ostiolata, Ell. \& Ev.

Ell. \& Ev. Bull. Torr. Bot. Club $11: 42$. d. April 1884.

Berl. \& Vogl. in Sacc. Syll. Add. 1-4: 45. d. 31 Dec. 1886.

Ell. in Cat. Pl. N. J. 527.1889.

Sacc. Syll. 9 : 508. d. 15 Sept. 1891. 
Ell. \& Ev. Pyren. 420. d. May I892.

Lindau in Engler \& Prantl, Pflanzenfam. 1' ${ }^{1}$. 452. Feb. 1897. Anthostoma ostiolatum, Cke.

Cke. Grev. 17 : 90 . June 1889.

Anthostomella palmacea, (Cke.) Sacc.

Sacc. Syll. 1 : 291. $d$. I 3 June I 882. Sphzria palmacea, Cke.

Cke. in Vize, Grev. 7 : 12. d. Sept. 1878.

Hark. \& Moore, Cat. Pac. Fung. 44. Feb. 1880. Didymospharella palmacea, Cke.

Cke. Grev. 18: 29. Dec. 1889. Didymospharia palmacea, Ell. \& Ev.

Ell. \& Ev. Pyren. 329. d. May 1892.

Anthostomella perfldiosa, (De Not.) Sacc.

Hark. Bull. Cal. Acad. 2: 445. I6 June 1887.

Anthostomella phæosticta, (B.) Sacc.

Sacc. Syll. $1:$ 279. $d$. I J June I882.* Spharia phaosticta, B.

Curtis, M. A., Bot. N. Car. 145. I867.

Anthostomella pholidigena, Ell.

*Ell. \& Ev. N. A. F. 1664. I886.

Berl. \& Vogl. in Sacc. Syll. Add. 1-4: 44. d. 31 Dec. 1886.

Sacc. Syll. 9 : 506. $d$. I 5 Sept. 1891.

Ell. \& Ev. Pyren. 420. $d$. May I 892 . Spharia pholidigena, Ell.

Ell. Bull. Torr. Bot. Club $10: 54$. d. May I883. Anthostoma pholidigena, Cke.

Cke. Grev. 17 : 9o. June 1889.

Anthostomella picacea, Sacc. See Anthostoma picaceum, (C. \& E.) Cke.

Anthostomella rostrospora, (Gerard) Sacc.

Sacc. Syll. $1: 287 . d$. 13 June 1882.

Cke. Grev, 18 : 28 . Dec. I889.

Ell. \& Ev. Pyren. 42 I. $d$. May 1892 . Spharia rostrospora, Gerard.

Gerard, Bull. Torr. Bot. Club. 5 : 26. d. June 1874 and Hedw. 13 : III. $d$. July 1874 .

Anthostomella sabalensioides, (Ell. \& Martin) Sacc.

Sacc. Syll. 2 : Add. p. xiii. $d$. I 3 June 1883 .

Cke. Grev. $18: 28$. Dec. 1889 .

Sacc. Syll. $9:$ 512. $d$. 15 Sept. 1891.

Ell. \& Ev. Pyren. 42I. d. May 1892.

Spharia sabalensioides, Ell. \& Martin.

Ell. \& Martin, Am. Nat. 16: 810. d. Oct. r882.

Ell. \& Martin, Jour. Myc. 1 : 99. $d$. Aug. 1885 and Hedw. $25: 42 . d$. Oct. 1886.

*Ell. \& Ev. N. A. F. 1964 . March I888.

Anthostomella sepelibilis, (B. \& C.) Sacc.

Sacc. Syll. $1: 28 \mathrm{I}, d$. 13 June 1882 .

Cke. Grev. 18 : 28 . Dec. 1889.

Ell. in Cat. PI. N. J. 527. 1889.

Ell. \& Ev. Pyren. 417.d. May I892.

Spharia sepelibilis, B. \& C.

B. Grev. 4 : 146 (184) no. 928. $d$. June 1876.

C. \& E. Grev. 5 : 94. March 1877.

*Ell. N. A. F. 1200 . 1884 . 
Anthostomella smilacinina, (Pk.) Sacc.

Sacc. Syll. 1: 281. $d$. 13 June 1882 .

Cke. Grev. 18: 28 . Dec. 1889.

Ell. \& Ev. Pyren. 419. d. May 1892. Spharia smilacinina, Pk.

Pk. Rept. N. Y. Mus. $29: 62$. d. 1878.

Anthostomella Sphærotheca, Earle.

Earle, Bull. Torr. Bot. Club 25 : 360 . d. 15 July 1898.

Earle in Mohr, Contr. U. S. Nat. Herb. 6 : 177. 3I July I90I.

Sacc. \& Syd. Syll. 16 : 440. d. I Feb. 1902.

Anthostomella suberumpens, Ell. \& Ev.

Ell. \& Ev. Proc. Phil. Acad. 1894: 338. d. 30 Nov. 1894.

Sacc. Syll. $11: 282$. $d$. July 1895 .

Lindau in Engler \& Prantl. Pflanzenfam. $1^{1}$ : 452. Feb. 1897.

Smyth, Tr. Kans. Acad. 15: 67 . March 1898.

Anthostomella thyridioides, Ell. \& Ev.

Ell. \& Ev. Jour. Myc. 9: 167. d. 29 Oct. 1903.

Sacc. Syll. 17 : 595. d. 25 May 1905.

Anthracoidea, Bref.

See Cintractia, Cornu.

Anthracoidea Caricis, Bref.

See Cintractia Caricis, (P.) Magn.

Anthracoidea subinclusa, Bref.

See Cintractia subinclusa, (Koern.) Magn.

Anthracophyllum Nigrita, (Lév.) Kalchb.

NoTE. The genus Anthracophyllum is by some recent writers kept distinct from Xerotus but it is considered by Patouillard, Essai Tax. 146. 1900, to be merely a subgenus of Xerotus. If kept distinct it is not easy to state what are the generic characters. Hennings in Engler and Prantl, Pflanzenfam. $I^{1 * *}:$ 222. 1898 makes a distinction on the spores which in Anthracophyllum are said to be black and in Xerotus colorless; but according to Patouillard the spores in both cases are colorless.

Although we have never seen living material, in all the herbarium material which we have seen of Xerotus Nigrita, the type of Anthracophyllum, the spores were certainly not black nor even opaque. The generic distinction founded on the relative position of the stipe and pileus hardly seems to be of importance in this case as there are so many transitional stages. It seems to us better then to retain the name Xerotus Nigrita, Lév., $q . v$.

Anthurus borealis, Burt.

Note. The differences between the genera Anthurus and Lysurus are certainly slight and the variety Klitzingii, Henn. of Anthurus borealis is by him placed in the genus Lysurus in Hedw. Beibl. 41: (173). Oct. 1902.

Burt, Mem. Boston Soc. 3 : 487-505. d. pl. 49, f. 1-14. pl. 50, f. 15-18, 20-23. Oct. 1894 .

Sacc. Syll. 11 : $155 . d$. July 1895 .

Burt, Bot. Gaz. 22 : 284. 20 Oct. 1896.

Burt, Bot. Gaz. 22 : 390. d. 23 Nov. 1896.

Pk. Rept. N. Y. Mus. 49: 35 (21). 5 Nov. 1897.

Pk. Rept. N. Y. Mus. 50: 132.28 March 1898.

Fischer, E., in Engler \& Prantl, Pflanzenfam. 1"** : 288. d. f. 136 A-D. Sept. 1898.

Griffiths, Bull. Torr. Bot. Club 26 : 628, f. 22 Dec. 1899 .

Underw. Moulds 133. I899.

Fischer, E., Neue Denkschr. Schweiz. Ges. $36^{3}$ : 41. d. 1900. 
Beardslee, Rept. Ohio Acad. 9 : 19. 1901.

Grout, Torreya 2: 49. I2 April 1902."

Henn. Hedw. Beibl. 41 : (I69), (171)-(173). f $1-8 . \quad$ I8 Oct. 1902.

Lloyd, Myc. Notes 183, 188. f. 74, 75. June 1904.

Lysurus borealis, Henn.

Sacc. Syll. $17: 214.25$ May 1905.

Aphanomyces lævis, D By.

D By. in Pringsh. Jahrb. Wis. Bot. $2: p l .20, f .17,18.1860 . *$

Humphrey, Tr. Am. Phil. Soc. 17 : 128. d. pl. 20, f. 105-107. 1893.

Aphanomyces scaber, DBy.

DBy. in Pringsh. Jahrb. Wis. Bot. 2 : pl. $20, f .14^{-16 .}$ 1860.*

Humphrey, Tr. Am. Phil. Soc. 17: 130. d. pl. 20, f. 108-111. 1893.

Apiospora apiospora, Underw. \& Earle.

See Apiospora Montagnei, Sacc.

Apiospora Montagnei, Sacc.

Sacc. Syll. $1:$ : 539. $d$. I3 June I 882.

Cke. Grev. 18:13. Sept. 1889.

Ell. \& Ev. Pyren. 31 I. d. May I892. Sphzria apiospora, Dur. \& Mont.

Curtis, M. A., Bot. N. Car. 147. 1867.

B. Grev. 4: 144 (182). June 1876.

*Rav. Fung. Am. 679. ${ }_{1882}$.

Detonina apiospora, O. Kuntze.

Kuntze, O., Rev. Gen. Pl. 2 : 851. I891. Apiosporium Montagnei, Tracy \& Earle.

Tracy \& Earle, Bull. Miss. Exp. Sta. 38: 140. May I896. Apiospora apiospora, Underw. \& Earle.

Underw. \& Earle, Bull. Ala. Exp. Sta. 80: 186. 28 June 1897.

Earle in Mohr, Contr. U.S. Nat. Herb. 6 : i74. 31 July I9oI.

Apiospora Polypori, Ell. \& Ev.

Ell. \& Ev. Pyren. 3i1. d. May 1892.

Sacc. Syll. 11 : 301. d. July 1895.

Lindau in Engler \& Prantl, Pflanzenfam. 1' ${ }^{1}$ : 430. Feb. 1897.

Apiospora Rosenvingel, Rostr.

Rostr. Meddelelser om Groeñl. 3 : 6I6. d. I89I.

Sacc. Syll. 11 : $301 . d$. July I 895 .

Apiosporium elongatum, Underw. \& Earle.

See Capnodium elongatum, B. \& Desm.

Apiosporium? erysiphoides, Sacc. \& Ell.

Sacc. Mich. $2: 566 . d$. I Dec. I 882.

Sacc. Syll. $2: 815 . d$. I3 June 1883.

*Ell. N. A. F. 1232 . 1884 .

Ell. in Cat. Pl. N. J. 509.1889.

Sacc. Syll. 9 : $375 . d$. 15 Sept. I 891.

Ell. \& Ev. Pyren. pl. 8, f. 6. May 1892.

Detonina erysiphoides, O. Kuntze.

Kuntze, O., Rev. Gen. Pl. 2: 851. I891.

Apiosporium maximum, (S.) n. n. Stillbospora maxima, S.

S. Syn. Car. 75 (49) no. 506. d. I 1822.

Lk., L. Sp. Pl. ed. Willd. $6^{2}: 93 . d . \quad 1825$.

Spreng. in L. Syst. Veg. 16 ed. $4{ }^{1}$ : 569. d. 1827.

Apiosporium stilbosporeum, $\mathrm{Fr}$.

Fr. S. M. $3: 256 . d$. $\quad$ I 832.

Curtis, M. A., Bot. N. Car. I 15. 1867.

Sacc. Syll. 1: 33. $d$. I3 June I882. 
Exosporium maximum, S.

S. Syn. Am. Bor. 306 no. 3083 . 1834 .

Aplosporium Montagnei, Tracy \& Earle.

See Apiospora Montagnei, (Dur. \& Mont.) Sacc.

Aplosporium salleinum, (P.) Kze.

Note. Brick, 1900 states that this is Capnodium salicinum, Mont. See also Apiosporium salicinum, (P.) Kze. in Lindau, Engler \& Prantl, Pflanzenfam. $x^{1}$ : 338. Jan. 1897 .

S. Syn. Am. Bor. 265 no. 2402.1834.

Brick, 3 Beih. Jahrb. Hamb. Wiss. Austalt. $17: 230$ (15). 1900.

Aplosporium, sp. indet.

Earle, Muhlenbergia 1 : Io. July rgor.

Earle in Mohr, Contr. U. S. Nat. Herb. $6:$ I68. 3I July I90I.

A podachlya, Pringsh.

Thax. Bot. Gaz. 21: 325. d. i7 June 1896 and Rev. Myc. 23: 97. July 1901 .

Apodachlya brachynema, (Hildebr.) Pringsh.

Thax. Bot. Gaz. 21: 325. I7 June I896 and Rev. Myc. 23: 97. July I90I.

Apodachlya? completa, Humphrey.

Humphrey, Tr. Am. Phil. Soc. 17: 137. d. pl. 20, f. 119-121. May I893.

Schrt. in Engler \& Prantl, Pflanzenfam. 11 : I02. d. July I893.

Sacc. Syll. 11 : 245 . July 1895.

Apodachlya pirifera, Zopf.

Thax. Bot. Gaz. 21: 325. I7 June I896.

Aponectria inaurata, (B. \& Br.) Sacc.

Sacc. Syll. 2: 516. $d$. I3 June I 883.

Millsp. \& Nutt. Publ. Field Columb. Mus. Bot. 1 : 144. d. Jan. 1896. Nectria inaurata, B. \& Br.

Pk. Rept. N. Y. Mus. 24: 98. I 872.

$\mathrm{Pk}$. in Day's Cat., Bull. Buf. Soc. 4: 226 (162). 1883.

Cke. Grev. 12 : I06. 'June I884.

Aporia obscura, Duby.

See Schizothyrium obscurum, (Duby) Sacc.

Aposphæria, B. emend. Sacc.

Note. We have kept here the Apospharia of Berkeley emend. Sacc. as distinct from Coniothyrium, using the latter as limited by Saccardo, not Corda. The spores in Apospharia are hyaline and in Coniothyrium dark as the two genera are distinguished by Saccardo, who has been followed by most recent writers.

Aposphæria acuta, B.

NoTE. In Cooke's Handbook of British Fungi 426 Apospharia acuta, B. is given as occurring in the United States and on page 901 it is said to be the pycnidial stage of Metaspharia coniformis, (Fckl.) Sacc.

See Phoma acuta, (B.) Sacc.

Aposphæria allantella, $\mathrm{Pk}$.

See Phoma allantella, Pk.

Aposphæria alpigena, Ell. \& Ev.

Ell. \& Ev. Proc. Phil. Acad. 1894: : 358. d. 30 Nov. 1894.

*Ell. \& Ev. N. A. F. 3159. Nov. 1894 .

Sacc. Syll. 11 : $497 . d$. July 1895 .

Allescher, Rabh. Krypt.-FI. 16: 395. I899. 
Coniothyrium alpigenum, $\mathrm{O}$. Kuntze.

Kuntze, O., Rev. Gen. Pl. 32 : 459. 1898.

Aposphæria Amaranti, Ell. \& Barthol.

Ell. \& Barthol. Erythea 4 : 4. d. 2 Jan. I 896.

Sacc. \& Lindau, Hedw. 36 : Rep. $7: x_{1}$. 30 April 1897.

Smyth, Tr. Kans. Acad. 15: 70. March I898.

Sacc. \& Syd. Syll. 14: 893. d. 20 Aug. 1899.

Aposphæria aranea, $\mathrm{Pk}$.

Pk. Rept. N. Y. Mus. 42 : 126 (30). d. Dec. 1889.

Sacc. Syll. $10: 209 . d$. 30 June 1892 .

Jelliffe, Flora of Long Island 33. 1899. Coniothyrium araneum, $O$. Kuntze.

Kuntze, O., Rev. Gen. PI. $3^{2}$ : 459. 1898.

Aposphæria elypeata, (C. \& E.) Sacc.

Sacc. Syll. 3: i70. $d$. i 5 Dec. I884.

Ricker, Univ. of Maine Studies 3: 25. 30 April 1902.

Phoma clypeata, C. \& E.

C. \& E. Grev. 5 : 33. d. pl. 75, f. 10. Sept. 1876.

Ell. in Cat. Pl. N. J. 579. 1889.

Conlothyrium clypeatum, $\mathrm{O}$. Kuntze.

Kuntze, O., Rev. Gen. Pl. 32: 459. $\quad 1898$.

Aposphæria collabens, (B. \& C.) Sacc.

Sacc. Syll. $3:$ 176. $d$. 15 Dec. 1884.

Jones, H. L., Oberlin College Lab. Bull. 9: 5. 15 June 1898. Sphropsis collabens, B. \& C.

Sprague, Proc. Boston Soc. 6: 319. 1859.

B. Grev. 3: $2(67)$ no. 419. d. Sept. 1874.

Frost in Cat. Pl. Amherst $80 . \quad 1875$.

Cobb, List Pl. Amherst 38. 1887.

Bennett, Cat. PI. R. I. 86. I 888.

Coniothyrium collabens, $\mathrm{O}$. Kuntze.

Kuntze, O., Rev. Gen. Pl. 32: 459. 1898.

Aposphæria complanata, B.

Note. In the Handbook of British Fungi 426 Cooke states that Apospharia complanata, B. occurs in the United States and on page 903 he states that it is the pycnidial stage of Metaspharia complanata, (Tode) Sacc:

See Phoma complanata, (Tode) Desm.

Aposphæria condensata, Ell. \& Ev.

Ell. \& Ev. Bull. Torr. Bot. Club 24: 463. d. 30 Oct. 1897.

Lindau \& Syd. Hedw. 37 : Beibl. 7 : xliii. 9 April 1898.

Sacc. \& Syd. Syll. 14: 893. d. 20 Aug. 1899.

Aposphæria conica, Sacc. sec. Pk.

Pk. Rept. N. Y. Mus. 40:60. May 1888.

Aposphæria cornuta, Cke.

Ell. in Cat. PI. N. J. 579. I889.

Aposphæria elevata, (Pk.) Berl. \& Vogl.

Berl. \& Vogl. in Sacc. Syll. Add. 1-4: 3I5. d. 3I Dec. I886.

Sacc. Syll. 10: 209, $d$. 30 June 1892 .

Phoma elevata, $\mathrm{Pk}$.

Pk. Rept. N. Y. Mus. $38: 95 . d .1885$.

Coniothyrium elevatum, $\mathrm{O}$. Kuntze.

Kuntze, O., Rev. Gen. Pl. 32: 459. $\quad$ I8g8. 
A posphreria fibricola, (B.) Sacc.

Sacc. Syll. 3: 176. $d$. I5 Dec. 1884 .

* Ell. \& Ev. N. A. F. 2573. 1801.

Allescher, Rabh. Krypt.-FI. 16: 386. d. 388. d. 1899. Phoma fibricola, B.

B. Grev. 2: 83 (59). Dec. 1873.

C. \& E. Grev. 6: 2. Sept. 1877.

Ell. in Cat. PI. N. J. 578 . 1889.

Coniothyrium fibricolum, O. Kuntze.

Kuntze, O., Rev. Gen. Pl. 3² : 459. 1898.

Aposphæria fibriseda, (C. \& E.) Sacc.

Sacc. Syll. 3: 173. $d$. 15 Dec. 1884. Phoma fibriseda, C. \& E.

C. \& E. Grev. 6: 2. d. Sept. 1877 .

Ell. in Cat. PI. N. J. 579. 1889.

Coniothyrium fibrisedum, O. Kuntze.

Kuntze, O., Rev. Gen. PI. $3^{2}$ : 459. 1898.

Aposphæria ? hemisphærica, (A. \& S.) Sacc.

Ricker, Univ. of Maine Studies 3: 25. 30 April 1902. Spharia hemispharica, A. \& S.

S. Syn. Car. 47 (21) no. 230.1822. Spharonema hemispharicum, Fr.

S. Syn. Am. Bor. 247 no. 2140.1834 .

Curtis, M. A., Bot. N. Car. 115. 1867.

Aposphæria herbicola, Ell. \& Ev.

Ell. \& Ev. Erythea 2: 24. d. 9 Feb. 1894 .

Sacc. Syll. 11 : 497 . d. July 1895 . Coniothyrium herbicola, O. Kuntze.

Kuntze, O., Rev. Gen. Pl. 32: 459. 1898.

Aposphreria hysterella, Sacc.

Sacc. Syll. 3 : 170. d. I5 Dec. 1884.

Allescher, Rabh. Krypt.-Fl. 16: 391. 1899.

Ricker, Univ. of Maine Studies 3: 25. 30 April 1902. Spharonema subtile, B. \& C.

Russell, J. L., Proc. Essex Inst. 1 : 194 (7). 1856.

Sprague, Proc. Boston Soc. 6 : 319.1859.

B. Grev. 2 : $178(63)$ no. 407. d. June 1874.

Coniothyrium hysterellum, $O$. Kuntze.

Kuntze, O., Rev. Gen. PI. 322: 459. $\quad$ I898.

Aposphæria Kansensis, Ell. \& Ev.

Ell. \& Ev. Erythea $2: 24 . d$. 9 Feb. 1894.

Sacc. Syll. $11: 497 . d$. July 1895 .

Allescher, Rabh. Krypt.FI. 16: 392. 1899.

Coniothyrium Kansense, O. Kuntze.

Kuntze, O., Rev. Gen. Pl. $3^{2}$ : 459. 1898.

Aposphæria librincola, (Cke. \& Hark.) Sacc.

Sacc. Syll. 3 : 173. d. 15 Dec. 1884.

Phoma librincola, Cke. \& Hark.

Cke. \& Hark. Grev. 9: 82. d. March 1881.

*Ell. N. A. F. 731 . 1882 .

Cke. \& Hark. Bull. Cal. Acad. 1 : 14. Feb. 1884. Coniothyrium librincola, O. Kuntze.

Kuntze, O., Rev. Gen. Pl. $3^{2}: 459.1898$.

Aposphæria macrosperma, (Cke. \& Hark.) Sacc.

Sacc. Syll. $3: 174$. d. 15 Dec. 1884 . 
Spharopsis macrosperma, Cke. \& Hark.

Cke. \& Hark. Grev. 12: 93. d. June 1884 .

Hark. Bull. Cal. Acad. 1 : 170. 7 March i885.

Coniothyrium macrospermum, $\mathrm{O}$. Kuntze.

Kuntze, O., Rev. Gen. Pl. $3^{2}: 459.1898$.

Aposphæria minutula, (Pk.) Sacc.

Sacc. Syll. 3 : $176 . d$. I5 Dec. I884.

Coniothyrium minutulum, $\mathrm{Pk}$.

Pk. Bot. Gaz. 5 : 33. d. March 1880.

Coniothyrium minutulum, $\mathrm{O}$. Kuntze.

Kuntze, O., Rev. Gen. Pl. $3^{2}$ : $459 . \quad$ I 898.

Aposphæria nucicola, Ell. \& Ev.

Ell. \& Ev. Bull. Torr. Bot. Club. $24: 287 . d$. 29 June 1897.

Lindau \& Syd. Hedw. 37 : Beibl. 7 : xliii. 9 April 1898.

Sacc. \& Syd. Syll. 14: 894. d. 20 Aug. I 899.

Aposphæria Ohiensis, Ell. \& Ev.

Ell. \& Ev. Proc. Phil. Acad. 1895 : 431 . d. Nov. 1895.

Sacc. Hedw. 35 : Rep. 7 : xli. 25 Feb. 1896.

Sacc. \& Syd. Syll. 14:: 894. d. 20 Aug. I899. Coniothyrium Ohiense, $O$. Kuntze.

Kuntze, O., Rev. Gen. Pl. $3^{2}: 459$. $\quad$ I 898.

Aposphæria 0xybaphi, Ell. \& Ev.

Ell. \& Ev. Erythea 2: 24. d. 9 Feb. 1894.

Sacc. Syll. 11: 498 . $d$. July 1895 . Coniothyrium Oxybaphi, $\bigcirc$. Kuntze.

Kuntze, O., Rev. Gen. Pl. $3^{2}: 459 . \quad$ I 898.

Aposphæria Petersil, (B. \& C.) Sacc.

Sacc. Syll. 3 : 176. d. I5 Dec. 1884.

Underw. \& Earle, Bull. Ala. Exp. Sta. 80: IG3. $d .28$ June 1897. Phoma Petersii, B. \& C.

B. Grev. $2: 81$ (58) no. 377. $d$. Dec. 1873. Coniothyrium Petersil, O. Kuntze.

Kuntze, O., Rev. Gen.'Pl. $3^{2}$ : 459. $\quad$ I898.

Aposphæria pezizoides, Ell. \& Ev.

Ell. \& Ev. Proc. Phil. Acad. 1894 : 358. d. 30 Nov. 1894.

*Ell. \& Ev. N. A. F. 3158 . Nov. 1894 .

* Ell. \& Ev. Fung. Columb. 570. 1894.

Sacc. Syll. 11: 497. $d$. July I 895 .

Millsp.- \& Nutt. Publ. Field Columb. Mus. Bot. 1 : 108. d. 'Jan. I896.

Allescher, Rabh. Krypt.-Fl. 16: 382, 388 . I899.

Coniothyrium pezizoides, $\mathrm{O}$. Kuntze.

Kuntze, O., Rev. Gen. PI. $3^{2}: 459 . \quad$ I 898.

Aposphæria Rhododendri, (S.) Sacc.

Sacc. Syll. 3: 171. d. I5 Dec. I884.

Jacz. Nouv. Mem. Soc. Imp. Nat. Mosc. $15: 355 . d$. 1898. Spharonema Rhododendri, S.

S. Syn. Am. Bor. 247. no. 2141.d. 1834.

Coniothyrium Rhododendri, $\mathrm{O}$. Kuntze.

Kuntze, O., Rev. Gen. Pl. $3^{2}$ : 459. 1898.

Aposphæria subtilis, (Fr.) Sacc.

Sacc. Syll. 3 : I7 I. $d$. I5 Dec. 1884.* Spharonema subtile, $\mathrm{Fr}$.

Pk. Rept. N. Y. Mus. 24:85. 1872.

Pk. in Day's Cat., Bull. Buf. Soc. 4: 198 (134). 1883. Coniothyrium subsubtile, $\mathrm{O}$. Kuntze.

Kuntze, O., Rev. Gen, Pl. 3²: 460\%, 1898. 
Aposphæria turmalis, Ell. \& Ev.

Ell. \& Ev. Jour. Myc. 8: 63. d. 30 June 1902.

Aposphæria, sp. indet.

Stevens, F. L., Rept. Ohio Acad. '5 : 63. 1897.

Sturgis, Rept. Conn. Exp. Sta. 24: 288.1901.

Appendicularia, $\mathrm{Pk}$.

See Stigmatomyces, Karst.

Appendicularia entomophila, $\mathrm{Pk}$.

See Stigmatomyces entomophila, (Pk.) Thax.

Appendiculina, Berl.

See Stigmatomyces, Karst.

Appendiculina entomophila, Berl.

See Stigmatomyces entomophila, (Pk.) Thax.

Apyrenium lignatile, $\mathrm{Fr}$.

Johnson, Bull. Minn. Acad. 1 : 283. 1877 and Rept. Minn. Surv. 5 :

81. 1877 .

Somers, Tr. N. Scot. Inst. $7: 465 . \quad 1800$.

MacKay, Tr. N. Scot. Inst. 11: 139. Aug. 1904.

Arachnion, $\mathrm{S}$.

NotE. Brongniart in Essai d'une Classification Nat. des Champ. places Acinophora of Rafinesque as a synonym of Arachnion, in which he has been followed by some later writers. Brongniart remarks however that it is impossible to tell from the description what Acinophora really is, an opinion in which we quite concur.

S. Syn. Car. 59 (33). d. 1822.

Fr. S. M. 2 : 303.d. 1823.

Fr. S. O. V. 1: 131. d. 1825 .

Spreng. in L. Syst. Veg. 16. ed. $4^{1}$ : 378. d. 1827.

Nees \& Henry, Syst. 1: 68. $d . \quad 1837$.

Endl. Gen. Pl. 29. 1840.

Cda. Icon. $5: 25 . d$. 1842.

Fr. Summa $1: 443$. d. 1849 .

Tul. Fungi Hypogrei $18 \mathrm{I}$. 1851 .

De Toni in Sacc. Syll. 7: 150. d. 15 March 1888.

Fischer, E., in Engler \& Prantl, Pflanzenfam. 11** : 339. d. Dec. 1899.

White, Bull. Torr. Bot. Club 29: 252. 26 May 1902.

Lloyd, Myc. Notes I4I. March I903.

PAcinophora, Raf.

Raf. Med. Repos. 2 hex. 5 : 355. 1808.

Raf. Précis 5i. $d$. I8I4.

Endi. Gen. Pl. 29. d. 1840.

Cda. Icon. 5 : 44 . $\quad 1842$.

Arachnion album, S.

S. Syn. Car. 59 (33) no. 334. d. - 1822.

Fr. S. M. 2 : 303.d. 1823.

Spr. in L. Syst. Veg. 16 ed. $4^{1}: 519 . d$. 1827.

S. Syn. Am. Bor. 253 no. 2221. 1834.

Fr. Nova Acta. R. Soc. Sci. Upsal. Iil. 1 : 134. 1851 .

* Rav. Fung. Car. 4: 75 . 1855 .

Curtis, M. A., Bot. N. Car. III. 1867.

Cke. Grev. 6: 133. June 1878.

* Rav. Fung. Am. 15. 1878.

*Ell. N. A. F. 941 . 1883 .

Cragin, Bull. Wash. Lab. 1: 4I. Jan. 1885

De Toni in Sacc. Syll. 7 : 151. d. I5 March 1888. 
Ell. in Cat. Pl. N. J. 499. I889.

Webber, Tr. St. Louis Acad. 6: 18. 12 March 1892 and Contr. Bot. Dept. Univ. Nebr. 3: I8. I4 June I892.

Atk. Bull. Cornell Univ. 3: 22. 25 June I 897.

Fischer, E., in Engler \& Prantl, Pflanzenfam. 11**: 339. Dec. I899.

Earle in Mohr, Contr. U. S. Nat. Herb. 6.: 234. 31 July I9or.

Lloyd, Gen. Gast. I I. pl. 5, f. 28. Jan. I902.

Lloyd, Myc. Notes I42. d. pl. 16. March 1903.

Arachnion? aurantiacum, (Raf.) De Toni.

De Toni in Sacc. Syll. 7: 15I. d. I5 March 1888.

Fischer, E., in Engler \& Prantl, Pflanzenfam. 11**: 339. Dec. I899. Acinophora aurantiaca, Raf.

Raf. Med. Repos. I1. 5 : 355. 1808.

Raf. Précis 5I. $d$. I8I4.

Arachniotus trachyspermus, Shear.

Shear, Science II. 16 : 138. d. 25 July 1902.

Arachnopeziza aurata, Rehm non Fckl.

See Arachnopeziza rhaphidospora, (Ell.) Rehm.

Arachnopeziza Aurelia, (P.) Fckl.

Lindau in Engler \& Prantl, Pflanzenfam. 11: 200. d. f. $157 \mathcal{B}-E$. Dec. I894.

Fairman, Proc. Rochester Acad. 3: 218. d. March 1900.

Durand, Bull. Torr. Bot. Club $27: 484 . d$. 29 Sept. 1900. Peziza Aurelia, P.

* Rav. Fung. Car. 5 : 4 I. 1860.

Curtis, M. A., Bot. N. Car. I 33. 1867.

Cke. Handbook 692. $d$. I87I.

Cke. Bull. Buf. Soc. 2 : 293. March 1875.

B. Grev. 3: 156 (134). June 1875 .

Frost in Cat. PI. Amherst 90.1875.

C. \& E. Grev. 5 : 33. Sept. 1876.

C. \& E. Grev. 6 : 7 . Sept. 1877 .

*Ell. N. A. F. 59. $\quad$ I878.

Pk. Rept. N. Y. Mus. 34: 5 I. Oct. I883.

Cragin, Bull. Wash. Lab. 1: 70. Jan. 1885.

Cobb, List Pl. Amherst 47. I887.

Ell. in Cat. PI. N. J. 547. I 889 .

Millsp. Bull. W. Va. Exp. Sta. 24: 512. June I892. Belonidium Aurelia, De Not.

Sacc. Syll. 8 : $499 . d$. 20 Dec. 1889.

Bessey, Contr. Bot. Dept. Univ. Nebr. 3: 48. I4 June 1892.

Millsp. \& Nutt. Publ. Field Columb. Mus. Bot. 1 : I 52 . Jan. I896.

Underw. \& Earle, Bull. Ala. Exp. Sta. 80: 200. 28 June i 897.

Arachnopeziza rhaphidospora, (Ell.) Rehm.

Note. A specimen under this name distributed by Ellis in N. A. F. 842 was referred by Rehm in 1893 to Arachnopeziza aurata, Fckl. but in 1904 he cites it under the name here accepted.

The species cited by Massee in Grev. 22: 101. June 1894 as Periza rhaphidospora, B. \& C. Fung. Cub. no. 683 is not the same as the present species. It is described in Fung. Cub. under the name Peziza (Daryscypha), raphidofera, B. \& C. See Erinella rhaphidophora, (B. \& C.) Sacc.

Rehm, Ann. Myc. 2 : 352. d. 31 July I904.

Peziza rhaphidospora, Ell.

Ell. Bull. Torr. Bot. Club 6 : 107. d. Sept. 1876.

* Ell. N. A. F. 842 . I 882 . 
Ell. in Cat. Pl. N. J. 547. 1889.

Massee, Grev. 22: 101. d. June 1894.

Erinella rhaphidospora, Sacc.

Sacc. Syll. 8 : $509 . d$. 20 Dec. 1889. Arachnopeziza aurata, Rehm non Fckl.

Rehm, Rabh. Krypt.-Fl. 13:691. 1893.

Araiospora, Thax.

Thax. Bot. Gaz. 21 : 326. d. 17 June 1896 and Rev. Myc. $23: 95$. July 1901 .

Sacc. \& Lindau, Hedw. 36 : Rep. 7 : xvii. d. 30 April 1897.

Sacc. \& Syd. Syll. 14: 454. d. 20 Aug. 1899.

Lindau in Engler \& Prantl, Pflanzenfam. 11** : 529. d. Feb. 1900.

Araiospora pulchra, Thax.

Thax. Bot. Gaz. 21: 328. d. pl. 23, f. 20-25. 17 June 1896. and Rev. Myc. 23: $96 . p l .217, f .15,22,23$. July 1901 .

Sacc. \& Lindau, Hedw. 36 : Rep. 7 : xvii. 30 April 1897.

Sacc. \& Syd. Syll. 14: 455. d. 20 Aug. 1899.

Lindau in Engler \& Prantl, Pflanzenfam. 11**: 529 . Feb. 1900.

King, Science II. 15: 456. 21 March 1902.

King, Proc. Boston Soc. 31 : 21 I. pl. 11-15. Nov. 1903 and Bot. Gaz. $37: 72.20 \mathrm{Jan} .1904$.

Areyodes, O. F. Cook.

Cook, O. F., Science II. 15 : 651. 25 April 1902.

Areyodes incarnata, (A. \& S.) O. F. Cook.

Note. In the reference given below the name Arcyodes is proposed to replace Lachnobolus as understood by Lister. Whether the type species which is based on the old Licea incarnata, A. \& S. referred to Lachnobolus circinans, $\mathrm{Fr}$. by Lister is found in North America is a point on which opinions differ. The species quoted from Iowa under Lachnobolus circinans, Lister is by Macbride considered to be a different species peculiar to America which he calls Lachnobolus occidentalis, Macbr. - See Lachnobolus, sp.

Cook, O. F., Science II. 15 : 651. 25 April 1902.

Areyria adnata, (Batsch) Rostf.

See Arcyria incarnata, P.

Areyria albida, $P$.

See Arcyria cinerea, (Bull.) P.

Areyria albida, var. globosa, Lister.

See Lachnobolus globosus, (S.) Rostf.

Areyria albida, var. pomiformis, Lister.

See Arcyria pomiformis, (Leers) Rostf.

Areyria aurantiaca, Raunkiær.

See Arcyria ferruginea, Sauter.

Arcyria bicolor, B. \& C.

See Arcyria digitata, S.

Arcyria einerea, (Bull.) P.

NoTe. See also Arcyria digitata, (S.) Rostf.

Muhl. Cat. Pl. Am. Sept. 1 ed. 104. 1813. 2 ed. 107. 1818.

S. Syn. Car. 64 (38) no. 397. 1822.

S. Syn. Am. Bor. 259 no. 2336.1834.

Lea, Cat. Pl. Cin. 69. I849.

Sprague, Proc. Boston Soc. 5 : 328.1856.

Curtis, M. A., Bot. N. Car. I13. 1867.

B. \& C. Jour. Linn. Soc. 10 : 349.1869.

Pk. Rept. N. Y. Mus, $22: 89 . \quad 1869$. 
Cke. Handbook 40I. d. I87I.

B. Grev. $2: 67(56)$. Nov. 1873 .

Rostf. Mycetozoa 272. d. 1875 .

Frost in Cat. Pl. Amherst 79. 1875.

Farl. Bull. Bussey Inst. 1: 43I. March 1876 .

C. \& E. Grev. 6: 82. March 1878 .

Cke. Ann. N. Y. Acad. 1 : I80. d. May r878.

James, Jour. Cin. Soc. 2 : 66 (25). April 1879.

Pk. Rept. N. Y. Mus. $31: 57 . \quad 1879$.

Pk. in Day's Cat., Bull. Buf. Soc. 4: 196 (1 32$) .1883$.

*Ell. N. A. F. 1115. I 884 .

Wint. \& Demet. Hedw. 24: 200 (24). Oct. 1885.

Arth. \& Holw. Bull. Minn. Surv. 3: 39. I Oct. I887.

Langlois, Cat. Pl. La. 27. I887.

Berl. in Sacc. Syll. $7: 427 . d . \quad 15$ March 1888.

Schrt. in Engler \& Prantl, Pflanzenfam. 11 : 22. d. June 1889.

Ell. in Cat. Pl. N. J. 600 . 1889.

Webber, Rept. Nebr. Agr. 1889: I82 (42). I89o.

Macbr. Bull. Univ. Iowa 2 : $123 . d . p l .2, f .3,3^{\mathrm{a}}$. June 1892 .

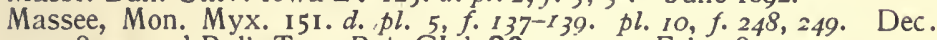
1892 and Bull. Torr. Bot. Club 20:77. 15 Feb. 1893.

Morg. Jour. Cin. Soc. 16: 20 (26). d. June I893.

Macbr. Bull. Univ. lowa $2: 379$. Nov. 1893.

Underw. Proc. lnd. Acad. 1893 : 30. Aug. I 894.

Kellerm. \& Werner, Geol. Ohio $7^{2}$ : 40I. Jan. I895.

Millsp. \& Nutt. Publ. Field Columb. Mus. Bot. 1 : 86. Jan. 1896.

Harvey, Bull. Torr. Bot. Club 23: 313. 20 Aug. 1896.

Macbr. \& Smith, C. L., Bull. Univ. Iowa $4: 73$. Dec. I8g6.

Macbr. Slime-moulds ro6. d. pl. $2, f .3,3^{a}$. Aug. I899.

Herbst, Fungal FI. Lehigh Valley 215.d. I899.

Jelliffe, Flora of Long Island I. I 899 .

Longyear, Rept. Mich. Agr. 1898: $95 . \quad$ I899.

Thomas, Proc. Ind. Acad. 1900 : i23. IgoI.

Ricker, Univ. of Maine Studies 3: I6. 30 April 1902.

Mutchler, Proc. Ind. Acad. 1902 : i 8. I903.

Macbr. in Sacc., Pk. \& Trel., Harriman Alaska Exp. 5 : 21. 5 April 1904 .

Sumstine, Torreya $4: 36$. I2 March 1904.

Farl. in Coker, Veg. Bahama 243. 1905.

Schaffner, Tr. Kans. Acad. 19: 204. 1905. Arcyria albida, P.

Olive, Proc. Ind. Acad. 1897 : I49. 1898.

Whitney, Rhodora 1: i28. I July i 899.

Mutchler, Proc. Ind. Acad. 1901: 292. 1902.

Mutchler, Proc. Ind. Acad. 1902 : Irg. I903.

A rcyria albida, Lister p. p.

Lister, Mycetozoa I86. d. I894. Arcyria stricta, Rostf.

Rostf. Mycetozoa Suppl. $36 . \quad$ r876.

Cke. Ann. N. Y. Lyceum 11: 406 . June 1877.

Bessey, Bull. lowa Agr. Coll. 1884 : 134. Jan. 1885. Arcyria Cookei, Massee.

Morg. Jour. Cin. Soc. 16: 21 (27). d. pl. 1, f. 16. June I893.

Kellerm. \& Werner, Geol. Ohio $7^{2}$ : 40I. Jan. I 895.

Clathrodes Cookei, Sheldon.

Sheldon, Minn. Bot. Studies 9: 467. 20 Nov. 1895 .

Clathrodes recutitum, Sheldon.

Sheldon, Minn. Bot. Studies 9: 468. 2o Nov, r895' 
Arcyria cinerea, Massee p. p.

See Arcyria pomiformis, (Leers) Rostf.

Arcyria clavata, Massee.

See Hemitrichla ciavata, (P.) Rostf.

Arcyria Cookei, Massee. See Arcyria cinerea, (Bull.) P.

Arcyria denudata, Macbr. See Arcyria punicea, P.

Arcyria digitata, (S.) Rostf.

NotE. This may probably be considered only a clustered variety of $A$. cinerea, (Bull.) P. An examination of the specimens of Arcyria pallida, B. \& C. and Arcyria bicolor, B. \& C. in Herb. Curtis shows that hoth are the same as Arcyria digitala, $\mathrm{S}$.

Pk. Rept. N. Y. Mus. $23: 53 . \quad 1872$.

Rostf. Mycetozoa 274. d. 1875.

Cke. Ann. N. Y. Lyceum 11: 406. June 1877.

Berl. in Sacc. Syll. 7 : 428. $d$. I 5 March 1888.

Schrt. in Engler \& Prantl, Pflanzenfam. $\mathbf{1}^{1}: 22 . d$. June 1889.

Massee, Mon. Myx. 153. d. Dec. I892.

Morg. Jour. Cin. Soc. 16 : 21 (27). d. June 1893.

Macbr. Bull. Univ. lowa 2: 379. Nov. 1893.

Kellerm. \& Werner, Geol. Ohio 72: 401. Jan. 1895.

Macbr. \& Smith, C. L. Bull. Univ. lowa 4: 73. Dec. 1896.

Macbr. Slime-moulds 197. $d$. Aug. 1899.

Longyear, Rept. Mich. Agr. 1898: $95 . \quad$ I 899.

Thomas, Proc. Ind. Acad. 1900: 123. 1901.

Mutchler, Proc. Ind. Acad. 1901 : 292.1902.

Duss, Champ. Guad. \& Martinique 85. 1903.

Mutchler, Proc. Ind. Acad. 1902 : I19. I903.

Sumstine, Torreya 4: 36. 12 March 1904.

Stemonitis digitata, $\mathrm{S}$.

S. Syn. Am. Bor. 260 no. 2350. d. 1834. Arcyria pallida, B. \& C.

Curtis, M. A., Bot. N. Car. 113.1867.

B. Grev. $2: 67$ (56) no. 365. d. Nov. 1873 .

Rostf. Mycetozoa 280. I 875 .

Arcyrla bicolor, B. \& C.

B. \& C. Jour. Linn. Soc. 10 : 349. d. 1869.

Rostf. Mycetozoa 280. 1875.

Arcyria albida, Lister p. p.

Lister, Mycetozoa I 86. d. I894.

Clathrodes digitatum, Sheldon.

Sheldon, Minn. Bot. Studies 9: 467. 20 Nov. 1895.

Arcyria ferruginea, Sauter.

Massee, Mon. Myx. 144. d. pl. 10, f. 250-253. Dec. I892.

Lister, Mycetozoa I84. d. pl. 66 B. I894.

Harvey, Bull. Torr. Bot. Club 23 : 313. 20 Aug. 1896.

Harvey, Bull. Torr. Bot. Club 24: 70. 28 Feb. 1897.

Underw. \& Earle, Bull. Ala. Exp. Sta. 80: 133. 28 June 1897.

Whitnev, Rhodora 1: 128. I July 1899.

Macbr. Slime-moulds 194. $d$. Aug. 1899.

Lister, Jour. Bot. $37: 463$. Nov. 1899.

Earle in Mohr. Contr. U. S. Nat. Herb. 6 : 139., 31 July rgor, Thomas, Proc. Ind. Acad. 1900: 123. roor.

Ricker, Univ, of Maine Studies 3: 16. 30 April 1902. 
Mutchler, Proc. Ind. Acad. 1901 : 292. 1902.

Mutchler, Proc. Ind. Acad. 1902 : i 8.1903. Arcyria macrospora, $\mathrm{Pk}$.

Pk. Rept. N. Y. Mus. 34: 43. d. Oct. 1883.

Durand, Bot. Gaz. 19 : 89. d. pl. $9, f .1,3$. pl. $10, f .9 .16$ March 1894.

Sturgis, Tr. Conn. Acad. $10: 488,489.5$ May 1900 and Bot. Centralbl. 86 : I I5. I7 April I9oI.

? Arcyria aurantiaca, Raunkiær.

Harvey, Bull. Torr. Bot. Club 23 : 313. 20 Aug. 1896.

Harvey, Bull. Torr. Bot. Club 24: 70. 28 Feb. 1897. Clathrodes ferrugineum, Sheldon.

Sheldon, Minn. Bot. Studies 9 : 466. 20 Nov. I 895. Clathrodes aurantiacum, Sheldon.

Sheldon, Minn. Bot. Studies 9: 467. 20 Nov. 1895 .

Arcyria flava, $P$.

See Arcyria nutans, (Bull.) Grev.

Arcyria globosa, S.

See Lachnoboius giobosus, (S.) Rostf.

Arcyria incarnata, P.

Note. See note under Arcyria minor, $\mathrm{S}$.

S. Syn. Car. 64 (38) no. $398 . \quad$ I822.

S. Syn. Am. Bor. 259 no. 2337.1834.

Sprague, Proc. Boston Soc. 5: 328. I 856.

Curtis, M. A., Bot. N. Car. i 3.1867.

Cke. Handbook 40I. d. f. 136. I871.

Pk. Rept. N. Y. Mus. 25 : 84. Sept. 1873.

Rostf. Mycetozoa 275. d. I875.

Cke. Ann. N. Y. Lyceum 11: 406. June 1877.

Pk. Rept. N. Y. Mus. 31 : 57.1879.

Pk. in Day's Cat., Bull. Buf. Soc. 4 : I96 (132). I883.

Farl. Appalachia 3: 238 . Jan. 1884.

Wint. \& Demet. Hedw. 24: 200 (24). Oct. 1885.

Massee, Mon. Myx. 145. d. Dec. 1892.

Cockerell, Bull. Torr. Bot. Club 20 : 296. I 5 July I 893.

Lister, Mycetozoa i 89. d. pl. 68 B. I894.

Macbr. Procr lowa Acad. 5 : 27. 1898.

Whitney, Rhodora 1 : $128 . \quad$ I July I899.

Macbr. Slime-moulds 193. d. Aug. 1899.

Thomas, Proc. Ind. Acad. 1900: 123. I901.

White, Bull. Torr. Bot. Club 29 : 55I. 26 Sept. I902.

Mutchler, Proc. Ind. Acad. 1901 : 292. 1902.

Mutchler, Proc. Ind. Acad. 1902 : II8. 1903.

Sumstine, Torreya 4: 36 . I2 March 1904.

Schaffner, Tr. Kans. Acad. 19 : 204. 1905.

Macbr. Proc. lowa Acad. 12 : 35. 1905.

Arcyria adnata, (Batsch) Rostf.

Rostf. Mycetozoa Suppl. 36. 1876.

Berl. in Sacc. Syll. 7 : 428. d. I5 March 1888.

Schrt. in Engler \& Prantl, Pflanzenfam. 11: 22. d. June 1889.

Ell. in Cat. Pl. N. J. 600 . I889.

Bessey, Contr. Bot. Dept. Univ. Nebr. $3: 45 . \quad$ I4 June I892.

Macbr. Bull. Univ. lowa 2: I24. $d$. June I892.

Morg. Jour. Cin. Soc. 16 : I9 (25). d. June 1893.

Kellerm. \& Werner, Geol. Ohio 72: 40r. Jan. I895.

Harvey, Bull. Torr. Bot. Club 23 : 31 3. 20 Aug. I896.

Ricker, Univ. of Maine Studies 3: I6. 30 April igo2. 
Clathrodes adnatum, Sheldon.

Sheldon, Minn. Bot. Studies 9: 466. 20 Nov. 1895.

Arcyria incarnata, P. var. nodulosa, Macbr.

Macbr. Slime-moulds i94. $d$. pl. $3, f . S$. Aug. 1899.

Mutchler, Proc. Ind. Acad. 1902 : 118.1903.

Sumstine, Torreya $4: 36.12$ March 1904.

Arcyria irregularis, Racib.?

Harvey, Bull. Torr. Bot. Club 26 : 323. 17 June 1899.

Ricker, Univ. of Maine Studies 3: 16. 30 April 1902.

Clathrodes irregulare, Sheldon.

Sheldon, Minn. Bot. Studies $9: 466.20$ Nov. 1895.

Areyria lelocarpa, Massee.

See Hemitrichia leiocarpa, (Cke.) Lister.

Areyria leiocarpoides, (Speg.) Massee.

Note. This species, founded on Cornwvia liciocarpoides, Speg. is said by Lister to be a form of Hemitrichia clavala, (P.) Rostf.

Massee, Kew Bull. I $38: 135$. June 1898.

Trinidad.

Arcyria leucocephala, Hoffm.

See Craterium leucocephalum, (P.) Ditm.

Arcyria lutea, S.

See Arcyria pomiformis, (Leers) Rostf.

Arcyria macrospora, $\mathrm{Pk}$.

See Arcyria ferruginea, Sauter.

Areyria magna, Rex.

Rex, Proc. Phil. Acad. 1893: 364. d. Marcl I894.

Macbr. Slime-moulds 190. $d$. Aug. 1899.

Mutchler, Proc. Ind. Acad. 1902: $119 . \quad$ I903.

Sumstine, Torreya 4: 36 . I2 March 1904.

Arcyria Oerstedtii, Lister p. p.

Lister, Mycetozoa I9o. $d$. 1894.

Arcyria magna, Rex, var. rosea, Rex.

Rex, Proc. Phil. Acad. 1893: 365. d. March I894.

Areyria minor, $\mathrm{S}$.

NoTE. Schweinitz remarks that this is closely related to Arcyria incarnata, $\mathrm{P}$. in which opinion he has been followed by some 'recent writers. It can hardly be regarded as a distinct species.

S. Syn. Am. Bor. 259 no. 2341. d. 1834 .

Rostf. Mycetozoa 280.1875.

Cke. Ann. N. Y. Lyceum 11: 407. June 1877 .

Morg. Jour. Cin. Soc. 16 : 19 (25). d. pl. 1, f. 17. June 1893.

Kellerm. \& Werner, Geol. Ohio $7^{2}: 401$. Jan. 1895 .

Underw. Proc. Ind. Acad. 1894 : 147. Oct. I895.

Harvey, Bull. Torr. Bot. Club 23 : 313 . 20 Aug. 1896.

Harvey, Bull. Torr. Bot. Club 26: 323. I7 June 1899.

Arcyria nutans, (Bull.) Grev.

Sprague, Proc. Boston Soc. 6 : 318.1859.

Curtis, M. A., Bot. N. Car. I 13. I867.

B. \& C. Jour. Linn. Soc. $10: 349$. 1869 .

Cke. Handbook $401 . d$. I87i.

Pk. Rept. N. Y. Mus. $26: 76$. April 1874.

Frost in Cat. PI. Amherst 79. 1875.

Farl. Bull. Bussey Inst. 1 : 43 I. March 1876. 
Cke. Ann. N. Y. Lyceum 11: 407. June I877.

Johnson, Bull. Minn. Acad. 1: 292. I877 and Rept. Minn. Surv. 5 : 84. I 877.

Phil. Grev. 7 : 21. Sept. 1878.

Pk. Rept. N. Y. Mus. $31:$ 57. 1879.

Hark. \& Moore, Cat. Pac. Fung. 20. Feb. I880.

Farl. Appalachia 3 : 238. Jan. I884.

Bessey, Bull. lowa Agr. Coll. 1884: I 34. Jan. I885.

Arth. \& Holw. Bull. Minn. Surv. 3: 39. I Oct. I887.

Langlois, Cat. Pl. La. 27. I887.

Berl. in Sacc. Syll. 7 : 429. d. I5 March I888.

Schrt. in Engler \& Prantl, Pflanzenfam. 1'1:22. d. f. 17, D-F. June I889.

Eli. in Cat. Pl. N. J. 6or. I889.

Bessey, Contr. Bot. Dept. Univ. Nebr. $3: 45$. 14 June 1892.

Macbr. Bull. Univ. Iowa 2: 125. d. pl. $2, f .6,6 a, 6 b$. June 1892.

Massee, Mon. Myx. I 5o. d. pl. 5, f. 140, 141. Dec. I892.

Morg. Jóur. Cin. Soc. $16: 20(26)$. d. June 1893.

Kellerm. \& Werner, Geol. Ohio $7^{2}: 401$. Jan. I895.

Millsp. \& Nutt. Publ. Field Columb. Mus. Bot. 1 : 86. Jan. I896.

Harvey, Bull. Torr. Bot. Club 23 : 313. 20 Aug. I8g6.

Harvey \& Knight, Bull. Torr. Bot. Club 24: 342. 29 July I 897.

*Ell. \& Ev. N. A. F. 3598. 24 Jan. I808.

Macbr. Proc. lowa Acad. 5 : 27. $\quad$ I898.

Macbr. Slime-moulds ig I. d. pl. $11, f .6,6^{a}, 6^{b}$. Aug. 1899 .

Fairman, Proc. Rochester Acad. 3: 2 i 2. March 1900.

Ricker, Univ. of Maine Studies 3: I6. 30 April 1902.

White, Bull. Torr. Bot. Club 29: 55 I. 26 Sept. 1902.

Mutchler, Proc. Ind. Acad. 1901 : 292. 1902.

Duss, Champ. Guad. \& Martinique 85. I903.

Mutchler, Proc. Ind. Acad. 1902: I I9. I903.

Sumstine, Torreya 4 : 36 . I2 March Igo4.

Macbr. Proc. lowa Acad. 12 : 35. I905.

Arcyria flava, $\mathrm{P}$.

S. Syn. Car. 63 (37) no. 395. 1822.

Grev. Scot. Crypt. Fl. $6:$ pl. 309. 1828.

S. Syn. Am. Bor. 259 no. $2335 . \quad 1834$.

Lister, Mycetozoa I9o. d. pl. 69 A. I894.

Olive, Proc. Ind. Acad. 1897 : I49. I898.

Whitney, Rhodora 1 : I 28 . I July 1899.

Mutchler, Proc. Ind. Acad. $1901: 292 . \quad$ I902.

Mutchler, Proc. Ind. Acad. 1902 : i I8. I903.

Clathrodes nutans, Sheldon.

Sheldon, Minn. Bot. Studies 9: 467. 20 Nov. I895. Arcyria Oerstedti, Farl. in Coker.

Farl. in Coker, Veg. Bahama 243. I905.

Arcyria ochroleuca, (Trent.) Fr.

See Arcyria pomiformis, (Leers) Rostf.

Areyria 0erstedtii, Rostf.

Arth. \& Holw. Bull. Minn. Surv. 3: 39. I Oct. I887.

Macbr. Bull. Univ. lowa 2 : I25. d. June I 892.

Lister, Mycetozoa Igo. d. pl. 69 B. I894. p. p.

Harvey, Bull. Torr. Bot. Club 23: 31 3. 20 Aug. I896.

Macbr. Slime-moulds I91. d. Aug. I 899 .

Thomas Proc. Ind. Acad. 1900 : I 23. IgoI.

Ricker, Univ. of Maine Studies 3 : I6. 30 April I902.

Mutchler, Proc. Ind. Acad. 1902 : II9. 1903.

Sumstine, Torreya 4 : 36. I2 March I9o4. 
Arcyria Oerstedtil, Lister p. p.

See Arcyria magna, Rex.

Arcyria Oerstedtil, Farl. in Coker.

See Arcyria nutans, (Bull.) Grev.

Arcyria pallida, B. \& C.

See Arcyria digitata, (S.) Rostf.

Arcyria pomiformis, (Leers) Rostf.

Rostf. Mycetozoa 271. d. 1875.

Cke. Ann. N. Y. Lyceum 11: 406 . June 1877

C. \& E. Grev. $6: 82$. March 1878.

Pk. Rept. N. Y. Mus. $31: 42,57.1879$.

Calkins, Jour. Myc. 3: 7. Jan. 1887.

Langlois, Cat. Pl. La. 27. I887.

Berl. in Sacc. Syll. $7: 427 . d$. I5 March 1888.

Ell. in Cat. Pl. N. J. 600.1889.

Webber, Rept. Nebr. Agr. 1889: 182 (42). 1890.

*Ell \& Ev. N. A. F. 3497 . 10 Dec. 1896.

Harvey, Bull. Torr. Bot. Club 24: 70.28 Feb. 1897.

Macbr. Proc. lowa Acad. 5 : $27 . \quad$ I898.

Macbr. Slime-moulds 197. $d$. Aug. I899.

Ricker, Univ. of Maine Studies 3: 16. 30 April 1902.

Mutchler, Proc. Ind. Acad. 1902 : I 19.1903.

Arcyria umbrina, Schum.

Curtis, M. A., Bot. N. Car. I13. I867.

Cke. Handbook 401. $d$. 1871 .

B. Grev. 2: $67(56)$. Nov. 1873 .

Phil. Grev. 5 : 115. March 1877.

Hark. \& Moore, Cat. Pac. Fung. 20. Feb. 1880.

Johnson, Bull. Minn. Acad. 1: 362. 1880.

Arcyria lutea, $\mathrm{S}$.

S. Syn. Car. 63 (37). d. no. 396. 1822.

Spreng. in L. Syst. Veg. 16 ed. $4^{1}$ : 530. d. 1827.

S. Syn. Am. Bor. 259 no. 2339. 1834.

Arcyria ochroleuca, (Trent.) Fr.

Fr. S. M. 3 : 18 I. 1832 .

Curtis, M. A., Bot. N. Car. 113. 1867.

Cke. Handbook $40 \mathrm{I} . d$. $187 \mathrm{I}$. Arcyria cinerea, Massee p. p.

Massee, Mon. Myx. 152. Dec. 1892. Clathrodes umbrinum, Sheldon.

Sheldon, Minn. Bot. Studies 9: 466. 20 Nov. 1895.

A rcyrta albida, var. pomiformis, Lister.

Lister, Mycetozoa 186. d. 1894.

Areyria punicea, $P$.

Muhl. Cat. Pl. Am. Sept. I ed. 104. 1813. 2 ed. 107. 1818.

S. Syn. Car. 64 (38) no. 399. 1822.

Grev. Scot. Crypt. Fl. 3 : pl. $130 . \quad 1825$.

Hitchcock, Cat. Pl. Amherst 59. 1829.

Hitchcock, Cat. Pl. Mass. 644. 1833. 2 ed. 646 (126). 1835.

S. Syn. Am. Bor. 259 no. 2338 . I834.

Lea, Cat. Pl. Cin. 60. $\quad$ I849.

Klotzsch, Linnæa 25: $364 . \quad 1852$ and Heller, Muhlenbergia 1 : 18.9 Aug. I901.

* Rav. Fung. Car. 2 : $80 . \quad 1853$.

Watt, Can. Nat. \& Geol. II. 2: 39 r. Oct. 1865. 
Curtis, M. A., Bot. N. Car. I13. 1867.

Cke. Handbook 400. $d$. I87I.

Pk. Rept. N. Y. Mus. 24: 84 . 1872.

Frost in Cat. Pl. Amherst 79. 1875 .

Rostf. Mycetozoa 268. $d$. 1875.

Farl. Bull. Bussey Inst. 1 : 43i. March 1876 .

Phil. Grev. 5 : I I5. March I877.

Cke. Ann. N. Y. Lyceum 11: 406. June 1877.

James, Jour. Cin. Soc. 2 : 66 (25). April 1879.

Pk. Rept. N. Y. Mus. $31:$ 57. 1879.

Hark. \& Moore, Cat. Pac. Fung. 20. Feb. I88o.

Johnson, Bull. Minn. Acad. 1 : 362.1880.

Bundy, Geol. Wis. I873-79. 1: 400.1883.

Pk. in Day's Cat., Bull. Buf. Soc. 4 : 196 (132). 1883.

Farl. Appalachia 3 : 238 . Jan. I 884 .

*Ell. N. A. F. III4. I884.

Wint. \& Demet. Hedw. 24: 200 (24). Oct. 1885.

Calkins, Jour. Myc. 3: 59. May 1887.

Arth. \& Holw. Bull. Minn. Surv. 3: 39. I Oct. 1887.

Langlois, Cat. PI. La. 27. I887.

Berl. in Sacc. Syll. $7: 426 . d$. I 5 March I 888.

Bennett, Cat. Pl. R. I. 85. I888.

Schrt. in Engler \& Prantl, Pflanzenfam. 11: 22. d. June I889.

Ell. in Cat. Pl. N. J. $600 . \quad 1889$.

Bessey, Contr. Bot. Dept. Univ. Nebr. 3: 45. I4 June I892.

Macbr. Bull. lowa 2 : I23. $d . p l .2, f .5,5 a$. June 1892 .

Massee, Mon. Myx. I42. d. pl. 10, f. 242-244. Dec. I892.

Atk. \& Schrenk, Jour. Mitch. Soc. 9: I06 (12). April I893.

Morg. Jour. Cin. Soc. 16: I9 (25). d. June 1893.

Macbr. Bull. Univ. Iowa 2: 379. Nov. 1893.

Underw. Proc. Ind. Acad. 1893 : 30. Aug. 1894.

Lister, Mycetozoa I88. d. pl. 68 A, f. $a, b . \quad$ I 894.

Kellerm. \& Werner, Geol. Ohio 72: 40I. Jan. I895.

Millsp. \& Nutt. Publ. Field Columb. Mus. Bot. 1:86. Jan. I896.

* Shear, N. Y. Fungi 400 . March 1896.

Harvey, Bull. Torr. Bot. Club 23: 3I2. 20 Aug. I 896.

Macbr. \& Smith, C. L., Bull. Univ. lowa 4: 73 . Dec. 1896.

Underw. \& Earle, Bull. Ala. Exp. Sta. 80: I33. 28 June 1897.

Olive, Proc. Ind. Acad. 1897 : I49. I898.

Harvey, Bull. Torr. Bot. Club 26: 324. I7 June I 899 .

Pk. Bull. N. Y. Mus. 6 : 234 . July 1899.

Whitney, Rhodora 1: I28. I July I899.

Herbst, Fungal Fl. Lehigh Valley 2I 5. d. 1899.

Longyear, Rept. Mich. Agr. 1898: 95. I 899.

Earle in Mohr. Contr. U.S. Nat. Herb. $6:$ 139. 31 July I9or.

Ricker, Univ. of Maine Studies 3: I6. 30 April r902.

Harvey, Bull. Torr. Bot. Club 26: 324. 17 June I902.

Mutchler, Proc. Ind. Acad. 1901 : 292. 1902.

Duss, Champ. Guad. \& Martinique 85. Ig03.

Mutchler, Proc. Ind. Acad. 1902: II8. I903.

Farl. in Coker, Veg. Bahama 243. I905.

Arcyria vernicosa, Rostf.

Rostf. Mycetozoa Suppl. 36. I876.

Clathrodes denudatum, Sheldon.

Sheldon, Minn. Bot. Studies 9: 466. 20 Nov. 1895 .

Arcyria denudata, Macbr.

Macbr. Slime-moulds 195. d. pl. 2, f. 5, $5^{a}$. Aug. 1899.

Fairman, Proc. Rochester Acad. 3: 212. March 1900. 
Macbr. Rhodora 2 : 79, 81. pl. 16, f. 6, 6a. 14 April 1900.

Sumstine, Torreya 4:36. 12 March 1904.

Macbr. in Sacc. Pk. \& Trel. Harriman Alaska Exp. 5 : 21. 5 April 1904.

Schaffner, Tr. Kans. Acad. 19: 204. 1905.

Arcyria rubiformis, Massee.

See note under Hemitrichia rubiform is, (P.) Lister.

Arcyria Serpula, Massee.

See Hemitrichia Serpuia, (Scop.) Rostf.

Arcyria stipata, Lister.

See Hemitrichia stipata, (S.) Macbr.

Arcyria stricta, Rostf.

See Arcyria cinerea, (Bull.) P.

Arcyria umbrina, Schum.

See Arcyria pomiformis, (Leers) Rostf.

Arcyria vernicosa, Rostf.

See Arcyria punicea, P.

Arcyria versicolor, Phil.

See Arcyria vileilina, Phil., var. versicolor, (Phil.) n. $\mathrm{n}$.

Arcyria vitellina, Phil.

Phil. Grev. 5 : 115. d. pl. 88, f. 7. March 1877 .

Hark. \& Moore, Cat. Pac. Fung. 20. Feb. 1880.

Berl. in Sacc. Syll. 7 : 432. d. I5 March 1888.

Macbr. Slime-moulds 192. $d$. Aug. 1899.

Macbr. Bull. lowa 2: 124. d. pl. 2, f. 4, 4a. June 1892 .

Mutchler, Proc. Ind. Acad. 1902 : 119.1903.

Clathrodes vitellinum, Sheldon.

Sheldon, Minn. Bot. Studies 9: 467. 20 Nov. I895.

Arcyria vitellina, Phil., var. versicolor, (Phil.) n. n.

Arcyria versicolor, Phil.

Phil. Grev. 5 : I1 $15 . d . p l .88$, f. 8. March 1877.

Cke. Ann. N. Y. Lyceum 11: 407. June i877.

Hark. \& Moore, Cat. Pac. Fung. 20. Feb. 1880.

Berl. in Sacc. Syll. 7 : 432. d. I5 March 1888.

Massee, Mon. Myx. 149. d.' Dec. 1892.

Lister, Mycetozoa I85. d. pl. 67 A. 1894.

Arcyria, sp. indet.

Massee, Journ. Bot. $30: 164$. June 1892 . West Indies.

Aregma, Fr.

Note. The genus Aregma was described by Fries in his Observationes 1 : 225. 1815. The genus Phragmidium was described by Link in Ges. Nat. Freunde Mag. 7: 30 . The date of this volume is given as 1816 on the titlepage of the volume. and this date has generally been accepted as correct. Fries however in Systema Mycologicum 3:495. 1832 makes the statement that Aregma and Phragmidium were published in the same year from which one might infer that that part of volume 7 including Link's paper was issued earlier than 1816 . The volume is in four parts of which part one including Phragmidium has the title "Erstes Quartal, Jan., Feh., März, 1813." It may therefore be the case that it is to an earlier edition of the Erstes Quartal issued in advance of the volume to which Fries refers. In the Summa, 507. 1849 Fries adopted the name Phragmidium including his Aregma. We have referred our species of Aregma to Phragmidium, that name being accepted by most writers. 
Aregma diseiflora, Arth.

Note. This name, founded on Ascophora disciflora, Tode Fung. Meckl. 1: 16. d.pl. $3, f .26,27.1790$ has been employed by Arthur in Indiana Plant Rusts, 1899 and 1904 to designate Phragmidium subcorticium, (Schrank) Wint.

The description and figures of Tode are wholly inadequate for the recognition of any one species and include forms on both Rubus and Rosa.

Tode's species is a composite considered by Streinz to be a synonym of Phragmidium incrassatum, Lk. a parasite of Rubus and by Arthur to designate one of the species parasitic on Rosa so that evidently it should not replace any of the more accurate names of modern usage.

Aregma Fragariæ, Arth. See Phragmidium Fragaria, (D C.) Wint.

Aregma mueronatum, Fr. See Phragmidium subcorticium, (Schrank) Wint.

Aregma mucronatum, Auct. Amer. p. p. See Phragmidium Rosa-alpina, (D C.) Wint.

Aregma mucronatum, Auct. Amer. p. p. See Phragmidium Rubi, (P.) Wint.

Aregma obtusatum, Fr. See Phragmidium Fragaria, (D C.) Wint.

Aregma speciosum, Fr. See Phragmidium speciosum, Fr.

Aregma triarticulatum, B. \& C.

See Phragmidium Fragaria, (D C.) Wint.

See Phragmidium Potentilla, (P.) Wint. See Phragmidium Potentilla-Canadensis, Diet.

Argynna, Morg

Morg. Jour. Cin. Soc. 18 : 41. d. Dec. 1895.

Sacc. Hedw. 35 : Rep. 7 : xxiv. d. 25 Feb. 1896.

Lindau in Engler \& Prantl, Pflanzenfam. $1^{1}: 338$. d. Jan. 1897.

Sacc. \& Syd. Syll. 14: 470. d. 20 Aug. 1899.

Argynna polyædron, (S.) Morg.

Note. The Spharia Curtisii, B. in Herb. Curtis 2174 which is stated by Cooke in Grev. 18: 60. March 1890 to be Spharia caryophaga, S. is not that species but Argynna polyadron, (S.) Morg.

It should be noticed that on page 144 of Curtis's Catalogue is a Nectria Curtisii, B. which is Calonectria Curtisii, (B.) Sacc.

Morg. Jour. Cin. Soc. 18 : 41. d. Dec. 1895.

Sacc. Hedw. 35 : Rep. 7 : xxiv. 25 Feb. 1896.

Lindau in Engler \& Prantl, Pflanzenfam. 11:338. Jan. 1897.

Sacc. \& Syd. Syll. 14: 470. d. 20 Aug. 1899.

Physarum polyadron, S.

S. Syn. Am. Bor. 257 no. 2300. $d . \quad 1834$.

Rostf. Mycetozoa II6. I875.

Cke. Ann. N. Y. Lyceum 11 : 387 . June 1877.

Berl. in Sacc. Syll. $7:$ 352. d. 15 March 1888.

Massee, Mon. Myx. 316. d. Dec. 1892.

Lister, Mycetozoa 62. d. 1804.

Spharia Curtisii, B. in Herb. Curtis.

Curtis, M. A., Bot. N. Car. 145. 1867.

Cke. Grev. 20: 84. March I892. 
Armillaria, $\mathrm{Fr}$.

Pk. Rept. N. Y. Mus. 43: 86 (40). d. Oct. I 890.

Pk. Rept. N. Y. Mus. 48: 262 (I64). d. March I897.

Armillaria appendiculata, $\mathrm{Pk}$.

Pk. Bull. Torr. Bot. Club 24: 140. d. 30 March 1897.

Underw. \& Earle, Bull. Ala. Exp. Sta. 80: 255. d. 28 June 1897.

Lindau \& Syd. Hedw. 37 : Beibl. 7: I. 9 April 1898.

Sacc. \& Syd. Syll. 14 : 70. d. 20 Aug. 1899.

Mcllvaine \& Macadam, Am. Fung. 54. d. 1900.

Earle in Mohr, Contr. U. S. Nat. Herb. 6: 227. 31 July I9or.

Armillaria aurantia, (Schæff.) Fr.

Atk. Mushrooms 84. d. f. 86.20 Oct. 1900.

Hay, Bull. Nat. Hist. Soc. N. B. 5: I 14.1903.

Agaricus aurantius, Schæeff.

S. Syn. Am. Bor. 146 no. 30. 1834 .

Agaricus Peckil, Howe.

Howe, Bull. Torr. Bot. Club $6: 66 . d$. Dec. 1875 .

Tricholoma Peckil, Howe.

Howe, Bull. Torr. Bot. Club 6 : 66. $d$. Dec. 1875 .

Pk. Rept. N. Y. Mus. $41: 82$. d. 1888.

Pk. Rept. N. Y. Mus. 44: I 55 (43). d. Dec. I89I.

Pk. Bull. Torr. Bot. Club 26: 253. I5 May 1899.

Atk. Mushrooms 86.20 Oct. 1900.

Pk. Rept. N.'Y. Mus. $55: 962 . d$. Nov. 1902. [Bull. $10:$ no. 54.]

Longyear, Rept. Mich. Acad. 4: 120.1904.

Mastoleucomyces Aurantius, O. Kuntze.

Kuntze, O., Rev. Gen. PI. 2 : 861. I891.

Armillaria bulbigera, (A. \& S.) Fr.

Pk. Rept. N. Y. Mus. 43: 91 (45). Oct. 1890.

Atk. Bull. Cornell Univ. 3: 24.25 June 1897.

Earle in Mohr, Contr. U. S. Nat. Herb. $6: 227$. 3I July Igor.

Selby, Rept. Ohio Acad. $10: 78.1902$.

Agarlcus bulblger, A. \& S.

S. Syn. Car. $80(54)$ no. 568 . 1822.

S. Syn. Am. Bor. I45 no. 18.1834.

Curtis, M. A., Bot. N. Car. 84. 1867.

Mastoleucomyces bulblger, $O$. Kuntze.

Kuntze, O., Rev. Gen. Pl. 2: 86I. I89I.

Armillaria cheimonophila.

N.B. On page I 6 the name cheimonophylla was erroneously written by me cheimonophila. A. B. S.

Armillaria cheimonophylla, B. \& C.

NOTE. This species which has no annulus as shown by an examination of original specimens is not a true Armillaria. It is placed by Patouillard (1887) in Mucidula, a genus based by him on Armillaria mucida, (Schrad.) Fr., a species reported in the United States but not common, which differs from $A$. cheimonophylla, B. \& C. in having an annulus although agreeing with it in some other respects.

Sacc. Cub. \& Manc. Syll. 5 : 86. d. 28 May I887.

Henn. in Engler \& Prantl, Pflanzenfam. 1 ${ }^{1 * *}: 270 . \quad$ I898. Agaricus chelmonophyllus, B. \& C.

B. \& C. Jour. Linn. Soc. 10: 284. d. I869.

Mastoleucomyces chelmonophyllus, O. Kuntze.

Kuntze, O., Rev. Gen. PI. 2: 86I. 1891.

West Indies. 
Mucidula cheimonophylla, Pat.

Pat. Bull. Soc. Myc. Fr. 15 : 192. d. 31 July I899.

Duss, Champ. Guad. \& Martinique 48. 1903.

Armillaria constricta, $\mathrm{Fr}$.

Sacc. Cub. \& Manc. Syll. 5 : 77. d. 28 May 1887.*

Pk. Rept. N. Y. Mus. 43 : 9I (45). Oct. I89o.

Agaricus constrictus, Fr.

Fr. S. M. $1: 28 . d$. I 821 .*

Hark. \& Moore, Cat. Pac. Fung. 2. Feb. I880.

Mastoleucomyces constrictus, $O$. Kuntze.

Kuntze, O., Rev. Gen. PI. 2 : 86ı. i 89 r.

Armillaria imperialis, Fr.

Hay, Bull. Nat. Hist. Soc. N. B. 5 : 115.1903.

Mastoleucomyces imperialis, $\mathrm{O}$. Kuntze.

Kuntze, O., Rev. Gen. Pl. 2 : 86r. I89r.

Armillaria luteovirens, (A. \& S.) Fr.

Agaricus luteovirens, A. \& S.

S. Syn. Car. $82(56)$ no. 622 . 1822 .

S. Syn. Am. Bor. 146 no. 31. 1834.

Curtis, M. A., Bot. N. Car. $84 . \quad 1867$.

Mastoleucomyces luteovirens, $\mathrm{O}$. Kuntze.

Kuntze, O., Rev. Gen. Pl. 2 : 86r. 189 r.

Armillaria macrospora, Pk.

Pk. Bull. Torr. Bot. Club 27 : 6ro. d. 29 Dec. 1900.

Sacc. \& Syd. Syll. 16: 19. $d$. I Feb. 1902.

Armillaria megalopoda, Bres.

Hay, Bull. Nat. Hist. Soc. N. B. 5 : I15. 1903. Mastoleucomyces megalopodus, $\mathrm{O}$. Kuntze.

Kuntze, O., Rev. Gen. PI. 2 : 86r. I89ז. .

Armillaria mellea, (Vahl) Fr.

Note. The sclerotioid condition of this species is the Rhizomorpha subcorticalis of older mycologists and we have for convenience inserted here the North American references to that Rhizomorpha but without intending to imply that in all cases cited the Rhizomorpha belongs beyond doubt to Armillaria mellea.

A difficulty in the synonymy arises from the fact that while Armillaria mellea normally has an annulus, specimens are occasionally found belonging undoubtedly to this species but in which the annulus is wanting.

There are other cases of forms referred to Clitocybe by some writers in consequence of the absence of an annulus, although in other respects certainly closely related to Armillaria mellea. Whether such forms are to be regarded as distinct species or merely as forms of Armillaria mellen is a matter of individual judgment. Peck in Rept. N. Y. Mus. 46: I34 (54). Dec. 1893 proposed the name Armillaria mellea, var. exannulata to include such forms. Later in Rept. N. Y. Mus. $5 \mathrm{I}^{1}: 284$, 302..$^{\circ}$ I Feb. I 899 he expresses the opinion that Clitocybe monadelpha, Morg. is distinct. We have here brought together all the references to Clitocybc monadelpha and clitocybe aquatica since they need to be consulted in connection with Armillaria mellea.

It should be remarked that this species was placed by Karsten (1879) in the genus Armillariella characterized by an annulus and decurrent gills as distinguished from forms like Armillaria robusta, (A.\& S.) Fr. in which the gills are not decurrent.

Sacc. Cub. \& Manc. Syll. 5 : 8o. d. 28 May 1887.

Pound, Bull. Nebr. Exp. Sta. 1: 360 (90). 18 Dec. 1889).

Pk. Rept. N. Y. Mus. 43: 88 (42). d. 9l (45). Oct. 1890 . 
Taylor, Rept. U. S. Agr. $1890: 367$ (6). pl. s,f. 4. 189o. [Reprint 1893.]

Banning in Pk. Rept. N. Y. Mus. 44: $179(67)$. Dec. I89 I.

Webber, Tr. St. Louis Acad. 6 : I8. 12 March 1892 and Contr. Bot. Dept. Univ. Nebr. 3: 18. 14 June 1892.

*Briosi \& Cav. Fung. Piante Colt. I66. $d$. $f$. April 1892.

Fairman, Proc. Rochester Acad. 2 : 155. Feb. 1893.

Atk. \& Schrenk, Jour. Mitch. Soc. 9 : 99 (5). April 1893.

Pk. Rept. N. Y. Mus. 46: 133 (53). Dec. 1893.

* Shear, N. Y. Fungi 102. 1893.

Taylor, Food Products 2 : 21. 1893.

Pk. Country Gent. 59: 582 (48). $f$. 9 Aug. 1894.

Underw. Proc. Ind. Acad. 1893: 6r. Aug. I894.

Pk. Rept. N. Y. Mus. 47: I72 (46), 20 Nov. I894.

Kellerm. \& Werner, Geol. Ohio 7²: 302. Jan. 1895.

* Ell. \& Ev. N. A. F. 3201. 24 April 1895.

McClatchie in Reid's Hist. Pasadena 619. 1895.

Bates, Tr. Mass. Hort. Soc. $1896: 185 . d$. 1896.

Webster, Am. Kitchen Mag. 6: 216. Feb. 1897.

Pk. Rept. N. Y. Mus. 48: 262 (164). d.pl. 20. March 1897.

Atl. Bull. Cornell Univ. $3: 24.25$ June i 897.

McClatchie, Proc. S. Cal. Acad. 1 : 385.5 June 1897.

Underw. \& Earle, Bull. Ala. Exp. Sta. 80: 256. 28 June 1897.

Taylor, Hubk. Mushrooms 3 : 2. pl. E, f. $t .1897$.

*Ell. \& Ev. N. A. F. 3501.24 Jan. 1898.

Williams, Asa Gray Bull. 6 : 106. Dec. 1898.

Henn. in Engler \& Prantl, Pflanzenfam. 11**: 270. d. f. 123 B. 1898.

[Underw.] N. Y. Myc. Club r6. 1898.

Pk. Bull. N. Y. Mus. 6: 193. July 1899.

Shear, Asa Gray Bull. 7 : 93. Oct. I 899.

Herbst, Fungal Fl. Lehigh Valley 36. d.pl. 1899.

Jelliffe, Flora of Long Island $42 . \quad$ I 899.

Longyear, Rept. Mich. Agr. 1898 : 92. 1899.

Underw. Moulds 121. 1899.

Webster, Rhodora 2: 32. 7 Feb. 1900.

Perry, Agricultural Student 6: 179. May 1900 and Ohio Univ. Bull. IV. 30: 7. May 1900.

Atk. Mushrooms, II.pl. 2, f. II. 83. d. pl. 27,f. 85. 20 Oct. 1900.

Hyams, Bull. N. Car. Exp. Sta. 177: 31. d. Dec. 1900.

Atk. in Bailey's Cycl. Am. Hort. I045. 1900.

Mcllvaine \& Macadam, Am. Fung. 55. d. pl. $16, f . t .1900$.

Wilcox; Bull. Okla. Exp. Sta. $49: 6$. pl. 4, f. 5. pl. $9, f .18$. Feb. 1901. Sweetzer, Bull. Pacific Univ. 14: 12. May 190I.

Earle in Mohr. Contr. U.S. Nat. Herb. $6: 227.31$ July I90I.

Jelliffe \& Underw. Proc. Nat. Sci. Assoc. Staten IsI. 8: no. 10. 14 Dec. 1901 .

Sturgis, Rept. Conn. Exp. Sta. 24: 272. I901.

Brown \& Fernekes, Bull. Wis. Nat. Hist. Soc. II. 2 : 46. Jan. 1902.

Murrill, Torreya 2: 25. 2 I Feb 1902.

Webster, Rhodora 4: 77.4 April I902.

Ricker, Univ. of Maine Studies $3: 55.30$ April 1902.

Ferguson, Bull. U. S. Agr. Pl. Industry 16:15. 14 June 1902.

White, Bull. Torr. Bot. Club 29: 562 . 26 Sept. I902.

Conant, Bull. Boston Myc. Club ig: 5 Feb. 1903.

Walker, Rept. Arkansas Exp. Sta. 1902 : 30. Feb. 1903.

Hay, Bull. Nat. Hist. Soc. N. B. 5 : 114.1903.

Longyear, Special Bull. Mich. Exp. Sta. 25: 45. March 1904.

Longyear, Rept. Mich. Acad. 4: II 19.1904. 
MacKay, Tr. N. Scot. Inst. 11 : I24. Aug. I904. Agaricus melleus, Vahl.

Muhl. Tr. Am. Phil. Soc. 4: 240. 1799.

Grev. Scot. Crypt. Fl. 6 : pl. 332.1828.

S. Syn. Am. Bor. 145 no. 20. 1834.

Lea, Cat. Pl. Cin. 49. 1849.

*Rav. Fung. Car. 2: $1 . \quad$ I853.

Sprague, Proc. Boston Soc. 6 : $316 . \quad$ I859.

Curtis, M. A., Bot. N. Car. 84. I867.

Pk. Rept. N. Y. Mus. 22 : 76.1869.

Curtis, M. A., Jour. Roy. Hort. Soc. Il. 2 : $73 . \quad$ I 870.

Cke. Handbook ig. pl. $2, f .3$. f. 36. d. $187 \mathrm{I}$.

Pk. Rept. N. Y. Mus. 23: 73. d. 1872.

Pk. Rept. N. Y. Mus. 24: I02. 1872.

Parker, Proc. Poughk. Soc. 1 : 84 . I Nov. 1875.

Frost in Cat. Pl. Amherst 62. 1875.

Farl. Bull. Bussey Inst. 1 : 436 . March 1876 .

Pk. Tr. Albany Inst. 8 : 164 . I876.

Johnson, Bull. Minn. Acad. 1 : 2 I2. 1877 and Rept. Minn. Surv. 5 : 67. 1877 .

Morg. Fl. Miami Valley 67. 1878.

James, Jour. Cin. Soc. $2: 63$ (22). April 1879.

Fowler, Rept. Agr. N. B. 1878 : Append. B: 63. 1879.

Hark. \& Moore, Cat. Pac. Fung. 2. Feb. 1880.

*Rav. Fung. Am. 407. I88I.

Morg. Jour. Cin. Soc. 6:64. d. April 1883.

Pk. Rept. N. Y. Mus. 33 : 36 . Oct. I883.

Bundy, Geol. Wis. $1873-79,1: 396.1883$.

Pk. in Day's Cat., Bull. Buf. Soc. 4: I75 (III). 1883.

Rav. S. Car. Resources 354. I883.

Porcher in Wood's Hdbk. Med. Sci. 3 : 274. pl. 12, f. 7. 1886.

Pk. Rept. N. Y. Mus. 39 : 57. Sept. 1887.

Cobb, List. Pl. Amherst 3I. I887.

Dudley, P. H., Bull. U. S. Agr. Forestry I : 44, 50. 1887.

Langlois, Cat. Pl. La. $27 . \quad$ I887.

Somers, Tr. N. Scot. Inst. $6: 286 . \quad$ I 887.

Scribner, Bot. Gaz. 13: 15. Jan. 1888.

Macbr. Bull. Univ. lowa 1: 38. d. Nov. I 888.

Bennett, Cat. Pl. R. I. 76. I888.

Pk. Rept. N. Y. Mus. 41: 9I, 92. 1888.

Ell. in Cat. Pl. N. J. 469 . 1880.

Scribner, Orchard \& Garden 12: I2. d. f. 1485, 1622. Jan. I89o.

Pammel, Proc. Soc. Prom. Agr. Sci. 1890: 9I. 1890.

Mayer, Waldungen von Nordamer. 2 I I, 434. 1890.

Scribner, Fung. Dis. $65 . f .1485,1622.1890$.

Pierce, Bull. U. S. Agr. Veg. Pathol. 2 : I 58.1892.

Taylor, Rept. U.S. Agr. 1891 : 412 (I2).d.pl. 2. I892. [Reprint I893.]

McCarthy, Bull. N. Car. Exp. Sta. 92: I I6, I22. f. 65. 22 Aug. I893.

Eby, Fungi of Lancaster Co. 7. I894.

Starnes, Bull. Ga. Exp. Sta. 28 : 282. Sept. 1895.

Anderson, Bot. Gaz. 22: 235. 23 Sept. 1896.

Graves, Asa Gray Bull. 5 : 28. March 1897.

Gall. \& Woods, Yearb. U.S. Agr. 1896: 249. I897.

Tavlor, Hdbk. Mushrooms 2: 13. d. pl. 6. 1897.

Tubeuf. Smith, Diseases of Plants 455. f. 285-288. 1897.

Schrenk, Bull. U. S. Agr. Veg. Pathol. 25:50. 1900.

Trel. Rept. Mo. Hort. Soc. 43: 234 (II). I900.

Wilcox, Bot. Gaz. 32 : 226. 24 Sept. Igor. 
Schrenk, Pacific Rural Press 61 : 324. 1901.

Schrenk, Yearb. U. S. Agr. 1900 : 200. 1901.

Piper \& Fletcher, Bull. Wash. Exp. Sta. 59: 3. f. 1-4. 1903. Agaricus polymyces, $\mathrm{P}$.

Muhl. Cat. Pl. Am. Sept. I ed. 104. 1813. 2 ed. 107. 1818.

S. Syn. Car. $80(54)$ no. 566.1822.

Mastoleucomyces melleus, $\mathrm{O}$. Kuntze.

Kuntze, O., Rev. Gen. PI. 2: 861. 1891. Rhlzomorpha subcorticalls, P.

Muhl. Cat. Pl. Am. Sept. I ed. 106. 1813. 2 ed. 108. 1818.

S. Syn. Car. I3I (105) no. 1369.1822.

Hitcheock, Cat. PI. Amherst 63. 1829.

Hitchcock, Cat. Pl. Mass. 647. ${ }_{1833 .} 2$ ed. 648 (128). 1835.

* Rav. Fung. Car. 3 : 47.1855.

Curtis, M. A., Bot. N. Car. 139. 1867.

Pk. Rept. N. Y. Mus. 24: 97.1872.

Frost in Cat. Pl. Amherst 93. 1875.

Lesquereux, Rev. Myc. $3: 74$. July 1881.

*Ell. N. A. F. 833. 1882 .

Cobb, List Pl. Amherst 45. 1887.

Webber, Rept. Nebr. Agr. 1889: $216(76) . \quad$ I890.

*Ell. \& Ev. N. A. F. 3100. April 1894 .

*Ell. \& Ev. Fung. Columb. 400. 1894.

Armillaria mellea, (Vahl) Fr., var. albida, Pk.

Pk. Rept. N. Y. Mus. 46: $134(54)$. $d$. Dec. 1893.

Pḱ. Country Gent. 59: 583 (50). 9 Aug. 1894.

Mcllvaine \& Macadam, Am. Fung. 56. d. 1900.

Armillaria mellea, (Vahl) Fr., var. bulbosa, Pk.

Pk. Rept. N. Y. Mus. $46: 133$ (53). d. Dec. 1893.

Pk. Country Gent. 59: 583 (50). 9 Aug. 1894.

Mcllvaine \& Macadam, Am. Fung. 56. d. 1900.

Hyams, Bull. N. Car. Exp. Sta. 177: 32. Dec. 1900.

?Piper \& Fletcher, Bull. Wash. Exp. Sta. $59:$ 13. d. f. 5. 1903.

Armillaria mellea, (Vahl) Fr., var. flava, Gillet.

Pk. Rept. N. Y. Mus. 46: 134 (54). Dec. 1893.

Pk. Country Gent. 59: 583 (50). 9 Aug. 1894.

Mcllvaine \& Macadam, Am. Fung. 56. d. 1900.

Hyams, Bull. N. Car. Exp. Sta. 177: 32. Dec. 1900.

Armillaria mellea, (Vahl) Fr., var. glabra, Gillet.

Pk. Rept. N. Y. Mus. 46: 134 (54). Dec. 1893.

Pk. Country Gent. 59: 583 (50). 9 Aug. 1894.

Mcllvaine \& Macadam, Am. Fung. 56. d. 1900.

Hyams, Bull. N. Car. Exp. Sta. 177: 32. Dec. 1900.

Armillaria mellea, (Vahl) Fr., var. nigripes, Pk. nom. nud.

McClatchie in Reid's Hist. Pasadena 619. I895.

Armillaria mellea, (Vahl) Fr., var. obscura, Gillet.

Pk. Rept. N. Y. Mus. 46: $134(54)$. Dec. 1893.

Pk. Country Gent. 59: 583 (50). 9 Aug. 1894. Mcllvaine \& Macadam, Am. Fung. 56. d. 1900.

Hyams, Bull. N. Car. Exp. Sta. 177: 32 . Dec. 1900.

Armillaria mellea, ( $\mathrm{Vahl}$ ) Fr., var. radicata, $\mathrm{Pk}$.

Pk. Rept. N. Y. Mus. 44: 150 (38). Dec. 1891.

Pk. Rept. N. Y. Mus. 46 : 134 (54). Dec. 1893.

Pk. Country Gent. 59 : $583(50)$. 9 Aug. I 894.

Mcllvaine \& Macadam, Am. Fung. 56. d. 1900. 
Armillaria mellea, (Vahl) Fr., var. exannulata, Pk.

Pk. Rept. N. Y. Mus. 46 : 134 (54). d. Dec. 1893.

Pk. Country Gent. 59: 583 (50). 9 Aug. I894.

Mcllvaine \& Macadam, Am. Fung. 56. d. pl. 16, f. 2. 1900.

Hyams, Bull. N. Car. Exp. Sta. 177: 32. Dec. 1900.

Wilcox, Bull. Okla. Exp. Sta. 49: 17. d. 18. pl. 4, f. 4. Feb. 1901. Clitocybe sociales, Fr.

Mcllvaine \& Macadam, Am. Fung. 89. d. 1900.

Clitocybe gymnopodia, Auct.

Langlois, Proc. La. Soc. Naturalists 1897-9 : 56. d. 1900. Agaricus monadelphus, Morg.

Morg. Jour. Cin. Soc. 6: 69. d. pl. 4. April 1883.

Cragin, Bull. Wash. Lab. 1 : 19. Sept. 1884.

Perry, Agricultural Student 6 : 179 . May 1900 and Ohio Univ. Bull. IV. 30: 7. May I900.

Clitocybe monadelpha, Morg.

Morg. Jour. Cin. Soc. 6: 69. d.pl. 4. April 1883.

Sacc. Cub. \& Manc. Syll. 5: 164. d. 28 May I887.

Kellerm. \& Werner, Geol. Ohio $7^{2}$ : 303. Jan. I895.

Pk. Rept. N. Y. Mus. 511: 284, 302. d. pl. 51,f. 1-5. II Feb. I 899.

Lloyd, Myc. Notes 17. $d$. April I899.

Herbst, Fungal Fl. Lehigh Valley 44. d. I899.

Mcllvaine \& Macadam, Am. Fung. 89. d. pl. 27. 1900.

Pk. Mem. N. Y. Mus. 3: 140. d. pl. 46,f. 7-12. Nov. 1900.

Hyams, Bull. N. Car. Exp. Sta. I 77 : 38. Dec. 1900.

Wilcox, Bull. Okla. Exp. Sta. 49 : 17. d. pl. 6, 9, f. 17. Feb. I9or.

Earle in Mohr, Contr. U. S. Nat. Herb. 6 : 226. 31 July I90I.

Williamson, Proc. Ind. Acad. 1900: i64. I90I.

Earle; Torreya 3: 60 . 18 April 1903.

Clitocybe aquatica, Banning.

Banning in Pk. Rept. N. Y. Mus. 44: I80 (68). d. Dec. I891.

Sacc. Syll. 11 : 14. $d$. July I 895.

Armillaria melleorubens, B. \& C.

B. \& C. Jour. Linn. Soc. $10: 283 . d$. 1869.

Sacc. Cub. \& Manc. Syll. 5 : 81. d. .28 May I887. Agaricus melleorubens, B. \& C.

B. \& C. Jour. Linn. Soc. $10: 283$. d. 1869. Mastoleucomyces melleorubens, $\mathrm{O}$. Kuntze.

Kuntze, O., Rev. Gen. Pl. 2: 86I. I89I.

Armillaria mueida (Schrad.) Fr.

Sacc. Cub. \& Manc. Syll. 5: 85. d. 28 May I 887.

Pk. Rept. N. Y. Mus. 43: 90 (44). Oct. I89o.

Banning in Pk. Rept. N. Y. Mus. 44: 187 (75). Dec. I891.

Mcllvaine \& Macadam, Am. Fung. 58. d. 1900.

Agaricus mucidus, Schrad.

S. Syn. Car. $80(54)$ no. 562 . I822.

S. Syn. Am. Bor. I 45 no. I9. I 834 .

Curtis, M. A., Bot. N. Car. 84. I867.

Cke. Handbook 20. d. 1871. Mastoleucomyces mucidus, $O$. Kuntze.

Kuntze, O., Rev. Gen. PI. 2 : 86i. I89ı.

Armillaria nardosmia, Eli.

Notr. The Agaricus rhagadiosus recorded by Peck, Rept. N. Y. Mus. 33: 18. Oct. I 883 is stated by him in Rept. $43: 87(4 \mathrm{I})$. Oct. 1890 to be the same as Armillaria nardosmia, Fill. Whether the latter should be considered distinct from the true Armillarin rhagadiosa, Fr. he thinks doubtful. Jelliffe states in a letter that his record of Clitocybe rhagadiosa is hased upun the above citation of Peck. A. B. S. 
Ell. Bull. Torr. Bot. Club $6: 75$. d. Feb. 1876.

Sacc. Cub. \& Manc. Syll. 5: 86. d. 28 May 1887.

Pk. Rept. N. Y. Mus. 43: 87 (4I). d. 9I (45). Oct. I890.

Hein. in Engler \& Prantl, Pflanzenfam. 11**: 270. 1898.

Herbst, Fungal Fl. Lehigh Valley 37. d. 1899.

Mcllvaine \& Macadam, Am. Fung. 57. d. I900.

* Shear, Ell. \& Ev. Fung. Columb. 1401. May r9or.

Agaricus nardosmius, Ell.

Ell. Bull. Torr. Bot. Club $6: 75$. d. Feb. 1876.

Ell. in Cat. Pl. N. J. 469 . I 889 .

Agaricus rhagadiosus, Pk. an Fr.?

Pk. Rept. N. Y. Mus. 33 : I8. Oct. 1883.

PArmillaria rhagadiosa.

Pk. Rept. N. Y. Mus. 43: 88 (42). Oct. 1890. Clitocybe rhagadiosus, Jelliffe.

Jelliffe, Flora of Long Island 42. I 899.

Mastoleucomyces nardosmius, O. Kuntze.

Kuntze, O., Rev. Gen. PI. 2 : 86ı. I89I.

Armillaria ponderosa, $\mathrm{Pk}$.

Pk. Bull. Buf. Soc. 1: 42. d. July 1873 .

Pk. Rept. N. Y. Mus. 26: 50. $d$. April 1874.

Sacc. Cub. \& Manc. Syll. 5: 78. d. 28 May 1887.

Pk. Rept. N. Y. Mus. 43:87 (4I) d. 91 (45). Oct. 1800.

Henn. in Engler \& Prantl, Pflanzenfam. 11**: 270. 1898.

Mcllvaine \& Macadam, Am. Fung. 54. d. 1900.

Hay, Bull. Nat. Hist. Soc. N. B. 5 : I I5. 1903. Agaricus ponderosus, $\mathrm{Pk}$.

Pk. Bull. Buf. Soc. $1: 42$. d. July 1873.

Pk. Rept. N. Y. Mus. 26: 50. $d$. April 1874.

Frost in Cat. Pl. Amherst 62. 1875.

Pk. Rept. N. Y. Mus. $29: 66.1878$.

Cobb, List PI. Amherst 3i. I887. Agaricus magnivelaris, $\mathrm{Pk}$.

Pk. Rept. N. Y. Mus. 29: 66. I 878. Mastoleucomyces ponderosus, $\mathrm{O}$. Kuntze.

Kuntze, O., Rev. Gen. Pl. 2 : 86I. I89I.

Armillaria ramentacea, (Bull.) Fr.

Sacc. Cub. \& Manc. Svll. 5: 76. d. 28 May 1887.

Pk. Rept. N. Y. Mus. 43: 9I (45). Oct. I89o. Agaricus ramentaceus, Bull.

Frost in Cat. PI. A mherst 62. 1875.

Cobb, List PI. Amlerst 3I. I887.

Mastoleucomyces ramentaceus, $\mathrm{O}$. Kuntze.

Kuntze, O., Rev. Gen. PI. 2 : 860. 1891.

Armillaria rhagadiosa, $\mathrm{Fr}$. See Armillaria nardosmia, Ell.

Armillaria robusta, (A. \& S.) Fr.

Pk. Rept. N. Y. Mus. 43: 91 (45). Oct. I89o.

Mcllvaine \& Macadam, Am. Fung. 52. d. I900.

Conant, Bull. Boston Myc. Club I9: 5 Feb. 1903.

Hay, Bull. Nat. Hist. Soc. N. B. 5 : II 5.1903. Agaricus robustus, A. \& S.

S. Syn. Car. 80 (54) no. 561. 1822 .

S. Syn. Am. Bor. 145 no. 17. 1834 .

Curtis, M. A., Bot. N. Car. 84. 1867.

Mastoleucomyces robustus, $\mathrm{O}$. Kuntze.

Kuntze, O., Rev. Gen. Pl. 2 : 86I. I89I. 
Armillaria solidipes, $\mathrm{Pk}$.

Pk. Bull. Torr. Bot. Club 27 : 611. d. 29 Dec. 1900.

Sacc. \& Syd. Syll. 16: 20. d. I Feb. 1902.

Armillaria umbilieata, (Pat.) Sacc. \& Syd.

Sacc. \& Syd. Syll. 16: 10. $d$. I Feb. I902.

Armillariella umbilicata, Pat.

Pàt. Bull. Soc. Myc. Fr. 15 : 191. d. 31 July 1899.

Duss, Champ. Guad. \& Martinique 52. 1903.

Armillaria viseidipes, $\mathrm{Pk}$.

Pk. Rept. N. Y. Mus. 44: : 28 (16). d.pl. 2, f. 1-3. Dec. 1891 .

Pk. Rept. N. Y. Mus. 46 : 134 (54). Dec. 1893.

Sacc. Syll. $11: 7 . d$. July I 895

Mcllvaine \& Macadam, Am. Fung. 53. d. 1900.

Armillariella mellea, Karst.

See Armillaria mellea, (Vahl) Fr.

Armillariella umbilicata, Pat. See Armillaria umbilicata, (Pat.) Sacc. \& Syd.

\section{Arrhenia tenella, Fr.}

Note. The genus Arrhenia is differently limited by different writers. The species given below is placed by Quélet in Dictyolus. Otto Kuntze substitutes Auriscalpium, S. F. Gray for Arrhenia, Fr. but Auriscalpium, S. F. Gray is based on Hydnum Auriscalpium, L. which is an entirely different plant from Arrhenia Auriscalpium, Fr., the type of Arrhenia.

Sacc. Cub. \& Manc. Syll. 5 : 499. d. 28 May I887.* Cantharellus tenellus, Fr.

S. Syn. Am. Bor. 153 no. 297 . 1834. Auriscalpium tenellum, O. Kuntze.

Kuntze, O., Rev. Gen. Pl. $3^{2}: 446 . \quad 1898$.

Aprhytidia, B. \& C.

Note. The genus Arrhytidia is placed by Patouillard, Essai Tax. 30. 1900 in Guepiniopsis, Pat. In regard to Arrhytidia fava given below an examination of the original specimen shows that although it belongs to the Dacryomycetinea it does not seem to be a genuine Guepiniopsis. Whether Arrhytidia can be regarded as a good genus can not be satisfactorily determined until its development is better known.

B. \& C. Hook. Jour. Bot. 1: 235 (I06). d. 1849.

B. \& C. Am. Jour. Sci. II. $10:$ I $87 . d$. Sept. I850.

Fr. Nova Acta R. Soc. Sci. Upsal. III. 1 : 114.1851.

Sacc. Cub. \& Manc. Syll. 6 : 804. $d$. I Aug. 1888.

Arrhytidia flava, B. \& C.

B. \& C. Hook. Jour. Bot. 1 : 235 (106). d. 1849. [Decades no. 224.]

B. \& C. Am. Jour. Sci. II. 10 : 187. d. Sept. 1850.

Curtis, M. A., Bot. N. Car. 101. 1867.

B. Grev. $1: 70$ (18). d. Nov. 1872 .

Sacc. Cub. \& Manc. Syll. 6 : 805. d. I Aug. I 888.

Underw. \& Earle, Bull. Ala. Exp. Sta. 80: 222. 28 June 1897.

Henn. in Engler \& Prantl, Pflanzenfam. 11**: 102. I898.

Earle in Mohr, Contr. U.S. Nat. Herb. 6 : 196. 31 July 1901.

Arrhytidia fulva, B: \& C.

Curtis, M. A., Bot. N. Car. 101. I867.

B. Grev. 1 : 70 (18) no. 171. d. Nov. 1872

Sacc. Cub. \& Manc. Syll. $6: 805 . d$. I Aug. 1888.

Underw. \& Earle, Bull. Ala. Exp. Sta. 80: 222. 28 June 1897.

Henn. in Engler \& Prantl, Pflanzenfam. 11**: 102.1898.

Earle in Molhr, Contr. U.'S. Nat. Herb. 6 : 196. 3I July Igor. 
Arthrinium caricicolum, Kze.

S. Syn. Am. Bor. 278 no. 2612 . 1834

Cke. Bull. Buf. Soc. 3 : 201. July 1877.

Arthrinium curvatum, Kze.

See Camptoum curvatum, (Kze.) Lk.

Arthrinium sporophlœum, Kze.

Sporophloum gramineum, Lk.

S. Syn. Am. Bor. 278 no. 2615.1834.

Cke. Bull. Buf. Soc. 3 : 201 . July 1877.

Arthonia confluens, Auct. Amer. p. p.

Note. The genus Arthonia has usually been considered to belong to lichens although some modern writers consider that at least part of the species should be regarded as fungi. In the two citations given below Peziza pru. inata, S. is said to be Arthonia confluens, a statement difficult to understand since $P$. pruinata is properly considered to be a synonym of Solenia poriaformis, (P.) Fckl. and is not related in the least to Arthonia.

Furthermore from the citations below it is not possible to say which of the several species known at different times under the name Arthonia confluens is referred to. Apparently as used by lichenologists at the present day that name is a synonym of different species of Opegrapha and Graphis.

For North American species of Arthonia from a lichenological point of view see Synopsis of the Genus Arthonia by Henry Willey, 1890.

Cke. Bull. Buf. Soc. 2 : 293. March 1875.

B. Grev. $3: 156$ (134). June 1875 .

Arthrobotrys, Cda.

Pound \& Clements, Minn. Bot. Studies 9 : 664. d. 30 Nov. 1896.

Arthrobotrys oligospora, Fres.

See Arthrobotrys superba, Cda., var. oligospora, (Fres.) Coem.

Arthrobotrys rosea, Massee.

Patterson, Bull. U. S. Agr. Pl. Industry 8: 27. 3 Feb. 1902.

Arthrobotrys superba, Cda., var. oligospora, (Fres.) Coem.

Pound, Bot. Surv. Nebr. 3: I I. 18 June I894. Arthrobotrys oligospora, Fres.

Farl. Bull. Bussey Inst. 1: 439. March 1876.

\section{Arthrobotryum, Ces.}

NoTE. The genus Arthrobotryum, attributed by various mycologists to Cesati, was originally described, although rather vaguely, in Klotzsch Herb. Viv. Myc. no. 1821. I854, under the generic name Arthrographium and the description was repeated in Bot. Zeit. 12: 186. 1854. In both places is quoted the figure in Hedwigia $1: p l .4, f .3$ (error for $f .1$ ) which is called Arthrobotryum stilboides without notes or description. As it is doubtful whether the generic characters as understood by Cesati could be recognized from the figure alone it might be questioned whether Arthrographium should not replace Arthrobotryum. Since the latter name has been constantly used by subsequent mycologists, not always perhaps in the same sense as used hy Cesati, we have not thought it best to abandon the name.

Some of the North American species which have been by some writers included in Arthrobotryum do not appear to us to be congeneric with A. stilboides, especially those here referred to Arthrosporium.

Whether Didymobotryum, Sacc. Syll. 4:626. d. Io April 1886 should be considered distinct from Arthrobotryum is so far as Nurth American species are concerned doubtful and we have placed the North American species of Didymobotryum under the present genus.

Pound \& Clements, Minn. Bot. Studies 9 ; 728, d. 31 May 1897. 
Arthrobotryum atrocephalum, (Ell.) Sacc.

Sacc. Syll. 4: 630. d. Io April i886.

Stilbum atrocephalum, Ell.

Ell. Bull. Torr. Bot. Club $6: 107 . d$. Sept. 1876.

Ell. in Cat. Pl. N. J. $572 . \quad 1889$.

Wettsteiniella atrocephala, $\mathrm{O}$. Kuntze.

Kuntze, O., Rev. Gen. Pl. 2 : 875. I89ז.

Arthrobotryum compositum, Pound \& Clements.

See Arthrosporium compositum, Ell.

Arthrobotryum didymum, (Cke.) Pound \& Clements.

NOTE. We have not been able to examine an authentic specimen of this species but the spures are said to be 1 -septate and for this reason it is placed by Saccardo in Didymobotryum. Since however $D$. pubescens is to be referred to Arthrobotryum we have preferred to place this species also in Arthrobotryum since, judging from the described species, the grounds on which Didymobotryum is separated from Arthrobotryum seem hardly adequate.

Pound \& Clements, Minn. Bot. Studies 9: 728. 31 May 1897. Stilbum didymum, Cke.

Cke. Grev. $7: 34$. $d$. Sept. 1878.

Didymobotryum Cookei, Sacc.

Sacc. Syll. $4: 626 . d$. 10 April 1886.

Lindau in Engler \& Prantl, Pflanzenfam. 11** $: 496$. Feb. 1900.

Arthrobotryum fusisporium, A. L. Smith.

Smith, A. L., Jour. Linn. Soc. 35: 13. d. pl. 1, f. 13-15. I April 1901.

Sacc. \& Syd. Syll. $16:$ i158. I Feb. 1902.

West Indies.

Arthrobotryum Melanoplaca, B. \& C.

NotE. This seems to be the conidia of a species of Meliola, possibly $M$. penicilliformis, Gaillard described on Psychotria from South America. See Gaillard, Le Genre Meliola 57. pl. 6, f. 2. 1892.

B. \& C. Jour. Linn. Soc. $10: 360 . d$. $1869 . \quad$ West lndies.

Sacc. Syll. 4: $629 . d$. 10 April 1886.

Wettsteiniella Melanoplaca, O. Kuntze.

Kuntze, O., Rev. Gen. Pl. 2: 875. I89I.

Arthrobotryum pubescens, (C. \& E.) Pound \& Clements.

Note. The spores of this species as figured in Grev. $6:$ pl. $q 6, f .3^{8}$ are two-celled but mature spores as shown in N. A. F. 2488 are four-celled and dark colored and the species belongs to Arthrobotryum rather than to Didy. mobotryum where it is placed by Saccardo.

Pound \& Clements, Minn. Bot. Studies $9: 728$. 31 May I 897. Graphium pubescens, C. \& E.

C. \& E. Grev. 6: 5. d. pl. 96, f. 38 . Sept. 1877 .

Ell. in Cat. Pl. N. J. 573. 1889.

*Ell. \& Ev. N. A. F. 2488.1890. Didymobotryum pubescens, Sacc.

Sacc. Syll. $4: 627 . d$. 10 April 1886.

Lindau in Engler \& Prantl, Pflanzenfam. 11** : 496. Feb. 1900.

Arthrobotryum robustum, C. \& E.

C. \& E. Grev. $7: 7$. d. Sept. 1878 .

Sacc. Syll. $4: 629$. d. 10 April 1886.

Ell. in Cat. Pl. N. J. 573.1889.

Wettsteiniella robusta, $\mathrm{O}$. Kuntze.

Kuntze, O., Rev. Gen. Pl. 2 : $875 . \quad$ i 891. 
Arthromitus, Leidy.

Leidy, Proc. Phil. Acad. 4: 227.d.9 Oct. 1849 and 5:36. 30 April 1850 .

Leidy, Smithsonian Contrib. 52:33. d. 34. April 1853 .

Arthromitus cristatus, Leldy.

Leidy, Proc. Phil. Acad. 4: 227 d. 9 Oct. 1849.

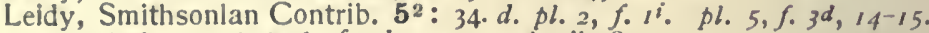
pl. $6, f .5^{e}$. pl. $8, f .1 f, 2^{d}, 3,4,5$. April 1853 .

Arthromitus nitidus, Leidy.

Leidy, Proc. Phil. Acad. 5: 35. d. 30 April 1850.

Arthromitus nitidus, Leidy.

See Arthromitus cristatus, Leidy.

Arthrosporium compositum, Ell.

*Ell. N. A. F. 415 . 1880 .

Ell. Bull. Torr. Bot. Club $8: 64 . d$. June 188 I.

Sacc. Syll. $4: 598 . d$. 10 April 1886.

Ell. in Cat. Pl. N. J. 573. 1889.

Morg. Bot. Gaz. 15 : 85. April 1890.

* Barthol., Ell. \& Ev. Fung. Columb. 1711. Feb. 1903. Arthrobotryum compositum, Pound \& Clements.

Pound \& Clements, Minn. Bot. Studies $9: 728$. 31 May 1897.

Artocreas Micheneri, B. \& C.

See Aleurodiscus Micheneri, (B. \& C.). Massee.

Artotrogus, Mont.

Note. We here refer the species of Artotrogus to Pythium, Pringsh. for the reason that by any other course we should increase bibliographical difficulties already very great. The original Artotrogus of Montagne in 13. Jour. Hort. Soc. London 1: 33. pl. 4, f. 27-29. 1846 and Gard. Chron. 1845: 640. 20 Sept. 1845 was very vaguely described and even with the mure detailed inforniation given by Deßary who examined Montagne's material one is not able to say with certainty what the type species, Artotrogzes hydnosporus, Mont. really was.

Otto Kuntze in Rev. Gen. Pl. $3^{2}: 443$. 1898 refers to Artotrogus, Fr. S. O. V. 497. 1825. Fries was not the author of the genus. The citation in Kuntze is an error and should have been Fries, Summa Veg. Scand. 497. 1849 in which place Fries refers to Montagne as the author of the genus adding a second species now known to be the conidial form of Nyctalis lycoperdoides, (Bull.) Schrt.

Pythium as defined by Pringsheim is a genus whose characters are sufficiently well recognized at the present day and the genus is adopted by nearly all modern writers. The intricacies of the synonymy of the genus Pythium as used by writers previous to Pringsheim are so great that it would be a fruitless labor to attempt to straighten them out and it seems to be the part of wisdom to retain the Pythium of Pringsheim which has been in general use since 1858 .

Artotrogus DeBaryanus, Atk. See Pythlum DeBaryanum, Hesse.

Artotrogus intermedius, Atk. See Pythium intermedium, D By.

Artotrogus, sp. indet.

See Pythium, sp. indet.

Aschersonia, Mont.

Note. For note on the validity of the genus Aschersonia see Hennings, 1904 .

Henn. Festschrift Ascherson 71, 72. 1904. 
Underwoodina, O. Kuntze.

Kuntze, O., Rev. Gen. Pl. $3^{2}$ : 538.1898.

Aschersonia Aleyrodis, Webber.

Webber, Proc. Fla. Hort. Soc. 9 : 73. 1896 ?

Webber, Bull. U. S. Agr. V. P. P. ${ }_{13}: 20,21 . d . p l .2, f .8$. pl. 3, f. 10-16. pl. 4, f. 17-23. 17 June 1897.

Lindau \& Syd. Hedw. 37: Beibl. 7 : xlix. 9 April 1898.

Sacc. \& Syd. Syll. 14: 991. $d$. 20 Aug. I 899.

Lindau in Engler \& Prantl, Pflanzenfam. 11*** 385. Jan. 1900.

Hume, Bull. Fla. Exp. Sta. $53: 164$. March 1900.

Gossard, Bull. Fla. Exp. Sta.67: 622. June 1903.

Henn. Festschrift Ascherson 69. 1904.

Aschersonia Tahitensis, Webber non Mont.

Cockerell, Jour. Inst. Jamaica 1:262. April I 893 .

Webber, Jour. Myc. $7: 364$. Nov. 1894.

Swingle \& Webber, Bull. U. S. Agr. V.P. P. 8: $27 . \quad$ pl. 7, f. 2. 1896.

Aschersonia basicystis, B. \& C.

NoTE. The original specimens which we have examined although young show that this is really an Aschersonia and can not be placed in the genus Bizzozeriella.

B. \& C. Jour. Linn. Soc. $10: 352 . d$. 1860.

Sacc. Syll. 3 : 6r9. d. I5 Dec. 1884 .

Sacc. Syll. 10: 717 . 30 June 1892 .

Henn. Festschrift Ascherson 68. 1904.

Bizzozeriella basicystis, $O$. Kuntze.

Kuntze, O., Rev. Gen. Pl. 3²: 538. 1898.

Aschersonia earpinicola, Ell. \& Dearn.

See Discella discoidea, C. \& P.

Aschersonia Cubensis, B. \& C.

B. \& C. Jour. Linn. Soc. $10: 351 . d$. 1869.

Sacc. Syll. $3: 620 . d$. I 5 Dec. 1884 .

Henn. Festschrift Ascherson 68. I904.

Aschersonia marginata, Ell. \& Ev.

Ell. \& Ev. Bull. Torr. Bot. Club 22 : 436. d. 31 Oct. I895. Hawaii.

Sacc. Hedw. 35 : Rep. 7 : xliv. 25 Feb. 1896.

Sacc. \& Syd. Syll. 14 : 989. d. 20 Aug. I899.

Henn. Festschrift Ascherson 68, 69. 1904.

West Indies.

Aschersonia oxyspora, B.

Nore. This name was originally written Aschersonia oxystoma, B. Hook. Jour. Bot. 6: 205 (1). d. 1854. [Decades 463] but for some reason unknown to us Berkeley and subsequent writers have written $A$.oxyspora.

Duss, Champ. Guad. \& Martinique 89. 1903.

West Indies.

Aschersonia paraphysata, Sacc.

Henn. Festschrift Ascherson 68. 1904.

West Indies.

Aschersonia Pittieri, Henn.

- Henn. Hedw. Beibl. 41: (IO4). d. 23 June 1902.

Henn. Festschrift Ascherson 69. 1904.

West Indies.

Aschersonia rufa, (B. \& Br.) Sacc.

*Smith, C. L., Cent. Amer. Fungi 46. 1896.

Cent. Amer.

West Indies.

Aschersonia Tahitensis, Webber non Mont.

Cent. Amer.

See Aschersonia Aleyrodis, Webber. 
Aschersonia turbinata, B.

B. Ann. Nat. Hist. II. 9: 199. d. March 1852.

Sacc. Syll. 3 : 619. $d$. I5 Dec. I884.

*Ell. \& Ev. N. A. F. 2172 . Feb. 1889.

Cockerell, Bull. Torr. Bot. Club 20: 296. I5 July 1893.

Webber, Bull. U. S. Agr. V. P. P. I3 : 31. d. pl. 4, f. 24, 25. pl. 5, f. 26. 17 June 1897 .

Duss, Champ. Guad. \& Martinique 89. 1903.

Henn. Festschrift Ascherson 68. I904.

Aschersonia viridans, (B. \& C.) Pat.

Pat. Bull. Soc. Myc. Fr. $7: 48(9), d$. I 89 I.

Henn. Festschrift Ascherson 68. I904.

Hypocrea viridans,' B. \& C.

B. \& C. Jour. Linn. Soc. $10: 376 . d$. 1860.

Sacc: Syll. $2: 532 . d$. I3 June 1883.

West Indies.

Clintoniella viridans, Sacc. \& Syd.

Sacc. \& Syd. Syll. 16 : 588. I Feb. 1902.

Aschersonia, sp. indet.

Webber, Proc. Fla. Hort. Soc. 8 : 49.1895.

Millsp. \& Nutt. Publ. Field Columb. Mus. Bot. 1 : I20. $d$. Jan. 1896. Massee, Textbk. PI. Diseases 102. 1899.

Ascobolus ærugineus, Fr.

Hark. \& Moore, Cat. Pac. Fung. 38. Feb. I88o.

Ascobolus atrofuseus, Phil. \& Plowr.

Hark. \& Moore, Cat. Pac. Fung. 38. Feb. 1880.

Lindau in Engler \& Prantl, Pflanzenfam. 11 : 193. Dec. I894.

Pound \& Clements, Bot. Surv. Nebr. 5: 22. 30 March 1901.

Durand, Bull. Torr. Bot. Club 29: 458. d. 25 July I902 and Rev. Myc. 24: (12). Oct. 1902.

Pk. Rept. N. Y. Mus. 564: 31. May 1903. [Bull. 67.]

Seaver, Bull. Univ. lowa 54: 240 (13), 283 (56). d. pl. 15, f. 1. Nov. 1904.

Seaver, Proc. lowa Acad. 12 : II4. July 1905.

Phaopeziza Nuttallii, Ell. \& Ev.

*Ell. \& Ev. N. A. F. 2908 . Oct. I893.

Ascobolus brunneus, Cke.

Cke. Grev. 5 : 153. June 1877.

Underw. \& Earle, Bull. Ala. Exp. Sta. 80: 200. 28 June 1897.

Earle in Mohr, Contr. U. S. Nat. Herb. 6 : 157. 31 July 1901.

Morg. Jour. Myc. 8 : 182. 20 Dec. 1902.

Seaver, Proc. lowa Acad. 12 : II 5. July 1905.

Ascobolus earbonarius, Karst.

Millsp. \& Nutt. Publ. Field Columb. Mus. Bot. 1 : 152. Jan. I8g6.

Ascobolus carneus, $\mathrm{P}$.

See Ascophanus carneus, (P.) Bond.

Ascobolus ciliatus, Schm.

See Lasiobolus equinus, (Muell.) Karst.

Ascobolus conglomeratus, $\mathrm{S}$.

See Angelina rufescens, (S.) Duby.

Ascobolus crenulatus, Karst.

Pk. Rept. N. Y. Mus. 30:62. Sept. 1878.

Sacc. Syll. 8 : 515. d. 20 Dec. 1889.

Ascobolus Cubensis, B. \& C.

B. \& C. Jour. Linn. Soc. 10 : 370. $d .1869$.

Sacc. Syll. 8 : 516. $d$. 20 Dec. I889.

West Indies. 
Ascobolus furfuraceus, $P$.

See Ascobolus stercorarius, (Bull.) Rehm.

Ascobolus glaber, $P$.

S. Syn. Car. 124 (98) no. 1269. 1822.

S. Syn. Am. Bor. 178 no. 958.1834.

Curtis, M. A., Bot. N. Car. 135. 1867.

Cke. Handbook 728. $d$. 1871 .

Cke. Bull. Buf. Soc. 2: 300 . March 1875 .

*Ell. \& Ev. N. A. F. 2333 . 1890.

Rehm, Rabh. Krypt.-FI. 13 : i122. d. May 1895.

Seaver, Bull. Univ. Iowa 54: 280 (53). d. pl. 16, f. 2. Nov. 1904.

Seaver, Proc. Iowa Acad. 12: I14. July igo5.

Ascobolus immersus, $\mathrm{P}$.

S. Syn. Am. Bor. 178 no. 959. 1834 .

Cke. Bull. Buf. Soc. 2: 300 . March 1875 .

Hark. \& Moore, Cat. Pac. Fung. 38. Feb. 1880.

Seaver, Bull. Univ. Iowa $5^{4}: 234$ (7), 238 (II), 28I (54). d. pl. 16, f. I. Nov. 1904.

Seaver, Proc. Iowa Acad. 12 : 114. July 1905.

Ascobolus incanus, Phil.

See Ascophanus incanus, (Phil.) Sacc.

Ascobolus Kerverni, Crouan.

See Saccobolus Kerverni, (Crouan) Boud.

Ascobolus Leveillei, Boud., var. Americanus, C. \& E.

C. \& E. Grev. 5 : 52. d. pl. $80, f .20$. Dec. 1876 .

Ell. in Cat. Pl. N. J. 554 . 1889.

Rehm, Rabh. Krypt.-Fl. $1^{3}$ : I I24. d. May 1895.

Ascobolus major, B. \& C.

Curtis, M. A., Bot. N. Car. 135. 1867.

B. Grev. 4: 6 (144) no. 772. d. Sept. 1875.

Cke. Bull. Buf. Soc. 3: 23. Sept. 1875.

Sacc. Syll. 8 : 519. d. 20 Dec. I889.

Ascobolus papillatus, Wallr.

See Lasiobolus equinus, (Muell.) Karst.

Ascobolus pilosus, Fr.

See Lasiobolus equinus, (Muell.) Karst.

Ascobolus polysporus, Karst.

See Rhyparobius polysporus, (Karst.) Sacc.

Ascobolus purpurascens, (P.) Calkins.

Note. This may possibly be Humaria purpurascens, (P.) Quél.

Calkins, Jour. Myc. 2 : ı66. Sept. 1886.

Ascobolus raripilus, Phil.

See Lasiobolus raripilus, (Phil.) Sacc.

Ascobolus stercorarius, (Bull.) Rehm.

Rehm, Rabh. Krypt.-Fl. 13: 1126. d. May 1895.

Pound \& Clements, Bot. Surv. Nebr. 5: 22. 30 March 1901.

Ascobolus furfuraceus, $\mathrm{P}$.

S. Syn. Car. $124(98)$ no. 1270. 1822.

Grev. Scot. Crypt. Fl. 6: pl. $307 . \quad 1828$.

S. Syn. Am. Bor. 178 no. $957 . \quad 1834$.

Curtis, M. A., Bot. N. Car. 135. 1867.

Cke. Handbook 727 . d. $f .338 .1871$.

Cke. Bull. Buf. Soc. 2 : 300 . March 1875 . 
B. Grev. 4: 6 (144). Sept. 1875.

Frost in Cat. PI. Amherst 9I. I875.

Farl. Bull. Bussey Inst. 1 : 438. March 1876.

Phil. Grev. 5: 36. Sept. 1876.

Cke. Grev. $7: 47$. Dec. 1878.

Pk. Rept. N. Y. Mus. $29: 56.1878$.

* Rav. Fung. Am. 312. 1879.

Hark. \& Moore, Cat. Pac. Fung. 38. Feb. 1880.

B. Jour. Linn. Soc. 17 : I6. 1880.

Rehm, Asc. Lojk. 31. d. 1882.

*Rav. Fung. Am. 632 . I882.

Pk. in Day's Cat., Bull. Buf. Soc. 4: 222 (158). 1883.

Bessey, Bull. lowa Agr. Coll. 1884: I42. Jan. 1885.

Cobb, List Pl. Amherst 47. 1887.

Langlois, Cat. PI. La. 27. 1887.

Bennett, Cat. PI. R. 1. 92. 1888.

Sacc. Syll. 8 : 516 . d. 20 Dec. 1889.

Ell. in Cat. PI. N. J. 554 . 1889.

Millsp. \& Nutt. Publ. Field Columb. Mus. Bot. 1 : 152. Jan. 1896.

Harper, Jahrb. Wiss. Bot. $29: 678$. pl. 12, f. 4t-46. 1896.

McClatchie, Proc. S. Cal. Acad. 1 : 364. 5 June 1897.

Harper, Ann. Bot. 13: 515. Dec. 1899.

Fairman, Proc. Rochester Acad. 3: 218. March 1900.

Durand, Bull. Torr. Bot. Club 27: 48I. 29 Sept. 1900.

Morg. Jour. Myc. 8 : 182. d. 20 Dec. 1902.

Seaver, Bull. Univ. lowa $5^{4}: 233(6), 282(55) . d . p l .15, f .2$. Nov. 1904.

Rostr. Meddelelser om Groenl. 30 : i 20.1904.

Seaver, Proc. lowa Acad. 12 : I14. July I905.

Ascobolus stercorarius, (Bull.) Rehm, var. retispora, Clements.

Pound \& Clements, Bot. Surv. Nebr. 5: 9. d. 30 March 1901.

Sacc. \& Syd. Syll. 16: i1 149. I Feb. 1902.

\section{Ascobolus Trifolii, Biv.}

See Pseudopeziza Trifolii, (Biv.) Fckl.

Ascobolus vicinus, Cke.

See Ascophanus vicinus, Boud.

Ascobolus vinosus, $B$.

*Ell. \& Ev. N. A. F. 2620.1891 .

Rehm, Rabh. Krypt.-Fl. $1^{3}$ : 1123. d. May 1895.

Ascobolus viridis, Curr.

Pk. Rept. N. Y. Mus. $30: 62$. Sept. 1878 .

Sacc. Syll. 8 : $519 . d .20$ Dec. 1889.

Seaver, Proc. lowa Acad. 12 : I14. July 1905.

Ascochyta, Lib.

Tassi, Bull. Lab. Bot. Siena $5:$ 40. pl. $1, f .1902$.

Ascochyta achlycola, Ell. \& Ev.

Ell. \& Ev. Proc. Phil. Acad. 1894: 364 . d. 30 Nov. 1894.

Sacc. Syll. $11: 522 . d$. July 1895 .

Ascochyta Alismatis, Ell. \& Ev.

See Ascochyta Alismatis, (Oud.) Trail.

Ascochyta Alismatis, (Oud.) Trail.

*Ell. \& Ev. N. A. F. 2380 . 1890.

Davis, J. J., Tr. Wis. Acad. 14: 94. Sept. 1903.

Ascochyta Alismatis, Ell. \& Ev.

Ell. \& Ev. Jour. Myc. 5 : 148. $d$. Sept. 1889.

Sacc. Syll. 10: 307. $d$. 30 June 1892. 
Ascochyta ampelina, Sacc.

Trel. Tr. Wis. Acad. 6: I2I (17). Nov. I884.

Ascochyta Asclepiadis, Ell. \& Ev.

Ell. \& Ev. Proc. Phil. Acad. 1894: 364. d. 30 Nov. I 894.

*Ell. \& Ev. N. A. F. 3265. 24 April r895.

Sacc. Syll. 11: 524. d. July I895.

Ascochyta Atriplicis, Lasch.

See Septoria Atriplicis, (Westd.) Fckl.

See also Phyllosticta Atriplicis, Desm.

Ascochyta baceæ, Rostr.

Rostr. Meddelelser om Groenl. 3 : 625. d. I89I.

Sacc. Syll. $11: 524 . d$. July 1895 .

Ascochyta Carthagenensis, Sacc.

Sacc. Mich. 2 : 144. d. April I880.

Sacc. Syll. 3 : $395 . d$. I 5 Dec. 1884.

Ascochyta Cassandræ, Pk.

Pk. Rept. N. Y. Mus. $38: 94$. d. 1885.

Berl. \& Vogl. in Sacc. Syll. Add. 1-4: 329. d. 31 Dec. I886.

Sacc. Syll. $10: 295 . d$. 30 June 1892.

Rostr. Meddelelser om Groenl. 18 : 69 (27). d. 1894.

Rostr. Meddelelser om Groenl. 18: $482 . \quad$ I 895.

Davis, J. J., Tr. Wis. Acad. 11: in I. Aug. 899.

Ascochyta Cephalanthi, Ell. \& Ev.

Sacc. Syll. 3 : 392. d. I5 Dec. I884.

Langlois, Cat. PI. La. $27 . \quad$ I 887.

Ascochyta clematidina, Thm.

Millsp. \& Nutt. Publ. Field Columb. Mus. Bot. 1 : i 6 . Jan. 1896.

Ascochyta colorata, Pk.

See Ascochyta Fragaria, Sacc.

Ascochyta confusa, Ell. \& Ev.

Ell. \& Ev. Jour. Myc. 10: 168. d. 24 June 1904.

Ascochyta cornicola, Sacc.

Ell. \& Ev. Jour. Myc. 5 : I48. d. Sept. I889.

Davis, J. J., Tr. Wis. Acad. 9 : 166. 21 Aug. 1893.

James, Jour. Cin. Soc. 18: 55. Dec. I 895.

Ascochyta coronaria, Ell. \& J. J. Davis.

Davis, J. J., Tr. Wis. Acad. 14: 94. d. Sept. I903.

* Barthol., Eli. \& Ev. Fung. Columb. 1807. 28 Dec. 1903.

Ascochyta Dianthi, (A. \& S.) Lib.

Rostr. Meddelelser om Groenl. 18: 69 (27). d. 1894 . Sphzria Dianthi, A. \& S.

S. Syn. Car. 47 (2I) no. 222. I 822.

S. Syn. Am. Bor. 226 no. I8i 7. I834.

Ascochyta Diapensiæ, Rostr.

Rostr. Meddelelser om Groenl. 18 : 70 (28). d. 1894.

Sacc. Syll. 11 : 524. d. July I895.

Lindau in Engler \& Prantl, Pflanzenfam. 11** : 367. Dec. I 899.

Ascochyta Ellisii, Thm.

See Guignardia Bidwellii, (Ell.) Viala \& Ravaz.

Ascochyta Fagopyri, Bres.

Note. This species reported on leaves of Fagopyrum is said to be distinct from Ascochyta Fagopyri, Thm. \& Boll. on stems which antedates it. Consequently the name given by Bresadola is changed in Sacc. \& Syd. Syll. 14: 948.' 20 Aug. I 899 to Ascochyta Bresadola, Sacc. \& Syd. but Thuemen's 
species is referred to Diplodina Fagropyri, (Thm. \& Boll.) Allescher in Rabh. Krypt.-Fl. $1^{6}: 643,686.1900$.

Orton, Rept. Vt. Exp. Sta. 12: 171. Dec. 1899 and Contr. Bot. Vt. 7: 171. Dec. 1899.

Ascochyta Fragariæ, Sacc.

Scribner, Rept. U. S. Agr. 1887 : 338. 1888.

Dudley, Bull. Cornell Exp. Sta. I4 $182,183 . f_{0}$ 10. Dec. 1889.

Halsted, Rept. N. J. Exp. Sta. 1893 : 329. I 894.

Tubeuf-Smith, Diseases of Plants 473. I 1897.

Sturgis, Rept. Conn. Exp. Sta. 24: 288.1901.

Clint. Rept. Conn. Exp. Sta. 27 : 360. May I904.

Ascochyta colorata, Pk.

Pk. Rept. N. Y. Mus. 38: 94. d.pl. 2, f. q, 10. 1885.

Berl. \& Vogl. in Sacc. Syll. Add. 1-4 : 331. d. 31 Dec. 1886.

Kelsey, Jour. Myc. 5 : 8I. June I 889.

Comes, Critt. Agr. 1 : $475 . \quad 1891$.

Sacc. Syll. $10: 302$. d. 30 June I 892.

Allescher in Rabh. Krypt.-Fl. 16:644. d. 1900.

Ascochyta Fraseræ, Ell. \& Ev.

See Diplodina Fraseræ, (Eli. \& Ev.) Tracy \& Earle.

Ascochyta Fremontiæ, Hark.

Hark. Bull. Cal. Acad. 2: 439 . d. I6 June i 887.

Sacc. Syll. $10: 297 . d$. 30 June 1802 .

Ascochyta graminicola, Sacc.

McClatchie in Reid's Hist. Pasadena 616. I895.

McClatchie, Proc. S. Cal. Acad. 1 : 374. 5 June 1897.

Ascochyta Hanseni, Ell \& Ev.

Ell. \& Ev. Bull. Torr. Bot. Club 24 : 464. d. 30 Oct. 1897.

*Ell. \& Ev. N. A. F. 3553. 24 Jan. I898.

Lindau \& Syd. Hedw. 37 : Beibl. 7 : xlvi. 9 April 1898.

* Ell. \& Ev. Fung. Columb. 1255. May 1898 .

Sacc. \& Syd. Syll. 14: 946. d. 20 Aug. 1899.

Ascochyta (?) infuscans, Ell. \& Ev.

Ell. \& Ev. Jour. Myc. 5 : 148 . d. Sept. 1889.

Sacc. Syll. 10: 301. $d$. 30 June 1892 .

Ascochyta Ledi, Rostr.

Rostr. Meddelelser om Groenl. 3: 570. d. 1888 and Bot. Centralbl. $36: 7 . d$. 1888 .

Rostr. Meddelelser om Groenl. $3: 625 . \quad$ I891.

Sacc. Syll. $10: 295$. d. 30 June 1892 .

Ascochyta Leonuri, Ell. \& Dearness.

Ell. \& Dearness, Proc. Can. Inst. 1 : $92 . d$. Sept. 1897.

Sacc. \& Syd. Syll. 14: 946. d. 20 Aug. I 899.

Ascochyta lethalis, Ell. \& Barthol.

*Barthol., Ell. \& Ev. Fung. Columb. 1808. d. 28 Dec. 1903.

Ascochyta Lophanti, J. J. Davis.

Davis, J. J., Tr. Wis. Acad. 14 : 95. d. Sept. 1903.

Ascochyta Ly copersici, Brun.

Stevens, F. L., Rept. Ohio Acad. 5 : 63. 1897.

Smith, C. O., Jour. Myc. $10: 98,99 . d$. f. 1-6. 31 May 1904.

Ascochyta Mali, Ell. \& Ev.

Ell. \& Ev. Bull. Torr. Bot. Club $27: 56 . d$. I7 Feb. 1900.

Sacc. \& Syd. Syll. 16 : 930. d. I Feb. I902. 
Ascochyta Menziesii, Ell. \& Ev.

McClatchie in Reid's Hist. Pasadena 616. 1895.

McClatchie, Proc. S. Cal. Acad. 1: 374. 5 June 1897.

Ascochyta Oxybaphi, Trel.

Trel. Tr. Wis. Acad. 6 : 121 (17). d. Nov. I884 and Jour. Myc. 1 : I4. $d$. Jan. 1885 .

Berl. \& Vogl. in Sacc. Syll. Add. 1-4 : 332. d. 31 Dec. 1886.

Sacc. Syll. $10: 306 . d$. 30 June 1892.

Ascochyta 0xytropidis, Schrt.

Schrt: Ber. Schl. Ges. 1887: 283. d. 1887 and Bot. Centralbl. 35 : 201. 1888 .

Sacc. Syll. $10:$ 304. d. 30 June 1892.

Ascochyta parasitica, Fautr.

Clint. Rept. Conn. Exp. Sta. 27 : 326. May I904.

Ascochyta Paulowniæ, Sacc. \& Brun.

Patterson, Bull. U. S. Agr. PI. Industry 8: 23. 3 Feb. 1902.

Ascochyta Petuniæ, Speg.

Halsted, Gard. \& Forest 5 : 141. d. 23 March 1892.

Ascochyta Pisi, Lib.

Hark. Bull. Cal. Acad. 1 : 160. 7 March 1885.

Hark. Bull. Cal. Acad. 1 : 261 (6). 19 Nov. 1885.

Gall. Bull. U. S. Agr. Veg. Pathol. i I : 88.1890.

Davis, J. J., Tr. Wis. Acad. 9 : 166.21 Aug. 1893.

Halsted, Rept. N. J. Exp. Sta. 1893 : 358, 36I. f. $3^{I}$. 1894.

Tracy \& Earle, Bull. Miss. Exp. Sta. 34 : Io9. d. May 1895.

Halsted, Rept. N. J. Exp. Sta. 1894: 351. 1895.

Halsted, Bull. Penn. Dept. Agr. 28 : 17. 1897.

Halsted, Rept. N. J. Exp. Sta. $1897: 323,360.1898$.

Powell, Bull. Del. Exp. Sta. 41 : 8. I898.

Selby, Bull. Ohio Exp. Sta. 121: 42. Sept. 1900.

Allescher in Rabh. Krypt.-Fl. ${ }^{6}:$ : 658. d. 1900.

Sturgis, Rept. Conn. Exp. Sta. 23 : 280. 1900.

Sturgis, Rept. Conn. Exp. Sta. 24: 278. I90I.

Clint. Rept. Conn. Exp. Sta. 27 : 337. May 1904.

Ascochyta Primulø, Trail.

Halsted, Rept. N. J. Exp. Sta. 1894: 380. f. 6I. 1895.

Ascochyta Quercus, Sacc. \& Speg.

Trel. Tr. Wis. Acad. 6: I2I (17). Nov. I884.

Ascochyta Quercuum, (Cke.) Sacc.

Sacc. Syll. 3 : 393. $d$. 15 Dec. 1884.

Allescher in Rabh Krypt.-Fl. 169:66I. 1900.

Spharellopsis Quercuum, Cke.

Cke. Grev. $12: 23$. d. Sept. 1883

?Diplodina Quercuum, Tracy \& Earle.

Tracy \& Earle. Bull. Torr. Bot. Club 26 : 494. d. 22 Sept. I8y9.

Sacc. \& Syd. Syll. $16: 940 . d$. I Feb. 1902.

Ascochyta Rhei, Ell. \& Ev.

Note. The name Phyllostictu Rhei has been twice used by Ellis and Everhart, first in Jour. Myc. 5: 145. 1889 and again in Proc. Phil. Acad. 189 I : 77. March 1891 . The former was made the basis of Ascochyta Rhei, Ell. \& Ev. 1893. An attempt has been made to connect the name l'hyllostict Halstediana with one of the descriptions and this has resulted in confusion. In Ell. \& Ev. Fung. Columb. 1254. May 1898 both descriptions are referred to Ascochytu Rhei. A. B. S.

Ell. \& Ev, Proc, Phil. Acad. 1893 : ı6o. d. 28 Feb. 1893. 
Sacc. Syll. $11: 525 . d$. July i 805.

- Smyth, Tr. Kans. Acad. 15 : 71 . Marcl 1898.

*Ell. \& Ev. Fung. Columb. 1254. May 1898.

Orton, Rept. Vt. Exp. Sta. 12 : 172. Dec. 1899. Contr. Bot. Vt. 7 : 172. Dec. 1899.

Sacc. \& Syd. Syll. 16 : 935. I Feb. 1902.

Davis, J. J., Tr. Wis. Acad. 14: 95. Sept. 1903.

Allescher in Rabh. Krypt.-FI. 17: 879. 1903.

Clint. Rept. Conn. Exp. Sta. 27 : 355. May 1904.

Phyllosticta Rhel, Ell. \& Ev.

Ell. \& Ev. Jour. Myc. 5: 145. d. Sept. 1880.

Ell. \& Ev. Proc. Phil. Acad. $1891: 77 . d$. March 1891.

Sacc. Syll. 10: 134. d. 30 June 1892.

Sacc. Syll. 10 : 135. $d$. 30 June 1892.

* Seymour \& Earle, E. F. 494. 15 Oct. 1897.

Allescher, Rabh. Krypt.-FI. $1^{6}: 144.1898$.

Allescher, Rabh. Krypt.-FI. $1^{6}: 348.1899$.

Ell. \& Ev. N. Am. Phyllostictas 70. Dec. 1900.

Phyllosticta Halstediana, Allescher.

Allescher, Rabh. Krypt.-Fl. $1^{6}$ : 144.1898.

Ascochyta Rhynchosiæ, (Thm.) Sacc.

Sacc. Syll. $3: 398$. $d$. I5 Dec. 1884.

Depazea Rhynchosia, Thm.

*Thm. Myc. Univ. 596. d. 1876.

Thm. Flora $60: 207$. d. May 1877.

Thm. Flora 61: 182 (6). $d$. 21 April 1878.

Ascochyta Rubi, Lasch.

Farl. Appalachia 3: 249. Jan. 1884.

Ascochyta salicifoliæ, Trel.

See Septoria salicifollax, (Trel.) Ell. \& Ev.

Ascochyta Silenes, EII. \& Ev.

Ell. \& Ev. Jour. Myc. 5 : 148. d. Sept. 1889.

Sacc. Syll. 10: 301. d. 30 June 1892.

Davis, J. J., Tr. Wis. Acad. 9: 166. 21 Aug. 1893.

Ascochy ta Sisymbril, Ell. \& Kellerm.

Ell. \& Kellerm. Jour. Myc. 5 : 142. d. Sept. 1889.

Sacc. Syll. $10: 302$. $d$. 30 June 1892 .

Tassi, Bull. Lab. Bot. Siena 5: 40. 1902.

Ascochyta Smilacis, Ell. \& Martin.

See Stagonospora Smilacis, (Ell. \& Martin) Sacc.

Ascochyta Solidaginum, (S.) Starb.

Note. We have given below the synonymy of this species founded on Sphreria Solidaginum, S. in accordance with the examination of Schweinitzian and other material inade by Starbäck, 1894. But we should say that our own examination of Spharia Solidaginum, S. and Dothidea Solidaginis, S. from Herb. Schweinitz does not agree in several respects with the observations of Starbäck.

The fungus instead of forming spots in the leaves is, in many cases at least, a parasite on the common Coleosporium of Solidago recently designated as Stichospora Solidaginis, (S.) Diet.

Amongst the synonyms enumerated by Starbäck is Septoria Solidaginis, Thm. An examination of the authentic specimen of Thuemen in Myc. Univ. 1399. 1879 shows however a Darluca parasitic on Coleosporium. Whether it can be referred to Darluca Filum, (Biv.) Cast. is not beyond doubt but it certainly is closely related to that species and it must be admitted that Starbäck's figure suggests possibly a Darluca. The specimen of Septoria 
Solidaginis, Cke. in Rav. Fung. Am. 784. 1882, shows no fungus which can be recognized. Specimens in other exsiccati resemble that in Mycotheca Universalis. In many cases there accompanies the pycnidial form a sclerotium form whose relation to the pycnidial form needs to be further studied.

It seems to us therefore that the true character of Spharia Solidaginum, $\mathrm{S}$. is not yet clearly settled but we are inclined to believe that it is a Darluca accompanying the common Coleosporium. If that is not the case it is doubtful whether it will ever be possible to determine from Schweinitzian specimens what Sphceria Solidaginum, S. really was.

Starb. Bot. Notiser 1893: 31. I5 Feb. I893.

Starb. Bihang till K. Sv. Vet. Akad. Handl. $19^{3}$ : no. 2 : 84. d. pl. $3, f$. $62 a-d$. 1804 .

Spharia Solidaginum, S.

S. Syn. Car. 47 (21) no. 225. d. I 822.

S. Syn. Am. Bor. 225 no. I802. 1834.

Curtis, M. A., Bot. N. Car. I50. I 867.

B. Grev. 4: I 54 (192). June I 876.

Sacc. Syll. $2: 436$. $d$. I 3 June I 883.

Starb. Bot. Notiser 1893: 3 I. 15 Feb. I 893.

Dothidea Solidaginis, Fr. p. p.

Fr. El. 2 : 125. 1828.

Dothidea Solidaginis, S. Am. Bor.

S. Syn. Am. Bor. 232 no. I908. I 834.

Watt, Can. Nat. \& Geol. II. 2 : 39I. Oct. I865.

* Rav. Fung. Am. 97. I 878.

Cke. Grev. 6 : I 45 . June I 878.

Cke. Grev. 13: 62. March I 885.

Ell. \& Ev. Proc. Phil. Acad. 1894: 345. d. 30 Nov. I 894.

Kellerm. \& Werner, Geol. Ohio $7^{2}$ : 360. Jan. I 895.

Spharia Soildaginis, S.

Fr. El. 2: 125. I 828.

S. Syn. Am. Bor. 315. I 834.

* Rav. Fung. Car. 2 : 60. I 853.

Pk. Rept. N. Y. Mus. 24: 99 . I 872.

C. \& E. Grev. 6 : 16. Sept. 1877.

Ell. \& Ev. Pyren. 399. May 1892. Septoria Solidaginis, Thm.

Thm. Flora 61 : I80 (4). d. 2 I April I 878.

Sacc. Syll. 3 : 546. d. 15 Dec. I 884.

Martin, Jour. Myc. 3 : 75. d. July I 887.

Roum. Rev. Myc. 11: I 33. I July I 889. Septoria Solidaginum, Thm.

*Thm. Mvc. Univ. 1399. I 879. ? Septoria Solidaginis, Cke.

*Rav. Fung. Am. 784. I 882.

Cke. Grev. 12: 25. d. Sept. 1883.

Halsted. Bull. lowa Agr. Coll. 1888: I09. April I888. Phyllachora Solidaginum, Sacc.

Sacc. Syll. 2 : 614. I 3 June I 883.

Ell. in Cat. Pl. N. J. 539. I 889.

Ascochyta Spartinæe, Trel.

Trel. Tr. Wis. Acad. 6 : I2I (I7).d. Nov. I884 and Jour. Myc. 1 : I4. d. Jan. I885.

Berl. \& Vogl. in Sacc. Syll. Add. 1-4: 332. d. 3 I Dec. I 886.

Sacc. Syll. 10: 307. d. 30 June I892.

Ascochyta teretiuseula, Sacc. \& Roum.

Ell. \& Ev. Jour. Myc. 4: 49. d. June I 888 . 
Ascochyta Thaspii, Elt. \& Ev.

Ell. \& Ev. Jour. Myc. 5 : 148. d. Sept. 1889.

*Ell. \& Ev. N. A. F. 2381. I890.

Sacc. Syll. 10 : 305. d. 30 June 1892.

Ascochyta Treleasei, Berl. \& Vogl.

Berl. \& Vogl. in Sacc. Syll. Add. 1-4: 332. d. 31 Dec. 1886.

Sacc. Syll. 10 : 305. d. 30 June 1892.

Davis, J. J., Tr. Wis. Acad. 9 : 159. 21 Aug. 1893. Ascochyta, sp.

Trel. Tr. Wis. Acad. $6: 121$ (17), d. Nov. 1884.

Ascochyta veratrina, Ell. \& Ev.

Ell. \& Ev. Proc. Phil. Acad. 1894: 364. d. 30 Nov. 1894.

Sacc. Syll. $11: 525 . d$. July 1895 .

Allescher in Rabh. Krypt.-Fl. 16:667. 1900.

Ascochyta Viciæ, Lib.

*Barthol., Ell. \& Ev. Fung. Columb. 1506. Dec. 1901.

Ascochyta Vleiæ, Trail.

See Ascochyta vicicola, Sace.

Ascochyta vicicola, Sacc.

Ascochyta Vicia, Trail.

*Ell. \& Ev. N. A. F. 3552. 24 Jan. 1898.

Ascochyta Violæ, Sacc. \& Speg.

Trel. Tr. Wis. Acad. 6 : 121 (17). Nov. 1884:

Halsted, Rept. N. J. Exp. Sta. 1891: 300. 1892.

Halsted, Rept. N. J. Exp. Sta. 1897 : 384. 1898.

Ascochyta zeicola, Ell. \& Ev.

Ell. \& Ev. Proc. Phil. Acad. 1895 : 433. d. Nov. 1895.

Sacc. Hedw. 35 : Rep. 7 : xliii. 25 Feb. 1896.

Sacc. \& Syd. Syll. 14: 947. d. 20 Aug. 1899.

Ascochyta, sp. indet.

Trel. Tr. Wis. Acad. 6 : I21 (17). Nov, I884.

Halsted, Gard. \& Forest 5: 353.27 July 1892.

Ell. \& Ev. Proc. Phil. Acad. 1893: 455. I893.

Clint. Rept. Conn. Exp. Sta. 27 : 310, 316, 322. May 1904.

Ascocorticium, Bref.

Earle, Bull. N. Y. Bot. Gard. 2 : 331. 25 April 1902.

Ascocorticium anomalum, (Ell. \& Hark.) Schrt.

Schrt. in Engler \& Prantl, Pflanzenfam. 11 : 161. d. f. 137. Oct. 1894.

Earle, Bull. N. Y. Bot. Gard. 2 : 331. 25 April 1902.

Ascomyces anomalus, Ell. \& Hark.

Ell. \& Hark. Bull. Torr. Club 8: 26. March 1881.

*Ell. N. A. F. 56t. 188I.

Exarscus anomalus, Sacc.

Sacc. Syll. $8: 820$. $d$. 20 Dec. 1 889.

Ascomyces aureus, Magn.

See Taphrina aurea, (P.) Fr.

Ascomyces aureus, Auct. Amer. p. p.

See Taphrina Johansonit, Sadebeck.

Ascomyces eærulescens, Mont. \& Desm.

See Taphrina carulescens, (Mont. \& Desm.) Tul.

Ascomyces deformans, B.

See Exaascus deformans, (B.) Fckl.

Ascomyees deformans, Hark. non B.

See Exaascus Aesculi, (Ell. \& Ev.) Patterson. 
Ascomyees deformans, var. Aeseuli, Ell. \& Ev.

See Exoascus Aesculi, (Ell. \& Ev.) Patterson.

Ascomyces deformans, var. purpurascens, Ell. \& Ev.

See Taphrina purpurascens, (Ell. \& Ev.) Robinson.

Ascomyces extensus, $\mathrm{Pk}$.

See Taphrina extensa, (Pk.) Sacc.

Aseomyees Pulgens, Cke. \& Hark.

Note. This is said by Harkness, I 885 , to be an aphidian gall and not a fungus.

Cke. \& Hark. Grev. 9 : 8. d. Sept. I880.

Cke. \& Hark. Bull. Cal. Acad. 1 : i8. Feb. 1884.

Hark. Bull. Cal. Acad. 1 : 265 (I0). Ig Nov. I885. Exoascus fulgens, Sacc.

Sacc. Syll. 8 : 820 . d. 20 Dec. 1889.

Patterson, Bull. Univ. Iowa $3^{3}$ : I I9. I4 May I 895.

Ascomyces letifer, $\mathrm{Pk}$.

See Taphrina letifer, (Pk.) Sacc.

Ascomyces Pruni, Hark.

See Exoascus communis, Sadebeck.

Ascomyces Quercus, Cke.

See Taphrina carulescens, (Mont. \& Desm.) Tul.

Ascomyees rubrobrunneus, Pk.

See Taphrina rubrobrunnea, (Pk.) Sacc.

Ascomyces Tosquinetii, Auct. Amer.

See Taphrina Alni-incanz, (Kuehn) Magn.

Ascomycetella, Pk.

Note. The genus Ascomycetella, Pk. Bull. Torr. Bot. Club 8; 50. May I 881 is, as far as the type species is concerned, the same as Cookella, Sacc. Mich. 1: 407 . 1878. The genus Ascomycetella is however retained by most mycologists and the author of the genus as given by Saccardo is Ellis. This is not strictly the case. The genus Ascomycetella in its restricted sense is based on A. Floridana, Ell. but this species was considered by Ellis to be congeneric with Ascomycetella, Pk. The distinction between Cookella and Ascomycetella was really first given by Saccardo in Syll. 8: 843, 846. 20 Dec. 1889 who separates them on the ground that in Cookella the spores are colored and in Ascomycetella they are subhyaline, a distinction upon which too much weight should not be placed. With regard to the limits of the genus see notes by Starbäck, Bih K. Sv. Vet. Akad. Handl. $25^{3}$ : no. I : 40, 4 I. 1899, Hennings, Engler's Bot. Jahrb. 28:276. 22 May 1900 and Hedw. 40: 354. 30 Dec. 1901 .

Ascomyeetella, Sacc.

Ascosorus, Henn. \& Ruhland.

Henn. Engler's Bot. Jahrb. 28:276. 22 May 'I900.'

Ascomycetella aurantiaca, Ell. \& Martin.

See Leptophyma aurantiacum, (Ell. \& Martin) Sacc.

Ascom yeetella filicina, Ell. \& Ev.

Ell. \& Ev. Jour. Inst. Jamaica 1: 247. d. April I893.

Ascomyeetella Floridana, Ell. \& Martin.

Ell. \& Martin, Am. Nat. 18: I 148. d. Nov. I884.

*Ell. \& Ev. N. A. F. 2069. I 888.

Sacc. Syll. 8 : 847. d. 20 Dec. I889.

Starb. Bih. K. Sv. Vet. Akad. Handl. $25^{3}$ : no. I : 41. 1899.

Henn. Engler's Bot. Jahrb. $28: 276.22$ May I900.

Henn. Hedw. $40: 354$. f. $2^{a-c}$. 30 Dec. Igol.' 
* Rehm, Ascoinyceten 1499. 1903. Ascosorus Floridanus, Henn. \& Ruhland.

Henn. Engler's Bot. Jahrb. 28 : 276. d. 22 May 1900.

Ascomycetella quercina, Pk.

See Cookella quercina, (Pk.) Sacc.

Ascomycetella sulphurea, Wint.

Wint. Hedw. 24: 23. d. 1885 .

Sacc. Syll. 8 : 846 . d. 20 Dec. 1889.

Mexico.

Lindau in Engler \& Prantl, Pflanzenfam. ${ }^{1}{ }^{1}:$ 243. Dec. 1894

Starb. Bih. K. Sv. Vet. Akad. Handl. $25^{3}$ : no. $1: 41 . d$. I899.*

Ascomycetella, sp. indet. Conidia.

* Seymour \& Earle, E. F. 182, 186, 190. I Sept. 1892.

Ascophanus Aurora, ( $\mathrm{Cr}$.) Boud.

Pound, Clements and others, Bot. Surv. Nebr. $4: 42.20 \mathrm{Jan} .1896$.

Ascophanus carneus, (P.) Boud.

Sacc. Syll. 8 : 534. d. 20 Dec. 1880.

Lindau in Engler \& Prantl, Pflanzenfam. 1' $:$ 190. d. Dec. I894.

Millsp. \& Nutt. Publ. Field Columb. Mus. Bot. 1: 152. Jan. 1896.

Arth. Proc. Ind. Acad. $1896: 216$ (3). 1897.

Seaver, Proc. lowa Acad. 12 : 113. July 1905. Ascoboius carneus, $\mathrm{P}$.

Phil. Grev. 5: 117. March 1877.

Clie. Grev. 6 : 142. June 1878.

Hark. \& Moore, Cat. Pac. Fung. 38. Feb. 1880.

*Ell. N. A. F. 853. 1882 .

Calkins, Jour. Myc. 2: 105. Sept. 1886.

*Ell. \& Ev. N. A. F. 2620 . Feb. I891.

Ascobolus carneus, Rav. Fung. Am.

See Ascoboius testaceus, (Moug.) Phil.

Ascophanus earneus, var. difformis, Pound \& Clements. See Ascophanus difformis, (Nyl.) Boud.

Ascophanus Cesatii, (Carest.) Sacc.

Rostr. Meddelelser om Groenl. 18: 59 (17). 1894.

Ascophanus einerellus, (Karst.) Speg.

Rostr. Meddelelser om Groenl. 18 : 59 (17). 1894.

Ascophanus cinereus, ( $\mathrm{Cr}$.) Boud.

Seaver, Bull. Univ. lowa $5^{4}: 276$ (49). d. pl. 14, f. 1. Nov. 1904.

Seaver, Proc. lowa Acad. 12 : 113 . July 1905.

Ascophanus difformis, (Nyl.) Boud.

A scophanus carneus, var. difformis, Pound \& Clements.

Pound \& Clements, Bot. Surv. Nebr. 5: 22. 30 March I90I.

Ascophanus granuliformis, (Cr.) Boud.

?Millsp. \& Nutt. Publ. Field Columb. Mus. Bot. 1 : 152. Jan. 1806.

Seaver, Proc. lowa Acad. 12: 113. July 1903.

Ascophanus humosoides, Pk.

Pk. Bull. N. Y. Mus. 12: 22. d. Jan. 1888.

Sacc. Syll. $8:$ 533. $d .20$ Dec. 1889.

Lindau in Engler \& Prantl, Pflanzenfam. 11: 190. Dec. 1894. Peziza humosoides, Pk.

Pk. Rept. N. Y. Mus. 32: 46. d. 1886.

Ascophanus incanus, (Phil.) Sacc.

Sacc. Syll. 8 : 529. $d$. 20 Dec. 1889. Ascobolus incanus, Phil.

Phil. Grev. 5: 117. d. pl. 8o, f. 10. March 1877.

Hark. \& Moore, Cat. Pac. Fung. 38, Fęb, 1880. 
Ascophanus isabellinus, Clements.

Pound \& Clements, Bot. Surv. Nebr. 5: 9. d. 30 March I9oI.

Sacc. \& Syd. Syll. 16: i149. I Feb. I902.

Ascophanus lilacinus, Cke.

Cke. Bull. Buf. Soc. 3 : 23. Sept: 1875 .

Cke. Grev. 21 : 74. $d$. March 1893.

Sacc. Syll. 11: 42I. d. July I895.

Ascophanus microsporus, (B. \& Br.) Phil.

Rostr. Meddelelser om Groenl. 3: 610. $d$. I891.

Seaver, Bull. Univ. lowa 5 $: 275$ (48). d. pl. 13, f. 2. Nov. 1904.

Seaver, Proc. lowa Acad. 12: 113 . July 1905.

Ascophanus papillatus, Boud.

See Laslobolus equlnus, (Muell.) Karst.

Ascophanus testaceus, (Moug.) Phil.

Phil. British Discomycetes 310. $d$. 1887.

Seaver, Bull. Univ. lowa $5^{4}: 239$ (12). 276 (49). d. pl. 13, f. I. Nov. I904.

Seaver, Proc. lowa Acad. 12 : II3. July 1905.

Ascobolus carneus, Rav. Fung. Am.

*Rav. Fung. Am. 176 . I878.

Ascophanus tetraonalis, $\mathrm{Pk}$.

Pk. Bull. N. Y. Mus. 12: 22. d. Jan. 1888.

Sacc. Syll. 8: 533. d. 20 Dec. 1889.

Pezlza tetraonalis, Pk.

Pk. Rept. N. Y. Mus. 32: 46. d. 1886.

Ascophanus vicinus, Boud.

Sacc. Syll. 8: 530.'d. 2o Dec. 1889.

Ascobolus viclnus, Cke.

C. \& E. Grev. $6: 8$. Sept. 1877 .

Ascophora chartarum, B. \&.C., nom. nud.

Note. As far as we know this name occurs only in M. A Curtis, Bot. N. Car. as cited below. The specimen in Herb. Curtis is a small Rhlzopus with spores $7 \mu$ in diameter. The material is not sufficient to determine the exact species.

Curtis, M. A., Bot. N. Car. 153. 1867.

Ascophora disciflora, Tode.

See Aregma disciflora, Arth.

Ascophora elegans, Cda.

See Thamnldlum elegans, Lk.

Ascophora fusca, B. \& C.

Note. The specimen of this species in Herb. Curtis shows that it is Rhlz. opus nlgricans, Ehrb.

Ascophora Mucedo, Tode. See Rhizopus nlgrlcans, Ehrb.

Ascophora nueuum, Cda.

Nore The American specimens of this species belong to Rhlzopus nlgrlcans, Ehrb. under which A. Fischer includes also the European plant of this name.

Ascophora perennis, $P$.

NotE. We have not been able to examine a Schweinitzian specimen of this species.

S. Syn. Car. 125 (99) no. I285. I822. 
Ascosorus, Henn. \& Ruhland.

See Ascomycetelia, Sacc.

Ascosorus Floridanus, Henn. \& Ruhland. See Ascomycetella Fioridana, Ell.

Ascospora. Fr.

NotE. The genus Ascospora has been understood in so many different senses by different authors that it is next to impossible to say to what genera some of the species probably belong.

The genus was originally founded by Fries on Spharia Aegopodii, P. but this has since been shown to agree more nearly in structure with the species placed by Fries in the genus Stigmatea, Fr. Summa 421 than with the species placell under Ascospora, Fr. S. O. V. 112. 1825, Summa 425. It seems to us better to retain the genus Stigmatea as used by modern writers than to complicate the subject still further by reviving the genus Ascospora.

Ascospora aterrima, Lev.

See Piacospharia aterrima, (Lev.) Sacc.

Ascospora Geranil, O. Kuntze. See Stigmatea Geranii, Fr.

Ascospora gregaria, O. Kuntze. See Stigmatea gregaria, Cke.

Ascospora Podophylli, M. A. Curtis. See Phyliosticta Podophyili, (M. A. Curtis) Wint.

Ascospora Polygoni, O. Kuntze. See Stigmatea Poiygoni, (Fr.) n. n.

Ascospora Ranunculi, O. Kuntze. See Stigmatea Ranuncuil, Fr.

Ascospora Robertiani, O. Kuntze. See Stigmatea Robertiani, Fr.

Ascospora selerotidea, O. Kuntze. See Stigmatea scierotidea, Cke.

Ascospora seminata, O. Kuntze. See Stigmatea seminata, (B. \& Rav.) Sacc.

Ascospora Sequoiæ, O. Kuntze.

See Stigmatea Sequoire, (Cke. \& Hark.) Sacc.

Ascospora submaculans, O. Kuntze. See Stigmatea submacuians, Mont.

\section{Aspergillus.}

Note. There are numerous references to species of Aspergillus scattered through medical and technical works which can not be included here where we have given those of interest to the systematic mycologist.

Aspergillus alutaceus, B. \& C.

Curtis, M. A., Bot. N. Car. 128. 1867.

B. Grev. 3 : 108 (118) $110.655 . d$. March 1875.

Sacc. Syll. 4: 69. d. 10 April 1886.

Wehmer, Mem. Soc. Phys. et Hist. Nat. Genève $33^{2}$ : no. 4: 124. 1901.

Aspergillus aurantiacus, B.

See Nematogonium aurantiacum, Desm.

Aspergillus aviarius, $\mathrm{Pk}$.

See Aspergillus fumigatus, Fres. 
Aspergillus eandidus, Lk.

Note. The synonymy of Aspergillus candidus, Lk. is very much confused. For notes on this subject see Wehmer, Mem. Soc. Phys. et Hist. Nat. Genève $33^{2}:$ no. 4: 95,98 . 1901. It is of course impossible to say whether the references below belong to the Aspergillus candidus, Sacc. or not, still less whether they belong to the Monilia candida, P. on which Aspergillus candidus, Lk. is based.

S. Syn. Am. Bor. 282 no. 2673.1834.

Mont. Pl. Cell. Cuba 305. $d$. I84I.

Andrews, Proc. Boston Soc. $5: 323$. April 1856.

B. \& C. Jour. Linn. Soc. 10: 362. 1869.

Hark. \& Moore, Cat. Pac. Fung. 28. Feb. I880.

Bundy, Geol. Wis. I873-79, 1: 400 . 1883.

Sacc. Syll. 4: 66. $d$. Io April 1886.

Rostr. Meddelelser om Groenl. 3 : $577 . \quad$ I 888.

Webber, Rept. Nebr. Agr. 1889 : I9I ( 51 I). 1890.

Rostr. Meddelelser om Groenl. $3: 628$. I801.

Lindau, Rabh. Krypt.-Fl. 18: I49. d. I 5 July 1904.

Aspergillus eimmerius, B. \& C.

Note. Examination of original specimens shows that this is not an Asper. gillus but a Periconia undistinguishable from the $P$. chlorocephala, Fres. in Rabh. Fung. Eur. 166r. 1873 .

Frost, Proc. Boston Soc. 12 : 80.1869.

B. Grev. 3: 108 (118) no. 656. d. March 1875.

Sacc: Syll. 4 : 7 I. $d$. IO April 1886.

Wehmer, Mem. Soc. Phys. et Hist. Nat. Genève $33^{2}:$ no. $4:$ Iog. $d$. 1901.

Aspergillus clavatus, Desm.

Sacc. Mich. 2 : 577. Dec. I 882.

Sacc. Syll. 4: 67 . d. Io April I886.

Ell. in Cat. Pl. N. J. 558.1889.

Webber, Rept. Nebr. Agr. 1889: 192 (52). 1890.

Massee \& Salmon, Ann. Bot. 16: 82. d. pl. $5, f .104$. March 1902.

Lindau, Rabh. Krypt.-Fl. $1^{8}: 130 . d$. 15 July 1904.

Rostr. Meddelelser om Groenl. 30: I2I. I904.

Aspergillus clavellus, $\mathrm{Pk}$.

Pk. Rept. N. Y. Mus. 34: 49. d.pl. 2, f. 1-5. Oct. 1883.

Sacc. Syll. $4: 67 . d$. 10 April 1886.

Wehmer, Mem. Soc. Phys, et Hist. Nat. Genève $33^{2}$ : no. $4: 9$ I. $d$. I90I.

Aspergillus clavellus, $\mathrm{Pk}$.

See Aspergillus clavatus, Desm.

\section{Aspergillus Cookei, Sacc.}

Note. This species, based on Aspergillus mucoroideus, Cke. non Cda. is Australian and is credited erroneously to North America.

Wehmer, Mem. Soc. Phys, et Hist. Nat. Genève $33^{2}:$ no. $4:$ Iog. $d$. IgOI.

Aspergillus eroeatus, B. \& C.

See Chondromyces crocatus, B. \& C.

Aspergillus cucurbitaceus, M. A. Curtis in Herb.

Choanephora Cucurbitarum, (B. \& Rav.) Thax.

Aspergillus Curtisii; B.

See Rhinotrichum Curtisii, B. 
Aspergillus dublus, Cda.

Hark. \& Moore, Cat. Pac. Fung. 29. Feb. 1880.

Aspergillus erythrocephalus, B. \& C.

B. \& C. Jour. Linn. Soc. $10: 362 . d$. 1869.

Sacc. Syll. 4: 68. d. Io April i 886.

West Indies.

Wehmer, Mem. Soc. Phys. et Hist. Nat. Genève 33": 126. d. 1901.

Aspergillus fimetarius, $\mathrm{Pk}$.

Pk. Rept. N. Y. Mus. 42: 128 (32). d. Dec. 1889.

Sacc. Syll. 10: 524. d. 30 June 1892 .

Wehmer, Mem. Soc. Phys. et Hist. Nat. Genève $33^{2}:$ no. $4:$ 102. $d$. I901.

Aspergillus flavus, Lk.

Pk. Rept. N. Y. Mus. $31: 45 . \quad 1879$.

Sacc. Syll. $4: 69 . d$. 10 April 1886.

Webber, Rept. Nebr. Agr. 1889 : 192 (52). 1890.

*Ell. \& Ev. N. A. F. 2870. March I893.

?Cockerell, Jour. Inst. Jamaica 1: 262. April 1893.

Millsp. \& Nutt. Publ. Field Columb. Mus. Bot. 1: $88 . \quad J a n$. 1896.

Ell. in Millsp. Publ. Field Columb. Mus. Bot. 1: 285 . Dec. 1896. [Publ. 1 5.]

*Ell. \& Ev. Fung. Columb. 108o. 22 Dec. 1896.

Clark, Bot. Gaz. 28 : 291, 307-314. 30 Nov. 1899. 399. Io Jan. 1900. Duggar, Bot. Gaz. 31: 44. 21 Jan. 1901.

Smith, M. H., Bot. Gaz. 31 : 126. 23 Feb. 1901.

*Barthol, Ell. \& Ev. Fung. Columb. 1507. Dec. 1901.

Clark, Bot. Gaz. 33 : 31.24 Jan. 1902.

O'Brien, Bull. Torr. Bot. Club 29: 170. 24 March $1902 .^{\circ}$

Ricker, Univ. of Maine Studies 3: 20. 30 April 1902.

Lindau, Rabh. Krypt.-Fl. 18: 129. $d$. 15 July 1904.

Aspergillus fuliginosus, $\mathrm{Pk}$.

Pk. Bull. Buf. Soc. 1: 69. d. July 1873 .

Pk. Rept. N. Y. Mus. 26: 79. d. April 1874.

Frost in Cat. PI. Amherst 86. 1875 .

Sacc. Syll. 4 : 70. d. 10 April 1886.

Cobb, List Pl. Amherst 40. 1887.

Webber, Rept. Nebr. Agr. 1889: 192 (52). 1890.

Ell. in Millsp. Publ. Field Columb. Mus. Bot. 1 : 285. . Dec. 1896. [Publ. 15.]

* Shear, Ell. \& Ev. Fung. Columb. 1492. May ıوо.

Wehmer, Mem. Soc. Phys. et Hist. Nat. Genève $33^{2}$ : no. $4: 109$. $d$. I901.

Aspergillus fumigatus, Fres.

NoTE. For notes on the ascosporic form of this species see Grijns, Centralbl. Bact. 2 Abt. 11 : 330-332. f. 1-6. 31 Dec. 1903.

We do not understand why the name fumigatus, Fres. (1863) is adopted by modern writers rather than nigrescens, Rob. a synonym dating from 1953 .

Chester, Rept. Del. Exp. Sta. 6 : 129. d. f. 3, 4. I 894.

Boston, Phila. Med. Jour. $7: 446$. f. $t$. 2 March 1901 .

Grijns, Centralbl. Bact. 2 Abt. 11: 330, 332. f. 1-6. 31 Dec. 1903. Aspergillus nigrescens, Robin.

Leidy, Proc. Phil. Acad. 4, I2. I9 Jan. 1875.

Aspergillus aviarius, $\mathrm{Pk}$.

Pk. Rept. N. Y. Mus. 44: $120(8), 137$ (25).d.pl. 4, f. 9-12. Dec. 1891. Sacc. Syll. $10: 524$. d. 30 June 1892.

Wehmer, Mem. Soc. Phys. et Hist. Nat. Genève $33^{2}$ : no. 4 : 91. d. I901. 
Aspergillus glaueus, Lk.

See Eurotium herbariorum, (Wigg.) Lk.

Aspergillus glaueus, var. oblongisporus, Ell. \& Ev.

Millsp. \& Nutt. Publ. Field Columb. Mus. Bot. 1: 88. d. Jan. 1896.

Aspergillus griseus, Lk.

S. Syn. Am. Bor. 282 no. 2679. I 834.

Webber, Rept. Nebr. Agr. 1889 : 192 (52). 1890.

Lindau, Rabh. Krypt.-Fl. 18: 135. d. 15 July I904.

Aspergillus herbariorum, E. Fischer.

See Eurotium herbariorum, (Wigg.) Lk.

Aspergillus laneus, S. Am. Bor.

See Rhinotrichum Curtisil, B.

Aspergillus maximus, Lk.

See Sporodinia Aspergillus, (Scop.) Schrt.

Aspergillus mollis, $\mathrm{B}$.

Hark. \& Moore, Cat. Pac. Fung. 29. Feb. 1880.

Aspergillus Myeobanche, Lk.

Note. This is referred by Streinz to Aspergillus glaucus, but it has apparently been ignored by modern writers. The Schweinitzian specimen examined showed no Aspergillus of any kind.

S. Syn. Am. Bor. 282 no. 2674. I834.

Aspergillus niger, $V$ an Tiegh.

See Sterigmatocystis nigra, Van Tiegh.

Aspergillus nigrescens, Robin.

See Aspergillus fumigatus, Fres.

Aspergillus nigrieans, Cke.

See Sterigmatocystis nigra, Van Tiegh.

Aspergillus nigriceps, B. \& C. nom. nud.

Cke. Grev. 17: 21. Sept. I888.

Aspergillus niveus.

NOTE. The material on which this determination was based is not in condition to warrant the record of this species as correct and it should be excluded from literature.

Farl. Bull. Bussey Inst: 2 : 25I. Jan. 1878.

Aspergillus oosporus, (Lk.) Wallr.

Aspergillus ovalispermus, Lk.

S. Syn. Am. Bor. 282 no. 2675. 1834.

Thm. Fungi Pomicoli 4I.d. 1879 .

Aspergillus Oryzæ, (Ahlburg) Cohn.

Note. The ascosporic condition of this species is Eurotium Oryze, Ahlburg.

Golden, Proc. Ind. Acad. 1898 : 189 (3). f. $1-12$. Dec. 1899.

Hiller, Proc. Ind. Acad. 1901: 272. 1902.

Lindau, Rabh. Krypt.-Fl. $1^{8}:$ i28. d. 30 June 1904.

Aspergillus ovalispermus, Lk.

See Aspergillus oosporus, (Lk.) Wallr.

Aspergillus phæocephalus, Dur. \& Mont. See Sterigmatocystis phæocephala, (Dur. \& Mont.) Sacc.

Aspergillus pulvinatus, B. \& C. nom. nud.

Curtis, M. A., Bot. N. Car. I29. I867.

* Ell. \& Ev. N.'A. F. 1648. 1886.

Ell. in Cat. PI. N. J. 558.1889. 
Aspergillus purpureofuseus, $\mathrm{S}$.

S. Syn. Am. Bor. 282 no. 2680 . d. 1834.

Sacc. Syll. 4: 68. $d$. Io April i 886.

Wehmer, Mem. Soc. Phys. et Hist. Nat. Genève $33^{2}:$ no. $4: 127 . d$. 1901. Aspergillus repens, (Cda.) Sacc.

NorE. It can not be told from the references given below whether the species intended is Aspergillus glaucus, var. repens, Cda. or Eurotium repens, DBy.

Sacc. Mich. 2 : 577. d. Dec. 1882.

* Ell. N. A. F. 1236. I 884 .

Sacc. Syll. 4: 64. d. Io April I886.

Webber, Rept. Nebr. Agr. 1889 : I92 (52). 1890.

Jelliffe, Druggists Circular 41: $95 . f$. $a-d$. April 1897.

Jelliffe, Bull. Torr. Bot. Club 24: 481 . 30 Oct. 1897.

Dresbach, Ohio Nat. 2 : 289. June 1902.

Aspergillus ? roseus, Lk.

S. Syn. Am. Bor. 282 no. 2677. 1834.

Andrews, Proc. Boston Soc. $5: 323$. $^{\circ}$ April 1850.

Curtis, M. A., Bot. N. Car. 128. 1867.

Cke. Handbook 588, $d$. 1871 .

B. Grev. 3: 107 (II7). March 1875 .

Frost in Cat. PI. Amherst 86.1875.

Cobb, List Pl. Amherst 40. 1887.

Aspergillus subgriseus, $\mathrm{Pk}$.

Pk. Bull. Torr. Bot. Club 22 : 2 Io. d. I5 May 1805.

Sacc. Hedw. 35 : Rep. 7 : xlvi. 25 Feb. 1896.

Sacc. \& Syd. Syll. 14 : I046. $d .20$ Aug. I899.

Wehmer, Mem. Soc. Phys. et Hist. Nat. Genève $33^{2}:$ no. $4: 149 . d$. Igor.

Aspergillus umbrinus, Patterson.

Patterson, Bull. Torr. Bot. Club 27 : 284. d. 26 May 1900.

Sacc. \& Syd. Syll. 16: 1028, $d$. I Feb. 1902.

Aspergillus virens, Lk.

S. Syn. Am. Bor. 282 no. $268 \mathrm{r}$. d. 1834 .

Johnson, Bull. Minn. Acad. 1: $340 . \quad 1878$.

Hark. \& Moore, Cat. Pac. Fung. 29. Feb. 1880.

Bundy, Geol. Wis. $1873-79,1: 400.1883$.

Webber, Rept. Nebr. Agr. 1889: 192 (52). I890.

Aspergillus, sp. indet.

Norton, Tr. St. Louis Acad. 7 : 240. 9 Nov. 1896.

Asterella, Sacc.

NotE. The genus Asterella, Sacc. does not seem to us to be really distinct from Asterina, Lev. and furthermore the fact that there is a prior genus of Hepatice of the same name would prevent the adoption of the name for a genus of Fungi.

See Asterina, Lev.

Asterella Bignoniæ, Sacc.

See Asterina Bignoniz, Ell. \& Ev.

Asterella capnoides, Sacc.

See Asterina capnoides, Ell.

Asterella carnea, Sacc.

See Asterina carnea, Ell. \& Martin.

Asterella Chamænerii, Rostr.

See Asterina Chamaneril, (Rostr.) Ell. \& Ev. 
Asterella clavuligera, Sacc.

See Asterina clavuligera, Cke.

Asterella crustacea, Ell. \& Ev.

See Asterina crustacea, (Ell. \& Ev.) n. n.

Asterella cuticulosa, Tracy \& Earle.

See Asterina cuticulosa, Cke.

Asterella discoidea, Sacc:

See Asterina discoidea, Ell. \& Martin.

Asterella Erithalidis, Ell. \& Ev.

See Asterina Erithalidis, (Ell. \& Ev.) n. n.

Asterella Gaultheriæ, Sacc.

See Asterina Gaultherize, M. A. Curtis.

Asterella graminicola, Sacc.

See Asterina graminicola; Ell. \& Ev.

Asterella intricata, Sacc.

See Asterina intricata, Ell. \& Martin.

Asterella nuda, Sacc.

See Asterina nuda, Pk.

Asterella oleina, Sacc.

See Asterina oleina, Cke.

Asterella patelloides, Sacc.

See Asterina patelloides, Ell. \& Martin.

Asterella paupercula, Sacc.

See Asterina paupercula, Ell. \& Ev.

Asterella Pearsoni, Sacc.

See Asterina Pearsoni, Ell. \& Ev.

Asterella Plantaginis, Sacc.

See Asterina Plantaginis, Ell.

Asterella Prosopidis, Ell. \& Ev.

See Asterina Prosopidis, (Ell. \& Ev.) n. n.

Asterella pustulata, Sacc.

See Asterina pustulata, Ell. \& Martin.

Asterella reptans, Lindau.

See Asterina reptans, B. \& C.

Asterella stomatophora, Sacc.

See Asterina stomatophora, Ell. \& Martin.

Asterella subcyanea, Sacc.

See Asterina subcyanea, Ell. \& Martin.

Asteridium anomalum, (Cke. \& Hark.) Lindau.

Lindau in Engler \& Prantl, Pflanzenfam. $1^{1}: 342$. Jan. 1897.

Asterina anomala, Cke. \& Hark.

Cke. \& Hark. Grev. 9: 87. d. March 1881.

Sacc. Syll. $1:$ 49. $d$. I3 June 1882.

Cke. \& Hark. Bull. Cal. Acad. 1: 20. Feb. 1884.

Martin, Jour. Myc. 1: 134. d. Nov. 1885.

Dimerosporium anomalum, Ell. \& Ev.

Ell. \& Ev. Pyren. 35. d. May 1892 .

Asteridium bicolor, Ell. \& Ev.

Ell. \& Ev. Erythea 5 : 5. d. 31 Jan. 1897.

Lindau \& Syd. Hedw. 37 : Beibl. 7 : xxii. 9 April 1898.

Sacc. \& Syd. Syll. 14: 700. d. 20 Aug. 1899. 
Asteridium Celastri, Sacc.

See Asterina Celastri, Ell. \& Kellerm.

Asteridium dothideoides, Ell. \& Ev.

Ell. \& Ev. Bull. Torr. Bot. Club 22 : 436. d. 31 Oct. 1895.

Sacc. Hedw. 35 : Rep. 7 : xxiv. 25 Feb. 1896.

Sacc. \& Syd. Syll. 14: 700. d. 20 Aug. 1899.

Asteridium Illicil, Tracy \& Earle.

Tracy \& Earle, Bull. Torr. Bot. Club 22 : 176. d. 18 April 1805.

Tracy \& Earle, Bull. Miss. Exp. Sta. 34: 100. d. May 1895.

Sacc. Hedw. 35 : Rep. 7 : xxiv. 25 Feb. 1896.

Sacc. \& Lindau, Hedw. 36 : Rep. 7 : xix. 30 April 1897.

Sacc. \& Syd. Syll. 14: 699. d. 20 Aug. 1899.

Asteridium lepidigenoides, (Ell. \& Ev.) Sacc.

Sacc. Syll. 9 : 435. d. 15 Sept. 1891.

Lindau in Engler \& Prantl, Pflanzenfam. 11 : 342. Jan. 1897. Asterina iepidigenoides, Ell. \& Ev.

Ell. \& Ev. Jour. Myc. 4: 121. d. Dec. 1888.

Ell. \& Ev. Pyren. 40. d. May 1892.

Asteridium lepidigenum, (Ell. \& Martin) Sacc.

Sacc. Syll. $9: 436$. $d$. I5 Sept. 1891.

Asterina lepidigena, Ell. \& Martin.

Ell. \& Martin, Am. Nat. 18: I1 48 . $d$. Nov. 1884.

Martin, Jour. Myc. 1 : 136. d. Nov. 1885.

*Ell. N. A. F. 136t, I885.

Berl. \& Vogl. in Sacc. Syll. Add. 1-4 : 8. d. 31 Dec. 1886.

*Wint. Fung. Eur. 3440 . 1886.

Ell. \& Ev. Pyren. 4I. d. May I 892.

Asteridium moniliforme, Ell. \& Ev.

Ell. \& Ev. in Millsp. Publ. Field Columb. Mus. Bot. 2: 16. March 1900. [Publ. 43.]

West Indies.

Asteridium purpureum, (Ell. \& Martin) Sacc.

Sacc. Syll. 9: 436. d. 15 Sept. 1891.

Asterina purpurea, Ell. \& Martin.

Ell. \& Martin, Jour. Myc. 2: 128. $d$. Nov. 1886.

Berl. \& Vogl. in Sacc. Syll. Add. 1-4 : 394. d. 3I Dec. 1886.

Calkins, Jour. Myc. 3: 7. Jan. 1887 .

Ell. \& Ev. Pyren. 43. d. May 1892.

Asteridium Xerophylli, (EII.) Sacc.

Sacc. Syll. 9 : 437. d. 15 Sept. 1891. Asterina Xerophyill, Ell.

Ell. Am. Nat. 17: 319. d. March 1883.

Sacc. Syll. 2 : Add. p. iii. d. I3 June 1883.

Martin, Jour. Myc. 1 : 135. d. Nov. 1885.

Ell. \& Ev. Pyren. 39. d. May 1892.

Asterina Agaves, Ell. \& Ev.

Ell. \& Ev. Bull. Torr. Bot. Club 27 : 571. d. 14 Nov. 1900. Mexico.

Sacc. \& Syd. Syll. 16 : 649. d. I Feb. 1902.

Asterina aliena, Ell. \& Gall.

Ell. \& Ev. Pyren. 36. d. May 1892.

Asterina anomala, Cke. \& Hark.

See Asteridium anomalum, (Cke. \& Hark.) Lindau.

Asterina asterophora, Ell. \& Martin, nom. nud.

Calkins, Jour. Myc. 2: 42. April 1886.

Calkins, Jour. Myc. $2: 127$. Nov. I 886. 
Asterina Bignoniæ, Ell. \& Ev.

Ell. \& Ev. Proc. Phil. Acad. 1890 : 220. d. 29 July 1890.

Ell. \& Ev. Pyren. 43. d. May I 892.

Asterella Bignoniz, Sacc.

Sacc. Syll. 9 : 393. d. I5 Sept. 1891.

Asterina bullata, B. \& C.

B. \& C. Proc. Am. Acad. 4 : 129. d. 14 Dec. 1858.

B. \& C. Jour. Linn. Soc. $10: 374$. d. 1869.

Cent. Amer.

Sacc. Syll. $1: 45 . d$. 51. $d$. I3 June 1882.

West Indies.

Ell. \& Ev. Pyren. 44. d. May 1892 .

Asterina capnoides, Ell.

Ell. Am. Nat. 17: 318. d. March 1883.

Sacc. Syll. 2 : Add. p. ii. $d$. I3 June I883.

Dimerosporium capnoides, Martin.

Martin, Jour. Myc. 1 : I45. d. Dec. 1885.

Ell. \& Ev. Pyren. 34. $d$. May 1892. Asterella capnoides, Sacc.

Sacc. Syll. 9 : 398. $d$. I 5 Sept. I891.

Asterina Celastri, Ell. \& Kellerm.

Ell. \& Kellerm. Jour. Myc. $1: 3 . d$. Jan. 1885 and Hedw. 24: 173. $d$. Aug. I 885 .

Martin, Jour. Myc. 1 : I34. d. Nov. I885.

Berl. \& Vogl. in Sacc. Syll. Add. 1-4: 12. d. 3I Dec. I886

*Ell. \& Ev. N. A. F. 1542. I 886.

Ell. \& Ev. Pyren. 39. d. May 1892.

Harvey, Bull. Torr. Bot. Club 23: 51. 29 Feb. 1896.

Ricker, Univ. of Maine Studies 3: 30. 30 April 1902. Asteridium Celastri, Sacc.

Sacc. Syll. $9: 436 . d$. 15 Sept. I 891.

Asterina carnea, Ell. \& Martin.

Ell. \& Martin, Am. Nat. 17: 1284, d. Dec. I883.

*Ell. N. A. F. 1290.1884.

Martin, Jour. Myc. 1 : I 34 . d. Nov. 1885.

Berl. \& Vogl. in Sacc. Syll. Add. 1-4: 9. d. 31 Dec. 1886.

Ell. \& Ev. Pyren. 38. d. May 1892.

*Pazschke, Fung. Eur. 4052. April I 895.

Lindau \& Syd. Hedw. 37: Beibl. 7: xxi. 9 April 1898. Asterella carnea, Sacc.

Sacc. Syll. 9 : 393. d. 15 Sept. I891.

Tracy \& Earle, Bull. Miss. Exp. Sta. 34 : 98. May 1895.

Asterina Chamæneril, (Rostr.) Ell. \& Ev.

Ell. \& Ev. Pyren. 36. d. May 1892. Asterella Chamænerii, Rostr.

Rostr. Meddelelser 'om Groenl. 3: 545. d. 1888 and Bot. Centralbl. $36: 5 . d$. 1888 .

Sacc. Syll. 9 : $399 . d$. 15 Sept. 1891.

Rostr. Meddelelser om Groenl. 3:614. I89I.

Rostr. Meddelelser om Groenl. 18: 6I (19). 1894.

Rostr. Meddelelser om Groenl. 30: i16. 1904.

Asterina elavuligera. Cke.

Cke. Grev. 6: 142. d. pl. sor, f. I. June 1878 and $7: 96$. March 1879.

* Rav. Fung. Am. 76.1878.

* Rav. Fung. Am. 758. I882.

Sacc. Syll. $1: 48$. d. I 3 June 1882 .

Berl. \& Vogl. in Sacc. Syll. Add. 1-4: 11. d. 3I Dec. 1886.

Ell. \& Ev. Pyren. 43. d. May 1892 . 
Dimerosporlum clavullgerum, Martin.

Martin, Jour. Myc. 1 : 145. d. Dec. 1885.

Asterella clavullgera, Sacc.

Sacc. Syll. 9 : 397. d. I 5 Sept. I891.

Asterina coffeicola, Ell. \& Ev.

Ell. \& Ev. Jour. Inst. Jamalca 1 : 248. d. April I893.

Asterina Colubrinæ, Ell. \& Kelsey.

Ell. \& Kelsey, Bull. Torr. Bot. Club 24: 207. d. 24 April 1897.

Sacc. \& Syd. Syll. 14:694. d. 20 Aug. 1899. West Indies.

Ell. \& Kelsey in Millsp. Publ. Field Columb. Mus. $1: 465$. Nov. 1902. [Publ. 68.]

Asterina comata, B. \& Rav.

B. Grev. 4: 10 (148) no. 791. d. Sept. 1875.

Cke. Grev. 6: 142. June 1878.

* Rav. Fung. Am. 73 . 1878.

Sacc. Syll. $1: 49 . d$. I3 June 1882.

Martin, Jour. Myc. $1:$ 137. $d$. Nov. 1885.

* Roum. Fung. Gall. 5036. 1889.

Roum. Rev. Myc. 11: I96. I Oct. I889.

*Ell. \& Ev. N. A. F. 2339. April 1890.

Ell. \& Ev. Pyren. 39. d. May I892.

Tracy \& Earle, Bull. Miss. Exp. Sta. 38 : 140. May 1896.

Underw. \& Earle, Bull. Ala. Exp. Sta. 80: 181. d. 28 June 1897.

Earle in Mohr. Contr. U. S. Nat. Herb. 6: I68. 31 July I90I.

Asterina conglobata, B. \& C.

See Dimerosporium conglobatum, (B. \& C.) Ell. \& Ev.

Asterina congregata, B. \& C.

B. \& C. Proc. Am. Acad. 4: 1 29. d. 14 Dec. $1858 . \quad$ Cent. Amer.

Sacc. Syll. $1: 48 . d$. 13 June 1882.

Ell. \& Ev. Pyren. 44. d. May 1892 .

Asterina connata, B. \& C.

B. \& C. Jour. Linn. Soc. $10: 374$. d. I869. West Indies.

Sacc. Syll. 1: 42. $d$. I3 June I882.

Asterina coriacella, Speg.

Duss, Champ. Guad. \& Martinique 83. 1903. West Indies.

Asterina erustacea, (Ell. \& Ev.) n. n.

A sterella crustacea, Ell. \& Ev.

Ell. \& Ev. in Millsp. Publ. Field Columb. Mus. Bot. 2 : 15. d. pl. 51. March 1900. [Publ. 43.]

Asterina cupressina, (Rehm) Cke.

C. \& E. Grev. 6: 17. d. Sept. 1877.

Rehm, Ascomyceten $96 . \quad$ I 88I.

Rehm, Hedw. 21: 57. April 1882.

Sacc. Syll. 1: 42 . $d$. 13 June 1882.

Martin, Jour. Myc. 1 : 138. d. Nov. 1885.

Ell. \& Ev. Pyren. 37. $d$. May 1892.

Venturia cupressina, Rehm in Exs.

*Rehm, Ascomyceten 394. I 877.

*Ell. N. A. F. 160. I878.

*Ell. N. A. F. 500.1880.

*Thm. Myc. Univ. 1543. I 880.

Ell. in Cat. PI. N. J. $516 . \quad 1889$.

* Roum. Fung. Gall. 5142 . 1890 .

Roum. Rev. Myc. 12 : 20. I Jan. I890. 
Asterina cuticulosa, Cke.

Cke. Grev. $7: 49 . d$. Dec. 1878.

*Rav. Fung. Am. 328.1879.

Sacc. Syll, $1: 44$. $d$. 13 June 1882.

Martin, Jour. Myc. 1 : I37. d. Nov. 1885.

Ell. \& Ev. Pyren. 40. d. May I 892 .

Asterella cuticulosa, Tracy \& Earle.

Tracy \& Earle, Bull. Miss. Exp. Sta. 34 : 99. d. May 1895.

Asterina decolorans, B. \& C.

B. Grev. 4: 9 (I47) no. 785. d. Sept. 1875 .

Sacc. Syll. $1: 46 . d$. 13 June 1882.

Martin, Jour. Myc. I : 137. d. Nov. I885.

Ell. \& Ev. Pyren. 40. $d$. May I892.

Asterina delitescens, Ell. \& Martin.

Ell. \& Martin, Am. Nat. 17 : 1284. d. Dec. 1883.

*Ell. N. A. F. I29I. 1884 .

Martin, Jour. Myc. 1 : I34. d. Nov. 1885.

Berl. \& Vogl. in Sacc. Syll. Add. 1-4: 10. d. 31 Dec. 1886.

Sacc. Syll. 9 : 383. d. 15 Sept. 1891.

Ell. \& Ev. Pyren. 36. d. pl. 6. May 1892.

Tracy \& Earle,-Bull. Miss. Exp. Sta. 34: 98. May 1895.

Earle, Bot. Gaz. 21: 226, 227. 25 April 1896.

Asterina diplodioides, B. \& C.

B. Grev. 4: 9 (I47) no. 787. d. Sept. 1875.

Sacc. Syll. $1: 4 \mathrm{I} . d$. 13 June 1882.

Martin, Jour. Myc. 1 : 137. d. Nov. 1885.

Ell. \& Ev. Pyren. 44. d. May 1892.

Underw. \& Earle, Bull. Ala. Exp. Sta. 80: 181. d. 28 June 1897.

Earle in Mohr, Contr. U. S. Nat. Herb. 6 : I68. 3i July I90I.

Asterina discoidea, Ell. \& Martin.

Note. It is possible that Asterina discoidea, Ell. \& Martin may be the same as A. oleina, Cke. but the specimen of the latter in Rav. Fung. Am. 757 is too immature to give satisfactory information.

Ell. \& Martin, Am. Nat. 18: I 148. d. Nov. 1884.

Ell. \& Martin, Jour. Myc. 1: I0I. $d$. Aug. I885 and Hedw. 25 : 44. d. Feb. 1886.

Martin, Jour. Myc. 1 : 135. d. Nov. 1885.

Calkins, Jour. Myc. $2: 42$. April 1886

Berl. \& Vogl. in Sacc. Syll. Add. 1-4:8. d. 31 Dec. 1886.

*Ell. \& Ev. N. A. F. $1784 . \quad$ I887.

Ell. \& Ev. Pyren. 37. d. May i 892. Asterella discoidea, Sacc.

Sacc. Syll. 9 : 395. $d$. 15 Sept. 1891.

Asterina dubiosa, Bomm. \& Rous.

Bomm. \& Rous. in Durand \& Pittier, Bull. Soc. Bot. Belg. $35^{1}$ : $157 . d$. 1896.

Sacc. \& Syd. Syll. 14: 696. d. 20 Aug. 1899.

Asterina Erithalidis, (Ell. \& Ev.) n. n. Asterella Erithalidis, Ell. \& Ev.

Ell. \& Ev. in Millsp. Publ. Field Ċolumb. Mus. Bot. 2 : 15. d.pl. 50. March 1900. [Publ. 43.]

West Indies.

Asterina erysiphoides, B. \& C. nom. nud. See Spharella nyssacola, Cke.

Asterina erysiphoides, Ell. \& Martin.

See Asterina patelloides, Ell. \& Martin. 
Asterina Eugeniæ, Mont.

Mont. Syll. Crypt. 255. d. 1856.

West Indies.

Sacc. Syll. $1: 49 . d$. 13 June 1882.

Dothidea Eugenia, Mont.

Mont. Ann. Sci. Nat. 11. 8: 359. d. 1837.

Asterina examinans, B. \& C.

B. \& C. Jour. Linn. Soc. 10:374. d. 1869. West Indies.

Sacc. Syll. $1: 45 . d$. 13 June 1882.

Asterina exasperans, B. \& C.

See Phacidium exasperans, S.

Asterina furcata, Pat.

Pat. Jour. de Bot. 2: I48 (7). d. f. 4. I May 1888.

West Irrdies.

Asterina Gaultheriæ, M. A. Curtis.

Pk. Rept. N. Y. Mus. 22 : 101. 1860.

Martin, Jour. Myc. $1:$ 1 $34 . d$. Nov. 1885.

*Ell. N. A. F. 1358 . 1885.

Berl. \& Vogl. in Sacc. Syll. Add. 1-4 : 7. d. 31 Dec. 1886.

Ell. in Cat. Pl. N. J. 5 10. 1889.

Ell. \& Ev. Pyren. 42. d. May 1892.

* Seymour \& Earle, E. F. 139. I Sept. 1892.

Davis, J. J., Tr. Wis. Acad. 9: 165. 21 Aug. 1893.

Kellerm. \& Werner, Geol. Ohio 72: 352. Jan. 1895.

Sacc. Syll. 11 : 256. $d$. July 1805 .

Harvey, Bull, Torr. Bot. Club 23: 51. 29 Feb. 1896.

Davis, J. J., Tr. Wis. Acad. 11 : 168. Aug. 1897.

Orton, Rept. Vt. Exp. Sta. 12: 167. Dec. 1899 and Contr. Bot. Vt. 7: 167: Dec. 1899.

Ricker, Univ. of Maine Studies 3: 30. 30 April 1902.

Asterella Gaultheria, Sarc.

Sacc. Syll. $9:$ 394. $d$. I5 Sept. I89I.

Asterina globifera, Ell. \& Ev.

Ell. \& Ev. Bull. Torr. Bot. Club 22: 435. d. 31 Oct. 1895. Hawaii. Sacc. Hedw. 35 : Rep. 7 : xxiv. 25 Feb. 1896.

Sacc. \& Syd. Syll. 14 : 696. $d .20$ Aug. 1899.

Asterina graminicola, Ell. \& Ev.

Ell. \& Ev. Proc. Phil. Acad. 1893: 128. d. 28 Feb. 1893. Asterella graminicola, Sacc.

Sacc. Syll. 11 : $257 . d$. July 1895.

Asterina granulosa, (Klotzsch) Hook. \& Arnott.

Tracy \& Earle, Bull. Miss. Exp. Sta. 34: 98. d. May 1895.

Tracy \& Earle, Bull. Miss. Exp. Sta. 38 : 140. May 1896.

Asterina Ilicis, Ell.

Norv. It seems to us from specimens examined that the structure of this species corresponds to that of the genus Microphyma in which it is placed by Spegazzini.

See Microphyma Ilicis, (Ell.) Speg.

Asterina inquinans, Ell. \& Ev.

Ell. \& Ev. Jour. Myc. 3: 41. d. April 1887.

Calkins, Jour. Myc. $3: 46$. April 1887.

Langlois, Cat. PI. La. 27. 1887.

*Ell. \& Ev. N. A. F. 1785 . I887.

Sacc. Syll. $9: 388 . d$. 15 Sept. 189 r.

Ell. \& Ev. Pyren. 41. d. May 1892 .

Tracy \& Earle, Bull. Miss. Exp. Sta. 38 : 140. May 1896.

Earle, Bull. Torr. Bot. Club 27 : 120. d. 24 March 1900. 
Hume, Rept. Fla. Exp. Sta. $1900: 40 . \quad$ I 900.

Patterson, Bull. U. S. Agr. Pl. Industry 8: I8. 3 Feb. I902.

Asterina intricata, Ell, \& Martin.

Ell. \& Martin, Am. Nat. 18 : 69. d. Jan. I884.

Martin, Jour. Myc. 1: 136. d. Nov. 1885.

Berl. \& Vogl. in Sacc. Syll. Add. 1-4: 8. d. 31 Dec. 1886.

Ell. \& Ev. Pyren. 38. $d$. May I892.

Asterella intricata, Sacc.

Sacc. Syll. 9 : 395. $d$. 15 Sept. I89 I.

Tracy \& Earle, Bull. Miss. Exp. Sta. 34 : 99. d. May 1895.

Asterina Lauri-Borboniæ, (S.) Cke.

Note. The Dothidea Lauri-Borbonice, S. has been placed in Asterina with doubt by Cooke in Grev. 13: 67. March 1885 and also in Asteroma, Cke. Grev. 13: 41. Dec. 1884. Saccardo places it in Phyllachora.

All the specimens of Dothidea Lauri-Borbonice in Herb. S. which have been examined by different writers have been imperfect and it is impossible to say what the species is and as there is no more reason for assigning it to Asterina than to Asteroma we give here all the references known to us. The species must be classed permanently with species ignota.

Cke. Grev. 13: 67. March 1885 . Dothidea Lauri-Borbonia, S.

S. Syn. Am. Bor. 232 no. $1899 . d$. 1834 .

Cke. Grev. 13: 4I. Dec. I884.

Ell. \& Ev. Pyren. 605. May I892. Phyilachora Lauri-Borboniz, Sacc.

Sacc. Syll. $2: 6$ Io. $d$. I 3 June I 883 . Asteroma Lauri-Borboniz, Cke.

Cke. Grev. 13:41. Dec. I884.

Asterina Leemingil, Ell. \& Ev.

NotE. The conidial stage of this species is probably Glenospora melioloides, M. A. Curtis, of which no description has been published. Compare also Dimerosporium Galactis, Ell. \& Ev.

Ell. \& Ev. Proc. Phil. Acad. 1893 : I28. d. 28 Feb. 1893.

Gaillard, Bull. Soc. Myc. 9: 97. pl. 7, f. 3. 3I March 1893 .

Small \& Vail, Mem. Torr. Bot. Club 4: 200. d.pl. 82. April 1894.

*Ell. \& Ev. N. A. F. 3108 . Nov. I894.

*Ell. \& Ev. Fung. Columb. 511. 1894.

Sacc. Syll. 11 : 256. d. July 1895 .

Millsp. \& Nutt. Publ. Field Columb. Mus. Bot. 1 : 132. Jan. I896. Glenospora meiloioides, M: A. Curtis, nom. nud.

Curtis, M. A., Bot. N. Car. I27. I867.

Cke. Bull. Buf. Soc. 3: I96. July.i 877 .

Asterina lepidigena, Ell. \& Martin.

See Asteridium iepidigenum, Sacc.

Asterina lepidigenoides, Ell. \& Ev.

See Asteridium iepidigenoides, (Ell. \& Ev.) Sacc.

Asterina Lepiniana, Mont. See Asterina peilicuiosa, B.

Asterina megalospora, B. \& C.

B. \& C. Jour. Linn. Soc. 10: 373. d. I869. West Indies.

Sacc. Syll. 1: 41. $d$. I3 June 1882 .

Asterina melioloides, B. \& C.

See Dimerosporium meiloioides, (B, \& C, ) Martin. 
Asterina Mexicana, Ell. \& Ev.

Ell. \& Ev. Bull. Torr. Bot. Club 27 : 51. d. 17 Feb. $1900 . \quad$ Mexico. Sacc. \& Syul. Syll. $16: 648 . d$. I Feb. 1902.

Asterina minor, Ell. \& Ev.

Ell. \& Ev. Jour. Myc. 2: 42. d. April 1886.

Berl. \& Vogl. in Sacc. Syll. Add. 1-4: 11. d. 31 Dec. 1886.

Sacc. Syll. 9 : $389 . d$. 15 Sept. 1891.

Ell. \& Ev. Pyren. 41. d. May 1892.

?Asterina nigerrima, Ell.

Notk. This species is placed by Lindau in the genus Asterula.

Ell. Bull. Torr. Bot. Club 8 : 91. d. Aug. 1881.

Sacc. Syll. $1: 48 . d$. 13 June 1882.

Martin, Jour. Myc. 1 : 137. d. Nov. 1885.

Ell. in Cat. Pl. N. J. 509. 1889.

Ell. \& Ev. Pyren. 43. May 1892.

Asterula nigerrima, Lindau.

Lindau in Engler \& Prantl, Pflanzenfain. 1': 339. Jan. 1897.

Asterina nuda, $\mathrm{Pk}$.

Martin, Jour. Myc. 1 : 134. d. Nov. 1885.

Pk. Rept. N. Y. Mus. 38: 102. d. pl. 2, f. 11-15. 1885.

Berl. \& Vogl. in Sacc. Syll. Add. 1-4: 9. d. 31 Dec. 1886.

Ell. \& Ev. Pyren. 36. d. May 1892.

Asterella nuda, Sacc.

Sacc.'Syll. 9 : $397 . d . \quad$ I 5 Sept. I891.

Asterina oleina, Cke.

Note. See note under Asterina discoidea, Ell. \& Martin.

Cke. Grev. 11 : 38. d. Sept. 1882.

*Rav. Fung. Am. 757. 1882.

Sacc. Syll. 2: Add. p. ii. $d$. 13 June 1885.

Ell. \& Martin, Jour. Myc. 1 : IoI. d. Aug. 1885.

Martin, Jour. Myc. 1 : 137. d. Nov. 1885.

Calkins, Jour. Myc. 2 : 126. Nov. 1886.

Ell. \& Ev. Pyren. 43. d. May 1892.

Asterella oleina, Sacc.

Sacc. Syll. 9 : 396. d. 15 Sept. 1891.

Asterina orbicularis, B. \& C.

See Dimerosporium orbiculare, (B. \& C.) Martin.

Asterina ostiolata, B. \& C.

B.\& C. Proc. Am. Acad. 4 : 129. d. 14 Dec. $1858 . \quad$ Cent. Amer.

Sacc. Syll. $1: 51 . d$. 13 June 1882.

Ell. \& Ev. Pyren. 44. d. May 1892.

Asterina patelloldes, Ell. \& Martin.

Martin, Jour. Myc. 1: 136. d. Nov. 1885.

Berl. \& Vogl. in Sacc. Syll. Add. 1-4: 7. d. 31 Dec. 1886.

Ell. \& Ev. Pyren. 38. d. May 1892.

Asterina erysiphoides, Ell. \& Martin.

*Ell. N. A. F. 1359. I885. Asterella patelloides, Sacc.

Sacc. Syll. 9 : 394. d. I5 Sept. I891.

Asterina paupercula, Ell. \& Ev.

Ell. \& Ev. Jour. Myc. 4: 121. d. Dec. I888.

Ell. \& Ev. Pyren. 37. d. May 1892.

Asterella paupercula, Sacc.

Sacc. Syll. 9: 396. d.' 15 Sept. 1891. 
Asterina Pearsoni, Ell. \& Ev.

Ell. \& Ev. Jour. Myc. 1 : 92. d. July 1885.

Martin, Jour. Myc. 1 : 135. d. Nov. 1885.

Berl. \& Vogl. in Sacc. Syll. Add. 1-4: Io. d. 31 Dec. I 886.

Ell. in Cat. Pl. N. J. 510.1889.

Ell. \& Ev. Pyren. 42. $d$. May 1892.

Asterella Pearsoni, Sacc.

Sacc. Syll. 9 : 395. $d$. I 5 Sept. I89 I.

Asterina pelliculosa, B.

Note. Under this name in Fungi Cubenses Asterina Lepiniana, Mont. is mentioned as a synonym. We have not been able to trace the latter name elsewhere and it is probably a manuscript name.

B. \& C. Jour. Linn. Soc. 10 : 373. I869.

B. Jour. Linn. Soc, 14: 352. I7 Oct. 1874.

B. Grev. 4: Io (148). Sept. I875.

Cke. Grev. 6 : 142. June 1878.

*Rav. Fung. Am. 75. I878.

Sacc. Syll. $1: 46 . d$. 13 June 1882 .

Martin, Jour. Myc. 1 : 138. d. Nov. 1885.

Cke. in Hemsley, Bot. Challenger $1:$ ro4. 1885.

Pat. Jour. de Bot. 3 : 342 (39). 16 Oct. I889.

Ell. \& Ev. Pyren. 37. d. May I 892 .

Tracy \& Earle, Bull. Miss. Exp. Sta. $34: 98$. d. May 1895.

Earle, Bot. Gaz. 21 : 228. 25 April 1896.

Tracy \& Earle, Bull. Miss. Exp. Sta. 38: I40, 143. May I896.

Underw. \& Earle, Bull. Ala. Exp. Sta. 80: 181. 28 June 1897.

Earle in Mohr, Contr. U.S. Nat. Herb. $6:{ }_{168} 68$ J July I90I.

Patterson, Bull. U. S. Agr. Pl. Industry 8: 18. 3 Feb. 1902.

Duss, Champ. Guad. \& Martinique, 83. 1903.

Dimerosporium orbiculare, Atk. non Martin.

Atk. Bull. Cornell Univ. $3: 4$. 25 June 1897.

Asterina picea, B. \& C.

B. \& C. Jour. Linn. Soc. $10: 374 . d .1869$.

Sacc. Syll. $1: 46 . d$. I3 June 1882 .

Ell. \& Ev. Pyren. 36. d. May 1892.

Asterina Pinastri, Sacc. \& Ell.

NotE. The attachments of the perithecia by hyphal discs such as characterize the Microthyriacea show that this species can not be a Parodiella.

Sacc. Mich. $2: 567$. d. Dec. 1882.

*Ell. N. A. F. 789 . 1882 .

Sacc. Syll. 2 : Add. p. ii. $d$. I 3 June 1883.

*Wint. Fung. Eur. 2942. 1883.

Martin, Jour. Myc. 1: I 35. d. Nov. 1885.

Sacc. Syll. $9: 387 . d$. 15 Sept. I 89 I.

Ell. \& Ev. Pyren. 39. d. May 1892.

*Ell. \& Ev. Fung. Columb. 615. 24 April 1895.

Parodiella rigida, Ell. \& Ev.

Ell. \& Ev. Jour. Myc. 4: 62. d.' July I 888.

Sacc. Syll. 9: 4 IO. $d$. 15 Sept. I89I.

Lindau in Engler \& Prantl, Pflanzenfam. 1' ${ }^{1}: 336$. Jan. I897.

Asterina Pittieri, Bomm. \& Rous.

Bomm. \& Rous. in Durand \& Pittier, Bull. Soc. Bot. Belg. $35^{1}$ : 156. d. I 806.

Sacc. \& Syd. Syll. 14: 695. d. 20 Aug. I 899.

est Indies.

Bermuda.

West Indies. 
Asterina Plantaginis, Ell.

Ell. Bull. Torr. Bot. Ćlub $9: 74$. $d$. June 1882.

*Ell. N. A. F. 791.1882.

Sace. Syll. 2 : Add. p. iii. $d$. 13 June 1883.

Hark. Bull. Cal. Acad. 1: 165. 7 March 1885.

Martin, Jour. Myc. 1 : 138. d. Nov. 1885.

Ell. in Cat. PI. N. J. 510.1880 .

Ell. \& Ev. Pyren. 4o. d. May 1892.

* Seymour \& Earle, E. F. 348. I May 1893.

Halsted, Rept. N. J. Exp. Sta. 1892 : 348.1893.

*Ell. \& Ev. Fung. Columb. 7'3. 24 April 1895.

Davis, J. J., Tr. Wis. Acad. 11 : 170. Aug. 1897.

Jones, H. L., Oberlin College Lab. Bull. 9: 5. 15 June 1898.

Orton, Rept. Vt. Exp. Sta. 12 : 167 . Dec. 1899 and Contr. Bot. Vt. 7: 167. Dec. 1899.

Astereila Plantaginis, Sacc.

Sacc. Syll. 9: 398. d. 15 Sept. 1891.

Asterina platasea, B. \& C.

B. \& C. Jour. Linn. Soc. 10 : 373. d. 1860.

Sacc. Syll. $1: 40 . d$. 13 June 1882.

Asterina Prosopidis, (Ell. \& Ev.) n. n.

Asterella Prosopidis, Ell. \& Ev.

Ell. \& Ev. Am. Nat. 31: 340. d. April 1897.

Lindau \& Syd. Hedw. 37 : Beibl. 7 : xxii. 9 April 1808.

Sacc. \& Syd. Syll. 14: 698 . d. 20 Aug. I 899 .

Asterina purpurea, Ell. \& Martin.

See Asteridium purpureum, (Ell. \& Martin) Sacc:

Asterina pustulata, Ell. \& Martin.

Ell. \& Martin, Am. Nat. 18: 1148. d. Nov. 1884.

Martin, Jour. Myc. 1: 136. d. Nov. 1885.

Berl. \& Vogl. in Sacc. Syll. Add. 1-4: 8. d. 31 Dec. 1886.

*Ell. \& Ev. N. A. F. 1543.1886.

Ell. \& Ev. Pyren. 41. d. May 1892.

Astereila pustuiata, Sacc.

Sacc. Syll. 9 : 395. d. 15 Sept. 1891.

Asterina radians, Ell.

Ell. Jour. Myc. $7: 276$. d. 15 May 1893.

Sacc. Syll. 11 : 255 . d. July 1895 .

Asterina ramularis, Ell. See Asteruia ramularis, (Ell.) Sacc.

Asterina reptans, B. \& C.

NotE. This species is made the type of Trichopeltis by Spegazzini but that name so far as we know has not yet appeared in North American literature except as stated below. Whether Asterind reptans, Ule, Myc. Bras. 65 is the true $A$. reptans, B. \& C. seems to us doubtful. We have examined Berkeley and Curtis specimens on which the species is founded and they do not seem to have the same structure as the specimen in Myc. Bras.

B. \& C. Jour. Linn. Soc. 10 : 373. d. 1869.

West Indies.

Sacc. Syll. $1: 46 . d$. 13 June 1882 .

Trichopeltis reptans, Speg.

Sacc. Syll. 9 : 1068.15 Sept. 1801.

Lindau in Engler \& Prantl, Pflanzenfam. ${ }^{1}{ }^{1}$ : 342. Jan. 1897. Asterella reptans, Lindau.

Lindau in Engler \& Prantl, Pflanzenfam. 1 ${ }^{1}$ : 340. Jan. 1897. Mlcrothyrlum reptans, Pat. in Duss.

Duss, Champ. Guad. \& Martinique 83. I903. 
Asterina rubicola, Ell. \& Ev.

Ell. \& Ev. Proc. Phil. Acad. 1890: 219. d. 29 July I890.

*Ell. \& Ev. N. A. F. 2340 . 1890.

Sacc. Syll. 9: $384 . d$. I5 Sept. I89r.

Ell. \& Ev. Pyren. 35. d. May 1892.

Davis, J. J., Tr. Wis. Acad. 9 : 165. 21 Aug. I893.

Orton, Rept. Vt. Exp. Sta. 12: I67. Dec. 1899 and Contr. Bot. Vt. 7: 167. Dec. I899.

*Vestergren, Micr. Sel. 815. March I904.

Asterina sabalicola, Earle.

Earle, Bull. Torr. Bot. Club 27 : I20. d. 24 March I900.

Sacc. \& Syd. Syll. $16: 648$. d. I Feb. I902.

Asterina Sidæ, Earle.

Earle, Bull. N. Y. Bot. Gard. 3 : 310. d. 30 June 1904. West Indies.

Asterina solanicola, B. \& C.

B. \& C. Jour. Linn. Soc. $10: 374 . d$. 1869.

Sacc. Syll. $1: 48 . d$. I 13 June 1882 .

Wint. Hedw. 25: 94 (3). June I 886.

Rehm, Hedw. Beibl. 34 :- (I6I). d. I9 Oct. I895.

Asterina sphærelloides, Ell. \& Ev.

Ell. \& Ev. Bull. Torr. Bot. Club 24: 126. d. 30 March 1897. Hawaii. Lindau \& Syd. Hedw. 37: Beibl. 7 : xxii. 9 April 1898.

Asterina Sphærotheca, Karst. \& Roum.

Bomm. \& Rous. in Durand \& Pittier, Bull. Soc. Bot. Belg. 351 : I 57. I 896.

Asterina sphenispora, Ell. \& Ev.

Ell. \& Ev. Jour. Inst. Jamaica 1: 248. d. 262. April I893.

Asterina spurea, B. \& C.

Note. The specific name is spelled spurca in Grev. 4: Io where the species is first described but in the two previous citations of Ravenel and Curtis it is spelled spuria.

*Rav. Fung. Car. 4: : 69. 1855.

Curtis, M. A., Bot. N. Car. I52. 1867.

B. Grev. 4: Io (148) ho. 789. d. Sept. 1875.

Sacc. Syll. $1: 50 . d$. 13 June 1882 .

Martin, Jour. Myc. 1 : 138. d. Nov. 1885.

Ell. \& Ev. Pyren. 44. d. May 1892.

Underw. \& Earle, Bull. Ala. Exp. Sta. 80: I8I. $d .28$ June I897.

Earle in Mohr, Contr. U.S. Nat. Herb. 6 : 168. 3 I July Igor.

Asterina spuria, B. \& C.

See Asterina spurca, B. \& C.

Asterina stomatophora, Ell. \& Martin.

Ell. \& Martin, Jour. Myc. 1: 98. d. Aug. 1885 and Hedw. $25: 4$ I. $d$. Feb. I886.

Martin, Jour. Myc. 1 : 136. $d$. Nov. I885.

Calkins, Jour. Myc. 2 : 127. Nov. I886.

Berl. \& Vogl. in Sacc. Syll. Add. 1-4: 9. d. 3 I Dec. 1886.

Ell. \& Ev. Pyren. 4r. d. May 1892.

Asterella stomatophora, Sacc.

Sacc. Syll. 9 : 394. $d$. I 5 Sept. I 89 r.

Asterina subcyanea, Ell. \& Martin.

Ell. \& Martin, Am. Nat. 18 : I I48. $d$. Nov. I884.

Martin, Jour. Myc. 1: 136. d. Nov. 1885.

*Ell. N. A. F. 1360.1885 .

Berl. \& Vogi. in Sacc. Syll. Add. 1-4: 7. d. 3i Dec. I886. 
Ell. \& Ev. Pyren. 42. d. May 1802 . Asterella subcyanea, Sacc.

Sacc. Syll. $9: 395 . d$. 15 Sept. 1891.

Lindau in Engler \& Prantl, Pflanzenfam. 1' ${ }^{1}$ : 340. Jan. 1897.

Asterina tenella, Cke.

Cke. Grev. 13:67. d. March 1885.

Martin, Jour. Myc. 1: 135. d. Nov. 1885 .

Berl. \& Vogl. in Sacc. Syll. Add. 1-4: 10. d. 31 Dec. 1886.

Sacc. Syll. 9: $383 . d$. 15 Sept. 1891 .

Ell. \& Ev. Pyren. 39. d. May 1892 .

Lindau in Engler \& Prantl, Pflanzenfam. 11: 341. Jan. 1897.

Asterina triloba, Earle.

Earle, Bull. N. Y. Bot. Gard. 3 : 310. d. 30 June 1904. West Indies.

Asterina Wrightil, B. \& C.

B. Grev. 4: IO (148) no. 790. d. Sept. 1875.

Clie. Ann. N. Y. Acad. 1 : 184,187 . May 1878.

Sacc. Syll. 1: 49. $d . \quad$ I3 June 1882.

Martin, Jour. Myc. 1 : $138 . d$. Nov. 1885.

Ell. \& Ev. Pyren. 44. d. May 1892.

Asterina Xerophylli, Ell. See Asteridium Xerophylli, (Ell.) Sacc.

Asterina Yucatanensis, Ell. \& Ev.

Ell. \& Ev. in Millsp. Publ. Field Columb. Mus. Bot. $1: 285 . d . p l .9, f$. 1-5. Dec. 1896. [Publ. 15.]

Sacc. \& Syd. Syll. 14: 695. d. 20 Aug. 1899.

Asterina, sp. indet.

Trel. Tr. Wis. Acad. 6 : 119. Nov. 1884.

Small \& Vail, Mem. Torr. Bot. Club 4: 201. April 1894.

Asterinula, Ell. \& Ev.

See Leptothyrella, Sacc.

Asterinula Dearnessil, Ell. \& Ev.

See Leptothyrella Dearnessii, (Ell. \& Ev.) Sacc.

Asterinula Langloisil, Ell. \& Ev.

See Leptothyrella Langloisii, (Ell. \& Ev.) Sace.

Asteroconium, Syd.

Syd. Ann. Myc. 1: $36 . d$. 30 Jan. 1903 and Jour. Myc. $10: 244 . d$. 14 Oct. 1904.

Asteroconium Saccardoi, Syd.

Syd. Ann. Myc. 1: 36. d. 30 Jan. 1903.

Asterodon, Pat.

Pat. Bull. Soc. Myc. Fr. 10 : 129. d. 1894.

Pat. Essai Tax. 121. d. 1900. Hydnochate, Pk.

Pk. Rept. N. Y. Mus. 50: I13. d. 28 March 1898. Hydnochatella, Sacc.

Sacc. \& Syd. Syll. 14: i1. 20 Aug. 1899.

Asterodon ferruginosum, Pat.

Pat. Essai Tax. 121. 1900. Hydnochate setigera, Pk.

Pk. Rept. N. Y. Mus. 50: II3. d. 28 March I 898.

Sacc. \& Syd. Syll. 14: 211 . d. 20 Aug. 1899. Asterodon setiger, Pk.

Pk. Bull. N. Y. Mus. $6: 221$. July 1899. [Bull. 28.]

Asteroma Aceris, Rob.

Thm. Nat. Can, 10: io. Jan. 1878 . 
Asteroma alpinum, Sacc.

Rostr. Meddelelser om Groenl. 3 : $570 . \quad$ I 888.

Rostr. Meddelelser om Groenl. 30 : 120. 1904.

Asteroma atramentarium, B.

B. Grev. 2: $98(79)$ no. $456 . d$. Jan. I874.

Sacc. Syll. 3 : 202. $d$. 15 Dec. 1884.

Asteroma Bartsiæ, Rostr.

Rostr. Meddelelser om Groenl. 3 : 570. $d$. I 888 and Bot. Centralbl. $36: 7 . d$. 1888 .

Rostr. Meddelelser om Groenl. 3 : 624. I89I.

Asteroma Brassicæ, Chev.

Rostr. Meddelelser om Groenl. 3: 570. 1888.

Asteroma Cerasi, Cke.

See Asteroma cerasicolum, Sacc.

Asteroma cerasicolum, Sacc.

Sacc. Syll. 3 : 202. $d$. I5 Dec. I884.

Allescher, Rabh. Krypt.-Fl. $1^{6}: 45 \%$. 1899.

Asteroma Cerasi, Cke.

*Rav. Fung. Am. 521. I $88 \mathrm{I}$.

Cke. Grev. $12:$ 24. d. Sept. I883.

*Thm. Myc. Univ. $2197 . \quad$ I883.

Asteroma erustaceum, M. A. Curtis.

Nore. M. A. Curtis in Bot. N. Car. I 18 gives this name to the Dothidea crustacea of Schweinitz which as far as Schweinitzian specimens examined indicate does not show the ascosporic condition but is in all probability, judging by the. structure of the young stages, either a Dothidea or a Phyllachora.

See Dothidea crustacea, S.

Asteroma Dianthi, Cke. \& Hark.

Cke. \& Hark. Grev. 9: 84. d. March I 88I.

Hark. Bull. Cal. Acad. 1 : 14. 29 Feb. 1884.

Sacc. Syll. 3: 204. $d$. I 5 Dec. I884.

Asteromá Diospyri, (S.) M. A. Curtis.

NoTE. We have brought together under this name all the references and synonyms of Xyloma Diospyri, S. The Schweinitzian specimens show only immature fungi and at the present day there is no one able to say what the species really is. It should be placed in species ignota.

Curtis, M. A., Bot. N. Car. I18. 1867.

Sacc. Syll. 3 : 207. $d$. I 5 Dec. I884.

Comes, Critt. Agr. $1: 468 . \quad$ I89 I. Xyloma Diospyri, $\dot{\mathrm{S}}$.

S. Syn. Car. $53(27)$ no. 272. $d . \quad 1822$. Dothidea Diospyri, Fr.

Fr. S. M. $2: 561 . d$. 1823.

S. Syn. Am. Bor. 232 no. 1907. I834.

Cke. Grev. 13: 44. Dec. 1884 .

Cke. Grev. 13: 62. March 1885.

Asteroma elegans, M. A. Curtis.

See Phyllachora elegans, (S.) Sacc.

Asteroma Epilobii, Fr.

Schrt. Ber. Schl. Ges. 1887 : 283. $\quad$ I887.

Dothidea Epilobil, Fr.

Pk. Rept. N. Y. Mus. 31 : 49. 1879.

Ell. \& Ev. Pyren. 615. d. May I892. 
Asteroma Euphorbiæe, Fcki.

Webber, Rept. Nebr. Agr. 1889 : 217 (77). 1890.

Asteroma Fraseræ, Ell. \& Ev.

Ell. \& Ev. Bull. Torr. Bot. Club 25 : 509. d. 10 Sept. 1808.

Sacc. \& Syd. Syll. 16 : 890. d. I Feb. 1902.

Asteroma geographicum, (DC.) Desm.

Curtis, M. A., Bot. N. Car. 118.1867.

Sacc. Syll. $3: 202 . d$. 15 Dec. 1884.

Tubeuf-Smith, Diseases of Plants 470. 1897.

Allescher, Rabh. Krypt.-Fl. 16: 472. d. 475. 1899.

Lindau in Engler \& Prantl, Pflanzenfam. 11** : 357. Dec. 1899.

*Barthol., Ell. \& Ev. Fung. Columb. 1508. Dec. 1901. Dothidea geographica, Fr.

S. Syn. Am. Bor. 232 no. 1904.1834.

* Rav. Fung. Car, 1: 67. 1852.

B. Grev. 4: 105 (175). March 1876.

*Rav. Fung. Am. 739. 1882.

Asteroma graminis, Westd. ?

*Ell. \& Ev. N. A. F. 2160. 1889.

Asteroma Himantia, Fr. See Spherella Hlmantia, (P.) Karst.

Asteroma Impatientis, (S.) M. A. Curtis.

Nore. We have brought together here all the references pertaining to Dothidea Impatientis, S. Schweinitzian material shows only undeveloped specimens and the plant should be placed with species ignota.

Curtis, M. A., Bot. N. Car. 118. 1867. Dothldea Impatlentls, S.

S. Syn. Am. Bor. 234 no. 1926. d. 1834.

Cke. Grev. 13 : 42. Dec. 1884 .

Phyllachora Impatlentis, Sacc.

Sacc. Syll. $2: 617$. d. 13 June 1883.

Asteroma inelegans, (S.) M. A. Curtis.

Note. This species should be classed with species ignota like the last and for similar reasons.

Curtis, M. A., Bot. N. Car. 118. 1867. Dothldea Inelegans, S.

S. Syn. Am. Bor. 233 no. 191 5. d. 1834.

Cke. Grev. $13: 42$. Dec. 1884 . Phyllachora Inelegans, Sacc.

Sacc. Syll. 2 : 619. d. 13 June 1883.

Asteroma infuscans, Ell. \& Ev.

Ell. \& Ev. Proc. Phil. Acad. 1895 : 43 I. d. Nov. 1895.

Sacc. Hedw. 35 : Rep. 7 : xlii. 25 Feb. 1896.

*Ell. \& Ev. N. A. F. 3359. Feb. 1896.

*Ell. \& Ev. Fung. Columb. 959. April 1896.

Sacc. \& Syd. Syll. 14: $902 . d$. 20 Aug. 1899. Asteroma lvacola, Ell. \& Ev.

Ell. \& Ev. Am. Nat. 31: 428. d. May 1897.

Ell. \& Ev. Bull. Torr. Bot. Club 24: 462.30 Oct. 1897.

*Ell. \& Ev. Fung. Columb. 1141. July 1897.

*Ell. \& Ev. N. A. F. 3543. 21 Jan. 1898.

Lindau \& Syd. Hedw. 37 : Beibl. 7 : xliii. 9 April 1898.

Asteroma Juncaginearum, Rabh.

Rostr. Meddelelser om Groenl. 3: 570. 1888. 
Asteroma Lauri-Borboniæ, Cke.

See Asterina Lauri-Borboniz, (S.) Cke.

Asteroma lineola, M. A. Curtis.

See Phoma lineola, (S.) Cke.

Asteroma Liriodendri, Cke.

*Rav. Fung. Am. 522. I88I.

Cke. Grev. $12:$ 25. d. Sept. 1883.

Sacc. Syll. $3: 203$. $d$. I 5 Dec. 1884 .

Patterson, Bull. U. S. Agr. PI. Industry 8: 23. 3 Feb. 1902.

Asteroma Panici, M. A. Curtis.

See Phyllachora Panici, (S.) Sacc.

Asteroma Parkinsoniæ, Ell. \& Ev.

Ell. \& Ev. Proc. Phil. Acad. 1895: 43I. d. Nov. 1895.

Sacc. Hedw. 35 : Rep. 7 : xli. 25 Feb. 1896.

Sacc. \& Syd. Syll. 14: 901. d. 20 Aug. I899.

Asteroma pomigenum, M. A. Curtis.

See Dothidea pomigena, S.

Asteroma Pringlei, Pk.

Pk. Bot. Gaz. 7 : 55. d. May I882.

Asteroma punctiforme, B.

B. Grev. 2: 97 (78) no. 455. d. Jan. 1874.

Sacc. Syll. 3: 203. d. I5 Dec. 1884 .

Asteroma reticulatum, (DC.) Chev.

Frost in Cat. Pl. Amherst 81. I875.

Cobb, List. Pl. Amherst 38. 1887.

Asteroma ribicolum, Ell. \& Ev.

Kelsey, Jour. Myc. 5 : 81. June I889.

Ell. \& Ev. Jour. Myc. 5 : I48. d. Sept. 1889.

Comes, Critt Agr. 1: 469. 189 r.

Sacc. Syll. 10 : 219. d. 30 June 1892.

Asteroma Robergei, Desm.

Curtis, M. A., Bot. N. Car. 118. 1867.

B. Grev. $2: 98(79)$. Jan. 1874 .

Sacc. Syll. 3 : 2 Io. d. I5 Dec. I884.

Rostr. Meddelelser om Groenl. 3: 569. 1888.

Rostr. Meddelelser om Groenl. 3 : 624. I89I.

Asteroma Robergei, Desm., var. Arundinariæ, Sacc.

Sacc. Syll. 3 : 2 Io. $d$. I 5 Dec. I884.

Asteroma Rosæ, Lib.

See Actinonema Rosæ, (Lib.) Fr.

Asteroma Salicis, Rob. \& Desm.

Desm. Ann. Sci. Nat. II. 19: 350. d. r843.*

Rostr. Meddelelser om Groenl. 3: $570 . \quad$ I888.

Rostr. Meddelelser om Groenl. 3 : 624. I891.

Asteroma Saxifragæ, Ell. \& Ev.

Ell. \& Ev. Proc. Phil. Acad. 1893 : I 58. d. 28 Feb. 1893.

Sacc. Syll. 11 : 50r. $d$. July r 895 .

Asteroma Senecionis, Ell. \& Ev.

Ell. \& Ev. Bull. Torr. Bot. Club 24: 287. d. 29 June 1897.

Lindau \& Syd. Hedw. 37 : Beibl. 7 : xliii. 9 April 1898.

Sacc. \& Syd. Syll. 14: $901 . d$. 20 Aug. I899. 
Asteroma tinctorium, Cke.

*Rav. Fung. Am. 523. I881.

Cke. Grev. $12: 25$. d. Sept. 1883.

Sacc. Syll. $3: 203$. d. 15 Dec. 1884 .

Asteroma vagans, Desm.

*Barthol., Ell. \& Ev. Fung. Columb. 2005. 20 March 1905.

Asteroma venulosum, (Wallr.) Fckl.

Davis, J. J., Tr. Wis. Acad. 9: 166. 21 Aug. 1893.

Asteroma vernicosum, (DC.) Fckl.

Note. The specimen of Dothidea vernicosa, Fr. of the Schweinitz herbarium shows only undeveloped fungi and therefore it is uncertain whether the Schweinitzian specimen is really the Asteroma vernicosum of (D C.) Fckl.

Curtis, M. A., Bot. N. Car. I18. 1867.

Dothidea vernicosa, Fr.

S. Syn. Am. Bor. 232 no. 1900.1834.

Cke. Grev. 13: 44. Dec. 1884 .

Asteroma Xanthil, M. A. Curtis.

Note. This manuscript name appears only in M. A. Curtis, Bot. N. Car. 118 and there is 10 specimen of it in the Curtis herbarium. It is therefore impossible to say whether it could be the Asteroma Xanthii, D C. referred by Cooke in Grev. to Puccinia Xanthil, S.

Asterophora agaricicola, Cda.

Note. This is the conidial form of Nyctalis lycoperdoides, (Bull.) Schrt. $q \cdot v$.

Asterophora agaricoides, Fr.

Note. This is the conidial form of Nyctalis lycoperdoides, (Bull.) Schrt. $q . v$.

\section{Asterophora effusa, S.}

NoTE. An examination of an authentic Schweinitzian specimen shows that this is not an Asterophora in the proper sense. It appears to be one of the conidial forms of Polyporus called Ptycogaster but the Schweinitzian material is not sufficient to enable one to say certainly what species it is.

S. Syn. Am. Bor. 263 no. 2384. d. 1834.

Asterophora nucuum, Rav.

Note. This is a misprint for Ascophora nucuum, Cda. $q . v$.

Asterophora Pezizæ, Cda.

NOTE. This species of which the earlier name is Stephanoma strigosum, Wallr. is the conidial form of a Hypomyces whose ascosporic condition has not yet been found, as far as we know, in North America. It is the species called by Tulasne, Carp. 3 : 52. 1865 Hypomyces Peziza. For the conidial stage alone the name should perhaps be Asterophora strigosa, (IVallr.) n. $n$.

Pk. Rept. N. Y. Mus. 35 : 142. 1885.

Ell. \& Ev. Jour. Myc. 2: 76. July 1886. Synthetospora electa, Morg.

Morg. Bot. Gaz. 17 : 192. d. f. 2. I5 June 1892.

Kellerm. \& Werner, Geol. Ohio 72: 371. Jan. 1895.

Sacc. Syll. 11 : 608 . d. July 1895 .

Lindau in Engler \& Prantl, Pflanzenfam. 11**: 451. f. $234 \mathrm{~A}$. Feb. 1900. 
Sepedonium tuberculiferum, Ell. \& Ev.

Ell. \& Ev. Am. Nat. 31 : 430. d. May 1897.

Lindau \& Syd. Hedw. 37 : Beibl. 7 : liii. 9 April 1898.

Sacc. \& Syd. Syll. 14: 1056. d. 20 Aug. I899.

Asterophora physaroides, Fr.

NOTE. This is the conidial form of Nyctalis lycoperdoides, (Bull.) Schrt. $q . v$.

Asterophora strigosa, (Wallr.) n. n.

See note under Asterophora Peziza, Cda.

Asterosporium betulinum, $\mathrm{Pk}$.

Pk. Rept. N. Y. Mus. $33: 26 . d$. pl. $1, f .4-5$. Oct. 1883.

Sacc. Syll. 3: 782. $d$. I5 Dec. I 884.

Ell. in Cat. Pl. N. J. 592.1889.

Asterosporium Hoffmanni, Kze.

Note. The Schweinitzian specimen of Asterosporium vulgare examined shows no definite structure. It is impossible to say to what genus it belongs.

Curtis, M. A., Bot. N. Car. I I9. I867.

Cke. Handbook 468. $d . f .181$. I871.

*Ell. N. A. F. 956 . I883.

Sacc. Syll. $3: 782$. $d$. I5 Dec. I 884.

Lindau in Engler \& Prantl, Pflanzenfam. 11**: 409. f. $214 C-E$. Jan. 1900.

Allescher, Rabh. Krypt.-Fl. 17: 663. d. f. a-d. 1902.

? Stilbospora asterosperma, $\mathrm{P}$.

S. Syn. Car. 75 (49) no. 507. I822.

? Asterosporium vulgare, S. Am. Bor.

S. Syn. Am. Bor. 299 no. 2980. I 834.

Asterosporium vulgare, S. Am. Bor. 2980.

See Asterosporium Hoffmanni, Kze.

Asterostroma, Massee.

Massee, Jour. Linn. Soc. 25 : I54. $d .8$ June I889.

Sacc. Syll. $9: 236 . d$. I 5 Sept. 1891.

Morg. Jour. Cin. Soc. 18: 38. d. Dec. 1895.

\section{Asterostroma albidocarneum, Massee.}

Note. This species is founded on the Corticium albidocarneum of Rav. Fung. Car. 4: 14 which was supposed by Ravenel to be the same as Thelephora albidocarnea, $\mathrm{S}$. This however is not the case as shown by Massee's examination and our own examination of authentic specimens of Thelephora albidocarnea, S. from Herb. S. which is not an Asterostroma. The true Thelephora albidocarnea, $\mathrm{S}$. which is the Corticium albidocarneum, (S.) Massee is not to be confounded with the present species. The references to Corticium albidocarneum in American literature relate in some cases to Corticium albidocarneum, (S.) Massee, $q . v$. We have given here those which belong properly to Asterostroma albidocarneum, Massee. The citations of Morgan and Kellerman \& Werner probably belong here but we have not seen specimens representing them.

Massee, Jour. Linn. Soc. 25 : I 55. d. pl. 46, f. 8-q. 8 June I 889.

Sacc. Syll. $9: 237$. I 5 Sept. I 89 I.

Corticium albidocarneum, Rav.

* Rav. Fung. Car. 4: $14 . \quad 1855$.

Curtis, M. A., Bot. N. Car. 107. 1867.

B. Grev. $1:$ i 78 (36). June I 873 .

Morg. Jour. Cin. Soc. 10: 200. d. Jan. I888. 
Sacc. Cub. \& Manc. Syll. 6 : 6zo. d. I Aug. I888. p. p.

Kellerm. \& Werner, Geol. Ohio $7^{2}: 333$. Jan. 1895.

Asterostroma bicolor, Ell. \& Ev.

Ell. \& Ev. Proc. Phil. Acad. 1893 : 441. d. 1893.

Sacc. Syll. 11 : I28. $d$. July I 895 .

Pk. Rept. N. Y. Mus. 55: 954. Nov. 1902. [Bull. $10:$ no. 54.]

Asterostroma cervicolor, (B. \& C.) Massee.

Massee, Jour. Llnn. Soc. 25 : 155. d. 8 June 1889.

Sacc. Syll. 9 : 237. I 5 Sept. I 89 I.

Millsp. \& Nutt. Publ. Field Columb. Mus. Bot. 1 : 17I. Jan. I896.

Corticium cervicolor, B. \& C.

B. Grev. 1 : 179 (37) no. 267. d. June 1873.

* Rav. Fung. Am. 228. 1879.

Calkins, Jour. Myc. 2 : 106. Sept. 1886.

Sacc. Cub. \& Manc. Syll. 6: 621. d. I Aug. I 888

Underw. \& Earle, Bull. Ala. Exp. Sta. 80 : 223. d. 28 June 1897.

Earle in Mohr, Contr. U.S. Nat. Herb. 6: 197. 31 July I90I.

Asterostroma corticola, Massee.

Massee, Jour. Linn. Soc. 25 : I 55. d. 8 June 1889.

Sacc. Syll. 9 : 236. $d$. is Sept. 1891.

Millsp. \& Nutt. Publ. Field Columb. Mus. Bot. 1 : I7 I. Jan. 1896.

Henn. in Engler \& Prantl, Pflanzenfam. 11**: 122.1898.

Asterostroma muscicola, (B. \& C.) Massee.

Massee, Jour. Linn. Soc. 25 : 155. d. 8 June 1889.

Sacc. Syll. $9: 237.15$ Sept. 1891.

Hymenochate muscicola, B. \& C.

B. \& C. Jour. Linn. Soc. $10: 334 \cdot d$. 1869.

West Indies.

Cke. Grev. 8: 149. June 1880.

Sacc. Cub. \& Manc. Syll. 6 : 602. $d$. I Aug. I 888.

Asterostroma pallidum, Morg.

Morg. Jour. Cin. Soc. 18: 38. d. Dec. 1895.

Sacc. Hedw. 35: Rep. 7 : x. 25 Feb. 1896.

McClatchie, Proc. S. Cal. Acad. 1: 378. 5 June I897.

Sacc. \& Syd. Syll. 14 : 223. d. 20 Aug. 1899.

Asterula nigerrima, Lindau.

See Asterina nigerrima, Ell.

Asterula ramularis, (Ell.) Sacc.

Sacc. Syll. 9 : $375 . d$. I5 Sept. I89i. Asterina ramularis, Ell.

Ell. Bull. Torr. Bot. Club 9 : 20. $d$. Feb. 1882.

*Ell. N. A. F. 790.1882.

Sacc. Syll. 2 : Add. p. ii. d. I3 June 1883.

Martin, Jour. Myc. 1 : 138. d. Nov. 1885.

Ell. \& Ev. Pyren. 4o.d. May 1892 .

Asterula Tracyi, Pk.

Pk. Rept. N. Y. Mus. 47: I 52 (26). d. Nov. I894.

Tracy \& Earle, Bull. Miss. Exp. Sta. $34: 98$. d. May 1895.

Sacc. Syll. 11 : 254. d. July 1895 .

Astræus, Morg.

Morg. Jour. Cin. Soc. 12 : 19. d. April 1889.

Fischer in Engler \& Prantl, Pflanzenfam. 11**: $341 . d$. Dec. I 899.

Hollos, Gast. Hung. 78. $d$. I60. I904.

Astræus hygrometricus, Morg.

See Astraus stellatus, (Scop.) E. Fischer. 
Astræus stellatus, (Scop.) E. Fischer.

Fischer, E., in Engler \& Prantl, Pflanzenfam. 11**: 341. d. f. 178. Dec. 1899.

Earle in Greene, Pl. Baker. 2: 5. 25 March IgoI.

Hollos, Gast. Hung. 78. d. 160. pl. 10, f. 26-29. 1904.

Geaster hygrometricus, P.

S. Syn. Car. 59 (33) no. 329. 1822.

Spreng. in L. Syst. Veg. I6 ed. 41: $518 . d$. 1827.

Hitchcock, Cat. Pl. Amherst 60. I829.

Hitchcock, Cat. PI. Mass. 646. I833. 2 ed. 647 (127). 1835.

Fr. S. M. 3 : 19. $d$. 1832.

Klotzsch, Linnæa 8 : 490. 1833.

S. Syn. Am. Bor. 256 no. 2250.1834.

B. Ann. Nat. Hist. 3 : $399 . \quad$ I839.

Bischoff, Lehrb. der Bot. $3^{2}$ : I I 58. I840.

* Rav. Fung. Car. 3: 75.1855.

Sprague, Proc. Boston Soc. 5 : 328. 1856.

Curtis, M. A., Bot. N. Car. I ro. 1867.

Pk. Rept. N. Y. Mus. 22:89. 1869.

Cke. Handbook 37I. $d$. i87I.

Featherman, Bot. Surv. La. 1871 : I58. 1872.

B. Grev. 2 : 35 (48). Sept. 1873.

Pk. in Hayden's Rept. U. S. Geol. Surv. 6: 792. 1873.

Frost in Cat. Pl. Amherst 78. 1875.

*Thm. Myc. Univ. t1o. 1875.

Cke. Grev. 5 : 150. June 1877.

Johnson, Bull. Minn. Acad. 1: 285. 1877 and Rept. Minn. Surv. 5 : 82. 1877.

Farl. Bull. Bussey Inst. 2 : 226. Jan. 1878.

Cke. Ann. N. Y. Acad. 1 : i79. May i878.

*Ell. N. A. F. 108.1878.

Hark. \& Moore, Cat. Pac. Fung. I8. Feb. 1880.

Gerard, Bull. Torr. Bot. Club 8: 34. March I88I.

*Rav. Fung. Am. 471. 1882.

Bundy, Geol. Wis. 1873-79, 1 : 399. I883.

Rav. S. Car. Resources 355.1883.

Morg. Am. Nat. 18: 969 . d. f. 12 . Oct. I884.

Cragin, Bull. Wash. Lab. 1 : 40. Jan. 1885.

Morg. Jour. Myc.'1: 8. d. Jan. 1885.

Calkins, Jour. Myc. 3: 70 . June 1887.

De Toni, Rev. Myc. 9 : I29 (2I). d. pl. 63, f. E. July 1887.

Cobb, List Pl. Amherst 37. I887.

De Toni in Sacc. Syll. 7 : $90 . d$. 15 March 1888.

Bennett, Cat. PI. R. I. 84. I888.

Trel. Tr. Wis. Acad. $7:$ ro8. $d$. pl. $t, f . t . \quad$ I 888.

Massee, Ann. Bot. 4: $: 81,83$. pl. 4, $f .70$. Nov. 1889.

Ell. in Cat. Pl. N. J. 498.

Britton \& Hollick, Proc. Nat. Sci. Assoc. Staten Isl. 2 : Special I I. Aug. I890.

Jelliffe, Brooklyn Daily Eagle Almanac 5 : 76. $\quad$ I890.

Webber, Tr. St. Louis Acad. 6: I8. I2 March 1892 and Contr. Bot. Dept. Univ. Nebr. 3: i8. I4 June 1892.

*Ell. \& Ev. Fung. Columb. 645.24 April 1895.

Macbr. \& Allin, Bull. Univ. lowa 4: 58. d. 22 Dec. I896.

Harvey, Bull. Torr. Bot. Club 24: 73. 28 Feb. 1897 .

Atk. Bull. Cornell Univ. 3: 22. 25 June 1897.

Jelliffe, Flora of Long Island 42. I899.

Longyear, Rept. Mich. Agr. 1898 : 94. $\quad$ I 899. 
Mcllvaine \& Macadam, Am. Fung. 580. d.pl. 160.1900.

Sweetser. Bull. Pacific Univ. 14: 12. May I9oI.

Lloyd, Gen. Gast. pl. $8, f .40$. Jan. 1902.

Ricker, Univ. of Maine Studies 3: 72. 30 April 1902.

Lloyd, Geastra 8. $d . f .5-11.40$. June 1902.

Conant, Bull. Boston Myc. Club 19: 5 Feb. 1903.

Cockerell, Jour. Myc. 10: 50. I9 March 1904.

Longyear, Rept. Mich. Acad. 4 : 123.1904. Geaster fibrillosus, S.

S. Syn. Car. 59 (33) no. 330. d. 1822.

Spreng. in L. Syst. Veg. 16 ed. $4^{1}: 518.1827$.

Fr. S. M. $3: 20$. d. 1832 .

S. Syn. Am. Bor. 256 no. 2251. 1834.

Curtis, M. A., Bot. N. Car. I Io. 1867.

Morg. Am. Nat. 18 : 969. d. f. Oct. 1884.

Morg. Jour. Myc. 1 : 9. d. Jan. I885.

De Toni, Rev. Myc. 9: 130 (22). d. July 1887.

De Toni in Sacc. Syll. 7 : 9o. d. 15 March 1888.

Morg. Jour. Cin. Soc. $12:$ 19. d. April 1889. Astræus hygrometricus, Morg.

Morg. Jour. Cin. Soc. 12: 20. pl. 2, $f B, 8-I I . d$. April 1889.

McClatchie in Reid's Hist. Pasadena 618. 1895.

McClatchie, Proc. S. Cal. Acad. 1: 386. 5 June I897.

Underw. \& Earle, Bull. Ala. Exp. Sta. 80: 266. 28 June 1897.

Underw. Moulds i 40. I 899.

Earle in Mohr, Contr. U. S. Nat. Herb. 6 : 234. 3I July 190 I.

Astræus stellatus, (Scop.) E. Fischer, var. giganteus, (Morg.) n. n. Geaster hygrometricus, P., var. giganteus, Morg.

Lloyd, Myc. Notes 68. $f$. 30 . Sept. I 901 .

Lloyd, Geastræ I0. $d . f .12,13$. June 1902.

Astrodochium, Ell. \& Ev.

Ell. \& Ev. Am. Nat. 31 : 430. $d$. May 1897.

Lindau \& Syd. Hedw. 37 : Beibl. 7 : Ivi. d. 9 April 1898.

Sacc. \& Syd. Syll. 14: i I17. d. 20 Aug. 1899.

Lindau in Engler \& Prantl, Pflanzenfam. $1^{1 * *}: 500 . d$. Feb. I900.

Astrodochium Coloradense, Ell. \& Ev.

Ell. \& Ev. Am. Nat. 31 : 430. d. May 1897.

Lindau \& Syd. Hedw. 37 : Beibl. 7 : Ivi. 9 April 1898.

Ell. \& Ev. Bull. Torr. Bot. Club 25: 514. 10 Sept. 1898.

Sacc. \& Syd. Syll. 14: i I 18. d. 20 Aug. 1899.

Lindau in Engler \& Prantl, Pflanzenfam. 11**: 500. Feb. 1900.

Astrosporina seabella, Schrt.

See Inocybe scabella, (A. \& S.) Fr.

Astryeum, Raf.

Note. So far as we can see this is not different from Actigea, $q . v$.

Raf. Med. Repos. 2 hex. 5 : 355. d. 1808.

Desv. Jour. de Bot. 2 : 175. d. I809.

Lloyd, Myc. Notes 129. Feb. 1903.

Athalia bombacina, $P$.

See Sporotrichum bombacinum, Lk.

Atractium coccigenum, B. \& C. nom. nud.

Featherman, Bot. Surv. La. 1870: I23. I87I.

Atractium flammeum, B. \& Rav. Tul.

NoTE. This is the conidial stage of Spharostilbe flammea, (B. \& Rav.) 
B. \& Rav. in B. \& Br. Am. Nat. Hist. 11. $13: 461$ (15). d. June 1854. [Notices no. 757.]

Cke. Handbook 555. d. f. 227. I87I.

Featherman, Bot. Surv. La. 1871: 159. 1872.

Pk. Rept. N. Y. Mus. 25: 92 . Sept. 1873.

B. Grev. 4: 16 (154). Sept. 1875.

Cke. Bull. Buf. Soc. 3 : 191. Feb. 1877.

Sacc. Syll. 4: $599 . d$. 10 April 1886.

Langlois, Cat. PI. La. 27. 1887.

*Ell. \& Ev. N. A. F. 3312. Feb. 1896.

Lindau in Engler \& Prantl, Pflanzenfam. 11** : 492. f. 255B. Feb. 1900.

Atractium flammeum, B. \& Rav., var. minor, Rav.

*Rav. Fung. Car. $5: 86$. 1860.

Atractium fusisporum, B. \& C. nom. nud.

Note. This species has never been described but it was said by Cooke,

1877 , to be probably a form of some Spharostilbe.

Curtis, M. A., Bot. N. Car. I26. I867.

Cke. Bull. Buf. Soc. 3 : 191. Feb. 1877.

Atractium pallidum, B. \& C.

Note. This is thought by some to be a form of Spharostilbe fammea, (B. \& Rav.) Tul.

B. Grev. 4: 47 (156). Dec. 1875 .

Ell. \& Ev. Jour. Myc. 3: 3. d. Jan. 1887.

Atractobolus, O. Kuntze.

See Dasyscypha, Fr.

\section{Atractobolus lutescens, S.}

NOTE. This is not a fungus but the eggs of an insect.

S. Syn. Am. Bor. 254 no. 2225. d. 1834.

B. Grev. 2 : $34(48)$. Sept. 1873.

De Toni in Sacc. Syll. 7 : 47. 15 March 1888.

\section{Atractobolus ubiquitarius, Fr.}

Note. Of the two species of the genus Atractobolus mentioned by Schweinitz, 1834, A. lutescens is not a fungus but insect eggs. Atractobolus ubiquitarius under which name Schweinitz quotes Fr. S. M. 2: 305. 1823 is a small Peziza belonging to some genus closely allied to Mollisia, which we are unable to determine specifically whereas in Summa 439. 1849 Fries states that his Atractobolus abiquitarius is insect eggs. See also Berkeley in Entomol. Monthly Mag. 1o: 183. Jan. 1874 .

Mycologists do not seem to be able to recognize the original Atractobolus ubiquitarius of Tode of which Tode gives P'eziza annularia, Batsch as a synonym. The plates of 'Tode and Batsch suggest quite as much the work of an insect as a fungus and the assumption of $\mathrm{O}$. Kuntze that Atractobolus includes Dasyscypha, Fr. seems to us quite unwarranted.

S. Syn. Am. Bor. 254 no. 2224 . 1834.

B. Grev. 2 : 34 (48). Sept. I873.

De Toni in Sacc. Syll. 7: 47. 15 March 1888.

Auerswaldia densa, Bomm. \& Rous.

Bomm. \& Rous. in Durand \& Pittier Bull. Soc. Bot. Belg. $35^{1}$ : 162. d. 1806 .

Sacc. \& Syd. Syll. 14: 675. d. 20 Aug. I899. 
Auerswaldia Pringlei, (Pk.) Sacc.

Sacc. Syll. $2: 626 . d$. I J June 1883. Dothidea Pringlei, Pl..

Pk. Bot. Gaz. $7:$ 37. $d$. May 1882.

Calkins, Jour. Myc. 3: 82. July 1887.

Ell. \& Ev. Pyren. 6io. d. May I892.

McClatchie, Proc. S. Cal. Acad. 1: 362. 5 June I897.

Phyllachora Pringiel, Cke.

Cke. Grev. 13 : 69. March 1885.

Aulographum aclcolum, Hark.

See Lembosia acicoia, (Hark.) Sacc.

Aulographum Arundinariæ, Cke.

Cke. Grev. 14: 14. 'Sept. I885.

Berl. \& Vogl. in Sacc. Syll. Add. 1-4 : 264. d. 3I Dec. 1886.

Sacc. Syll. 9: $1102 . d$. 15 Sept. 1891.

Ell. \& Ev. Pyren. 678. d. May I892.

Aulographum eæspitosum, Ell. \& Ev.

See Lembosia hysterioides, Sacc. \& Syd.

Aulographum ciliatum, B. \& C.

B. \& C. Jour. Linn. Soc. $10: 373 . d$. 1869.

Sacc. Syll. $2: 731 . d$. I3 June 1883.

West Indies.

Aulographum confluens, Earle.

Earle, Bull. Torr. Bot. Club 25: $365 . d$. 15 July 1898.

Earle in Mohr, Contr. U. S. Nat. Herb. $6: 162.31$ July 1901.

Sacc. \& Syd. Syll. $16: 659 . d$. I Feb. 1902.

Aulographum eulmigenum, Ell.

Ell. Bull. Torr. Bot. Club 8: 65. $d$. June i88I.

Sacc. Syll. $2: 729 . d$. I J June 1883.

Ell. in Cat. Pl. N. J. $542 . \quad 1889$.

*Ell. \& Ev. N. A. F. 2379 . 1890.

Ell. \& Ev. Pyren. 679. d. May 1892.

Lindau in Engler \& Prantl, Pflanzenfam. 11 : 272. May 1896.

Aulographum gracile, Ell. \& Martin.

Ell. \& Ev. Pyren. 678. d. May 1892.

Sacc. Syll. 11: 386. $d$. July 1895 .

Aulographum Lituræ, Cke.

See Lembosia Lituræ, (Cke.) Sacc.

Aulographum lucens, Hark.

See Lembosia iucens, (Hark.) Sacc.

Aulographum maculare, B. \& Br.

B. \& C. Jour. Linn. Soc. 10: 373. I869.

Cke. Ann. N. Y. Acad. 1 : 184. May 1878.

West Indies.

Sacc. Syll. 2 : $730 . d$. 13 June 1883.

Aulographum Pinorum, Desm.

Ell. \& Ev. Pyren. 677. d. May I892.

Underw. \& Earle, Bull. Ala. Exp. Sta. 80: 196. 28 June 1897.

Earle in Mohr, Contr. U. S. Nat. Herb. 6 : 162. 31 July I90I.

Aulographum quereinum, Ell. \& Martin.

Ell. \& Martin, Am. Nat. 17: 1283. d. Dec. I883.

*Ell. N. A. F. 1284. 1884.

Calkins, Jour. Myc. 2 : 105. Sept. 1886.

Lanǵlois, Cat. Pl. La. 27. 1887.

*Ell. \& Ev. N. A. F. 2066. March 1888.

*Pazschke, Fung. Eur. 3675. 1890.

Ell. \& Ev. Pyren. 678. d. May 1892. 
Rehm, Ann. Myc. 2 : 354. 31 July 1904.

Sacc. Syll. $17: 895 . d$. 25 May 1905. Lembosia quercina, Tracy \& Earle.

Tracy \& Earle, Bull. Miss. Exp. Sta. 34: 105. d. May 1895.

Sacc. Hedw. 35 : Rep. 7 : xxxv. 25 Feb. 1896.

Sacc. \& Syd. Syll. 14: 714. $d$. 20 Aug. 1899.

Aulographum reticulatum, Phil. \& Hark.

Phil. \& Hark. Bull. Cal. Acad. 1: 25 (5).d. Feb. I884.

*Wint. Fung. Eur. 3265. 1885.

Berl. \& Vogl. in Sacc. Syll. Add. 1-4: 263. d. 31 Dec. 1886.

*Ell. \& Ev. N. A. F. $2065 . \quad$ I 888.

Sacc. Syll. 9: I IOI. $d$. I 5 Sept. I89 I.

Ell. \& Ev. Pyren. 678. $d$. May I892.

Aulographum subconfluens, $\mathrm{Pk}$.

Pk. Rept. N. Y. Mus. 28 : 70. $d$. 1876.

Sacc. Syll. $2:$ 729. $d$. I3 June 1883.

Ell. \& Ev. Pyren. 679. $d$. May 1892 .

Aulographum vagum, Desm.

Ell. in Cat. Pl. N. J. 542. 1889.

Ell. \& Ev. Pyren. 677. d. May I892.

Aureobasidium Vitis, Viala \& Boyer.

Sturgis, Rept. Conn. Exp. Sta. 24: 272. I901.

Auricula.

See Auricularia.

Auricula auriformis, O. Kuntze.

See Auricularia auriformis, (S.) Earle.

Auricula blepharistoma, O. Kuntze.

See Auricularia blepharistoma, (B. \& C.) n. n.

Auricula coffelcolor, O. Kuntze.

See Auricularia coffeicolor, (B.) n. n.

Auricula delicata, $O$. Kuntze.

See Auricularia delicata, (Fr.) Henn.

Auricula fuscosuceinea, O. Kuntze.

See Auricularia fuscosuccinea, (Mont.) n. n.

Auricula hispidula, O. Kuntze.

See Auricularia hispidula, (B.) n. n.

Auricula Judæ, Batt.

See Auricularia Auricula-Judz, (L.) Schrt.

Auricula minuta, $O$. Kuntze.

See Auricularia Wrightil, (B. \& C.) n. n.

Auricula nigra, O. Kuntze.

See Auricularia nigrescens, (Swartz) n. n.

Auricula polytricha, O. Kuntze.

See Auricularia polytricha, (Mont.) Sacc.

Auricula protracta, O. Kuntze.

See Auricularia auriformis (S.) Earle.

Auricula scutelliformis, O. Kuntze.

See Exidia scutelliformis, (B. \& C.) n. n.

Auricula tenuis, O. Kuntze.

See Auricularia tenuis, (Lev.) n. n.

Auricula tremellosa, O. Kuntze.

See Auricularia tremellosa, (Fr.) Pat. 
Auricula velutina, O. Kuntze.

See Auricularia velutina, (Lév.) Pat.

Auricula Wrightil, O. Kuntze.

See Auricularia Wrightii, (B. \& C.) n. n.

Auricularia, Bull.

Nort. We have included under Auricularia species of Hirncola in accordance with the arrangement of Lindau in Engler \& Prantl, P'flanzenfam. I.** $^{1 *}$ 85. Oct. 1897 and Patouillard, Essai Tax. I4. 1900. It should be remarked however with regard to some of the species which authors have referred to Auricularia and Hirneola that their microscopic structure is not sufficiently well known to enable one to say whether they really belong to this group or not. In certain instances however an examination of authentic specimens shows that they do not belong to the Auriculariacea.

IVe have given under Auricularia all the North American species known to us which have been described under Auricularia, Hirneola and Exidia which an examination of specimens proves to belong really to the genus Auricularia as well as certain species of Hirneola which probably belong to Auricularia but of which we have been unable to examine specimens and of which data in published references are insufficient to settle the genus clefinitely.

Hirneola, Fr.

Fr. S. O. V. 93. d. 1825 .

Fr. Fung. Natal. 24. $d$. 1848.

Auricularia Auricula, Underw.

See Auricularia Auricula-Judz, (L.) Schrt.

Auricularia Auricula-Judæ, (L.) Schrt.

Bres. Henn. \& Magn. in Engl. Jahrb. 17 : 492. I893.

Henn. Hedw. 37: 279. 31 Dec. 1898.

Perry, Agricultural Student 6: 178. May 1900 and Ohio Univ. Bull. IV. $30: 4$. May igoo.

Heller, Muhlenbergia 1: 20. 9 Aug. 1900.

Pat. Essai Tax. 15.f. $8^{a}$. 1900.

Earle in Mohr, Contr. U.S. Nat. Herb. 6 : 194. 31 July 1901.

White, Bull. Torr. Bot. Club 29: 552. 26 Sept. 1902.

Duss, Champ. Guad. \& Martinique 8. 1903. Auricula Juda, Batt.

Kuntze, O., Rev. Gen.Pl. 2 : 844. I89r. Tremella Auricula-Juda, L.

S. Syn. Car. ì I5 (89) no. 1136. I822. Peziza Auricula, L.

Muhl. Tr. Am. Phil. Soc. 3 : I84. I793. Tremella Auricula, Mart.

Muhl. Cat. Pl. Am. Sept. I ed. I05. I8I3. 2 ed. I08. I8I8. Auricularia sambucina, Mart.

Wint. \& Demet. Hedw. 24: 185 (9). Oct. 1885.

Underw. Proc. Ind. Acad. 1893: 58. Aug. I894. Exidia Auricula-Juda, Fr.

Fr. S. M. 2 : 221. d. I822.

S. Syn. Am. Bor. 185 no. I091. 1834.

Lea, Cat. Pl. Cin. 68. I849.

B. Ann. Nat. Hist. II. 9 : I99. March 1852.

* Rav. Fung. Car. 1 : 34.1852.

Curtis, M. A., Bot. N. Car. I09. 1867.

Pk. Rept. N. Y. Mus. 22: 88. I 869.

Fowler, Rept. Agr. N. B. 1878 : Append B : 63. 1879.

Hark. \& Moore, Cat. Pac. Fung. I6. Feb. I880. 
*Ell. N. A. F. 519 . I88I.

Bessey, Bull. lowa Agr. Coll. 1884: i48. Jan. 1885.

Hirneola Auricuia $=$ Juda, B.

B. \& C. Jour. Linn. Soc. 10: 340. 1869 .

Cke. Handbook 349. d.f.97. I87I.

B. Grev. 2 : $19(45)$. Aug. 1873 .

Frost in Cat. PI. Amherst 77. 1875.

Johnson, Bull. Minn. Acad. 1: 283. 1877 and Rept. Minn. Surv. 5 : 81. 1877.

Cke. Ann. N. Y. Acad. 1: 179. May I878.

Cke. Grev. 7 : 2. Sept. 1878.

James, Jour. Cin. Soc. 2 : 66 (25). April 1879.

Hark. \& Mcore, Cat. Pac. Fung. 17. Feb. I880.

*Rav. Fung. Am. 463. I88I.

Rav. S. Car. Resources 355. I883.

Farl. Appalachia 3: 248 . Jan. 1884 .

Cragin, Bull. Wash. Lab. 1: 28. Sept. I884.

Arth. \& Holw. Bull. Minn. Surv. 3 : 29. I Oct. I887.

Cobb, List Pl. Amherst 37. 1887.

Langlois, Cat. Pl. La. 30. 1887.

Morg. Jour. Cin. Soc. 11 : 94. d. July 1888.

Sacc. Cub. \& Manc. Syll. 6: 766. d. I Aug. I 888.

Somers, Tr. N. Scot. Inst. $7:$ i $8 . \quad$ I 888.

Ell. in Cat. Pl. N. J. 497. 1889.

Morg. Bot. Gaz. 15:85. April 1890.

Hollick \& Britton, Proc. Nat. Sci. Assoc. Staten Isl. 2 : Special II. Aug. 1890.

Webber, Rept. Nebr. Agr. 1889 : 23 I (9I). 1890.

Banning in Pk. Rept. N. Y. Mus. 44: 187 (75). Dec. I89I.

Massee, Journ. of Bot. 30: 163. June I 892 .

Taylor, Food Products 2: 22. 1893.

Pk. Country Gent. $59: 685$ (80). 20 Sept. 1894.

Pk. Rept. N. Y. Mus. 47: $173(47)$. 20 Nov. 1804.

Kellerm. \& Werner, Geol. Ohio $7^{2}: 336$. Jan. 1895.

Millsp. \& Nutt. Publ. Field Columb. Mus. Bot. 1 : 173. Jan. 1896.

Underw. \& Earle, Bull. Ala. Exp. Sta. 80: 220. 28 June 1897.

Harvey \& Knight, Bull. Torr. Bot. Club 24: 341. 29 July I 897.

Pk. Bull. N. Y. Mus. 6: 224. July i 899. [Bull. no. 28.]

Herbst, Fungal FI. Lehigh Valley I86. $d$. I899.

Hess \& Vandivert, Proc. lowa Acad. $7:$ i 86.1900.

Mcllvaine \& Macadam, Am. Fung. 528. d.pl. 143. 1900.

* Shear, Ell. \& Ev. Fung. Columb. 1411. May 1901.

Pk. Rept. N. Y. Mus. 541 : 172. Dec. I90I.

Ricker, Univ. of Maine Studies 3 : 71. 30 April 1902.

Weems \& Hess, Proc. Soc. Prom. Agr. Sci. 23: 167. 1902.

Kellerm. Ohio Myc. Bull. 1 : 20. f. 26. I2 June 1903.

Hay, Bull. Nat. Hist. Soc. N. B. 5 : I 19.1903.

MacKay, Tr. N. Scot. Inst. 11: 139. Aug. I904.

Auricularia Auricuia, Underw.

Underw. in Northrop, Mem. Torr. Bot. Club 12: I5. Io Dec. Igoz.

Auricularia auriformis, (S.) Earle.

Note. The specimens in Wright's Cuban Fungi no. 286 referred to IIirneola auriformis in Fungi Cubenses p. 340 appear not to be the same as the Schweinitzian specimen on which the species is founded.

The Exidia protracta of Léveille seems to us so far as the description shows to belong to this species as suggested by Fries in Fung. Natal. 26. 1848. The grounds of distinction given by Berkeley and Montagne in Ann. Sci. Nat. III. II : 242 (74). 1849 in view of our examination of Schweinitzian 
specimens of Pesiza auriformis do not appear to indicate a real specific difference.

In the citation given it is stated that the Auricularia Lesuerii, Bory manuscript is the same as Exidia protracta, Lév.

Earle in Molir, Contr. U. S. Nat. Herb. 6 : 194. 3 I July I 901. Peziza auriformis, $\mathrm{S}$.

S. Syn. Car. I16 (90) no. II55. d. 1822.

Hitchcock, Cat. Pl. Amherst 62. 1829.

Hitchcock, Cat. Pl. Mass. 647. I833. 2 ed. 648 (128). 1835. Exidia auriformis, $\mathrm{S}$.

Fr. S. M. $2: 223$. d. 1822 .

S. Syn. Am. Bor. I85 no. Io92. 1834 .

Fr. Epicr. 590. d. 1836-38.

B. \& Mont. Ann. Sci. Nat. III. $11: 242 . \quad$ I 849 .

Klotzsch, Linnza $25: 365 . \quad$ I 852.

Curtis, M. A., Bot. N. Car. 109. 1867.

Heller, Mulılenbergia 1 : I9. 9 Aug. 1900. Tremelia auriformis, Spreng.

Spreng. in L. Syst. Veg. 16 ed. $4^{1}$ : 535. d. 1827. Exidia protracta, Lév.

Lév. Am. Sci. Nat. IIl. 2 : 218. d. I844.

B. \& Mont. Am. Sci. Nat. III. $11: 242$ (74). d. 1849. Hirneola auriformis, $\mathrm{Fr}$.

Fr. Fung. Natal. 26. d. I848.

Morg. Jour. Cin. Soc. 11: 95. d. July 1888.

Sacc. Cub. \& Manc. Syll. 6: 765. d. I Aug. I 888.

Kellerm. \& Werner, Geol. Ohio $7^{2}: 336$. Jan. 1895.

Atk. Bull. Cornell Univ. 3: 24. 25 June I897. Hirneola protracta, B.

Sacc. Cub. \& Manc. Syll. 6 : 766. d. I Aug. I 888. Auricula auriformis, O. Kuntze.

Kuntze, O., Rev. Gen. Pl. 2: 844. I891. Auricula protracta, O. Kuntze.

Kuntze, O., Rev. Gen. PI. 2 : 844. I89I.

Auricularia blepharistoma, (B. \& C.) n. n. Hirneola blepharistoma, B. \& C.

B. \& C. Jour. Linn. Soc. 10: 340.d. 1869.

Sacc. Cub. \& Manc. Syll. 6: 760. d. I Aug. I 888.

West Indies. Auricuia blepharistoma, O. Kuntze.

Kuntze, O., Rev. Gen. Pl. 2 : 844. I89I.

Auricularia Cantharellus, Fr. See Craterellus Canthareilus, (S.) Fr.

Auricularia coffeicolor, (B.) n. n. Hirneola coffeicoior, $\mathrm{B}$.

B. Jour. Linn. Soc. $15: 49$. d. 3 March 1876.

Cke. in Hemsley, Bot. Challenger 1: 102. 1885.

Bermuda. Auricula coffeicoior, O. Kuntze.

Kuntze, O., Rev. Gen. Pl. 2: 844. I 89 I.

Auricularia delieata, (Fr.) Henn.

Bres. Henn. \& Magn. in Engl. Jahrb. 17 : 492. I893.

Henn. Hedw. $37: 279.31$ Dec. 1898.

Heller, Muhlenbergia 1 : 20. 9 Aug. igor. Laschia dellcata, Fr.

Fr. Nova Acta R. Soc. Sci. Upsal. III. 1 : I05. d. I85 I.

B. Ann. Nat. Hist. I1. 9: 197. March 1852.

Mexico. Auricuia delicata, $\mathrm{O}$. Kuntze.

Kuntze, O., Rev. Gen. Pl. 32: 446. I 898 . 
Auricularia flava, (Swartz) n. n.

NoTE. In connection with Hirneola, Fr. S. O. V. 93 consult Fr. S. M. 2 : I6I.

Peziza flava, Swartz.

Swartz, Prodr. 150. d. 1788.

Swartz, Fl. Ind. Occ. 3 : 1939. d. 1806.

West Indies.

Fr. S. M. 2 : 161. d. 1822.

Sacc. Syll. 8: 511.20 Dec. 1889.

Hirneola flava, Fr.

Fr. S. O. V. 93. 1825 .

Fr. Fung. Natal. 24. 1848.

Auricularia fuscosuceinea, (Mont.) n. n.

NoTE. The Exidia fuscosuccinea, Mont. has been placed by recent writers almost universally in Ifirneola and hence as interpreted by us it belongs to the genus Auricularia. The only specimen of this species in Herb. Curtis is of such doubtful authenticity that we do not feel like basing any definite conclusions upon it. See note under Auricularia Wrightii, (B. \& C.) n. n.

Exidia fuscosuccinea, Mont.

Mont. Pl. Cell. Cuba 364, $d$. 1841 .

Mont. Ann. Sci. Nat. I1. $17:$ 125. d. 1842.

West Indies.

B. \& Mont. Ann. Sci. Nat. Ill. $11: 242$. I 849

Hirneola nigra, Fr., var. fuscosuccinea, Fr.

Fr. Fung. Natal. 27. d. 1848.

Hirneola fuscosuccinea, Mont.

Mont. Syll. Crypt. 181. $d$. 1856.

B. \& C. Jour. Linn. Soc. 10: 340. I869.

Sacc. Cub. \& Manc. Syll. $6: 768 . d$. I Aug. 1888.

Auricula fuscosuccinea, $\mathrm{O}$. Kuntze.

Kuntze, O., Rev. Gen. Pl. 2 : 844. I891.

Auricularia grisea, Fr.

See Stereum porrectum, Fr.

Auricularia hispidula, (B.) n. n.

Hirneola hispidula, $\mathrm{B}$.

B. 'Jour. Linn. Soc. 14 : 352. I7 Oct. I 874.

Sacc. Cub. \& Manc. Syll. $6: 769$. d. I Aug. I 888.

West Indies. Auricula hispidula, O. Kuntze.

Kuntze, O., Rev. Gen. Pl. 2 : 844. 189 I.

Auricularia inconeinna, Cke. nom. nud.

Note. The name Stereum inconcinnum of Herb. Berkeley, apparently a manuscript name, is said by Cooke, I $89 \mathrm{I}$, to be an Auricularia.

Cke. Grev. $20:$ 34. Dec. 1891 .

Stereum inconcinnum, Herb. B.

Hark. Bull. Cal. Acad. 1: 256 (I). 19 Nov.o 1885.

Auricularia lobata, Sommf.

Mont. Pl. Cell. Cuba 373. d. 1841.

B. \& C. Jour. Linn. Soc. $10: 335.1869$.

Sacc. Cub. \& Manc. Syll. $6: 762$. d. I Aug. 1888.

Ell. \& Macbr. Bull. Univ. lowa 3: 190. Feb. 1896.

*Smith, C. L., Cent. Amer. Fungi 148. 1896.

Patila lobata, O. Kuntze.

Kuntze, O., Rev. Gen. Pl. 2 : 864. I89I.

West Indies.

Cent. Amer. 
Auricularia mesenterica, (Dicks.) Fr.

- Sprague, Proc. Boston Soc. 6 : 318.1859.

Curtis, M. A., Bot. N. Car. I06. 1867.

Cke. Handbook 319 . d. f. 83. 1871 .

Frost in Cat. Pl. Amherst 75. 1875.

Bundy, Geol. Wis. I873-79, 1 : 399. I883.

Cobb, List PI. Amherst 36. 1887.

Sacc. Cub. \& Manc. Syll. 6: 762. d. I Aug. 1888.

Bennett, Cat. PI. R. 1.82. 1888.

Pat. Essai Tax. 15. 1800.

Massee, Jour. of Bot. 30: 163. June 1892 .

Bomm. \& Rous. in Durand \& Pittier, Bull. Soc. Bot. Belg. $35^{1}$ : 154. I 806.

Duss, Champ. Guad. \& Martinique 8. 1903.

Thelephora mesenterica, P.

S. Syn. Car. $106(80)$ no. 1018. 1822.

Phlebia mesenterica, Fr.

S. Syn. Am. Bor. 165 no. 598.1834.

B. \& C. Jour. Phil. Acad. 11. 3: 218.1856.

Patila mesenterica, O. Kuntze.

Kuntze, O., Rev. Gen. Pl. 2: 864. I89r.

Auricularia nigra, Earle.

See Auricularia nigrescens, (Swartz) n. n.

Auricularia nigrescens, (Swartz) n. n.

Note. This species was originally named nigrescens by Swartz but later for some unknown reason he changed the name to nigricans. Fries in turn changed it to nigra, which name appears in most modern writings.

Peziza nigrescens, Swartz.

Swartz, Prodr. I50. d. 1788.

Peziza nigricans, Swartz.

West Indies.

Swartz, Fl. Ind. Occ. 3 : 1938. d. 1806. Hirneola nigra, Fr.

Fr. Fung. Natal. 27. $d$. 1848.

Fr. Nova Acta R. Soc. Sci. Upsal. III. 1 : I18. I85I. Auricula nigra, O. Kuntze.

Kuntze, O., Rev. Gen. Pl. $2: 844$. I89I. Auricularia nigra, Earle.

Earle, Bull. Torr. Bot. Club 26 : 633. 22 Dec. 1899.*

Atk. in Coker, Veg. Bahama 245. I905.

Auricularia polytricha, (Mont.) Sacc.

Pat. Jour. de Bot. 3 : 34I (39). I6 Oct. 1889.

Pat. Essai Tax. I 5. 1890.

Duss, Champ. Guad. \& Martinique 8. 1903. Exidia polytricha, Mont.

Mont. PI. Cell. Cuba 365. d. I84I. Hirneola polytricha, Fr.

Fr. Nova Acta R. Soc. Sci. Upsal. III. 1 : I17. 1851. Cent. Amer. B. \& C. Jour. Linn. Soc. 10 : 340 . 1869.

Polakowsky, Jahresb. Ver. Erdkunde Dresden 16: 73. 1879.

Hark. \& Moore, Cat. Pac. Fung. 17. Feb. 1880.

Hawaii.

Sacc. Cub. \& Manc. Syll. 6 : 766. d. I Aug. I 888.

Massee, Journ. of Bot. 30: 163. June I892.

Tonduz, Bull. Herb. Bois. 4: 160. March I896.

Bomm. \& Rous. in Durand \& Pittier, Bull. Soc. Bot. Belg. $35^{1}$ : 154. I896.

*Smith, C. L., Cent. Amer. Fungi 150. 1896. 
Smith, A. L., Jour. Linn. Soc. 35 : I I. I April I90I.

Auricula polytricha, O. Kuntze.

Kuntze, O., Rev. Gen. Pl. 2 : 844. I89I.

Auricularia reflexa, Bull.

See Stereum hlrsutum, (Willd.) Fr.

Auricularia regularis, Fr.

See Thelephora regularls, S.

Auricularia sambucina, Mart.

See Auricularia Aurlcula-Judz, (L.) Schrt.

Auricularia scutelliformis, Earle.

NoTE. In authentic specimens of this species which we have examined the basidia are divided vertically and not transversely and this species can not be placed in the Auriculariacea.

See ExIdla scutelliformis, (B. \& C.) n. n.

Auricularia tenuis, (Lév.) n. n.

Exidla tenuis, Lév.

Lév. Ann. Sci. Nat. III. 5 : ı60. I846. HIrneola tenuis, Fr.

Fr. Nova Acta R. Soc. Sci. Upsal. III. 1 : II8. 185 I. Aurlcula tenuls, O. Kuntze.

Kuntze, O., Rev. Gen. Pl. 2: 844. I891.

Auricularia tremellosa, (Fr.) Pat.

NoTE. All the North American specimens of Laschia tremellosa, $\mathrm{Fr}$. which we have been able to examine except that of C. L. Smith, Cent. Am. Fungi 142. 1896 show the structure of Auricularia as already stated by Patouillard, 1887. The specimen of Smith no. 142 is not mature but it does not appear to be quite the same as the other specimens examined.

Pat. Jour. de Bot. 1: 226, 23I. pl. 4, f. 8-10. I 5 Sept. I 887. Laschla tremellosa, Fr.

B. \& C. Jour. Linn. Soc. $10: 323$. I 869.

Sacc. Cub. \& Manc. Syll. $6: 407$. $d$. I Aug. I 888.

* Smith, C. L., Cent. Amer. Fungi 142. I896.

Smith, A. L., Jour. Linn. Soc. 35: 7. I April I9oI.

West Indies.

Hawaii. Auricula tremellosa, $\mathrm{O}$. Kuntze.

Kuntze, O., Rev. Gen. Pl. 32: 446. 1898.

Auricularia velutina, (Lév.) Pat.

Pat. Jour. de Bot. 1 : 226, 23 I. pl. 4, f. 6, 7. I 5 Sept. I 887.

Pat. Essai Tax. I5. $f .8^{b}, c$. 1900 . Laschla velutina, Lév.

B. Ann. Nat. Hist. II. 9: 197. March 1852. Auricula velutina, $\mathrm{O}$. Kuntze.

Kuntze, O., Rev. Gen. Pl. $3^{2}: 446.1898$.

Auricularia Vespertilio, Fr.

Fr. Nova Acta R. Soc. Sci. Upsal. III. 1 : I I2. $d$. I85I. Cent Amer.

Polakowsky, Jahresb. Ver. Erdkunde Dresden 16: 73. 1879.

Sacc. Cub. \& Manc. Syll. 6 : 763. $d$. I Aug. I 888.

Patlla Vespertillo, O. Kuntze.

Kuntze, O., Rev. Gen. Pl. 2 : 864. I 89 I.

Auricularia Wrightil, (B. \& C.) n. n.

Note. It seems to us that Hirneolu Wrightii, B. \& C. and Hirneolu minuta, B. \& C. are the same species. The specimens examined were not in very good fruiting condition but they probably though not certainly belong to the genus Auricularia. It would be interesting to know the relation of this species to Auricularia fuscosuccinea. 
Hirneola Wrightii, B. \& C.

B. \& C. Jour. Linn. Soc. 10: 340. d. 1869.

Sacc. Cub. \& Manc. Syll. 6: 767. d. I Aug. 1888.

West Indies. Hirneola minuta, B. \& C.

B. \& C. Jour. Linn. Soc. 10:340. d. 1869.

Sacc. Cub. \& Manc. Syll. 6: 767. d. I Aug. I 888.

West Indies. Auricula minuta, $\mathrm{O}$. Kuntze.

Kuntze, O., Rev. Gen. Pl. 2: 844. 1891.

Auricula Wrightii, O. Kuntze.

Kuntze, O., Rev. Gen. Pl. 2 : 844. 189 I.

Auriscalpium tenellum, O. Kuntze.

See Arrhenia tenella, Fr.

Aylographum.

See Aulographum.

Bactridium, Kze.

Pound \& Clements, Minn. Bot. Studies $9: 734$. d. 3I May 1897.

Bactridium carneum, Kze.

Note. The Schweinitzian specimen of this species, which we have exam. ined, does not show any definite structure.

S. Syn. Am. Bor. 276 no. 2576. 1834 .

Bactridium clavatum, B. \& Br. See Bactridium flavum, Kze.

Bactridium effusum, $\mathrm{S}$.

Note. We have not been able to examine a specimen of this species.

S. Syn. Am. Bor. 276 no. 2577. d. 1834 .

Sacc. Syll. 4 : 693. d. 10 April 1886.

Bactridium Ellisil, B.

See Bactridium flavum, Kze.

Bactridium flavum, Kze.

S. Syn. Am. Bor. 276 no. 2575. 1834 .

Curtis, M. A., Bot. N. Car. 120. 1867.

Frost, Proc. Boston Soc. 12 : 79. 1869.

Cke. Handbook 479. d. f. 188.1871.

Pk. Rept. N. Y. Mus. 26: 77. April 1874 .

Frost in Cat. Pl. Amherst 82. 1875.

Pk. in Day's Cat., Bull. Buf. Soc. 4: 203 (139). 1883.

Sacc.Syll. $4: 691 . d$. Io April 1886.

Cobb, List Pl. Amherst 39. 1887.

Millsp. Bull. W. Va. Exp. Sta. 24: 517 . June 1892.

Kellerm. \& Werner, Geol. Ohio 72 : 374. Jan. 1895.

Millsp. \& Nutt. Publ. Field Columb. Mus. Bot. 1 : 100. Jan. 1896.

Lindau in Engler \& Prantl, Pflanzenfam. 11**: 508. f. $260 \mathrm{E}$. Feb. I900.

Duss, Champ. Guad. \& Martinique 94. 1903.

Oidium aureum, S. Am. Bor.

S. Syn. Am. Bor. 285 no. 2744 . 1834.

Curtis, M. A., Bot. N. Car. I30. 1867.

Hark. \& Moore, Cat. Pac. Fung. 30. Feb. 188a Bactridium Ellisii, B.

B. Grev. 3: 51 (93) no. 538. d. Dec. 1874.

Pk. Rept. N. Y. Mus. 31: 58. I879.

Cragin, Bull. Wash. Lab. 1: 68. Jan. 1885.

Sacc. Syll. 4: 602. d. 10 April I886.

Arth. \& Holw. Bull. Minn. Surv. 3: 34 . I Oct. 1887. 
Morg. Bot. Gaz. 15 : 86. April 1890.

McClatchie in Reid's Hist. Pasadena 617. 1895.

Ricker, Univ. of Maine Studies 3: 24. 30 April 1902.

Bactridium clavatum, B. \& Br.

Morg. Bot. Gaz. 15: 86. April I 890.

McClatchie, Proc. S. Cal. Acad. 1: 377. 5 June 1897.

\section{Bactridium fulvellum, $B$.}

NoTE. This was originally described apparently in Fungi Cubenses where it is said to occur also in Venezuela. An exanination of Wright's Cuban specimen and Fendler's from Venezuela in Herb. Curtis shows that the Venezuela and Cuban plants are not the same species. The Cuban specimen in habit and microscopic structure is certainly very near to, if not identical with, Bactridium favum.

The Venezuela specimens are branching, coralloid masses about $6 \mathrm{~mm}$. in height and of a yellowish color. They are composed of a dense branching mass of hyphre which branch dichotomo-corymbosely at the surface and have tips bearing spores composed of 8 to 14 cells and measuring $280 \mu$ to $300 \mu$ by $28 \mu$ to $35 \mu$. It is doubtful whether this species is properly placed in the genus Bactridium.

B. \& C. Jour. Linn. Soc. $10:$ 354. $d .1869 . \quad$ West Indies. Sacc. Syll. $4: 692$. d. Io April 1886.

Bactrodesmium elavulatum, Cke. \& Hark.

See Clasterosporium clavulatum, (Cke. \& Hark.) Sacc.

Bactrodesmium opacum, Cke. \& Hark.

See Clasterosporium Harknessii, Sacc.

Badhamia, B.

Including Scyphium, Rostf.

Badhamia affinis, Rostf.

Rostf. Mycetozoa 143. $d$. 1875.

Pk. Rept. N. Y. Mus. 31 : 41,56. 1879.

Berl. in Sacc. Syll. 7: 331. d. I5 March 1888.

Lister, Mycetozoa $36 . d$. I894.**

Morg. Jour. Cin. Soc. 19: 35 (107). d.pl. 3 (15), f. 7o. 23 Aug. 1896.

Macbr. Slime-moulds 66. $d$. Aug. 1899.

Badhamia capsulifera, (Bull.) B.

NoTE. It seems to he generally agreed that Badhamia capsulifera, (Bull.) B. and Badhamia hyalina, (P.) B. should be united. Didymium simulans, Howe is united with this species on the authority of Peck. See also note under Badhamia papaveracea, B. \& Rav.

Morg. Jour. Cin. Soc. 19 : 34 (106). d. 23 Aug. I896.

Harvey, Bull. Torr. Bot. Club 24: 67. d. 28 Feb. 1897.

Macbr. Slime-moulds 68. d. Aug. 1899.

Ricker, Univ. of Maine Studies 3: 12. 30 April 1902.

Physarum hyalinum, P.

S. Syn. Car. 62 (36) no. 371. 1822.

S. Syn. Am. Bor. 257 no. 2276.1834.

Curtis, M. A., Bot. N. Car. I 12. 1867.

Badhamia hyalina, B.

Cke. Handbook 392. d. 1871 .

Cke. Ann. N. Y. Lyceum 11: 390. June 1877.

Pk. Rept. N. Y. Mus. 29: 46. ${ }_{1878 .}$

Pk. Rept. N. Y. Mus. 31: 56.1879.

? Bessey, Bull. lowa Agr. Coll. $1884^{\circ}$ : I 34. Jan. 1885.

Berl. in Sacc. Syll. 7: 332. d. I5 March I 888.

Ell. in Cat. PI. N. J. 595. I 889. 
Schrt. in Engler \& Prantl, Pflanzenfam. 1' ${ }^{1}$ :35. d. Aug. 1892.

McClatchie, Bot. Gaz. 19: 246. 20 June 1894.

McClatchie in Reid's Hist. Pasadena 609. 1895.

Harvey, Bull. Torr. Bot. Club 23 : 307. 20 Aug. 1896.

Olive, Proc. Ind. Acad. 1897 : 149.1898.

Ricker, Univ. of Maine Studies 3: 12. 30 April 1902.

Didymium simulans, Howe.

Howe, Bull. Torr. Bot. Club 6: 30. d. April 1875 and Hedw. 14: 188. $d$. Dec. 1875 .

Clie. Ann. N. Y. Lyceum 11: 392. June 1877.

Pk. Rept. N. Y. Mus. 31: 57. 1879.

Berl. in Sacc. Syll. $7: 332$. d. 15 March 1888.

Badhamia varia, Massee p. p.

Massee, Mon. Myx. 319. d. Dec. 1892 and Bull. Torr. Bot. Club 20 : 79. Feb. 1893 .

Badhamia chrysotricha, Rostf.

See Badhamia decipiens, (M. A. Curtis) B.

Badhamia decipiens, (M. A. Curtis) B.

Note. Physarum subglobostm, Berl. in Sacc. Syll. $7: 352$ is an error for Physarum chrysotrichum, B. \& C. which is again quoted on page 333 of the Sylloge under its proper name. See also note under Physarum Auriscalpium, Cke.

B. Grev. 2: $66(55)$. Nov. 1873 .

Berl. in Sacc. Syll. 7 : 333 . d. 15 March 1888.

Lister, Mycetozoa 32. d. pl. $3 B, f . a-i .1894$.

Morg. Jour. Cin. Soc. 19: $36(108) d . p l .3(15), f .7 l .23$ Aug. 1896.

Underw. \& Earle, Bull. Ala. Exp. Sta.80: 133.d. 28 June 1897.

Lister, Jour. Bot. 35: 210. June 1897.

Macbr. Slime-moulds 63. d. Aug. 1899.

Earle in Molyr. Contr. U. S. Nat Herb. $6: 141$. 3I July 1901.

Sumstine, Torreya $4: 36.12$ March 1904.

Lister, Jour. of Bot. 42: 129. d. May 1904.

Physarum decipiens, M. A. Curtis.

Curtis, M. A., Am. Jour. Sci. Il. $6: 352$. d. Nov. 1848.

Curtis, M. A., Bot. N. Car. 112. 1867.

Rostf. Mycetozoa I15. I875.

Physarum chrysotrichum, B. \& C.

B. Grev. 2: 66 (54) no. 357. d. Nov. 1873 .

Rostf. Mycetozoa II 5. I875.

Massee, Mon. Myx. 300. d. Dec. I892.

Badhamia chrysotricha, Rostf.

Rostf. Mycetozoa, Suppl. 4. d. 1876.

Cke. Ann. N. Y. Lyceum 11: 390. June I877.

Physarum subglobosum, Berl.

Berl. in Sace. Syll. $7: 352$. d. 15 March 1888.

Physarum gyrosum, Massee p. p.

Massee, Mon. Myx. 307. 1892.

Badhamia Pasciculatà, (Jungh.) Rostf.

Nork. We have not been able to examine specimens of this species.

Harvey, Bull. Torr. Bot. Club 23 : 308. 20 Aug. 1896.

Ricker, Univ. of Maine Studies 3: 12. 30 April 1902.

Badhamia granulifera, Massee.

NotE. See Didymium granuliferum, Phil. and note in Lister, Mycetozoa 106 under Lepidoderma. 
. 

2 Farlow, William Gilson

5356 Bibliographical index of F97F34 North Anerican Fungi

PLEASE DO NOT REMOVE CARDS OR SLIPS FROM THIS POCKET

UNIVERSITY OF TORONTO LIBRARY 


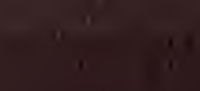

1

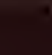

